Michael Ahrens

Experimentelle

Analyse der Wirkungen ausgewählter

DirektmarketingInstrumente

Ein Beitrag zur Effektivitäts- und

Effizienzmessung im Direktmarketing 
Michael Ahrens

\section{Experimentelle Analyse der Wirkungen ausgewählter Direktmarketing-Instrumente}

Trotz der in den letzten Jahren stetig gewachsenen Bedeutung des Einsatzes des Direktmarketings zur Erreichung der Marketing-Ziele vieler Unternehmen wird der systematischen und umfassenden Analyse der zentralen, die Effektivität und Effizienz des Direktmarketing-Einsatzes beeinflussenden Determinanten nur geringe Aufmerksamkeit gewidmet. Vor diesem Hintergrund ist das Ziel dieser Arbeit, einen Beitrag zur experimentellen Analyse der Wirkungsweise der Direktmarketing-Instrumente Mailing, Email und SMS zu leisten. Aufbauend auf einer theoretischen Fundierung des direktmarketing-spezifischen Wirkungsprozesses werden zunächst die Wirkungen der zentralen endogenen und exogenen Determinanten des Direktmarketing-Einsatzes analysiert und in einem Wirkungsmodell zusammengefasst. Auf Basis einer umfangreichen empirischen Analyse verschiedener Direktmarketing-Aktionen eines Unternehmens aus dem Verkehrsdienstleistungsbereich werden zusammenfassend Einflussrichtung und -stärke der identifizierten Determinanten untersucht sowie Implikationen für Wissenschaft und Praxis abgeleitet.

Michael Ahrens wurde 1975 in Oldenburg geboren. Er studierte Betriebswirtschaftslehre in Münster und Berkeley, USA. Ab 2001 arbeitete er als wissenschaftlicher Mitarbeiter am Marketing Centrum Münster. In dieser Zeit war er Geschäftsführer der Wissenschaftlichen Gesellschaft für Marketing und Unternehmensführung e.V. Die Promotion erfolgte Ende 2004. 
Experimentelle Analyse der Wirkungen ausgewählter Direktmarketing-Instrumente 


\section{SCHRIFTEN ZU MARKETING UND MANAGEMENT}

Herausgegeben von Prof. Dr. Dr. h.c. mult. Heribert Meffert

Band 51

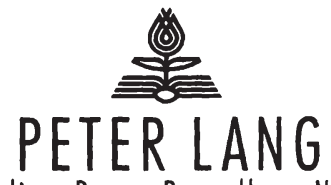

Frankfurt am Main - Berlin. Bern - Bruxelles - New York - Oxford - Wien 


\section{Michael Ahrens}

\section{Experimentelle Analyse der Wirkungen ausgewählter Direktmarketing-Instrumente}

Ein Beitrag zur Effektivitäts-

und Effizienzmessung im Direktmarketing

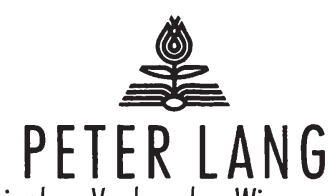

Europäischer Verlag der Wissenschaften 
Bibliografische Information der Deutschen Nationalbibliothek Die Deutsche Nationalbibliothek verzeichnet diese Publikation in der Deutschen Nationalbibliografie; detaillierte bibliografische Daten sind im Internet über <http://www.d-nb.de> abrufbar.

Open Access: The online version of this publication is published on www.peterlang.com and www.econstor.eu under the international Creative Commons License CC-BY 4.0. Learn more on how you can use and share this work: http://creativecommons.org/licenses/ by/4.0.

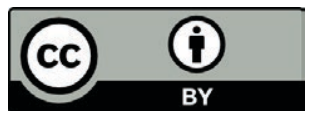

This book is available Open Access thanks to the kind support of ZBW - Leibniz-Informationszentrum Wirtschaft.

Zugl.: Münster (Westfalen), Univ., Diss., 2004

Gedruckt auf alterungsbeständigem, säurefreiem Papier.

\author{
D 6 \\ ISSN 0176-2729 \\ ISBN-10: 3-631-53747-6 \\ ISBN-13: 978-3-631-53747-3 \\ ISBN 978-3-631-75048-3 (eBook) \\ (c) Peter Lang GmbH \\ Europäischer Verlag der Wissenschaften \\ Frankfurt am Main 2007 \\ Alle Rechte vorbehalten.
}

Das Werk einschließlich aller seiner Teile ist urheberrechtlich geschützt. Jede Verwertung außerhalb der engen Grenzen des

Urheberrechtsgesetzes ist ohne Zustimmung des Verlages unzulässig und strafbar. Das gilt insbesondere für Vervielfältigungen, Übersetzungen, Mikroverfilmungen und die Einspeicherung und Verarbeitung in elektronischen Systemen.

Printed in Germany 124567

www.peterlang.de 
Meinen Eltern,

meiner Großmutter, meinem Großvater ( $\dagger$ )

und

\section{Nadine}


Michael Ahrens - 978-3-631-75048-3

Downloaded from PubFactory at 01/11/2019 08:52:24AM

via free access 


\section{Vorwort des Herausgebers}

Die vielerorts zu beobachtende Unverhältnismäßigkeit von Aufwendungen und Wirkungen traditioneller Massenmedien hat in den letzten Jahren vermehrt zu einer Umverteilung der Marketingaufwendungen in Richtung direkter Kommunikationsformen geführt. Aufgrund ihrer individualisierbaren Inhalte und ihrer Interaktivität versprechen hierbei insbesondere die Instrumente des Direktmarketing einen höheren Wirkungsgrad im Sinne der Effektivität bei gleichem Investitionsvolumen und damit eine höhere Effizienz. Doch trotz der aus dieser Entwicklung resultierenden wachsenden Bedeutung des Direktmarketing für die Erreichung der Marketing- und Unternehmensziele wird der systematischen und umfassenden Analyse der zentralen, die Effektivität und Effizienz der einzelnen Direktmarketing-Instrumente beeinflussenden Determinanten vonseiten der Unternehmen nur geringe Aufmerksamkeit gewidmet. Stattdessen werden direktmarketingbezogene Entscheidungen häufig ohne eine strategische Einordnung in die Zielhierarchie des Unternehmens vollzogen und scheinen zudem auf operativer Ebene vielfach das Resultat intuitiver Entscheidungsprozesse der MarketingVerantwortlichen zu sein. Angesichts der rasanten Entwicklung interaktiver Medien des Direktmarketing sind die theoretische Fundierung und empirische Absicherung der Wirkung des Instrumenteeinsatzes eine besondere Herausforderung. Der Verfasser der vorliegenden Arbeit hat sich zum Ziel gesetzt, für den Einsatz ausgewählter Direktmarketing-Instrumente aus wissenschaftlicher und praktischer Sicht einen Beitrag zu leisten.

Ausgangspunkt der Untersuchung bildet die theoretische Konzeption eines integrierten Modells zur Erfassung und Erklärung der Wirkungen des Einsatzes der drei Direktmarketing-Instrumente Mailing, Email und SMS. In diesem Zusammenhang werden im Rahmen einer detaillierten Analyse des direktmarketingspezifischen Wirkungsprozesses die aus Unternehmenssicht steuerbaren Determinanten anhand eines eigenen Strukturierungsrasters in Entscheidungen hinsichtlich zielgruppen-, angebots-, medien- sowie gestaltungsbezogener Parameter unterteilt. In Ergänzung hierzu erfolgt eine konzeptionelle Analyse des Einflusses exogener Determinanten in Form von personen- und situationsbedingten Einflussfaktoren, welche ebenfalls in das Wirkungsmodell einfließen.

Als zentrale Datenbasis der empirischen Überprüfung des konfigurierten Modells dienen dem Autor die Ergebnisse einer feldexperimentellen Untersuchung in Form verschiedener Direktmarketing-Aktionen eines Unternehmens aus dem Verkehrsdienstleistungsbereich, welches dem Segment der sog. Low Cost Airli- 
nes zuzuordnen ist. An dem Feldexperiment nahmen knapp 3.000 Personen teil. Die Auswertung der empirischen Daten erfolgte dabei auf Basis eines dreigeteilten Vorgehens: Den ersten Untersuchungsschritt bildete die Analyse der Rezipientenreaktionen auf die 28 verschiedenen Direktmarketing-Stimuli, welche sich im Hinblick auf die Zielgruppe, das bzw. die eingesetzten Instrumente sowie das enthaltene Angebot unterschieden. In dem sich anschließenden zweiten Schritt der empirischen Untersuchung widmete sich der Verfasser der Analyse des Einflusses ausgewählter exogener Determinanten in Form von personen- und situationsbezogenen Determinanten auf das Response- und Buchungsverhalten der angesprochenen Personen. Der dritte Schritt fokussierte auf die Analyse der relativen Bedeutung der im ersten Schritt untersuchten endogenen und der im zweiten Schritt als signifikant identifizierten exogenen Determinanten im Zusammenspiel ihrer Wirkungen auf das Rezipientenverhalten. Mit Hilfe des Rechenansatzes der logistischen Regression konnte gezeigt werden, dass das Response- und $\mathrm{Bu}-$ chungsverhalten von den aufgezeigten Determinanten unterschiedlich stark beeinflusst wird. Die Ergebnisse der drei durchgeführten Analyseschritte bilden den Ausgangspunkt für die abschließende Formulierung konkreter Implikationen für die Gestaltung des Direktmarketing im untersuchten Unternehmen sowie für die Gestaltung des Direktmarketing im Allgemeinen. Ansatzpunkte für weitere Forschungsarbeiten schließen die Arbeit.

Insgesamt stellt die Arbeit eine Bereicherung der wissenschaftlichen Forschung auf dem Gebiet des Direktmarketing dar. So gelingt es dem Verfasser, das anspruchsvolle Ziel einer experimentellen Analyse der Wirkungen ausgewählter Instrumente des Direktmarketing zu strukturieren, theoretisch-systematisch zu fundieren und mit einer umfassenden experimentellen Untersuchung empirisch zu analysieren. Hervorzuheben ist insbesondere die komplexe empirische Untersuchung, im Rahmen derer neben der Konfiguration, Durchfuhrung und Auswertung zahlreicher Direktmarketing-Aktionen auch eine Vor- und Nachbefragung der angesprochenen Kundengruppen durchgeführt wurde. Darüber hinaus gelingt es dem Verfasser, unter Einbeziehung ökonomischer Kosten-Nutzen-Überlegungen fundierte Aussagen über die Vorteilhaftigkeit der untersuchten Direktmarketing-Aktionen abzuleiten.

Das empirische Datenmaterial der vorliegenden Arbeit beruht auf einem Forschungsprojekt des Centrums für interaktives Marketing und Medienmanagement mit der Germanwings GmbH. Ohne die Förderung des Projektes durch die Germanwings $\mathrm{GmbH}$ sowie die Deutsche Post AG wäre die Durchführung der empirischen Untersuchung in dieser Form nicht möglich gewesen. Hierfür gilt mein besonderer Dank.

Münster, im Januar 2006

Prof. Dr. Dr. h. c. mult. H. Meffert 


\section{Vorwort des Autors}

Vor dem Hintergrund der in zahlreichen Studien nachgewiesenen Wirkungsvorteile des Direktmarketing gegenüber klassischen Werbeformen haben viele Unternehmen in den letzten Jahren begonnen, ihr kommunikatives Instrumentarium in Richtung direkter Kommunikationsformen zu verschieben, und forcieren immer häufiger den Einsatz von Direktmarketing-Instrumenten, insbesondere Postwurfsendungen (Mailings), Emails und SMS. Doch obwohl in vielen Unternehmen Direktmarketing-Kampagnen mittlerweile den Einsatz klassischer Werbemaßnahmen in punkto Einsatzintensität und Investitionsvolumen übertreffen, scheint eine systematische Analyse der die Effektivität und Effizienz der eingesetzten Direktmarketing-Instrumente determinierenden Faktoren sowohl in Wissenschaft als auch in der Praxis nur äußerst fragmentiert ausgeprägt zu sein.

Um aber die Instrumente des Direktmarketing im Sinne der Unternehmensziele noch effektiver und effizienter einsetzen zu können, sind weiterführende Erkenntnisse über die Bedeutung und das Zusammenspiel der im Rahmen des Direktmarketing-Einsatzes vom Unternehmen steuerbaren endogen sowie exogener Einflussfaktoren notwendig. Mit der vorliegenden Arbeit wird daher das Ziel verfolgt, die Einflussrichtung und -stärke ausgewählter Einflussfaktoren auf den Erfolg des Direktmarketing-Einsatzes zu untersuchen und damit einen Beitrag zur theoretischen Fundierung und experimentellen Analyse der Wirkungen ausgewählter Direktmarketing-Instrumente zu leisten.

Die vorliegende Arbeit wurde im September 2004 von der Wirtschaftswissenschaftlichen Fakultät der Westfälischen Wilhelms-Universität als Dissertationsschrift angenommen. Ihre Erstellung war nur mit der Unterstützung zahlreicher Personen und Institutionen möglich. Mein besonderer Dank gilt zunächst meinem akademischen Lehrer und Doktorvater Prof. Dr. Dr. h. c. mult. Heribert Meffert, der die Themenstellung bereits frühzeitig anregte und laufend unterstutzte. $\mathrm{Zu}$ dem förderte er meine fachliche und persönliche Entwicklung in vielfältiger Weise. Herrn Prof. Dr. Dr. h. c. Klaus Backhaus danke ich an dieser Stelle ausdrücklich für die Übernahme des Zweitgutachtens.

Das Datenmaterial der vorliegenden Arbeit resultiert aus einem Forschungsprojekt mit der Germanwings GmbH. Danken möchte ich daher insbesondere Herrn Dr. Andreas Bierwirth und seinen Mitarbeitern für die gemeinsame Abstimmung der Erhebungsstruktur und die organisatorische Unterstützung im Rahmen der Befragung, die zu einem Großteil an Bord der Germanwings- 
Maschinen stattfand. Darüber hinaus möchte ich der Deutschen Post AG für die umfassende Förderung des Projektes danken.

Bedanken möchte ich mich auch bei allen aktuellen und ehemaligen Kollegen und Freunden am Lehrstuhl, die mich wăhrend der Verfassung der Arbeit in vielfältiger Weise unterstützt, motiviert und von anderweitigen Aufgaben weitestgehend entlastet haben. Namentlich hervorheben möchte ich Herrn Prof. Dr. Dr. Helmut Schneider, der mir in allen Phasen der Dissertation wertvolle Anregungen gab und mir immer diskussionsbereit und aufmunternd zur Seite stand. Einen besonderen Anteil am Gelingen der vorliegenden Arbeit hat darüber hinaus mein Freund, Dr. Markus Krummenerl, der - trotz eigener Arbeitsbelastung - zu jeder Tag- und Nachtzeit für Fragen und Diskussionen bereitstand und mit dem ich sowohl im beruflichen wie auch im privaten eine unterhaltsame Zeit in Münster verbinde. Ebenso ist es mir ein besonderes Anliegen, meiner langjährigen Kollegin in der Wissenschaftlichen Gesellschaft für Marketing und Unternehmensführung e. V., Frau Eva-Maria Figge, für ihre Unterstützung bei der Erstellung der Dissertation zu danken. Sie hat mir stets den Rücken freigehalten und mich mit ihrer frischen und fröhlichen Art immer wieder aufgemuntert. Danken möchte ich ferner Herrn Jens Reich für die Unterstutzung bei der formellen Gestaltung der Arbeit. Nicht zuletzt ist es mir ein besonderes Anliegen, meinen Freundinnen und Freunden außerhalb des Instituts für die zahlreichen aufmunternden Gespräche zu danken, die mir auch in Krisenzeiten geholfen haben, den Kopf für das Wesentliche wieder frei zu bekommen.

Darüber hinaus möchte ich meinen Eltern danken, die einen weitaus größeren Anteil am Entstehen und Gelingen dieser Arbeit gehabt haben, als sie selbst vielleicht vermuten. Erst durch ihre liebevolle Unterstützung und ihr großes Verständnis konnte ich die vorliegende Arbeit mit großer Entschlossenheit, Ausdauer und Konzentration angehen. Aber auch während meines Studiums und während meiner Schulzeit gaben sie mir immer den nötigen Rückhalt und die notwendige Unterstützung, um die auftretenden Herausforderungen zu bewältigen. Sie haben so meinen bisherigen Werdegang erst ermöglicht. Hierfur möchte ich ihnen zutiefst danken.

Ebenso danken möchte ich meiner Großmutter und meinem Großvater $(\dagger)$, die mich ebenfalls während meiner Studien- und Promotionszeit begleitet und in vielfältiger Weise unterstützt haben. Danken möchte ich insbesondere meiner Großmutter für die täglichen mitternächtlichen Telefonate, in denen sie mich während der Promotionszeit regelmäßig von Neuem aufgemuntert und motiviert hat. Meinen Eltern und meinen Großeltern möchte ich daher die vorliegende Arbeit widmen.

Dir, liebe Nadine, möchte ich von ganzem Herzen für Deine liebevolle Unterstützung und Deine Nachsicht danken. Trotz răumlicher Distanz ist es Dir immer 
wieder gelungen, meine Blicke auf das Wesentliche zu lenken. Neben Deinen fachlichen Anregungen habe ich insbesondere durch Deine ausgeglichene und erfrischende Art immer wieder Kraft schöpfen und mich ganz auf die Fertigstellung der Arbeit konzentrieren können. Deine liebevolle Zuneigung und Deine grenzenlose Rücksichtnahme haben mir auch in schwierigen Tagen Kraft und Motivation gegeben. Daher möchte ich auch Dir diese Arbeit widmen.

Münster, im Januar 2006

Michael Ahrens 
Michael Ahrens - 978-3-631-75048-3

Downloaded from PubFactory at 01/11/2019 08:52:24AM

via free access 
Inhaltsverzeichnis

Abbildungsverzeichnis

Tabellenverzeichnis

Abkürzungsverzeichnis

A. Effektivitäts- und Effizienzorientierung als Herausforderung an das Direktmarketing

1 Zunehmende Bedeutung von Wirkungsanalysen im Direktmarketing

2 Effektivität und Effizienz im Direktmarketing als Untersuchungsgegenstand

2.1 Entwicklungsstand und Abgrenzung des Direktmarketing

2.2 Effektivität und Effizienz als Beurteilungskriterien des Direktmarketing-Einsatzes

2.3 Bestandsaufnahme von Forschungsarbeiten zur Effektivität und Effizienz des Direktmarketing

3 Zielsetzung und Gang der Untersuchung

B. Konzeptionelle Analyse des Einflusses endogener und exogener Determinanten des Direktmarketing-Erfolgs

1 Theoretische Ableitung eines Modells zur Beurteilung des Direktmarketing-Erfolgs

1.1 Analyse der Wirkungskategorien des Direktmarketing

1.2 Ansatzpunkte der Erfolgsmessung im Direktmarketing

1.2.1 Effektivitätsmessung als Ausgangspunkt der Erfolgsbeurteilung

1.2.2 Effizienzmessung als komplementäres Element der Erfolgsbeurteilung

2 Analyse des Einflusses endogener Determinanten auf die Effektivität und Effizienz

2.1 Entscheidungsprozess des Direktmarketing als Strukturierungsraster zur Identifikation zentraler endogener Determinanten

2.2 Endogene Determinanten der Effektivität und Effizienz

2.2.1 Zielgruppenbezogene Determinanten 
2.2.2 Angebotsbezogene Determinanten

2.2.3 Medienbezogene Determinanten

2.2.3.1 Trägermedium

2.2.3.2 Rückkanalmedium

2.2.4 Gestaltungsbezogene Determinanten

3 Analyse des Einflusses exogener Determinanten auf die Effektivität und Effizienz

3.1 Personenbezogene Determinanten

3.2 Situative Determinanten

4 Synoptische Darstellung eines Bezugsrahmens zur experimentellen Analyse des Einflusses endogener und exogener Determinanten des Direktmarketing-Erfolgs

C. Experimentelle Untersuchung der Effektivität und Effizienz des Direktmarketing-Einsatzes

1 Anforderungen an die Durchführung von Feldexperimenten

2 Design der experimentellen Untersuchung

2.1 Auswahl des Untersuchungsobjekts

2.2 Aufbau der experimentellen Untersuchung

2.2.1 Operationalisierung der abhängigen Variablen

2.2.2 Operationalisierung der unabhängigen Variablen

2.2.3 Operationalisierung der kontrollierten Variablen

2.2.4 Operationalisierung ausgewählter exogener Determinanten

2.2.5 Zusammensetzung der Testgruppen

2.3 Ablauf der experimentellen Untersuchung

2.4 Methoden der Datenauswertung

3 Ergebnisse der experimentellen Untersuchung

3.1 Einfluss endogener Determinanten des Direktmarketing-Einsatzes

3.1.1 Auswertung des Gesamtexperiments

3.1.1.1 Effektivitätsbeurteilung

3.1.1.2 Effizienzbeurteilung

3.1.2 Vergleichende Analyse von Neukundenakquisition und Bestandskundenbearbeitung

3.1.2.1 Effektivitätsbeurteilung

3.1.2.2 Effizienzbeurteilung

3.1.3 Vergleichende Analyse verschiedener verkaufsorientierter Angebote im Rahmen der Bestandskundenbearbeitung

3.1.3.1 Effektivitätsbeurteilung

3.1.3.2 Effizienzbeurteilung 
3.1.4 Vergleichende Analyse des Einsatzes verschiedener Instrumente und Instrumentekombinationen 3.1.4.1 Effektivitätsbeurteilung

3.1.4.2 Effizienzbeurteilung

3.1.5 Synoptische Darstellung der Ergebnisse der Effektivitätsund Effizienzanalyse

3.2 Einfluss exogener Determinanten des Direktmarketing-Einsatzes

3.2.1 Analyse des Einflusses personenbezogener Determinanten

3.2.2 Analyse des Einflusses situationsbezogener Determinanten

3.2.3 Synoptische Darstellung der Ergebnisse der Analyse ausgewählter exogener Determinanten

3.3 Integrative Analyse des Einflusses ausgewählter endogener und exogener Determinanten auf das Rezipientenverhalten

3.3.1 Vergleich von Nicht-Reagierern und Reagierern

3.3.2 Vergleich von Interessenten und Buchern

3.3.3 Vergleich der Nicht-Reagierern und Buchern

3.3.4 Synoptische Darstellung der Ergebnisse der integrativen Analyse

D. Zusammenfassung und Implikationen

1 Zusammenfassende Würdigung der Forschungsergebnisse

2 Spezifische Implikationen für die Optimierung des DirektmarketingEinsatzes im untersuchten Unternehmen

3 Allgemeine Implikationen für den Direktmarketing-Einsatz und die Effektivitäts- und Effizienzmessung

4 Ansatzpunkte für weiterführende Forschungsarbeiten 
Michael Ahrens - 978-3-631-75048-3

Downloaded from PubFactory at 01/11/2019 08:52:24AM

via free access 
Abbildungsverzeichnis

Abb. 1: Die Instrumente des Direktmarketing gegliedert nach der Art des Kundenkontaktes

Abb. 2:

Allgemeiner Bezugsrahmen der Untersuchung

Abb. 3:

Teil- und Zielwirkungen des Direktmarketing-Einsatzes

Abb. 4:

Tauglichkeitsprüfung potenzieller Wirkungskategorien des Direktmarketing-Einsatzes

Abb. 5: Kontakt- und wirkungsbasierte Effektivitätsmessung im Direktmarketing

Abb. 6: Kontakt- und wirkungsbasierte Erfolgsmessung im Direktmarketing

Abb. 7: Ausgewählte Entscheidungstatbestände im Rahmen des Direktmarketing-Einsatzes

Abb. 8: Ausgewählte Angebotsformen des verkaufsorientierten Direktmarketing-Einsatzes und zugrunde liegende Kundenvorteile

Abb. 9: Integriertes Modell zur Beurteilung der Effektivität und Effizienz des Direktmarketing

Abb. 10: Übersicht über die 28 versendeten Direktmarketing-Stimuli

Abb. 11: Zeitlicher Ablauf der empirischen Untersuchung

Abb. 12: Zeitlicher Ablauf der Experimente

Abb. 13: Mehrstufige Analyse der Zusammenhänge zwischen ausgewählten exogenen Determinanten und dem Rezipientenverhalten

Abb. 14: Effektivitätskennzahlen der Experimente auf Gesamtaktionsebene

Abb. 15: Effektivitätsvergleich der Bestandskundenbearbeitung und der Neukundenakquisition

Abb. 16: Effektivitätsvergleich der drei verkaufsorientierten Angebote

Abb. 17: Effektivitätskennzahlen der 28 versendeten DirektmarketingStimuli im Überblick

Abb. 18: Abweichung der Responsequote von der durchschnittlichen Responsequote der verschiedenen Zielgruppen und Angebote

Abb. 19: Verlustquote von der Anzahl zugestellter Stimuli zur Anzahl Responses 
Abb. 20: Abweichung der Buchungsquoten der Instrumente und Instrumentekombinationen von der durchschnittlichen $\mathrm{Bu}$ chungsquote

Abb. 21: Verlustquote von der Anzahl Responses zur Anzahl Buchungen

Abb. 22: Synoptische Darstellung der Analyse des Einflusses ausgewählter exogener Determinanten auf das Rezipientenverhalten

Abb. 23: Analysierte endogene und exogene Variablen im Hinblick auf das Responseverhalten (Analyse 1)

Abb. 24: Sensitivitätsanalyse der signifikanten Variablen im Rahmen der ersten Analyse

Abb. 25: Analysierte endogene und exogene Variablen im Hinblick auf das Buchungsverhalten (Analyse 2)

Abb. 26: Sensitivitätsanalyse der signifikanten Variablen im Rahmen der zweiten Analyse

Abb. 27: Analysierte endogene und exogene Variablen im Hinblick

Abb. 28: $\quad$ auf das Buchungsverhalten (Analyse 3)

\section{der dritten Analyse}

Abb. 29: Synoptische Darstellung der Einflussstärke der signifikanten endogenen und exogenen Determinanten auf Basis der drei Analysen 


\section{Tabellenverzeichnis}

Tab. 1: Bestandsaufnahme ausgewählter Forschungsarbeiten zur (feld-)experimentellen Analyse der Effektivität und Effizienz des Direktmarketing

Tab. 2: $\quad$ Direktmarketingspezifische Kostenkategorien

Tab. 3: Effektivitätskennzahlen im Rahmen der empirischen Untersuchung

Tab. 4: Kostenkategorien im Rahmen der Direktmarketing-Experimente

Tab. 5: Effizienzkennzahlen im Rahmen der empirischen Untersuchung

Tab. 6: Inhaltliche Gestaltung der Direktmarketing-Stimuli im Rahmen der Experimente

Tab. 7: Kriterien zur Herstellung der Strukturgleichheit der Stichproben

Zusammensetzung der Testgruppen

Tab. 8: Informationsverhalten der Bucher auf Gesamtaktionsebene

Tab. 9: Kostenkategorien der Experimente auf Gesamtaktionsebene_-

Tab. 10: Kostenorientierte Effizienzkennzahlen auf Gesamtaktions-

Tab. 11: ebene

Tab. 12:

Deckungsbeitragsrechnung auf Gesamtaktionsebene

Tab. 13: Informationsverhalten der Bestands- und Neukunden während des Buchungsvorgangs

Tab. 14: Durchschnittlich realisierte Ticketpreise sowie Preisbereitschaften in Abhängigkeit der unterschiedlichen Zielgruppen

Tab. 15: Gesamtkosten der Bestandskundenbearbeitung und der Neukundenakquisition

Tab. 16: Kostenorientierte Effizienzkennzahlen der Bestandskundenbearbeitung und der Neukundenakquisition

Tab. 17: Realisierte Deckungsbeiträge der Bestandskundenbearbeitung und der Neukundenakquisition

Tab. 18: Informationsverhalten der buchenden Personen in Abhängigkeit des versendeten Angebots

Tab. 19: Durchschnittlich realisierte Ticketpreise sowie Preisbereitschaften in Abhängigkeit der unterschiedlichen Angebote 
Tab. 20: Gesamtkosten der drei Angebote unterteilt nach den zentralen Kostenkategorien

Tab. 21: Kostenorientierte Effizienzkennzahlen der drei Angebote im Vergleich

Tab. 22: Realisierte Deckungsbeiträge der drei Angebote im Vergleich

Tab. 23: Vergleich der durchschnittlichen Responsequoten des isolierten und des integrativen Einsatzes der DirektmarketingInstrumente

Tab. 24: Vergleich der durchschnittlichen Buchungsquoten des isolierten und des integrativen Einsatzes der DirektmarketingInstrumente

Tab. 25: Informationsbedarf der Rezipienten beim isolierten Instrumenteeinsatz

Tab. 26: Werbeerinnerung beim isolierten und integrativen Instrumenteeinsatz

Tab. 27: Kostenorientierte Effizienzkennzahlen und Deckungsbeiträge in Abhängigkeit der eingesetzten Instrumente

Tab. 28: Vergleich der Effizienzkennzahlen des isolierten und des integrativen Instrumenteeinsatzes

Tab. 29: Zusammenfassende Darstellung der Effektivität und Effizienz der eingesetzten Direktmarketing-Stimuli auf Basis von Rangplätzen

Tab. 30: Zusammenhang zwischen soziodemographischen Merkmalen und Rezipientenverhalten

Tab. 31: Zusammenhang zwischen psychographischen Merkmalen und Rezipientenverhalten

Tab. 32: Zusammenhang zwischen verhaltensorientierten Merkmalen und dem Rezipientenverhalten

Tab. 33: Analyse des Zusammenhangs zwischen situationsbezogenen Determinanten und Rezipientenverhalten

Tab. 34: Klassifikationsmatrix des Modells zum Vergleich von NichtReagierern und Reagierern

Tab. 35: Empirische Analyse des Responseverhaltens auf Basis der Logistischen Regression (Analyse 1)

Tab. 36: Klassifikationsmatrix des Modells zum Vergleich von Interessenten und Buchern

Tab. 37: Empirische Analyse des Buchungsverhaltens auf Basis der Logistischen Regression (Analyse 2) 
Tab. 38: Klassifikationsmatrix des Modells zum Vergleich von NichtReagierern und Reagierern

Tab. 39: Empirische Analyse des Buchungsverhaltens auf Basis der Logistischen Regression (Analyse 3) 
Michael Ahrens - 978-3-631-75048-3

Downloaded from PubFactory at 01/11/2019 08:52:24AM

via free access 
Abkürzungsverzeichnis

a. a. O. am angegebenen Ort

a. M. am Main

Abb. Abbildung

ACTA Allensbacher Computer- und Technik-Analyse

AG Aktiengesellschaft

Aufl. Auflage

Bd. Band

bspw. beispielsweise

B Bucher

BQ Buchungsquote

bzgl. bezüglich

bzw. beziehungsweise

ca. circa

CIM Centrum fur interaktives Marketing und Medienmanagement, Münster

CPC Cost per contact

CPI Cost per interest

CPO Cost per order

CRM Customer Relationship Management

d. h. das heißt

DBA Deckungsbeitrag pro Aktion

DBS Deckungsbeitrag pro Stichprobe

DBZ Deckungsbeitrag pro Zielgruppe

DEA Data Envelopment Analyse

DDV Deutscher Direktmarketing Verband

Diff. Differenz

DMA Direct Marketing Association

DRTV Direct Response Television

e. V. eingetragener Verein

eCRM Electronic Customer Relationship Management 


$\begin{array}{ll}\text { EDV } & \text { Elektronische Datenverarbeitung } \\ \text { et al. } & \begin{array}{l}\text { et alii, et alia, et alteri } \\ \text { etc. }\end{array} \\ \text { et cetera } \\ \text { f. } & \text { folgende } \\ \text { ff. } & \text { fortfolgende } \\ \text { FAZ } & \text { Frankfurter Allgemeine Zeitung } \\ \text { GfK } & \text { Gesellschaft für Konsum- und Absatzforschung } \\ \text { ggf. } & \text { gegebenenfalls } \\ \text { ggü. } & \text { gegenüber } \\ \text { GmbH } & \text { Gesellschaft mit beschränkter Haftung } \\ \text { GT } & \text { Gesamt-Ticketanzahl } \\ \text { Hrsg. } & \text { Herausgeber } \\ \text { I } & \text { Interessenten } \\ \text { i. d. R. } & \text { in der Regel } \\ \text { i. e. S. } & \text { im engeren Sinne } \\ \text { i. H. v. } & \text { in Höhe von } \\ \text { i. S. } & \text { im Sinne } \\ \text { i. w. S. } & \text { im weiteren Sinne } \\ \text { insb. } & \text { insbesondere } \\ \text { Jg. } & \text { Jahrgang } \\ \text { Kap. } & \text { Kapitel } \\ \text { MCC } & \text { Maximum chance criterium } \\ \text { Mio. } & \text { Millionen } \\ \text { MP } & \text { Maximale Preisbereitschaft } \\ \text { Mrd. } & \text { Milliarde(n) } \\ \text { n. s. } & \text { nicht signifikant } \\ \text { No. } & \text { Number } \\ \text { Nr. } & \text { Nummer } \\ \text { PCC } & \text { Proportional chance criterium } \\ \end{array}$


R Reagierer

RQ Responsequote

S. Seite

Sign. Signifikanz

SMS Short Message Service

sog. $\quad$ sogenannte $(n, r, s)$

SPSS Superior Performing Software Systems (ehemals Statistical

Package for the Social Sciences)

STK Stückkosten

Tab. Tabelle

u. a. und andere / unter anderem

u. U. unter Umständen

U Umsatz

usw. und so weiter

VATM Verband der Anbieter von Telekommunikations- und Mehrwertdiensten e. V.

vgl. vergleiche

Vol. Volume

vs. versus

WWW World Wide Web

z. B. zum Beispiel

ZfB Zeitschrift furr Betriebswirtschaft

ZFP Zeitschrift für Forschung und Praxis

ZQ Zustellquote 
Michael Ahrens - 978-3-631-75048-3

Downloaded from PubFactory at 01/11/2019 08:52:24AM

via free access 


\section{A. Effektivitäts- und Effizienzorientierung als Herausforderung an das Direktmarketing}

\section{Zunehmende Bedeutung von Wirkungsanalysen im Direktmarketing}

Seit mehreren Jahren sehen sich viele Unternehmen mit stagnierenden Märkten, zunehmend homogenen Leistungen und steigender Wettbewerbsintensität konfrontiert. ${ }^{1}$ Diese Entwicklung geht in vielen Branchen mit einem immer schlechter zu prognostizierenden Konsumentenverhalten sowie sinkenden Loyalitätsraten einher. ${ }^{2}$ Im Zuge dieser Entwicklungen haben viele Firmen begonnen, ihre marktgerichteten Aktivitäten bezüglich des Beitrags zur Erreichung der Unternehmensziele kritisch zu analysieren. ${ }^{3}$ Einem auf Basis dieser Analyse häufig identifizierten Bedarf an Kostensenkungsmaßnahmen wird seit geraumer Zeit immer häufiger auch mit Kürzungen der Marketing-Budgets entsprochen. ${ }^{4}$ Insbesondere wegen ihres hohen Anteils an den Gesamtkosten und des häufig nur schwer fassbaren Nutzens sind Marketing-Ausgaben bei rückläufigen Umsätzen primäres Ziel von Sparmaßnahmen. ${ }^{5}$

1 Vgl. Meffert, H., Konsumgütermarketing: Vom Massen- zum Individualmarketing, in: Frankfurter Allgemeine Zeitung, 15. April 2002, Nr. 87, S. 25.

2 Vgl. Meffert, H., Giloth, M., Aktuelle markt- und unternehmensbezogene Herausforderungen an die Markenführung, in: Meffert, H., Burmann, C., Koers, M. (Hrsg.), Markenmanagement. Grundlagen der identitätsorientierten Markenführung, Wiesbaden 2002, S. 101.

3 Vgl. Reinecke, S., Tomczak, T., Einsatz von Instrumenten und Verfahren des Marketingcontrollings in der Praxis, in: Reinecke, S., Tomczak, T., Geis, G. (Hrsg.), Handbuch Marketingcontrolling: Marketing als Motor für Wachstum und Erfolg, Frankfurt a. M., Wien 2001, S. $76 \mathrm{ff}$.

4 Im europäischen Vergleich reagierten die Unternehmen in Deutschland am stärksten auf die konjunkturellen Veränderungen der Jahre 2000 bis 2002 und reduzierten ihre Kommunikationsaufwendungen im Durchschnitt in dieser Zeit um 15,3\%. Vor allem im Bereich der Telekommunikation (-33\%) und der Finanzdienstleistungen (-18\%) wurden die Werbeetats verringert, während andere Branchen, wie bspw. die Automobilindustrie $(+1 \%)$, die Budgets erhöhten. Vgl. Göttgens, O., Wastl, S., Kein Marketing - Kein Konsum - Kein Wachstum, in: Insight, Nr. 4, 2003, S. 56.

5 Eine überproportionale Senkung des Anteils der Kommunikationsaufwendungen am Gesamt-Marketing-Budget im Vergleich zur Umsatzentwicklung lässt sich für 2001/2002 bspw. bei der Deutschen Telekom AG (-59\%) und bei Viag Interkom $(-46 \%)$ beobachten. Gleichzeitig wurde auch die Höhe der Gesamtaufwendungen reduziert. Vgl. Mercer Management Consultants (Hrsg.), Communications Benchmark 2003, München 2003, S. 1. 
Viele Marketing-Verantwortliche stehen damit vor der Herausforderung, ein geringeres Budget optimal auf die unterschiedlichen Marketinginstrumente verteilen zu müssen. ${ }^{6}$ Erschwert werden Budgetentscheidungen dieser Art aufseiten der Unternehmen durch die tief greifende Umbruchphase, in der sich die Kommunikationslandschaft derzeit befindet. ${ }^{7}$ In vielen Bereichen wird die Wirkung klassischer Mediawerbung zunehmend in Frage gestellt. ${ }^{8}$ So hat bspw. der Umfang an Kommunikationsinhalten, die täglich über die unterschiedlichen Kanäle auf den Konsumenten einströmen, trotz rückläufiger Werbeumsätze ${ }^{9}$ in den letzten Jahren aufgrund einer verstärkten zielgruppenspezifischen Marktbearbeitung nicht ab-, sondern vielmehr zugenommen. ${ }^{10}$ In Verbindung mit der gleich bleibenden Aufnahmefähigkeit und Verarbeitungskapazität des Konsumenten erzielen die versendeten Botschaften immer seltener die gewünschte Wirkung. Zudem kommt es aufgrund einer ständig steigenden Anzahl empfangbarer TV-

6 Vgl. stellvertretend Meffert, H., Marketing: Grundlagen marktorientierter Unternehmensführung, 9. Aufl., Wiesbaden 2000, S. 555.

7 Vgl. u. a. Bruhn, M., Kommunikationspolitik, München 1997, S. 1; Sistenich, F., Eventmarketing. Ein innovatives Instrument zur Metakommunikation in Unternehmen, Wiesbaden 1999, S. 5.

8 Zwar besitzt die kommunikative Profilierung von Produkten und Dienstleistungen nach wie vor eine zentrale Rolle im Wettbewerb. Doch trotz erheblicher Werbeinvestitionen nimmt der Wirkungsgrad traditioneller Massenmedien, wie TV, Radio und Print, immer stärker ab. Vor allem die Wirkung klassischer Mediawerbung im Rahmen der Markenführung wird zunehmend in Frage gestellt, da unter den beschriebenen Umständen sowohl die Kontaktchance als auch die Wirkung einer einzelnen Botschaft tendenziell sinkt. Vgl. Joachimsthaler, E. A., Aaker, D. A., Aufbau von Marken im Zeitalter der Post-Massenmedien, in: Esch, F. R. (Hrsg.), Moderne Markenführung, 3. Aufl., Wiesbaden 2001, S. 539.

9 Insgesamt gingen die Nettoeinnahmen der Werbung für die erfassbaren Werbeträger vom Jahr 2000 auf 2001 um 7,2\%, von 2001 auf 2002 um weitere 7,5\% zurück. Damit liegen die Netto-Werbeeinnahmen in Deutschland mit einer Höhe von knapp $20 \mathrm{Mrd}$. Euro unter den Werten des Jahres 1999. Hierbei sind insbesondere die Umätze für klassische TV-Werbung (von 2001 auf 2002 -11,5\%) und Printmedien (Tageszeitungen $-12,5 \%$, Fachzeitschriften $-10,1 \%$ und Publikumszeitschriften -7,5\%) betroffen. Vgl. Zentralverband der deutschen Werbewirtschaft (Hrsg.), Werbung in Deutschland 2003, Bonn 2003, S. 13 ff. Im gleichen Zeitraum nahm der Anteil direkter Kommunikationsformen, wie bspw. Postwurfsendungen und Onlinewerbung, stetig zu.

10 Allein zwischen 1985 und 1995 hat sich die Anzahl der TV-Spots von 0,15 Mio. auf 1,3 Mio. Stück mehr als verneunfacht. Ebenso steigerte sich die Anzahl der RadioSpots im gleichen Zeitraum von 0,34 Mio. auf 1,86 Mio. Im Printanzeigenbereich war in dieser Zeit immerhin noch eine Zunahme von knapp $40 \%$ zu verzeichnen. Vgl. Nielsen S + P 1996, zitiert bei: Bruhn, M., Kommunikationspolitik, München 1997, S. 77. 
Angebote und Print-Titel zu einer zunehmenden Zersplitterung der Medien ${ }^{11}$ und damit zu immer kleiner werdenden Zielgruppen, deren Erreichung aus Sicht der Unternehmen immer aufwendiger wird. ${ }^{12}$ Um dennoch zum Konsumenten durchzudringen, erfolgt vielfach eine überproportionale Steigerung der Kommunikationsaufwendungen für einzelne Produkte und Zielgruppen. ${ }^{13}$ Viele Branchen bzw. Unternehmen sind in den letzten Jahren dieser Entwicklung gefolgt und haben mit ihrem Verhalten dazu beigetragen, dass Aufwendungen und Wirkungen im Rahmen der Kommunikation immer weiter auseinander klaffen. Die Unverhältnismäßigkeit von Aufwendungen und Wirkungen im Rahmen des Einsatzes traditioneller Massenmedien hat daher vermehrt zu einer Umverteilung der Medienausgaben zugunsten direkter Kommunikationsformen gefürt. ${ }^{14}$ Aufgrund ihrer individualisierbaren Inhalte und ihrer Interaktivität versprechen hierbei vor allem die Instrumente des Direktmarketing einen höheren Wirkungsgrad im Sin-

11 Waren 1984 lediglich 11 TV-Sender in Deutschland verfügbar, sind es im Jahr 1994 bereits über 79. Im Jahr 2003 können mittlerweile über 400 Sender pro Haushalt empfangen werden. Ebenso hat sich die Anzahl der Print-Titel (Publikumszeitschriften, Tageszeitungen, Fachzeitschriften etc.) mehr als verzehnfacht. In diesem Zusammenhang spricht Bruhn auch von einer „Atomisierung der Medien“. Vgl. Bruhn, M., Kommunikationspolitik, a. a. O., S. 76.

12 Diese Informationsüberlastung hat gleichzeitig zu einer Positionierungsenge in der Psyche des Konsumenten geführt, die es den Unternehmen erschwert, ein klares Vorstellungsbild ihrer Produkte und Dienstleistungen bei ihren Zielgruppen zu verankern. Bereits im Jahr 1987 schätzte Kroeber-Riel den Anteil der aus der Werbung verarbeiteten Informationen auf lediglich $2 \%$. Vgl. Kroeber-Riel, W., Informationsüberlastung durch Massenmedien und Werbung in Deutschland, in: Die Betriebswirtschaft, Jg. 47, Nr. 3, 1987, S. 257-264.

13 Vgl. Bruhn, M., Kommunikationspolitik, a. a. O., S. 71. Für ausgewählte Zielgruppen werden allerdings trotz gekürzter Kommunikations-Budgets steigende Aufwendungen verzeichnet. In diesem Kontext erscheint bspw. die Ansprache junger Zielgruppen mit Hilfe des Einsatzes von SMS-Werbung attraktiv. Vgl. Tran, T., How to trap into the teen marketing, in: Advertising \& Marketing to Children, JulySeptember 2003, 31-38.

14 Setzten Anfang der 90er Jahre lediglich $20 \%$ der Unternehmen im Rahmen ihrer Kommunikation die Instrumente des Direktmarketing ein, wuchs dieser Anteil im Jahr 2002 bereits auf $69 \%$ an. Auch in Zukunft wird mit einem steigenden Einsatz der Direktmarketing-Instrumente gerechnet. Vgl. hierzu Deutsche Post AG (Hrsg.), Direktmarketing Deutschland 2003 - Fakten, Trends und Hintergründe zum Direktmarketing 2002/2003, Direkt Marketing Monitor, Studie 15, Bonn 2003, S. 17. Neben wirtschaftlichen Prognosen der Direktmarketingverbände deuten auch Befragungen unter Marketing-Verantwortlichen auf ein weiteres Wachstum des Direktmarketing hin. Im Rahmen einer Befragung unter Managern sahen $53 \%$ der Teilnehmer einen weiteren Anstieg der Bedeutung des Direktmarketing in den nächsten Jahren. Vgl. Mercer Management Consultants (Hrsg.) Communications Benchmark 2003, a. a. O. 
ne der Effektivität bei gleichem Investitionsvolumen und damit eine höhere Effizienz. ${ }^{15}$ So werden die Instrumente des Direktmarketing zunehmend eingesetzt, um durch geringere Streuverluste bei gleichzeitig erhöhter Wirkung im Vergleich zu klassischen Kommunikationsaktivitäten Kostenvorteile zu generieren. ${ }^{16}$ Darüber hinaus weist die direkte Kommunikation aufgrund ihrer charakteristischen Individualisierbarkeit eine hohe Flexibilität und Skalierbarkeit auf und erscheint daher auch für den Einsatz in Unternehmen mit geringerem MarketingBudget attraktiv. ${ }^{17}$ Neben den klassischen Instrumenten des Direktmarketing, wie bspw. der Postwurfsendung und dem Telefonmarketing, finden mittlerweile auch die neuen interaktiven Medien, wie etwa Email und SMS, einen hohen Verbreitungsgrad ${ }^{18}$ und werden vielfach auch in Kombination eingesetzt. ${ }^{19}$

In diesem Zusammenhang hat sich gezeigt, dass der Einsatz des Direktmarketing immer häufiger über eine primär auf die Vermittlung kommunikativer Botschaften abzielende „Direktwerbung“ hinausgeht. So werden die Instrumente des Direktmarketing in verschiedenen Branchen zunehmend auch im Rahmen der Distributionspolitik zum direkten Vertrieb von Produkten und Dienstleistungen eingesetzt, ${ }^{20}$ wobei insbesondere die Instrumente Email und SMS in den letzten Jahren in der Unternehmenspraxis verstärkt Verwendung gefunden haben. Vereinzelt wird durch den Direktmarketing-Einsatz auch der Bereich der Sortimentspolitik tangiert, indem Unternehmen zunehmend die Möglichkeit zur Ansprache

15 Vgl. Holland, H., Direktmarketing, München 1992, S. $17 \mathrm{ff}$.

16 Vgl. Meffert, H., Direktmarketing und marktorientierte Unternehmensführung, in: Dallmer, H. (Hrsg.), Das Handbuch Direct Marketing \& More, 8. Aufl., a. a. O., S. $44 \mathrm{f}$.

17 Vgl. Evans, M., O'Malley, L., Patterson, M., Direct Marketing: rise and rise or rise and fall, in: Marketing Intelligence and Planning, Vol. 13, No. 6, 1995, S. 16-23; Holland, H., Direktmarketing-Aktionen professionell planen, Wiesbaden 2001, S. 16.

18 Im Zeitraum von 1996 bis 2002 stiegen die Brutto-Aufwendungen im Direktmarketing in Deutschland von 15,3 Mrd. Euro auf 25,0 Mrd. Euro an. Werden hierbei Aufwendungen für Bannerwerbung im Internet, die erstmalig 2002 erhoben wurden, mitgerechnet, steigt diese Zahl für das Jahr 2002 sogar auf 29,0 Mrd. Euro. Insgesamt entfielen hiervon im Jahr 2002 ca. $39 \%$ auf adressierte, $6 \%$ auf unadressierte und $1 \%$ auf teiladressierte Werbesendungen. Damit steigt der prozentuale Anteil des Mediums „Werbesendungen“ im Vergleich zum Jahr 2001 von $42 \%$ auf $46 \%$ der gesamten Direktmarketingaufwendungen. Vgl. hierzu Deutsche Post (Hrsg.), Direktmarketing Deutschland 2003, a. a. O., S. 23.

19 Mittlerweile setzen $77 \%$ der Unternehmen, die Postwurfsendungen verwenden, gleichzeitig auch das Internet als direktes Kommunikationsinstrument ein. In $58 \%$ der Fälle werden Werbesendungen mit Telefonmarketing kombiniert. Vgl. hierzu Deutsche Post (Hrsg.), Direktmarketing Deutschland 2003, a. a. O., S. 54.

20 Vgl. Iyer, R. T., Hill, J. S., International direct marketing strategies: a US-European comparison, in: European Journal of Marketing, Vol. 30, No. 3, 1996, S. 65-83. 
der Kunden mit personalisierten und individualisierten Angeboten anstreben, die mit Hilfe der Direktmarketing-Instrumente direkt an bestehende und potenzielle Kunden versendet werden, wie bspw. in der Luftverkehrsbranche. ${ }^{21}$

Doch trotz der aus dieser Entwicklung resultierenden wachsenden Bedeutung des Direktmarketing für die Erreichung der Marketing- und Unternehmensziele, wird der systematischen und umfassenden Analyse der zentralen, die Effektivität und Effizienz der einzelnen Direktmarketing-Instrumente beeinflussenden Determinanten vonseiten der Unternehmen nur geringe Aufmerksamkeit gewidmet. ${ }^{22}$ Stattdessen werden direktmarketingbezogene Entscheidungen häufig ohne eine strategische Einordnung in die Zielhierarchie des Unternehmens vollzogen und scheinen zudem auf operativer Ebene vielfach das Resultat intuitiver Entscheidungsprozesse der Marketing-Verantwortlichen zu sein. ${ }^{23}$ In den meisten Fällen fehlt es sowohl an Tests der einzusetzenden Direktmarketing-Stimuli im Vorfeld von Kampagnen als auch an detaillierten Auswertungen der Ergebnisse im Anschluss an durchgeführte Aktionen.

Ein möglicher Hauptgrund für das vielfach unstrukturierte Vorgehen in der Unternehmenspraxis kann in dem noch immer dünnen theoretischen Fundament vermutet werden, welches die betriebswirtschaftliche Forschung in diesem Bereich liefert. ${ }^{24}$ Vor dem Hintergrund der beschriebenen zunehmenden Wertorientierung im Marketing sowie den aufgezeigten Veränderungen in der Kommunikationslandschaft stellt sich daher auch vermehrt aus wissenschaftlicher Sicht die Frage nach den wirkungstheoretischen Grundlagen des Direktmarketing und den damit verbundenen ökonomischen Konsequenzen des Einsatzes der verschiedenen Instrumente. ${ }^{25}$ Schon seit längerer Zeit gefordert, finden sich bislang aber nur vereinzelt Forschungsarbeiten, die sich in einer umfassenden Perspektive dem Einfluss unterschiedlicher Determinanten auf die Wirkungen

21 Vgl. Ahrens, M., Münstermann, M., Schneider, H., Direktmarketing in der wissenschaftlichen Diskussion, Arbeitspapier des Centrum für interaktives Marketing und Medienmanagement, Münster 2002, S. 15.

22 Vgl. ebenda, S. $15 \mathrm{ff}$.

23 Vgl. u. a. Löffler, H., Scherfke, A., Praxishandbuch Direct Marketing, Berlin 2000, S. 42, sowie Ahrens, M., Münstermann, M., Schneider, H., Direktmarketing in der wissenschaftlichen Diskussion, a. a. O., S. $21 \mathrm{ff}$.

24 Vgl. Meffert, H., Direktmarketing und marktorientierte Unternehmensführung, in: Dallmer, H. (Hrsg.), Das Handbuch Direct Marketing \& More, a. a. O., S. 36 f.

25 Vgl. Reinecke, S., Tomzcak, T., Einsatz von Instrumenten und Verfahren des Marketingcontrollings in der Praxis, in: Reinecke, S., Tomczak, T., Geis, G. (Hrsg.), Handbuch Marketingcontrolling: Marketing als Motor für Wachstum und Erfolg, Frankfurt a. M., Wien 2001, S. $76 \mathrm{ff}$. 
des Direktmarketing wissenschaftlich fundiert widmen. ${ }^{26}$ Die Mehrzahl der aktuellen Publikationen zu diesem Themenbereich beschränkt sich auf praktische Leitfäden, die aus einer konzeptionellen Perspektive mögliche Einflussfaktoren der Direktmarketingwirkung aufzeigen, ohne diese einer empirischen Untersuchung zu unterziehen. Der Großteil der empirischen Arbeiten fokussiert bislang auf die Analyse einiger weniger Einflussfaktoren und deren Wirkung auf die Response- und Kaufquoten von Mailings, wobei insbesondere die Auswirkungen gestalterischer Aspekte wie Farbe und Layout der einzelnen Instrumente sowie der Einfluss ausgewählter demographischer und soziodemographischer Rezipientenmerkmale auf die Wirkungsstärke des Direktmarketing-Stimulus diskutiert werden. ${ }^{27}$

Offen bleibt aber bis heute die Analyse von Wirkungsunterschieden zwischen verschiedenen Direktmarketing-Instrumenten auf Basis einer systematischen Analyse der endogenen, d. h. vom Unternehmen steuerbaren, sowie den Wirkungsprozess beeinflussenden exogenen Determinanten in Form personen- und situationsbezogener Einflussfaktoren. Zudem fehlt es noch immer an umfassenden empirischen Erkenntnissen zur Einflussstärke der einzelnen endogenen und exogenen Determinanten im Verhältnis zueinander und hinsichtlich der Bedeutung zum Erreichen eines spezifischen Wirkungsziels. Die aus einer derartigen Analyse resultierende Identifikation der für den Erfolg einer DirektmarketingAktion verantwortlichen Einflussfaktoren könnte dem unstrukturierten Vorgehen entgegenwirken, mit dem viele Unternehmen heute ihre Direktmarketing-Strategie entwickeln und gleichermaßen wertvolle Hinweise geben, auf welche Faktoren sich eine Optimierung unter Effektivitäts- und/oder Effizienzgesichtspunkten konzentrieren sollte.

26 Vielmehr hat sich in den letzten Jahren gemäß der US-amerikanischen Forschungstradition das Interesse auf die Untersuchung einzelner Einflussfaktoren verlagert, die im Rahmen von Experimenten untersucht werden. Ein Vergleich des Standes der Forschungsaktivitäten im Direktmarketing in Deutschland und den USA findet sich bei Münstermann, M., Stand der Direktmarketing-Forschung und -Praxis in den USA, Dokumentation einer Forschungsreise zur DMA Annual Conference and Exhibition 2002 und DMEF Educators' Conference 2002, Arbeitspapier des Centrum für interaktives Marketing und Medienmanagement, Münster 2002.

27 Vgl. James, E. L., Li, H., Why Do Consumers Open Direct Mail?, Journal of Direct Marketing, Vol. 7, No. 2, S. 34-40, Vögele, S., Handbook of Direct Mail,: The Dialogue Method of Direct Written Sales Communication, New York 1992; Vriens, M., van der Scheer, H. R., Hoekstra, J. C., Bult, J. R., Conjoint Experiments for Direct Mail Response Optimization, in: European Journal of Marketing, Vol. 32, No. 3, 1998, S. 323-339. 


\section{Effektivität und Effizienz im Direktmarketing als Untersuchungsgegens- tand}

\subsection{Entwicklungsstand und Abgrenzung des Direktmarketing}

Die historische Entwicklung des Direktmarketing zeigt starke Analogien zur Evolution des klassischen Marketing. ${ }^{28}$ In den 50er Jahren war das Direktmarketing weitestgehend durch die verschiedenen Ausgestaltungsformen des direkten Verkaufs der großen Versandhandelshäuser geprägt und fand in der wissenschaftlichen Literatur kaum Beachtung. ${ }^{29}$ Neben dem direkten Verkauf ermöglichten in den 60er Jahren die Anfänge der automatisierten Adressverwaltungen den Einsatz von adressierten Werbesendungen als neuartiges Kommunikationsinstrument. ${ }^{30}$ Direktmarketing wurde in dieser als „separativ-instrumental“ bezeichneten Phase ${ }^{31}$ in der wissenschaftlichen Literatur intensiver, wenngleich noch losgelöst vom klassischen Absatz lediglich als Ergänzung der Kommunikationsaktivitäten betrachtet. ${ }^{32}$ Als vorherrschendes Direktmarketing-Instrument dieser Dekade lässt sich die klassische Werbesendung nennen, aber auch der persönliche Verkauf rückte stärker in den Mittelpunkt des wirtschaftlichen Interesses. ${ }^{33}$

Erst mit einer erneuten Ausweitung der medialen Anwendungsbreite um das Telefon in den 70er Jahren wuchs die Erkenntnis, dass die verschiedenen Formen des Direktmarketing synergetisch mit den anderen Marketing-Mix-Elementen einzusetzen sind. ${ }^{34}$ Mit Beginn der „Computerisierung des Marketing“ in den frühen 80er Jahren wurde Direktmarketing mit Hilfe des Einsatzes rechnergestützter Datenbanken zu „Kundenstamm-Marketing“ weiterentwickelt. ${ }^{35}$ Ziel war hierbei der systematische Aufbau und die Auswertung von Datenbanken zur intensiveren Betreuung bestehender Kunden und im Rahmen der Neukundenakquisition. ${ }^{36}$ Mit Beginn der 90er Jahre setzte sich allmählich die Erkenntnis

28 Vgl. Meffert, H., Direktmarketing und marktorientierte Unternehmensführung, a. a. O., S. $38 \mathrm{ff}$.

29 Vgl. Löffler, H., Scherfke, A., Praxishandbuch Direktmarketing, a. a. O.

30 Vgl. Holland, H., Direktmarketing, 2. Aufl., München 2004, S. 11.

31 Vgl. Dallmer, H., Thedens, R. (Hrsg.), Handbuch des Direct-Marketing, Wiesbaden 1981, S. 74.

32 Vgl. Meffert, H., Direktmarketing und marktorientierte Unternehmensführung, a. a. O., S. 43.

33 Vgl. Dallmer, H., Thedens, R. (Hrsg.), Handbuch des Direct-Marketing, a. a. O., S. 70.

34 Vgl. ebenda, S. 74.

35 Vgl. Weinhold-Stünzi, H., Grundsätze des Kundenstamm-Marketing, in: Thexis, Heft 2, 1987, S. 8 ff.

36 Vgl. Dallmer, H., Handbuch Direct Marketing, 1987, S. 26 ff. 
durch, Direktmarketing im Sinne eines „Integrated Directmarketing“'37 nicht allein funktional zu betrachten, sondern stärker in den strategischen Marketingplanungs- und Marketingentscheidungsprozess einzubeziehen, ${ }^{38}$ indem es auf konzeptioneller Ebene ein Höchstmaß an persönlicher Kundennähe im Sinne eines Customized Marketing propagierte. ${ }^{39}$ Hierbei standen sowohl die Möglichkeiten innovativer Produktionstechnologien (Mass Customization) als auch verbesserte Verfahren der Vertriebsunterstützung im Vordergrund. Mit der schnellen Diffusion internetbasierter Technologien in der zweiten Hälfte der 90er Jahre beginnt die vorläufig letzte Entwicklungsstufe des Direktmarketing. Vor dem Hintergrund der nun erstmals möglichen unmittelbaren Vernetzung von Informations- und Interaktionsparametern gewinnen Konzepte, die eine ganzheitliche, personalisierte und dialogorientierte Kundenbearbeitung ermöglichen, vermehrt an Bedeutung. Insofern wird das Konzept des Customer Relationship Management (CRM) als Leitgedanke eines modernen Direktmarketing aufgefasst. ${ }^{40}$

Die verschiedenen unter dem Begriff des Direktmarketing subsumierten Inhalte sind im Rahmen der beschriebenen Entwicklung geprägt von einer Begriffsausweitung in Richtung eines das instrumentelle Spektrum überschreitenden Philosophieansatzes, die auch im klassischen Marketing in den letzten Jahrzehnten zu beobachten war. ${ }^{41}$ Zusammenfassend spiegeln sich die geschilderten Entwicklungsstufen in drei einander ergänzenden Begriffsbestimmungen wider:

- Vorwiegend in der Unternehmenspraxis wird Direktmarketing in erster Linie als Kommunikationsinstrument definiert, das i. d. R. flankierend im Rahmen des Marketing-Mix zum Einsatz kommt und messbare Reaktionen bei der Zielperson auslöst. ${ }^{42}$ Während die klassische Marketingkommunikation

37 Roman, E., Integrated Direct Marketing, Techniques and Strategies for Success, New York 1988.

38 Vgl. Nash, E. L., Direct Marketing - Strategy, Planning, Execution, 4th edition, New York 2000; Brown, H. E., Buskirk, B., Readings \& Cases in Direct Marketing, Lincolnwood, Chicago 1989.

39 Vgl. Kotler, Ph., Development and Tendencies of Marketing in the 1990's, in: Meffert, H., Wagner, H. (Hrsg.), Marketing - Quo vadis?, Arbeitspapier Nr. 49 der Wissenschaftlichen Gesellschaft für Marketing und Unternehmensführung e. V., Münster 1988; Meffert, H., Kirchgeorg, M., Marketing - Quo Vadis? - Herausforderungen und Entwicklungsperspektiven des Marketing aus Unternehmenssicht, in: Meffert, H., Wagner, H., Backhaus, K. (Hrsg.), Arbeitspapier Nr. 89 der Wissenschaftlichen Gesellschaft für Marketing und Unternehmensführung e. V., Münster 1994.

$40 \mathrm{Vgl}$. Meffert, H., Direct Marketing und marktorientierte Unternehmensführung, a. a. O., S. 42.

41 Vgl. ebenda, S. 10.

42 Vgl. stellv. Gerardi, A., zit. nach Kirchner, G., Sobeck, S., Lexikon des Direktmarketing, Landsberg/Lech 1989, S. 144; Deutscher Direktmarketing Verband (Hrsg.), Facts \& Figures, Wiesbaden 2000. 
überwiegend einkanalig ausgestaltet ist, führt der Einsatz des Direktmarketing i. d. R. zu einer zweikanaligen, interaktiven Kommunikation von Unternehmen und Konsumenten. ${ }^{43}$

- Im Zuge der rasanten technologischen Entwicklungen und der starken Diffusion des Internet wird Direktmarketing insbesondere im Rahmen des ECommerce in einer breiter gefassten Definition interpretiert und als strategischer Ansatz verstanden, in dessen Rahmen sämtliche direkte Kommunikations- und Vertriebskanäle mit dem Ziel der individuellen Einzelansprache nicht mehr nur flankierend, sondern als zentrale Aktionsparameter eingesetzt werden. ${ }^{44}$ In diesem Zusammenhang ergibt sich zunehmend auch die Möglichkeit zur Individualisierung von Produkten und Preisen i. S. der vielfach postulierten individualisierten (Massen-) Marktbearbeitung. ${ }^{45}$

- Die bisher am weitesten gefasste Definition des Begriffs interpretiert Direktmarketing als Philosophieansatz und beschreibt es als eine ,spezifische Ausprägung des Marketing-Konzepts, das auf Basis moderner Informations- und Kommunikationstechnologien die Orientierung an individualisierten Kundenbeziehungen im Sinne eines echten Dialogs durch eine effiziente Personalisierung sämtlicher Marketing-Parameter in den Mittelpunkt der unternehmerischen Handlungen stellt. “46

Trotz der steten Ausweitung des Anspruchsspektrums wird Direktmarketing in Wissenschaft und Praxis primär auf der Ebene unterschiedlicher Kommunikationsinstrumente analysiert. Damit erfolgt eine Interpretation des Direktmarketing

43 Vgl. Kirchner, G., Die neue Praxis der Direktwerbung. Wie Sie Ihre Verkaufs- und Werbeprobleme selbst lösen, Wiesbaden 1991, S. 9. Weitere Vertreter dieser Definitionsrichtung sind Bird, D., Commonsense Direct Marketing, Landsberg/Lech, New York 1990; Roberts, M. L., Berger, P. D., Direct Marketing Management, 2nd edition, Upper Saddle River, New Jersey 1999; Stone, B., Jacobs, R., Successful Direct Marketing Methods, 7th edition, New York 2001.

44 Vgl. Iyer, R. T., Hill, J. S., International direct marketing strategies: a US-European comparison, a. a. O., S. 65-83.

45 Vgl. Schnäbele, P., Mass Customized Marketing - Effiziente Individualisierung von Vermarktungsobjekten und -prozessen, Wiesbaden 1997, S. 13 ff.; Dorner, B., Versandhandelsmarketing. Ansätze zur Kundengewinnung und Kundenbindung, Wiesbaden 1999, S. 54 ff. sowie Bruns, J., Direktmarketing, Ludwigshafen 1998, S. 319340. Im Rahmen der Produktion kundenindividueller Produktion finden insbesondere die sog. Flexiblen Fertigungssysteme Einsatz. Zum Begriff der Flexiblen Fertigungssysteme (FFS) vgl. Adam, D., Produktionsmanagement, 9. Aufl., Wiesbaden 1997, S. 90 ff.; Piller, T., Kundenindividuelle Massenproduktion. Die Wettbewerbsstrategie der Zukunft, München, Wien 1998, S. 124.

46 Meffert, H., Direct Marketing und marktorientierte Unternehmensführung, a. a. O., S. 43; Löffler, H., Scherfke, A., Praxishandbuch Direktmarketing, a. a. O., S. 41 ff.; Bruns, J., Direktmarketing, a. a. O., S. 53 f. 
i. d. R. aus Sicht eines instrumentellen Marketing-Verständnisses. Vor dem Hintergrund der Zielsetzung der vorliegenden Arbeit soll Direktmarketing allerdings in Anlehnung an die strategische Interpretation verstanden werden, im Rahmen derer die individuelle Kommunikation über die Instrumente des Direktmarketing das Kernstück sämtlicher Aktivitäten bildet und die gezielte Einzelansprache als konstitutives Merkmal des Direktmarketing-Einsatzes angesehen werden kann. ${ }^{47}$ Gleichzeitig verfolgt der Einsatz des Direktmarketing das Ziel, die individuelle Ansprache des Konsumenten durch die Individualisierung der übrigen MixBereiche zu forcieren, wobei die kommunikative Individualisierung den Schwerpunkt dieser Bemühungen bildet. ${ }^{48}$

$\mathrm{Zu}$ den wichtigsten Instrumenten des Direktmarketing zählt hierbei die klassische Werbung, die durch Einzelansprache in schriftlicher, mündlicher oder fernmündlicher Form "direkt" ausgestaltet werden kann. ${ }^{49}$ In diesem Zusammenhang ergibt sich die Möglichkeit zur direkten Kundenkommunikation auf Basis von Postwurfsendungen (im Folgenden: Mailing), Telefonmarketing oder dem Einsatz neuer Medien, die unterschiedlich stark personalisiert ausgestaltet sein können. ${ }^{50} \mathrm{Zu}$ den in den letzten Jahren am stärksten gewachsenen Instrumenten gehört aufgrund seiner charakteristischen Merkmale Individualität und Dialogorientierung das Internet, ${ }^{51}$ und hierbei insbesondere der Bereich der elektroni-

47 Vgl. Hilke, W., Kennzeichnung und Instrumente des Direkt-Marketing, in: Hilke, W. (Hrsg.), Direkt-Marketing, Schriften zur Unternehmensführung, Bd. 47, Wiesbaden 1993, S. 11.

48 „Direktmarketing hat das Ziel, Reaktionen bei den Empfängern auszulösen“. Holland, H., Direktmarketing, 2. Aufl., München 2004, S. 20. Daher sollen „... basierend auf der Initiierung eines Kontaktes, einen nicht beobachtbaren individuellen Verarbeitungs- und Bewertungsprozess beim Rezipienten in Gang zu setzen, in dessen Konsequenz Wirkungen entstehen, die letztendlich in der Kaufhandlung münden.“ In diesem Zusammenhang liegen die Ziele des Direktmarketing in der Beeinflussung von Gedächtnis- und Verhaltenswirkungen. Während im Rahmen kognitiv-orientierter Ziele i. d. R. angestrebt wird, die Wahrnehmung des Rezipienten auf das Leistungsangebot des Unternehmens zu lenken bzw. Informationen über mögliche Leistungsangebote zu vermitteln, fokussieren affektiv-orientierte Ziele auf den Aufbau oder die Veränderung von Einstellungen, Images und Präferenzen beim Rezipienten. Jede Art des äußeren Verhaltens wird letztendlich durch die konativ-orientierten Ziele verfolgt. Vgl. Bruhn, M., Kommunikationspolitik, a. a. O., S. 503.

49 Vgl. Hilke, W., Kennzeichnung und Instrumente des Direkt-Marketing, a. a. O., S. 5-30.

50 Vgl. Tapp, A., Principles of Direct and Database Marketing, 2nd edition, Harlow, Essex 2000, S. $273 \mathrm{ff}$.

51 Institut für Demokopie (Hrsg.), Allensbacher Computer- und Technik-Analyse, Ungebrochene Dynamik der Internetnutzung, Allensbach 2003. 


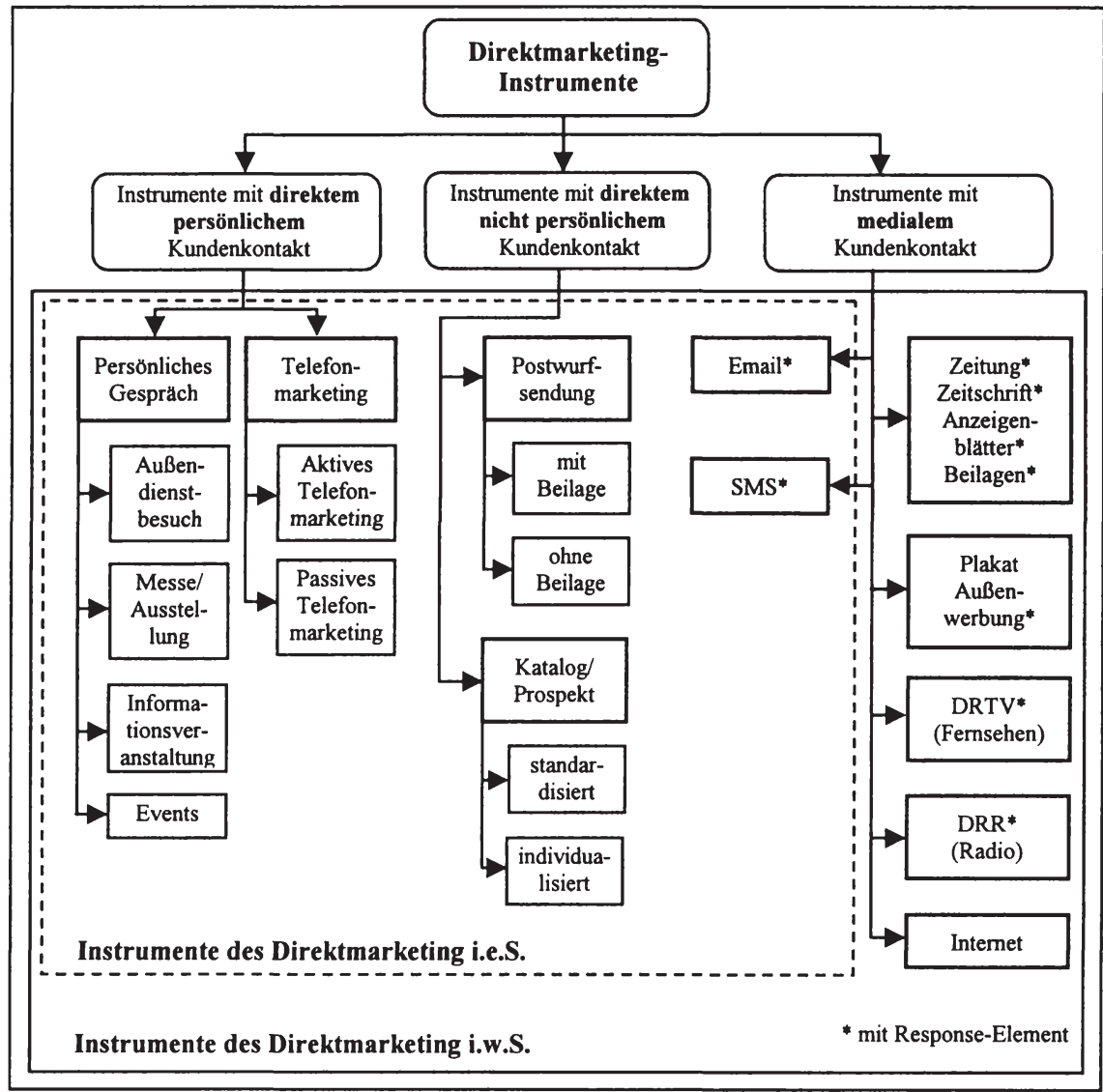

Abb. 1: Die Instrumente des Direktmarketing gegliedert nach der Art des Kundenkontaktes

(Quelle: in Anlehnung an Link, J., Schleuning, C., Das neue interaktive Direktmarketing, a. a. $O$., $S .48 \mathrm{ff}$.) 
schen Post (Emails). ${ }^{52}$ Gleichzeitig haben die elektronischen Medien mit dem Aufkommen spezieller mobilfunkbasierter Dienste, allen voran dem Short Message Service (SMS), zusätzlich an Bedeutung gewonnen. ${ }^{53}$ Daneben werden aber auch im Rahmen von klassischen Coupon-Anzeigen, Werbe- und Antwortkarten, Katalogen und telekommunikativen Medien, wie TV und Funk, weitgehend unpersönliche Medien eingesetzt, die durch das Angebot eines ,direkten Responses" direkte Kommunikationsmaßnahmen darstellen. ${ }^{54}$ Im Printbereich hat sich diese Werbeform bereits seit längerem etabliert, während die Direkt-ResponseWerbung in den elektronischen Medien (z. B. Teleshopping) erst mit dem Auftreten der Privatsender stark an Bedeutung gewonnen hat. ${ }^{55}$ Vor diesem Hintergrund soll zwischen Direktmarketing-Instrumenten i. e. S. und DirektmarketingInstrumenten i. w. S. unterschieden werden. ${ }^{56} \mathrm{Zu}$ ersteren zählen die „klassischen" Direktmarketing-Instrumente, wie Mailing, aber auch neuere Medien, wie etwa Email und SMS. Dieser Instrumentengruppe ist gemein, dass sie individualisiert gestaltet und zielgerichtet, d. h. personengenau, adressiert werden können. $\mathrm{Zu}$ den Direktmarketing-Instrumenten i. w. S. zählen zusätzlich insbesondere klassische Marketing-Instrumente mit Response-Elementen (vgl. Abb. 1).

Die folgenden Ausführungen beziehen sich vor dem Hintergrund des Direktmarketing-Verständnisses der vorliegenden Arbeit ausschließlich auf diejenigen

52 Bei der Verwendung von Emails im Rahmen der direkten Kundenkommunikation zeigt sich zum aktuellen Zeitpunkt noch ein heterogenes Bild. Während im verarbeitenden Gewerbe Email-Marketing von ca. $15 \%$ der Unternehmen und im Handel von ca. $16 \%$ eingesetzt wird, sind dies bei Dienstleistern sogar $23 \%$ der Unternehmen. Im Vergleich zum Vorjahr stiegen die Umsätze im Bereich Email-Marketing deutschlandweit auf 1,7 Mrd. Euro in 2002. Hinzu kommen Aufwendungen für das Internet i. H. v. 3,67 Mrd. Euro sowie zusätzlich Bannerwerbung i. H. v. 0,32 Mrd. Euro für das Jahr 2002. Vgl. hierzu Deutsche Post AG (Hrsg.), Direktmarketing Deutschland 2003, a. a. O., S. 26 ff.

53 Allein in Deutschland wurden im Jahr 2003 ca. 36 Mrd. SMS verschickt. Vgl. VATM (Hrsg.), Neue Zahlen zum Mobilfunkmarkt. Mehr Kunden nutzen immer mehr SMS, Pressemitteilung vom 18.03.2004, Abruf am 12.06.2004 unter www.vatm.de.

54 Vgl. Link, J., Schleuning, C., Das neue interaktive Direktmarketing: die neuen elektronischen Möglichkeiten der Kundenanalyse und Kundenbindung, Ettlingen 2001, S. $48 \mathrm{ff}$.

55 Vgl. Hilke, W., Kennzeichnung und Instrumente des Direkt-Marketing, a. a. O., S. 5-30.

56 In anderen Systematisierungsansätzen wird eine Unterscheidung im Hinblick auf den Grad der Kundenaktivierung (passiv, reaktionsorientiert, interaktionsorientiert) bzw. nach der Phase im Interaktionsprozess vorgenommen. Vgl. Bruhn, M., Kommunikationspolitik, a. a. O., S. 484 f.; Link, J., Schleuning, C., Das neue interaktive Direktmarketing: die neuen elektronischen Möglichkeiten der Kundenanalyse und Kundenbindung, a. a. O., S. $48 \mathrm{ff}$. 
Instrumente, die eine individuelle, vonseiten des Unternehmens induzierte, gezielte Ansprache bestehender und potenzieller Kunden ermöglichen. ${ }^{57}$ Von weiteren Instrumenten, insbesondere klassischen Kommunikationsinstrumenten wie TV- und Plakat-Werbung mit Response-Elementen, wird abstrahiert, weil sie zum gegenwärtigen Zeitpunkt weder aktiv auf einzelne Personen im Sinne einer individuellen Kommunikation gerichtet, noch ihre Inhalte bzw. die damit verbundenen Angebote personalisiert und individualisiert werden können. ${ }^{58}$ Gleichzeitig wird auch das Internet als Direktmarketing-Medium von der weiteren Betrachtung ausgeschlossen, da es sich hierbei ebenfalls um ein Pull-Medium handelt und ein zielgerichteter Einsatz nicht gewährleistet ist. ${ }^{59}$ Aus diesem Grund beziehen sich die folgenden Ausfuhrungen mit der Postwurfsendung (im Folgenden Mailing) auf eines der klassischen sowie mit Email und SMS auf zwei „neuere“ Direktmarketing-Instrumente.

Neben der individuellen Skalierbarkeit der drei genannten Instrumente besitzt der Einsatz dieser Instrumente zum gegenwärtigen Zeitpunkt für die Unternehmenspraxis einen hohen Stellenwert bzw. wird dieser in der Zukunft noch steigen. In diesem Zusammenhang besitzt das klassische Mailing aufgrund seiner vielfältigen Einsatzmöglichkeiten aktuell den größten Anteil an den Direktmarketing-Aufwendungen deutscher Unternehmen und gehört auch hinsichtlich der Einsatzhäufigkeit zu den meistgenutzten Medien dieser Kategorie. ${ }^{60}$ Ebenso sind Emails in den letzten Jahren zu einem unverzichtbaren Element der Unternehmenskommunikation geworden, die aufgrund ihrer guten zeitlichen Steuerbarkeit bei gleichzeitig niedrigen Kosten auch in Zukunft weiterhin an Bedeutung ge-

57 Vor dem Hintergrund des der Arbeit zugrunde liegenden Direktmarketing-Verständnisses ist Hilke zuzustimmen, der die gezielte Einzelansprache des Konsumenten als konstitutives Merkmal des Direktmarketing ansieht. Vgl. Hilke, W., Kennzeichnung und Instrumente des Direkt-Marketing, a. a. O., S. 11.

58 So besitzen die genannten Instrumente lediglich medialen und damit einen „klassisch-indirekten Charakter". Der Rezipient dieser Trägermedien kann vom Unternehmen ex-ante nicht ausgewählt und individuell adressiert werden. Vgl. hierzu u. a. Link, J., Schleuning, C., Das neue interaktive Direktmarketing, a. a. O., S. 55 ff. sowie die dort angegebene Literatur.

59 Vgl. u. a. Bauer, H. H., Mäder, R., Fischer, C., Determinanten der Werbewirkung von Markenhomepages, Arbeitspapier des Instituts für Marktorientierte Unternehmensführung, Mannheim 2001, S. 15; Kroeber-Riel, W., Weinberg, P., Konsumentenverhalten, München 1992, S. 99.

60 Nach dem Internet wurden adressierte Werbesendungen als zweithäufigstes Direktmarketing-Instrument in Deutschland eingesetzt (ca. 300.000 Verwender in Deutschland). Gemessen an den Aufwendungen war die adressierte Werbesendung mit Gesamtaufwendungen i. H. v. 11,2 Mrd. Euro das bedeutendste Medium. Vgl. hierzu Deutsche Post AG (Hrsg.), Direktmarketing Deutschland 2003, a. a. O., S. 47. 
winnen werden. ${ }^{61}$ Eine ähnlich positive Zukunft wird dem Einsatz von SMS vorausgesagt. So werden vor allem die technologischen Entwicklungen im Bereich der mobilen Endgeräte zu neuen, umfassenderen Darstellungsmöglichkeiten bei gleichzeitig sinkenden Transaktionskosten führen. ${ }^{62}$ Darüber hinaus wird das Handy wegen der zunehmenden Medienkonvergenz zu einem immer wichtigeren Instrument im Rahmen der privaten und beruflichen Kommunikation aber auch im Bereich des M-Commerce. Es wird vermutet, dass die Anzahl der über das Handy abgewickelten Transaktionen in Form des Erwerbs von Produkten und Dienstleistungen in den nächsten Jahren stark wachsen wird. ${ }^{63}$ Der derzeit vorherrschende hohe Anteil von jungen Leuten an den Handy- sowie Email-Nutzern wird sich in den nächsten Jahren gleichmäßig auf sämtliche Bevölkerungsschichten ausweiten. ${ }^{64}$

\subsection{Effektivität und Effizienz als Beurteilungskriterien des Direktmarketing- Einsatzes}

In der betriebswirtschaftlichen Literatur werden die Begriffe „Effektivität“ und „Effizienz" seit Mitte des letzten Jahrhunderts sowohl in wissenschaftlichen Publikationen als auch in Praktikerleitfäden intensiv diskutiert. ${ }^{65} \mathrm{Ihr}$ hoher Stellenwert als zentrale Beurteilungskriterien des Marketing-Erfolgs zeichnet sich be-

61 In diesem Zusammenhang sei auf die vielfältigen künftigen technischen Entwicklungsmöglichkeiten im multimedialen Umfeld hingewiesen. Vgl. Münstermann, M., Stand der Direktmarketing-Forschung und -Praxis in den USA, a. a. O., S. $23 \mathrm{ff}$.

62 Vgl. Picot, A., Neuburger, R., Mobile Business - Erfolgsfaktoren und Voraussetzungen, in: Reichwald, R. (Hrsg.), Mobile Kommunikation. Wertschöpfung, Technologien, neue Dienste, Wiesbaden 2002, S. $55 \mathrm{ff}$.

63 Prognostiziert werden in diesem Zusammenhang Steigerungen der Umsätze im Rahmen des M-Commerce von derzeit 60 Mio. Euro in den nächsten Jahren auf über das Zehnfache i. H. v. 663 Mio. Euro weltweit. Vgl. hierzu Forrester (Hrsg.), MCommerce in 2003, New York 2003.

64 Der derzeit noch hohe Anteil an jungen Nutzern wird sich in den kommenden Jahren in Richtung einer ausgeglichenen Verteilung auf sämtliche Altersgruppen verlagern. Allen voran ist gegenwärtig die Altersgruppe der 21-25jährigen Nutzer gefolgt von den 26-30jährigen Nutzern, am häufigsten vertreten. Vgl. StarWap AG, unveröffentlichte Studie 2003, S. 1-3.

65 Vgl. hierzu eine der ersten Definitionen von Effektivität im Kontext der Kommunikationsforschung bei Dichter, der den Begriff im Jahr 1949 als ,the kind of effect it was set out to achieve" definiert. Dichter, E., A Psychological View of Advertising Effectiveness, in: Journal of Marketing, Vol. 14, No. 1, 1949, S. $61 \mathrm{ff}$. 
reits seit geraumer Zeit ab. ${ }^{66}$ Doch trotz der zentralen Bedeutung der beiden Begriffe in der wissenschaftlichen Diskussion werden sie vielfach immer noch heterogen verwendet. Zudem besteht häufig keine Einigkeit darüber, in welchem Verhältnis die beiden Begriffe zueinander stehen. ${ }^{67}$

Die Mehrzahl der wissenschaftlichen Publikationen bezieht den Begriff der Effektivität auf den Output der Leistungserstellung und versteht hierunter eine Maßgröße, die somit die Erreichung vorgegebener Ziele angibt. ${ }^{68}$ In diesem Kontext kann zwischen zwei Arten der Effektivitätsbeurteilung im Marketing unterschieden werden: ${ }^{69}$ Mit dem Begriff der Effektivität i. w. S. wird eine durch den Einsatz eines (Direktmarketing-) Stimulus verursachte Veränderung eines Outputs im Sinne von Wirksamkeit bezeichnet. Effektivität i. e. S. ist dagegen strenger zu interpretieren und meint zusätzlich die Überprüfung der Höhe der Wirksamkeit in Bezug auf ein vorab festzulegendes Zielniveau. Eine Maßnahme kann demnach als effektiv i. w. S. bezeichnet werden, wenn sie zu einer signifikanten Wirkung im Hinblick auf ein direktmarketingspezifisches Ziel führt, bspw. die messbare Steigerung der Bekanntheit eines neu eingeführten Produktes durch die Verschickung von Postwurfsendungen mit Warenproben um $5 \%$ innerhalb der relevanten Zielgruppe. Eine Maßnahme ist effektiv i. e. S., wenn der damit verbundene Wirksamkeitsgrad über einem vorab formulierten Zielniveau liegt, d. h. wenn das vorab definierte Zielniveau bspw. eine Bekanntheitssteigerung um $3 \%$ darstellte. Im weiteren Verlauf der Arbeit soll der Begriff der Effektivität in Anlehnung an die erste Begriffsauffassung definiert werden und bezeichnet damit jede messbare Reaktion eines Rezipienten in Folge eines Direktmarketing-Stimulus, unabhängig davon, ob es sich um eine psychographische (z.

66 Vgl. Bonoma, T. V., Clark, B. H., Marketing Performance Assessment, Boston 1988; Frazier, G. et al. (Hrsg.), 1988 AMA Educators Proceedings. Efficiency and Effectiveness in Marketing, Chicago 1988.

67 Vgl. stellvertretend für eine Reihe von Autoren Gzuk, R., Messung der Effizienz von Entscheidungen, Tübingen 1975, S. 12; Bünting, H. F., Organisatorische Effektivität von Unternehmen. Ein zielorientierter Ansatz, Wiesbaden 1995, S. 73; Grabatin, G., Effizienz von Organisationen, Berlin 1981, S.18 f.; Scholz, C., Effektivität und Effizienz, organisatorische, in: Frese, E. (Hrsg.), Handwörterbuch der Organisation, 3. Aufl., Stuttgart 1992, S. 533.

68 Cunningham, J. B., Approaches to the Evaluation of Organizational Effectiveness, in: Academy of Management Review, Vol. 5, 1977, S. 463-474; Bluedorn, A. C., Cutting the Gordian Knot: A Critique of the Effectiveness Tradition in Organizational Research, in: Sociology and Social Research, Vol. 64, 1980, S. 479; Price, J. L., The Study of Organizational effectiveness, in: The Sociological Quarterly, Vol. 13, 1972, S. 3-15.

69 Vgl. Lasslop, Effektivität und Effizienz von Events - Wirkungstheoretische Analyse und empirische Befunde, Wiesbaden 2004, S. 11. 
B. eine Einstellungsänderung) oder verhaltensorientierte Reaktionen (z. B. Kauf) handelt.

Der Begriff der Effizienz stellt im Allgemeinen ein Beurteilungskriterium dar, mit dem sich beschreiben lässt, ob eine Maßnahme geeignet ist, ein vorgegebenes Ziel in einer bestimmten Art und Weise (z. B. unter Wahrung der Wirtschaftlichkeit) zu erreichen. ${ }^{70}$ I. d. R. beschreibt der Effizienzbegriff somit eine Output/Input-Relation, die sich auf das Verhältnis aus Ressourceneinsatz und der durch diesen erzielte Wirkung bezieht. ${ }^{71}$ Im Gegensatz zur Effektivität wird nicht die absolute Höhe des Outputs betrachtet, sondern diese relativ zum Input analysiert. Es reicht demnach nicht aus, bspw. den Saldo von direktmarketingspezifischen Aufwand zu direktmarketingspezifischen Ertrag zu betrachten; erst durch die Berechnung des Verhältnisses zeigt sich der leistungswirtschaftliche Zusammenhang von Mittel und Zweck ${ }^{72}$ und lässt so Aussagen zur „Ergiebigkeit" ${ }^{673}$ in diesem Fall - des Direktmarketing-Einsatzes zu. Damit orientiert sich der Effizienzbegriff primär an der Beurteilung der Wirtschaftlichkeit und damit maßgeblich am ökonomischen Prinzip. ${ }^{74}$ Vor dem Hintergrund knapper Ressourcen ist es demnach im ökonomischen Sinne rational, das Verhältnis von Input und Output zu optimieren. Hierzu lassen sich entweder bestimmte Outputmengen mit minimalen Ressourcen erzeugen (Minimalprinzip) oder maximale Outputmengen (bzw. Wirkungen) mit gegebenen Ressourcen realisieren (Maximalprinzip). Da i. d. R. die minimalen Input- und maximalen Outputmengen unbekannt sind, steht die Optimierung der Relation durch einen Vergleich aller möglichen Kombinationen von Input und Output im Mittelpunkt der Bemühungen. ${ }^{75}$

70 Vgl. Gabler Wirtschaftslexikon, Wiesbaden 1997, S. 1006. Erste Definitionsansätze zur Effizienz lassen sich bei Phillips finden, der bereits 1941 Effizienz als ,the ratio of results achieved to the means used" definierte. Vgl. Phillips, C. F., A critical Analysis of recent Literature dealing with Marketing Efficiency, in: Journal of Marketing, Vol. 5, No. 4, 1941, S. 360.

71 Vgl. Scholz, C., Effektivität und Effizienz, organisatorische, a. a. O. S. 533; Gzuk, R., Messung der Effizienz von Entscheidungen, a. a. O., S. 14. Bei Betrachtung von Faktorinput- und Faktoroutputgrößen wird auch von Produktivität i. S. einer technischen Effizienz gesprochen. Werden die eingesetzten Faktormengen z. B. mit Kosteninformationen bewertet, handelt es sich um ökonomische Effizienz. Vgl. Adam, D., Produktionsmanagement, 9. Aufl., a. a. O., S. 1.

72 Vgl. Meyer, P. W., Die Werbeerfolgskontrolle, Düsseldorf 1963, S. 71.

73 Vgl. Dellmann, K., Eine Systematisierung der Grundlagen des Controlling, in: Spremann, K., E. Zur (Hrsg.), Controlling, Wiesbaden 1992, S. 121.

74 Vgl. Horváth, P., Controlling, 4. Aufl., München 1991, S. 139.

75 Vgl. hierzu Greißinger, P., Wirtschaftlichkeitsanalysen im Gesundheitswesen. Analyse und beispielhafte Anwendung der Data Envelopment Analysis, Wiesbaden 2000, S. 11. 
Von Effizienz kann daher im Kontext des Direktmarketing nur gesprochen werden, wenn es zu einem gegebenen Output/Input-Verhältnis einer Direktmarketingmaßnahme keine alternative Maßnahme gibt, die über ein besseres Verhältnis bei gleichem Ressourceneinsatz verfügt. In der wissenschaftlichen Literatur wird in diesem Zusammenhang häufig die Nebenbedingung festgelegt, dass der Output größer als der Input sein muss, da sonst ein Verlust knapper Ressourcen entsteht. ${ }^{76}$

Allerdings stellt ein positives Output-/Input-Verhältnis nur eine notwendige, aber keine hinreichende Bedingung für das Vorliegen von Effizienz dar. Vielmehr werden die Suche und Festlegung von geeigneten Vergleichsobjekten zu zentralen Aspekten der Effizienzbeurteilung. ${ }^{77}$

\subsection{Bestandsaufnahme von Forschungsarbeiten zur Effektivität und Effizienz des Direktmarketing}

Sowohl Fragen zur Effektivität als auch zur Effizienz des Direktmarketing wurden in der Vergangenheit aus unterschiedlichen Forschungsperspektiven betrachtet. Mittlerweile sind beide Aspekte von Betriebswirtschaftlern vermehrt aufgegriffen und in verschiedenen theoretischen und empirischen Untersuchungen diskutiert worden. ${ }^{78}$ Ziel der meisten zum aktuellen Zeitpunkt vorliegenden Arbeiten ist es, Gestaltungsempfehlungen für den optimierten DirektmarketingEinsatz abzuleiten, der zu Effektivităts- und ggf. auch Effizienzsteigerungen führt. ${ }^{79}$ Ebenso wie in der Unternehmenspraxis wird der Begriff des Direktmarketing in den meisten Arbeiten instrumentell interpretiert, so dass die Mehrheit

76 In diesem Zusammenhang stellt Meffert fest, dass sich die Betriebswirtschaftslehre seit jeher mit dem "zweckgerechten Einsatz bzw. der Verteilung knapper Mittel, d. h. mit dem rationalen einzelwirtschaftlichen Handeln" beschäftigt. Meffert, H., Die Flexibilität in betriebswirtschaftlichen Entscheidungen, unveröffentlichte Habilitationsschrift, München 1968, S. 1.

77 Vgl. Danne, S., Messebeteiligungen von Hochschulen: Ziele und Erfolgskontrolle, Frankfurt a. M. 2000, S. 95 ff., Ueding, R., Management von Messebeteiligungen, Frankfurt a. M., S. 259 ff.; Burmann, Ch., Erfolgskontrolle im Eventmanagement, IST-Studieninstitut für Kommunikation GmbH (Hrsg.), Düsseldorf 1998, S. 100.

78 Einen Überblick über ausgewählte Arbeiten zur Wirkungskontrolle des Direktmarketing i. S. der Effektivität findet sich bei Ahrens, M., Münstermann, M., Schneider, H., Direktmarketing in der wissenschaftlichen Diskussion, a. a. O., S. $47 \mathrm{ff}$.

79 Vgl. bspw. Bezjian-Avery, A., Calder, B., Iacobucci, D., New Media Interactive Advertising vs. Traditional Advertising, in: Journal of Advertising Research, Vol. 38, No. 4, July-August 1998. 
der Arbeiten die Instrumente des Direktmarketing in ihrer Funktion als Teil der Kommunikationspolitik analysiert. ${ }^{80}$

Ein erster Schwerpunkt in der direktmarketingspezifischen Literatur beschäftigt sich im Rahmen von theoretisch-konzeptionellen Analysen mit den vermeintlich zentralen Einflussfaktoren der einzelnen Instrumente auf die Effektivität und Effizienz ihres Einsatzes sowie mit der Suche nach der optimalen Gestaltung dieser Determinanten. Den hieraus generierten, i. d. R. in Praktikerleitfäden zusammengefassten Empfehlungen fehlt es allerdings zumeist an einer empirischen Validierung der Ergebnisse, so dass Aussagen zur Einflussrichtung und -stärke der beschriebenen Determinanten auf die Effektivität bzw. die Effizienz des Direktmarketing sowie zur relativen Bedeutung der einzelnen Determinanten nur partiell getroffen werden können. Zudem beruhen die Empfehlungen in vielen Fällen auf spezifischen Erfahrungen der Autoren und beziehen sich daher häufig auf einzelne Branchen ${ }^{81}$ oder Instrumente, ${ }^{82}$ was ihr Verallgemeinerungspotenzial in erheblichem Maße begrenzt.

Mit dem Ziel der empirischen Analyse konzeptionell abgeleiteter Gestaltungsempfehlungen des Direktmarketing-Einsatzes sind insbesondere experimentelle Wirkungsanalysen seit geraumer Zeit zum Gegenstand der wissenschaftlichen Diskussion des Direktmarketing geworden. ${ }^{83}$ Ein Schwerpunkt der Forschung beschäftigt sich hierbei mit der Durchführung von Laborexperimenten, im Rahmen deren die Auswirkungen einer gezielten Variation eines oder mehrerer Gestaltungsparameter des Direktmarketing-Stimulus unter ansonsten konstanten Umfeldbedingungen analysiert werden. ${ }^{84}$ Laborexperimente werden insbesondere für Untersuchungen eingesetzt, bei denen die Gefahr besteht, dass personenbezogene oder in der Kontaktsituation begründete Einflussfaktoren eine Erhebung unter realen Bedingungen unmöglich oder zu aufwendig erscheinen lassen bzw. das Messergebnis gravierend verfälschen würden. ${ }^{85}$ Durch die gezielte

80 Vgl. Deutscher Direktmarketing Verband (Hrsg.), Facts \& Figures, Wiesbaden 2000.

81 Bamler bezieht seine Ausführungen bspw. auf die Wirkungsanalyse von Mailings im Business-to-Business-Bereich und arbeitet intensiv die Besonderheiten heraus. Vgl. Bamler, G., Zur Wirkung von Direkt-Werbung im Industriegüterbereich, Frankfurt a. M. u. a. 1987.

82 Vgl. Aschoff, M., Professionelles Direkt- und Dialogmarketing per E-Mail, München, Wien 2002. Der Autor bezieht seine Ausführungen explizit nur auf den Bereich von Emails.

83 Vgl. Ahrens, M., Münstermann, M., Schneider, H., Direktmarketing in der wissenschaftlichen Diskussion, a. a. O., S. $47 \mathrm{ff}$.

84 Zum Begriff des klassischen Laborexperiments vgl. u. a. Meffert, H., Marketingforschung und Käuferverhalten, 2. Aufl., Wiesbaden 1992, S. $207 \mathrm{ff}$.

85 Vgl. Weis, H. Ch., Steinmetz, P., Marktforschung, Ludwigshafen, Kiel 1991, S. 111; Berekoven, L., Eckert, W., Ellenrieder, P., Marktforschung. Methodische Grundla- 
Auswahl der Probanden sowie die Schaffung von ähnlichen Wahrnehmungssituationen können im Rahmen von Laborexperimenten vielfältige exogene Faktoren als Ursache für unterschiedliche Reaktionen der Probanden ausgeschlossen werden; dies erleichtert die Erhebung von Ursache-Wirkungszusammenhängen zwischen endogenen und damit aus Unternehmenssicht beeinflussbaren Determinanten und dem Rezipientenverhalten. ${ }^{86}$

Vorrangiges Ziel der meisten Laborexperimente im Bereich des Direktmarketing ist ebenfalls die Messung momentaner Gedächtnis- und Verhaltensreaktionen der Rezipienten nach Darbietung eines spezifischen DirektmarketingStimulus. In diesem Kontext finden häufig die in der klassischen Werbewirkungsforschung verwendeten apparativen Verfahren wie Hautwiderstandsmessungs- oder Blickaufzeichnungsgeräte Anwendung. ${ }^{87}$ Der inhaltliche Schwerpunkt der Untersuchungen hat sich hierbei im Bereich des Direktmarketing zumeist auf die Analyse des Einflusses gestalterischer Aspekte konzentriert. Mit der Durchführung von Laborexperimenten können vielfach wertvolle Informationen für die Optimierung des Direktmarketing gewonnen werden. Jedoch erweist sich die Übertragbarkeit der Ergebnisse aus der künstlichen Umgebung in die Unternehmenspraxis - ebenso wie im Bereich der klassischen Kommunikationsforschung - als problematisch. ${ }^{88}$ So werden zwar konstante Umfeldbedingungen geschaffen, was gleichzeitig die Zuordnung einer beim Probanden gemessenen Wirkung auf die Darbietung eines bestimmten Stimulus zulässt, eine Überprüfung von tatsächlichem Response- oder Kaufverhalten unter realen Rahmenbedingungen ist auf Basis von Laborexperimenten zumeist nicht möglich. ${ }^{89}$

Vor dem Hintergrund der Möglichkeit einer kundenindividuellen Messung des Rezipientenverhaltens eignen sich insbesondere die Instrumente des Direktmarketing für eine empirische Analyse des Einflusses ausgewählter endogener Determinanten auf die Effektivität und Effizienz im Rahmen von Feldexperimenten, da sich die aus der klassischen Werbewirkungsforschung bekannten $\mathrm{Zu}$ ordnungsprobleme von Ursache und Wirkung größtenteils umgehen lassen. Aufgrund der individuellen Skalierbarkeit der einzelnen Direktmarketing-Stimuli in Verbindung mit der personenindividuellen Erhebung der Rezipientenreaktionen nach Erhalt eines spezifischen Direktmarketing-Stimulus wird es ebenso möglich, residuale Wirkungen im Verhalten der Rezipienten als Ergebnis der Variation ei-

gen und praktische Anwendung, Wiesbaden 1989, S. 151; Cox, E. P., Marketing Research, New York u. a. 1979, S. $154 \mathrm{ff}$.

86 Vgl. Meffert, H., Marketingforschung und Käuferverhalten, a. a. O., S. 207.

87 Vgl. Green, P. E., Tull, D. S., Methoden und Techniken der Marketingforschung, 4. Aufl., Stuttgart 1982, S. $141 \mathrm{ff}$.

88 Vgl. Meffert, H., Marketingforschung und Käuferverhalten, a. a. O., S. 208 f.

89 Vgl. ebenda. 
nes spezifischen Einflussfaktors zuzuordnen..$^{90}$ Als Voraussetzung für die Validität der Ergebnisse gilt allerdings die Bedingung, dass ein Großteil der übrigen Umfeldfaktoren möglichst kontrolliert bzw. konstant gehalten werden kann, was gleichermaßen die zentrale Herausforderung der meisten in der Wissenschaft diskutierten Arbeiten darstellt. ${ }^{91}$

Mit Ausnahme einiger Forschungsarbeiten stand im Bereich der (feld-)experimentellen Studien in der Vergangenheit die isolierte Analyse einzelner Einflussfaktoren im Mittelpunkt der Betrachtung. Ein Schwerpunkt der Forschungsarbeiten lag auf der Untersuchung der Effektivität und Effizienz einzelner Instrumente des Direktmarketing und der Optimierung dieser in gestalterischer Hinsicht. ${ }^{92}$ In der Mehrzahl der Untersuchungen werden im Rahmen von Feldexperimenten die Wirkungen einzelner endogener oder exogener Faktoren auf eine einzelne abhängige Variable, wie bspw. Response- oder Bestellquoten, analysiert. ${ }^{93}$ Neben objekt- bzw. stimulusbezogenen Einflussfaktoren wie Farbe oder Form eines Mailings ${ }^{94}$ beschäftigen sich andere Forschungsarbeiten auch mit der Wirkung einzelner situations- und personenbezogener Einflussfaktoren. ${ }^{95}$ Während im Rahmen situationsbezogener Einflussfaktoren z. B. die Auswirkung des Klimas auf die Responsequote von Werbesendungen untersucht wird, widmet sich die Untersuchung personenbedingter Einflussfaktoren dem Einfluss rezipientenbezogener Merkmale, wie etwa Einstellungen ggü. dem Direktmarketing, und deren Einfluss auf die Effektivität der Instrumente. ${ }^{96}$ In den meisten dieser Arbei-

90 Vgl. Green, P. E., Tull, D. S., Methoden und Techniken der Marketingforschung, a. a. O., S. $319 \mathrm{ff}$.

91 Vgl. ebenda, S. 322.

92 Vgl. Wulf, K. de, Hoekstra, J. C., Commandeur, H. R., The Opening und Reading Behavior of Business-to-Business Direct Mail, in: Industrial Marketing Management, Vol. 29, S. 133-145.

$93 \mathrm{Zu}$ den endogenen Einflussfaktoren zählen hierbei sämtliche vom Unternehmen bewusst steuerbare Determinanten, die einen Einfluss auf die Effektivität und damit auch auf die Effizienz besitzen. Exogene Faktoren hingegen haben ebenso einen Einfluss, ihre Wirkung kann aber vonseiten der Unternehmen nicht beeinflusst, lediglich berücksichtig werden.

94 Bspw. prüfen Smith/Berger den Einfluss unterschiedlich gestalteter Werbebriefe beim Einsatz im Rahmen des Fundraising und deren Einfluss auf das Spendenaufkommen. Vgl. Smith, J., Berger, P. D., The Impact of Direct Marketing Appeals on Charitable Marketing Effectiveness, in: Journal of the Academy of Marketing Science, Vol. 24, No. 3, 1996, S. 219-231.

95 Dallmer nennt die personen- und situationsbezogenen Faktoren auch den „Bedingungsrahmen für die Wirkung der Marketing-Stimuli"“. Dallmer, H., Erfolgsbedingungen der Kommunikation im Direct-Marketing, Wiesbaden 1979, S. 73.

96 Bei der zuletzt genannten Gruppe von Einflussfaktoren werden vor allem explorative Verfahren zur Datenauswertung eingesetzt, die Reaktionsdaten auf eine bestimmte 
ten werden allerdings monokausale Zusammenhänge zwischen einer spezifischen unabhängigen und einer abhängigen Variablen untersucht. ${ }^{97}$ Diesen zumeist eindimensionalen Erklärungsansätzen fehlt es daher häufig an verallgemeinerungsfähigen Aussagen..$^{98}$ So besitzen einige Untersuchungsergebnisse nur in ihrem Untersuchungskontext Gültigkeit oder weisen aufgrund einer kleinen Stichprobe bzw. eines engen Branchenfokus nur ein geringes Verallgemeinerungspotenzial auf. ${ }^{99}$

Die zentrale Herausforderung bei der Durchführung von Feldexperimenten liegt in methodischer Hinsicht in der Schaffung konstanter Umweltbedingungen, die sich in einer möglichst großen Zahl kontrollierter endogener und exogener Variablen widerspiegeln sollte. ${ }^{100} \mathrm{Im}$ Rahmen zahlreicher Forschungsarbeiten wird allerdings aus Komplexitätsgründen vielfach auf die Konstanthaltung exogener Einflussgrößen verzichtet, obwohl diese einen erheblichen Einfluss auf die Konsumentenreaktion besitzen können. ${ }^{101}$ Zusätzlich zu den beschriebenen Defiziten und des geringen Verallgemeinerungspotenzials erweisen sich daher einige der Ergebnisse als zweifelhaft. So bleibt vielfach fraglich, ob tatsächlich der experimentelle Input in Form eines modifizierten Direktmarketing-Stimulus oder

Direktmarketing-Aktion mit psychographischen Profilen verknüpfen. Vgl. bspw. die Arbeit von Chittenden/Rettie zum Responseverhalten im Email-Marketing. Chittenden, L., Rettie, R., An evaluation of e-mail marketing and factors affecting response, in: Journal of Targeting, Measurement and Analysis for Marketing, Vol. 11, No. 3, S. 203-217.

97 In vielen Studien zur Wirkungsmessung des Direktmarketing, speziell im Bereich des Industriegütermarketing, wird die Wirkung von Direktmarketing-Aktivitäten durch Response-Größen gemessen. Vgl. Bamler, G., Zur Wirkung von Direkt-Werbung im Industriegüterbereich, a. a. O., S. $66 \mathrm{ff}$. und die dort angegebene Literatur.

98 In Bezug auf das methodische Vorgehen ist zu bemerken, dass der Begriff der Effektivität vielfach ohne einen strengen Zielbezug analysiert wird und eine operationale Zielformulierung i. d. R. nicht vorliegt. Stattdessen wird mit Effektivität in einem meist experimentellen Kontext die Hypothese eines kausalen Zusammenhangs zwischen Stimulus und Ergebnisdimension geprüft.

99 So kann vermutet werden, dass sich die Ergebnisse der Effektivitätsanalyse von Mailings im Rahmen des US-Kongress-Wahlkampfes nur partiell auf andere Bereiche übertragen lassen. Vgl. Weaver-Lariscy, R. A., Tinkham, S. F., Use of impact of direct mail in the context of integrated marketing communications: U.S. Congressional campaigns in 1982 and 1990. in: Journal of Business Research, Vol. 37, No. 5, 1996, S. 233-244.

$100 \mathrm{Vgl}$. Meffert, H., Marketingforschung und Käuferverhalten, a. a. O., S. 207.

$101 \mathrm{Vgl}$. Steffenhagen, H., Wirkungen der Werbung. Konzepte - Erklärungen - Befunde, Aachen, Mainz 2000, S. 13; Dallmer, H., Erfolgsbedingungen der Kommunikation im Direct-Marketing, a. a. O., S. 73. 
Effektivitäts- und Effizienzorientierung als Herausforderung

exogene Determinanten zu den gemessenen Wirkungsunterschieden geführt haben. ${ }^{102}$

Neben methodischen Defiziten können auch aus inhaltlicher Perspektive die Erkenntnisse auf einzelnen Themengebieten trotz hoher wissenschaftlicher und praktischer Relevanz zum aktuellen Zeitpunkt noch als äußerst rudimentär bezeichnet werden. ${ }^{103}$ Ein zentrales Forschungsdefizit liegt im Bereich von Intramediavergleichen begründet, die sich mit der Analyse der Effektivität und Effizienz unterschiedlicher Direktmarketing-Instrumente im Vergleich zueinander beschäftigen, um Aussagen über die optimale Auswahl des effektivsten bzw. effizientesten Kommunikationsinstruments treffen zu können. ${ }^{104}$ Einer Vielzahl konzeptionell-theoretischer Arbeiten, die eine Bewertung der einzelnen Instrumente anhand unterschiedlicher Kriterien, wie etwa Darstellungsmöglichkeiten, regionaler und zeitlicher Steuerbarkeit etc., vornehmen, stehen zwar zahlreiche empirische Effektivitäts- und Effizienzvergleiche gegenüber. ${ }^{105}$ Doch während die überwiegende Zahl der vorliegenden empirischen Arbeiten die postulierten Wirkungsvorteile der direkten Kommunikationsmedien ggü. den klassischen Kommunikationsformen bestätigt, liegen vergleichsweise wenige Arbeiten zu Intramediavergleichen im Bereich des Direktmarketing vor. ${ }^{106}$ Sowohl im Hinblick

102 Nur wenige Untersuchungen im Bereich der Feldexperimente genügen dieser Anforderung. Dieses Defizit trifft häufig auf unternehmensbezogene Studien zu, die zwar nicht den Anspruch einer wissenschaftlichen Untersuchung erheben, aber häufig als einzige Informationsquelle fur Wissenschaft und Praxis dienen.

$103 \mathrm{Vgl}$. Meffert, H., Direktmarketing und marktorientierte Unternehmensführung, a. a. O., S. $36 \mathrm{f}$.

104 Zur Unterscheidung der Begriffe Inter- bzw. Intramediavergleiche vgl. bspw. Bruhn, M., Kommunikationspolitik, a. a. O., S. 83 ff. Zu einer detaillierten Darstellung unterschiedlicher Formen von Intermediavergleichen vgl. Dallmer, H., Erfolgsbedingungen der Kommunikation im Direct-Marketing, a. a. O., S. $155 \mathrm{ff}$.

105 Vgl. bspw. Bezjian-Avery, A., Calder, B., Iacobucci, D., New Media Interactive Advertising vs. Traditional Advertising, a. a. O., S. 23-32. In der wissenschaftlichen Literatur zum Direktmarketing lässt sich eine Vielzahl von konzeptionellen Arbeiten identifizieren, die sich mit dem Vergleich der Wirkungen der klassischen Kommunikationsinstrumente und der Instrumente des Direktmarketing beschäftigen und Intramediavergleiche vornehmen. Vgl. hierzu bspw. Schefer, D., Medien des Direct Marketing im Intermedia-Vergleich, in: Dallmer, H. (Hrsg.), Das Handbuch Direct Marketing \& More, 8. Aufl., a. a. O., S. 105-123.

106 Seit einigen Jahren steht vor allem das Internet im Mittelpunkt der Betrachtung. Hierbei beschäftigen sich verschiedene Untersuchungen mit der Frage nach den Einsatzmöglichkeiten und der Wirksamkeit des Internets als Werbemedium unter Effektivitäts- und Effizienzgesichtspunkten und mit der Eignung zur Beeinflussung psychographischer Zielgrößen des Unternehmens, wie Bekanntheit, Image usw. im Vergleich zu den klassischen Kommunikationsinstrumenten. Vgl. bspw. Yoon, S.-J., 
auf die Art der untersuchten Medien als auch die verwandten Vergleichskriterien sind in den letzten Jahren nur wenige Publikationen erschienen, die eine umfassende empirische Basis besitzen. ${ }^{107}$ Die vorhandenen Arbeiten fokussieren dabei häufig nur auf zwei ausgewählte Instrumente oder beschränken sich bei ihrem Vergleich auf eine Zielgröße, wie bspw. die Responsequote. So fehlt es zum aktuellen Zeitpunkt noch an umfassenden Vergleichen mehrerer DirektmarketingInstrumente, im Rahmen derer eine Betrachtung der Wirkungsunterschiede anhand mehrerer Zielgrößen sowie unter Beachtung personen- und situationsbezogener Aspekte erfolgt. ${ }^{108}$

Ein weiteres inhaltliches Defizit kann in einem Mangel an empirisch fundierten Aussagen zu den Wirkungen des kombinierten Einsatzes mehrerer Direktmarketing-Instrumente gesehen werden. ${ }^{109}$ In diesem für die Praxis höchst relevanten Forschungsfeld fehlt es noch immer an umfassenden Erkenntnissen zur Bestimmung der optimalen Kombination verschiedener Direktmarketing-Instrumente. ${ }^{110}$ Aufbauend auf den bekannten Stärken- und Schwächen-Analysen liegt zwar den meisten konzeptionellen Arbeiten die Hypothese zugrunde, dass sich

Kim, J.-H., Is the Internet More Effective than Traditional Media? Factors Affecting the Choice of Media, in Journal of Advertising Research, Vol. 41, 2001, S. 53-60.

107 Während die traditionellen Kommunikationsinstrumente auf empirischer Basis bereits häufiger mit den Instrumenten des Direktmarketing verglichen wurden, sind insbesondere Vergleiche von mehreren Direktmarketing-Instrumenten zum gegenwärtigen Zeitpunkt noch die Ausnahme.

108 Durch den hohen Verbreitungsgrad der allgemein bekannten Effektivitäts- und Effizienzkennzahlen erscheint eine Vergleichbarkeit der Instrumente auf Basis der Ergebnisse unabhängiger Experimente auf den ersten Blick möglich. In diesem $\mathrm{Zu}-$ sammenhang ist allerdings zu bemerken, dass die Interpretationsspielräume der Effizienzkennzahlen nicht vollständig aufgelöst werden. So liegt ein zentrales Problem der Vergleichbarkeit der Werte insbesondere in der willkürlichen Aufschlüsselung einzelner Kostenarten, die u. U. bei der Berechnung berücksichtigt werden. Eine objektive Vergleichbarkeit der Instrumente anhand von Effizienzkennzahlen erscheint durch in unterschiedlichen Versuchen gemessenen Kennzahlen daher nicht aussagekräftig. Vielmehr ist es notwendig, die Instrumente des klassischen und des Direktmarketing unter gleichen Bedingungen im Rahmen von Intramediavergleichen gegenüberzustellen.

109 Zur Notwendigkeit der integrierten Kommunikation vgl. bspw. Bruhn, M., Kommunikationspolitik, a. a. O., S. 86 f. Zum Konzept der integrierten Kommunikation vgl. auch Busch, R., Dögl, R., Unger, F., Integriertes Marketing, Wiesbaden 1995, S. 317. Hinsichtlich der grundlegenden Wirkungsweise integrierter Kommunikation im Bereich der klassischen Werbung vgl. u. a. Esch, F.-R., Wirkung integrierter Kommunikation, Wiesbaden 1998.

110 Dies betrifft nicht nur den Bereich der einstufigen Kampagnen, sondern auch den Bereich der mehrstufigen Kampagnen. Zum Begriff der mehrstufigen DirektmarketingKampagnen vgl. Holland, H., Direktmarketing, 2. Aufl., München 2004, S. 61 ff. 
die Effektivität und ggf. auch die Effizienz des Medieneinsatzes durch den integrativen zielgerichteten Einsatz mehrerer Direktmarketinginstrumente steigern lassen. ${ }^{11}$ Bislang wurde dies aber weder auf Basis verschiedener Instrumentekombinationen noch unter Verwendung verschiedener Effektivitäts- und Effizienzkennzahlen untersucht. Als Schwerpunkt der vergangenen Jahre lassen sich in der wissenschaftlichen Diskussion vielmehr Untersuchungen zum integrativen Einsatz klassischer Kommunikationsinstrumente und direkter Kommunikationsinstrumente identifizieren. ${ }^{112}$

Als weiterer Mangel ist zum gegenwärtigen Zeitpunkt zu konstatieren, dass es an umfassenden Aussagen zur relativen Bedeutung der einzelnen Wirkungsdeterminanten im Hinblick auf die verfolgten Zielwirkungen und somit im Verhältnis der Einflussstärke zueinander fehlt. Zu den komplexeren Arbeiten innerhalb der (feld-) experimentellen Untersuchungen zählen zwar auch Arbeiten, die den Einfluss mehrerer, meist unterschiedlicher Determinanten hinsichtlich Effektivität und Effizienz analysieren. ${ }^{113}$ Diese Untersuchungen bilden allerdings zum aktuellen Zeitpunkt noch die Ausnahme. So fehlt es immer noch an Untersuchungen, die sich einer umfassenderen Perspektive bedienen und endogene sowie exogene Determinanten in Relation zueinander untersuchen und ihre Bedeutung in einem Gesamtzusammenhang würdigen. ${ }^{114}$

Neben der reinen Wirkungsbetrachtung im Rahmen von Effektivitätsanalysen existieren darüber hinaus zahlreiche Arbeiten, die sich mit der Effizienz des Direktmarketing-Einsatzes befassen. Im Hinblick auf die ökonomische Perspektive ist allerdings festzuhalten, dass nur wenige, umfassende theoretisch-konzeptio-

$111 \mathrm{Vgl}$. Schulz, D. E., Kitchen, P. J., A Multi-country comparison of the drive for IMC, in: Journal of Advertising Research, Vol. Jan.-Feb., 1997, S. 21-37; zur Betrachtung von Coupons als Rückkanalmedium vgl. Jain, C. L., Broadcast support to newspaper ads, Journal of Advertising Research, Vol. 15, No. 5, 1975, S. 69-72; WeaverLariscy, R. A., Tinkham, S. F., Use of impact of direct mail in the context of integrated marketing communications: U.S. Congressional campaigns in 1982 and 1990, a. a. O., S. 233-244.

$112 \mathrm{Vgl}$. zu den grundsätzlichen Kombinationsmöglichkeiten bspw. Zorn, D., Integrierte Kommunikation - Grundlagen und zukünftige Entwicklung, a. a. O., S. 53 ff.; Bruhn, M., Kommunikationspolitik, a. a. O., S. $530 \mathrm{ff}$.

113 Als eine zentrale Arbeit kann in diesem Zusammenhang die Analyse von Smith/ Berger angesehen werden, die anhand von Mailings im Business-to-BusinessBereich zeigen, welche Faktoren für das Öffnen, das Lesen sowie das Antworten auf einen Werbebrief verantwortlich sind. Vgl. Smith, J., Berger, P. D., The Impact of Direct Marketing Appeals on Charitable Marketing Effectiveness, a. a. O., S. 219231.

114 Vgl. Wulf, K. de, Hoekstra, J. C., Commandeur, H. R., The Opening und Reading Behavior of Business-to-Business Direct Mail, a. a. O., S. 133-145. 


\begin{tabular}{|c|c|c|c|}
\hline Autoren & $\begin{array}{c}\text { Forschungsfrage / } \\
\text { Zielgrøße }\end{array}$ & $\begin{array}{l}\text { Experimenteller Input / } \\
\text { unabhängige Variable }\end{array}$ & Empirische Basis \\
\hline $\begin{array}{l}\text { Hennessy } \\
\text { (1987) }\end{array}$ & $\begin{array}{l}\text { Analyse des Responsever- } \\
\text { haltens bei Mailings }\end{array}$ & $\begin{array}{l}\text { Mailings mit unterschied- } \\
\text { lichen Absendernamen }\end{array}$ & Feldexperiment \\
\hline $\begin{array}{l}\text { Gordon / } \\
\text { Tellerman } \\
(1990)\end{array}$ & $\begin{array}{l}\text { Analyse des Responsever- } \\
\text { haltens bei Mailings }\end{array}$ & $\begin{array}{l}\text { Mailings mit Mengen- } \\
\text { prämien und Prămien fur } \\
\text { schnellen Response }\end{array}$ & Feldexperiment \\
\hline $\begin{array}{l}\text { Sherman / } \\
\text { Greene / Plank } \\
\text { (1991) }\end{array}$ & $\begin{array}{l}\text { Analyse des Responsever- } \\
\text { haltens bei Mailings }\end{array}$ & $\begin{array}{l}\text { Mailings mit unterschied- } \\
\text { licher Argumentation im } \\
\text { Rahmen des Angebots }\end{array}$ & Feldexperiment \\
\hline $\begin{array}{l}\text { Williams / } \\
\text { Beard / Kelly } \\
\text { (1991) }\end{array}$ & $\begin{array}{l}\text { Analyse des Responsever- } \\
\text { haltens bei Mailings }\end{array}$ & $\begin{array}{l}\text { Lesbarkeit von Werbebrie- } \\
\text { fen auf Basis unterschied- } \\
\text { licher Mailings }\end{array}$ & Feldexperiment \\
\hline $\begin{array}{l}\text { James / Li } \\
\text { (1993) }\end{array}$ & $\begin{array}{l}\text { Analyse des Responsever- } \\
\text { haltens bei Mailings }\end{array}$ & $\begin{array}{l}\text { Verschiedene Mailings } \\
\text { mit unterschiedlichen } \\
\text { Umschlagcharakteristika }\end{array}$ & Feldexperiment \\
\hline $\begin{array}{l}\text { Berger / Smith } \\
\text { (1996) }\end{array}$ & $\begin{array}{l}\text { Analyse des Response- und } \\
\text { Spendenverhaltens bei } \\
\text { Mailings }\end{array}$ & $\begin{array}{l}\text { Verschiedene Formen von } \\
\text { Spendenanfragen per Mai- } \\
\text { lings }\end{array}$ & Feldexperiment \\
\hline $\begin{array}{l}\text { Weaver- } \\
\text { Lariscy / } \\
\text { Tinkham } \\
(1996)\end{array}$ & $\begin{array}{l}\text { Analyse des Einflusses der } \\
\text { Verwendung von Mailings } \\
\text { im Hinblick auf das Wahl- } \\
\text { ergebnis }\end{array}$ & $\begin{array}{l}\text { Einsatz von Mailings im } \\
\text { Rahmen des integrierten } \\
\text { Marketing }\end{array}$ & Feldexperiment \\
\hline $\begin{array}{l}\text { Berger / Smith } \\
\text { (1997) }\end{array}$ & $\begin{array}{l}\text { Analyse des Response- und } \\
\text { Spendenverhaltens bei } \\
\text { Mailings }\end{array}$ & $\begin{array}{l}\text { Verschiedene Mailings } \\
\text { mit unterschiedlichen } \\
\text { Gestaltungsvarianten; per- } \\
\text { sonenbezogene Daten }\end{array}$ & Feldexperiment \\
\hline $\begin{array}{l}\text { Vriens / van } \\
\text { der Scheer / } \\
\text { Hoekstra / } \\
\text { Bult (1998) } \\
\end{array}$ & $\begin{array}{l}\text { Analyse des Response- und } \\
\text { Spendenverhaltens bei } \\
\text { Mailings }\end{array}$ & $\begin{array}{l}\text { Verschiedene Mailings } \\
\text { mit unterschiedlichen } \\
\text { Umschlag- und Briefcha- } \\
\text { rakteristika }\end{array}$ & $\begin{array}{l}\text { Feldexperiment } \\
\text { und Befragung }\end{array}$ \\
\hline $\begin{array}{l}\text { De Wulf / } \\
\text { Hoekstra / } \\
\text { Commandeur } \\
(2000)\end{array}$ & $\begin{array}{l}\text { Analyse des Offnungs-, Le- } \\
\text { se- und Responseverhaltens } \\
\text { bei Mailings }\end{array}$ & $\begin{array}{l}\text { Verschiedene Mailings } \\
\text { mit unterschiedlichen } \\
\text { Gestaltungsvarianten; per- } \\
\text { sonenbezogene Daten }\end{array}$ & $\begin{array}{l}\text { Feldexperiment } \\
\text { und Befragung }\end{array}$ \\
\hline $\begin{array}{l}\text { Yun / Trumbo } \\
(2000)\end{array}$ & $\begin{array}{l}\text { Analyse des Einsatzes von } \\
\text { Mailings, Emails und In- } \\
\text { ternet im Rahmen von Be- } \\
\text { fragungen }\end{array}$ & $\begin{array}{l}\text { Versand identischer Fra- } \\
\text { gebogen per Mailing und } \\
\text { Email sowie Download- } \\
\text { moglichkeit im Internet }\end{array}$ & Feldexperiment \\
\hline $\begin{array}{l}\text { Chittenden/ } \\
\text { Rettie (2002) }\end{array}$ & $\begin{array}{l}\text { Analyse des Responsever- } \\
\text { haltens bei Emails }\end{array}$ & $\begin{array}{l}\text { Verschiedene Emails, u. a. } \\
\text { mit variierter Lange und } \\
\text { Lănge der Betreffzeile }\end{array}$ & $\begin{array}{l}\text { Feldexperimente } \\
\text { und Expertenbe- } \\
\text { fragung }\end{array}$ \\
\hline $\begin{array}{l}\text { Helgeson / } \\
\text { Voss / } \\
\text { Terpening } \\
(2002)\end{array}$ & $\begin{array}{l}\text { Analyse des Responsever- } \\
\text { haltens bei mailingsbasier- } \\
\text { ten Fragebögen }\end{array}$ & $\begin{array}{l}\text { Verschiedene Mailings } \\
\text { mit unterschiedlichen ge- } \\
\text { stalterischen Charakteris- } \\
\text { tika }\end{array}$ & Feldexperimente \\
\hline $\begin{array}{l}\text { Chittenden / } \\
\text { Rettie (2003) }\end{array}$ & $\begin{array}{l}\text { Analyse des Responsever- } \\
\text { haltens bei Emails }\end{array}$ & $\begin{array}{l}\text { Verschiedene Emails mit } \\
\text { unterschiedlichen Ange- } \\
\text { boten }\end{array}$ & Feldexperiment \\
\hline
\end{tabular}

Tab. 1: Bestandsaufnahme ausgewählter Forschungsarbeiten zur (feld-)experimentellen Analyse der Effektivität und Effizienz des Direktmarketing 
nelle Arbeiten vorliegen, die sich eingängig mit dem Effizienzbegriff auseinandersetzen und damit über den Erkenntnisstand der erwähnten Praktikerleitfäden hinausgehen. Der Großteil der Erkenntnisse wird zumeist mit Hilfe der Analyse von realen Direktmarketing-Kampagnen in der Unternehmenspraxis untersucht. So existieren zahlreiche Arbeiten, die mit Hilfe der bekannten Wirtschaftlichkeitskennzahlen, wie z. B. Kosten pro Kontakt, Kosten pro Response oder kampagnenbezogener Gewinn, die Effizienz des Direktmarketing untersuchen. Unter wissenschaftlichen Gesichtspunkten erweist sich die Aussagekraft derartiger Untersuchungen aufgrund von Zuordnungsproblemen bzw. Abgrenzungsproblematiken aber häufig als unzulänglich. ${ }^{115}$ Zudem gehen wenige Arbeiten über die alleinige Darstellung der Effizienzkennzahlen hinaus und analysieren den direktmarketingspezifischen Wirkungsprozesses.

Tabelle 1 gibt einen Überblick über ausgewählte Forschungsarbeiten, die sich auf den angesprochenen Gebieten mit der experimentellen Analyse der unterschiedlichen Direktmarketing-Instrumente sowie ausgewählter endogener und exogener Determinanten des Direktmarketing-Einsatzes auseinandergesetzt haben.

Zusammenfassend zeigt eine Bestandsaufnahme der Literatur eine Reihe offener Punkte bei der experimentellen Analyse der Wirkungen ausgewählter Direktmarketing-Instrumente:

- Dies betrifft zum einen die potenziellen Wirkungen des DirektmarketingStimulus, die als Output eines direktmarketingspezifischen Wirkungsprozesses beim Rezipienten identifiziert werden müssen. So fehlt es zum gegenwärtigen Zeitpunkt an einem allgemeinen Bezugsrahmen, im Rahmen dessen eine Abgrenzung der direktmarketingspezifischen Teil- und Zielwirkungen erfolgt. Erst auf Basis eines theoretisch fundierten Bezugsrahmens, der für verschiedene Direktmarketing-Instrumente Gültigkeit besitzt, ist die Identifikation von Effektivitätsmaßstäben und daraus abzuleitender Messverfahren für den Direktmarketing-Einsatz sinnvoll möglich.

- Als ein zentrales Defizit der bisherigen Forschungsbemühungen kann festgehalten werden, dass es den vorliegenden Arbeiten an einer konsistenten Verdichtung der untersuchten Einzelaspekte in Form eines integrierten Modells und damit an einer umfassenden Systematisierung der zentralen endogenen und exogenen Determinanten im Rahmen des beschriebenen Wirkungsprozesses fehlt.

- Während in konzeptionellen und empirischen Intermediavergleichen die Effektivität und Effizienz der klassischen Kommunikationsmedien und der Ins-

115 Vgl. Ahrens, M., Münstermann, M., Schneider, H., Direktmarketing in der wissenschaftlichen Diskussion, a. a. O., S. $47 \mathrm{ff}$. 
trumente des Direktmarketing miteinander verglichen werden, mangelt es zum gegenwärtigen Zeitpunkt immer noch an umfassenden Intramediavergleichen, die mehrere Direktmarketing-Instrumente zum Gegenstand haben. Ein umfassender Vergleich der Effektivität und Effizienz unterschiedlicher Direktmarketing-Instrumente im Rahmen eines empirischen Intramediavergleiches, der verschiedene Zielgrößen berücksichtigt, existiert bislang nicht.

- Die Untersuchung des Einflusses des eingesetzten Trägermediums auf die Effektivität und Effizienz erfolgt in den meisten Fällen im isolierten Einsatz. Dabei erscheinen Aussagen zu den Wirkungen des integrativen Einsatzes mehrerer Direktmarketing-Instrumente sowie der daraus resultierenden ökonomischen Konsequenzen noch nicht ausreichend empirisch untersucht.

- In den bisherigen Vergleichen wird zudem primär der Einfluss der ausgewählten Trägermediums auf eine Zielgröße untersucht, während es an der Untersuchung weiterer Wirkungsgrößen fehlt. Die Mehrzahl der Untersuchungen bezieht sich hierbei auf Mailings; nur vereinzelt sind Arbeiten zum Bereich der Emails zu finden. Neben weiteren endogenen Determinanten, wie bspw. angebotsbezogenen Determinanten, ist ebenfalls der Einfluss exogener Faktoren in Form personen- und situationsbezogener Determinanten auf die Zielwirkungen des Direktmarketing-Einsatzes zu untersuchen.

- Gleichzeitig wird im Rahmen der bisherigen Ansätze nicht deutlich, welche Bedeutung den verschiedenen endogenen und exogen Determinanten im Rahmen des Wirkungsprozesses in Relation zueinander zukommt. Erst durch eine auf Basis einer umfassenden Perspektive basierenden empirischen Analyse der zentralen Determinanten werden Aussagen zur relativen Bedeutung der einzelnen Entscheidungstatbestände für die Erreichung der unterschiedlichen Zielgrößen des Direktmarketing möglich.

\section{Zielsetzung und Gang der Untersuchung}

Vor dem Hintergrund der vorangegangenen Ausführungen besteht das übergeordnete Ziel der vorliegenden Arbeit darin, einen Beitrag zur experimentellen Analyse der Wirkungsweise ausgewählter Direktmarketing-Instrumente zu leisten. Teilziele liegen in diesem Zusammenhang in der theoretischen Fundierung des Wirkungsprozesses der verschiedenen Instrumente, anhand dessen der Einfluss zentraler endogener und exogener Determinanten auf die Effektivität und Effizienz untersucht werden soll. Aus den genannten Zielen leiten sich folgende Forschungsschwerpunkte ab: 
- Die theoretische Konzeption eines Modells zur Erfassung und Erklärung der Wirkungen des Einsatzes ausgewählter Direktmarketing-Instrumente auf Basis fundierter wissenschaftlicher Erkenntnisse.

- Die Systematisierung endogener und exogener Einflussfaktoren, die den aufgezeigten Wirkungsprozess beeinflussen, sowie der Analyse der Relevanz dieser Faktoren hinsichtlich der Effektivität und Effizienz des Direktmarketing-Einsatzes.

- Die empirische Untersuchung der Einflussrichtung und Einflussstărke ausgewählter endogener Determinanten der Direktmarketing-Stimuli im Hinblick auf ausgewählte Zielgrößen sowie die Analyse der Relevanz ausgewăhlter exogener Determinanten im Rahmen des aufgezeigten Wirkungsprozesses.

- Die empirische Analyse der relativen Bedeutung der untersuchten endogenen und exogenen Determinanten auf die Wirkungen des Direktmarketing.

- Die Ableitung von Implikationen für die Ausgestaltung und Erfolgskontrolle des Direktmarketing.

Mit den beschriebenen Zielsetzungen und Forschungsschwerpunkten ist der Gang der Untersuchung bereits vorgezeichnet: Teil B der Arbeit widmet sich zunächst der theoretischen Konzeption eines Modells zur Beurteilung der Effektivität und Effizienz des Direktmarketing-Einsatzes. Hierzu werden die Teil- und Zielwirkungen der Wirkungsprozess des Direktmarketing detailliert analysiert und auf Basis der Ergebnisse Möglichkeiten zur Messung der Effektivität und Effizienz aufgezeigt. Auf Grundlage des abgeleiteten Wirkungsprozesses wird in einem weiteren Schritt eine Untersuchung des Einflusses zentraler endogener und exogener Determinanten vorgenommen. In diesem Zusammenhang werden die einzelnen Wirkungsdeterminanten isoliert untersucht, etwaige Wirkungsbeziehungen untereinander konkretisiert sowie in einem weiteren Schritt in den Gesamtkontext des Wirkungsprozesses eingeordnet. ${ }^{116}$ Sämtliche Erkenntnisse flieBen in ein integriertes Modell ein, welches die zentralen, den Wirkungsprozess des Direktmarketing beeinflussenden Determinanten systematisch aufzeigt.

Die empirische Analyse des Einflusses ausgewählter endogener Determinanten auf Effektivität und Effizienz des Direktmarketing-Einsatzes erfolgt im Rahmen eines Feldexperiments bei einem Unternehmen aus dem Bereich des Verkehrsdienstleistungsbereichs und ist in dem sich anschließenden Teil $\mathbf{C}$ der Arbeit dargestellt. Auf Basis einer umfassenden Effektivitäts- und Effizienzanalyse werden Einflussrichtung und -stärke ausgewählter endogener Determinanten des

116 Die Auswahl relevanter Wirkungsdeterminanten des Direktmarketing basiert auf verhaltenswissenschaftlichen Ansätzen der Marketing-Wissenschaft. Vgl. Kroeber-Riel, W., Marketingtheorie - eine verhaltensorientierte Erklärung von Marktreaktionen, Köln 1972. 
Direktmarketing im Rahmen mehrerer Direktmarketing-Aktionen analysiert. Durch die gezielte Variation einzelner Stellhebel eines Direktmarketing-Stimulus (experimenteller Input) und unter Konstanz weiterer endogener Determinanten (kontrollierte Variablen) werden die Auswirkungen dieser auf die Rezipientenreaktionen untersucht. In diesem Zusammenhang erfolgt ebenfalls eine Analyse des Einflusses ausgewählter exogener Determinanten aus den Bereichen der personen- und situationsbezogenen Determinanten im Hinblick auf ihre Bedeutung für das Response- und Kaufverhalten der Probanden. Abschließend die als relevant erachteten endogenen und exogenen Determinanten in ein Gesamtmodell integriert, welches die Generierung von Aussagen über die relative Bedeutung der Faktoren für die Erreichung der intendierten Zielwirkungen ermöglicht (vgl. Abb. 2).

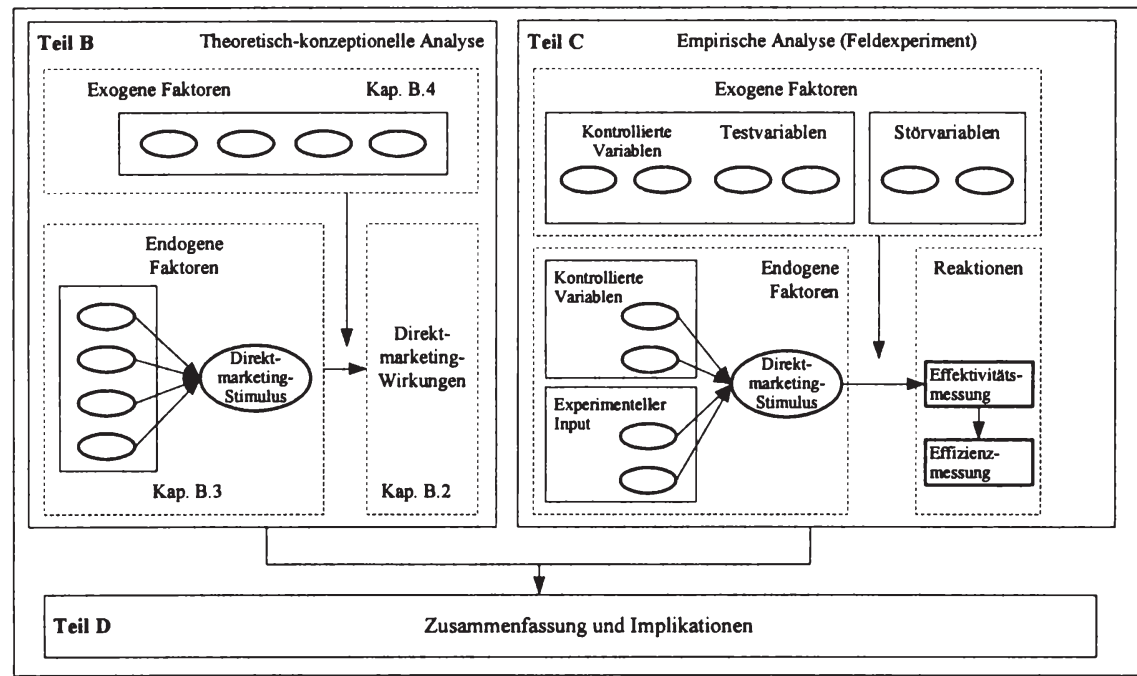

Abb. 2: Allgemeiner Bezugsrahmen der Untersuchung 
Teil D fasst die zentralen Ergebnisse der Untersuchung zusammen. Darauf aufbauend werden Implikationen für die Optimierung des DirektmarketingEinsatzes in der Praxis abgeleitet. ${ }^{117}$ Anregungen für weitere Forschungsarbeiten beschließen die Ausführungen.

117 Die Ableitung von Gestaltungsempfehlungen ergibt sich aus der Tradition des entscheidungsorientierten Paradigmas der Betriebswirtschaftslehre. Vgl. Heinen, E., Einführung in die Betriebswirtschaftslehre, 9. Aufl., Wiesbaden 1985, S. 259 ff.; Meffert, H., Die Leistungsfähigkeit der entscheidungs- und systemorientierten Marketinglehre, in: Kortzfleisch, G. v. (Hrsg.), Wissenschaftsprogramm und Ausbildungsziele der Betriebswirtschaftslehre, Berlin 1971, S. 167 ff.; Witt, F. H., Theorietradition der betriebswirtschaftlichen Forschung, Wiesbaden 1995, S. 29. 


\section{B. Konzeptionelle Analyse des Einflusses endogener und exogener Determinanten des Direktmarketing-Erfolgs}

\section{Theoretische Ableitung eines Modells zur Beurteilung des Direktmarke- ting-Erfolgs}

\subsection{Analyse der Wirkungskategorien des Direktmarketing}

Den Ausgangspunkt und damit die logische Ursache sämtlicher DirektmarketingWirkungen bildet ein von Unternehmen gestalteter Direktmarketing-Stimulus, der eingesetzt wird, um ein gewünschtes psychographisches und/oder verhaltensorientiertes Ziel bei den Rezipienten zu erreichen. ${ }^{118} \mathrm{Im}$ Wesentlichen besteht der Direktmarketing-Stimulus aus einem Werbemittel sowie einem Werbeträger. Die Werbemittelgestaltung beinhaltet hierbei die inhaltliche Werbebotschaft sowie deren gestalterische Umsetzung auf dem entsprechenden Werbeträger. ${ }^{119}$

In Abhängigkeit der konkreten Gestaltung des Direktmarketing-Stimulus kann es aufseiten des Rezipienten zu unterschiedlichen Wirkungen kommen, die sich anhand verschiedener Kriterien systematisieren lassen. In der wissenschaftlichen Literatur existiert eine Vielzahl von allgemeinen Modellen, die sich mit der Systematisierung von Kommunikations- und Werbewirkungen beschäftigen. ${ }^{120}$ Bislang sind jedoch nur vereinzelt Modelle in den Kontext des Direktmarketing übertragen worden, so dass es noch immer an einer allgemein anerkannten Sys-

118 Dallmer unterscheidet im Zusammenhang mit den Wirkungen der MarketingKommunikationen zwischen rezipientenbezogenen Wirkungen, wie bspw. Wirkungen auf das Wissen, auf Einstellungen oder Meinungen, sowie anbieterbezogenen Wirkungen, wie etwa Wirkungen auf den Umsatz, die Kosten und den Gewinn. Die verhaltensbezogenen Wirkungen stehen zwischen diesen unmittelbaren (rezipientenbezogenen) und den mittelbaren (anbieterbezogenen) Wirkungen, da das Verhalten des Rezipienten letztendlich zu den ökonomischen Konsequenzen auf Seiten des Unternehmens führt. Vgl. Dallmer, H., Erfolgsbedingungen der Kommunikation im Direct-Marketing, a. a. O., S. 66; Behrens, K. Ch., (Hrsg.), Absatzwerbung, 2. Aufl., Wiesbaden 1976, S. $107 \mathrm{ff}$.

$119 \mathrm{Vgl}$. Steffenhagen, H., Wirkungen der Werbung, a. a. O., S. 14.

120 Vgl. ebenda, S. 6 ff.; zu einer umfassenden Taxonomie unterschiedlicher Werbewirkungsmodelle Vakratsas, D., Ambler, T., How Advertising Works: What Do We Really Know, in: Journal of Marketing, Vol. 63, No. 1, 1999, S. 26-43. 
tematisierung der direktmarketingspezifischen Wirkungen fehlt. ${ }^{121}$ Vielmehr werden in der wissenschaftlichen Literatur instrumentespezifische Partialmodelle diskutiert, die dem Ziel der vorliegenden Arbeit jedoch nicht gerecht werden. ${ }^{122}$ Als Grundlage der folgenden Ausführungen soll daher zunächst auf das Modell von Steffenhagen zurückgegriffen werden, das eine Unterscheidung der Kommunikationswirkungen hinsichtlich der Kriterien Art der Wirkung sowie der zeitlichen Nähe zwischen Stimulus und Wirkung ermöglicht. ${ }^{123}$

- Unter den momentanen Gedächtniswirkungen werden sämtliche Rezipientenreaktionen subsumiert, die in unmittelbarer zeitlicher Nähe zum originären Direktmarketingimpuls stehen. Insbesondere nicht beobachtbare innere Vorgänge affektiver und kognitiver Natur, die während des Kommunikationskontaktes auftreten, werden berücksichtigt. ${ }^{124}$

- Zu dauerhaften Gedächtniswirkungen kommt es, wenn die Wirkungen eines Direktmarketing-Stimulus das Langzeitgedächtnis beeinflussen. Konstitutives Merkmal der dauerhaften Gedăchtniswirkungen ist es, dass die Wirkungen auch noch längere Zeit nach dem Kontakt mit einer Kommunikationsbotschaft nachweisbar sind. ${ }^{125}$ Hierunter werden alle Arten von wertfreien Kenntnissen und Assoziationen, wertenden Einstellungen und Verhaltensabsichten gegenüber einem Meinungsgegenstand subsumiert. ${ }^{126}$

121 Das Modell von Steffenhagen ist ursprünglich dem Bereich der klassischen Werbung zuzuordnen und wurde nur vereinzelt in den Kontext des Direktmarketing gestellt. So greifen bspw. Wiedmann/Schwab das Modell von Steffenhagen auf, eine detaillierte Auseinadersetzung im Hinblick auf direktmarketingspezifische Wirkungen erfolgt jedoch nicht. Vgl. Wiedmann, R., Schwab, K., Erfolgsmessung und optimierung von Online-Marketing-Aktivitäten, in: Dallmer, H. (Hrsg.), Das Handbuch Direct Marketing \& More, a. a. O., S. $849 \mathrm{f}$.

122 Vgl. bspw. Wulf, K. de, Hoekstra, J. C., Commandeur, H. R., The Opening und Reading Behavior of Business-to-Business Direct Mail, a. a. O., S. 133-145; Helgesson, J. G., Voss, K. E., Terpening, W. D., Determinants of Mail-Survey Response: Survey Design Factors and Respondent Factors, in: Psychology \& Marketing, Vol. 19, No. 3, S. 302-328.

123 Vgl. Steffenhagen, H., Wirkungen der Werbung, a. a. O., S. 8 f.; und im Folgenden auch Steffenhagen, H., Werbeziele, in: Berndt, R., Hermanns, A. (Hrsg.), Handbuch Marketing-Kommunikation, Wiesbaden 1993, S. 286 ff. Die Unterscheidung zwischen kurz- und langfristigen Verhaltenswirkungen wird bei Steffenhagen nicht explizit vorgenommen. Ihre Relevanz für das Direktmarketing ergibt sich aber durch die Kategorisierung der einzelnen, unter den Zielen des Direktmarketing subsumierten Inhalte, wie etwa „Verschicken von Einladungen“ (kurzfristig) sowie „Änderungen des Images“ (langfristig). Vgl. Holland, H., Direktmarketing, München 2004, S. 20.

$124 \mathrm{Vgl}$. Steffenhagen, H., Wirkungen der Werbung, a. a. O., S. $8 \mathrm{f}$.

125 Vgl. Behrens, G., Werbewirkungsanalyse, Opladen 1976, S. 47 ff.

126 Vgl. Steffenhagen, H., Wirkungen der Werbung, a. a. O., S. 74 ff. 
- Bei den momentanen Verhaltenswirkungen handelt es sich um in kurzem zeitlichem Abstand zum Stimulus folgende (Impuls-)Handlungen. ${ }^{127} \mathrm{Zu}$ diesen momentanen Wirkungen zăhlen bspw. das Öffnen eines Werbebriefes, die Informationssuche im Internet nach Erhalt einer Werbe-Email oder der Anruf im Call-Center nach Erhalt einer Werbe-SMS.

- Dauerhafte Verhaltenswirkungen beziehen sich auf sämtliche Arten des längerfristig beobachtbaren Konsumentenverhaltens, insb. des Informationsund Kaufverhaltens, das sich häufig erst in einem erheblichen zeitlichen Abstand zum Werbekontakt äußert. ${ }^{128}$

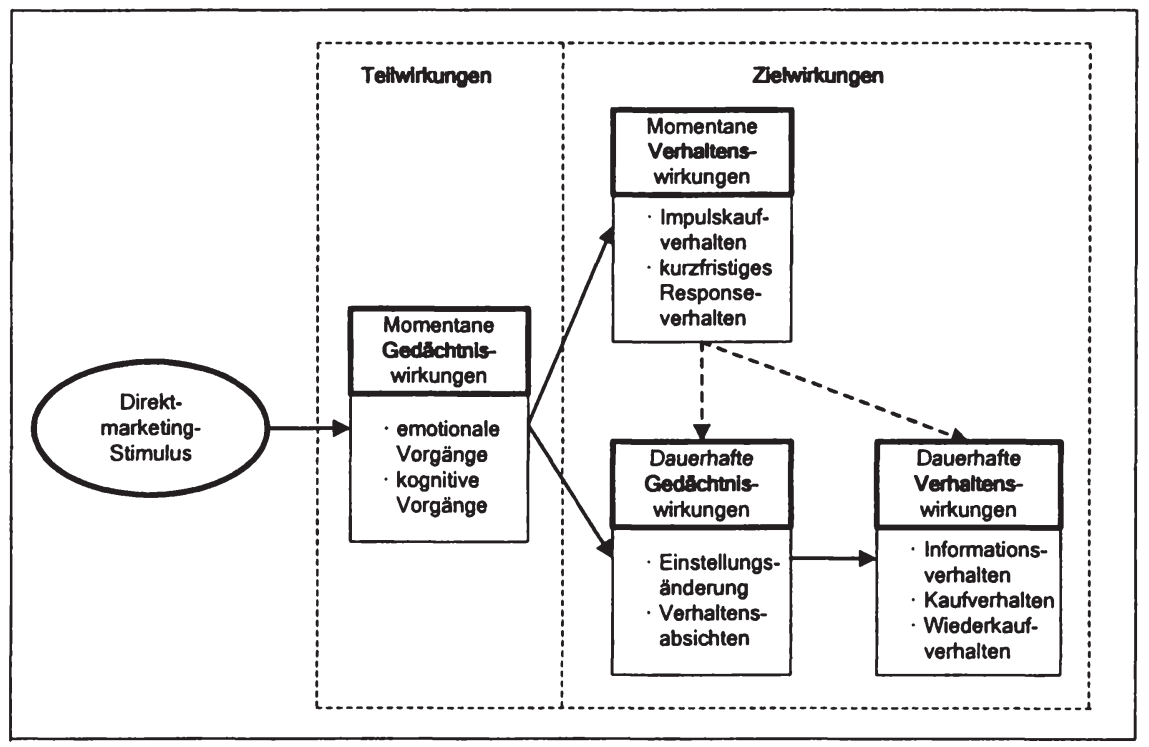

Abb. 3: Teil- und Zielwirkungen des Direktmarketing-Einsatzes

(Quelle: in Anlehnung an Steffenhagen, H., Wirkungen der Werbung, a. a. O., S. 13.)

In Anlehnung an die Ziele des Direktmarketing lassen sich die aufgezeigten Kategorien danach unterscheiden, ob es sich um Teilwirkungen oder um Zielwirkungen handelt. Teilwirkungen sind als sog. Modalziele den eigentlichen Zielen vorgelagert und gelten damit als eine notwendige Bedingung für die Erreichung

$127 \mathrm{Vgl}$. Steffenhagen, H., Wirkungen der Werbung, a. a. O., S. 8.

128 Häufig äußern sich die finalen Verhaltenswirkungen auch Aktivitäten nach dem Kauf, wie etwa in einem veränderten Gebrauchs-, Verbrauchs- oder Umweltverhalten. Vgl. Mayer, H., Illmann, T., Markt- und Werbepsychologie, a. a. O., S. 14 f. 
der gewünschten Zielwirkungen. Im Fall der beschriebenen Wirkungskategorien handelt es sich bei den momentanen Gedächtniswirkungen um modale Zielsetzungen, da diese vorliegen, wenn es zum Kontakt zwischen DirektmarketingStimulus und Rezipient kommt. Hierbei handelt es sich insbesondere um emotionale und kognitive Prozesse, die erfolgen, wenn der Rezipient den Direktmarketing-Stimulus wahrnimmt. Da sie aus Unternehmenssicht noch keine originären Ziele des Direktmarketing-Einsatzes darstellen, handelt es hierbei um Teilwirkungen des Direktmarketing-Einsatzes. Sowohl momentane Verhaltenswirkungen in Form von spontanem Response- oder Kaufverhalten als auch dauerhafte Gedächtnis- und Verhaltenswirkungen stellen dagegen finale Ziele des Direktmarketing-Einsatzes dar und gehören daher zu der Kategorie der Zielwirkungen des Direktmarketing-Einsatzes. Die folgende Abbildung zeigt die aufgezeigten Wirkungskategorien im Überblick (vgl. Abb. 3).

Interessant erscheint hierbei, dass zwischen den einzelnen Wirkungskategorien verschiedene Interdependenzen bestehen. So ist bspw. zu beachten, dass die momentanen Verhaltenswirkungen zwar Zielwirkungen darstellen, i. d. R. aber auch einen Einfluss auf die dauerhaften Zielwirkungen - sowohl Gedächtnisals auch Verhaltenswirkungen - besitzen können.

Vor dem Hintergrund der beschriebenen Rezipientenreaktionen erscheint eine detaillierte Auseinandersetzung mit den dargestellten Wirkungskategorien erforderlich, um die im Kontext des Direktmarketing für die Effektivitäts- und Effizienzmessung relevanten Zielwirkungen herauszuarbeiten. Trotz des Schwerpunktes verhaltensorientierter Zielsetzungen in der Unternehmenspraxis ist deshalb zunächst die Eignung der unterschiedlichen Wirkungskategorien zur Beurteilung der Effektivität des Direktmarketing im Hinblick auf die Erfüllung verschiedener Anforderungen zu untersuchen, die an eine umfassende Effektivitätsmessung definierten Ziele zu stellen sind. ${ }^{129}$ Im Einzelnen bestehen folgende Anforderungen:

- Reagibilität: Zwischen den gemessenen Direktmarketing-Wirkungen und dem eingesetzten Direktmarketing-Stimulus muss eine eindeutige Zuordnung i. S. einer Mittel-Zweck-Beziehung möglich sein.

- Selektive Steuerungskraft: Ein zielgerichteter Einsatz des Direktmarketing ist nur dann möglich, wenn sich aus den Zielen konkrete Impulse für die Ausgestaltung des Stimulus ableiten lassen. Erst die selektive Steuerungskraft er-

129 Vgl. Bruhn, M., Kommunikationspolitik, a. a. O., S. 244 ff.; Steffenhagen, H., Werbeziele, a. a. O., S. $287 \mathrm{ff}$. Gleichzeitig sollte das betrachtete Wirkungsziel hinsichtlich Ausmaß, Inhalt, Zeit- und Segmentbezug formuliert sein. Vgl. auch Meffert, H., Marketing. Grundlagen marktorientierter Unternehmensführung, a. a. O., S. 76; Lasslop, I., Effektivität und Effizienz von Events, a. a. O., S. 57. 
möglicht eine zielgerechte Auswahl der Instrumente sowie die Definition und Ausrichtung konkreter Direktmarketing-Maßnahmen.

- Validität der Messung und Erhebungsaufwand: Im Rahmen der Effektivitätsbeurteilung einer Direktmarketing-Wirkung erfordert deren Erfassung die Anwendung eines validen Messverfahrens. Gleichzeitig ist im Rahmen des Einsatzes in der Praxis sicherzustellen, dass der mit der Messung verbundene Erhebungsaufwand in einem ausgewogenen Verhältnis zum Nutzen steht.

Es ist davon auszugehen, dass sich die Wirkungen eines DirektmarketingStimulus auf sämtliche der beschriebenen Wirkungskategorien beziehen können. Zwar wurde vielfach nachgewiesen, dass eine Beeinflussung dauerhafter Gedächtniswirkungen durch die Instrumente des Direktmarketing möglich ist, ${ }^{130}$ allerdings schränken einige Autoren ihre Aussagen dahingehend ein, dass zum Zweck einer messbaren Wirkung auf die dauerhaften Gedächtnisinhalte i. d. R. mehrere Wiederholungen des Direktmarketing-Stimulus und somit ein erhöhter Werbedruck vorangehen müssen, ${ }^{131}$ was allerdings auch zu Reaktanzen fuhren kann. ${ }^{132}$ Eine Zuordnung eines einzelnen Direktmarketing-Stimulus und einer Veränderung dauerhafter Gedächtnisinhalte i. S. der Reagibilität ist kaum möglich. Die Wirkungen eines Direktmarketing-Stimulus äußern sich häufig lediglich in momentanen Gedächtniswirkungen, ohne dabei eine "dauerhafte Spur" in der Psyche des Konsumenten zu hinterlassen. Darüber hinaus ist auch eine valide Messung im Rahmen der Unternehmenspraxis nur schwerlich möglich. Aufgrund der notwendigen Isolierung der Kommunikationswirkung gegenüber weiteren endogenen und exogenen Einflussfaktoren würde der Erhebungsaufwand übermäBig hoch sein, da laborähnliche Verhältnisse erforderlich wären. Aus diesem Grund sind dauerhafte Gedächtniswirkungen als Beurteilungsmaßstab der Effek-

$130 \mathrm{Vgl}$. Schlosser, A. E., Shavitt, S., Kanfer, A., Survey of Internet users' attitudes toward Internet advertising, in: Journal of Interactive Marketing, Vol. 13, No. 3, 1999, S. 1-21.

131 Vgl. Mayer, H., Illmann, T., Markt- und Werbepsychologie, a. a. O., S. 543.

132 Diamond/Noble analysierten Reaktanzerscheinungen infolge eines zu hohen Werbedrucks bei Direct Mail-Aktionen am Beispiel von Spendenorganisationen. Sie kommen zu dem Ergebnis, dass die Anzahl der empfangenen Mailings in der Spendenbranche positiv korreliert ist mit defensivem Verhalten gegenüber den Spendenaufforderungen. Vgl. Diamond, W. D., Noble, S. M., Defensive Responses to Charitable Direct Mail Solicitations, Journal of Interactive Marketing, Vol. 15, No. 3, 2001, S. 2-12. Dieses Ergebnis wird ebenfalls bestätigt durch eine Untersuchung von Farley/Harvey (ebenfalls im Versandhandel), im Rahmen derer eine negative Korrelation zwischen der Anzahl von versendeten Katalogen und dem Responseverhalten nachgewiesen werden konnte. Farley, J. U., Harvey, P. D., Assessing a "Real World" Mail Order Experiment, Current Issues \& Research in Advertising, Vol. 7, No. 1, 1984, S. 123-139. 
tivität des Direktmarketing nur eingeschränkt einsetzbar. Wenngleich eine selektive Steuerungskraft gegeben scheint, stellt sich jedoch die Frage, mit welcher Häufigkeit und Frequenz die einzelnen Direktmarketing-Stimuli eingesetzt werden müssten, um die gewünschte Veränderung dauerhafter Gedächtnisinhalte zu erreichen. Im Folgenden soll daher aufgrund der genannten Aspekte von dauerhaften Gedächtnisinhalten als Zielkategorie des Direktmarketing abstrahiert werden.

Gleichermaßen problematisch gestaltet sich die eindeutige Zuordnung von dauerhaften Verhaltenswirkungen zu einem vorausgehenden DirektmarketingStimulus. ${ }^{133}$ Trotz der offensichtlichen Vorteile der Effektivitătsmessung im Direktmarketing treten einige methodische Problemfelder der klassischen Kommunikationsforschung auch hier auf. So gestaltet sich die Zurechenbarkeit bei Überschneidung zweier oder mehrerer Direktmarketing-Stimuli insbesondere bei langfristigen Verhaltenswirkungen zunehmend kompliziert. ${ }^{134}$ Zudem wird das in längerer zeitlicher Distanz zum Direktmarketing-Stimulus liegende Kaufverhalten durch eine Reihe anderer Einflussfaktoren beeinflusst, die nicht primär einem einzelnen Direktmarketing-Stimulus zuzuordnen sind und deren Messung im Rahmen von Feldexperimenten - außer in langfristig angelegten Testmärkten nur schwerlich möglich ist. Ihr Wirkungsbeitrag wäre ebenso wie im Falle der dauerhaften Gedächtnisinhalte, wenn überhaupt, nur mit erheblichem Aufwand zu separieren. Aus diesem Grund werden dauerhafte Verhaltenswirkungen als Effektivitätsmaßstab des Direktmarketing-Einsatzes ebenfalls von der weiteren Betrachtung ausgeschlossen.

An dieser Stelle wird deutlich, dass im Kontext des Direktmarketing, nicht nur aufgrund der hohen Bedeutung in der Unternehmenspraxis, primär momentane Verhaltenswirkungen für die Beurteilung der Effektivität relevant sind. Insbesondere durch ihre zeitliche Nähe zum Kommunikationsakt ist bei dieser Verhaltenskategorie eine hohe Reagibilität gegeben. Die in vielen Fällen vorliegende Identität von Ansprache- und Rückkopplungskanal erleichtert hierbei die

133 Steffenhagen konstatiert in diesem Zusammenhang, dass insbesondere finale Verhaltenswirkungen auf vielfältige Beeinflussungsquellen zurückzuführen sind und daher der „Beeinflussungsbeitrag der Werbung“ i. d. R. nur schwer separiert werden kann. Vgl. Steffenhagen, H., Wirkungen der Werbung, a. a. O., S. $220 \mathrm{f}$.

134 Werden neben den Instrumenten des Direktmarketing bspw. auch klassische Kommunikationsinstrumente eingesetzt, erscheint es im Rahmen einer Effektivitätsbeurteilung notwendig, die durch die indirekte Kommunikation verursachten Verhaltenswirkungen von anderen Einflüssen zu eliminieren, was i. d. R. zu den bekannten Separierungsproblemen der klassischen Kommunikationsforschung fuhrt. Gleichzeitig besteht auch im Rahmen der Wirkungsforschung im Direktmarketing das Problem der Vielzahl potenzieller Einflussfaktoren auf den Wirkungsgrad der Direktmarketing-Instrumente sowie der Interdependenzen verschiedener Faktoren. 
Zuordnung von Direktmarketing-Stimulus zur Rezipientenreaktion und lässt damit sofortige Aussagen zur Effektivität von einzelnen Maßnahmen zu. ${ }^{135}$ Gleichzeitig besitzt ihre Messung im Direktmarketing den Vorteil, dass sowohl die Effektivität als auch die Effizienz einer Maßnahme in den meisten Fällen isoliert von anderen kommunikationspolitischen Aktivitäten und darüber hinaus auf kundenindividueller Basis analysiert werden können. ${ }^{136}$ Der Erhebungsaufwand ist aufgrund der genannten Vorteile als gering einzuschätzen; die Messung erfolgt zumeist valide.

\begin{tabular}{|c|c|c|c|c|}
\hline Wirkungs- $\begin{array}{c}\text { Anforderungs- } \\
\text { kriterien } \\
\text { kategorie }\end{array}$ & Reagibilität & $\begin{array}{l}\text { Selektive } \\
\text { Steuerungs- } \\
\text { kraft }\end{array}$ & $\begin{array}{l}\text { Valide } \\
\text { Messung }\end{array}$ & $\begin{array}{l}\text { Vertretbarer } \\
\text { Erhebungs- } \\
\text { aufwand }\end{array}$ \\
\hline $\begin{array}{l}\text { Momentane } \\
\text { Gedächtniswirkungen }\end{array}$ & ja & ja & nein & nein \\
\hline $\begin{array}{l}\text { Dauerhafte } \\
\text { Gedächtniswirkungen }\end{array}$ & nein & ja & nein & nein \\
\hline $\begin{array}{l}\text { Momentane } \\
\text { Verhaltenswirkungen }\end{array}$ & $\mathrm{ja}$ & ja & ja & ja \\
\hline $\begin{array}{l}\text { Dauerhafte } \\
\text { Verhaltenswirkungen }\end{array}$ & nein & nein & $\mathrm{Ja}$ & nein \\
\hline
\end{tabular}

Abb. 4: Tauglichkeitsprüfung potenzieller Wirkungskategorien des DirektmarketingEinsatzes

(Quelle: in Anlehnung an Bruhn, M., Kommunikationspolitik, a. a. O., S. 245; Lasslop, I., Effektivität und Effizienz von Events, a. a. O., S. 58.)

Momentane Verhaltenswirkungen werden insbesondere durch momentane Gedächtniswirkungen hervorgerufen, die sich allerdings nur schwer in Feldexperimenten messen lassen. Eine valide Messung erfordert theoretisch eine Erhebung während der Kontaktsituation, was technisch durchaus möglich erscheint, allerdings mit erheblichem Aufwand verbunden ist. $\mathrm{Zu}$ einer Messung bedarf es i. d. R. einer laborähnlichen Untersuchungssituation, wenn nicht auf eine direkte Befragung des Rezipienten nach dem Kommunikationsakt zurückgegriffen werden kann, welche ebenfalls mit Messproblemen behaftet sein kann. Aufgrund der skizzierten Problembereiche soll im weiteren Verlauf auf momentane Gedächt-

135 Vgl. Holland, H., Direktmarketing, 2. Aufl., München 2004, S. 19; Schaller, G., Organisation der Erfolgskontrolle im Direct Marketing, in: Dallmer, H. (Hrsg.), Handbuch Direct Marketing, 7. Aufl., Wiesbaden 1997, S. 580; Fraser-Robinson, J., The Secret of Effective Direct Mail, London 1989, S. 3 f.

136 Vgl. Kehl, R. E., Controlling mit Database Marketing - Effizienzmessung absatzpolitischer Instrumente, Ettlingen 2000, S. $254 \mathrm{ff}$. 
niswirkungen nur als Vorstufe der momentanen Verhaltensreaktionen eingegangen werden. Letztere bilden damit die zentralen Zielwirkungen der vorliegenden Arbeit (vgl. Abb. 4).

\subsection{Ansatzpunkte der Erfolgsmessung im Direktmarketing}

\subsubsection{Effektivitätsmessung als Ausgangspunkt der Erfolgsbeurteilung}

Vor dem Hintergrund der Zielsetzung der vorliegenden Arbeit soll eine Systematisierung der für die Effektivitätsmessung relevanten Kennzahlen in Anlehnung an den Wirkungsprozess des Direktmarketing erfolgen, in dessen Zentrum die zur Erfassung der momentanen Verhaltenswirkungen identifizierten Effektivitätskennzahlen stehen. Aufgrund der komplexen Wirkungszusammenhänge erscheint es daher sinnvoll, den Wirkungsprozess weiter zu verfeinern und an verschiedenen Stellen die momentanen Gedächtniswirkungen in die Untersuchung mit einzubeziehen.

Notwendige Bedingung für das Eintreten jeglicher Art von Wirkung durch das Direktmarketing ist ein Kontakt zwischen Direktmarketing-Stimulus und Rezipient. ${ }^{137}$ I. d. R. wird angenommen, dass eine Zielperson mit einem Direktmarketing-Stimulus in Kontakt gekommen ist, wenn der Versand des Direktmarketing-Stimulus aus Sicht des Unternehmens erfolgreich vollzogen wurde. ${ }^{138}$ Wenngleich der erfolgreiche Versand bzw. eine erfolgreiche Zustellung des Direktmarketing-Stimulus noch nicht mit einem Rezipientenkontakt gleichzusetzen ist, besteht dennoch eine hohe Wahrscheinlichkeit eines Kontaktes zwischen der adressierten Zielperson und dem Direktmarketing-Stimulus. Das Verhältnis der

137 Dallmer konstatiert in diesem Zusammenhang, dass es zunächst das Ziel einer jeden Direktmarketing-Kampagne ist, „Zielgruppen zu erreichen bzw. einen direkten Kontakt herzustellen“. Dallmer, H., Das System des Direct Marketing - Entwicklungsfaktoren und Trends, in: Dallmer, H. (Hrsg.), Das Handbuch Direct Marketing \& More, a. a. O., S. 11.

138 Probleme im Rahmen der Kontaktschaffung entstehen bspw. dann, wenn aufgrund einer fehlerhaften Adresse der potenzielle Rezipient des Stimulus nicht erreicht werden kann oder sich seine Adressdaten nach einmaliger Erfassung durch das Unternehmen geändert haben. $\mathrm{Zu}$ den typischen kundenbezogenen Fehlerquellen im Rahmen der Adresseingabe im Internet, die sich auch auf den Offlinebereich übertragen lassen, zählen bspw. die Eingabe von Phantasieadressen oder unvollständigen Adressen sowie die unbewusste Eingabe von falschen Daten aufgrund mangelnder Sorgfalt. Vgl. hierzu bspw. Bachmann, M., Adressvalidierung und Adressanreicherung via Internet, in: Dallmer, H. (Hrsg.), Das Handbuch Direct Marketing \& More, a. a. O., S. $676 \mathrm{f}$. 
erfolgreich zugestellten Direktmarketing-Stimuli im Vergleich zur Anzahl der versendeten Direktmarketing-Stimuli kann durch die sog. Zustellquote erfasst werden. Diese stellt damit einen ersten Indikator für die Effektivität des Direktmarketing-Einsatzes im Hinblick auf die Kontaktchancen mit den Rezipienten dar. ${ }^{139}$

Ist der Direktmarketing-Stimulus in den Wahrnehmungsbereich des Rezipienten eingedrungen, können aufseiten des Rezipienten verschiedene emotionale und kognitive Verarbeitungsprozesse ausgelöst werden, aus denen eine spontane Verhaltenswirkung resultieren kann. Zur Vereinfachung der Darstellung dieses Wirkungszusammenhangs wird häufig auf zeitlich aufeinander aufbauende Wirkungsstufen zurückgegriffen. ${ }^{140}$ So erfolgt in der präkommunikativen Phase, d. h. vor Beginn der eigentlichen psychischen Verarbeitungsprozesse, zunächst die Selektion des Werbemediums und damit auch der Werbebotschaft. ${ }^{141}$ In dieser Phase entscheidet der Rezipient, ob er das Werbemedium zu seiner Informationsaufnahme heranzieht und die inhaltlichen Aussagen zur Kenntnis nimmt. ${ }^{142}$

139 Eine Berechnung erfolgt durch die Subtraktion der nicht zugestellten Werbesendungen von der Gesamtzahl der ausgesendeten Stimuli und die anschließende Division durch die Anzahl der versandten Stimuli. Im Fall der elektronischen Medien kann dies bspw. durch die Auswertung der Fehlerprotokolle der Email- und SMS-Server erfolgen; im Rahmen der physischen Trägermedien erfolgt zumeist eine direkte Rücksendung fehlerhaft adressierter Stimuli. Durch die Möglichkeiten des Datenabgleichs sowie den sich anschließenden Plausibilitätschecks bei freiwilliger Angabe der Adresse, Email-Adresse und Handy-Nummer bei potenziellen Kunden lassen sich heutzutage vor allem bei adressierten Werbesendungen fehlerhafte Adressen frühzeitig erkennen und ggf. die Zustellquote verbessern. In diesem Zusammenhang können Unternehmen auf eine Vielzahl von Dienstleistungsanbietern zur Adressvalidierung und -aktualisierung zurückgreifen.

140 In Anlehnung an die klassische Werbewirkungsforschung werden bspw. Wirkungsmodelle wie das AIDA-Modell von Lewis verwendet. Eine umfassende Darstellung der klassischen Kommunikationsmodelle findet sich bei Bruhn, M., Kommunikationspolitik, a. a. O., S. 27. Aufgrund der Heterogenität der Instrumente lassen sich in der direktmarketing-spezifischen Literatur aber auch instrumentespezifische Wirkungsmodelle finden. Vgl. für Postwurfsendungen Vriens, M. et al., Conjoint Experiments for Direct Mail Response Optimization, in: European Journal of Marketing, Vol. 32, No. 3, S. 323-339, sowie für Emails Chittenden, L., Rettie, R., An evaluation of e-mail marketing and factors affecting response, in: Journal of Targeting, Measurement and Analysis for Marketing, Vol. 11, No. 3, S. 203-217.

$141 \mathrm{Vgl}$. Ronneberger, F., Sozialisation durch Massenkommunikation, in: Ronneberger, F. (Hrsg.), Sozialisation durch Massenkommunikation - Der Mensch als soziales und personales Wesen (Band IV), Stuttgart 1971, S. 73. Diese Phase wird häufig auch als Selektionsphase bezeichnet.

142 Vgl. Bamler, G., Zur Wirkung von Direkt-Werbung im Industriegüterbereich, a. a. O., S. $73 \mathrm{ff}$. In diesem Kontext entscheidet der Rezipient implizit auf Basis des ökonomischen Prinzips, welche Informationen er zu seiner Information heranzieht. 
In diesem Kontext wird häufig die Öffnungsquote von Mailings, Werbe-Emails oder SMS als weiterer Indikator für die Effektivität der unterschiedlichen Trägermedien i.S. einer erzielten Wirkung beim Rezipienten angesehen. ${ }^{143}$ Die Messung dieser Kennzahl gestaltet sich aus Sicht der Praxis gleichsam aufwendig und ist daher zumeist an ein experimentelles Umfeld gebunden. ${ }^{144}$

Erst im Anschluss an die Öffnung des Werbemediums erfolgt in der sog. kommunikativen Phase eine Wahrnehmung und Verarbeitung der Werbebotschaft. ${ }^{145}$ Der Verarbeitung vorgelagert ist die Wahrnehmung des Direktmarketing-Stimulus, die ihrerseits primär durch die Aktivierung und Aufmerksamkeit des Rezipienten während des Werbekontaktes determiniert wird. ${ }^{146}$ Diese Verarbeitungsstufe beinhaltet auf Seiten des Rezipienten zumeist eine Verständnis- und

Vgl. Schramm, W., The nature of communication between humans, in: Schramm, W., Roberts, P. (Hrsg.), The process and effects of mass communication, Urbana u. a. 1971, S. 3 ff. und S. 32. Die Gefahr einer bewussten Selektion besteht mittlerweile insbesondere bei Emails darin, dass die Werbung gelöscht wird. In einigen Untersuchungen stellte sich heraus, dass die Öffnungsrate bei Emails mittlerweile unter $50 \%$ liegt. In diesem Zusammenhang wird häufig auf die unbewusste Selektion von Werbemitteln hingewiesen, die zum Wegwerfen bzw. Löschen von DirektmarketingStimuli bereits vor Öffnung führen kann. Eine unbewusste Selektion im Rahmen des Direktmarketing würde bspw. dann vorliegen, wenn der Rezipient den Direktmarketing-Stimulus nicht explizit lesen möchte, aber die Werbeaussage bzw. den Absendernamen zur Kenntnis nimmt.

143 Eine Öffnungsquote lässt sich nur bei denjenigen Direktmarketing-Instrumenten ermitteln, die vor einer Auseinandersetzung mit den Inhalten aktiv vom Rezipienten physisch oder elektronisch geöffnet werden müssen. Dies ist z. B. bei Mailings, Emails und SMS der Fall.

144 Im Hinblick auf Mailings wird die Öffnungsquote bspw. im Rahmen des GfK Direktmarketing Panels über die sog. Attention-Rate gemessen. Wăhrend die AttentionRate der hier beschrieben Öffnungsquote entspricht, bezeichnet die Trash-Rate den Anteil an Mailings, der bereits vor dem Öffnen weggeworfen wird. Die Höhe der Trash-Rate liegt im Branchendurchschnitt bei $20 \%$. Vgl. GfK (Hrsg.), GfK Direktmarketing Panel, in: GfK Newsletter 02/2003, Nürnberg 2003.

145 Vgl. Hermanns, A., Konsument und Werbewirkung - Das phasenorientierte Werbewirkungsmodell, Band 14 der Schriftenreihe: Schwerpunkt Marketing, Bielefeld, Köln 1979, S. 92; Kaiser, A., Werbung - Theorie und Praxis werblicher Beeinflussung, München 1980, S. $86 \mathrm{f}$.

146 Vgl. Steffenhagen, H., Wirkungen der Werbung. Konzepte - Erklärungen - Befunde, a. a. O., S. $47 \mathrm{ff}$. Die Analyse der Wahrnehmung von Direktmarketing-Werbung wird seit Jahrzehnten in Experimenten untersucht. Interessant erscheint hierbei insbesondere die Frage nach dem Blickverlauf der Augen, wenn der Rezipient die Werbung das erste Mal wahrnimmt. Zu den Pionieren auf dem Gebiet der Blickaufzeichnung zählt Vögele. Vgl. Vögele, S., Bidmon, R. K., Psychologische Aspekte der Dialogmethode, in: Dallmer, H. (Hrsg.), Das Handbuch Direct Marketing \& More, a. a. O., S. 444. 
Bewertungsdimension, in der Inhalte des Direktmarketing-Stimulus zur Kenntnis genommen und ggf. Leistungsangebote bewertet werden. ${ }^{147}$ Als momentane Wirkungen des Werbekontaktes können hieraus bspw. momentane Kenntnisse bzw. momentanes Wissen bis hin zu momentanen Verhaltensbereitschaften resultieren. ${ }^{148}$ Die Kenntnis der psychologischen Verarbeitungsprozesse ist für viele Unternehmen interessant, eine Messung erscheint aber aufgrund eines hohen Erhebungsaufwandes ökonomisch in den meisten Fällen nicht sinnvoll. ${ }^{149}$ Daher wird die Erfassung emotionaler und kognitiver Prozesse während der Wahrnehmung und Verarbeitung von Werbebotschaften vielfach im Rahmen von Laborexperimenten oder mittels nachträglicher Befragung vorgenommen. ${ }^{150}$

Nach Selektion, Wahrnehmung und Verarbeitung erfolgt in der sog. postkommunikativen Phase die beim Rezipienten zu beobachtende Verhaltenswirkung auf den Direktmarketing-Stimulus. ${ }^{151}$ Die Bandbreite möglicher Reaktionen reicht hierbei von keiner Reaktion bis zu direkten Spontanhandlungen wie schriftlichem oder mündlichem Response- oder Kaufverhalten. Je nach Ausgestaltung des Responsekanals können diese Reaktionen zeitnah oder zeitversetzt erfolgen. ${ }^{152}$ Unabhängig vom Responsekanal dienen für eine Einschätzung der Effektivität der versendeten Direktmarketing-Stimuli sog. Responsequoten. ${ }^{153}$ Responsequoten bezeichnen das Verhältnis der Anzahl der Kundenreaktionen zur Anzahl der ausgesandten Stimuli. ${ }^{154}$ Sie finden als Kontrollinstrument insbe-

147 In diesem Zusammenhang verwendet Bamler die Begriffe der Perzeption (Aufnahme und Identifizierung von Kommunikationssignalen) und der Apperzeption (Verstehensprozess). Vgl. Bamler, G., Zur Wirkung von Direkt-Werbung im Industriegüterbereich, a. a. O., S. $125 \mathrm{f}$. und $134 \mathrm{ff}$.

$148 \mathrm{Vgl}$. Steffenhagen, H., Wirkungen der Werbung, a. a. O., S. $64 \mathrm{ff}$.

149 Die Veränderung einer psychographischen Erfolgsgröße lässt sich in den meisten Fällen nur schwer vom Einfluss anderer Maßnahmen, wie bspw. TV-Werbung, separieren. Insbesondere in der Direktmarketing-Forschung lassen sich primär verhaltensorientierte Reaktionsmessungen finden. Vgl. Bruhn, M., Kommunikationspolitik, a. a. O., S. 537.

150 In diesem Kontext kommen bspw. apparative Messmethoden wie die Blickaufzeichnungskamera zum Einsatz. Vgl. Vögele, S., Bidmon, R. K., Psychologische Aspekte der Dialogmethode, a. a. O., S. 435-457.

151 Vgl. Bamler, G., Zur Wirkung von Direkt-Werbung im Industriegüterbereich, a. a. O., S. 59.

$152 \mathrm{Vgl}$. bspw. Holland, H., Direktmarketing, München 1992, S. $120 \mathrm{ff}$.

153 Vgl. Holland, H., Direktmarketing, 2. Aufl., München 2004, S. 361; Elsner, R., Optimiertes Direkt- und Databasemarketing unter Einsatz mehrstufiger dynamischer Modelle, Wiesbaden 2003, S. 75.

154 Mit Hilfe einer sog. Eingangsstatistik kann neben der absoluten Höhe des Rücklaufs zusätzlich aufgezeichnet werden, wann welcher Empfänger in welcher Art und Weise auf den Direktmarketing-Stimulus reagiert hat. Damit erhalten Unternehmen nicht nur Hinweise über das Reaktionsverhalten der Zielgruppe, sondern auch über den 
sondere im Rahmen von Mailing-Aktionen, Zeitungsbeilagen mit Antwortkarte und Coupon-Aktionen, Emails und SMS Anwendung. ${ }^{155}$ Die Berechnung erfolgt je nach Art des Response mittels manueller Erhebungen bzw. im Fall elektronischer Medien zumeist aufgrund automatisierter Responsemessungen. ${ }^{156}$

Das Responseverhalten des Rezipienten in Form von Informationsanfragen an das Unternehmen kann zum einen als finale Zielgröße des DirektmarketingEinsatzes angesehen werden. Zum anderen kann das dem eigentlichen Kaufakt vorgelagerte Responseverhalten des Rezipienten auch nur eine notwendige Voraussetzung für die Erreichung weiterer verhaltensorientierter Ziele darstellen und

zeitlichen Aufwand, der für die Beantwortung erforderlich ist. In diesem Zusammenhang wird häufig auch die sog. Halbwertszeit einer Direktmarketing-Maßnahme ermittelt. Die Halbwertszeit bezeichnet die Zeit, nach der $50 \%$ der Reaktionen eingegangen sind. Die Kenntnis der Halbwertszeit ist vor allem für die Prognose der Reaktionen von ähnlichen Aktionen von Interesse. Vgl. Kairies, P., Direktmarketing für technische Produkte und Dienstleistungen: mit neuen Methoden effizient Kunden finden und binden, Renningen-Malmsheim 2001, S. 141; Holland, H., Direktmarketing, 2. Aufl., München 2004, S. 362. Rücklaufquoten können auch im Rahmen mehrstufiger Mailings eingesetzt werden. Vgl. Ceyp, M., Erfolgskontrollen im Direktmarketing, in: Direkt Marketing. Zeitschrift für Dialogmarketing und Integrierte Kommunikation, Nr. 4, 2002, S. 26-31.

155 Vgl. Greff, G., Telefonmarketing und Call-Center, in: Dallmer, H. (Hrsg.), Das Handbuch Direct Marketing \& More, a. a. O., S. 255. I. d. R. wird die Responsequote ex-post gemessen. Seit geraumer Zeit beschäftigen sich aber immer mehr Ansätze mit der Vorhersage von Responsequoten beim Einsatz unterschiedlicher Direktmarketing-Instrumente. Vgl. Levin, N., Zahavi, J., Continous predictive modeling, in: Journal of Interactive Marketing, Vol. 12, No. 2, 1998, S. 5-22. In den letzten Jahren haben sich die Anwendungsbereiche der Responsemessungen kontinuierlich auf die neuen Medien, bspw. auf den Bereich von Emails, ausgeweitet. Auch bei SMS kann der Response in Form eines Anrufes einer in der SMS angegebenen Telefonnummer als Reaktionsquote direkt gemessen werden. Vgl. Hinrichs, Ch., Lippert, I., Kosten und Wirkungen mobiler Werbung, in: Silberer, G., Wohlfahrt, J., Wilhelm, T. (Hrsg.), Mobile Commerce. Grundlagen, Geschäftsmodelle, Erfolgsfaktoren, Wiesbaden 2002, S. $265 \mathrm{ff}$.

$156 \mathrm{Zu}$ den typischen Informationsquellen zählen in diesem Zusammenhang Analysen von Logfiles, Session-IDs, Cookies, Logins, Emails, Formulareinträgen und Transaktionsdaten. Vgl. Bachem, Ch., Marketingcontrolling im E-Commerce, in: Reinecke, S., Tomczak, T., Geis, G. (Hrsg.), Handbuch Marketingcontrolling: Marketing als Motor für Wachstum und Erfolg, a. a. O., S. 568 ff.; Striegl, T., Effizientes Direktmarketing - Mit der richtigen Email-Strategie Absatz fördern, Kunden binden, Kosten senken, Bonn 2003, S. 154 ff. Mit Hilfe eines Datenbanksystems und entsprechender Auswertungssoftware lassen sich diese Daten nicht nur nutzen, um den Erfolg einzelner Maßnahmen oder Aktivitäten beurteilen zu können, sondern die gewonnenen Informationen können ebenfalls zur Gestaltung zukünftiger Maßnahmen genutzt werden. Vgl. Roberts, M. L., Expanding the role of direct marketing database, in: Journal of Interactive Marketing, Vol. 11, No. 4, 1997, S. 26-35. 


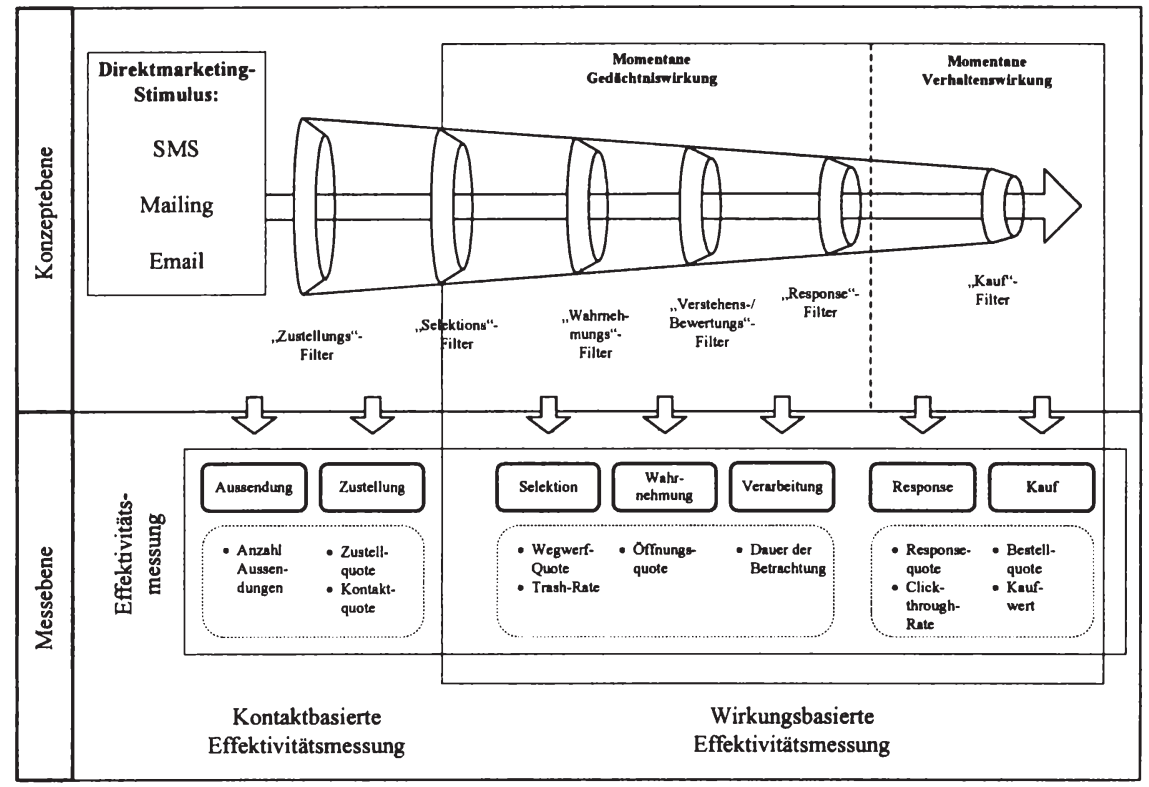

\section{Abb. 5: Kontakt- und wirkungsbasierte Effektivitätsmessung im Direktmarketing}

wird damit als modale Zielgröße interpretiert. ${ }^{157}$ Um den ökonomischen Erfolg einer Direktmarketing-Aktion beurteilen zu können, ist daher neben der Auswertung der Responsequote eine Erhebung der dem Versand der DirektmarketingStimuli direkt zuzuordnenden Transaktionen nach Anzahl und Wert vorzunehmen. ${ }^{158}$ Die Bestell- bzw. Kaufquote drückt dabei aus, wie viele Personen im Vergleich zur Anzahl der angeschriebenen Personen ein konkretes Angebot erworben haben. ${ }^{159}$

157 Bei beratungsintensiven Kaufprozessen wird häufig auf ein Call-Center zur Bestellentgegennahme zurückgegriffen. In diesem Zusammenhang stellt der Response eine notwendige Voraussetzung für den Kauf des Produktes oder der Dienstleistung dar. Ein Kaufakt ist jedoch nicht zwangsläufig die Folge eines Anrufes. So können bspw. lediglich Informationen zu den angebotenen Produkten oder Dienstleistungen abgefragt werden.

158 Vgl. Gerth, N., Die Bedeutung des Online Marketing für die Distributionspolitik, in: Link, J. (Hrsg.), Wettbewerbsvorteile durch Online Marketing. Die strategischen Perspektiven elektronischer Märkte, 2. Aufl., Berlin, Heidelberg, New York 2000, S. 181 ff. Der Autor bezieht seine Ausführungen primär auf den Bereich des ECommerce.

159 Das Verhältnis zwischen zwei Effektivitätskennzahlen, bspw. zwischen der Response- und der Kaufquote, kann mit Hilfe sog. Umwandlungsquoten (hier: von Reagie- 
Die dargestellten Effektivitätskennzahlen lassen sich zusammenfassend in einem Teilmodell darstellen, welches sich auf die momentanen Verhaltenswirkungen sowie die vorgelagerten momentanen Gedächtniswirkungen konzentriert. ${ }^{160}$ Hierbei lassen sich eine kontakt- und eine wirkungsbasierte Effektivitätsmessung voneinander unterscheiden (vgl. Abb. 5). ${ }^{161}$

\subsubsection{Effizienzmessung als komplementäres Element der Erfolgsbeurteilung}

In der wissenschaftlichen Literatur zur Erfolgsbeurteilung von Marketing-Aktivitäten wird in vielen Fällen die Meinung vertreten, dass eine umfassende Erfolgsbeurteilung des Einsatzes der Marketing-Instrumente nur durch die gleichzeitige Betrachtung der Effektivität und des für die Erreichung der gewünschten Ziele notwendigen Aufwands möglich ist. ${ }^{162}$ Die für die Veränderung der Zielgrößen eingesetzten Ressourcen bestimmen die Effizienz des DirektmarketingEinsatzes. ${ }^{163}$ Vor dem Hintergrund des ökonomischen Prinzips ist es daher auch generisches Ziel des Direktmarketing, die aktive Gestaltung der Marktbeziehungen so effizient wie möglich vorzunehmen. ${ }^{164}$ Von Effizienz kann daher im Kontext des Direktmarketing auch nur dann gesprochen werden, wenn es zu einem gegebenen Output/Input-Verhältnis einer Direktmarketingmaßnahme keine alternative Maßnahme gibt, die über ein besseres Verhältnis verfügt. ${ }^{165}$ Durch die

rern zu Kunden) ausgedrückt werden. Vgl. Belz, C., Strategisches Direktmarketing, a. a. O., S. 52.

160 Im Rahmen des dargestellten Modells der Untersuchung werden die momentanen Gedächtniswirkungen als „Filter“ angesehen, die es auf dem Weg zur gewünschten Wirkung zu passieren gilt. Vgl. hierzu bspw. Broadbent, D. E., Perception and communication, London 1958.

161 Wie in Abb. 5 dargestellt, bezieht sich die kontaktbasierte Effektivitätsmessung auf die Effektivität eines Direktmarketing-Stimulus vor dem Kontakt bzw. auf den Kontakt selbst. Die wirkungsbasierte Effektivitätsmessung setzt ihrerseits an den momentanen Gedächtnis- und Verhaltenswirkungen an.

$162 \mathrm{Vgl}$. Lasslop, I., Effektivität und Effizienz von Events, a. a. O., S. 13.

$163 \mathrm{Zu}$ trennen ist der hier verwendete Begriff der instrumentellen Effizienzanalyse (Effizienzkontrolle auf instrumenteller Ebene) von einer häufig als inhaltsorientierten Effizienzanalyse bezeichneten Begriff, dem sog. Marketing-Audit, das allerdings i. d. R das marktbezogene Führungssystem zum Untersuchungsgegenstand hat.

164 Vgl. Kehl, R. E., Controlling mit Database Marketing, a. a. O., S. 247.

165 Als geeignete Vergleichsobjekte erweisen sich i. d. R. andere DirektmarketingAktivitäten des Unternehmens, die sich im Hinblick auf die jeweils zu untersuchende endogene Determinante unterscheiden. Der Schwerpunkt der Effizienzvergleiche liegt hierbei auf den kommunikationspolitischen Aktivitäten des Unternehmens zumeist auf einem Vergleich unterschiedlicher Instrumente. 
gleichzeitige Messung der Effektivität und Effizienz kann somit eine umfassende Erfolgsbeurteilung der Direktmarketing-Maßnahme erfolgen. ${ }^{166}$

Zur Bestimmung der im Rahmen der Effizienzbewertung heranzuziehenden Inputgrößen gilt es, die Kosten sämtlicher Sach- und Dienstleistungen, die zur Planung und Durchführung einer Direktmarketing-Aktion sowie zur abwicklungsbezogenen Kommunikation mit dem Rezipienten anfallen, systematisch zu erfassen. Im Einzelnen lassen sich im Rahmen des mit dem DirektmarketingEinsatz verbundenen Ressourcenverbrauchs verschiedene Kostenkategorien identifizieren: ${ }^{167}$

- Unter die sog. Konzeptionierungskosten fallen sämtliche Kosten der Rahmenplanung des Direktmarketing-Einsatzes hinsichtlich der Auswahl der Zielgruppe, der Sortiments- und der Werbeplanung sowie der SituationsAnalyse vor dem Einsatz des Direktmarketing. ${ }^{168}$ Zumeist bilden hierbei Personalkosten den Hauptbestandteil dieser Kostenkategorie.

- Neben den Konzeptionierungskosten fallen im Vorfeld einer Direktmarketing-Aktion ggf. Kosten für die Zusammenstellung von Informationen an, wie bspw. für die Zusammenstellung der anzuschreibenden Adressen oder zur Konfiguration individualisierter Angebotsbündel. Diese Informationskosten betreffen sowohl Kosten im Rahmen der Aufbereitung von Bestandskundendaten (z. B. in Form von Personalkosten) als auch für den Zukauf von Adressen zur Neukundenakquisition (z. B. beim Kauf von Fremdlisten bei einem Adressbroker).

- Die Werbemittelerstellung erfordert zumeist Investitionen im Bereich der Layoutgestaltung, der Reproduktion etc. und wird unternehmensintern oder durch spezialisierte Dienstleister erbracht. ${ }^{169}$ Dieser Teilbereich der sog. Werbemittelkosten wird ggf. ergänzt Reproduktionskosten. ${ }^{170}$

166 Eine Direktmarketing-Maßnahme kann damit als erfolgreich bezeichnet werden, wenn sie sowohl effektiv i. e. S. ist und der dafür notwendige Ressourceneinsatz gleichermaßen effizient war. Vgl. zur Diskussion dieser Begriffsdefinition u. a. Lasslop, I., Effektivität und Effizienz von Events, a. a. O., S. 9 ff.

167 Zur instrumentespezifischen Darstellung potenzieller Kostenblöcke können die Arbeiten verschiedener Autoren herangezogen werden. Für eine vergleichende Darstellung unterschiedlicher Kostenarten im Bereich von Mailings und Telefonmarketing vgl. u. a. Hölscher, U., Kalkulation einer Direktwerbe-Aktion, in: Dallmer, H. (Hrsg.), Das Handbuch Direct Marketing \& More, a. a. O., S. 459 ff.

168 Vgl. Kehl, R. E., Controlling mit Database Marketing, a. a. O., S. 66 f.

169 Vgl. bspw. für eine Wertschöpfungskette im internetbasierten Direktmarketing inkl. der outgesourcten Wertschöpfungsstufen Meffert, H., Direct Marketing und marktorientierte Unternehmensführung, a. a. O., S. $49 \mathrm{f}$.

170 Dieser Schritt entfällt bei den elektronischen Medien Email und SMS. 
- Versandbezogene Abwicklungskosten entstehen insbesondere bei der Versendung physischer Trägermedien, wie Mailing oder Coupons. Im Falle der elektronischen Medien lassen sich zwar noch deutliche Kostenunterschiede bspw. zwischen Email und SMS verzeichnen, die absolut auftretenden Kosten sind aber im Vergleich zu einer postalischen Versendung relativ gering.

- Letztendlich können in Abhängigkeit der angebotenen Produkte und Dienstleistungen des Unternehmens unterschiedlich hohe kundenbezogene Abwicklungskosten entstehen. Hierunter werden i. d. R. sämtliche Kostenblöcke subsumiert, die im Rahmen der Auftragsabwicklung sowie im Bereich von Informationsanfragen anfallen.

Die folgende Tabelle fasst die wichtigsten Kostenkategorien auf Basis der zugrunde liegenden Prozesse zu unterschiedlichen Kategorien zusammen (vgl. Tab. 2): ${ }^{171}$

\begin{tabular}{|l|l|l|}
\hline Kostenkategorie & Sub-Kategorie & Prozesse \\
\hline \multirow{4}{*}{$\begin{array}{l}\text { Konzeptionierungs- } \\
\text { kosten }\end{array}$} & Ist-Analyse & Analyse der Ausgangssituation \\
\cline { 2 - 3 } & Rahmenplanung & $\begin{array}{l}\text { Kunden-/Zielgruppenplanung, Ange- } \\
\text { botsplanung, Werbeplanung etc. }\end{array}$ \\
\hline \multirow{4}{*}{ Informationskosten } & Datenbankeinrichtung & Software, Hardware, Wartung etc. \\
\cline { 2 - 3 } & Informationserlangung & $\begin{array}{l}\text { Kauf von Fremdlisten, Formatierung, } \\
\text { Konvertierung, Dublettenabgleich etc. }\end{array}$ \\
\cline { 2 - 3 } & Datenbankpflege & Adresserfassung, Adressänderung etc. \\
\hline \multirow{3}{*}{$\begin{array}{l}\text { Versandbezogene } \\
\text { Abwicklungskosten }\end{array}$} & Werbemittelerstellung & $\begin{array}{l}\text { Layout, Fotographie, Text, Fotosatz, } \\
\text { Reproduktion etc. }\end{array}$ \\
\cline { 2 - 3 } & Vervielfältigung & $\begin{array}{l}\text { Druckabwicklung, Druck (Katalog, } \\
\text { Hülle, Karte usw.), Verarbeitung etc. }\end{array}$ \\
\cline { 2 - 3 } $\begin{array}{l}\text { Kundenbezogene } \\
\text { Abwicklungskosten }\end{array}$ & Auftragsbestätigung & $\begin{array}{l}\text { Packmittel, Kuvertieren, Portokosten, } \\
\text { Versandabwicklung etc. }\end{array}$ \\
\cline { 2 - 3 } & Aackmittel, Rechungsabwicklung etc. \\
\cline { 2 - 3 } & Kundenbetreuung & $\begin{array}{l}\text { Auftragsannahme (telefonisch/ schrift- } \\
\text { lich), Rechungsstellung etc. } \\
\text { telefonischer Anfragen, Mahnwesen } \\
\text { etc. }\end{array}$ \\
\hline
\end{tabular}

Tab. 2: Direktmarketingspezifische Kostenkategorien

(Quelle: in Anlehnung an Kehl, R. E., Erfolgsmessung im Direktmarketing, a. a. O., S. $66 f$.)

171 Nicht mit einbezogen in die Betrachtung der Effizienzbeurteilung werden an dieser Stelle die das konkret beworbene Produkt oder die Dienstleistung betreffenden Produktionskosten, da sie in keinem ursächlichen Verhältnis zum DirektmarketingEinsatz stehen. 
Die in der vorliegenden Tabelle enthaltenen Kostenblöcke werden je nach Art der betrachteten Kennzahl bei einer Erfolgsbeurteilung mit in die Berechnung einbezogen oder nicht. Die Höhe der einbezogenen Kosten richtet sich wiederum nach der Art der Schlüsselung. Auf Basis des dargestellten Wirkungsprozesses lassen sich den aufgezeigten Effektivitätskennzahlen entsprechende Effizienzkennzahlen gegenüberstellen. So beschreibt die Kennzahl „Kosten pro Kontakt" (CPC: Cost per contact) die Effizienz eines Direktmarketing-Instruments hinsichtlich des Ziels „Kontaktschaffung“. ${ }^{172}$ Eine Berechung erfolgt durch die Belegung der Zustellquote mit den angefallenen Kosten. ${ }^{173}$ Im Rahmen der Berechnung werden die vor Versendung der Direktmarketing-Stimuli angefallenen Kosten sowie die Versandkosten zur Anzahl der erreichten Personen ins Verhältnis gesetzt.

Wird dieser Kostenblock um die für die Entgegennahme der Reaktionen der Rezipienten notwendigen Kosten, bspw. durch Betreiben eines Call-Centers, ergänzt und zur Anzahl der gewonnenen Interessenten bzw. der Reagierer ins Verhältnis gesetzt, lassen sich Kennzahlen, wie Kosten pro Interessent oder Reagierer (CPI: cost per interest), berechnen. ${ }^{174}$ Unabhängig von der Höhe der getätigten Umsätze beleuchten Kennzahlen wie Kosten pro Auftrag (CPO: cost per order) die Effizienz der Auftragsgewinnung des jeweiligen Direktmarketing-

172 Vgl. Pepels, W., Marketing, München 1996, S. 598; Ruland, J., Werbeträger: Einführung in die Praxis des Werbeträgereinsatzes, Bad Homburg 1972, S. 19. Die Kosten variieren in Abhängigkeit der eingesetzten Instrumente in erheblichem Maße. Während bspw. 1.000 ausgehende Telefonanrufe im Rahmen des Telefonmarketing (Outbound Calls) bis zu 6.000 Englische Pfund kosten, liegen die Tausenderkontaktpreise für Emails bei unter 2 Pfund. Vgl. Tapp, A., Principles of Direct and Database Marketing, 2nd edition, Harlow, Essex 2000, S. 278. Beim Vergleich der Medien fällt hierbei auf, dass insbesondere Emails und SMS wegen ihrer geringen Versandkosten Mailings und Telefon-Aktionen überlegen scheinen. Vgl. Hinrichs, Ch., Lippert, I., Kosten und Wirkungen mobiler Werbung, a. a. O., S. 273.

173 In diesem Zusammenhang wird häufig die Frage diskutiert, ob im Rahmen der Berechnung lediglich bis zu diesem Zeitpunkt angefallene Kostenblöcke Berücksichtigung finden dürfen oder auch Kosten, die mit der Abwicklung und dem Management des Responses in Verbindung stehen. In der vorliegenden Arbeit wird der erstgenannten Meinung gefolgt.

174 In diesem Zusammenhang lässt sich eine Vielzahl von Kostenarten identifizieren, die im Rahmen des Einsatzes der unterschiedlichen Direktmarketing-Instrumente für die Erfolgskontrolle Relevanz besitzen. Eine detaillierte Auflistung von Kostenarten, die im Zusammenhang mit Mailings anfallen, liefert Hölscher. Vgl. Hölscher, U., Entscheidungsprozess bei der Anmietung von Adressen im Business-Bereich, in: Dallmer, H. (Hrsg.), Das Handbuch Direct Marketing \& More, a. a. O., S. 559-568; Geller, L. K., Response. Die unbegrenzten Möglichkeiten des Direktmarketing, Landsberg/Lech 1997, S. 191. 
Instrumentes bzw. einer Aktion. ${ }^{175}$ Im Rahmen der Berechnung werden die Kosten für eine Aussendung sowie die Auftragsentgegennahme mit der Anzahl der aus dieser Maßnahme generierten Aufträge bzw. Bestellungen dividiert. ${ }^{176}$ Eine weitere kostenorientierte Kennzahl stellt hierbei die Kennzahl Kosten pro Neukunde (CPC: cost per customer) dar, bei der zusätzlich zu den Kosten der Bestandskundenbearbeitung ggf. auch Kosten für den Kauf von Neukundenadressen in die Berechnung mit einbezogen werden. ${ }^{177}$ Die beschriebenen Kennzahlen ermöglichen einen guten Vergleich unterschiedlicher Instrumente und Angebotsformen des Direktmarketing, die im Rahmen verschiedener Aktionen eingesetzt wurden.

Die auf Basis der kostenorientierten Kennzahlen generierten Aussagen zur Effizienz des Direktmarketing-Einsatzes können durch eine gewinn- bzw. rentabilitätsorientierte Berechnung erweitert werden, ${ }^{178}$ die erst unter Auswertung der aus dem Direktmarketing-Einsatz resultierenden Transaktionen nach Anzahl und Wert der Bestellungen/Verkäufe möglich ist. ${ }^{179}$ Werden die generierten Umsätze den angefallenen Kosten gegenüber gestellt, kann der aktions- oder kampagnenbezogene Gewinn berechnet werden. ${ }^{180}$ Darüber hinaus lässt sich im

175 Vgl. Holland, H., Direktmarketing, 2. Aufl., München 2004, S. 366.

176 Die so gewonnene Kennzahl gibt Aufschluss darüber, welche Kosten zur Gewinnung eines Auftrags nötig waren. Aussagen zur Rentabilität lassen sich jedoch noch nicht treffen, da die Ertragsseite zunächst keine Berücksichtigung findet. Vgl. Holland, H., Direktmarketing, München 1992, S. $181 \mathrm{ff}$.

177 Vgl. Wiedemann, R., Schwab, K., Erfolgsmessung und -optimierung von OnlineMarketing-Aktivitäten, in: Dallmer, H. (Hrsg.), Das Handbuch Direct Marketing \& More, a. a. O., S. $844 \mathrm{ff}$. Aber auch im Rahmen des Telefonmarketing werden die kostenorientierten Ansätze der Erfolgskontrolle verwendet. Den Hauptanteil an den Gesamtkosten zur Betreibung eines Call-Centers bilden Personalkosten. Vgl. Greff, G., Telefonmarketing und Call-Center, in: Dallmer, H. (Hrsg.), Das Handbuch Direct Marketing \& More, a. a. O., S. 251 f. Daneben kommen aber auch weitere Kennzahlen, wie bspw. Telefonkosten pro Stunde, Telefonkosten pro Anruf oder Telefonkosten pro Bestellung zum Einsatz. Vgl. bspw. Stone, B., Jacobs, R., Successful Direct Marketing Methods, a. a. O., S. 210 ff.; Hölscher, U., Kalkulation einer Direktmarketing-Aktion, in: Dallmer, H. (Hrsg.), Das Handbuch Direct Marketing \& More, a. a. O., S. 460.

178 Grundsätzlich zählt dieser Bereich nicht zur klassischen Effizienzmessung; er soll aber im Folgenden der Vollständigkeit halber aufgeführt werden.

179 Vgl. Ceyp, M., Erfolgskontrollen im Direktmarketing, a. a. O., S. 26-31; Geller, L. K., Response. Die unbegrenzten Möglichkeiten des Direktmarketing, a. a. O., S. 199 ff. sowie Gerth, N., Die Bedeutung des Online Marketing für die Distributionspolitik, a. a. O., S. $181 \mathrm{ff}$.

180 Aussagen über den Beitrag einer Direktmarketing-Aktion zum Unternehmensgewinn lassen sich nur treffen, wenn auch die entsprechenden Kosten furr die Herstellung des Produktes bzw. der Dienstleistung mit in die Kalkulation einbezogen werden. An- 
Rahmen einer Gegenüberstellung des Gewinns zum eingesetzten Kapital die Rentabilität einer Aktion ermitteln. ${ }^{181}$ Im Bereich der rein finanzorientierten Ansätze zur Erfolgskontrolle im Direktmarketing wird in der Unternehmenspraxis die Break-Even-Analyse eingesetzt. ${ }^{182}$ Durch den Vorteil einer kundenindividuellen Zurechenbarkeit von Umsätzen und Kosten werden teilweise auch Ansätze zur Berechnung des Kundenwertes verwandt. ${ }^{183}$ In diesem Kontext finden auch traditionelle Kundenbewertungsverfahren, wie die ABC-Analyse ${ }^{184}$, Scoring-Modelle, wie die Recency-Frequency-Monetary-Ratio-Methode ${ }^{185}$, und Kundenstrukturportfolios ${ }^{186}$ Anwendung. ${ }^{187}$ Die dargestellten Effizienzkennziffern und Messansätze komplettieren damit die zuvor beschriebene kontakt- und

sonsten ist die Differenz der durch den Einsatz des Direktmarketing generierten Umsätze und den dieser Aktivität zurechenbaren Kosten als Deckungsbeitrag zu bezeichnen.

181 Stone/Jacobs sprechen im Zusammenhang mit finanzorientierten Kennzahlen des Direktmarketing von Key Performance Indicators (KPI), die im Rahmen des gesamten Marketing-Mix zum Einsatz kommen können. Vgl. Stone, B., Jacobs, R., Successful Direct Marketing Methods, a. a. O., S. 457 ff. Neben einzelnen rentabilitätsorientierten Kennzahlen finden darüber hinaus auch Kennzahlensysteme Anwendung. Primäre Zielgröße solcher Kennzahlensysteme ist i. d. R. eine rentabilitätsbezogene Spitzenkennzahl, die in weitere Unterkennzahlen aufgesplittet wird. Zu den bekanntesten und am häufigsten in der Unternehmenspraxis des Direktmarketing eingesetzten rentabilitätsorientierten Ansätzen zählt das sog. DuPont-System of Financial Control. Vgl. Kehl, R. E., Controlling mit Database Marketing - Effizienzmessung absatzpolitischer Instrumente, a. a. O., S. 247.

$182 \mathrm{Vgl}$. Kirchner, G., Sobeck, S., Lexikon des Direktmarketing, a. a. O., S. 81 f.

183 Vgl. u. a. Link, J., Schleuning, C., Das neue interaktive Direktmarketing, a. a. O., S. 119 ff.; Gierl, H., Koncz, J., Customer Lifetime Value, in: Dallmer, H. (Hrsg.), Das Handbuch Direct Marketing \& More, a. a. O., S. 939-956.

$184 \mathrm{Vgl}$. Ceyp, M., Erfolgskontrollen im Direktmarketing, a. a. O., S. 30. In derartigen Untersuchungen konnte vielfach belegt werden, dass nur ein geringer Prozentsatz der Kunden für einen Großteil des Umsatzes verantwortlich ist.

185 Vgl. Belz, C., Strategisches Direktmarketing, a. a. O., S. 269.

186 Hierbei werden die bestehenden und potenziellen Kunden zumeist anhand mehrerer Dimensionen klassifiziert und auf diese Weise unterschiedlichen Clustern zugeordnet. Vgl. Belz, C., Strategisches Direktmarketing, a. a. O., S. 270; Heinemann, Ch., Werbung im interaktiven Fernsehen, Wiesbaden 1998, S. $158 \mathrm{ff}$.

$187 \mathrm{Vgl}$. Kehl, R. E., Controlling mit Database Marketing - Effizienzmessung absatzpolitischer Instrumente, a. a. O., S. 242; Scholzen, J. N., Schubert, A., Richtlinien für eine erfolgreiche Kundenanalyse in: Dallmer, H. (Hrsg.), Das Handbuch Direct Marketing \& More, a. a. O., S. 709. Eine Würdigung verschiedener Scoring-Modelle erfolgt bei Malthouse. Vgl. Malthouse, E., Assessing the performance of direct marketing scoring models, in: Journal of Interactive Marketing, Vol. 15, No.1, 2001, S. 4962. 
wirkungsbasierte Effektivitätsmessung zu einem umfassenden Modell der Erfolgsbeurteilung des Direktmarketing-Einsatzes (vgl. Abb. 6).

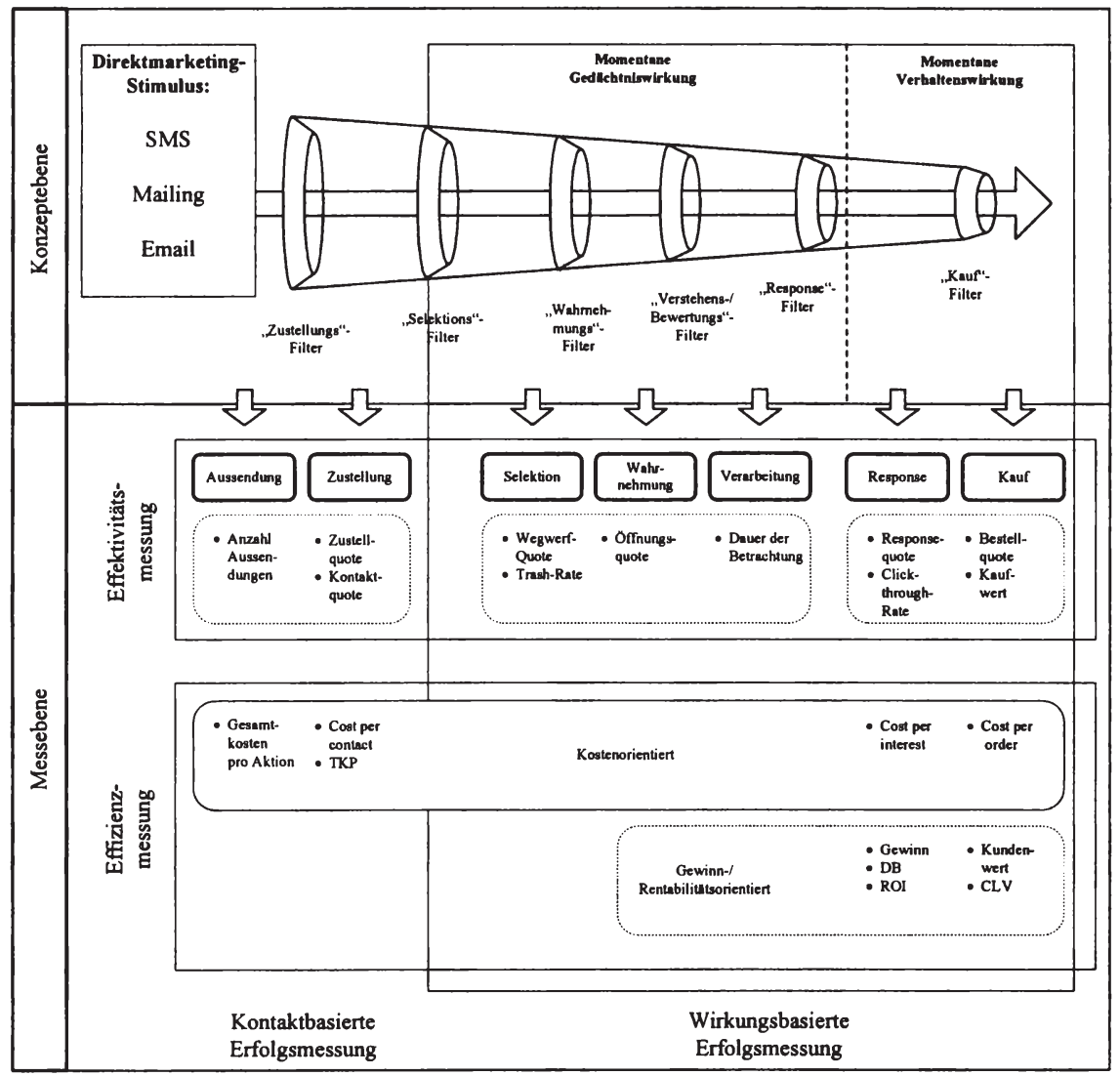

Abb. 6: Kontakt- und wirkungsbasierte Erfolgsmessung im Direktmarketing

\section{Analyse des Einflusses endogener Determinanten auf die Effektivität und Effizienz}

Im Mittelpunkt der vorliegenden Arbeit steht die experimentelle Analyse ausgewählter Direktmarketing-Instrumente im Hinblick auf die Wirkungen beim Rezipienten. Aus Unternehmenssicht stellt allerdings die Auswahl eines spezifischen Direktmarketing-Instruments lediglich eine endogene Determinante des Direkt- 
marketing-Stimulus dar. Um den Einfluss der Auswahl eines Trägermediums in Relation zum Einfluss anderer endogener Determinanten des DirektmarketingEinsatzes einschätzen zu können, bedarf es der Untersuchung weiterer zentraler endogener Determinanten des Direktmarketing-Einsatzes. Aus diesem Grund werden anhand eines direktmarketingspezifischen Entscheidungsprozesses weitere endogene Determinanten in die Untersuchung miteinbezogen, von denen anzunehmen ist, dass unterschiedliche Ausprägungen dieser Variablen von Bedeutung für den Wirkungsprozess des Direktmarketing-Stimulus sind.

\subsection{Entscheidungsprozess des Direktmarketing als Strukturierungsraster zur Identifikation zentraler endogener Determinanten}

Der Entscheidungsprozess des Direktmarketing setzt sich aus verschiedenen Stufen zusammen, die i. d. R. aufeinander aufbauen und als interdependent anzusehen sind: ${ }^{188}$ Vor dem Hintergrund des Ziels der vorliegenden Arbeit, den Einfluss zentraler endogener Determinanten auf Effektivität und Effizienz des Direktmarketing zu untersuchen und dabei insbesondere momentane Verhaltenswirkungen als primäre Zielgröße heranzuziehen, sind hierfür verschiedene, primär operative Entscheidungstatbestände zu berücksichtigen. In Anlehnung an Elsner soll daher ein auf dem „System des Direktmarketing“ aufbauender Entscheidungsprozess aufgezeigt werden, im Rahmen dessen die zentralen endogenen Determinanten auf unterschiedlichen Ebenen Berücksichtigung finden (vgl. Abb. 7). ${ }^{189}$

188 Ähnliche Abläufe von Planungsprozessen finden sich bspw. bei Holland, der u. a. für die Planung des Mailingeinsatzes neben den Zielsetzungen die Identifikation der Zielgruppe, das Angebot, das Timing sowie gestalterische Aspekte nennt. Vgl. Holland, H., Direktmarketing, 2. Aufl., München 2004, S. 292 ff. Elsner folgt hierbei der Reihenfolge: Bestimmung von Werbeziel und Werbestrategie, Festlegung einer Aufbau- und Ablaufplanung, Zielgruppenauswahl, Einsatz der Response-Medien und Werbemittel sowie Bestimmung der Versandtechnik. Vgl. Elsner, R., Optimiertes Direkt- und Databasemarketing unter Einsatz mehrstufiger dynamischer Modelle, a. a. O., S. $48 \mathrm{f}$.

189 Trotz der primär kommunikativen Interpretation des Direktmarketing in der Unternehmenspraxis und der tendenziell ähnlichen Entscheidungstatbestände im Vergleich zur klassischen Kommunikationsplanung ergab eine Befragung des Marktforschungsinstituts TNS Emnid, dass sowohl strategische Entscheidungen, wie bspw. die Zielgruppenbestimmung, als auch operative Entscheidungen, bspw. die Auswahl eines geeigneten Direktmarketing-Instruments, in den überwiegenden Fällen durch die Geschäftsleitung bzw. die Inhaber des Unternehmens vorgenommen werden. Vgl. TNS Emnid, unveröffentlichte Studie im Auftrag des Deutschen Direktmarketing Verbandes, 2003, S. 1-5. 


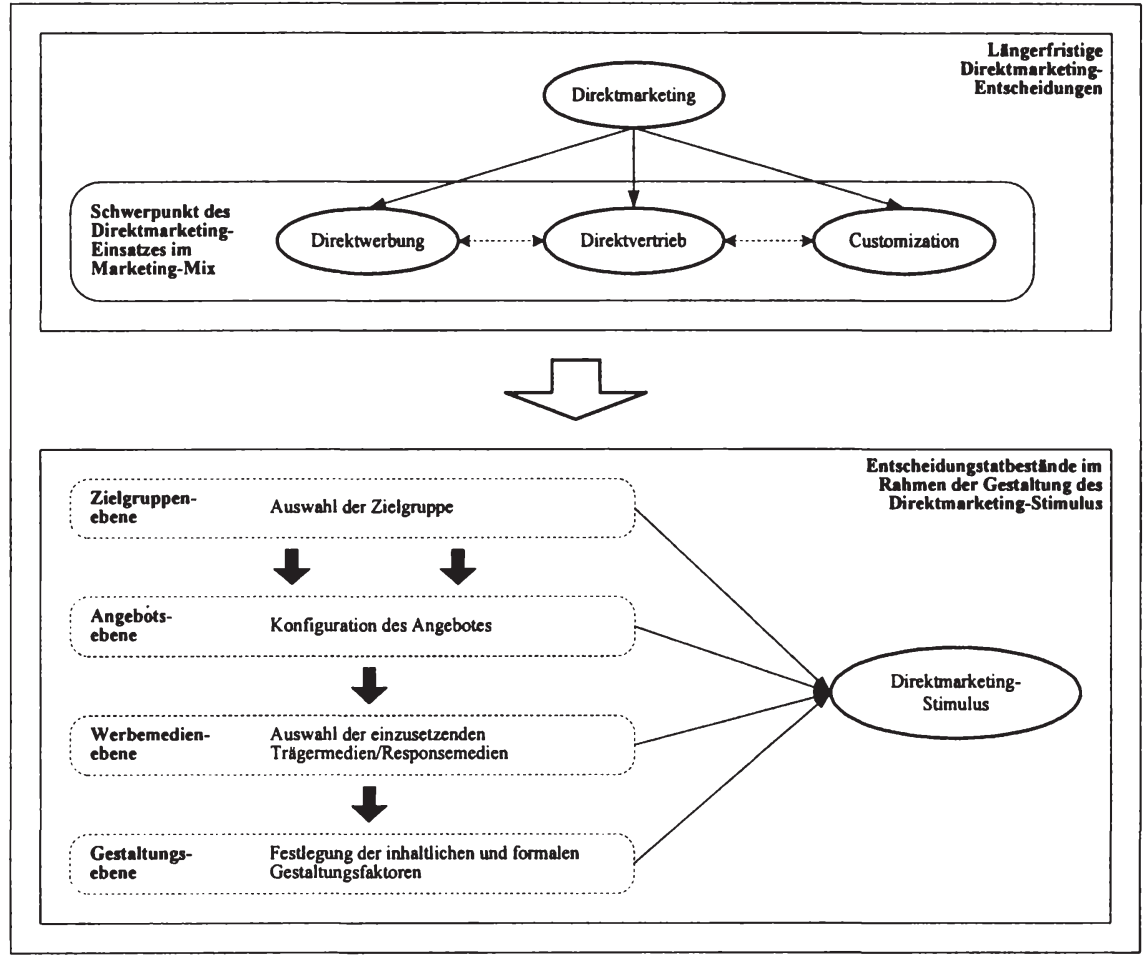

Abb. 7: Ausgewählte Entscheidungstatbestände im Rahmen des Direktmarketing-Einsatzes

Je nach Branche, Markt- und Wettbewerbsumfeld wird der Einsatz des Direktmarketing mit unterschiedlicher strategischer Schwerpunktsetzung verfolgt. $\mathrm{Zu}$ nennen sind in diesem Zusammenhang der primär direkte Werbung fokussierte Direktmarketing-Einsatz („Direktwerbung“), der Direktmarketing-Einsatz, der darüber hinaus auch den Direktvertrieb forciert („Direktvertrieb"), sowie der Direktmarketing-Einsatz, der zusätzlich eine personenindividuelle Gestaltung von Produkten bzw. Dienstleistungen und Preisen beinhaltet („Customization“). 190 Während die Festlegung einer Grundausrichtung des Direktmarketing-Einsatz zum Bereich der strategischen Planung zählt, erweisen sich im Rahmen der hier fokussierten umsetzungsorientierten Perspektive des Direktmarketing-Einsatzes verschiedene Entscheidungstatbestände für die Gestaltung des DirektmarketingStimulus als relevant. Hierzu zählen im einzelnen:

190 Dieser Zusammenhang ist im oberen Teil von Abb. 7 dargestellt. 
1. Vor dem Hintergrund der Unternehmensziele und der Ziele des Direktmarketing-Einsatzes sind zunächst die Zielgruppen des Direktmarketing-Stimulus festzulegen. Hierbei stellt sich die grundlegende Frage, ob Bestandskunden oder Neukunden angesprochen sowie weitere Differenzierungen innerhalb der einzelnen Gruppen vorgenommen werden sollen. ${ }^{191}$

2. In einem weiteren Schritt sind die angebotsbezogenen Komponenten des Direktmarketing-Stimulus festzulegen, d. h. das Leistungsangebot, welches dem Kunden offeriert wird, bzw. die Informationen, die dem Kunden übermittelt werden sollen. Dieser Schritt ist zumeist eng mit der Auswahl der Zielgruppe verbunden, an die der Direktmarketing-Stimulus gesendet werden soll. Beide Entscheidungen sind daher i. d. R. nur simultan zu treffen.

3. In Abhängigkeit des konkreten Angebots sowie der adressierten Zielgruppe erfolgt in Abhängigkeit der Eignung zur Erreichung der intendierten Ziele die Auswahl eines oder mehrerer Direktmarketing-Instrumente. ${ }^{192}$ In diesem Zusammenhang ist zu untersuchen, ob der isolierte Einsatz eines Instruments oder der integrative Einsatz mehrerer Direktmarketing-Instrumente erfolgen soll. Gleichzeitig gilt es festzulegen, welches Medium als Rückkanal zur Erfassung und Übermittlung der Kundenreaktionen an das Unternehmen eingesetzt wird.

4. Nach der Festlegung der einzusetzenden Träger- und Rückkanalmedien sind vor dem Hintergrund der ausgewählten Angebots-/ Zielgruppenkombinationen die gestalterischen Parameter unter Beachtung der instrumentespezifischen Besonderheiten zu konfigurieren. ${ }^{193}$ Hierbei ist es notwendig, neben formellen Parametern, wie Layout und Design, auch inhaltliche Faktoren, wie bspw. die Argumentation des Werbetextes, zu bestimmen.

In Abhängigkeit des jeweils eingesetzten Instruments sind abschließend der konkrete Versandzeitpunkt der Direktmarketing-Stimuli festzulegen sowie im Zuge der Erfolgskontrolle geeignete Messansätze auszuwählen, mit deren Hilfe eine Direktmarketing-Aktion hinsichtlich Effektivităt und Effizienz beurteilt werden kann. ${ }^{194}$

191 Elsner nennt diesen Schritt der Planung des Direktmarketing-Einsatzes auch Systemplanung. Vgl. Elsner, R., Optimiertes Direkt- und Databasemarketing unter Einsatz mehrstufiger dynamischer Modelle, a. a. O., S. $48 \mathrm{f}$.

192 In diesem Zusammenhang erfolgt - ggf. unter Rückgriff auf entsprechende Intramediavergleiche - eine Mediaselektion eines oder mehrerer Trägermedien. Vgl. Schweiger, G., Grundlagen der Streuplanung, in: Kaiser, A. (Hrsg.), Werbung. Theorie und Praxis werblicher Beeinflussung, München 1980, S. 156.

193 Dieser Prozess wird i. d. R. als Werbemittelgestaltung bezeichnet. Vgl. Steffenhagen, H., Wirkungen der Werbung, a. a. O., S. 14.

194 Vgl. Holland, H., Direktmarketing, 2. Aufl., München 2004, S. 46 f. In der vorliegenden Arbeit soll das Timing allerdings in Zusammenhang mit dem integrierten 
Konzeptionelle Analyse des Direktmarketing-Erfolgs

\subsection{Endogene Determinanten der Effektivität und Effizienz}

\subsubsection{Zielgruppenbezogene Determinanten}

Aufgrund der mit dem Direktmarketing-Einsatz verbundenen Vorteile erfolgt die Kommunikation mit den Bestandskunden in vielen Branchen zunehmend unter Einsatz der Instrumente des Direktmarketing. ${ }^{195}$ In Abhängigkeit der konkreten Ziele des Direktmarketing-Einsatzes werden in der Unternehmenspraxis auf Bestandskundenseite häufig weitere Untersegmente gebildet, anhand derer eine spezifische Marktbearbeitung vorgenommen wird. ${ }^{196}$ In vielen Fällen erfolgt die Segmentierung der Bestandskunden zusätzlich auf Basis der ökonomischen Bedeutung des Kunden für das Unternehmen, bspw. gemessen an der Höhe des Umsatzanteils oder anhand des Kundenwertes. ${ }^{197}$ Ebenfalls Anwendung finden im Kontext der Segmentierung auch soziodemographische, psychographische oder verhaltensbezogene Kriterien. ${ }^{198}$ Darüber hinaus werden die Instrumente des Direktmarketing aber auch vielfach zur Akquisition neuer Kunden eingesetzt. Im Rahmen der Neukundenakquisition kommt es ebenfalls zunehmend zu einem segmentspezifischen Direktmarketing-Einsatz, der neben regionalen Segmentierungskriterien vermehrt auf soziodemographische Informationen zurückgreift. ${ }^{199}$

Einsatz der Instrumente diskutiert werden und bildet daher keine eigenständige Stufe im Entscheidungsprozess.

$195 \mathrm{Zu}$ den Chancen und Risiken eines CRM-gestützten Direktmarketing vgl. bspw. Crux, R., Scherfke, A., Chancen und Risiken im CRM-gestützten Direct Marketing, in: Dallmer, H. (Hrsg.), Das Handbuch Direct Marketing \& More, a. a. O., S. $1119 \mathrm{ff}$.

196 Holland weist in diesem Zusammenhang auf das Konzept der Loyalitätsleiter von Kreutzer hin. Demnach können Kunden hinsichtlich ihrer Beziehung zum Unternehmen in verschiedene Klassen von „keine Kenntnisse“ bis zu „Stammkunde“ eingeteilt und entsprechend bearbeitet werden. Vgl. Holland, H., Direktmarketing, 2. Aufl., München 2004, S. 68 ff. Zum ursprünglichen Konzept vgl. Kreutzer, R. T., Die Basis für den Dialog, in: Absatzwirtschaft, Nr. 4, 1990, S. 106.

197 Vgl. zum Einsatz des Kundenwerts im CRM bspw. Brown, S., Customer Relationship Marketing: A Strategic Imperative in the World of E-business, Ontario 2000, S. 13. Zum Konzept des Customer Lifetime Value im Kontext des Direktmarketing vgl. Gierl, H., Koncz, J., Customer Lifetime Value, in: Dallmer, H. (Hrsg.), Das Handbuch Direct Marketing \& More, a. a. O., S. 939-956.

198 Vgl. Böhler, H., Marksegmentierung auf Basis eines Direct-Marketing-Konzepts, in: Dallmer, H. (Hrsg.), Das Handbuch Direct Marketing \& More, a. a. O., S. 921-937.

199 In diesem Zusammenhang bieten verschiedene Anbieter, wie bspw. Schober oder AZ Bertelsmann, mittlerweile eine Vielzahl „psychologischer Kriterien“ zur Anreicherung der Kundendateien zum Kauf an. So können bspw. Präferenzen gegenüber bestimmten Automarken als Kriterien ausgewählt werden. 
Aus Unternehmenssicht stellt sich aufgrund zu erwartender unterschiedlicher Reaktionswahrscheinlichkeiten von Bestands- und Neukunden zunächst die Frage nach dem Einfluss der Zielgruppenauswahl auf die Effektivität und Effizienz des Direktmarketing-Einsatzes. ${ }^{200}$ In hohem Maße verantwortlich für die unterschiedliche Responsewahrscheinlichkeit von Bestands- und Neukunden dürften hierbei die Kenntnis und die Vertrautheit der Bestandskunden mit dem angebotenen Produkt oder der Dienstleistung sein, die sich durch konkrete Erfahrungen mit dem Leistungsangebot des Unternehmens manifestiert. ${ }^{201}$ Demnach neigen Konsumenten mit Produkterfahrungen eher zum Kauf eines Produktes bzw. einer Dienstleistung im Gegensatz zu unerfahrenen und insofern mit der Leistung unvertrauten Konsumenten, insbesondere wenn es sich um Verbrauchsgüter handelt. ${ }^{202}$ Eigene Erfahrungen können vielmehr dazu führen, dass die konkret übermittelte Werbebotschaft aus Sicht der Bestandskunden durch dessen Selbsterfahrung auch realisierbar und damit glaubwürdig erscheint. ${ }^{203}$ Die zentrale Rolle von Erfahrungen im Kontext der Werbewirkungsforschung wird hierbei durch die Annahme verstärkt, dass einmal gemachte Erfahrungen kaum durch nachträgliche werbliche Aussagen modifiziert werden können. ${ }^{204}$ Vielmehr kann die aus der konkreten Produkterfahrung resultierende Zufriedenheit mit einem Produkt oder einer Dienstleistung die Wiederkaufabsicht des Konsumenten positiv beeinflussen, was per definitione bei den Neukunden nicht erfolgen kann. ${ }^{205}$ Produkterfahrungen können somit als zentrale Prädikatoren des Konsumentenverhaltens angesehen werden. ${ }^{206}$ Darüber hinaus sind Bestandskunden neben

200 Holder et al. beziehen sich explizit in ihrer Definition von Direktmarketing sowohl auf bestehende als auch potenzielle neue Kunden. Demnach ist Direktmarketing ,... the planned recording, analysis and tracking of consumer and business direct response behaviour to develop marketing strategies for current and future customers". Holder, D., Davies, D., Stone, M., Part of IDM Diploma course material, Institute of Direct Marketing, Teddington/Richmond-upon-Thames 1992, S. 1 ff. Vögele konstatierte zur Bedeutung der Zielgruppenauswahl für das Direktmarketing hierzu Folgendes: „Besser ein schlechtes Mailing an die richtige Zielgruppe als ein gutes an die falsche." Vögele, S., 99 Erfolgsregeln des Direktmarketing, 5. Aufl., Landsberg/Lech 2003, S. 88.

201 Vgl. Mayer, H., Illmann, T., Markt- und Werbepsychologie, a. a. O., S. 87.

202 Vgl. Morwitz, V. G., Schmittlein, D., Using Segmentation to Improve Sales Forecasts Based on Purchase Intent. Which "Intenders" Actually Buy?, in: Journal of Marketing Research, Vol. 12, No. 2, 1992, S. 396.

203 Vgl. Mayer, H., Illmann, T., Markt- und Werbepsychologie, a. a. O., S. 87.

204 Vgl. Nemetz, K., Wie lernt der Konsument? Lernen aus Werbung und/oder Produktverwendung, in: Marketing Journal, Jg. 25, Nr. 6, S. $574 \mathrm{f}$.

205 Vgl. Mayer, H., Illmann, T., Markt- und Werbepsychologie, a. a. O., S. 236 f.

$206 \mathrm{Vgl}$. Mayer, H., Werbewirkung und Kaufverhalten. Unter ökonomischen und psychologischen Aspekten, Stuttgart 1990, S. 175. 
dem Leistungsangebot auch mit der Verkaufsabwicklung des Unternehmens vertraut. Dieser Aspekt dürfte vor allem im Kontext des Direktverkaufs - wenn eine vorherige Besichtigung der Waren nicht möglich ist - die Kaufwahrscheinlichkeit erhöhen. Vor diesem Hintergrund stellen viele Käufe der Bestandskunden Wiederholungskäufe dar, denen - im Vergleich zu den Neukunden - i. d. R. eine limitierte Kaufentscheidung vorausgeht. ${ }^{207}$

In diesem Zusammenhang scheint auch der Einsatz der DirektmarketingInstrumente aus Sicht der Bestandskunden ein Bestandteil der gewöhnlichen Kundenbearbeitung darzustellen, in die sie ggf. freiwillig zugestimmt haben. Auch der Einsatz bislang nicht eingesetzter Direktmarketing-Instrumente scheint hierbei nicht als Störung wahrgenommen zur werden. Im Gegensatz dazu liegt die Erlaubnis zum Bewerben häufig im Rahmen der Neukundenakquisition nicht vor und so werden insbesondere direkte Kundenansprachen unter Einsatz von Direktmarketing-Instrumenten als Eingriff in die Privatsphäre gewertet, welche in der Folge zu einer geringeren Kaufwahrscheinlichkeit führen kann. ${ }^{208}$ Insgesamt ist daher zu vermuten, dass sich die Bearbeitung der Bestandskunden mit Hilfe des Direktmarketing-Einsatzes bei Gleichheit des Angebotes effektiver gestaltet als die der Neukundenakquisition, insbesondere dann, wenn es sich um typisch standardisierte Produkte und Dienstleistungen des Unternehmens handelt.

Im Hinblick auf einen Vergleich der mit der Bestandskundenbearbeitung bzw. der Neukundenakquisition verbundenen Kosten kann zunächst bei Versendung gleicher Angebote auf gleichen Trägermedien von einer ähnlichen Kostenstruktur für die Gestaltung, ggf. den Druck und die Konfektionierung sowie den Versand des Direktmarketing-Stimulus ausgegangen werden. Während allerdings die für die Versendung der Stimuli benötigten Adressdaten der Bestandskunden dem Unternehmen i. d. R. vorliegen und lediglich Kosten für die Aufbereitung in Form von Personalkosten anfallen, wird bei der Neukundenakquisition zumeist eine Neuerhebung bzw. ein Kauf von Adressdaten notwendig, der zusätzliche Kosten verursachen kann. Gleichzeitig kann angenommen werden, dass Neukunden - sollten sie das Leistungsangebot des Unternehmens noch nicht kennen u. U. zu einem intensiveren Informationsverhalten vor dem Kauf neigen und daher höhere Kosten bei der Bearbeitung von Anfragen z. B. im Rahmen der Kaufabwicklung anfallen. Die unterschiedliche Höhe der Gesamtkosten zwischen Bestandskundenbearbeitung und der Neukundenakquisition auf der Ebene der einzelnen Instrumente ergibt sich daher zumeist aus einem zusätzlichen Kostenblock für die Bereitstellung und Nutzung von Neukundenadressdaten. In Verbindung

207 Vgl. Mayer, H., Illmann, T., Markt- und Werbepsychologie, a. a. O., S. $98 \mathrm{ff}$.

$208 \mathrm{Vgl}$. Chittenden, L., Rettie, R., An evaluation of e-mail marketing and factors affecting response, a. a. O., S. $203 \mathrm{f}$. 
mit anzunehmenden geringeren Response- und Kaufquoten ist daher zu vermuten, dass sich die Bestandskundenbearbeitung gegenüber der Neukundenakquisition effizienter gestaltet und damit grundsätzlich auch im Kontext des Direktmarketing der These von Reichheld/Sasser gefolgt werden kann, nach der die Bindung bestehender Kunden günstiger ist als die Akquirierung neuer. ${ }^{209}$

\subsubsection{Angebotsbezogene Determinanten}

Ein Großteil der eingesetzten Direktmarketing-Stimuli zielt darauf ab, den Rezipienten zu Reaktionen zu veranlassen, die nicht automatisch in einem Kauf- oder Bestellprozess münden, sondern deren Ziel in der Gewinnung von Informationen über den Rezipienten liegt. ${ }^{210}$ Eine Incentivierung zur Preisgabe persönlicher Informationen erfolgt häufig über eine Teilnahme an Verlosungen oder Gewinnspielen. ${ }^{211}$ Den primär auf Informationsgenerierung abzielenden Angeboten steht in der Unternehmenspraxis allerdings auch eine Vielzahl verkaufsorientierter Direktmarketing-Stimuli gegenüber, mit denen der direkte Verkauf von Produkten und Dienstleistungen angestrebt wird. ${ }^{212}$ Im Vergleich zu den auf Informationsgenerierung abzielenden Angeboten erfolgt die Reaktion des Rezipienten auf verkaufsorientierte Angebote aufgrund des Interesses an der angebotenen Leistung bzw. einer konkreten Kaufabsicht und stellt damit das Ergebnis eines persönlichen Abwägungsprozesses zwischen Nutzen aus der konkreten Leistung sowie dem meist monetären Nutzenverzicht dar. Aus Unternehmenssicht sind die verkaufsorientierten Angebote von besonderer Relevanz für eine Effektivitätsund Effizienzanalyse, da sie mit ihrer Konfiguration und dem sich anschließenden Versand nicht nur Kosten verursachen, sondern i. d. R. auch einen direkten

209 Vgl. Reichheld, F., Sasser, W. E., Zero-Migration: Dienstleister im Sog der Qualitätsrevolution, in: Harvard Business Manager, 13. Jg., Heft 4, 1991, S. 108-116.

210 Vgl. Holland, H., Direktmarketing, 2. Aufl., München 2004, S. 22.

211 Vgl. Schefer, D., Schuler, R., Adressgenerierung im Internet, in: Dallmer, H. (Hrsg.), Das Handbuch Direct Marketing \& More, a. a. O., S. 590 ff.; Bachmann, M., Adressvaldierung und Adressanreicherung via Internet, in: Dallmer, H. (Hrsg.), Das Handbuch Direct Marketing \& More, a. a. O., S. $597 \mathrm{ff}$.

212 Diese beiden grundlegenden Ziele spiegeln sich auch in den Kategorien der einzelnen Direktmarketing-Instrumente wider, wie bspw. der Unterscheidung von Werbeund Verkaufsemails, Werbe- und Verkaufs-SMS etc., wie sie in der Literatur häufig $\mathrm{zu}$ finden sind. Vgl. die unterschiedlichen Ausgestaltungsformen von EmailWerbung (bspw. Email-Newsletter, Werbeemails, Verkaufsemails etc.) bei Deutscher Direktmarketing Verband (Hrsg.), eMail-Marketing - Dialog pur, Best Practice Guide Nr. 4, Bonn 2003; zu den unterschiedlichen Formen mobiler Werbung in Form inhalts-, transaktions- und responseorientierter Werbung etc. vgl. Hinrichs, Ch., Lippert, I., Kosten und Wirkungen mobiler Werbung, a. a. O., S. 270 f.; Deutscher Direktmarketing Verband (Hrsg.) Mobile-Marketing - Dialog on Tour, a. a. O., S. 3 f. 
Einfluss auf die Erlössituation des Unternehmens besitzen. Aus diesem Grund sollen die verkaufsorientierten Angebote im Mittelpunkt der folgenden $\mathrm{Be}$ trachtung stehen.

Von besonderer Relevanz für die Analyse der Effektivität und Effizienz des Direktmarketing-Einsatzes im Rahmen einer feldexperimentellen Untersuchung erscheinen insbesondere drei Angebotstypen, denen eine Vielzahl der in der Praxis zu beobachtenden Ausprägungen der verkaufsorientierten Direktmarketing-Stimuli zugeordnet werden können. So werden die Instrumente des Direktmarketing in vielen Fällen eingesetzt, um standardisierte Produkte und Dienstleistungen des Unternehmens zusätzlich $\mathrm{zu}$ den bestehenden, meist stationären Verkaufskanälen über den Direkt-Verkauf zu vertreiben. ${ }^{213}$ In den meisten Fällen erfolgt im Rahmen dieser „standardisierten Angebotsform“ keine Modifikation des Leistungsangebots in Richtung Individualisierung - weder in preislicher noch in produktionstechnischer Hinsicht. Das Nutzenpotenzial aus Kundensicht und damit der zentrale Kaufanreiz können hierbei gegenüber dem stationären Verkauf primär in einem distributionspolitischen Mehrwert bspw. in einer bequemeren Bestellabwicklung oder Lieferung und damit in erhöhter Convenience gesehen werden. ${ }^{214}$ Letztendlich ist auch eine Steigerung des Kundenvertrauens möglich, z. B. durch Entanonymisierung infolge personalisierter Ansprache über sämtliche Kanäle. ${ }^{215}$

Eine wirkungsvolle Modifikation der standardisierten Angebotsform kann durch die Variation des Preises erfolgen. In diesem Kontext kann der Direktvertrieb von Produkten und Dienstleistungen dazu beitragen, dass kostenintensive Intermediäre wie Groß- und Einzelhändler umgangen und die generierten Kostenvorteile durch Preissenkungen teilweise an die Kunden weitergegeben werden können. ${ }^{216}$ Gleichsam finden die Instrumente des Direktmarketing Einsatz, um preislich reduzierte Waren und Dienstleistungen in möglichst kurzer Zeit zu ver-

213 Die Einbeziehung distributionspolitischer Parameter im Bereich des Direktmarketing zeigte sich insbesondere am Beispiel der Versandhändler. So wurde Direktmarketing lange Zeit mit Versandhauswerbung gleichgesetzt, da direkte Kommunikation und Direktvertrieb/-verkauf i. d. R. zusammen auftraten. Vgl. Donelly, L., Geradi, A., Direkt-Marketing ist nicht Versandhauswerbung, in: D \& G Direkt Marketing, Ettlingen 1987, S. 8.

214 Vgl. bspw. Feinberg, R. A., Eastlick, M. A., Direct Marketing in the USA: past failures and future promises, in: International Journal of Retail and Distribution Management, Vol. 25, No. 8, 1997, S. 256-261.

215 Vgl. Fassot, G., eCRM-Instrumente: Ein beziehungsorientierter Überblick, in: Eggert, A., Fassot, G. (Hrsg.): eCRM - Electronic Customer Relationship Management, Stuttgart 2001, S. 131-157.

$216 \mathrm{Vgl}$. Meffert, H., Direktmarketing und marktorientierte Unternehmensführung, a. a. O., S. 44. 
kaufen. ${ }^{217}$ Im Rahmen dieser zweiten Form der verkaufsorientierten Angebote wird dem Kunden zusătzlich zu den Vorteilen des Direktvertriebs als wichtiger Kaufanreiz ein monetärer Vorteil offeriert, wenn er das beworbene Angebot kauft. Diese Form des „rabattierten Angebots“ wird häufig von Fluglinien und anderen Dienstleistungsunternehmen zum Verkauf von Restkontingenten eingesetzt, wenn kurzfristig verfügbare Kapazitäten zeitnah verkauft werden sollen.

Von einigen Autoren wird die Meinung vertreten, dass trotz den beschriebenen Kundenvorteilen primär die Befriedigung individueller Bedürfnisse als zentraler Nutzen des Direktmarketing-Einsatzes anzusehen ist. ${ }^{218}$ Aus diesem Grund werden unter zu Hilfenahme der Instrumente des Direktmarketing ,individualisierte Leistungsangebote" vertrieben, die häufig exklusiv nur über einen direkten Vertriebsweg zu beziehen sind. ${ }^{219}$ Wegen der zunehmend kostengünstigeren Möglichkeiten, neben der Individualisierung der Kommunikation auch Produkte und Dienstleistungen in einer individualisierten Form anzubieten, erscheint die Analyse dieser Angebotsform ebenfalls geboten. ${ }^{220}$ In diesem Kontext reichen die Angebote von „quasi“-individualisierten Leistungen, wie etwa dem Angebot von Urlaubsreisen, bis hin zu eigens für den Rezipienten angefertigten Produkten bzw. Dienstleistungen, wie etwa im Bereich von Textilien und Schuhen. ${ }^{221}$ Die Individualisierung kann hierbei auch in einem zeitpunktbezogenen Bezug des Angebotes liegen.222 Gleichzeitig ist zudem eine „direkte Preispolitik“ möglich, die zur Festlegung eines individuellen Preises zwischen Anbieter und Nachfrager

217 Insbesondere in konjunkturell schwierigen Zeiten werden im Zuge der Kürzungen der Marketing-Budgets die Investitionen in die Kommunikation reduziert und zeitgleich häufig Preissenkungen in unterschiedlichen Bereichen des Sortiments mit dem Ziel durchgeführt, den kurzfristigen Absatz zu fördern und Kunden zu Impulskäufen zu animieren. Vgl. Göttgens, O., Wastl, S., Kein Marketing - Kein Konsum - Kein Wachstum, a. a. O., S. 53.

218 Link, J, Gerth, N., eCRM als strategische und organisatorische Herausforderung, in: Eggert, A., Fassot, G. (Hrsg.), eCRM - Electronic Customer Relationship Management, a. a. O., S. 305-328.

$219 \mathrm{Vgl}$. Meffert, H., Direktmarketing und marktorientierte Unternehmensführung, a. a. O., S. 44.

220 Vgl. zum Konzept des (Mass) Customization u. a. Piller, T., Kundenindividuelle Massenproduktion, a. a. O.

221 Die Folge hiervon kann nicht nur in der steigenden Anzahl von adressierten und personalisierten Mailings gesehen werden. Auch hat die Zahl an individualisierten Produkten und Preisen in den letzten Jahren weiter zugenommen. Vgl. Piller, T., Kundenindividuelle Massenproduktion, a. a. O.

222 Zur Bestimmung des optimalen Versandzeitpunkts vgl. bspw. Elsner, der einen Ansatz zur Ertragsoptimierung durch optimale Werbeaktionsfrequenz am Beispiel eines Versandhandelsunternehmens vorstellt. Vgl. Elsner, R., Optimiertes Direkt- und Database-Marketing unter Einsatz mehrstufiger dynamischer Modelle, a. a. O. 
führen kann. ${ }^{223}$ Die Kaufanreize aus Sicht des Konsumenten können bei den individuellen Angeboten im zusätzlichen Mehrwert durch eine zumeist individuelle Problemlösung gesehen werden. ${ }^{224}$ In der folgenden Abbildung sind die drei im Rahmen der vorliegenden Arbeit relevanten Angebotsformen und die jeweils spezifischen Kundenvorteilsdimensionen dargestellt (vgl. Abb. 8).

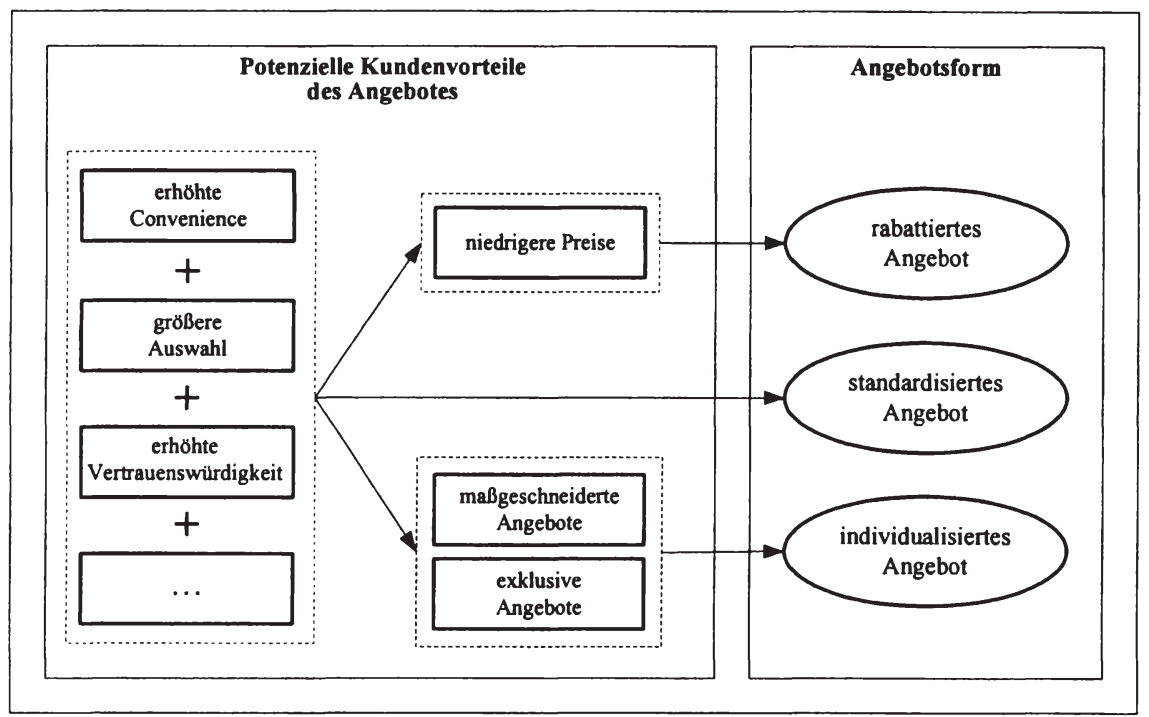

Abb. 8: Ausgewählte Angebotsformen des verkaufsorientierten Direktmarketing-Einsatzes und zugrunde liegende Kundenvorteile

Ob die verkaufsorientierten Direktmarketing-Stimuli allerdings die intendierten Wirkungen beim Konsumenten hervorrufen, hängt von einer Vielzahl weiterer Einflussfaktoren ab, wie etwa dem persönlichen Bedarf zum Zeitpunkt des Angebots, situativen Komponenten etc. 225 Daruber hinaus ist vor dem Hintergrund der drei aufgezeigten Angebotsformen davon auszugehen, dass die jeweiligen Angebote zu individuellen psychischen Verarbeitungsprozessen und in der

223 Hierbei lassen sich unterschiedliche Formen der Preisdifferenzierung erkennen, die bspw. kundengruppenbezogen oder zeitlich befristet ausgestaltet sein kann. Vgl. Elsner, R., Optimiertes Direkt- und Databasemarketing unter Einsatz mehrstufiger dynamischer Modelle, a. a. O., S. 15 f.

$224 \mathrm{Vgl}$. Kehl, R. E., Controlling mit Database Marketing - Effizienzmessung absatzpolitischer Instrumente, a. a. O., S. 15 f.

225 Eine Diskussion der Relevanz weiterer Einflussfaktoren erfolgt im weiteren Verlauf der Arbeit. 
Folge zu differierenden Verhaltensreaktionen beim Konsumenten führen können, die sich nach der jeweiligen konkreten Ausgestaltung der Inhalte richten. ${ }^{226}$ Dringt der Direktmarketing-Stimulus letztendlich zum Rezipienten durch, ohne am Selektions- bzw. Wahrnehmungsfilter zu scheitern, entscheidet im konkreten Fall - in Anlehnung an das klassische Marketing - die subjektiv wahrgenommene „Attraktivität der Leistung““.227

Vor dem Hintergrund der vielfältigen Ausgestaltungsmöglichkeiten der drei Angebotsformen lassen sich lediglich Tendenzaussagen zu den Auswirkungen des Angebots auf die Effektivität des Direktmarketing-Einsatzes treffen. So ist zu vermuten, dass individualisierte Produkte und Dienstleistungen, wenn diesen die richtigen Informationen hinsichtlich der Angebotspräferenzen zugrunde liegen, aus Sicht des Rezipienten eine höhere Attraktivität besitzen und daher eher zum Kauf führen können als standardisierte Leistungen. Ein Vergleich der Angebotsformen hinsichtlich des Informationsverhaltens des Rezipienten und der mit der Bearbeitung der Informationsanfragen verbundenen Beanspruchung von Unternehmensressourcen lässt vermuten, dass standardisierte Angebote, die der $\mathrm{Be}-$ standskunde bereits zuvor im Leistungsangebot des Unternehmens wahrgenommen hat, bei Interesse zu weniger Informationsbedarf aufseiten der Rezipienten führen als individualisierte Angebote. ${ }^{228}$ Während standardisierte Angebote der überwiegenden Zahl von Bestandskunden des Unternehmens bekannt sein dürften, führen die individualisierten Angebote aufgrund neuartiger Leistungskomponenten ggf. zu Verunsicherung bei den Kunden und demzufolge zu einem erhöhten Informationsbedarf. Insbesondere im Rahmen des verkaufsorientierten Direktmarketing, bei dem eine direkte Besichtigung der Produkte zumeist nicht möglich ist - ergeben sich u. U. Fragen bei potenziellen Käufern. Nicht-stan-

226 Holland spricht in diesem Zusammenhang vom Produkt, das einen Anteil von 25$40 \%$ am Erfolg einer Direktmarketing-Maßnahme besitzt. Bei diesem Wert handelt es sich allerdings um einen geschätzten Wert, der nicht empirisch belegt wurde. Vgl. Holland, H., Direktmarketing, 2. Aufl., München 2004, S. 68.

227 Vgl. bspw. Dallmer, H., Direct-Marketing, in: Handbuch des Marketing, München 1989, S. 544; Holland, H., Direktmarketing, 2. Aufl., München 2004, S. 68. Darüber hinaus stellen Vriens et al. die Attraktivität des Angebots als zentralen Einflussfaktor auf das Responseverhalten von Postwurfsendungen dar, die letztendlich nach der Selektion und dem Öffnen des Mailings das Verhalten des Rezipienten im Hinblick auf das konkret beworbene Leistungsangebot determiniert. Vgl. Vriens, M. et al., Conjoint Experiments for Direct Mail Response Optimization, a. a. O., S. 323-339. Auch bei Emails erscheint die Attraktivität des Angebots als zentraler Einflussfaktor für die Effektivität einer Aktion zu sein. Vgl. Chittenden, L., Rettie, R., An evaluation of email marketing and factors affecting response, a. a. O., S. 203-217.

228 In diesem Zusammenhang lässt sich konstatieren, dass die bereits im vorangegangenen Kapitel erwähnte Produktvertrautheit auch in diesem Zusammenhang wiederum relevant erscheint. 
dardisierte Angebote führen daher im Vergleich zu standardisierten Angeboten i. d. R. zu einem intensiveren Informationsverhalten, was aufseiten des Unternehmens zu höheren Kosten in der Bearbeitung von Kundenanfragen münden kann. Dieser Sachverhalt trifft ggf. auf rabattierte Anagebote ebenfalls dann zu, wenn die Rabattart nicht selbsterklärend ist.

Die Betrachtung der Erlöswirkungen zeigt weitere Unterschiede der drei ausgewählten Angebotsformen. So kann vielfach beobachtet werden, dass die zunehmende Individualisierung von Produkten und Dienstleistungen auch von einer höheren Preisbereitschaft der Konsumenten insbesondere im Dienstleistungsbereich getragen wird, da diese in einer Individualisierung einen höheren persönlichen Nutzen sehen, welcher i. d. R. teurer entlohnt wird. ${ }^{229}$ Es ist daher zu vermuten, dass abhängig von den Vorteilen der Individualisierung im Vergleich zum Standardsortiment des Unternehmens nicht nur höhere Preise für die Leistungen realisiert werden können, sondern auch die Bereitschaft zur Bezahlung höherer Preise vorhanden ist. Im Rahmen des rabattierten Angebots kann hingegen von einem positiven Mengeneffekt ausgegangen werden, der allerdings per definitione zu Lasten der durchschnittlichen Preise pro verkaufter Einheit geht.

\subsubsection{Medienbezogene Determinanten}

Neben Entscheidungen die Konfiguration zielgruppen- und angebotsbezogener Determinanten betreffend, erfolgt im Zuge der Mediaselektion die Auswahl eines bzw. mehrerer Direktmarketing-Instrumente, mittels derer der Versand der Angebotsbündel an die Adressaten erfolgt. Aufgrund des hohen Anteils der medienbezogenen Kosten an den Gesamtkosten einer Direktmarketing-Aktion ist die Auswahl der Trägermedien von besonderer ökonomischer Relevanz. ${ }^{230}$ Vor diesem Hintergrund soll die Mediaselektion mit dem Ziel erfolgen, bei einer Zielgruppe eine bestimmte direktmarketingspezifische Wirkung mit minimalen Kosten (Minimalprinzip) oder eine maximale direktmarketingspezifische Wirkung mit gegebenem Budget (Maximalprinzip) zu erreichen. ${ }^{231}$ Der Auswahlprozess eines oder mehrerer geeigneter Trägermedien nimmt damit eine Schlüsselfunk-

229 Vgl. u. a. Meffert, H., Marketing. Grundlagen marktorientierter Unternehmensführung, a. a. O., S. $500 \mathrm{ff}$.

230 In diesem Zusammenhang sind die in Kapitel B.2.2 dargestellten Kosten für die Gestaltung, die Konfiguration, die Informationsaufbereitung und -beschaffung, den Versand sowie die Informationsbearbeitung zu berücksichtigen.

231 Der zweite geschilderte Fall dürfte in der Praxis am häufigsten auftreten. Vgl. zum Begriff der Mediaselektion Meffert, H., Marketing. Grundlagen marktorientierter Unternehmensführung, a. a. O., S. 811. 
tion in der Kommunikationsplanung vieler Unternehmen ein. ${ }^{232}$ Neben der Auswahl eines geeigneten Trägermediums ist ebenfalls die Entscheidung hinsichtlich des Rückkanal-Mediums zu treffen. ${ }^{233}$

\subsubsection{Trägermedium}

Die Intramediaselektion stellt einen zentralen Teilschritt im Rahmen des Direktmarketing-Planungsprozesses dar, ${ }^{234}$ da angenommen werden kann, dass sich die einzelnen Direktmarketing-Instrumente in unterschiedlichem Maße für die Erreichung der vom Unternehmen intendierten Ziele eignen. ${ }^{235}$ Die Eignung der Instrumente zur Erzeugung der gewünschten Gedächtnis- und Verhaltenswirkungen hängt von einer Reihe von Faktoren ab, die in trägerspezifischen Merkmalen auf der einen und daraus resultierenden instrumentespezifischen Wirkungsprozessen beim Rezipienten auf der anderen Seite begründet liegen. $\mathrm{Zu}$ den zentralen Determinanten der Effektivität zählen insbesondere die quantitative (globale) und qualitative (zielgruppenspezifische) Reichweite, die zeitliche und regionale Steuerbarkeit sowie das Image des Mediums. ${ }^{236}$ Vor dem Hintergrund der Verbreitung und der Beschaffenheit der verschiedenen Medien entstehen zudem unterschiedliche trägerspezifische Kosten, die sich auf die Effizienz der einzelnen Trägermedien auswirken können. ${ }^{237}$

Im Rahmen der Analyse der Eignung des Trägermediums, eine ausgewählte Zielgruppe zu erreichen, fällt zunächst auf, dass sich sämtliche der ausgewählten Medien aufgrund ihrer guten Skalierbarkeit für die individualisierte Ansprache von Rezipienten eignen. ${ }^{238}$ So können alle Trägermedien - bei Kenntnis der postalischen Adresse, der Emailadresse bzw. der Mobilfunknummer - zu einer ziel-

232 Vgl. Schefer, D., Medien des Direct Marketing im Intermedia-Vergleich, a. a. O., S. $105 \mathrm{ff}$.

233 Häufig wird der Einfluss des Rückkanalmediums im Zuge der gestaltungsbezogenen Determinanten behandelt. Im Rahmen der vorliegenden Arbeit wird die Auffassung vertreten, dass die Entscheidung, welches Medium als Rückkanal eingesetzt werden soll, bereits im Rahmen dieses Auswahlprozesses getroffen wird.

234 Zum Begriff der Intramediaselektion vgl. Meffert, H., Marketing. Grundlagen marktorientierter Unternehmensführung, a. a. O., S. 811.

$235 \mathrm{Vgl}$. Schefer, D., Medien des Direct Marketing im Intermedia-Vergleich, a. a. O., S. $114 \mathrm{ff}$.

236 In Anlehnung an Schefer, D., Medien des Direct Marketing im Intermedia-Vergleich, a. a. O., S. $105 \mathrm{ff}$.

$237 \mathrm{Vgl}$. ebenda, S. $114 \mathrm{ff}$.

238 Dies war letztendlich auch ein Auswahlkriterium bei der Beschränkung auf Mailing, Email, SMS. Vgl. Kap. B.3.2.3. und Schefer, D., Medien des Direct Marketing im Intermedia-Vergleich, a. a. O., S. $105 \mathrm{ff}$. 
genauen Personenansprache genutzt werden. ${ }^{239}$ Während die drei Instrumente zunehmend in der Kommunikation mit den Bestandskunden eingesetzt werden, kann es ggf. von Interesse sein zu wissen, ob die Medien von potenziellen Neukunden des Unternehmens genutzt werden und ob im Falle des Einsatzes im Direktmarketing die Möglichkeit eines Werbekontaktes grundsätzlich gegeben ist. ${ }^{240} \mathrm{Um}$ Streuverluste zu vermeiden, wird das Kriterium „Penetration in der

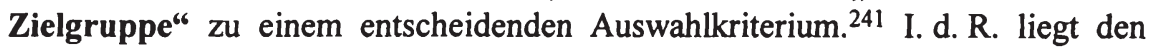
Unternehmen zur ökonomischen Bewertung der Reichweite und damit zur Beurteilung der Medienwirkung zusätzlich der Tausenderkontaktpreis des Mediums vor, der neben der Beurteilung der Reichweite i. S. der Effektivität auch eine Beurteilung der Wirtschaftlichkeit i. S. der Effizienz zulässt. ${ }^{242}$

Neben der Eignung zur Ansprache ausgewählter Zielgruppen unterscheiden sich die Instrumente auch im Hinblick auf die Kommunikationsträgerqualität. ${ }^{243}$ Unter diesem Begriff wird im Allgemeinen die Eignung von Medien ver-

239 Vgl. zu Medien mit „direktem Kundenkontakt“ bei Link, J., Schleuning, C., Das neue interaktive Direktmarketing: die neuen elektronischen Möglichkeiten der Kundenanalyse und Kundenbindung, a. a. O., S. $49 \mathrm{ff}$.

240 Bedingung für den Einsatz ist eine möglichst hohe Übereinstimmung zwischen den Merkmalen der Rezipienten der Werbung und den typischen Mediennutzern.

241 In diesem Zusammenhang werden sowohl die Zielgruppenaffinität als auch die Nettoreichweite als zentrale Indikatoren für den Penetrationsgrad herangezogen. Vgl. Unger, F. et al., Mediaplanung, Methodische Grundlagen und praktische Anwendungen, 3. Aufl., Heidelberg 2002, S. 15 f. Im Zuge einer allgemeinen Betrachtung lassen sich bei den unterschiedlichen Direktmarketing-Instrumenten in Abhängigkeit der Betrachtungsebene (bspw. regional, demographisch, psychographisch) unterschiedliche Penetrationsraten feststellen. So können bspw. über Postwurfsendungen oder aktives Telefonmarketing (Outbound Calls) nahezu sämtliche Personen bzw. Haushalte erreicht werden, wenn die entsprechenden Adress- bzw. Telefondaten vorliegen. Insbesondere bei den elektronischen Medien wie Email und SMS lässt sich zwar eine steigende Diffusion innerhalb der Bevölkerung erkennen, die vollständige Abdeckung sämtlicher Bevölkerungsschichten ist aktuell aber noch nicht gegeben. Vgl. MediaTransfer AG Netresearch \& Consulting (Hrsg.), Status quo und Trends im Kommunikations-Markt in Europa und Nord-Amerika, Hamburg 2003.

242 Der Tausenderkontaktpreis zählt zu den in der Praxis am meisten verbreiteten Bewertungsverfahren und ergibt sich als Quotient aus den Kosten einer Einschaltung x1000 und den erreichten Rezipienten. Vgl. Rogge, H.-J., Werbung, 4. Aufl., Ludwigshafen 1996, S. 245 ff.

243 Der Notwendigkeit zur Beachtung weiterer Faktoren neben der genannten Penetrationsquote folgt auch Schweiger. Dies wird u. a. in seiner Definition von Mediaselektion deutlich, in der er das Ziel der Mediaselektion darin sieht, ,jene Medien auszuwählen und die Zahl der Einschaltungen so festzulegen, dass ein möglichst hoher Anteil der Angehörigen der Zielgruppe die für notwendig erachtete Zahl an Kontakten erhält, wobei die Eignung der Werbeträger zur Präsentation der Werbebotschaft 
standen, eine spezifische Kommunikationsbotschaft zu transportieren, um ein gewünschtes Kommunikations- oder Verhaltensziel zu erreichen. ${ }^{244}$ Von besonderer Bedeutung ist hierbei der trägerspezifische Umfang der Darstellungsmöglichkeiten der Werbeinhalte. ${ }^{245}$ Das Mailing bietet in diesem Zusammenhang vielfältige Möglichkeiten der Darstellung der Werbebotschaft, welche durch beigefügte Informationsbroschüren sowie Warenproben ergänzt werden. Dies kann u. U. zu einer längeren und intensiveren Auseinandersetzung mit dem Direktmarketing-Stimulus führen. ${ }^{246}$ Demgegenüber ermöglicht der Einsatz von Emails die Verwendung von multimedialen Inhalten sowie das Beifugen von Filmen oder sonstigen elektronischen Daten, was ebenfalls einen positiven Einfluss auf Aufmerksamkeit und Betrachtungsdauer besitzen kann. ${ }^{247}$ Den multimedialen Darstellungsmöglichkeiten der Email stehen bei der SMS gegenwärtig noch technische Restriktionen gegenüber, wie bspw. eine Beschränkung der Länge der Textnachrichten, die einen vielfältigeren Einsatz möglich machen würden. ${ }^{248}$ Es kann daher angenommen werden, dass insbesondere die Rezipienten von SMS - sollten sie am beworbenen Leistungsangebot Interesse zeigen - zusätzliche Informationen beim Unternehmen anfordern werden.

zu berücksichtigen ist." Schweiger, G., Grundlagen der Streuplanung, in: Kaiser, A. (Hrsg.), Werbung. Theorie und Praxis werblicher Beeinflussung, a. a. O., S. 156.

$244 \mathrm{Vgl}$. Meffert, H., Marketing. Grundlagen marktorientierter Unternehmensführung, a. a. O., S. 697. Im Fall des Direktmarketing-Stimulus bezieht sich die Eignung der einzusetzenden Instrumente auf die Dimension des Botschaftstransportes und hängt u. a. davon ab, welche Funktionen das Medium im Rahmen des Kaufentscheidungsprozesses besitzt, wie hoch der Schwierigkeitsgrad der Kommunikation aus Sicht des Konsumenten mit diesem Instrument ist, welches Image das jeweilige Medium besitzt, welches die spezifischen Nutzungspotenziale beim Empfänger bzw. welche Kosten auf Seiten des Unternehmens entstehen usw. Dallmer nennt im Zusammenhang mit den Funktionen des Mediums die Frage nach einer statusverleihenden bzw. risikominimierenden Funktion. Vgl. Dallmer, H., Erfolgsbedingungen der Kommunikation im Direct-Marketing, a. a. O., S. 80.

245 In diesem Zusammenhang untersuchte Rogers im Rahmen einer VerbraucherLangzeitstudie die Wirkungen bei Erhalt von Briefwerbung beim Konsumenten. Vgl. Rogers, J. L., Consumer Response to Advertising Mail, in: Journal of Advertising Research, Vol. 29, No. 6, 1989, S. 18-24.

246 Hierbei wird häufig der Begriff des „Verstărkers“ benutzt. Vgl. Kirchner, G., Sobeck, S., Lexikon des Direktmarketing, a. a. O., S. 422; Holland, H., Direktmarketing, 2. Aufl., München 2004, S. 296 ff.

247 Vgl. zu den Möglichkeiten der Email-Gestaltung bspw. Aschoff, M., Professionelles Direkt- und Dialogmarketing per E-Mail, a. a. O.

248 Dies bezieht sich auf den herkömmlichen SMS-Standard, der eine Begrenzung auf 160 Zeichen besitzt. Insbesondere neue Standards lassen hier eine Erweiterung der Möglichkeiten erwarten. Vgl. Deutscher Direktmarketing Verband (Hrsg.) MobileMarketing - Dialog on Tour, DDV Best Practice Guide Nr. 7, Mainz 2003, S. 8. 
Während die potenziellen Gestaltungsparameter der einzelnen Medien inkl. der Darstellungsform den Mediaplanern bereits vor der Aussendung bekannt sind, zeichnen sich die einzelnen Medien durch eine unterschiedlich gute Steuerbarkeit der Kontaktsituation aus. So wird die Verarbeitung der Werbebotschaft beim Rezipienten durch die trägerspezifische Nutzungssituation und den zumeist trägerspezifischen Nutzungszeitpunkt determiniert. ${ }^{249}$ Ohne die genauen personenbezogenen Umstände der ,,medienspezifischen Empfangssituation“250 zu kennen, kann jedoch vermutet werden, dass durch die notwendigen technischen Voraussetzungen des Email-Empfangs die Kontakt- und damit die primäre Wirkungssituation an einen Computer gebunden ist, während beim Mailing der Erstkontakt je nach privater oder beruflicher Ausrichtung zu Hause oder am Arbeitsplatz erfolgt. ${ }^{251}$ Am schlechtesten lässt sich der Wahrnehmungsort von SMS prognostizieren, da der Empfang an keinen spezifischen Ort gebunden ist und damit generell an jedem beliebigen Ort stattfinden kann.

Die trägerspezifischen Merkmale sowie die daraus resultierenden unterschiedlichen Nutzungssituationen können aber - unabhängig vom Trägermedium - dazu führen, dass der Direktmarketing-Stimulus den Rezipienten in einer ungünstigen Situation erreicht. ${ }^{252}$ In Verbindung mit der Tatsache, dass der Konsument zum gegenwärtigen Zeitpunkt aufgrund der weiten Verbreitung in der Praxis an den Erhalt von Werbemailings und Werbe-Emails, aber noch nicht an den Erhalt von Werbe-SMS gewöhnt ist, sondern diese vielmehr als störend empfindet, besteht daher insbesondere bei SMS die Gefahr, Reaktanzen beim Rezipienten auszulösen. ${ }^{253}$

$249 \mathrm{Zu}$ trennen ist die medienspezifische Nutzungssituation von allgemeinen situativen Faktoren während des Kontaktes, die sich durch Personeneinflüsse oder andere nicht-trägermedienspezifische Einflussfaktoren unabhängig vom eingesetzten Medium ergeben können. Der Einfluss sozialer (z. B. Einzel- vs. Gruppenempfang), sozioökonomischer (z. B. von der Familienphase abhängige Faktoren) und psychologischer Wirkelemente (z. B. Ablenkungseffekte) wird in Kap. B.3 näher beleuchtet.

$250 \mathrm{Vgl}$. Bessler, H., Bledjian, F., Systematik der Massenkommunikationsforschung, München, Basel 1967, S. 52.

251 Interessant erscheint in diesem Zusammenhang, dass Emails von nahezu jeder zweiten Person sofort oder innerhalb der ersten Stunden nach Erhalt gelesen werden. Vgl. Allensbacher Computer- und Technik-Analyse (ACTA 2003), Ungebrochene Dynamik der Internetnutzung, a. a. O.

$252 \mathrm{Vgl}$. Bamler, G., Zur Wirkung von Direkt-Werbung im Industriegüterbereich, a. a. O., S. $96 \mathrm{ff}$.

253 Dies liegt u. a. daran, dass im Hinblick auf SMS-Werbung vonseiten der Verbraucher noch keine Werbeüberflutung beklagt, wird wie dies bei Emails beim sog. Spamming der Fall ist. Zur intensiven Diskussion des Begriffs „Spam“ vgl. Aschoff, M., Professionelles Direkt- und Dialogmarketing per E-Mail, a. a. O., S. 137. Aber auch die klassische Postwurfsendung findet immer noch Beachtung. Vgl. GfK (Hrsg.), GfK 
Es ist daher anzunehmen, dass der Einsatz der verschiedenen Trägermedien bei gleichen Angeboten und Zielgruppen aufgrund der beschriebenen Aspekte zu unterschiedlichen Responsequoten führt; es ist weiterhin anzunehmen, dass die Frage, ob letztendlich auch ein Kauf erfolgt, allerdings nur partiell vom eingesetzten Trägermedium abhängt. ${ }^{254}$ Insofern können zwar in Abhängigkeit des Trägermediums unterschiedliche Response- und Kaufquoten erreicht werden, das Verhältnis der Kaufquote gegenüber der Responsequote scheint aber unabhängig vom eingesetzten Trägermedium und damit über die Instrumente tendenziell konstant zu sein.

Neben diesen Faktoren, die sich primär auf die Effektivität der Instrumente beziehen, sind unterschiedlich hohe Kosten für die Gestaltung und Versendung der betrachteten Trägermedien im Rahmen des Effektivitäts- und Effizienzvergleichs erkennbar. ${ }^{255}$ Das Mailing kann aufgrund der relativ hohen Materialkosten als das vergleichsweise teuerste Trägermedium angesehen werden, wobei die absolute Höhe der Gesamtkosten stark von der Ausgestaltung und ggf. beigefügten Verstärkern wie Broschüren oder Warenproben abhängig ist. ${ }^{256}$ Tendenziell werden die Kontaktkosten für Emails und SMS geringer ausfallen als bei Mailings. Aufgrund der Vielzahl potenzieller Einflussfaktoren auf die Wirkung der Medien bei den Rezipienten sowie die mit der Konfiguration, Gestaltung und dem Versand verbundenen Kosten und nicht zu verallgemeinernder Kostendegressionseffekte sind Aussagen über die Effizienz kaum möglich und wenig plausibel.

Direktmarketing Panel, in: GfK Newsletter 02/2003, Nürnberg 2003. So zeigen die Ergebnisse des Panels der GfK, dass die Attentionrate, d. h. der Anteil von Rezipienten, der eine Postwurfsendung öffnet und liest, zwar branchenspezifisch schwankt, aber insgesamt bei über $80 \%$ liegt. Starke Schwankungen lassen sich bspw. zwischen der Automobil- (91,6\%) und der Kosmetikbranche (66,0\%) feststellen. Vgl. GfK (Hrsg.), GfK Direktmarketing Panel, a. a. O., S. 4.

254 So wird im Regelfall die Responsequote als Indikator für das Interesse des Konsumenten angesehen. Die Kaufquote zeigt hingegen den Anteil der tatsächlich kaufenden Personen an den adressierten Personen. Nur wenn keinerlei Rückfragen stattfinden und der Kaufakt sofort bspw. per E-Commerce-Angebot durchgeführt werden kann, ergeben sich nahezu identische Response- und Kaufquoten. Dieser Fall ist allerdings nicht als typisch anzusehen, insbesondere wenn die Bestellungen über ein Call-Center abgewickelt werden.

255 Vgl. Dallmer, H., Erfolgsbedingungen der Kommunikation im Direct-Marketing, a. a. O., S. 172.

256 Die Versandkosten von Emails und SMS haben sich in den letzten 5 Jahren um mehr als $90 \%$ reduziert, während die Kosten für die Versendung von Mailings nahezu konstant geblieben sind. Vgl. Schefer, D., Medien des Direct Marketing im Intermedia-Vergleich, a. a. O., S. 123. 
Vor dem Hintergrund der steigenden Effektivitäts- und Effizienzorientierung im Direktmarketing werden daher neben instrumentespezifischen Besonderheiten im isolierten Einsatz auch zunehmend Fragen zum integrativen Einsatz verschiedener Direktmarketing-Instrumente diskutiert. ${ }^{257}$ Ausgangspunkt der Überlegungen ist hierbei die Annahme, dass durch den kombinierten Einsatz mehrerer Trägermedien eine Wirkungsverstärkung und damit ggf. auch eine Steigerung der Effizienz erfolgen kann. ${ }^{258}$ Die Höhe der Wirkungsverstärkung hängt maßgeblich vom Grad der „Orchestrierung der Marketing-Instrumente“ 259 ab, der sich über die formale, inhaltliche sowie zeitliche Integration determiniert. ${ }^{260}$ Beim Einsatz der vorliegenden Instrumente des Direktmarketing lassen sich unterschiedliche Arten des kombinierten Einsatzes unterscheiden. ${ }^{261}$ So findet vielfach der Einsatz mehrerer Direktmarketing-Instrumente ohne jegliche Differenzierung im Hinblick auf eine zeitliche Abstimmung statt. Aus Unternehmenssicht bilden diese Aktionen die unterste Komplexitätsstufe möglicher Kombinationsformen, im Rahmen derer lediglich der zeitgleiche Versand eines Angebotes über mehrere Direktmarketing-Instrumente erfolgt. ${ }^{262}$ Die übrigen Gestaltungsparameter sind hierbei inhaltlich und formell gleich gestaltet; die zusătzliche Wirkung und damit der Einfluss auf die Effektivität des Einsatzes ergibt sich primär durch den „erhöhten Werbedruck“ gegenüber dem isolierten Einsatz. ${ }^{263}$ In

257 Vgl. bspw. Andersen, H. et al., The CRM Handbook, Hellerup 1999, S. 51.

258 Zorn spricht hierbei von einem möglichen Gesamtergebnis der Kommunikation, das „größer ist als die Summe der Einzelwirkungen“. Zorn, D., Integrierte Kommunikation - Grundlagen und zukünftige Entwicklung, a. a. O., S. 53 ff. Vgl. hierzu auch Busch, R., Dögel, R., Unger, F., Integriertes Marketing, 3. Aufl., Wiesbaden 2001, S. 451.

259 Holland, H., Direktmarketing, 2. Aufl., München 2004, S. 44.

$260 \mathrm{Vgl}$. Bruhn, M., Kommunikationspolitik, a. a. O., S. 100 ff. Dem simultanen Einsatz mehrerer Medien wird aus Sicht der Praxis ein immer höherer Stellenwert eingerăumt. Vgl. Dettmar, H., Thema Mediamix wird wichtiger, in: Horizont, Nr. 30, 1998, S. 41; Gill, K. H., Optimierung durch Media-Mix-Strategien, in: Horizont, Nr. 24, 1997, S. 37.

$261 \mathrm{Im}$ Folgenden soll allerdings unter dem integrativen Einsatz der Einsatz verschiedener Instrumente verstanden werden. Der Fall der einfachen Wiederholung einer Werbebotschaft mit Hilfe des gleichen Instruments soll nicht betrachtet werden, zumal der integrative Einsatz unterschiedlicher Instrumente eine höhere Effektivität verspricht als die Wiederholung mit dem gleichen Medium. Vgl. Springer-Verlag (Hrsg.), Lernprozesse im Test, aus der Reihe „Marken im Bild“, o. J., o. O.

262 So werden von einigen Unternehmen zeitgleich Emails und SMS versendet. Vgl. bspw. die Direktmarketing-Aktionen der Lufthansa AG.

263 Der Begriff des Werbedrucks im Kontext des Direktmarketing bezeichnet i. d. R. die Intensität bzw. Stärke eines Direktmarketing-Stimulus. Hierbei wird der Werbedruck durch vielfältige Faktoren, wie bspw. der Art der Werbeträger, der Anzahl von Schaltungen sowie der zeitliche Distanz zwischen zwei Schaltungen, beeinflusst. Zur Be- 
diesem Zusammenhang lassen sich als mögliche Zielwirkungen beim Rezipienten eine erhöhte Kontakt- sowie ggf. eine erhöhte Response- und Kaufwahrscheinlichkeit vermuten. ${ }^{264}$

In vielen Fällen reicht der zeitgleiche Versand mehrerer Werbebotschaften mittels unterschiedlicher Instrumente allerdings nicht zur Erreichung der angestrebten Effektivitätssteigerung aus. ${ }^{265}$ So ist es möglich, dass der eigentliche Kaufakt häufig erst am Ende einer mehrstufigen Kette von unterschiedlichen Direktmarketing-Aktionen steht, im Rahmen derer mehrfache, auch zeitversetzte Kontakte zwischen einem konkreten Angebot und dem Rezipienten stattfinden. ${ }^{266}$ Sog. mehrstufige Direktmarketing-Aktionen finden seit mehreren Jahren zunehmend Verwendung. ${ }^{267}$ Im Rahmen der Gestaltung dieser hat es sich als zunehmend effektiv erwiesen, im Zuge nachfolgender Stimuli auf vorangegangene Stimuli explizit Bezug zu nehmen. ${ }^{268}$ Ein mehrstufiges Vorgehen hat - im Vergleich zum zeitgleichen Aussenden eines Angebots mit Hilfe mehrerer Direktmarketing-Instrumente - den Vorteil, dass weitere Aktionen in Abhängigkeit des Erfolges vorheriger Stufen eingeleitet und inhaltlich abgestimmt werden können. ${ }^{269}$ Der positive Einfluss mehrstufiger Aktionen für die Effektivität konnte vielfach nachgewiesen werden. ${ }^{270}$

urteilung des Werbedrucks wird in der Unternehmenspraxis üblicherweise ein Vergleich mit Maßnahmen der Konkurrenz herangezogen. Vgl. Dögl, R., Unger, F., Taschenbuch der Werbepraxis, Heidelberg 1995, S. $134 \mathrm{ff}$.

264 Vgl. Mayer, H., Illmann, T., Markt- und Werbepsychologie, a. a. O., S. 499 f. Gleichzeitig können sich durch eine steigende Anzahl von Werbemittelkontakten die Erinnerung, die Einstellung sowie die Kaufabsicht verändern. Ray/Sawyer bestätigten nicht nur diese Annahme, sondern verglichen auch die Auswirkungen einer steigenden Anzahl von Werbemittelkontakten bei shopping und convenience goods. Vgl. Ray, M. L., Sawyer, A. G., Repetition in media models. A laboratory technique, in: Journal of Market Research, February 1971, S. 20-29.

$265 \mathrm{Vgl}$. zu den grundlegenden Problemen koordinierter Werbemaßnahmen bspw. Esch, F.-R., Wirkung integrierter Kommunikation. a. a. O., S. 7 ff.

266 Vgl. Mayer, H., Illmann, T., Markt- und Werbepsychologie, a. a. O., S. 543.

267 Vgl. Holland, H., Direktmarketing, 2. Aufl., München 2004, S. 61 ff. Der kombinierte Einsatz mehrerer Direktmarketing-Instrumente wird häufig auch unter dem Begriff der Crossmedia-Strategien subsumiert. Mittlerweile lassen sich unter diesem Begriff zunehmend komplexere Kampagnen identifizieren, die vor allem im Rahmen des CRM und ähnlichen Kundenbindungsaktivitäten eingesetzt werden.

268 Hierbei entscheiden vor allem die Anzahl der Wiederholungen sowie die zeitliche Abstimmung über die Effektivität einer Maßnahme. Vgl. bspw. Ray, M. L., Sawyer, A. G., Repetition in media models. A laboraty technique, a. a. O., S. 240.

269 In Zusammenhang mit mehrstufigen Aktionen müssen allerdings auch sog. WearoutEffekte beachtet werden, die dazu führen, dass es nach Überschreitung eines Idealpunkts der Werbewirkung durch zusätzliche Wiederholungen zu einer Übersättigung (Wearout) kommt. Unter bestimmten Umständen kann eine ablehnende Haltung 
In diesem Kontext haben sich verschiedene Formen mehrstufiger Direktmarketing-Aktionen herauskristallisiert, die sich hinsichtlich der Reihenfolge der eingesetzten Instrumente sowie inhaltlicher Werbeaussagen unterscheiden.271 Häufig findet ein Erstkontakt zwischen Konsument und Unternehmen auf Basis einer schriftlichen Ansprache unter Einsatz von Mailings statt. Sollten sich vonseiten des Rezipienten keine Reaktionen zeigen, wird dieser mit Hilfe von aktivem Telefonmarketing einen zweites Mal angesprochen. ${ }^{272}$ Hierbei kann es sein, dass der Call-Center-Mitarbeiter ggf. auch nach den Gründen für die ausgebliebene Reaktion fragt. Aber auch die Integration des Internet sowie des EmailMarketing in mehrstufige Direktmarketing-Aktionen wird vonseiten der Praxis aufgrund der kostengünstigen Einsatzmöglichkeiten zunehmend forciert. ${ }^{273}$

Die Frage nach dem Einfluss des integrierten Einsatzes verschiedener Direktmarketing-Instrumente auf die Effektivität und Effizienz kann zum aktuellen Zeitpunkt nur in Ansätzen beantwortet werden. ${ }^{274}$ Es ist jedoch zu vermuten, dass

gegenüber einem Unternehmen und dessen werblichen Aktivitäten zu heftigen Reaktanzen auf Seiten der Empfänger führen. Vgl. zum idealtypischen Verlauf einer Werbewirkungskurve Corkindale, D., Newall, J., Advertising thresholds and wearout, in: European Journal of Marketing, Vol. 12, No. 5, 1978, S. 332. In diesem Zusammenhang lassen sich vielfältige Untersuchungen identifizieren, die sich mit der Responsewahrscheinlichkeit auf mehrmalige Spendenanfragen für wohltätige Zwecke beschäftigen. Vgl. Diamond, W. D., Noble, S. M., Defensive Responses to Charitable Direct Mail Solicitations, a. a. O., S. 2-12.

270 Vgl. Striegl, T., Effizientes Direktmarketing, a. a. O., S. 173 ff. Loro zeigt unterschiedliche Einsatzmöglichkeiten von Mailings im B2B-Bereich in Kombination mit dem Einsatz des Internet. Vgl. Loro, L., No, Direct Mail is not Dead, in: Business Marketing, Vol. 82, No. 10, S. 27 und 29.

$271 \mathrm{Vgl}$. zu einem Überblick der Möglichkeiten Holland, H., Direktmarketing, 2. Aufl., München 2004, S. 61 ff. Ein typisches Beispiel einer komplexen mehrstufigen Direktmarketing-Konzeption findet sich bei Kotler/Bliemel. Vgl. hierzu Kotler, Ph., Bliemel, F., Marketing-Management. Analyse, Planung, Umsetzung und Steuerung, 8. Aufl., Stuttgart 1995, S. 1098.

272 Dass der Einsatz mehrstufiger Direktmarketing-Aktionen von Bedeutung für die Effektivität einer Aktion ist, zeigte bspw. Belz am Beispiel der Eurocard. In einem Experiment führte der integrierte Einsatz von klassischem Mailing und Telefonmarketing zu einer Steigerung des Absatzerfolgs. Vgl. Belz, Ch., Strategisches Direktmarketing, a. a. O., S. $54 \mathrm{ff}$. Jedoch wurde nicht untersucht, ob der zeitgleiche Versand gekoppelt mit einem entsprechenden Angebot, nicht die gleiche oder sogar eine höhere Wirkung gezeigt hätte.

273 Vgl. Hünerberg, R., Bedeutung von Online-Medien für das Direktmarketing, in: Link, J. (Hrsg.), Wettbewerbsvorteile durch Online Marketing. Die strategischen Perspektiven elektronischer Märkte, 2. Aufl., Berlin, Heidelberg, New York 2000, S. 129.

274 Als zentrales Defizit der bisherigen Forschungsbemühungen lassen sich zum einen die noch fehlenden Kombinationsmöglichkeiten von zwei oder mehr Instrumenten 
auch im Rahmen des Direktmarketing-Einsatzes die Integration mehrerer Instrumente einen positiven Einfluss auf die Effektivität besitzt. Demgegenüber lassen sich insbesondere aufgrund der vielfältigen Kombinations- und Ausgestaltungsmöglichkeiten kaum Aussagen über die Effizienz treffen. Aufgrund ihrer geringen Kontaktkosten erscheint allerdings unter Effizienzgesichtspunkten insbesondere die Kombination von Email und SMS von besonderem Interesse.

\subsubsection{Rückkanalmedium}

Einen wichtigen Gestaltungsparameter sämtlicher Direktmarketing-Stimuli stellt das Rückkanalmedium dar. Die zumeist als Response-Elemente bezeichneten Medien bieten dem Rezipienten des Direktmarketing-Stimulus die Möglichkeit, mit dem Absender in Kontakt zu treten. In Abhängigkeit der Ausgestaltung des Responsekanals werden die Reaktionen des Rezipienten in schriftlicher (per Post, Email oder SMS) oder mündlicher Form (per Anruf) registriert. Aus Sicht der Rezipienten kann es sich bei dem Inhalt des Responses bspw. um die Zusendung von Informationen im Rahmen von Gewinnspielen oder um Zusendung von direkten Bestellungen handeln. ${ }^{275}$

Als ein zentraler Vorteil des Direktmarketing gegenüber den klassischen Kommunikationsinstrumenten wurde die genaue Zuordnung von Stimulus und Rezipientenverhalten hervorgehoben, welche letztendlich mit Hilfe des Rückkanalmediums erfolgt. ${ }^{276}$ Vielfach beeinflusst daher die Auswahl des Trägermediums gleichermaßen auch die Auswahl eines spezifischen Rückkanals, da sich i. d. R. bei Verwendung desselben Trägermediums Kostenvorteile generieren lassen. ${ }^{277}$ Als potenzieller Rückkanal für die unterschiedlichen Medien erscheinen somit zunächst sämtliche Trägermedien des Direktmarketing einsetzbar, die diese Zuordnung zulassen. In den meisten Fällen kommt daher zunächst das Träger-

erkennen. Gleichzeitig werden die Instrumente nur auf Basis einzelner Zielgrößen verglichen. Vgl. Schultz, D. E., Kitchen, P. J., Integrated Marketing Communications in U.S. Advertising Agencies: An Exploratory Study, in: Journal of Advertising Research, Vol. 37, No. 5, September-October 1997, S. 7-18.

$275 \mathrm{Zu}$ den typischen Response-Elementen zählen hierbei insbesondere bei Mailings Rücksendekarten mit Rückumschlägen, Antwortkarten sowie Fax-Bestellscheine. Vgl. auch Kirchner, G., Sobeck, S., Lexikon des Direktmarketing, a. a. O., S. 48 ff. Bei den elektronischen Medien stellen die Trägermedien des Direktmarketing selbst das Antwortmittel dar, wie bspw. eine Antwort-Email, Antwort-SMS etc.

276 Vgl. Ceyp, M., Erfolgskontrollen im Direktmarketing, a. a. O., S. 26-31.

277 Vgl. Tapp, A., Principles of Direct and Database Marketing, 2nd edition, Harlow, Essex 2000, S. 273 und 276. 
medium selbst in Betracht, das im Rahmen des Direktmarketing-Stimulus eingesetzt wird. ${ }^{278}$

Der Einfluss auf die Effektivität einer Direktmarketing-Maßnahme ergibt sich insbesondere durch den beim Rezipienten vermuteten Aufwand zur Initiierung des Responsevorgangs. So stellt das Responsemedium in den meisten Fällen in seiner Funktion als „Enabler“ der Responseweiterleitung lediglich eine Art „Hygienefaktor" dar, da eine Entscheidung für oder gegen die Reaktion auf den Direktmarketing-Stimulus in den meisten Fällen bereits im Vorfeld gefallen ist. Zum Aufwand zählen der mit der Nutzung verbundene monetäre Aufwand (z. B. durch notwendiges Frankieren des Rückumschlages oder durch Telefonkosten beim Anruf in einem Call-Center) sowie der persönliche Aufwand (zeitlich, physisch etc.). Bei Überschreiten einer kritischen Höhe kann der Aufwand den Responsevorgang negativ beeinflussen und ggf. zu einem Abbruch der Kommunikation führen. ${ }^{279}$ Die mit der Nutzung des Responsemediums verbundenen technischen Voraussetzungen für einen reibungslosen Ablauf des Responsevorgangs stellen aus Sicht des Rezipienten ebenfalls Hygienefaktoren dar, die unternehmensseitig bei „Versagen“ einen erheblichen negativen Einfluss auf die Effektivität und Effizienz besitzen können. So kann z. B. der Ausfall einer Buchungshotline in der Zeit nach dem Versand einer Werbe-Email einer Fluglinie für das Unternehmen einen unwiderruflichen Verlust von Umsätzen bedeuten. ${ }^{280}$

Vor diesem Hintergrund hat sich gezeigt, dass es sich positiv auf die Effektivität einer Direktmarketing-Maßnahme auswirken kann, wenn der Rezipient seinen „Response“ in Form von Anfragen oder Bestellungen „ohne Medienbruch“ an das Unternehmen weitergeben kann. ${ }^{281}$ So scheint aus Sicht der Rezipienten die Umgehung eines Medienbruchs einen Conveniencefaktor darzustellen, der

278 Vgl. Holland, H., Direktmarketing, 2. Aufl., München 2004, S. 315.

279 Mittlerweile gehören bspw. in einigen Branchen frankierte Rückumschläge zum Standard-Response-Element. Während branchenübergreifend bei der Versendung von Mailings in 26,8 \% der Fälle frankierte Rückumschläge beigefügt werden, liegt dieser Wert in der Banken- bzw. Finanzdienstleistungsbranche bei über $40 \%$. Vgl. GfK, Finanzdienstleister verzeichnen Anstieg, in: Deutscher Direktmarketing Verband (Hrsg.), Dialog, Heft Juli, 2003, S. 9.

280 Wird das Homeshopping-Angebot nicht auf anderen Kommunikationswegen beworben, werden die konkreten Produkte bzw. Dienstleistungen i. d. R. auch nicht nachgefragt. Es zeigt sich hierbei die besondere Relevanz des einmaligen Werbe-Stimulus bspw. einer Dauerwerbesendung.

281 So wird ein Medienbruch bspw. durch direktes Anklicken einer Email oder Anrufen der Absendernummer einer SMS vermieden. Insbesondere auf in einer Email eingebundene Links wird in vielen Fällen direkt zum Erhalt weiterer Informationen geklickt. Damit ausgelöste Bestellungen sind schneller, unmittelbarer und bequemer. Vgl. Hünerberg, R., Mann, A., Einsatzgebiete und -probleme des Internet im Marketing, in: Industriemanagement, Nr. 1, 1999, S. 31. 
die Verhaltensabsicht positiv beeinflussen kann. ${ }^{282}$ Allerdings wird ein Medienbruch häufig in Kauf genommen, wenn sich mit einem Wechsel des Mediums spezifische Vorteile ergeben, bspw. eine einfachere Responseerfassung wie im Fall des Einsatzes elektronischer Medien oder eine kostengünstigere Abwicklung der Responses. Die Frage nach der Auswahl eines geeigneten Responsekanals muss auch in Abhängigkeit der Produkt- bzw. Dienstleistungskategorie erfolgen, in der das Unternehmen tätig ist. ${ }^{283}$ So hängt die Festlegung eines geeigneten Responsemediums von der Art der beworbenen Produkte und Dienstleistungen, insbesondere von ihrer Komplexităt, d. h. aus Sicht des Rezipienten von deren Erklärungsbedürtigkeit, $a b .{ }^{284}$ Aus diesem Grund setzen immer mehr Unternehmen bei beratungsintensiven Dienstleistungen, wie Flug- und Finanzdienstleistungen, Call-Center im Rahmen des Responsemanagements von Direktmarketing-Aktionen ein. Als besondere Herausforderung an das Responsekanalmanagement ist hierbei nicht nur die steigende Zahl der Kommunikationskanäle in den letzten Jahren, sondern auch die steigende Zahl möglicher Responsekanäle zu konstatieren. ${ }^{285}$ Die sich aus den unterschiedlichen Kombinationsmöglichkeiten ergebende zunehmende Gefahr eines Medienbruchs führt bei fehlender Identität von Kommunikations- und Rückkopplungskanal zu zahlreichen Problemen im Rahmen der Erfolgskontrolle, wie bspw. der lückenlosen Identifikation der Kunden über sämtliche Kommunikations- und Responsekanäle. In vielen Fällen wer-

282 Vgl. Hünerberg, R., Bedeutung von Online-Medien für das Direktmarketing, in: Link, J. (Hrsg.), Wettbewerbsvorteile durch Online Marketing. Die strategischen Perspektiven elektronischer Märkte, 2. Aufl., Berlin, Heidelberg, New York 2000, S. 135.

$283 \mathrm{Vgl}$. zu den gegenwärtigen Einsatzbereichen des Direktmarketing u. a. Deutsche Post AG (Hrsg.), Direktmarketing Deutschland 2003, a. a. O., S. $27 \mathrm{ff}$.

284 Vor dem Hintergrund der steigenden Komplexität von Produkten und Dienstleistungen sowie dem zunehmenden Anteil an Retouren und dem damit verbundenen Handling von Rücknahmen und Beschwerden ist der Einsatz des Telefonmarketing in zahlreichen Branchen unumgänglich geworden. Während sich Outbound Calls im Rahmen der Neukundenakquisition noch immer als schwierig und im Rahmen des Privatkundengeschäfts als unzulässig erweisen, erscheinen gerade bei erklärungsbedürftigen Produkten Call-Center im Sinne von Inbound Calls als Responsemedium geeignet. Der positive Einfluss einer persönlichen Kommunikation zeigte sich in einer Untersuchung von Simmel/Berger, die einen positiven Zusammenhang zwischen Gesprächsführung und Spendenverhalten eruierten. Vgl. Simmel, L. L., Berger, P. D., The art of the ask: Maximizing verbal compliance in telefundraising, in: Journal of Interactive Marketing, Vol. 14, No. 3, 2000, S. 12-40 sowie die dort angegebene Literatur.

285 Vgl. Ceyp, M., Erfolgskontrollen im Direktmarketing, a. a. O., S. $26 \mathrm{ff}$. 
den komplexe EDV-Systeme zur notwendigen Erfolgsvoraussetzung für eine valide Messung der Effektivität. ${ }^{286}$

Der Einfluss des Responseelements auf die Effizienz des DirektmarketingEinsatzes kann je nach Auswahl und Gestaltung des Rückkanalmediums ebenso heterogen ausfallen wie die Auswahl und Gestaltung des Trägermediums. ${ }^{287}$ In diesem Zusammenhang sind für eine genaue Beurteilung vor allem die Kosten für die Bereitstellung des Responsemediums (Personalkosten im Call-Center, Druckkosten bei Rückumschlägen etc.) zu berücksichtigen.

\subsubsection{Gestaltungsbezogene Determinanten}

Im Rahmen der Werbemittelforschung werden die Auswirkungen der formalen und inhaltlichen Gestaltung der Werbestimuli auf die Beachtung und das Verständnis der Werbung seit Jahrzehnten untersucht. ${ }^{288}$ Ziel der meisten Forschungsbemühungen ist es, Erfolgsfaktoren der Gestaltung für eine bessere Aufnahme und Verarbeitung der Werbebotschaft abzuleiten, die in der Folge auch die Wahrscheinlichkeit der gewünschten Rezipientenreaktion erhöhen. Die Wirkung der Gestaltung des Direktmarketing hängt in besonderem Maße von der Kommunikationsmittelqualität der einzelnen Trägermedien $\mathrm{ab},{ }^{289}$ unter der die Summe aller formalen und inhaltlichen Kriterien verstanden wird, die die Gestaltung einer Kommunikationsbotschaft determinieren. ${ }^{290}$ Im Zusammenhang mit dem Direktmarketing sprechen Gottschling/Rechenbauer von der Herausforderung des „gehirngerechten Gestaltens“ des Direktmarketing-Stimulus. ${ }^{291}$

286 Vgl. bspw. Zorn, D., Integrierte Kommunikation - Voraussetzungen und Wirkungsweise, a. a. O., S. $816 \mathrm{ff}$.

287 So existieren umfangreiche Checklisten, die Kriterien zum Vergleich unterschiedlicher Responsekanäle bieten. Vgl. bspw. Schefer, D., Medien des Direct Marketing im Intermedia-Vergleich, a. a. O., S. $105 \mathrm{ff}$.

288 Ein Überblick über die vielfältigen Ansätze der Werbemittelforschung findet sich bei Mayer, H., Illmann, T., Markt- und Werbepsychologie, a. a. O., S. 476 ff. In diesem Zusammenhang stellen die Autoren die Frage: „Mit welchen gestalterischen Komponenten aus dem vielfältigen Repertoire an verbalen, visuellen, akustischen sowie u. U. auch olfaktorischen Stimuli und deren Kombinationen lassen sich die gesetzten Ziele am ehesten und effizientesten erreichen?“

$289 \mathrm{Vgl}$. Meffert, H., Marketing. Grundlagen marktorientierter Unternehmensführung, a. a. O., S. 697.

$290 \mathrm{Vgl}$. ebenda.

291 Vgl. Gottschling, St., Rechenbauer, H. O., Direktmarketing, a. a. O., S. 124 ff. Mit diesem Ausdruck betonen die Autoren die Relevanz gestaltungsbezogener Faktoren und deren Einfluss auf die Wahrnehmungs- und Verarbeitungsprozesse des Rezipienten, die im menschlichen Gehirn ablaufen. 
Mit Bezug auf die trägerspezifischen Merkmale ergeben sich bei den verschiedenen Direktmarketing-Instrumenten unterschiedliche formelle Gestaltungsmöglichkeiten und damit unterschiedliche Optimierungspotenziale, von denen ein Einfluss auf die Effektivität bzw. Effizienz vermutet werden kann. ${ }^{292}$ Während die klassischen (papierbasierten) Medien aufgrund ihrer physischen Existenz eine Vielzahl von Gestaltungsoptionen hinsichtlich Optik, Haptik und Olfaktorik bieten, sind die Möglichkeiten im Bereich der elektronischen Medien primär auf den graphischen und akustischen Bereich begrenzt. ${ }^{293}$

Die Analyse gestaltungsbezogener Determinanten im Rahmen des MailingEinsatzes konzentriert sich in erster Linie auf die formelle Gestaltung des Briefumschlages, des darin enthaltenen Werbebriefs sowie ggf. des Rückumschlages bzw. der Antwortkarte. ${ }^{294}$ Im Rahmen des trägerspezifischen Wirkungsprozesses steht zumeist die Gestaltung des Umschlages im Mittelpunkt der Betrachtung, da das Öffnen als notwendige Voraussetzung für die weiteren Reaktionen beim Re-

292 Vgl. Dallmer, H., Erfolgsbedingungen der Kommunikation im Direct-Marketing, a. a. O., S. 80. In diesem Zusammenhang darf die inhaltliche Gestaltung nicht mit der angebotsbezogenen Gestaltung, d. h. dem eigentlichen Leistungsangebot, verwechselt werden, welches im Rahmen von Kap. B.3.2 behandelt wurde. Vielmehr stellt die inhaltliche Gestaltung die codierte Überbringerin der Leistungskomponenten dar.

$293 \mathrm{Vgl}$. Schefer, D., Medien des Direct Marketing im Intermedia-Vergleich, a. a. O., S. 122.

294 Stellvertretend für eine Reihe von Autoren sei im Zusammenhang mit Mailings auf Hatch verwiesen, der eine umfassende Liste zur erfolgreichen Gestaltung von Mailings vorstellt. Der Autor identifiziert ca. 200 Determinanten, die einen Einfluss auf die Effektivität besitzen. Vgl. Hatch, D., The Ultimate Direct Mail Checklist, in: Target Marketing, Vol. 12, No. 8, 1998. Die Analyse des Autoren basiert auf der Auswertung eines Konsumenten-Panels, im Rahmen dessen verschiedene Gestaltungskategorien, wie bspw. das Format, die Größe, die enthaltenen Angebote, die Bestellmöglichkeit oder die Bezahloptionen, auf den Wirkungsgrad des Mailings analysiert wurden. Während die Untersuchung von Hatch zu den umfangreicheren gehört, lassen sich verschiedene Ausarbeitungen zu einzelnen Gestaltungsparametern finden. Eine ähnliche Untersuchung im Zusammenhang mit Mailings und Katalogen haben Hitchcock/Ragosin durchgeführt. Dabei verfolgten die Autoren das Ziel, die erfolgsrelevanten Gestaltungselemente von Mailings und Katalogen zu identifizieren, die im Rahmen der Ansprache des Konsumenten von diesem in besonderem Maße wahrgenommen werden. Die Autoren kommen zu dem Ergebnis, dass vor allem der Einsatz von Bildern und Photographien einen signifikant positiven Einfluss auf den Erfolg von Katalogen und Mailings besitzt. Vgl. Hitchcock, T., Ragosin, Y., Creating Power Pictures, in: Target Marketing, Vol. 21, No. 6, 1998, S. 54-57. Gleichzeitig werden aber auch die vorgelagerten Verarbeitungsstufen durch verschiedene Faktoren determiniert, die das Öffnen des Briefumschlages beeinflussen. Vgl. Vriens, M. et al., Conjoint Experiments for Direct Mail Response Optimization, a. a. O., S. 323-339. 
zipienten angesehen wird. ${ }^{295}$ Als besonders positiv wirken sich auf die „Öffnungswahrscheinlichkeit" normalgroße Briefumschläge sowie aufgeklebte Briefmarken. ${ }^{296}$ Der Gestaltung des Werbebriefes wird hierbei i. d. R. die meiste Aufmerksamkeit gewidmet, da von ihm der größte Einfluss auf die Effektivität vermutet wird, obwohl der Rezipient zunächst mit dem äußeren Teil des Mailings - dem Briefumschlag - in Kontakt kommt. Bei der Gestaltung des Werbebriefes haben sich insbesondere die graphische Anordnung der Textbausteine sowie die Platzierung eingebundener Bilder als relevant für die Wirkung erwiesen. ${ }^{297}$ Darüber hinaus können im Rahmen von Mailings Warenproben mitgeliefert werden, die einen positiven Einfluss auf das Rezipientenverhalten besitzen können. ${ }^{298}$

295 Vgl. Wulf, K. de, Hoekstra, J. C., Commandeur, H. R., The Opening and Reading Behavior of Business-to-Business Direct Mail, a. a. O., S. 135.

$296 \mathrm{Vgl}$. ebenda, S. $138 \mathrm{f}$.

297 Als eine der zentralen Arbeiten der deutschen Direktmarketing-Wissenschaft kann in diesem Zusammenhang die Arbeit von Vögele bezeichnet werden, der sich im Rahmen seiner 1976 entwickelten Dialog-Methode u. a. der Blickaufzeichnungskamera zur Optimierung von Mailings bedient. Vögele geht davon aus, dass sich die Reaktionen auf den Erhalt einer Postwurfsendung in verschiedenen Wellen abbilden lassen. So kommt es bspw. zur ersten „Ablagewelle“ des Mailings, wenn das Werbemedium nicht in den ersten 20 Sekunden die Aufmerksamkeit des Rezipienten auf sich zieht. In dieser Zeit entscheidet sich, ob sich der Rezipient mit dem Mailing weiterbeschäftigt, es wegwirft oder lediglich weglegt, um es zu gegebener Zeit wieder anzuschauen. Vgl. Kirchner, G., Sobeck, S., Lexikon des Direktmarketing, a. a. O., S. 10 ff; Vögele, S., Handbook of Direct Mail,: The Dialogue Method of Direct Written Sales Communication, New York 1992.

$298 \mathrm{Ihr}$ Einsatz ist an die Versendung eines physischen Trägermediums, wie bspw. das Mailing, gekoppelt. Vgl. Kirchner, G., Sobeck, S., Lexikon des Direktmarketing, a. a. O., S. 422. So nehmen im Rahmen von Produktneueinführungen Mailings mit angefügten Warenproben einen bedeutenden Stellenwert im Rahmen der Bekanntmachung ein. Fiore $/ Y u$ zeigten im Zusammenhang von Warenhauskatalogen, dass kostenlose Produktsamples einen positiven Einfluss auf die Einstellung gegenuber dem Produkt und die Kaufbereitschaft haben können. Vgl. Fiore, A. M., Yu, H., Effects of imagery copy and product samples on responses towards the product, in: Journal of Interactive Marketing, Vol. 15, No. 2, 2001, S. 37-47. Seaver/Simpson konnten diesen Zusammenhang in ihrem Experiment nicht bestătigen. Vgl. Seaver, B. L., Simpson, E., in: Journal of Direct Marketing, Vol. 9, No. 3, Summer 1995, S. 8-20. Vgl. hierzu auch Kwon, Y.-H., Paek, S. L., Arzeni, M., Catalog vs. non-catalog shoppers of apparel: Perceived risks, shopping orientations, demographics, and motivations, in: Clothing and Textiles Research Journal, Vol. 10, Nr. 1, 1991, S. 13-19. Vgl. zu den unterschiedlichen Formen der Warengutscheine bei SMS u. a. Hinrichs, Ch., Lippert, I., Kosten und Wirkungen mobiler Werbung, a. a. O., S. 276 f. Bspw. werden über SMS Gutscheine für Kinos oder Konzerte verschickt, die am Eingang zum preiswerteren Erwerb von Tickets berechtigen. In diesem Zusammenhang untersuchten Gordon/Tellerman den Response in Abhängigkeit von unterschiedlichen Mengen-Prämien und Prämien für eine zeitliche Beschleunigung des Responses. Vgl. 
Doch trotz des nachgewiesenen Einflusses der Gestaltung auf die Effektivität können nur in den wenigsten Fällen verallgemeinerungsfähige Aussagen getroffen werden. ${ }^{299}$ Vielmehr ergeben sich vor dem Hintergrund der angesprochenen Zielgruppen, der betrachteten Branche, des beworbenen Produkte bzw. Dienstleistungen etc. erhebliche Unterschiede. Die Nutzung von Mailings als Responseelement erfordert zudem eine Auseinandersetzung mit gestalterischen Aspekten von Rückumschlägen. Bei klassischen Mailings werden einer Sendung häufig vorgefertigte Briefumschläge als Response-Elemente beigefügt, von denen bei entsprechender Portoübernahme durch das Unternehmen ebenfalls ein positiver Einfluss auf die Effektivität ausgehen kann. ${ }^{300}$ Hierbei zeigten sich zudem Unterschiede in der Responsehöhe in Abhängigkeit der Form und der Farbe des Rückumschlages. ${ }^{301}$

Gordon, P. J., Tellerman, B. J., Impact of prompt-Response and Quantity Premiums on Direct-Mail Response Rates, in: Journal of Direct Marketing, Vol. 4, No. 3, S. 41 45. Bei Church findet sich eine entsprechende Zusammenfassung sämtlicher Untersuchungen bis zum Jahr 1993, die sich mit dem Einfluss des Incentives zur Steigerung der Responsequote beschäftigen. Vgl. Church, A. H., Estimating the Effect of Incentives on Mail Survey Resonse Rates: A Meta-Analysis, in: Public Opinion Quarterly, Vol. 57, 1993, S. 62. Zur Akzeptanz von Warenproben im Bereich von Mailings. Vgl. Deutsche Post AG (Hrsg.), Verbraucherbefragung zur Akzeptanz von Produktproben, unveröffentlichte Studie, Bonn 2000.

299 So wird immer wieder konstatiert, dass die identifizierten Erfolgsfaktoren vor dem Hintergrund des spezifischen Unternehmenskontextes individuell überprüft werden sollten. Vgl. Szugger, A., Text im Mailing, in: Holland, H. (Hrsg.), Das Mailing Planung, Gestaltung, Produktion, Wiesbaden 2002, S. $89 \mathrm{ff}$.

300 Für eine umfassende Übersicht zu potenziellen Gestaltungsparametern vgl. bspw. Gottschling, St., Rechenbauer, H. O., Direktmarketing, a. a. O., S. 72 ff. So wurde insbesondere bei kostenlosen Briefmarken ein positiver Einfluss auf das Responseverhalten verzeichnet und sich die Responsezeit durch einen beigefugten, freigestellten Umschlag signifikant erhöht. Als Grund furr die schnelle Antwort identifizierte Jardine den monetären Wert der Briefmarken, den es aus Sicht der Empfänger nicht zu verschenken galt. Vgl. Jardine, F., The Power of Promotional Stamps and Donors through these Powerful Response Boosters, in: Target Marketing, Vol. 20, No. 10, 1997. Für eine umfassende Übersicht zu potenziellen Gestaltungsparametern vgl. bspw. Gottschling, St., Rechenbauer, H. O., Direktmarketing, a. a. O., S. 72 ff.

301 In einer umfassenden Arbeit untersuchte Schoenleber über 30 unterschiedlich gestaltete Rückkanäle auf Basis von unterschiedlichen Rückantwortkarten, die sich im Hinblick auf Farbe, Typographie, Format etc. unterschieden und zeigte die Auswirkungen auf den Erfolg der Instrumente auf. Vgl. Schoenleber, H., Response-Building Reply Devices - more than 30 ways to make your reply mechanism work harder, in: Target Marketing, Vol. 20, No. 12, 1997. Darüber hinaus analysierte Bawa verschiedene Einflussfaktoren, die das Responseverhalten auf Coupons von Werbebriefen determinieren. Vgl. Bawa, K., Influences on Consumer Response to Direct Mail Cou- 
Auch die formelle Gestaltung elektronischer Trägermedien - insbesondere von Emails - ist in den letzten Jahren zunehmend zum Gegenstand der wissenschaftlichen Diskussion geworden. ${ }^{302}$ Das starke Wachstum von Werbe-Emails und die häufig zu beobachtende „Überschwemmung“ des Konsumenten mit emailbasierter Werbung hat aufseiten der Rezipienten zu einem selektiveren Umgang mit Emails geführt, ${ }^{303}$ der sich immer häufiger in der Abwehrstrategie des konsequenten Löschens von Werbe-Emails bereits vor dem Lesen niederschlägt. $\mathrm{Ob}$ eine Werbe-Email überhaupt gelesen wird, hängt daher mittlerweile bei der Mehrzahl der Rezipienten von der Kenntnis des Absenders ${ }^{304}$ sowie von der Frage $a b$, ob dem Versender der Email die Erlaubnis zum Bewerben grundsätzlich gegeben wurde. ${ }^{305}$ Insbesondere in Zusammenhang mit dem letztgenannten Aspekt kann eine Steigerung der Effektivität verbunden sein, wenn explizit nach der Erlaubnis zum Bewerben gefragt wurde. ${ }^{306}$ So werden permission-basierte Emails zumeist von den Empfängern erwartet und in den meisten Fällen auch gelesen. ${ }^{307}$ Gleichzeitig haben sich im Rahmen der Gestaltungsoptimierung von

pons: An Integrative Review, in: Psychology and Marketing, Vol. 13, No. 2, 1996, S. $129 \mathrm{ff}$.

$302 \mathrm{Vgl}$. Chittenden, L., Rettie, R., An evaluation of e-mail marketing and factors affecting response, a. a. O., S. 203-217.

303 Im Rahmen mehrerer Studien konnte belegt werden, dass die Öffnungsrate bei Emails unter $50 \%$ liegt. In Deutschland werden bspw. nur $35 \%$ der Emails geöffnet, auf $9,5 \%$ der Emails wird ferner der angegebene Link besucht. Vgl. hierzu bspw. Doubleclick, eMail Trend-Report 2003.

304 Insbesondere die Vertrautheit mit dem Adressaten, die sich in der Kenntnis des Absenders widerspiegelt, hat einen erheblichen Einfluss auf die Art und Stärke der Werbewirkung. Vgl. Steffenhagen, H., Wirkungen der Werbung, a. a. O., S. 23. Zu den grundlegenden Arbeiten zum Einfluss des Kommunikators auf die Effektivität des Direktmarketing vgl. bspw. Köhler, R., Der Einfluß des Kommunikators auf die Wirkung direkter Marktkommunikation, in: Dallmer, H. (Hrsg.), Direct Marketing, 7. Aufl., a. a. O., S. 149-170.

305 Vgl. Schwarz, T., Permission Marketing macht Kunden süchtig, 2. Aufl., Würzburg 2000, S. $132 \mathrm{ff}$.

306 Der positive Effekt einer erlaubnisbasierten Werbung wurde vielfach nachgewiesen. Vgl. Kent, R., Brandal, H., Improving email response in a permission marketing context, in: International Journal of Market Research, Vol. 45, No. 3, 2003. Im Rahmen zahlreicher Studien konnte gezeigt werden, dass das Einholen der Permission einen positiven Einfluss auf den Response hat. So steigen die Responseraten von klassischen Printmailings von ca. $1 \%$ Response auf 5 bis $18 \%$ Response bei permissionbasierten Mailings, in Ausnahmefällen sogar auf bis zu 35\% Vgl. MessageMedia/emnid, unveröffentlichte Studie, 2001.

$307 \mathrm{Vgl}$. Kent, R., Brandal, H., Improving email response in a permission marketing context, a. a. O., S. 497 ff. Zum Konzept des Permission Marketing vgl. Godin, S., Permission Marketing. Turning strangers into friends, and friends into customers, New York 1999. 
Emails die Betreffzeile sowie die Anordnung potenzieller Links innerhalb einer Email als wichtige Determinanten der Effektivität erwiesen. ${ }^{308}$ Ebenso besitzt auch die Länge der Email, die Anzahl der eingebauten Bilder sowie mitgeschickte „Verstärker“309 einen Einfluss auf die Effektivität. ${ }^{310}$ Allerdings sind in diesem Zusammenhang - neben den Aussagen zu den Vorteilen einer permissionbasierten Versendung von Emails - nur vereinzelt allgemein gültige Aussagen zum Einfluss auf die Effektivität möglich, zumal die Gestaltung branchen- und produktabhängig ebenfalls stark variiert. ${ }^{311}$

Aufgrund der steigenden Anzahl verfügbarer Endgeräte mit erweiterten multimedialen Darstellungsmöglichkeiten scheint der Short Message Service (SMS) bereits in naher Zukunft über ein hohes werberelevantes Potenzial zu verfügen. ${ }^{312}$ Die derzeit verfügbaren Darstellungsmöglichkeiten bieten zwar noch begrenzte Gestaltungsmöglichkeiten; ${ }^{313}$ trotzdem haben sich bereits unterschiedliche For-

308 Vgl. Bachem, Ch., Erfolgskontrolle im E-Commerce, a. a. O., S. 59. Insbesondere vor dem Hintergrund des starken Wachstums sog. Spam-Mails hat sich das Interesse auf die formalen Kriterien zur Optimierung von Emails konzentriert. Im Allgemeinen bezeichnet der Begriff des Spam unerwünschte Werbe-Emails. Schätzungen zufolge werden täglich über $2 \mathrm{Mrd}$. Spam-Emails verschickt. Mit der Werbeinflation durch Spam-Mails gehen Schätzungen zufolge jährlich Produktivitätseinbußen i. H. v. 2,5 Mrd. Euro einher.

309 Vgl. Münstermann, M., Stand der Direktmarketing-Forschung und -Praxis in den USA, a. a. O., S. 1 ff. Bereits die Verwendung von HTML-Emails erweist sich in vielen Fällen reinen Textnachrichten gegenüber als effektiver. So liegen bspw. ca. dreimal so hohe Click-Through-Raten bei HTML- im Vergleich zu reinen Text-Emails vor. Vgl. Doubleclick, eMail Trend-Report 2003. Hierbei verspricht das Anhängen von Audio- und Videodateien neue Möglichkeiten zur Kundenbearbeitung bzw. ansprache und damit eine gezielte Differenzierungsmöglichkeit gegenüber anderen Unternehmen. Vgl. Gunn, E. P., Marketers keen on enriched email, in: Advertising Age, Vol. 71, No. 43, 2000, S. 512-514.

$310 \mathrm{Vgl}$. Chittenden, L., Rettie, R., An evaluation of e-mail marketing and factors affecting response, a. a. O., S. 203-217. In diesem Zusammenhang stellen Chittenden/Rettie in Anlehnung an das Wirkungsmodell von Vriens et al. ein Modell zur Wirkung von Emails auf. Die hierin beschriebene Wirkungskette mündet in einem Response, der durch die Stufen „Öffnen der Email“ und „Lesen der Email“ dem Modell von Vriens et al. nachempfunden wurde. Vgl. Vriens, M. et al., Conjoint Experiments for Direct Mail Response Optimization, a. a. O., S. 323-339.

311 So sind insbesondere die Produktkategorie sowie die Zielgruppe relevant für die konkrete Ausgestaltung. Vgl. Kent, R., Brandal, H., Improving email response in a permission marketing context, a. a. O.

312 Am wenigsten Beachtung in der wissenschaftlichen Diskussion wird derzeit noch gestalterischen Aspekten im Bereich der SMS-Werbung geschenkt.

313 Trotz dieser Beschränkung hat sich aber eine Vielzahl von unterschiedlichen Formen der SMS-Werbung herauskristallisiert. $\mathrm{Zu}$ den wichtigsten vgl. Hinrichs, $\mathrm{Ch}$., Lippert, I., Kosten und Wirkungen mobiler Werbung, a. a. O., S. 276 f. 
men von Werbe-SMS herauskristallisiert, wobei im Rahmen der Gestaltung weder farbliche Aspekte, wie bei Mailings und Emails, noch beigefügte Verstärker in Form von Informationsbroschüren und angehängten Audio-Dateien eine Rolle spielen. Zentraler Einflussfaktor auf die Effektivität bleibt zumeist das Einholen der Erlaubnis zum Bewerben des Rezipienten, aber auch gestalterische Aspekte bei der Responsekonfiguration sind zunehmend von Bedeutung. ${ }^{314}$

Aber nicht nur die formale sondern auch die inhaltliche Gestaltung des Werbemittels besitzt einen Einfluss auf den Wirkungsgrad. ${ }^{315}$ Insbesondere der Argumentation, der Form des Appells an den Rezipienten, der Glaubwürdigkeit sowie der Lesbarkeit der Aussagen wird eine hohe Bedeutung beigemessen. ${ }^{316}$ Immer häufiger wird hierbei auch der Zusammenhang zwischen der inhaltlichen Gestaltung und ökonomischen Größen analysiert, wobei sich jedoch zumeist kaum instrumenteübergreifende Aussagen treffen lassen. ${ }^{317}$ Eine Ausnahme bildet hierbei die im Rahmen des Einsatzes der betrachteten Instrumente Mailing, Email und SMS von vielen Unternehmen genutzte Möglichkeit zur kostengünsti-

$314 \mathrm{Vgl}$. zu ausgewählten gestalterischen Aspekten im Rahmen der Responsemöglichkeiten Holland, H., Direktmarketing, München 1992, S. 126 f.

315 Dallmer fasst hierbei unter der Kommunikationsaussage der Werbung formale und inhaltliche Faktoren zusammen und bezieht zusätzlich kontextuelle sowie technischformale Einflussfaktoren in seine Untersuchung mit ein. Vgl. Dallmer, H., Erfolgsbedingungen der Kommunikation im Direct-Marketing, a. a. O., S. $79 \mathrm{f}$.

316 Eine umfassende Arbeit findet sich bspw. bei Sherman/Greene/Plank, die in ihrer Studie analysieren, welchen Einfluss die Argumentation in Werbebriefen auf die Responsequote hat. Vgl. Sherman, E., Greene, J. N., Plank, R. E., Exploring Business-to Business Direct Mail Campaigns: Comparing One-Sided, Two-Sided and Comparative Message Structures, in: Journal of Direct Marketing, Vol. 5, No. 2, 1991, S. 25-30. Die Autoren kommen u. a. zu dem Ergebnis, dass Werbebriefe mit vergleichender Argumentation nicht zwangsläufig zu signifikant höheren Responseraten fuhrten als Werbebriefe mit einseitiger Argumentation. Eine Analyse des Einflusses der Länge der Nachricht auf die Responsequote wurde von Beard/Williams/Kelley durchgeführt. Vgl. Beard, J. D., Williams, D. L., Kelly, J. P., The Long versus the Short Letter, in: Journal of Direct Marketing, Vol. 5, 1991, S. 27-34.

317 Im Zusammenhang mit dem Fundraising analysierten bspw. Smith/Berger den Zusammenhang zwischen der inhaltlichen Gestaltung von Spendenbriefen und dem Responseverhalten. Vgl. Smith, J., Berger, P. D., The Impact of Direct Marketing Appeals on Charitable Marketing Effectiveness, a. a. O., S. 219-231. In der Untersuchung anhand einer Stichprobe von 18.144 Personen wurde deutlich, dass die auf dem Spendenbrief vorgeschlagene Spendenhöhe lediglich die Responserate beeinflusste aber nicht die tatsächliche Höhe der jeweiligen Spende. Hingegen haben umgekehrt Zusatzinformationen, wie bspw. statistisches Material zum Spendenverhalten, zwar einen signifikanten Einfluss auf die Höhe der Spende aber eben nicht auf die Responserate. 
gen Personalisierung der Anrede. ${ }^{318}$ Während unpersonalisierte Nachrichten meist der Weitergabe einer Information an möglichst viele Empfänger dienen, wird durch die Personalisierung einer Nachricht mit Hilfe einer persönlichen Anrede des Empfängers zumeist eine deutliche Aufmerksamkeitssteigerung erreicht, die sich auch positiv auf die Response- und Kaufquote auswirken kann. ${ }^{319}$

Im Hinblick auf die mit der gestalterischen Umsetzung verbundenen Kostenunterschiede zwischen den einzelnen Trägermedien lassen sich nur Tendenzaussagen treffen. So ist lediglich zu vermuten, dass die physischen Trägermedien wegen der höheren Stück- und Versandkosten sowie etwaiger beigefügter Warenproben zu höheren Aufwendungen führen. ${ }^{320}$ Die eventuell positiven Effekte auf die Effektivität müssen daher ebenfalls unter Effizienzgesichtspunkten analysiert werden. Insgesamt bleibt festzuhalten, dass sich im Hinblick auf die gestalterische Umsetzung des konkreten Direktmarketing-Stimulus eine Vielzahl von Determinanten identifizieren lassen, deren Analyse auf den ersten Blick interessant erscheint. Im Vergleich der Wirkungen der aufgezeigten gestalterischen Stellhebel zu den bereits vorgestellten zielgruppen-, angebots- und medienbezo-

318 Vor diesem Hintergrund wurden vor allem in der anglo-amerikanischen Literatur vermehrt die Wirkungen personalisierter und individualisierter DirektmarketingStimuli untersucht. So konnte im Rahmen verschiedener Arbeiten gezeigt werden, dass die Effektivităt der Direktmarketing-Instrumente durch zusătzliche Personalisierung und Individualisierung steigt. In diesem Zusammenhang analysierte Hennessy bspw. die Auswirkungen der Personalisierung von Mailings im Hinblick auf die Responsequote. Vgl. Hennessy, H. D., Comparing Personalized Name to Title Only Labels in a Business Mailing, in: Journal of Direct Marketing, Vol. 1, No. 2, 1987, S. 99-102. Der Autor konnte nachweisen, dass die Verwendung einer persönlichen Anrede im Vergleich zu einer unpersönlichen Ansprache einen signifikant positiven Einfluss auf den Response von Werbebriefen besitzt. In diesem Fall dürfen aber die zusätzlich gewonnenen Konsumentenreaktionen nicht durch die zusätzlichen Kosten überkompensiert werden. Vgl. auch Belz, Ch., Strategisches Direktmarketing, a. a. O., S. 63.

$319 \mathrm{Vgl}$. bspw. furr den Bereich der Emails Di Ianni, A., The e-business enterprise and the "Web First" principle of e-marketing, in: Journal of Interactive Marketing, Vol. 2, No. 3, 2000, S. 215-218. Für den Bereich von Mailings vgl. bspw. Helgesson, J. G., Voss, K. E., Terpening, W. D., Determinants of Mail-Survey Response: Survey Design Factors and Respondent Factors, a. a. O., S. 302-328. Holland weist aber auch darauf hin, dass eine persönliche Ansprache mitunter ,anbiedernd“ und „überspitzt“ wirken kann. Die Herausforderung aus Unternehmenssicht liegt demnach in der Einschätzung des richtigen „Personalisierungsgrades“. Vgl. Holland, H., Direktmarketing, 2. Aufl., München 2004, S. 310. Zur Abgrenzung der Begriffe Personalisierung und Individualisierung vgl. Pickhardt, M., Mehrwert durch Personalisierung, in: Dallmer, H. (Hrsg.), Das Handbuch Direct Marketing \& More, a. a. O., S. 969 ff.

$320 \mathrm{Vgl}$. Schefer, D., Medien des Direct Marketing im Intermedia-Vergleich, a. a. O., S. 123. 
genen Determinanten erscheint der absolute Einfluss tendenziell geringer auszufallen. Aus diesem Grund sollen die gestalterischen Determinanten im Folgenden von der Betrachtung ausgeschlossen werden.

\section{Analyse des Einflusses exogener Determinanten auf die Effektivität und Effizienz}

Neben den stimulusbezogenen Determinanten existieren mit den personen- und situationsbezogenen Determinanten exogene Faktoren, die einen Einfluss auf die Effektivität und Effizienz des Direktmarketing-Einsatzes besitzen können und deren Untersuchung vor dem Hintergrund einer experimentellen Wirkungsanalyse geboten scheint. ${ }^{321}$

\subsection{Personenbezogene Determinanten}

Die besondere Stellung des Rezipienten und seiner individuellen Merkmale ergibt sich im Direktmarketing nicht zuletzt durch den responseorientierten, zweiseitigen Kommunikationsprozess, den durch den Einsatz der Instrumente anstrebt wird. ${ }^{322}$ Im Rahmen dieser zweiseitigen Kommunikation erscheint es allerdings nahe liegend, dass unterschiedliche Personen auf gleiche Werbereize unterschiedliche Reaktionen zeigen. ${ }^{323}$ Vor diesem Hintergrund sollen im Folgenden die wichtigsten personenbezogener Determinanten identifiziert werden, die einen potenziellen Einfluss auf das Response- und Kaufverhalten ausüben können. ${ }^{324}$

321 In der wissenschaftlichen Literatur werden die exogenen Faktoren unterschiedlich systematisiert und bezeichnet. Während bspw. Steffenhagen Kontaktsituation sowie Personenmerkmale unterscheidet, bezeichnet Dallmer die personenbezogenen Einflussfaktoren als Rezipientenmerkmale und die situationsbezogenen Determinanten als Stör- und Stützungsfaktoren. Vgl. Steffenhagen, H., Wirkungen der Werbung, a. a. O., S. 13; Dallmer, H., Erfolgsbedingungen der Kommunikation im Direct-Marketing, a. a. O., S. 73.

$322 \mathrm{Vgl}$. Dallmer, H., Erfolgsbedingungen der Kommunikation im Direct-Marketing, a. a. O., S. 104.

$323 \mathrm{Vgl}$. Mayer, H., Reitmeier, A.-F., Empfängerabhängige Bedingungen werblicher Kommunikation, in: GfK (Hrsg.), Jahrbuch der Absatz- und Verbrauchsforschung, Jg. 27, Heft 3, 1981, S. 197; Steffenhagen, H., Wirkungen der Werbung, a. a. O., S. 17.

$324 \mathrm{Vgl}$. Dallmer, H., Erfolgsbedingungen der Kommunikation im Direct-Marketing, a. a. O., S. 104. Die personenbezogenen Determinanten unterscheiden sich hierbei 
Die personenbezogenen Determinanten lassen sich grundsätzlich in individuelle Zustandsmerkmale und in individuelle Verhaltensmerkmale unterteilen. $\mathrm{Zu}$ den wichtigsten personenbezogenen Zustandsmerkmalen zählen demographische, sozioökonomische sowie psychologische Merkmale. ${ }^{325}$ Der Bereich der individuellen Verhaltensmerkmale bezieht sich auf generelle Verhaltensmerkmale sowie das situationsspezifische Verhalten des Rezipienten. ${ }^{326}$

Der Schwerpunkt der Analyse personenbezogener Zustandsmerkmale lag in der Vergangenheit auf den demographischen und soziodemographischen Größen Alter, Geschlecht, Einkommen und Bildung. ${ }^{327}$ In Anlehnung an eine Pionierstudie von Gillett aus dem Jahr 1970 zeichneten sich die damaligen Versandhandelskäufer insbesondere durch eine überdurchschnittliche Bildung und ein überdurchschnittliches Einkommen aus. ${ }^{328}$ Als zentraler Grund hierfür wurde die in Abhängigkeit der sozialen Schicht angenommene höhere Kontaktintensität mit Direktmarketing-Medien aufgezeigt. ${ }^{329}$ Die Rolle des Alters wurde im Rahmen der allgemeinen Werbewirkungsforschung in vielen Studien ebenfalls untersucht. In diesem Kontext wird häufig auf Basis biologischer Überlegungen postuliert, dass sich mit zunehmendem Alter die Fähigkeit zur Informationsverarbeitung und -speicherung verlangsamt. ${ }^{330} \mathrm{Im}$ Gegensatz dazu scheint die Mediennutzung mit steigendem Alter, insbesondere im Bereich TV und Printmedien, zuzunehmen; ein Umstand, der die Gruppe der älteren Menschen auch vor dem Hintergrund ihrer wachsenden volkswirtschaftlichen Bedeutung für TV- und Printwerbetreibende im Zuge des demographischen Wandels zunehmend interessant erscheinen

von den momentanen und dauerhaften Gedächtnisinhalten des Rezipienten dadurch, dass sie selbst - entsprechend dem der Arbeit zugrunde liegenden Verständnis - keine Zielgrößen des Direktmarketing-Einsatzes darstellen, aber dennoch als Merkmale des Rezipienten einen Einfluss auf die Wirkungen eines Direktmarketing-Stimulus besitzen.

$325 \mathrm{Vgl}$. ebenda.

326 Vgl. Mayer, H., Illmann, T., Markt- und Werbepsychologie, a. a. O., S. 610 f.

327 Vgl. Meffert, H., Marketing. Grundlagen marktorientierter Unternehmensführung, a. a. O., S. $690 \mathrm{ff}$.

328 Vgl. Gillett, P. L., A Profile of Urban In-home Shoppers, in: Journal of Marketing, Vol. 34, No. 3, 1970, S. 43.

329 Vgl. IMR (Hrsg.), Bedeutung und Nutzeffekt der Direktwerbung, in: Direkt-Marketing, 10. Jg., 1975, S. 376-377, zitiert bei: Dallmer, H., Erfolgsbedingungen der Kommunikation im Direct-Marketing, a. a. O., S. 136.

330 Vgl. z. B. Salthouse, T. A., A theory of cognitive aging. New York 1985; Craik, F. I. M., Byrd, M., Aging and cognitive deficits: The role of attentional resources, in: Craik, F. I. M., Trehub, S. E. (Hrsg.), Aging and cognitive processes, New York 1982, S. $191 \mathrm{ff}$. 
lässt. ${ }^{331} \mathrm{Im}$ Bereich der Mediennutzung ist hierbei allerdings zwischen den klassischen und den neuen Medien zu differenzieren, da letztere insbesondere von jüngeren Zielgruppen genutzt werden. So ist anzunehmen, dass der Einsatz der Instrumente Email und SMS insbesondere bei jüngeren Rezipienten zu einer erhöhten Kontakt- und in der Folge zu einer höheren Responsewahrscheinlichkeit führt.

Auch im Hinblick auf das Geschlecht lassen sich Unterschiede in der Mediennutzung erkennen, die in konzeptioneller Hinsicht auf der Annahme basieren, dass sich das Informationsverarbeitungsverhalten sowie die Beeinflussbarkeit von Mann und Frau grundsätzlich unterscheiden. ${ }^{332}$ Während angenommen wird, dass sich Frauen hinsichtlich der Informationsverarbeitung intensiver und detaillierter mit Kommunikationsbotschaften auseinandersetzen ${ }^{333}$ und diese primär emotional, subjektiv und intuitiv verarbeiten, ${ }^{334}$ wird Männern eher eine analytische, logische und stärker objektive und selektiv geprägte Informationsverarbeitung unterstellt. ${ }^{335}$ Es wird allerdings angenommen, dass diese Unterschiede aufgrund der allmählichen Konvergenz der Geschlechterrollen im Laufe der Zeit zurückgehen. ${ }^{336}$ Weitere geschlechterspezifische Unterschiede lassen sich hinsichtlich der Mediennutzung feststellen. So äußern sich die geschlechterspezifischen Präferenzen bspw. in Bezug auf die Inhalte des Programmangebots. ${ }^{337}$ Hingegen zeigt sich ein zunehmend ausgeglichenes Bild bei der Nutzung einzelner Me-

331 Zudem gestaltet sich die Beeinflussbarkeit älterer Menschen durch die zumeist verfestigten Einstellungen schwieriger als die junger Menschen, was die Chance eines Spontankaufs i. d. R. reduziert. Vgl. Kroeber-Riel, W., Konsumentenverhalten, München 1975, S. 118; McGuire, W. J., Nature of attitudes and Attitude Change, in: Lindzey, G., Aronson, E. (Hrsg.), Handbook of Social Psychology, London 1969, S. 233-346.

332 Vgl. Darley, W. K., Smith, R. E., Gender differences in information processing strategies: An empirical test of the Selectivity Model in Advertising Response, in: Journal of Advertising, Vol. 24, No. 1, 1995, S. 41 und die dort zitierte Literatur.

333 Vgl. z. B. Mayer, H., Illmann, T., Markt- und Werbepsychologie, a. a. O. S. 614 und die dort zitierte Literatur.

334 Vgl. z. B. Broverman, D. M, Klaiber, E., Kobayashi, Y., Vogel, W., Roles of Activation and Inhibition in Sex Differences in Cognitive Abilities, in: Psychological Review, Vol. 75, No. 1, 1968, S. 23 ff.; Gleser, G., Gottschalk, L. A., Gottschalk, J. W., The Relationship of Sex and Intelligence to Choice of Words: A Normative Study of Verbal Behavior, in: Journal of Clinical Psychology, Vol. 15, No. 2, 1959, S. $182 \mathrm{ff}$.

335 Vgl. Broverman, D.M., Klaiber, E., Kobayashi, Y., Vogel, W., Roles of Activation and Inhibition in Sex Differences in Cognitive Abilities, a. a. O., S. $23 \mathrm{ff}$; Darley, W. K., Smith, R. E., Gender differences in information processing strategies, a. a. O., S. 70.

336 Vgl. Mayer, H., Reitmeier, A. F., Empfängerabhängige Bedingungen werblicher Kommunikation, a. a. O., S. $203 \mathrm{ff}$.

337 Männer präferieren im Fernsehen Sport-, Technik- und Finanzsendungen. 
diengattungen. Während die geschlechterspezifischen Unterschiede hinsichtlich der Internet- und Emailnutzung vor einigen Jahren noch erheblich waren, hat sich diese ebenso wie die Nutzung von Mobiltelefonen in den letzten Jahren zunehmend angeglichen. Für den Einsatz im Direktmarketing erscheint daher nicht die Mediengattung als differenzierend zwischen den Geschlechtern, sondern primär die beworbene Produktkategorie bzw. das beworbene Sortiment. Insgesamt ist hinsichtlich des Einflusses des Geschlechts zu konstatieren, dass immer noch keine allgemeingültigen Aussagen getroffen werden können. ${ }^{338}$ Mit Blick auf die heute bspw. im Versandhandel verwendeten Käufertypologien wird allerdings zunehmend deutlich, dass es in allen sozialen Schichten, unabhängig vom Einkommen und Bildungsniveau sowie unabhängig von Alter und Geschlecht direktmarketing-affine Personen gibt. ${ }^{339}$

Vor diesem Hintergrund und dem sich abzeichnenden Wertewandel ist es zudem in den letzten Jahren zunehmend zu einer Inkonsistenz der Konsummuster gekommen. ${ }^{340}$ Als Folge dieser Entwicklungen hat sich der Schwerpunkt des forscherischen Interesses in Richtung der psychologischen Kriterien verschoben. Diese Entwicklung kann als Resultat der Erkenntnis gesehen werden, dass in vielen Fällen der Erklärungsbeitrag von Persönlichkeitsmerkmalen zur Erreichung der gewünschten Direktmarketing-Wirkung höher ist als der von soziodemographischen Merkmalen. ${ }^{341}$ In die Kategorie der psychologischen Merkmale fallen sämtliche nicht beobachtbare, psychographische Konstrukte, die sich zum einen in allgemeine Persönlichkeitsmerkmale, zum anderen in produkt- und medienabhängige Merkmale unterteilen lassen. ${ }^{342} \mathrm{Zu}$ den allgemeinen Persönlichkeitsmerkmalen zählen überdauernde Charakteristika des Menschen wie Intelligenz, Selbstvertrauen und Risikobereitschaft. Direktmarketingunspezifisch gestaltet sich der Einfluss von Intelligenz, welche im Allgemeinen als begünstigender Faktor für das Verständnis einer Direktmarketing-Werbung angesehen werden kann, gleichzeitig aber auch die Resistenz gegenüber Beeinflussungsversuchen und Spontanhandlungen erhöht. ${ }^{343}$ Die gereingere Wahrscheinlichkeit eines Impulskaufs trifft insbesondere auf Personen mit einem hohen Selbstbewusstsein $\mathrm{zu}^{344} \mathrm{Im}$ Falle der Risikobereitschaft ist zum einen die Besorgnis des Konsu-

$338 \mathrm{Vgl}$. Darley, W. K., Smith, R. E., Gender differences in information processing strategies, a. a. O., S. 41 und die dort zitierte Literatur.

339 Vgl. Holland, H., Direktmarketing, 2. Aufl., München 2004, S. 75.

$340 \mathrm{Vgl}$. Meffert, H., Giloth, M., Aktuelle markt- und unternehmensbezogene Herausforderungen an die Markenführung, a. a. O., S. 99-132.

$341 \mathrm{Vgl}$. Unger, F. et al., Mediaplanung, a. a. O., S. 9.

342 Vgl. Meffert, H., Marketing, a. a. O., S. $192 \mathrm{ff}$.

$343 \mathrm{Vgl}$. Mayer, H., Illmann, T., Markt- und Werbepsychologie, a. a. O., S. 619.

$344 \mathrm{Vgl}$. ebenda, S. 625. 
menten zu nennen, dass persönliche Daten beim Kauf weitergegeben werden. ${ }^{345}$ Dieser Aspekt ist insbesondere in Zusammenhang mit Email-Werbung zu erwähnen. Demgegenüber zeigen sich risikoaffine Personen nicht nur der Werbung gegenüber aufgeschlossener, sondern schätzen auch das Kaufrisiko im Direktmarketing - trotz der fehlenden Möglichkeit der vorherigen Besichtigung der Ware kleiner ein als risikoaverse Personen. ${ }^{346}$

Weitaus wichtiger wird die Rolle der produkt- und medienspezifischen Merkmale eingeschätzt, unter denen u. a. Motive, Bedürfnisse und Interessen subsumiert werden ${ }^{347}$ und die sich auf die beworbenen Produkte bzw. eingesetzten Medien richten. Als zentraler Einflussfaktor dieser Kategorie wird vor allem das Interesse des Rezipienten an den beworbenen Produkten oder Dienstleistungen gesehen, da es sich zumeist in einem hohen Involvement des Rezipienten widerspiegelt. ${ }^{348}$ Ein hohes Interesse an einem Leistungsangebot fürt i. d. R. zu einer höheren Wahrscheinlichkeit der Beachtung einer Direktmarketing-Werbung sowie zu einer intensiveren Auseinandersetzung mit dem Botschaftsinhalt, welche weiterhin die Responseabsicht verstärken kann. ${ }^{349}$ Insbesondere bei Mailings konnte dieser Zusammenhang nachgewiesen werden. ${ }^{350}$

Gleichzeitig werden in Wissenschaft und Praxis vielfach Adressatenmerkmale diskutiert, die eine Beziehung zur Bildung und Aktualisierung von Motiven, Entscheidungskriterien und Einstellungen haben und damit ebenfalls indirekt auf das Kaufverhalten wirken. ${ }^{351}$ In diesem Zusammenhang wird darauf hingewiesen,

345 Vgl. Schweiger, A., Wilde, K.-D., Database-Marketing - Aufbau und Management, in Hilke, W. (Hrsg.), Direkt-Marketing, Wiesbaden 1993, S. 107.

346 Vgl. Bruns, J., Direktmarketing, a. a. O., S. 247.

347 Vgl. ebenda., S. 617.

348 Vgl. zum Begriff des Involvement u. a. Kroeber-Riel, W., Weinberg, P., Konsumentenverhalten, a. a. O., S. 92 f; Meffert, H., Marketing: Grundlagen marktorientierter Unternehmensführung, a. a. O., S. $111 \mathrm{f}$.

349 Vgl. Grimm, C., Tietz, W., Die Einstellung von Werbeempfängern zur Direktwerbung, Arbeitspapier Nr. 83, Lehrstuhl für Marketing der Universität ErlangenNürnberg, Nürnberg 1999, S. 32.

$350 \mathrm{Vgl}$. Deutsche Post AG (Hrsg.), Werbeerfolgsmessung von Werbesendungen, Direkt Marketing Monitor, Studie 4, Bonn 1997, S. 45. Dies trifft nach den Ergebnissen der Studie neben der allgemeinen Akzeptanz des Direktmarketing-Mediums auch auf die produktspezifische Akzeptanz zu. So konnte eine weitaus höhere Responsebereitschaft bei Personen mit überdurchschnittlicher ausgeprägter Akzeptanz im Vergleich zu Personen mit unterdurchschnittlich ausgeprägter Akzeptanz nachgewiesen werden.

351 In der klassischen Kommunikationsforschung findet in diesem Zusammenhang häufig der Begriff der Personenqualität Verwendung. Dieser beschreibt die Fähigkeit des Empfängers, Kommunikationsbotschaften aufzunehmen und diese in einem zweiten 
dass auch Direktmarketing-Wirkungen in hohem Maße von der Prädisposition des Rezipienten beeinflusst werden, die bereits vor dem zu analysierenden Kommunikationskontakt existieren. ${ }^{352}$ Auf die zentrale Bedeutung dieser Prädispositionen wird in einer Reihe von Studien aus dem Bereich der Werbewirkungsforschung verwiesen. ${ }^{353} \mathrm{Zu}$ den wichtigsten empfängerbezogenen Einflussfaktoren dieser Gruppe gehört die Einstellung des Rezipienten gegenüber dem Unternehmen, gegenüber dem Direktmarketing im Allgemeinen sowie gegenüber einzelnen Direktmarketing-Instrumenten im Speziellen. ${ }^{354}$ Unabhängig vom eingesetzten Trägermedium kann zunächst vermutet werden, dass eine positive Einstellung gegenüber dem Direktmarketing die Aufnahmebereitschaft für Direktmarketing-Angebote erhöht und daraus resultierend ein Response bzw. Kauf wahrscheinlicher wird. ${ }^{355}$ Negative Erfahrungen in der Vergangenheit resultieren zumeist in einer negativen Einstellung gegenüber dem Direktmarketing im Allgemeinen, die weiterhin dazu führt, dass Direktmarketing-Werbung von den Rezipienten als Belästigung aufgefasst und ggf. vor dem Öffnen weggeworfen bzw. gelöscht wird. ${ }^{356}$

Der Einfluss von Einstellungen gegenüber einzelnen Trägermedien besitzt im Direktmarketing ebenfalls eine besondere Relevanz. So wurde vielfach nachgewiesen, dass die Einstellung gegenüber Mailings keinen Einfluss auf die Öffnungswahrscheinlichkeit besitzt. ${ }^{357}$ Jedoch hat sich gezeigt, dass der sich an-

Schritt zu verarbeiten. Vgl. Meffert, H., Marketing. Grundlagen marktorientierter Unternehmensführung, a. a. O., S. 694.

$352 \mathrm{Vgl}$. Steffenhagen, H., Wirkungen absatzpolitischer Instrumente: Theorie und Messung der Marktreaktion, a. a. O., S. 87; Kroeber-Riel, W., Weinberg, P., Konsumentenverhalten, a. a. O., S. $494 \mathrm{f}$.

353 Vgl. z. B. Sujan, M., Consumer Knowledge: Effects on Evaluation Strategies Mediating Consumer Judgments, in: Journal of Consumer Research, Vol 12, No. 1, 1985, S. 31 ff.; Alba, J. W., Hutchinson, J., Dimensions of Consumer Expertise, in: Journal of Consumer Research, Vol. 13, No. 4, 1987, S. 411 ff.; Brucks, M., The Effect of Product Class Knowledge on Information Search Behavior, in: Journal of Consumer Research, Vol. 12, No. 1, 1985, S. 1 ff.

354 Vgl. u. a. Hermanns, A., Konsument und Werbewirkung, a. a. O., S. 134.

355 Gleichzeitig existieren aber auch Untersuchungen, bei denen eine negative Einstellung der Rezipienten zu einer besonders intensiven Beachtung der Werbung geführt hat. Der Grund hierfür liegt darin, dass diese dadurch versuchten, ihre negativen Meinungen zu bestätigen. Diese Ergebnisse bilden allerdings die Ausnahme. Vgl. Ernst, O., Meinungen über die Werbung - was bedeuten sie?, in: $\mathrm{ZV}+\mathrm{ZV}$, 71. Jg., Nr. 19/20, 1974, S. 514 ff.

356 Vgl. Akaah, I. P., Korgaonkar, P. K., Lund, D., Direct Marketing Attitudes, in: Journal of Business Research, Vol. 34, No. 3, 1995, S. 217.

357 Vgl. Wulf, K. de, Hoekstra, J. C., Commandeur, H. R., The Opening and Reading Behavior of Business-to-Business Direct Mail, a. a. O., S. 138. 
schließende Prozess der inhaltlichen Auseinandersetzung sowie das Responseverhalten maßgeblich durch die trägerspezifische Einstellung beeinflusst werden. ${ }^{358}$ Gleichzeitig kann sich diese nicht nur auf das zur Vermittlung der Werbebotschaft eingesetzte Instrument beziehen, da auch mit dem Rückkanal ein weiteres Medium im Einsatz ist, welches als potenzieller Einflussfaktor die Response- und Kaufwahrscheinlichkeit beeinflussen kann. ${ }^{359}$

Doch trotz der positiven Entwicklungen der Akzeptanz sowie des Images der verschiedenen Direktmarketing-Instrumente stehen immer noch viele Konsumenten dem Direktmarketing mit ablehnender Haltung gegenüber. ${ }^{360}$ Liegt eine ablehnende Haltung gegenüber einem spezifischen Medium vor, wird dies häufig kommuniziert bzw. gegenüber den Werbetreibenden dokumentiert. ${ }^{361}$ Die Missachtung solcher Hinweise kann u. U. zu erheblichen Reaktanzen gegenüber dem werbetreibenden Unternehmen führen. Die Gründe für die strikte Ablehnung von Direktmarketing-Werbematerialien sind hierbei vielfältiger Natur und können bspw. in nachbarschaftlichem Konformitătsdruck und Prestigekommunikation begründet liegen. ${ }^{362}$

$358 \mathrm{Vgl}$. ebenda, S. $138 \mathrm{ff}$.

359 Während die Einstellungen von Konsumenten gegenüber den klassischen Direktmarketing-Instrumenten schon vielfältig untersucht wurden, sind Untersuchungen zu den Einstellungen der Konsumenten gegenüber den neuen Medien, insbesondere der Internet-Werbung erst in den letzten Jahren zunehmend zum Gegenstand der Betrachtung geworden. In diesem Zusammenhang konnte die postulierte positive Korrelation von Einstellungen und Verhalten häufig bestätigt werden. Vgl. Schlosser, A. E., Shavitt, S., Kanfer, A., Survey of Internet users' attitudes toward Internet advertising, a. a. O., S. 1-21.

360 Obwohl das Image des Direktmarketing teilweise noch immer mit dem Image von Hauswurfsendungen gleichgesetzt wird, hat es sich im Zuge der letzten Jahrzehnte nicht zuletzt mit dem Aufkommen der neuen Medien - deutlich verbessert und steigende Akzeptanz in der Bevölkerung gefunden. Wăhrend bis Mitte der neunziger Jahre die überwiegende Zahl der Konsumenten Werbung durch DirektmarketingInstrumente ablehnten, ist die Zustimmung und damit auch der gewünschte Erhalt in den letzten Jahren stark gestiegen. Dies zeigt sich in der noch immer steigenden Anzahl von Personen, die sich in die sog. Robinson-Liste eintragen. Den in der Liste eingetragenen Personen werden von Seiten der direktmarketingaktiven Unternehmen keinerlei Werbematerialien zugeschickt.

361 Dies erfolgt bspw. durch die Anbringung entsprechender Schilder am Briefkasten,

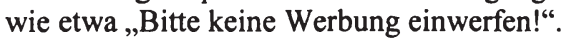

362 In diesem Zusammenhang untersuchten bspw. Weitkunat/Weitkunat anhand einer Stichprobe von 731 Wohneinheiten die Gründe für die Verweigerung von Werbeprospekten durch entsprechende Kennzeichnung der Briefkästen. Hierbei zeigte sich, dass nicht diejenigen Haushalte Werbeprospekte am ehesten verweigerten, die auf günstige Einkaufsgelegenheiten angewiesen waren. Es zeigte sich vielmehr, dass sozialpsychologische Ursachen für die Ablehnung verantwortlich waren. So wurde her- 
Während die beschriebenen Rezipientenmerkmale i. d. R. Zustandscharakter aufweisen, zählen zu den allgemeinen Verhaltensmerkmalen des Rezipienten „relativ zeitstabile Verhaltensweisen wie das Produktnutzungs-, das Kauf- und Innovationsverhalten sowie das Informationsverhalten.“"363 Insbesondere die Medienvertrautheit sowie das Responseverhalten auf bestimmte DirektmarketingStimuli in der Vergangenheit sind dabei als zentrale Treiber zu nennen. Es konnte gezeigt werden, dass insbesondere bei Mailings ein in der Vergangenheit überdurchschnittlich ausgeprägtes Bestellverhalten die Responsewahrscheinlichkeit auch in der Zukunft erhöht. ${ }^{364}$ Eine hohe Zufriedenheit mit früheren Leistungen kann hierbei als „Guthaben“ die Reaktion auf ein weiteres Angebot stimulieren und somit die Effektivität einer Maßnahme steigern. In diesem Zusammenhang hat sich ebenfalls gezeigt, dass das mehrmalige Zusenden der gleichen Direktmarketingangebote mitunter zum Erfolg führen kann. ${ }^{365}$ So verweisen verschiedene Autoren darauf, dass es im Rahmen der Durchführung einer Direktmarketing-Kampagne von besonderer Bedeutung für den Erfolg sein kann zu wissen, ob der potenzielle Empfänger einer Direktwerbung bereits Verwender

ausgearbeitet, dass nachbarschaftlicher Konformitätsdruck und Prestigekommunikation die Wahrscheinlichkeit signifikant erhöhten, dass eine Verweigerung der Werbeprospekte vorlag. In diesem Zusammenhang lassen sich weitere Untersuchungen zur Ablehnung von Werbeprospekten und korrespondierenden Fragestellungen identifizieren. Vgl. Weitkunat, R., Weitkunat, T., Effektivität von Direktmarketing: Sozialpsychologische Ursachen der Verweigerung von Prospekten durch Konsumenten, in: GfK (Hrsg.), Jahrbuch der Absatz- und Verbrauchsforschung, Jg. 4, 2001, S. 406419.

363 Mayer, H., Illmann, T., Markt- und Werbepsychologie, a. a. O., S. 626. Insgesamt wird den Verhaltensmerkmalen im Vergleich zu den Zustandsmerkmalen weniger Aufmerksamkeit geschenkt, da aus diesen Zustandsmerkmalen i. d. R. ein bestimmtes Verhalten resultiert, d. h. die genannten Faktoren ursächlich sind für das Zustandekommen.

$364 \mathrm{Vgl}$. Bamler, G., Zur Wirkung von Direkt-Werbung im Industriegüterbereich, a. a. O., S. 94. Insbesondere Erfahrungen können im Falle einer ,positiven Richtung“ auch wiederholt positive Reaktionen auslösen. Im Rahmen des Direktmarketing, bei dem häufig kein persönlicher, sondern lediglich ein medialer Kontakt zwischen Käufer und Verkäufer einer Ware oder Dienstleistung besteht, sind in der Vergangenheit gesammelte positive Erfahrungen von besonderer Bedeutung für das zukünftige Verhalten auf Unternehmensangebote.

$365 \mathrm{Vgl}$. hierzu auch die mehrstufigen Direktmarketing-Aktionen, die in den vorangegangenen Kapiteln diskutiert wurden. In diesem Zusammenhang lassen sich verschiedene Untersuchungen finden, die sich mit der Responsewahrscheinlichkeit auf mehrmalige Anfragen, bspw. für wohltätige Zwecke beschäftigen. Vgl. Diamond, W. D., Noble, S. M., Defensive Responses to Charitable Direct Mail Solutions, a. a. O., S. 2-12 sowie die dort angegebene Literatur. 
eines bestimmten Produktes oder einer Marke ist. ${ }^{366}$ Auch hier wird ein positiver Zusammenhang zur Responsewahrscheinlichkeit und zum Kaufverhalten angenommen.

In zahlreichen Untersuchungen konnte belegt werden, dass die Kenntnis personenbezogener Determinanten von Seiten der Unternehmen im Rahmen des Direktmarketing-Einsatzes gezielt zu einer Steigerung des Response- und Kaufverhaltens tragen kann. ${ }^{367}$ So ist es häufig unter ökonomischen Gesichtspunkten sinnvoll, vor der Durchführung einer Direktmarketing-Aktion zusätzliche Informationen über die Zielgruppe selbständig zu erheben bzw. die Informationen von externen Dienstleistern zu erwerben, um die eigene Datenbasis für eine gezieltere Marktbearbeitung anzureichern. ${ }^{368}$ Allerdings sei abschließend darauf hingewiesen, dass die personenbezogenen Faktoren einen entscheidenden Einfluss auf die Effektivität des Direktmarketing besitzen können. Hinsichtlich der diskutierten Faktoren fehlt es bislang immer noch an umfassenden Aussagen zur relativen Bedeutung der untersuchten Determinanten zueinander sowie in Relation zu den endogenen Determinanten. Erkenntnisse in diesem Bereich wären allerdings eine zentrale Voraussetzung für das Ableiten von Handlungsempfehlungen für die Praxis.

366 Insbesondere vor dem Hintergrund einer Neueinführung einer dem Empfänger bekannten bzw. im Haushalt verwendeten Markenfamilie oder Produktkategorie gehörenden Produktinnovation erscheint die Information von Bedeutung, da sich durch diese Empfängereigenschaft die Wahrscheinlichkeit des Interesses signifikant erhöht. Dies und andere relevante Informationen können bei Adressverlagen gekauft werden. So verfügen diese Unternehmen mittlerweile über große Datenbestände potenziell relevanter Informationen, die im Rahmen einer Mikrosegmentierung oder eines gänzlich individualisierten Angebotes eingesetzt werden können.

367 Vgl. Wheaton, J., How demographic and psychographic Database overlays can boost your Response, in: Target Marketing, Vol. 21, No. 10, 1998, S. 107 und 111.

368 Ein Zukauf von Informationen kann gezielt zur Anreicherung der eigenen Kundendatenbank beitragen und ermöglicht in einem zweiten Schritt ggf. den Aufbau detaillierter Kundenprofile, sowie Kundensegmente und in Einzelfällen auch die Anwendung statistischer Prognosemodelle zur Schätzung des möglichen Responses. Schlussendlich können die Investitionen in zusätzliche Kundendaten zu einer höheren Rentabilität sämtlicher Aktivitäten beitragen. Vgl. Lehr, G., Entscheidungsprozesse bei der Anmietung von Adressen im Consumer-Bereich, in: Dallmer, $\mathrm{H}$. (Hrsg.), Direct Marketing \& More, 8. Aufl., Wiesbaden 2002, S. 509-543. Aber auch klassische Media-Analysen, die bspw. zeigen, welche Medien von welcher Personengruppe zu welcher Zeit genutzt werden, enthalten vielfach schon die benötigten Informationen. Vgl. Unger, F. et al., Mediaplanung, a. a. O., S. 9. 


\subsection{Situative Determinanten}

Die Wirkung eines Direktmarketing-Stimulus wird durch eine Reihe kommunikationsexterner Stör- und Stützungsvariabeln beeinflusst, die unter dem Begriff der Situationsqualität subsumiert werden. ${ }^{369}$ Die situationsbezogenen Determinanten sind weder vom Unternehmen zu kontrollieren, noch können sie aufgrund ihres episodenhaften Charakters zu dauerhaften Persönlichkeitsmerkmalen gezählt werden. ${ }^{370}$ Im Allgemeinen fallen in diese Kategorie sämtliche Begleitumstände der Botschaftsaufnahme, ${ }^{371}$ die sowohl aus der konkreten Kontaktsituation mit dem Direktmarketingmedium als auch aus der komplexen Umwelt des Empfängers resultieren können. ${ }^{372}$ Dallmer nennt die Situation eine „Restkategorie“, unter der alle sonstigen Einflüsse zusammengefasst werden, die nicht dem Kommunikator, der Aussage, dem Medium oder dem Rezipient zugeordnet werden können. ${ }^{373}$ Grundsätzlich wird in diesem Zusammenhang die Hypothese erhoben, dass die Wahrnehmung und Verarbeitung von Werbeaussagen qualitativ, $d . h$. aus wirkungstheoretischer Sicht, umso effektiver abläuft, je weniger Stör- und Unterbrechungsquellen vorliegen. ${ }^{374}$

$\mathrm{Zu}$ den wichtigsten Situationsfaktoren gehören Störeinflüsse während der Botschaftsaufnahme durch andere Personen, andere Medien, Lärm etc. Als Einflussfaktoren können aber auch ein möglicher Zeitdruck des Empfängers, Ablen-

369 Vgl. Dallmer, H., Erfolgsbedingungen der Kommunikation im Direct-Marketing, a. a. O., S. 89.

370 Vgl. Lasslop, I., Effektivität und Effizienz von Events, a. a. O., S. 116. Die situationsbezogenen Determinanten können vom Unternehmen für gewöhnlich nicht beeinflusst werden, sie können aber mitunter prognostiziert und ihre Ausprägung eventuell in die Entscheidungen über die anderen Gestaltungsparameter einbezogen werden. $\mathrm{Zu}$ nennen sind hierbei räumliche, zeitliche und klimatische Bedingungen, genauso wie Einflussfaktoren, die sich aus der sozialen Umwelt des Empfängers ergeben.

$371 \mathrm{Vgl}$. Meffert, H., Marketing. Grundlagen marktorientierter Unternehmensführung, a. a. O., S. 696.

372 Vgl. Dallmer, H., Erfolgsbedingungen der Kommunikation im Direct-Marketing, S. 89.

373 Vgl. Dallmer, H., Erfolgsbedingungen der Kommunikation im Direct-Marketing, S. 89. Belk betont ebenfalls den „Sammelposten”-Charakter dieser Kateogrie und beschreibt die situationsbezogenen Determinanten als ,...all those factors particular to a time and place of observation which do not follow up from a knowledge of personal and stimulus (choice alternative) attributes and which have a demonstrable and systematic effect on current behavior". Belk, R. W., An Explanatory Assessment of Situational Effects in Buyer Behavior, in: Journal of Marketing Research, Vol. 11, 1974, S. 157.

374 Vgl. Hermanns, A., Konsument und Werbewirkung, a. a. O., S. 175. 
kungseffekte oder Gruppeneinflüsse wirken. ${ }^{375}$ Viele dieser Störfaktoren liegen dabei in sozioökonomischen Aspekten, wie bspw. der Familienphase oder den Wohnverhältnissen, begründet und werden hinsichtlich ihrer Wirkung auf die Reaktion eines Rezipienten häufig unterschätzt. ${ }^{376}$ Darüber hinaus kann die Aufnahme der Werbeaussage durch eigene Verhaltensaktivitäten, sog. Parallelbeschäftigungen, beeinflusst werden. ${ }^{377}$ Dies muss nicht immer zu einem die Wirkung des Werbereizes abschwächenden Impuls führen, sondern kann sich auch positiv auswirken, wie es bspw. im Fall des Essens während des Lesens nachgewiesen wurde. ${ }^{378}$ Solche Tätigkeiten tragen oftmals zu einer positiven Stimmung des Rezipienten während der Botschaftsaufnahme bei, was wiederum in einer positiveren Beurteilung der Anzeigen und Produkte resultieren kann. ${ }^{379}$

Neben dem Vorliegen dieser allgemeinen Störfaktoren ist die subjektive Entscheidungssituation des Rezipienten fur das Verhalten auf einen Direktmarketing-Stimulus ebenfalls relevant. ${ }^{380}$ Hierunter făllt bspw. die Frage nach dem Bedarf an einem spezifischen Produkt oder einer Dienstleistung zum Zeitpunkt des Werbekontaktes; aber auch die Frage des Entscheidungsgremiums, bspw. Partner, Familie etc., ist für das konkrete Verhalten auf einen DirektmarketingStimulus mitverantwortlich. Es kann insofern aus Unternehmenssicht von zentraler Bedeutung bei auf Spontanhandlungen abzielenden Direktmarketing-Aktionen sein zu wissen, inwieweit der Partner bzw. die Familie am Kaufentscheidungsprozess teilnimmt und wie gro $\beta$ das Buying Center ist. ${ }^{381}$ Die Frage nach einem möglichen Response richtet sich hierbei auch nach der Zeitdauer, die benötigt wird, die Entscheidung bzgl. eines konkreten Angebotes zu treffen. Insbe-

$375 \mathrm{Vgl}$. ebenda.

376 Vgl. Dallmer, H., Erfolgsbedingungen der Kommunikation im Direct-Marketing, S. 89. $\mathrm{Zu}$ unterscheiden sind diese Faktoren von den trägerspezifischen Faktoren, die bspw. die Nutzungssituation aufgrund der Spezifika des Instrumentes bedingen.

377 Vgl. Janis, I. L., Kaye, D., Kirschner, P., Facilitating effects of „Eating-WhileReading" on responsiveness to persuative communications, in: Journal of Personality and Social Psychology, Vol. 1, No. 2, S. 181 ff.; Dallmer, H., Thedens, R. (Hrsg.), Handbuch des Direct-Marketing, a. a. O., S. 90.

378 Vgl. Janis, I. L., Kaye, D., Kirschner, P., Facilitating Effects of "Eating-WhileReading" on Responsiveness to Persuasive Communications, a. a. O., S. 181-186.

379 Vgl. Müller, H., Khazaka, D., Stimmungseinflüsse auf die Wirkung informativer und emotionaler Werbung, in: Marketing ZFP, 17. Jg., Heft 3, 1995, S. 190.

380 Vgl. Holland, H., Direktmarketing, 2. Aufl., München 2004, S. 289.

381 Dieser Frage wurde im Hinblick auf Mailings in konzeptioneller Hinsicht u. a. bei Bamler nachgegangen, der den Einfluss der Größe des Buying Centers beim Einsatz von Direktwerbung im Business-to-Business-Bereich untersucht. Vgl. Bamler, G., Zur Wirkung von Direkt-Werbung im Industriegüterbereich, a. a. O., S. 170. Für den Bereich der Emails vgl. Chittenden, L., Rettie, R., An evaluation of e-mail marketing and factors affecting response, a. a. O., S. 205. 
sondere bei Mailings, die oft sämtlichen Familienmitgliedern vorliegen, sind häufig von der Leerung des Briefkastens bis zum finalen Kaufentscheid mehrere Personen beteiligt. ${ }^{382}$ Dies kann, wenn bspw. eine Werbesendung von einem Familienmitglied vorgelesen und daruber in der Gruppe diskutiert wird, zu einem positiven, aber auch negativen Einfluss auf die Entscheidung führen. ${ }^{383}$

Im Kontext des Direktmarketing wurden in diesem Zusammenhang weitere Einflussfaktoren untersucht, die sich auf die zeitlichen Umstände während der Kontaktsituation beziehen. Insbesondere im Falle der Zusendung von Mailings werden verschiedene zeitliche Determinanten wie Wochen- bzw. Jahreszeiten sowie besondere Anlässe und Ereignisse in ihrer Relevanz für die Effektivităt des Direktmarketing-Einsatzes unterschätzt. So beeinflussen bspw. Fest- und Feiertage zunächst die Lesewahrscheinlichkeit von Direktmarketing-Werbung in hohem Maße positiv. ${ }^{384}$ Ebenso begünstigen bestimmte klimatische Verhältnisse, bspw. in Form von schlechtem Wetter, die Verweildauer des DirektmarketingStimulus in „häuslicher Sphäre“ 385 und erhöhen damit die Kontakt- und in der Folge die Response- bzw. Kaufwahrscheinlichkeit. ${ }^{386}$ Zur Auswahl der Wochentage liegen bislang zwar keine allgemeingültigen Ergebnisse vor, allerdings werden von Seiten der Direktmarketing-aktiven Unternehmen einzelne Wochentage präferiert, an denen ein erhöhtes Werbeaufkommen und damit eine verringerte Kontakt- und Lesewahrscheinlichkeit vorliegt. In diesem Kontext konnte gezeigt werden, dass insbesondere Personen höherer Einkommensschichten, die vermehrt mit Werbesendungen angesprochen werden, diese rigoroser aussortieren als Per-

382 Vgl. Dallmer, H., Erfolgsbedingungen der Kommunikation im Direct-Marketing, a. a. O., S. 139

$383 \mathrm{Vgl}$. ebenda, S. $89 \mathrm{ff}$.

384 In einer Untersuchung der Deutschen Post gaben $75 \%$ der Befragten an, dass sie Werbesendungen an solchen Tagen „oft bis immer" lesen. Vgl. Deutsche Post AG (Hrsg.), Verbraucher und Direktwerbung, a. a. O., S. 14.

385 Vgl. Dallmer, H., Erfolgsbedingungen der Kommunikation im Direct-Marketing, a. a. O., S. 103.

386 Im Falle der Prospekte bzw. Haushaltswerbung erscheinen die empfängerseitige Auseinandersetzung mit Werbematerialen bei schlechtem Wetter aufgrund reduzierter Freizeitmöglichkeiten und deshalb die Kontaktwahrscheinlichkeit wesentlich höher zu sein als bei gutem Wetter. Dies hat ebenfalls positive Auswirkungen auf die Responsequote. In diesem Zusammenhang konnte am Beispiel japanischer Hauswurfsendungen gezeigt werden, dass die Responsequoten an regenreichen Tagen signifikant steigen. Aber auch im Falle von Emails ist die Nutzungswahrscheinlichkeit im privaten Gebrauch wesentlich wahrscheinlicher, wenn das Wetter schlecht ist. Vgl. bspw. Komori, K., Successful Marketing in Japan, in: Target Marketing, No. 5, 1997. 
sonen niedrigerer Einkommensklassen. ${ }^{387}$ Unabhängig vom Einkommen wurde dieser Zusammenhang im Fall von Business-to-Business-Werbesendungen bestätigt. So konnte gezeigt werden, dass der allgemeine Werbedruck, gemessen in der Anzahl unterschiedlicher Mailings, keinen Einfluss auf die Öffnungswahrscheinlichkeit besaß. Ebenso wie eine wochenbezogene Analyse kann auch die Tageszeit einen Einfluss auf die Wahrnehmung besitzen, welches im Fall der ausgewählten drei Instrumente jedoch noch nicht untersucht wurde. ${ }^{388}$

Im Rahmen der klassischen Werbewirkungsforschung konnte über die o. g. Einflussfaktoren hinaus mehrfach gezeigt werden, dass der physische Ort des Werbekontaktes einen zentralen Einflussfaktor in diesem Bereich darstellt, weil durch ihn u. a. unterschiedliche Aktivierungswirkungen ausgelöst werden. ${ }^{389}$ Aus diesem Grund unterscheiden sich die Wahrnehmung einer Werbebotschaft und die daraus resultierenden Verarbeitungsprozesse in Abhängigkeit danach, ob der Werbekontakt zu Hause oder außer Haus, bspw. während der Arbeit, stattfindet. ${ }^{390}$ Allgemein betrachtet werden Informationen aus ähnlichen Informationsträgern im privaten Umfeld schneller und häufiger aufgenommen als an öffentlichen Orten. ${ }^{391}$ Ein Vergleich des privaten Umfelds mit der Arbeitsstätte führt allerdings zu dem Ergebnis, dass am Arbeitsplatz Werbesendungen weniger stark vor dem Öffnen selektiert werden und das Aufmerksamkeitsniveau zumeist höher ist. ${ }^{392}$ Das Aktivierungsniveau des Rezipienten wird hierbei maßgeblich beeinflusst durch das Involvement einer Person gegenüber einer Werbeaussage. ${ }^{393}$ So

387 Vgl. Rogers, J. L., Consumers' Response to Advertising, a. a. O., S. 21 ff.; Kahn, H. L., How to Get Customers to Read What You Send Them, in: Sales and Marketing, Vol. 16, Heft 7, 1991, S. 23.

388 In einer Untersuchung von Danaher/Green zur Effektivität von Werbung im Direct Response Television Advertising (DRTV) zeigte sich zudem, dass der Response im Form von Anrufen und Bestellungen auf bestimmte Werbespots maßgeblich von der Tageszeit abhängt. Weiterhin kommen als bedeutende Einflussfaktoren bspw. das Programm, in die die Commercials eingebettet sind, in Betracht. Vgl. Danaher, P. J., Green, B. J., A Comparison of Media Factors that influence the Effectiveness of Direct response Television, in: Journal of Direct Marketing, Vol. 11, No. 2, 1997, S. 46-58.

389 Vgl. Riedmüller, F., Der Einfluss situationsspezifischer Faktoren auf die Werbequalität, in: Transfer. Werbeforschung \& Praxis, Vol. 1, 2000, S. 22-26.

$390 \mathrm{Vgl}$. Steffenhagen, H., Wirkungen der Werbung, a. a. O., S. 15.

391 Vgl. Hill, R. J., Bonjean, C. M., News Diffusion: A Test of the Regularity Hypothesis, in: Journalism Quarterly, Vol. 41, Summer 1964, S. 336-342, zitiert bei: Dallmer, H., Erfolgsbedingungen der Kommunikation im Direct-Marketing, a. a. O., S. 104.

392 Vgl. Deutsche Post AG (Hrsg.), Verbraucher und Direktwerbung - Verkaufen per Post 1995, Direkt Marketing Monitor, Studie 2, Bonn 1995, S. 23.

393 Vgl. Kroeber-Riel, W., Esch, F.-R., Strategie und Technik der Werbung, Verhaltenswissenschaftliche Ansätze, a. a. O., S. $188 \mathrm{ff}$. 
determiniert letztendlich der Grad der Ich-Beteiligung, wie stark situationsbedingte Störungen den Rezeptionsvorgang beeinflussen. ${ }^{394}$ Der Einfluss situationsbedingter Faktoren während des Rezeptionsvorgangs ist demnach umso wirkungsloser i. S. der verfolgten unternehmensseitigen Ziele, je stärker der IchBezug der Aussage für den Rezipienten ist. ${ }^{395}$

Im Vergleich zu den personenbezogenen Faktoren erscheinen die situativen Determinanten schwieriger prognostizierbar. Ihr Einfluss wird daher - wenn möglich mittels Prognosen (wie im Fall des Wetters) - mit in die Konfiguration einer Kampagne einbezogen. Fraglich bleibt allerdings, ob die Verbesserung der Effektivität mittels Prognosen auch unter Effizienzgesichtspunkten sinnvoll ist. Vielmehr erscheint die Bedeutung situativer Determinanten im Fall des Direktmarketing von geringerer Bedeutung zu sein als im Fall der klassischen Werbung. So konstatierte Vögele in Zusammenhang mit der Wirkung von Mailings, dass eine Besonderheit der Direktwerbung darin bestehe, dass es zu einer „Ablage" der Werbebotschaft kommen kann, an die sich - in einem gewissen zeitlichen Abstand - eine zweite Wahrnehmungs- und Verarbeitungsphase anschließen kann. Im Vergleich beispielsweise zu einer Plakatwerbung kann daher die Auseinandersetzung mit einem Direktmarketing-Stimulus mit einem spezifischen Angebot nach entsprechender Unterbrechung bzw. Ablage fortgesetzt werden. Dies führt dazu, dass situative Faktoren u. U. weniger Bedeutung für das Responseund Kaufverhalten des Konsumenten besitzen. Darüber hinaus bleibt festzuhalten, dass der Einfluss der situationsbezogenen Faktoren auf Response- und Kaufwahrscheinlichkeit gegeben ist, unternehmensseitig die Richtung und Stärke der situativen Komponenten jedoch nur geschätzt werden kann.

\section{Synoptische Darstellung eines Bezugsrahmens zur experimentellen Analy- se des Einflusses endogener und exogener Determinanten des Direktmar- keting-Erfolgs}

Als Basis einer empirischen Analyse der Effektivität und Effizienz des Direktmarketing lassen sich die bisherigen Ausführungen zu den Zielwirkungen und Wirkungsdeterminanten des Direktmarketing in einem integrierten Modell zusammenfassen (vgl. Abb. 9).

394 Vgl. Sherif, C. W., Sherif, M., Nebergall, R. E., Attitude and Attitude Change, Philadelphia, London 1965.

395 Vgl. Bamler, G., Zur Wirkung von Direkt-Werbung im Industriegüterbereich, a. a. O., S. $66 \mathrm{ff}$. 


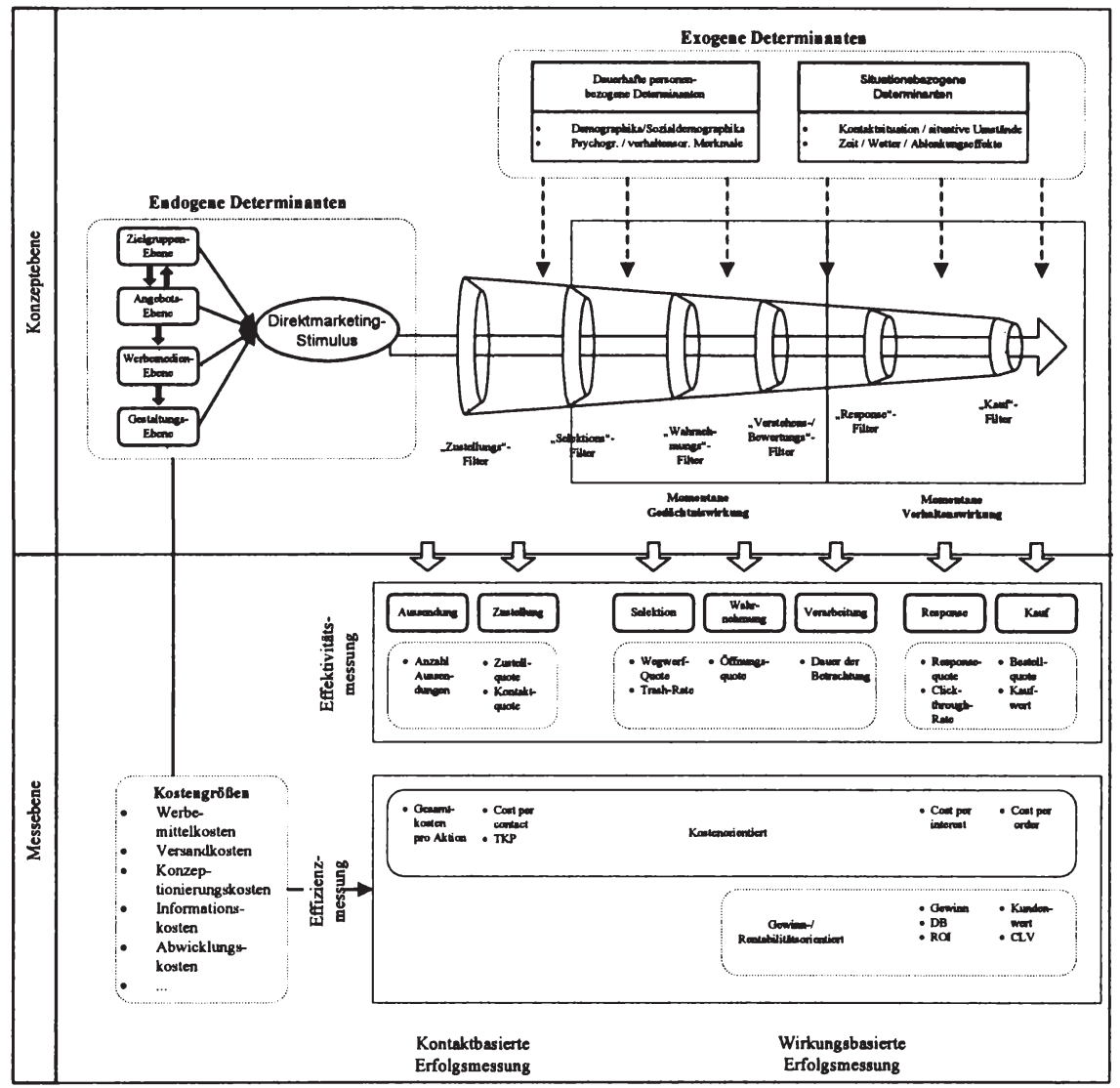

Abb. 9: Integriertes Modell zur Beurteilung der Effektivität und Effizienz des Direktmarketing

Der Schwerpunkt der folgenden empirischen Analyse des DirektmarketingEinsatzes basiert auf einem explorativen Vorgehen, in dessen Mittelpunkt die Messung der Effektivität und Effizienz unterschiedlich gestalteter Direktmarketing-Stimuli im Rahmen verschiedener Direktmarketing-Aktionen steht. Analog zu dem skizzierten Modell sollen dabei die zentralen endogenen Determinanten des Direktmarketing-Einsatzes mit dem Schwerpunkt auf den ausgewählten Instrumenten analysiert werden. Ebenso ist es das Ziel, die Relevanz ausgewăhlter exogener Determinanten auf die Effektivität und Effizienz des DirektmarketingEinsatzes nachzuweisen und ihre Bedeutung in Relation zu den endogenen Determinanten aufzuzeigen. 


\section{Experimentelle Untersuchung der Effektivität und Effizienz des Direktmarketing-Einsatzes}

\section{Anforderungen an die Durchführung von Feldexperimenten}

Mit dem Einsatz von Experimenten in der Marketingforschung wird in den meisten Fällen die Aufdeckung von Kausalzusammenhängen angestrebt. ${ }^{396}$ Unter einem Experiment wird dabei eine wiederholbare, unter kontrollierten, vorher festgelegten Umweltbedingungen durchgefürte Versuchsanordnung verstanden. ${ }^{397}$ Mit Hilfe dieser Versuchsanordnung erfolgt die Messung der Wirkungen einer oder mehrerer unabhängiger Variablen auf eine abhängige Variable, so dass letztendlich Aussagen darüber möglich sind, „ob und inwieweit der Einsatz oder die Variation einer Marketing-Variablen in einer ursächlichen Beziehung zu der Veränderung der gemessenen abhängigen Variablen steht.“"398 Die unabhängigen Variablen stellen hierbei den experimentellen Input dar, deren Einfluss auf eine abhängige Variable, z. B. das Responseverhalten, gemessen werden soll. ${ }^{399} \mathrm{Zu}$ den weiteren Bestandteilen eines experimentellen Designs gehören ebenso die Testelemente bzw. Testeinheiten, welche die Objekte repräsentieren, an denen die Experimente durchgeführt werden. Hierbei kann es sich um Geschäfte oder Produkte handeln; zumeist werden allerdings Probanden als Testelemente eingesetzt. ${ }^{400}$

Notwendige Voraussetzung für die erfolgreiche Aufdeckung von UrsacheWirkungs-Zusammenhängen mit Hilfe von Experimenten ist es, dass sich sowohl Ursache als auch Wirkungen von anderen Einflussfaktoren isolieren lassen. ${ }^{401}$ Um eine Zuordnung von Ursache und Wirkung zu gewährleisten, sollten weitere Einflussfaktoren eliminiert bzw. konstant gehalten werden. ${ }^{402}$ Dies betrifft insbesondere den Bereich der exogenen, d. h. nicht vom Unternehmen direkt steuerba-

396 Vgl. Meffert, H., Marketingforschung und Käuferverhalten, a. a. O., S. 206; Weis, H. Ch., Steinmetz, P., Marktforschung, a. a. O., S. 111.

397 Vgl. Kinnear, T. C., Taylor, J. R., Marketing Research. An Applied Approach, 2nd edition, New York, S. 361.

398 Vgl. Meffert, H., Marketingforschung und Käuferverhalten, a. a. O., S. 207.

399 Abzugrenzen von experimentellen Versuchen sind daher insbesondere Tests, bei denen eine Variation einer unabhängigen Variable zufällig erfolgt. Vgl. Berekoven, L., Eckert, W., Ellenrieder, P., Marktforschung, a. a. O., S. 151.

400 Vgl. Meffert, H., Marketingforschung und Kăuferverhalten, a. a. O., S. 207.

401 Vgl. Berekoven, L., Eckert, W., Ellenrieder, P., Marktforschung. a. a. O., S. 151.

402 Vgl. ebenda, S. 151. 
ren Determinanten, die den Wirkungsprozess des Direktmarketing beim Rezipienten beeinflussen können. Da aber eine Kontrolle sämtlicher, den Wirkungsprozess beeinflussender Determinanten im Rahmen eines experimentellen Versuchsaufbaus kaum gewährleistet werden kann, wird angestrebt, neben den nicht beeinflussbaren Störvariablen eine möglichst hohe Anzahl von Einflussfaktoren auf einem bestimmten Ausprägungsniveau zu fixieren. Diese als kontrollierte Variablen bezeichneten Faktoren zeichnen sich dadurch aus, dass sie vom Unternehmen i. d. R. zwar beeinflussbar sind, aber eine Untersuchung ihres Einflusses auf die abhängige Variable nicht erfolgen soll. Durch Beibehaltung eines jeweils vorhandenen Ausprägungsgrades werden damit weitestgehend Ceteribusparibus-Bedingungen geschaffen, die die angestrebte Zuordnung von variierter unabhängiger und abhängiger Variable erleichtern. ${ }^{403}$

Um den Einfluss der angesprochenen nicht-beeinflussbaren Störvariablen möglichst gering zu halten, kommen daher häufig künstliche Versuchsanlagen in Form von Laborexperimenten zum Einsatz. Im Rahmen dieser speziellen Form der experimentellen Untersuchung erfolgt die Analyse von Ursache-Wirkungszusammenhängen unter künstlichen, d. h. speziell für den Zweck der Untersuchung und damit zumeist konstant gehaltenen Umfeldbedingungen. ${ }^{404}$ So lassen sich Einflüsse externer Störgrößen, wie bspw. Lärm von Familienmitgliedern, Konkurrenzwerbung sowie sonstige situative Aspekte, auf ein Minimum reduzieren. Als nachteilig erweist sich hingegen der fehlende Bezug zum finalen, real beobachtbaren Kaufverhalten, da die künstliche Umgebung in den meisten Fällen erheblich von der realen Kaufsituation abstrahiert. ${ }^{405}$

Aufgrund der mangelnden Realitătsnăhe der Laborexperimente werden daher zunehmend Feldexperimente eingesetzt, um den Einfluss einer oder mehrerer unabhängiger Variablen auf eine abhängige Variable in einer ,natürlichen Umgebung" zu untersuchen und Aussagen über mögliche Ursache-Wirkungszusammenhänge zu generieren. ${ }^{406}$ Die mit der vorliegenden Arbeit verfolgten Zielsetzungen erfordern die realitätsnahe Überprüfung der Wirkungen der unterschiedlichen Direktmarketing-Instrumente und damit die Durchführung eines Feldexperiments. Dies gilt umso mehr, als dass zu vermuten ist, dass das Response- und Kaufverhalten aufgrund der Komplexität nicht im Rahmen einer Laboruntersuchung nachgebildet und die Relevanz verschiedener endogener und exogener Determinanten im Rahmen eines künstlichen Umfelds nicht umfassend untersucht werden können. Gleichzeitig erscheint es mit Blick auf die eingesetzten Direkt-

403 Vgl. Meffert, H., Marketingforschung und Käuferverhalten, a. a. O., S. 208. 404 Vgl. Weis, H. Ch., Steinmetz, P., Marktforschung, a. a. O., S. 111.

405 Vgl. Berekoven, L., Eckert, W., Ellenrieder, P., Marktforschung. a. a. O., S. 154. 406 ebenda, S. 154. 
marketing-Instrumente aufgrund einer mangelnden wissenschaftlichen Fundierung von besonderer Relevanz zu wissen, in welcher konkreten Alltagssituation der Rezipient einen Direktmarketing-Stimulus erhält und wie er darauf reagiert. Im Falle der Durchführung eines Laborexperiments würde eine Untersuchung des situativen Kontextes als Einflussvariable nur mittels Befragung möglich sein, was zu den bekannten nicht validen Aussagen über die Relevanz führen kann.

Die Durchführung von Feldexperimenten ist ebenfalls an einige Besonderheiten geknüpft, deren Berücksichtigung gleichsam als Erfolgsvoraussetzungen einer validen Messung gelten. Diese sollen im Folgenden kurz dargestellt werden:

- Bereits vor der Durchführung des Experiments sind sämtliche Variablen des Versuchsaufbaus zu analysieren. Insbesondere die Gestaltung des sog. experimentellen Inputs in Form der unabhängigen Variablen, deren Einfluss auf eine abhängige Variable untersucht werden soll, ist mit großer Sorgfalt durchzuführen, um letztendlich mit Hilfe des Experiments auch die zugrunde liegenden Forschungsfragen beantworten zu können..$^{407}$

- Ebenso ist es das Ziel, möglichst viele kontrollierte Variablen, d. h. aus Unternehmenssicht manipulierbare unabhängige Variablen, deren Einfluss nicht Gegenstand der Analyse ist, konstant zu halten und zufällige Schwankungen dieser zu vermeiden. 408

- Als zentrale Herausforderung bei der Durchführung von Feldexperimenten ist die Konstanthaltung möglichst vieler exogener, d. h. nicht-manipulierbarer Variablen anzustreben, die als Störvariablen auftreten und einen Einfluss auf die abhängige Variable ausüben können. Neben einer möglichen Konstanthaltung lassen sich die Störvariablen alternativ auch in das experimentelle Design einbauen und als unabhängige Variable des Wirkungsprozesses betrachten. Eine weitere Möglichkeit der Berücksichtigung liegt in der zufälligen Zuordnung von Untersuchungseinheiten auf die einzelnen Experimentalbedingungen (,Randomisierung "). ${ }^{409}$

- $\mathrm{Zu}$ beachten ist weiterhin, dass Experimente zumeist nur die Messung kurzfristiger Wirkungen erlauben. ${ }^{410}$ Langfristige Wirkungen lassen sich nur unter größerem Aufwand über einen längeren Zeitraum mit einem experimentellen Versuchsaufbau messen, wozu Panelerhebungen geeigneter erscheinen. Aus diesem Grund ist der Einsatz von Experimenten zur Entscheidungsfindung bei strategischen Problemen nur bedingt möglich. ${ }^{411}$

407 Vgl. Meffert, H., Marketingforschung und Käuferverhalten, a. a. O., S. 28.

408 Vgl. Green, P. E., Tull, D. S., Methoden und Techniken der Marketingforschung, a. a. O., S. 323.

409 Vgl. Berekoven, L., Eckert, W., Ellenrieder, P., Marktforschung. a. a. O., S. 152 ff.

410 Vgl. Meffert, H., Marketingforschung und Käuferverhalten, a. a. O., S. 212.

411 ebenda. 
Bei Berücksichtigung dieser Besonderheiten lassen sich trotz der teilweise „künstlich“ konstant gehaltenen Variablen mit Hilfe von Feldexperimenten wertvolle Informationen gewinnen, die einen Beitrag zu einer ,rationalen und stärker fundierten Entscheidungsfindung ${ }^{\star 4 / 12}$ nicht nur im klassischen Marketing, sondern auch im Direktmarketing leisten können.

\section{Design der experimentellen Untersuchung}

\subsection{Auswahl des Untersuchungsobjekts}

Die vorliegenden empirischen Daten basieren auf einer umfassenden Untersuchung, die das Centrum für interaktives Marketing und Medienmanagement (CIM) in der Zeit von Oktober 2003 bis März 2004 zusammen mit einem Verkehrsdienstleistungsunternehmen der Luftfahrt-Branche durchfuhrte. Als Untersuchungsobjekt dienen im Rahmen der experimentellen Analyse der Wirkungen ausgewählter Direktmarketing-Instrumente verschiedene DirektmarketingAktionen, sowie eine vor- und eine nachgelagerte Befragung der Rezipienten der entsprechenden Direktmarketing-Stimuli.

Für die Auswahl der Luftverkehrsbranche sprach zunächst die Tatsache, dass der Einsatz der ausgewählten Direktmarketing-Instrumente Mailing, Email und SMS im Rahmen der Kundenbearbeitung branchenweit zunehmend Verwendung findet, und damit eine für die Analyse der Wirkungen nötige Grundakzep$\operatorname{tanz}$ der Instrumente bei den Konsumenten vorliegt. ${ }^{413} \mathrm{Um}$ instrumentespezifische Wirkungsunterschiede ableiten zu können, setzt eine derartige Analyse zudem voraus, dass die untersuchten Instrumente über ein Mindestmaß an Relevanz im Rahmen informations- und kaufbezogener Handlungen verfugen, da die Effektivität und Effizienz rein zu Imagezwecken eingesetzter DirektmarketingInstrumente im Rahmen der vorliegenden Analyse nicht untersucht werden soll. Dies ist letztendlich in der vorliegenden Branche gegeben; während insbesondere die klassischen Kommunikations-Instrumente, wie TV-Werbung und Hörfunkwerbung, zum Einsatz kommen, um den Bekanntheitsgrad des Unternehmens zu erhöhen, werden die Instrumente des Direktmarketing immer hăufiger eingesetzt, um zeitnah Restkontingente an freien Tickets zu verkaufen. Dies prädestiniert die

\section{2 ebenda.}

413 Mittlerweile setzen nahezu sämtliche der deutschen Luftverkehrsunternehmen EmailNewsletter ein, um ihre Kunden regelmäßig über die Angebote des Unternehmens zu informieren. Ein Großteil der Unternehmen versendet zusătzlich bei Bedarf konkrete Angebote per Email. Teilweise werden auch SMS zur Informationsverbreitung eingesetzt. 
Branche vor allem für die Durchfuhrung von Experimenten, mit denen das Ziel verfolgt wird, kurzfristigen Verhaltensreaktionen zu messen

Zum anderen lässt sich derzeit beobachten, dass es in der Luftverkehrsbranche mit Hilfe des Einsatzes der unterschiedlichen Direktmarketing-Instrumente zu einer zunehmenden differenzierten Marktbearbeitung kommt, welche sich in unterschiedlichen Angebotsformen widerspiegelt. Nach Auswertung der in der Vergangenheit gebuchten Flüge können individuelle Angebote zusammengestellt und bspw. per Email direkt an Bestandskunden des Unternehmens versendet werden. So können mit Hilfe des Einsatzes des Direktmarketing die Ticket-Angebote ohne größeren produktionstechnischen Aufwand individualisiert und den Kunden direkt angeboten werden. Dieser Umstand erlaubt damit auch die Untersuchung des Einflusses angebotsbezogener Determinanten, die zwar primär den Bereich der Bestandskundenbearbeitung zunehmend aber auch die Neukundenakquisition betreffen.

Das ausgewählte Unternehmen zählt innerhalb der Luftverkehrsbranche zu dem Segment der sog. Low Cost Airlines, die häufig auch als Billigflieger oder Billig-Airlines bezeichnet werden. Kennzeichnend für die Zugehörigkeit zu diesem Segment ist das Angebot von - im Branchendurchschnitt - günstigen Flugtickets auf ausgewählten Strecken innerhalb Deutschlands, Europas und teilweise in die USA. ${ }^{414}$ Die angebotenen Niedrigpreise beziehen sich zumeist auf ein bestimmtes Kontingent an Tickets pro Flug, welche sich in Abhängigkeit der im Zeitablauf steigenden Kapazitätsauslastung stufenweise erhöhen. Während die zuerst buchenden Personen ihre Tickets mit hoher Wahrscheinlichkeit für die in der Werbung kommunizierten Preise von 19 Euro erwerben können, werden die letzten verfügbaren Tickets eines Fluges häufig für deutlich mehr als das Zehnfache der ursprünglich kommunizierten Preise verkauft. Als zentrales Merkmal der Low Cost Airlines ist in diesem Zusammenhang das No-Frills-Konzept zu nennen. ${ }^{415}$ So beinhalten die kommunizierten Preise zwar sämtliche der üblicherweise anfallenden Steuern und Gebühren, beziehen sich aber ausschließlich auf die Kernleistung. Nicht in den Preisen eingeschlossen sind demnach Speisen und Getränke während des Fluges, die in den meisten Fällen allerdings käuflich erworben werden können.

414 Der Begriff „tiefpreisig“ bezieht sich hierbei insbesondere auf den Vergleich mit Airlines wie Lufthansa oder Air France, deren günstigste Tarife bereits deutlich über den Einstandspreisen der Low Cost Airlines liegen.

415 Mit dem Begriff „No-Frills“ wird im Allgemeinen ein Konzept bezeichnet, das außer der eigentlichen Kernleistung (in diesem Fall der Transportleistung) keine weiteren Services anbietet (Value Added Services). Vgl. Meffert, H., Dienstleistungsmarketing, 3. Aufl., Wiesbaden 2003, S. 232. 
Die Kommunikationsaktivitäten des betrachteten Unternehmens beschränkten sich zum Zeitpunkt vor und während der Untersuchung primär auf den Bereich der Plakat- und Hörfunk-Werbung mit regionalen Schwerpunkten. So wurde im Einzugsgebiet von $100 \mathrm{~km}$ um den Heimat-Flughafen an exponierten Stellen Plakatwerbung sowie in den dort ansässigen regionalen Radiosendern 15sekündige Hörfunkspots gesendet. Zusätzlich kam an einem Großteil der Zielflughäfen ebenfalls Plakat-, in ausgewählten Zielflughäfen auch Hörfunkwerbung zum Einsatz. Im Mittelpunkt dieser klassischen Marketing-Aktivitäten steht der Verweis auf die genannten 19 Euro-Tickets, die für ein begrenztes Sitzplatzangebot gelten. Das ausgewählte Unternehmen bot zum Zeitpunkt der Untersuchung Flüge zu 23 Zieldestinationen im europäischen In- und Ausland an, wobei der überwiegende Teil der Tickets über die unternehmenseigene Homepage verkauft wird. ${ }^{416}$

Der Bereich der klassischen Kommunikation wird zeitweise durch ausgewählte Direktmarketing-Aktionen ergänzt. Kernelement der DirektmarketingAktivitäten vor der Durchführung der Experimente stellte der wöchentlich bzw. vierzehntägig versandte Email-Newsletter dar, der den Verkauf von größeren Ticketkontingenten zum Preis von 19 Euro bewarb. Wesentliches Merkmal dieser Aktionen war die exklusive Buchungsmöglichkeit über die Homepage des Unternehmens und der kurze Buchungszeitraum. Die regelmäßig stattfindenden Aktionen wurden durch themenbezogene Events, wie bspw. eine „Karnevals“- oder eine „Oster"-Aktion, ergänzt, bei denen zusätzliche Kontingente zu Sonderpreisen angeboten wurden.

\subsection{Aufbau der experimentellen Untersuchung}

Im Kern der Untersuchung stehen die Ergebnisse einer Effektivitäts- und Effizienzbetrachtung verschiedener Direktmarketing-Aktionen, die im Namen der Low Cost Airline im Dezember 2003 an Bestandskunden des Unternehmens sowie potenzielle Neukunden versendet wurden. Im Rahmen dieser Aktionen wurden verschiedene Direktmarketing-Stimuli eingesetzt, um den Einfluss der Variation einzelner endogener Determinanten auf die Verhaltenswirkungen bei den Rezipienten zu analysieren. Die verschiedenen Direktmarketing-Aktionen wurden durch eine vor- und eine nachgelagerte Befragungswelle ergänzt. Auf

416 Insgesamt verkaufte das Unternehmen in den ersten 12 Monaten seit der Aufnahme der Geschäftstätigkeit im Oktober 2002 über 3 Mio. Tickets. Ca. $90 \%$ der Tickets wurden hierbei über die Buchungsmöglichkeit auf der Homepage, $7 \%$ der Tickets über die Buchungshotline sowie die verbleibenden $3 \%$ über Reisebüros verkauft. 
diese Weise war es möglich, über die rein quantitativen Ergebnisse der Effektivitäts- und Effizienzanalyse hinaus zusätzliche Informationen über personen- und situationsbezogene Determinanten zu erhalten. ${ }^{417}$

\subsubsection{Operationalisierung der abhängigen Variablen}

Als zentrale Zielkategorie des Direktmarketing-Einsatzes wurden im Verlauf der konzeptionellen Analyse die momentanen Verhaltenswirkungen identifiziert. Im Rahmen des Feldexperiments wurden daher als abhängige Variablen die kurzfristigen Verhaltenswirkungen in Form des Response- und Buchungsverhalten der Rezipienten ausgewählt. Diesem Verhalten vorgelagert ist zunächst die erfolgreiche Zustellung des Direktmarketing-Stimulus. Im Hinblick auf jeden der eingesetzten verschiedenen Direktmarketing-Stimuli wurden dabei für jede der 28 Stichproben mit einem Umfang von jeweils 100 Personen folgende Informationen erhoben (vgl. Tab. 3):

\begin{tabular}{|l|l|}
\hline Kennzahl & Inhalt \\
\hline Zustellquote (ZQ) & $\begin{array}{l}\text { Anzahl der tatsächlich zugestellten Direktmarketing- } \\
\text { Stimuli }\end{array}$ \\
\hline Responsequote (RQ) & $\begin{array}{l}\text { Anzahl von Personen, die auf den konkreten Direkt- } \\
\text { marketing-Stimulus reagiert und im Call-Center an- } \\
\text { gerufen haben (ohne Angabe, ob eine Buchung er- } \\
\text { folgte oder nicht) }\end{array}$ \\
\hline Buchungsquote (BQ) & $\begin{array}{l}\text { Anzahl von Personen, die ein entsprechendes Ange- } \\
\text { bot gebucht haben (unabhängig von der Anzahl der } \\
\text { gebuchten Tickets) }\end{array}$ \\
\hline Gesamt-Ticketanzahl (GT) & $\begin{array}{l}\text { Die Gesamtanzahl von Tickets, die sämtliche Bucher } \\
\text { einer Stichprobe über das Call-Center gebucht haben }\end{array}$ \\
\hline Gesamtumsatz (U) & $\begin{array}{l}\text { Gesamtumsatz der durch die Gesamt-Ticketanzahl } \\
\text { und den jeweiligen Preisen pro Stichprobe generiert } \\
\text { wurde }\end{array}$ \\
\hline
\end{tabular}

Tab. 3: Effektivitätskennzahlen im Rahmen der empirischen Untersuchung

Die Erfassung der Zustellquote erfolgte auf Basis der Auswertung der Fehlerprotokolle der Email- und SMS-Provider des Unternehmens sowie der zurück gesendeten Mailings. ${ }^{418}$ Die Messung der Responses und der Buchungen wurde über das unternehmenseigene Call-Center bzw. das Buchungssystem erhoben.

417 Der Fragebogen der Ex-ante-Befragung und der Ex-post-Befragung sind im Anhang dargestellt.

418 Für Direktmarketing-Stimuli, die fehlerhafte Adressen beinhalteten, wurden vor dem Hintergrund forschungsökonomischer Gründe keine Ersatz-Stimuli versendet. Viel- 


\begin{tabular}{|l|l|l|l|}
\hline Kostenkategorie & Sub-Kategorie & Erfasste Kosten & Informationsquellen \\
\hline \multirow{4}{*}{ Werbemittelkosten } & $\begin{array}{l}\text { Werbemitteler- } \\
\text { stellung }\end{array}$ & $\begin{array}{l}\text { Layout, Fotogra- } \\
\text { phie, Text, Foto- } \\
\text { satz, Reprodukti- } \\
\text { on etc. }\end{array}$ & $\begin{array}{l}\text { Externe Finanzbuch- } \\
\text { haltung } \\
\text { Interne Kostenrech- } \\
\text { nung }\end{array}$ \\
\cline { 2 - 5 } & Vervielfältigung & $\begin{array}{l}\text { Druckabwicklung, } \\
\text { Druck (Katalog, } \\
\text { Hülle, Karte, ...), } \\
\text { Verarbeitung etc. }\end{array}$ & $\begin{array}{l}\text { Externe Finanzbuch- } \\
\text { haltung } \\
\text { Interne Kostenrech- } \\
\text { nung }\end{array}$ \\
\hline $\begin{array}{l}\text { Versandbezogene } \\
\text { Abwicklungskosten }\end{array}$ & Werbemittel & $\begin{array}{l}\text { Packmittel, Ku- } \\
\text { vertieren, Porto- } \\
\text { kosten, Versand- } \\
\text { abwicklung etc. }\end{array}$ & $\begin{array}{l}\text { Externe Finanzbuch- } \\
\text { haltung } \\
\text { Interne Kostenrech- } \\
\text { nung }\end{array}$ \\
\hline $\begin{array}{l}\text { Kundenbezogene } \\
\text { Abwicklungskosten }\end{array}$ & $\begin{array}{l}\text { Kundenbetreu- } \\
\text { ung }\end{array}$ & $\begin{array}{l}\text { Kundenkorres- } \\
\text { pondenz, telefoni- } \\
\text { sche Anfragen, } \\
\text { Mahnwesen etc. }\end{array}$ & $\begin{array}{l}\text { Interne Kostenrech- } \\
\text { nung }\end{array}$ \\
\hline Informationskosten & $\begin{array}{l}\text { Informationser- } \\
\text { langung }\end{array}$ & $\begin{array}{l}\text { Ankauf von Post- } \\
\text { und Email- Adres- } \\
\text { sen sowie Handy- } \\
\text { nummern }\end{array}$ & $\begin{array}{l}\text { Externe Finanzbuch- } \\
\text { haltung }\end{array}$ \\
\hline
\end{tabular}

Tab. 4: Kostenkategorien im Rahmen der Direktmarketing-Experimente

Darüber hinaus konnten auf kundenindividueller Basis Daten zur Menge der abgerufenen Informationen innerhalb eines Buchungsvorgangs erhoben werden. ${ }^{419}$ Auf Basis der Intensität dieses Informationsverhaltens, welches sich primär in einer unterschiedlichen Länge der Gespräche zwischen Kunden und CallCenter-Mitarbeitern widerspiegelte, wurde die Verteilung der fixen Call-CenterKosten vorgenommen, die zur Kategorie der kundenbezogenen Abwicklungskosten zu zählen sind. ${ }^{420}$ Des Weiteren wurden Anrufer, die auch Flüge gebucht hat-

mehr wurde die Zustellquote explizit als Teil der Effektivitäts- und Effizienzanalyse angesehen.

419 In diesem Zusammenhang bewerteten die Call-Center-Mitarbeiter den Umfang der abgerufenen Informationen zum Angebot auf einer Skala (wenig, mittel, viel) und vermerkten diese Informationen kundenindividuell im Buchungssystem.

420 Aufgrund technischer Restriktionen wurde nicht die zeitliche Dauer der einzelnen Gespräche aufgezeichnet, sondern es erfolgte eine kundenindividuelle Bewertung der Intensität des Informationsbedarfs durch die Call-Center-Mitarbeiter. Die Einstufung eines Buchers erfolgte hierbei in eine der drei Klassen „wenig“, "mittel" und „viel“" auf Basis der Einschätzung derjenigen Call-Center-Mitarbeiter, die das Gespräch führten. Zeitlich lassen sich die drei Klassen mit durchschnittlichen Gesprächsdauern von <4 Minuten (,wenig"), 6 Minuten (,mittel") und >8 Minuten (,viel") beziffern. Dieses Vorgehen hat den Vorteil, dass etwaige technische Probleme vonseiten des Unternehmens, die zu längeren Gesprächsdauern führten, aber nicht unbedingt in ei- 
ten, nach Durchfuhrung der Buchung bzgl. ihrer maximalen Preisbereitschaft im Hinblick auf das erhaltene Angebot befragt.

In Ergänzung zur Effektivitătsmessung erfolgte die Berechnung der verschiedenen Effizienzkennzahlen auf Basis der angefallenen Kosten für die Bereitstellung einzelner, für die Durchführung der Direktmarketing-Experimente notwendiger Dienstleistungen, die größtenteils durch externe Unternehmen bereitgestellt wurden. Tab. 4 zeigt einen Überblick über die berücksichtigten Kostenblöcke sowie die dazugehörigen Informationsquellen.

Die Effizienz der unterschiedlichen Direktmarketing-Stimuli erfolgte - je nach betrachteter Untersuchungsebene - anhand entsprechender Kennzahlen (vgl. Tab. 5).

\begin{tabular}{|l|l|}
\hline Kennzahl & Inhalt \\
\hline Stückkosten (STK) & $\begin{array}{l}\text { Kosten der Konfiguration und des Versands pro Sti- } \\
\text { mulus }\end{array}$ \\
\hline Cost per contact (CPC) & Kosten pro Rezipientenkontakt \\
\hline Cost per interest (CPI) & Kosten pro Interessent (Anrufer im Call-Center) \\
\hline Cost per order (CPO) & Kosten pro Bucher \\
\hline $\begin{array}{l}\text { Deckungsbeitrag pro } \\
\text { Direktmarketing-Stimulus } \\
\text { (DBS) }\end{array}$ & $\begin{array}{l}\text { Gesamt-Deckungsbeitrag resultierend aus der } \\
\text { Versendung eines Direktmarketing-Stimulus an 100 } \\
\text { Personen }\end{array}$ \\
\hline $\begin{array}{l}\text { Deckungsbeitrag pro Aktion } \\
\text { (DBA) }\end{array}$ & $\begin{array}{l}\text { Gesamt-Deckungsbeitrag einer Aktion bestehend aus } \\
7 \times 100 \text { Personen }\end{array}$ \\
\hline $\begin{array}{l}\text { Deckungsbeitrag pro Ziel- } \\
\text { gruppe (DBZ) }\end{array}$ & $\begin{array}{l}\text { Gesamt-Deckungsbeitrag aus der Ansprache einer be- } \\
\text { stimmten Zielgruppe }\end{array}$ \\
\hline $\begin{array}{l}\text { Maximale Preisbereitschaft } \\
\text { (MP) }\end{array}$ & $\begin{array}{l}\text { Maximale Preisbereitschaft eines Buchers nach Rück- } \\
\text { frage durch den Call-Center-Mitarbeiter }\end{array}$ \\
\hline
\end{tabular}

\section{Tab. 5: Effizienzkennzahlen im Rahmen der empirischen Untersuchung}

In diesem Zusammenhang sei darauf hingewiesen, dass in die Berechnung der Stückkosten sowie in die Berechnung der Kennzahl „Cost per contact" sämtliche der aufgezeigten Kostenkategorien mit Ausnahme der kundenbezogenen Abwicklungskosten einfließen. ${ }^{421}$ Hingegen erfolgt die Berechnung der Kennzahlen

nem extensiveren Informationsbedarf des Anrufers begründet lagen, nicht in die $\mathrm{Be}-$ wertung einflossen.

421 Während Gestaltungs-, Versand- und Informationskosten im Rahmen der Produktion gemäß dem Verursachungsprinzip der jeweiligen Zielgruppe zugeordnet werden können, erfolgt eine Einbeziehung der Call-Center-Kosten entsprechend der tatsächlichen Kapazitătsbeanspruchung der Call-Center-Ressourcen. Die kapazitätsmäßige Zurechung i. S. des der Prozesskostenrechung zugrunde liegenden Gedankens der Ressourcenbeanspruchung erfolgte auf Basis der erhobenen "Informationsmenge“. 
„Cost per interest“ und „Cost per order“ sowie die Deckungsbeitragsrechnung unter Hinzuziehung dieses Kostenblocks. ${ }^{422}$

\subsubsection{Operationalisierung der unabhängigen Variablen}

Der Schwerpunkt der vorliegenden Untersuchung liegt auf der experimentellen Analyse der Wirkungen ausgewählter Direktmarketing-Instrumente, die damit als experimenteller Input kontrolliert variiert werden, während die übrigen unabhängigen Variablen konstant gehalten werden. Um die relative Bedeutung des Einflusses der Auswahl des Trägermediums für die intendierten Wirkungen des Direktmarketing aufzuzeigen, sollen zusätzlich zur Variation der Instrumente zwei, der Auswahl der Medien bzw. Medienkombinationen vorgelagerten Entscheidungen ebenfalls als unabhängige Variablen variiert werden. Demnach galt es für jeden Direktmarketing-Stimulus im Rahmen seiner Konfiguration Entscheidungen auf den drei aufgezeigte Ebenen hinsichtlich der konkreten Ausprägung zu treffen.

Auf der ersten Ebene musste zunächst entschieden werden, welche Zielgruppe den Stimulus erhalten sollte. In Anlehnung an die beiden grundlegenden Zielsetzungen „Neukundenakquisition“ und „Bestandskundenbearbeitung“ wurde im Rahmen der Zielgruppenauswahl eine entsprechende Adressierung potenzieller bzw. bestehender Kunden vorgenommen.

Auf der zweiten Ebene wurden die Direktmarketing-Stimuli im Hinblick auf das zugrunde liegende Angebot konfiguriert. Angesichts der hohen Relevanz für die Unternehmenspraxis standen hierbei ein standardisiertes, ein individualisiertes sowie ein rabattiertes Angebot zur Auswahl.

- Vor dem Hintergrund der in den regelmäßig stattfindenden NewsletterAktionen kommunizierten Preise wurde zum einen ein standardisiertes Angebot zum Preis von 19 Euro für einen einfachen Flug in zehn ausgewählte Destinationen offeriert. ${ }^{423} \mathrm{Ab}$ einer festgelegten Uhrzeit am Folgetag nach Zusendung des Angebots konnten sich die Rezipienten über eine Kundenhotline über das Angebot bzw. die Verfügbarkeit freier Tickets informieren und eine Buchung vornehmen. Eine Buchung war innerhalb eines Zeitraums von

Personen, die im Call-Center angerufen, aber nicht gebucht haben, wurden in diesem Zusammenhang wie Personen mit geringem Informationsbedarf eingestuft.

422 Den Call-Center-Kosten werden in diesem Kontext aussschließlich Personalkosten zugerechnet.

423 Zu den ausgewählten Destinationen zählten im Einzelnen: Rom, London, Paris, Mailand, Madrid, Lissabon, Zürich, Wien, Barcelona und Nizza. 
24 Stunden möglich und folgte - wie im Rahmen der bekannten Sonderaktionen - dem First-come-first-serve-Prinzip. ${ }^{424}$

- Das zweite Angebot umfasste ein rabattiertes Angebot, das bei gleichzeitiger Buchung von vier einfachen Flügen einen Rabatt von $25 \%$ auf den Gesamtpreis gewährte. ${ }^{425}$ Dieses „4 für 3“-Angebot konnte von einzelnen Personen für vier one-way Strecken, von Paaren aber auch von Gruppen mit mehr als zwei Personen gebucht werden. ${ }^{426}$

- Das dritte Angebot stellte ein hinsichtlich Destination, Preis und Anrede auf den jeweiligen Rezipienten individualisiertes Angebot dar. Die Auswahl der Zieldestination richtete sich nach dem am häufigsten in den letzten zwölf Monaten angeflogenen Ziel. ${ }^{427}$ Die Preise für ein einfaches Ticket lagen je nach Zieldestination bei 29 Euro, 39 Euro oder 49 Euro und damit über denen des standardisierten Angebotes. ${ }^{428}$ Im Vergleich zu den ersten beiden Angeboten wurde den Personen, die ein individualisiertes Angebot erhielten, der Preis als Garantiepreis offeriert. Dabei hatte die Preisgarantie Gültigkeit für die angesprochene und eine weitere Person für einen Hin- und Rückflug. Gleichzeitig wurde das Angebot für einen Zeitraum von 24 Stunden nach Buchungsbeginn für die angesprochene Person reserviert. ${ }^{429}$

Auf der dritten Ebene erfolgte die Auswahl des Instrumenteeinsatzes. In diesem Kontext wurde auch die Frage nach dem kombinierten Einsatz mehrerer Trägermedien entschieden. Möglich waren hierbei der Einsatz eines einzelnen Instrumentes (Mailing, Email oder SMS) sowie der kombinierte Einsatz von zwei (Mailing und Email, Mailing und SMS sowie Email und SMS) bzw. drei Instrumenten (Mailing, Email und SMS). Vor dem Hintergrund der drei Instrumen-

424 Diese Angebotsform entsprach in seiner Grundform dem standardisierten Angebot, welches bereits im konzeptionellen Teil der Arbeit beschrieben wurde.

425 Grundsätzlich wurde bei vier gleich teuren Tickets ein Rabatt von $25 \%$ gewährt. Im Fall unterschiedlicher hoher Ticketpreise erfolgte die Reduktion des Gesamtbetrages um die Höhe des günstigsten Tickets.

426 Das Angebot entsprach damit dem im konzeptionellen Teil der Arbeit dargestellten zweiten Angebotsform: dem rabattierten Angebot.

427 Diese Angabe wurde von den Beworbenen im Rahmen der Ex-ante-Befragung abgefragt. Eine Auswertung des Buchungssystems hätte alternativ erfolgen können.

428 Die Einordnung einer Zieldestination in eine Preisklasse richtete sich nach dem durchschnittlich auf der Strecke erlösten Preis sowie dem durchschnittlichen Ladefakor, d. h. dem Auslastungsgrad der Flugzeuge. Damit wurden die Tickets auf Strecken mit einer hohen Auslastung, wie Wien, Zürich und Barcelona, für 49 Euro angeboten, Strecken mit mittlerer Auslastung, wie bspw. Madrid, für 39 Euro und Strecken mit tendenziell geringerer Auslastung für 29 Euro, wie bspw. London.

429 Diese Angebotsform kann insofern in seiner Grundform der im konzeptionellen Teil dargestellten dritten Angebotsform des individualisierten Direktmarketing zugeordnet werden. 
te liegen insgesamt sieben Kombinationsmöglichkeiten hinsichtlich der Medienauswahl vor.

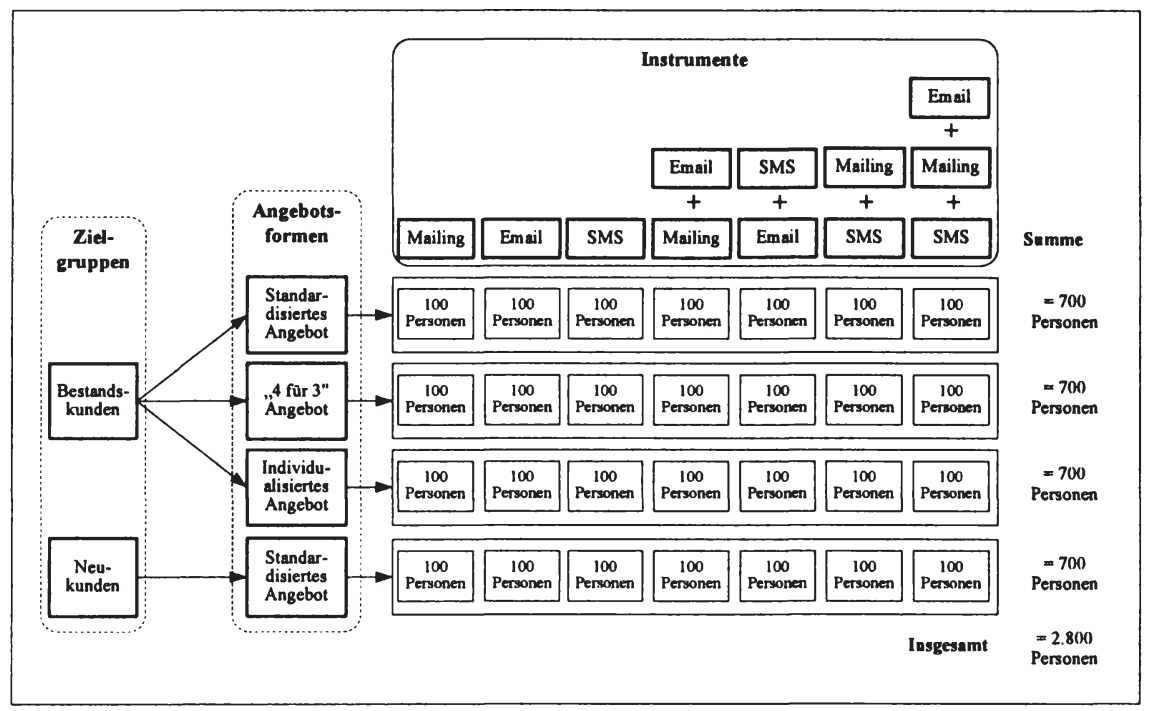

Abb. 10: Übersicht über die 28 versendeten Direktmarketing-Stimuli

Darüber hinaus konnten auf kundenindividueller Basis Daten zur Menge der abgerufenen Informationen innerhalb eines Buchungsvorgangs erhoben werden. ${ }^{430}$ Auf Basis der Intensität dieses Informationsverhaltens, welches sich primär in einer unterschiedlichen Länge der Gespräche zwischen Kunden und CallCenter-Mitarbeitern widerspiegelte, wurde die Verteilung der fixen Call-CenterKosten vorgenommen, die zur Kategorie der kundenbezogenen Abwicklungskosten zu zählen sind. ${ }^{431}$ Des Weiteren wurden Anrufer, die auch Flüge gebucht hat-

430 In diesem Zusammenhang bewerteten die Call-Center-Mitarbeiter den Umfang der abgerufenen Informationen zum Angebot auf einer Skala (wenig, mittel, viel) und vermerkten diese Informationen kundenindividuell im Buchungssystem.

431 Aufgrund technischer Restriktionen wurde nicht die zeitliche Dauer der einzelnen Gespräche aufgezeichnet, sondern es erfolgte eine kundenindividuelle Bewertung der Intensität des Informationsbedarfs durch die Call-Center-Mitarbeiter. Die Einstufung eines Buchers erfolgte hierbei in eine der drei Klassen „wenig“, „mittel“" und „viel“" auf Basis der Einschätzung derjenigen Call-Center-Mitarbeiter, die das Gespräch führten. Zeitlich lassen sich die drei Klassen mit durchschnittlichen Gesprächsdauern von $<4$ Minuten (,wenig"), 6 Minuten (,mittel“) und >8 Minuten (,viel“) beziffern. Dieses Vorgehen hat den Vorteil, dass etwaige technische Probleme vonseiten des 


\begin{tabular}{|c|c|c|c|}
\hline & Email / Mailing & SMS \\
\hline \multirow{2}{*}{ 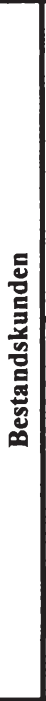 } & 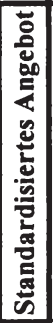 & $\begin{array}{l}\text { „Lieber Airline X-Kunde, } \\
\text { als Dank fur die Teilnahme an unserer Befragung vom Oktober/No- } \\
\text { vember } 2003 \text { bieten wir Ihnen exklusiv ein zusatzliches Kontingent } \\
\text { an } 19 € \text {-Tickets an. Die Tickets gelten auf allen Airline X-Strecken } \\
\text { im Zeitraum zwischen 5. Januar und 28. März 2004. Die Tickets } \\
\text { konnen Sie ab Mittwoch, 17. Dezember 2003, ab } 10 \text { Uhr fur } 24 \\
\text { Stunden ausschließlich uber unsere Kundenhotline mit der Tele- } \\
\text { fonnummer XXX. Nahere Informationen erhalten Sie ebenfalls dort. } \\
\text { Mit freundlichen Grußen } \\
\text { Ihr Airline X-Team“ }\end{array}$ & $\begin{array}{l}\text { "Lieber Airline X- } \\
\text { Kunde, als Dank } \\
\text { fur die Teilnahme } \\
\text { an unserer Befra- } \\
\text { gung bieten wir Ih- } \\
\text { nen exkl. 19€- } \\
\text { Tickets an. Buchbar } \\
\text { ab morgen 14Uhr } \\
\text { nur unter XXX." }\end{array}$ \\
\hline & 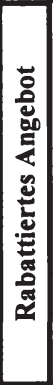 & $\begin{array}{l}\text { "Lieber Airline X-Kunde, } \\
\text { als Dank fur die Teilnahme an unserer Befragung vom Oktober/ No- } \\
\text { vember } 2003 \text { bieten wir Ihnen exklusiv } 4 \text { Tickets zum Preis von } 3 \\
\text { an. Bei einer Buchung von } 4 \text { Tickets fur Sie bzw. Ihre Freunde uber- } \\
\text { nimmt Airline-X die Kosten des gunstigsten Fluges. Dieses Angebot } \\
\text { gilt fur sămtliche Fluge auf allen Airline X-Strecken im Zeitraum } \\
\text { vom 5. Januar bis 28. März 2004. Die Tickets konnen Sie ab Mitt- } \\
\text { woch, 17. Dezember 2003, ab } 15 \text { Uhr fur } 24 \text { Stunden ausschließlich } \\
\text { uber unsere Kundenhotline unter der Telefonnummer XXX buchen. } \\
\text { Nahere Informationen erhalten Sie ebenfalls dort. } \\
\text { Mit freundlichen Grußen } \\
\text { Ihr Airline X-Team" }\end{array}$ & $\begin{array}{l}\text { „Lieber Airline X- } \\
\text { Kunde, als Dank } \\
\text { fur die Teilnahme } \\
\text { an unserer Befra- } \\
\text { gung konnen Sie } 4 \\
\text { Tickets zum Preis } \\
\text { von } 3 \text { buchen. Ab } \\
\text { morgen } 15 \mathrm{Uhr} \text { nur } \\
\text { unter xxx“ }\end{array}$ \\
\hline & 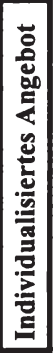 & $\begin{array}{l}\text { „Sehr geehrter Herr Ahrens, } \\
\text { als Dank fur die Teilnahme an unserer Befragung vom Oktober/ No- } \\
\text { vember } 2003 \text { hat Airline X exklusiv fur Sie } 2 \text { Tickets nach Rom furr } \\
\text { je } 39 \text { Euro reserviert. Dieses Angebot gilt für ein oder zwei Tickets } \\
\text { auf den besagten Strecken im Zeitraum vom 5. Januar bis } 28 \text {. Mărz } \\
\text { 2004. Sie konnen diese Tickets ab Mittwoch, 17. Dezember 2003, } \\
\text { ab } 14 \text { Uhr fur } 24 \text { Stunden ausschließlich uber unsere Kundenhotline } \\
\text { mit der Telefonnummer XXX buchen. Năhere Informationen erhal- } \\
\text { ten Sie ebenfalls dort. } \\
\text { Mit freundlichen Grußen } \\
\text { lhr Airline X-Team“ }\end{array}$ & $\begin{array}{l}\text { "Sehr geehrter Herr } \\
\text { Ahrens, Airline X } \\
\text { hat } 2 \text { Tickets nach } \\
\text { Rom fur je } 39 \text { Eur } \\
\text { fur Sie reserviert. } \\
\text { Buchbar ab morgen } \\
\text { 14Uhr fur 24h nur } \\
\text { unter XXX“ }\end{array}$ \\
\hline 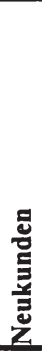 & 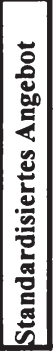 & $\begin{array}{l}\text { "Sehr geehrte Damen und Herren, } \\
\text { Airline X bietet seit mehr als einem Jahr preisgunstige Fluge nach } \\
\text { London, Paris, Rom und } 20 \text { weiteren europassche Stadte an. Im } \\
\text { Rahmen einer Sonderaktion konnen Sie Tickets zum Preis von } 19 € \\
\text { erwerben. Die Tickets gelten auf allen Airline X-Strecken im Zeit- } \\
\text { raum vom 5. Januar bis } 28 \text {. März 2004. Buchbar sind die Tickets ab } \\
\text { Mittwoch, 17. Dezember 2003, ab } 13 \text { Uhr fur } 24 \text { Stunden aus- } \\
\text { schließlich uber unsere Kundenhotline mit der Telefonnummer } \\
\text { XXX. Nahere Informationen erhalten Sie ebenfalls dort. } \\
\text { Mit freundlichen Grußen } \\
\text { Ihr Airline X-Team“ }\end{array}$ & $\begin{array}{l}\text {,Airline X bietet } \\
19 € \text {-Tickets fur Flu- } \\
\text { ge nach London, } \\
\text { Paris, Rom und } 20 \\
\text { weitere Stadte an. } \\
\text { Ab morgen } 15 \mathrm{Uhr} \\
\text { sind } 100019 €- \\
\text { Tickets unter XXX } \\
\text { buchbar" }\end{array}$ \\
\hline
\end{tabular}

Tab. 6: Inhaltliche Gestaltung der Direktmarketing-Stimuli im Rahmen der Experimente

Unternehmens, die zu längeren Gesprächsdauern führten, aber nicht unbedingt in einem extensiveren Informationsbedarf des Anrufers begründet lagen, nicht in die Bewertung einflossen. 
ten, nach Durchführung der Buchung bzgl. ihrer maximalen Preisbereitschaft im Hinblick auf das erhaltene Angebot befragt.

Abschließend werden die im Rahmen der Experimente verwendeten Formulierungen der unterschiedlichen Angebote in Abhängigkeit der Zielgruppe und des Instrumenteeinsatzes dargestellt. Inhaltlich orientierte sich die Formulierung des Angebots - neben der Zeichenbegrenzung aufseiten der SMS - an den unternehmenstypischen Formulierungen i. S. der Corporate Identity. Insgesamt konnten die Formulierungen der Angebote auf Basis von Mailings und Emails identisch formuliert werden. Aufgrund der Zeichenbegrenzung wurde das Angebot im Fall des SMS-Einsatzes entsprechend gekürzt. Die folgenden Tabelle zeigt die Texte der unterschiedlichen Angebote mit der entsprechenden Unterscheidung der Formulierungen hinsichtlich Email und Mailing zum einen sowie SMS zum anderen (vgl. Tab. 6). ${ }^{432}$

\subsubsection{Operationalisierung der kontrollierten Variablen}

Neben der Gestaltung des experimentellen Inputs in Form der zu variierenden unabhängigen Variablen ist es ebenfalls Ziel der systematischen Gestaltung von experimentellen Versuchsanlagen, bereits im Vorfeld der Untersuchung möglichst viele der potenziellen endogenen Einflussfaktoren auf die untersuchte abhängige Variable zu identifizieren und ihren Einfluss bspw. durch Konstanthaltung zu minimieren. ${ }^{433}$ Aus diesem Grund sollen zunächst weitere kontrollierte Variablen des Experimentes vorgestellt werden, die dem Bereich der endogenen Determinanten zuzuordnen sind. ${ }^{434}$ Als kontrollierte Variablen konnten vor allem gestalterische Determinanten, Timingaspekte sowie das Management des Rückkanals im Versuchsaufbau berücksichtigt werden.

Während sich der experimentelle Input auf die Bereiche zielgruppen-, angebotsform- und trägermedienbezogene Aspekte konzentrierte, wurden einige der im Rahmen des Entscheidungsprozesses des Direktmarketing-Einsatzes vorgestellten Determinanten explizit aus der Untersuchung ausgeschlossen. So sollte insbesondere der Einfluss gestalterischer Aspekte im Rahmen der Untersuchung nicht analysiert werden, da angenommen wurde, dass die Einflussstärke der Gestaltung im Verhältnis zu den drei variierten Determinanten von untergeordneter Bedeutung für das Erreichen der intendierten Wirkungen ist. Ziel war es daher, die graphischen Elemente über die unterschiedlichen Instrumente hinweg ähnlich

432 Aufgrund der Zeichenbegrenzung der SMS auf 160 Zeichen wurde der Werbetext für die SMS gekürzt. Bei sämtlichen Texten wurde der Name des Unternehmens durch „Airline X“ ersetzt, um die Anonymität sicherzustellen.

433 Vgl. Berekoven, L., Eckert, W., Ellenrieder, P., Marktforschung. a. a. O., S. 152.

434 Vgl. Meffert, H., Marketingforschung und Käuferverhalten, a. a. O., S. 208. 
zu gestalten, um möglichst wenig Varianz zu erhalten. Daher orientierte sich die formelle und inhaltliche Aufbereitung der Direktmarketing-Stimuli bei sämtlichen Zielgruppen, Angeboten und Instrumenten am bekannten Corporate Design des Unternehmens und wurde in Abhängigkeit der instrumentespezifischen graphischen und gestalterischen Darstellungsmöglichkeiten ähnlich umgesetzt. So wurde das Mailing auf den entsprechenden Briefbögen der Low Cost Airline gedruckt. Als Absender firmierte ebenso wie bei Email und SMS das ausgewählte Unternehmen. Entsprechend der Betreffzeile im Mailing, in der auf das Angebot hingewiesen wurde, gestaltete sich auch die Betreffzeile der Email. Im Fall der SMS entfiel dieser Schritt.

Darüber hinaus erfolgte eine detaillierte zeitliche Planung des Experiments, um möglichst wenige Überschneidungen im Timing verschiedener Marketingund Direktmarketing-Aktionen zu erzeugen und damit die Zuordnung von Stimulus und Wirkung nicht zu erschweren. Dieser Aspekt bezog sich auf verschiedene Ebenen:

- Jeder Proband erhielt nur ein Angebot über eines bzw. gleichzeitig über mehrere der drei Trägermedien und konnte demnach nur einmal an den Experimenten teilnehmen. Dieses Vorgehen vermied unerwünschte Wiederholungswirkungen sowie Wearouteffekte durch mehrere Direktmarketing-Stimuli. ${ }^{435}$

- Durch die nahezu zeitgleiche Versendung der 28 Direktmarketing-Stimuli an die 2.800 Probanden innerhalb von drei aufeinander folgenden Werktagen konnten zudem zeitlich saisonale Einflüsse über sämtliche Probanden hinweg als konstant angesehen werden.

- Ebenso wurde sichergestellt, dass sämtliche Direktmarketing-Stimuli den Rezipienten am Vorabend der eigentlichen Buchungsmöglichkeit zugingen. Die Zustellung der Emails und SMS erfolgte jeweils um 17.00 Uhr. Bei der Versendung der Mailings wurde ebenfalls eine Zustellung am Vortag des $\mathrm{Bu}$ chungstages vollzogen, wobei die Zustellung der Mailings mit der Auslieferung der Haus- bzw. Geschäftspost durch die Deutsche Post AG erfolgte.

- Darüber hinaus wurde auch im Falle des kombinierten Einsatzes mehrerer Direktmarketing-Instrumente von einer zusätzlichen Timing-Entscheidung abstrahiert. Aufgrund der vielfältigen Gestaltungsmöglichkeiten im Rahmen

435 Die eindeutige Zuordnung einer Verhaltenswirkung zu einem Stimulus wird erschwert, wenn mehrere verschiedene Stimuli in kurzem zeitlichen Abstand auf den Rezipienten einströmen. So ist grundsätzlich von einer erhöhten Werbewirkung auszugehen, wenn ein wiederholter Werbekontakt erfolgt. Vgl. bspw. Wimmer, R.-M., Wiederholungswirkungen der Werbung - Saarbrücken 1979. Nach Überschreiten eines bestimmten Schwellenwertes erfolgt aber bei zu häufiger Wiederholung ein absoluter Rückgang der Werbewirkung, ein sog. Wearout. Corkindale, D., Newall, J., Advertising thresholds and wearout, a. a. O., S. 327-378. 
des Timings (Parallelversand vs. zeitversetztes Versenden) erfolgte eine zeitgleiche Zustellung des Direktmarketing-Stimulus über mehrere Medien.

- Ebenfalls war es das Ziel, bereits bei der Planung des Experiments den Einfluss weiterer kommunikativer Aktivitäten des Unternehmens - insbesondere im Bereich des Direktmarketing - zu minimieren. Als besonders relevante Einflussgröße wurde vor allem der wöchentlich versendete Email-Newsletter angesehen, welcher zudem nur von einem Teil der Probanden abonniert wurde und die Vergleichbarkeit der Probanden weiter einschränkt. Daher fanden die Experimente an drei aufeinander folgenden Tagen statt, an denen neben dem Einsatz der klassischen Werbung keine Sonderaktionen durchgefuhrt wurden.

- Im Hinblick auf das Responsemanagement wurde für sämtliche Responseund Buchungsvorgänge ein Call-Center als Rückkanal eingesetzt. Vorteilhaft erscheint in diesem Zusammenhang insbesondere die Tatsache, dass der zeitliche und der monetäre Aufwand für sämtliche Anrufer ähnlich hoch waren, auch wenn dafür bei Email und Mailing ein Medienbruch zwischen Hin- und Rückkanal entstand.

\subsubsection{Operationalisierung ausgewählter exogener Determinanten}

$\mathrm{Zu}$ den sog. Störvariablen zählen sämtliche Größen, die neben den unabhängigen Variablen einen Einfluss auf die abhängige Variable - in diesem Fall das Response- und Buchungsverhalten der Rezipienten - besitzen. Diese Störvariablen sind jedoch als nichtkontrollierbare Parameter anzusehen und demnach dem $\mathrm{Be}-$ reich der exogenen Determinanten zuzuordnen. ${ }^{436} \mathrm{Zu}$ den wichtigsten Kategorien dieser exogenen Determinanten zählen sowohl personen- als auch situationsbezogene Determinanten. ${ }^{437}$ Die Erhebung der als relevant angesehenen exogenen Determinanten erfolgte mit Hilfe einer den Experimenten vor- und einer nachgelagerten Befragung. Ziel der Befragungen war es, personen- und situationsbezogene Einflussfaktoren zu identifizieren, die als potenzielle Wirkungsdeterminanten des Response- und Buchungsverhaltens fungieren.

In der den Experimenten vorgelagerten Ex-ante-Befragung im Oktober/November 2003 wurden daher zunächst Kunden des Unternehmens zu ausgewählten Themenbereichen befragt, die einer Anreicherung ihres persönlichen Profils dienten. In diesem Zusammenhang lag ein zentrales Ziel in der Erhebung von Aussagen über das allgemeine Informations- und Buchungsverhalten der Kunden

436 Vgl. Meffert, H., Marketingforschung und Käuferverhalten, a. a. O., S. 207.

437 Vgl. Steffenhagen, H., Wirkungen der Werbung, a. a. O., S. 13; Dallmer, H., Erfolgsbedingungen der Kommunikation im Direct-Marketing, a. a. O., S. 73. 
und weiterer für die vermuteten Reaktionsmuster verantwortlichen personenbezogenen Determinanten, vor allem soziodemographischer, psychographischer und verhaltensorientierter Merkmale. $\mathrm{Zu}$ diesem Zweck wurden die Befragungsteilnehmer gebeten, zu unterschiedlichen Themenkomplexen, wie Reisegewohnheiten, Buchungsverhalten, soziodemographischen Informationen etc., Angaben zu machen. 438

Die Durchführung der Ex-ante-Befragung fand an unterschiedlichen Orten statt, wobei der Großteil der Fragebögen (ca. $85 \%$ ) von den Befragten vor bzw. während des Fluges an Bord der Flugzeuge des Unternehmens ausgefullt wurden. Die Teilnahme an der Befragung wurde mit der Teilnahme an einer Verlosung von $5 \times 2$ Freiflügen in ausgewählte europäische Hauptstädte incentiviert. ${ }^{439}$ Die Befragung wurde im Zeitraum vom 23. Oktober 2003 bis 12. November 2003 durchgeführt. Insgesamt nahmen 3.319 Personen an der Ex-anteBefragung teil.

Ergänzt wurden die derart gewonnenen Informationen durch eine zweite Befragungswelle, an der 798 Personen drei Monate nach der Durchführung der Direktmarketing-Experimente im Februar/März 2004 teilnahmen. ${ }^{440}$ Der Ex-postFragebogen wurde per Post bzw. per Email an sämtliche Personen verschickt, die bei der Ex-ante-Befragung eine Post- oder Emailadresse angegeben hatten und sowohl an der Ex-ante-Befragung als auch an den Experimenten teilgenommenen hatten. Diese wurden gebeten, einige spezifische, auf die versendeten Direktmar-

438 Ein Unterziel des Ex-ante-Fragebogens lag in der Erhebung von Daten, die für die Durchführung der sich anschließenden Experimente notwendig waren. Zu den benötigten Daten zählten die postalische Adresse, eine gültige Emailadresse sowie eine aktuelle Mobilfunknummer der befragten Personen. In diesem Zusammenhang war es den Befragten freigestellt, sämtliche oder nur einzelne dieser Daten anzugeben. Darüber hinaus war die Erhebung der Daten mit der Frage verbunden, ob die befragten Personen einer Teilnahme an den Direktmarketing-Experimenten zustimmten und sich daher mit der Zusendung von Angeboten mittels verschiedener DirektmarketingInstrumente einverstanden erklärten. Durch die ausdrückliche Erlaubnis zur Durchführung verschiedener Experimente konnte damit auch der Philosophie des Unternehmens i. S. d. Permission Marketing entsprochen werden.

439 Die Angabe der persönlichen Daten sowie die Einwilligung zur Teilnahme an den Experimenten bildete keine Voraussetzung für die Teilnahme an der Verlosung. Lediglich die Angabe eines eindeutigen Identifikationsmerkmals (Name, Emailadresse oder Mobilfunknummer) war zur Gewinnbenachrichtigung notwendig.

440 Eine Ausnahme stellten diejenigen Personen dar, die im Rahmen der Ex-anteBefragung lediglich ihre Handy-Nummer angegeben hatten (89 Personen). So konnten 2.011 der insgesamt 2.100 im Rahmen der unterschiedlichen Aktionen angesprochenen Personen angeschrieben werden. Insgesamt konnten 798 Ex-post-Fragebögen generiert werden, was einer Rücklaufquote von ca. $40 \%$ entspricht. 
keting-Stimuli bezogene Fragen zu beantworten. ${ }^{441}$ Ziel der zweiten Befragung war es, weitere personenbezogene Determinanten zur Erklärung des tatsächlichen Verhaltens der angesprochenen Personen auf den erhaltenen Direktmarketing-Stimulus zu erheben, die vor der Durchführung der DirektmarketingAktionen allerdings nicht erhoben werden konnten, wie bspw. Informationen zum situativen Umfeld des Rezipienten während der Empfangssituation des Direktmarketing-Stimulus.

\subsubsection{Zusammensetzung der Testgruppen}

Insgesamt wurde jeder der 28 konfigurierten Direktmarketing-Stimuli an eine Testgruppe von 100 Personen verschickt, die vor Durchführung der Direktmarketing-Aktionen in einem mehrstufigen Prozess zusammengestellt wurden. Um Reaktionsunterschiede der Rezipienten möglichst vollständig auf die Variation einer unabhängigen Variablen zurückführen zu können, wurde auf eine zufällige Bildung zugunsten einer gezielten Zusammensetzung der Stichproben verzichtet. ${ }^{442}$ Die Bildung strukturgleicher Testgruppen gilt vielmehr als notwendige Voraussetzung, um den potenziellen Einfluss personenbezogener Faktoren zu minimieren. ${ }^{443}$ Der Aufbau strukturgleicher Stichproben erfolgte auf Basis verschiedener Kriterien, die im Rahmen des Ex-ante-Fragebogens erhoben wurden.

Als zentrale Determinanten des Buchungsverhaltens bei Flugreisen werden grundsätzlich der im Zeitpunkt der Zusendung herrschende Bedarf an Flügen sowie die Attraktivität des Angebotes hinsichtlich des Preises, der angebotenen Strecken sowie des Flugzeitraums angesehen. ${ }^{444} \mathrm{Da}$ weder der individuelle $\mathrm{Be}$ darf im Vorfeld der Untersuchung bei allen Bestandskunden hätte erhoben werden können, noch die Durchführung von Tests zur Bestimmung der Attraktivität der jeweiligen Angebots- und Instrumentekombinationen aufgrund von Kom-

441 Das Ausfüllen des Fragebogens wurde wiederum durch die Teilnahme an einer Verlosung von Freiflügen incentiviert.

442 Dieses Vorgehen folgt dabei dem grundlegenden Ziel von Experimenten, möglichst viele Einflussfaktoren auf die unabhängige Variable vor der Durchführung der Analyse zu eliminieren. Vgl. Green, P. E., Tull, D. S., Methoden und Techniken der Marketingforschung, a. a. O., S. 322.

$443 \mathrm{Vgl}$. Wolf, J., Marktforschung: praktische Anwendung mit zahlreichen Arbeitsblättern, a. a. O., S. 96.

444 Die Auswahl der für die Strukturgleichheit verwendeten Kriterien orientierte sich an der zentralen Zielgröße des Direktmarketing-Einsatzes: dem Buchungsverhalten. 
plexitätsüberlegungen als sinnvoll erschien, wurde auf andere Indikatoren zurückgegriffen, zu denen Informationen vorlagen: ${ }^{445}$

- Das Alter bestimmt insbesondere beim Buchungsverhalten die Reaktion auf kurzfristige Angebote, die sich - wie im vorliegenden Fall - auf einen kurzen Entscheidungszeitraum bezogen, allerdings für einen Reisezeitraum galten, der noch weiter entfernt lag. Junge Leute reisen zwar spontaner, planen aber Reisen nicht solange im Voraus wie ältere Personen, die Buchungen i. d. R. mit größerem zeitlichen Vorlauf vornehmen. Die Wahrscheinlichkeit einer Buchung ist daher im vorliegenden Fall tendenziell bei älteren Rezipienten vorhanden.

- Weiterhin determiniert das Einkommen das Buchungsverhalten, insbesondere vor dem Hintergrund verschiedener Angebote mit unterschiedlichen Preisen. So hat sich auch im Flugverkehrsbereich mehrfach gezeigt, dass mit steigendem Einkommen die Preissensibilität nachlässt und damit die Buchungswahrscheinlichkeit steigt.

- Schließlich hat der Reisegrund einen besonderen Einfluss auf die Buchungswahrscheinlichkeit. Während beruflich Reisende i. d. R. relativ preisunsensibel sind, reagieren Privatreisende äußerst stark auf Preisunterschiede. Darüber hinaus erscheint die Verfügbarkeit der Tickets zu bestimmten Flugzeiten insbesondere für beruflich Reisende von elementarer Bedeutung für die $\mathrm{Bu}$ chung zu sein und der Preis nicht als kaufentscheidendes Kriterium zu fungieren. Die Wahrscheinlichkeit einer Buchung ist demnach bei gleichzeitig beruflicher und privater Nutung des Flugzeugs als Verkehrsmittel am größten.

Weitere soziodemographische Kriterien wurden bei Konfiguration der Testgruppen nicht explizit berücksichtigt, da anzunehmen ist, dass sie sich teilweise implizit in den herangezogenen Merkmalen widerspiegeln, bspw. die Schulbildung im Einkommen, bzw. von untergeordneter Bedeutung sind. Tab. 7 gibt einen Überblick über die ausgewählten Kriterien zur Herstellung der Strukturgleichheit sowie den vermuteten Einfluss auf die Buchungswahrscheinlichkeit.

Die unterschiedlichen Ausprägungen der drei Merkmale (zwei Altersklassen, drei Einkommensklassen, drei Reiseklassen) ergeben insgesamt achtzehn unterschiedliche Kombinationsmöglichkeiten. Auf Basis unternehmensinterner Untersuchungen konnte der Anteil der verschiedenen Merkmalskombinationen im Hinblick auf den Kundenstamm des Unternehmens ermittelt werden. Die einhun-

445 Die Auswahl der Strukturvariablen erfolgte auf Basis einer umfassenden Literaturauswertung. Die derart gewonnenen Erkenntnisse wurden im Rahmen mehrerer Expertengespräche sowie auf Basis der Auswertungen vergangener DirektmarketingAktionen im Unternehmen intensiv diskutiert. 


\begin{tabular}{|l|l|c|}
\hline \multicolumn{1}{|c|}{ Kriterium } & \multicolumn{1}{|c|}{ Ausprägung } & \multicolumn{1}{c|}{$\begin{array}{c}\text { Vermuteter Einfluss auf die } \\
\text { Buchungswahrscheinlichkeit }\end{array}$} \\
\hline \multirow{2}{*}{ Alter } & jung (<35 Jahre) & 0 \\
\cline { 2 - 3 } & alt $(>35$ Jahre & + \\
\hline \multirow{3}{*}{ Einkommen } & niedrig (bis 1.500 $€)$ & - \\
\cline { 2 - 3 } & mittel $(1.500 €$ bis 3.000 $€)$ & 0 \\
\cline { 2 - 3 } & hoch (ab 3.000 $€)$ & + \\
\hline \multirow{3}{*}{ Reisegrund } & beruflich & - \\
\cline { 2 - 3 } & privat & 0 \\
\cline { 2 - 3 } & privat und beruflich & + \\
\hline $\begin{array}{l}+=\text { positiver Einfluss auf die Buchungswahrscheinlichkeit } \\
0=\text { neutraler Einfluss auf die Buchungswahrscheinlichkeit } \\
-=\text { negativer Einfluss auf die Buchungswahrscheinlichkeit }\end{array}$ \\
\hline
\end{tabular}

Tab. 7: Kriterien zur Herstellung der Strukturgleichheit der Stichproben

\begin{tabular}{|c|c|c|c|c|c|c|}
\hline $\begin{array}{l}\text { Struktur- } \\
\text { klasse }\end{array}$ & Alter & Einkommen & $\begin{array}{l}\text { Reise- } \\
\text { grund }\end{array}$ & $\begin{array}{c}\text { Buchungs- } \\
\text { wahrschein- } \\
\text { lichkeit }\end{array}$ & $\begin{array}{c}\text { Anteil bei den } \\
\text { Bestandskunden }\end{array}$ & $\begin{array}{l}\text { Anteil in } \\
\text { den Test- } \\
\text { gruppen }\end{array}$ \\
\hline 1 & alt & hoch & beides & +++ & $20,20 \%$ & $20,00 \%$ \\
\hline 2 & alt & hoch & privat & + & $1,20 \%$ & $1,00 \%$ \\
\hline 3 & alt & mittel & beides & ++ & $4,10 \%$ & $4,00 \%$ \\
\hline 4 & alt & hoch & privat & ++ & $1,10 \%$ & $1,00 \%$ \\
\hline 5 & jung & hoch & beides & ++ & $7,00 \%$ & $7,00 \%$ \\
\hline 6 & alt & hoch & beruflich & + & $7,20 \%$ & $7,00 \%$ \\
\hline 7 & alt & hoch & beruflich & + & $11,40 \%$ & $11,00 \%$ \\
\hline 8 & jung & mittel & beides & + & $10,00 \%$ & $10,00 \%$ \\
\hline 9 & alt & niedrig & beides & + & $0,90 \%$ & $1,00 \%$ \\
\hline 10 & alt & mittel & privat & + & $1,90 \%$ & $2,00 \%$ \\
\hline 11 & alt & niedrig & privat & 0 & $1,00 \%$ & $1,00 \%$ \\
\hline 12 & alt & mittel & beruflich & 0 & $6,60 \%$ & $7,00 \%$ \\
\hline 13 & jung & niedrig & beides & 0 & $1,00 \%$ & $1,00 \%$ \\
\hline 14 & jung & mittel & privat & 0 & $15,60 \%$ & $16,00 \%$ \\
\hline 15 & jung & niedrig & privat & - & $6,60 \%$ & $7,00 \%$ \\
\hline 16 & jung & mittel & beruflich & - & $2,20 \%$ & $2,00 \%$ \\
\hline 17 & alt & niedrig & beruflich & - & $0,80 \%$ & $1,00 \%$ \\
\hline \multirow[t]{2}{*}{18} & jung & niedrig & beruflich & - & $1,00 \%$ & $1,00 \%$ \\
\hline & & & & & $100,00 \%$ & $100,00 \%$ \\
\hline \multicolumn{7}{|c|}{$\begin{array}{ll}+++ & \text { stark uberdurchschnittliche Buchungswahrscheinlichkeit } \\
++ & \text { uberdurchschnittliche Buchungswahrscheinlichkeit } \\
+ & \text { leicht uberdurchschnittliche Buchungswahrscheinlichkeit } \\
\text { o } & \text { durchschnittliche Buchungswahrscheinlichkeit } \\
- & \text { leicht unterdurchschnittliche Buchungswahrscheinlichkeit } \\
-- & \text { unterdurchschnittliche Buchungswahrscheinlichkeit }\end{array}$} \\
\hline
\end{tabular}

Tab. 8: Zusammensetzung der Testgruppen 
dert Personen umfassenden Stichproben wurden anschließend auf Basis dieser Anteile quotiert. Die folgende Tabelle zeigt die Anteile der in der Grundgesamtheit der Bestandskunden vorhandenen Personen auf Basis der drei Kriterien Alter, Einkommen und Reisegrund sowie die realisierten Anteile dieser Strukturklassen in den Testgruppen. Hierbei konnten die Testgruppen hinsichtlich der Merkmale Alter, Einkommen und Reisegrund identisch mit der jeweiligen Quotierung bei den Bestandskunden gebildet werden (vgl. Tab. 8). Darüber hinaus konnte bei jeder Testgruppe eine geschlechterspezifische Quotierung von jeweils $50 \%$ realisiert werden.

Für die Bildung der Bestandskunden-Testgruppen (21 x 100 Personen) wurde auf die Daten aus der Ex-ante-Befragung zurückgegriffen. So lagen aus der Ex-ante-Befragung insgesamt 3.319 Fragebögen vor, von denen 215 Personen dem Unternehmen keine Erlaubnis zur Zusendung ausgewählter Angebote mittels der Instrumente Mailing, Email oder SMS gegeben hatten und folglich von der weiteren Testgruppenbildung ausgeschlossen wurden. Die angegebenen Daten der verbleibenden 3.104 Personen wurden weiterhin auf Vollständigkeit und Richtigkeit überprüft. Bei 317 Personen lagen unvollständige bzw. fehlerhafte Daten vor. Die betroffenen Personen wurden ebenfalls von der weiteren Betrachtung ausgeschlossen. Die verbleibenden 2.878 Personen konnten schließlich zu 21 Testgruppen à 100 Personen auf Basis der genannten Quotierung zusammengestellt werden.

Weiterhin erfolgte zum Zweck der Neukundenansprache die Konfiguration von weiteren sieben Testgruppen à 100 Personen anhand der Quotierung auf Basis der beschriebenen Kriterien. In diesem Zusammenhang konnten sämtliche der ausgewählten personenbezogenen Merkmale zur Herstellung der Strukturgleichheit beim Erwerb der Neukundendaten von einem Adressanbieter berücksichtigt werden. ${ }^{446}$ Die geschlechterspezifische Quotierung i. H. v. $50 \%$ konnte ebenfalls eingehalten werden. Zur Berücksichtigung weiterer Einflussfaktoren, die nicht kontrolliert werden konnten bzw. nicht prognostizierbar sind und demnach als potenzielle Störgrößen der Untersuchung angesehen werden, wurde daruber hinaus eine Kontrollgruppe von 100 Personen mit gleicher Quotierung hinsichtlich der Strukturklassen und des Geschlechts gebildet. ${ }^{447}$ Die Kontrollgruppe erhielt während der Durchführung der Experimente keine Direktmarketing-Stimuli. ${ }^{448}$

446 In diesem Kontext konnten Adressen bestehend aus Post- und Emailadresse sowie Mobilfunknummer im Hinblick auf sämtliche der ausgewählten Kriterien mit den entsprechenden Merkmalsausprägungen gekauft werden.

447 Die nicht zugeordneten Personen fanden keinen Eingang in die Untersuchung.

448 Die Personen der Kontrollgruppe entstammen ebenfalls dem Segment der Bestandskunden. 


\subsection{Ablauf der experimentellen Untersuchung}

Die Durchführung der verschiedenen Direktmarketing-Aktionen sowie der zwei Befragungswellen fanden in der Zeit vom Oktober 2003 bis zum März 2004 statt. Die Auswahl der Personen, die im Rahmen dieser Experimente ein Angebot erhielten, basierte auf der Teilnahme an einer Vorab-Befragung („Ex-anteBefragung"). Die Ergebnisse der Direktmarketing-Aktionen wurden im Hinblick auf verschiedene Zielgrößen ausgewertet und darüber hinaus mit Informationen aus einer zweiten Befragungswelle („Ex-post-Befragung“) angereichert. Die folgende Abbildung fasst den zeitlichen Ablauf der empirischen Untersuchung zusammen (vgl. Abb. 11).

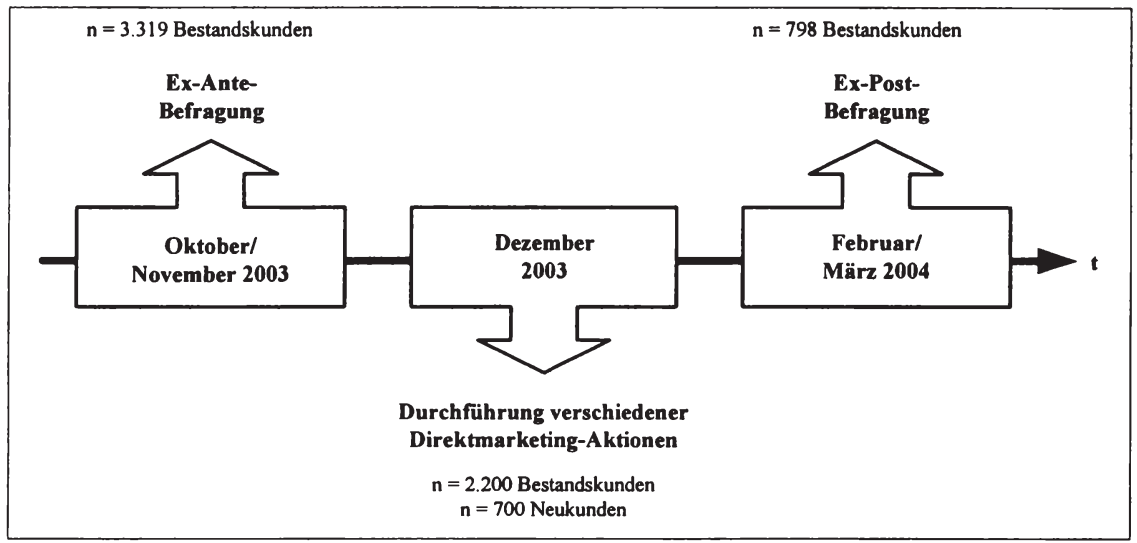

Abb. 11: Zeitlicher Ablauf der empirischen Untersuchung

Neben der Berücksichtigung der schwankenden Nachfrage im Jahresverlauf wurde der Zeitpunkt der Versendung der Direktmarketing-Aktionen in Abhängigkeit weiterer Direktmarketing-Aktivitäten des Unternehmens wie bereits erläutert vorgenommen. So wurden die Direktmarketing-Aktionen an drei aufeinander folgenden Tagen versendet, an denen keine sonstigen Direktmarketing-Aktionen stattfanden. In kurzem zeitlichen Abstand zu den Experimenten standen lediglich die regelmäßig durchgeführten Direktmarketing-Aktionen, die jeweils donnerstags durchgeführt wurden. Die folgende Abbildung zeigt abschließend die zeitliche Einbettung der durchgeführten Experimente vor dem Hintergrund weiterer direktmarketingbezogener Aktionen (vgl. Abb. 12). 


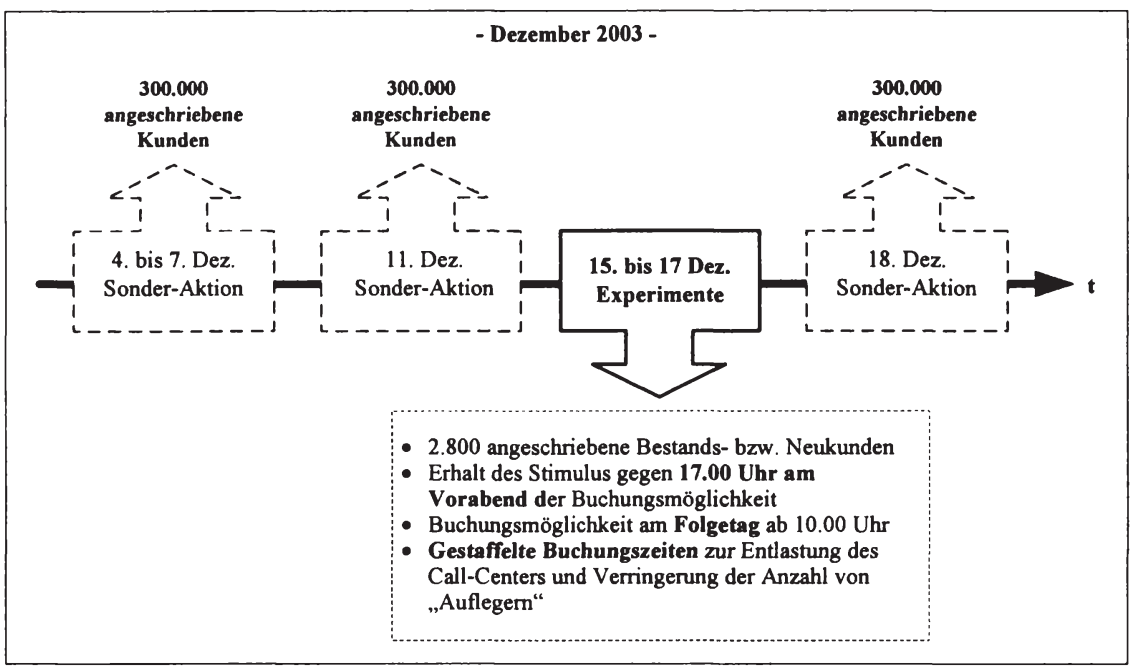

Abb. 12: Zeitlicher Ablauf der Experimente

\subsection{Methoden der Datenauswertung}

Kernelement der Datenauswertung bilden die Effektivitäts- und Effizienzanalysen der durchgefuhrten Direktmarketing-Aktionen. Von besonderer Relevanz sind in diesem Zusammenhang Aussagen über die Höhe der Zustell-, der Response- und der Buchungsquoten der Rezipienten sowie der entsprechenden Effizienzkennzahlen. Durch die gezielte Variation einzelner endogener Determinanten können mit Hilfe dieser Analysen die jeweils zu beobachtenden residualen Auswirkungen auf die abhängige Variable gemessen und damit der Einfluss spezifischer Ausprägungen der unabhängigen Variablen auf die Zielgröße quantifiziert werden. ${ }^{449}$

Neben den betrachteten endogenen Determinanten soll in einem zweiten Schritt der Einfluss ausgewählter exogener Determinanten auf das Response- und Buchungsverhalten des Rezipienten untersucht werden. Um zu überprüfen, ob ein beobachteter Zusammenhang zwischen einer unabhängigen exogenen Variable, wie bspw. dem Alter, und der abhängigen Variablen in Form der Rezipienten-

449 Dieses Vorgehen ermöglicht eine genaue Messung der Wirkungsunterschiede in quantitativer Hinsicht, bspw. in Form einer um $5 \%$ höheren Responsequote von Emails ggü. dem Einsatz von SMS. 
reaktion statistisch abgesichert oder vielmehr rein zufällig ist, kommen daruber hinaus verschiedene statistische Verfahren zum Einsatz. ${ }^{450}$

Die abhängige Variable hat drei mögliche kategoriale Ausprägungen: „keine Reaktion“, „Response, aber keine Buchung“ (Interessent) und „Buchung“. Die unabhängigen Variablen haben sowohl metrisches als auch nominales Skalenniveau und entstammen dem Bereich der personen- und situationsbezogenen Determinanten. Im Fall nominalskalierter unabhängiger Variablen, wie bspw. das Merkmal Beruf, findet aufgrund der ebenfalls nominalskalierten abhängigen Variablen die Kontingenzanalyse Anwendung. ${ }^{451}$ Als relevante Methode zur Überprüfung des Zusammenhangs zwischen den beiden Variablen wird der ChiQuadrat-Test herangezogen, der Aussagen über die Existenz oder Nicht-Existenz eines Zusammenhangs zwischen den unabhängigen und abhängigen Variablen zulässt. Mit Hilfe des Softwarepakets SPSS 12.0 werden die Kontingenzanalysen durchgeführt, die unter Berücksichtigung der Freiheitsgrade und aufgrund der Vergleichsgröße (Signifikanz) eine Ablehnung oder Annahme der Nullhypothese ermöglichen, dass die zwei betrachteten Variablen voneinander unabhängig sind. ${ }_{0}^{452}$ Die Stärke eines nachgewiesenen Einflusses lässt sich anhand verschiedener Maßzahlen erkennen, wobei dieser Schritt nicht im Mittelpunkt der Ausführungen stehen soll..$^{453}$

Im Fall metrisch skalierter unabhängiger Variablen kommen zur Prüfung der Existenz potenzieller Zusammenhänge entweder T-Tests für Mittelwertdifferenzen bzw. - bei Annahme einer fehlenden Normalverteilung - sog. nichtparametrische Tests für zwei unabhängige Stichproben zum Einsatz. ${ }^{454}$ Als zentrales Gütekriterium der T-Tests gilt hierbei das Signifikantniveau, welches einem Niveau von $5 \%$ genügen sollte. Liegen zwei unabhängige Stichproben vor

$450 \mathrm{Vgl}$. Janssen, J., Laatz, W., Statistische Datenanalyse mit SPSS für Windows, a. a. O., S. 236.

$451 \mathrm{Vgl}$. Backhaus, K. et al., Multivariate Analysemethoden. Eine anwendungsorientierte Einführung, 10. Aufl., Berlin u a. 2003, S. $229 \mathrm{ff}$.

452 Vgl. ebenda, S. 240.

453 Als mögliche Kennzahl wird der sog. Phi-Koeffizient eingesetzt, wobei Werte größer als 0,3 auf einen Zusammenhang hinweisen, der „mehr als trivial ist“. Vgl. Fleiss, J. L., Statsitical Methods for Rates and Proportions, 2nd edition, New York 1981, S. 60. Liegen mehr als zwei Ausprägungen einer Variablen vor, ist der Kontingenzkoeffizient zu berücksichtigen. Unabhängig von der Anzahl der Dimensionen lässt sich zudem Cramer's V einsetzen, dass - ebenso wie der ebenfalls einsetzbare Kontingenzkoeffizient - einen Wert von 1 annehmen kann und im Fall binär ausgeprägter Variablen mit dem Phi-Koeffizient übereinstimmt, wobei sich Unterschiede hinsichtlich des Vorzeichens ergeben können.

454 Vgl. Janssen, J., Laatz, W., Statistische Datenanalyse mit SPSS für Windows, a. a. O., S. $310 \mathrm{ff}$. und S. $497 \mathrm{ff}$. 
(bspw. Nicht-Reagierer vs. Reagierer) kommt zur Überprüfung des Zusammenhangs im Rahmen nicht-parametrischer Tests der sog. Mann-Whitney-Test zum Zuge. ${ }^{455}$ Als Test für $\mathrm{k}$ unabhängige Stichproben wird hingegen der KruskilWallis-Test verwendet, wobei dieser sowohl für den Zwei-Gruppen- als auch für den Drei-Gruppen-Fall einsetzbar wăre. Zentrales Gütekriterium ist hierbei ebenfalls das Signifikanzniveau. ${ }^{456}$

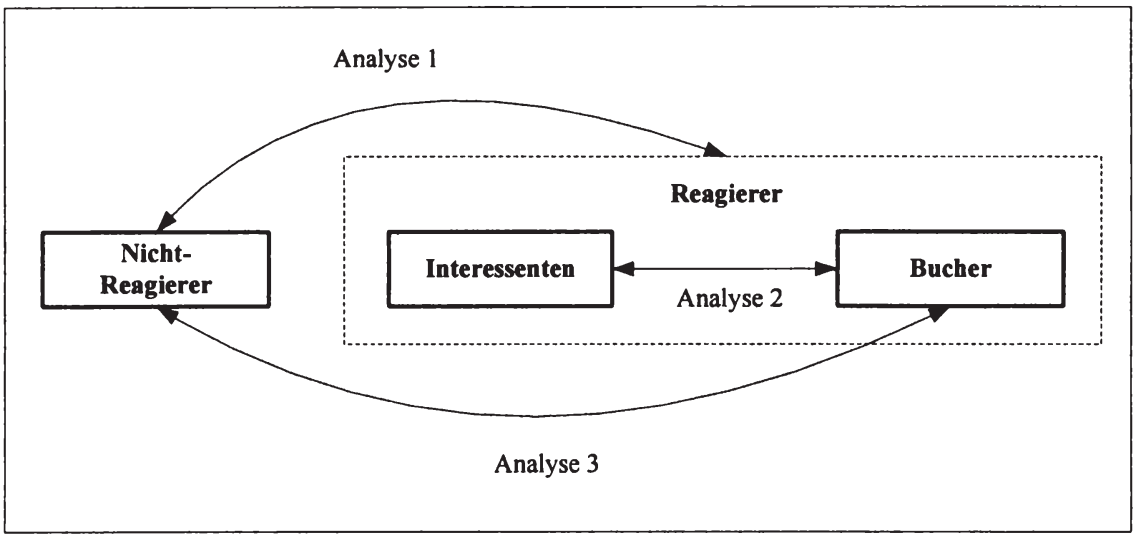

Abb. 13: Mehrstufige Analyse der Zusammenhänge zwischen ausgewählten exogenen Determinanten und dem Rezipientenverhalten

Im Rahmen dieses zweiten Analyseschrittes sollen die ausgewählten exogenen Variablen in einem mehrstufigen Vorgehen mit verschiedenen statistischen Tests untersucht werden. So wird zunächst geprüft, ob die betrachtete exogene Variable mit ihren verschiedenen Ausprägungen einen Zusammenhang zu der Entscheidung des Rezipienten aufweist, auf den Direktmarketing-Stimulus zu reagieren und damit eine Verhaltensreaktion in Form eines Anrufes im Call-Center zu zeigen (Analyse 1). Unabhängig von den Ergebnissen dieser Prüfung wird weiterhin untersucht, ob die betrachtete Variable in Zusammenhang mit Entscheidung von Anrufern im Call-Center steht, im Rahmen des Responsevorgangs auch einen Flug zu buchen (Analyse 2). Anschließend soll analysiert werden, ob die entsprechende exogene Variable grundsătzlich in Zusammenhang mit der Buchungsentscheidung steht, wobei nicht die Anrufer im Call-Center sondern die Nicht-Reagierer und die Bucher als Grundgesamtheit fur die Untersuchung dienen (Analyse 3). Der letztgenannte Vergleich liefert mitunter die wichtigste Er- 
kenntnis, da die Ergebnisse Aussagen darüber zulassen, welche Variablen NichtReagierer und Bucher voneinander trennen und in Zusammenhang mit der Buchungsentscheidung stehen, während Analyse 2 sich auf bereits reagierende Personen begrenzt. ${ }^{457}$ Die folgende Abbildung zeigt zusammenfassend das beschriebene Vorgehen der Analyse der exogenen Determinanten vor dem Hintergrund der beobachteten Rezipientenreaktion (vgl. Abb. 13).

Mit dem Ziel, die relative Bedeutung der in den vorangegangenen Schritten untersuchten exogenen und endogenen Determinanten für das Response- und Buchungsverhalten zu analysieren, wird in einem dritten Analyseschritt das Verfahren der Logistischen Regression eingesetzt. ${ }^{458}$ Ziel der Logistischen Regression ist die Erklärung der Zugehörigkeit von Beobachtungen zu Gruppen durch unabhängige Variablen. ${ }^{459} \mathrm{Ihr}$ Einsatz bietet sich insbesondere für qualitative Fragestellungen an, bei denen der Zusammenhang von verschiedenen unabhängigen Variablen auf eine dichotom ausgeprägte abhängige Variable (hier: Response $\mathrm{ja} /$ nein bzw. Buchung ja/nein) untersucht werden soll. ${ }^{460}$ Aufgrund der im vorliegenden Fall binär ausgeprägten abhängigen Variablen führt der Einsatz der herkömmlichen Regressionsanalyse zu einer Verletzung der Prämisse normalverteilter Residuen, so dass folglich inferenzstatistische Aussagen nicht möglich sind. ${ }^{461}$ Ebenso gestaltet sich im vorliegenden Fall auch der Einsatz der ZweiGruppen-Diskriminanzanalyse als schwierig, da zahlreiche kategorial-ausgeprägte unabhängige Variablen vorliegen, die zu einer Verletzung der Prämisse metrisch-skalierter unabhängiger Variablen im Rahmen der Diskriminanzanalyse führen. ${ }^{462}$ Hingegen ermöglicht der Einsatz der Logistischen Regression sogar

457 Das Responseverhalten wird nicht als zentrale sondern nur als modale Zielgröße angesehen. So hängt insbesondere der ökonomische Erfolg des DirektmarketingEinsatzes von der Erzielung eines möglichst hohen Umsatzes ab, der sich aus der absoluten Anzahl von Buchungen multipliziert mit den erzielten Ticketpreisen ergibt.

458 Das Vorgehen, bereits vor der Durchfuhrung der Logistischen Regression die Vielzahl potenzieller Einflussfaktoren auf die signifikanten Variablen zu beschränken, wird in der Literatur immer wieder vorgeschlagen. Vgl. bspw. Wulf, K. de, Hoekstra, J. C., Commandeur, H. R., The Opening und Reading Behavior of Business-toBusiness Direct Mail, a. a. O., S. 138.

459 Das Ergebnis einer derartigen Prüfung ergibt die Wahrscheinlichkeit der Zugehörigkeit einer Beobachtung zu einer Gruppe aufgrund der mit Regressionskoeffizienten gewichteten Ausprägungen der unabhängigen Variablen.

460 Vgl. Krafft, M., Logistische Regression, a. a. O., S. 239.

461 Vgl. Aldrich, J., Nelson, F. D., Linear Probability, Logit and Probit Models, a. a. O., S. 13 f.; Urban, D., Logit-Analyse: Statistische Verfahren zur Analyse von Modellen mit qualitativen Responsevariablen, 1993, S. $16 \mathrm{ff}$.

462 Vgl. Press, S. J., Wilson, S., Choosing Between Logsitic Regression and Discriminant Analysis, in: Journal of the American Statistical Association, No. 73, 1978, S. 700; Aldrich, J., Cnudde, C. F., Probing the Bounds of Conventional Wisdom: A 
Aussagen über die Veränderung der Gruppenzugehörigkeitswahrscheinlichkeit, wenn eine beobachtbare unabhängige Variable einen anderen Wert annimmt. ${ }^{463}$

Der Rechenansatz der Logistischen Regression unterscheidet sich in verschiedenen Punkten von dem der herkömmlichen Regressionsanalyse. So modelliert die Logistische Regression den Wahrscheinlichkeitsübergang einer kategorial ausgeprägten Variablen in Abhängigkeit der verschiedenen unabhängigen Variablen unter der Annahme, dass die Residuen einer logistischen Verteilung genügen. ${ }^{464}$ In diesem Zusammenhang ist es das Ziel, die Koeffizienten der zugrunde liegenden Funktion derartig zu schätzen, dass eine optimale Trennung der zwei Ausprägungen der abhängigen Variablen erreicht wird. Hierzu wird die Maximum-Likelihood-Methode verwendet, die das Produkt der Wahrscheinlichkeiten der Zuordnung zur jeweiligen Gruppe aller Beobachtungen maximiert. ${ }^{465}$ Auf Basis dieses Ansatzes erfolgt zunächst die Schätzung eines Modells, das sämtliche unabhängige Variablen enthält.

Als notwendige Voraussetzung für den Einsatz der Logistischen Regression ist das Vorliegen unabhängiger Regressoren zu nennen, $d$. $h$. die betrachteten Variablen dürfen keine Multikollinearität aufweisen. ${ }^{466}$ Zudem ist es erforderlich, dass eine möglichst große Stichprobe vorliegt, wobei die Fallzahl pro Gruppe, d. h. pro Ausprägung der unabhängigen Variablen, nicht kleiner als 25 sein sollte. ${ }^{467}$ Insgesamt kann das Verfahren der Logistischen Regression insbesondere ggü. der Diskriminanzanalyse als robuste Analysemethode angesehen werden. ${ }^{468}$

In der vorliegenden Arbeit soll das Verfahren der Logistischen Regression eingesetzt werden, um die Relevanz ausgewählter endogener und exogener Determinanten für das Response- und das Buchungsverhalten zu analysieren. In diesem Zusammenhang ermöglicht die Logistische Regression Aussagen über die Bedeutung der einzelnen unabhängigen Variablen zum einen für die Entscheidung des Rezipienten, Response bzw. keinen Response auf den Direktmar-

Comparison pf Regression, Probit, Discriminant Analysis, in: American Journal of Political Science, No. 19, August 1975, S. 579 ff.

$463 \mathrm{Vgl}$. Backhaus, K. et al., Multivariate Analysemethoden. Eine anwendungsorientierte Einführung, 9. Aufl., Berlin u. a. 2000, S. $104 \mathrm{ff}$.

464 Vgl. Backhaus, K. et al., Multivariate Analysemethoden. Eine anwendungsorientierte Einführung, 10. Aufl., Berlin u. a. 2003, S. $418 \mathrm{ff}$.

465 Vgl. Fahrmeir, L., Hamerle, A., Grundlegende multivariate Schätz- und Testprobleme, in: fahrmeir, L, Hamerle, A. (Hrsg.): Multivariate statistische Verfahren, Berlin, New York 1984, S. 59.

466 Vgl. Aldrich, J., Nelson, F. D., Linear Probability, Logit and Probit Models, a. a. O., S. 49.

467 Vgl. Backhaus, K. et al., Multivariate Analysemethoden. Eine anwendungsorientierte Einführung, 10. Aufl., Berlin u. a., S. 470.

468 Vgl. Krafft, M., Logistische Regression, a. a. O., S. 244. 
keting-Stimulus zu zeigen, sowie zum anderen eine Buchung bzw. keine Buchung vorzunehmen. Als unabhängige Variablen kommen insbesondere die im zweiten Schritt hinsichtlich eines möglichen Einflusses als relevant identifizierten exogenen Determinanten in Betracht. ${ }^{469}$

Für die Beurteilung der Aussagekraft eines derart geschätzten Modells kann allerdings nicht auf die herkömmlichen Maße und Tests der Regressionsanalyse zurückgegriffen werden, da die Koeffizienten mit Hilfe der MaximumLikelihood-Methode geschätzt werden. ${ }^{470}$ Aus diesem Grund erfolgt die Beurteilung der Güte des Gesamtmodells auf Basis verschiedener anderer Gütemaße. Eine perfekte Schätzung der Koeffizienten hätte einen Likelihood-Wert von 1 zur Folge. ${ }^{471}$ Es hat sich jedoch durchgesetzt, das geschätzte Modell mit -2 mal log (Likelihood) zu rechnen. Dieser Wert wird auch als Devianz oder -2LL bezeichnet. Gut geschätzte Modelle weisen dabei hohe Likelihood-Werte nahe 1 und damit Devianzen nahe Null auf; schlechte Schätzungen führen zu geringen Likelihood-Werten und Devianz-Werten nahe $1 .{ }^{472}$ Neben der ebenfalls häufig verwendeten Goodness-of-Fit-Statistik, bei der ein niedriger Wert und ein hohes Signifikanzniveau für eine gute Modellgüte stehen, ${ }^{473}$ wird i. d. R. auf den Likelihood-Ratio-Test und McFaddens- $\mathrm{R}^{2}$ zurückgegriffen. ${ }^{474}$ Beide Kriterien repräsentieren die Güte des geschätzten Modells gegenüber einem Ausgangsmodell, welches in den meisten Fällen nur aus einem Schätzwert der Konstanten besteht. Der Likelihood-Ratio-Test, der häufig auch als Model-Chi-Square bezeichnet wird, entspricht dem T-Test der Regressionsanalyse, wobei hohe Werte und niedrige Signifikanzniveaus auf eine hohe Modellgüte hinweisen. McFaddens- $\mathbf{R}^{2}$ entspricht in etwa dem $R^{2}$ der Regressionsanalyse, ${ }^{475}$ wobei McFaddens- $R^{2}$ generell niedriger ausfällt als bei der linearen Regressionsanalyse. Werte zwischen 0,2

469 Vgl. zu einem ähnlichen Vorgehen bspw. Wulf, K. de, Hoekstra, J. C., Commandeur, H. R., The Opening und Reading Behavior of Business-to-Business Direct Mail, a. a. O., S. 138.

$470 \mathrm{Vgl}$. Kraff, M., Der Ansatz der Logistischen Regression und seine Interpretation, in: ZfB, 67. Jg., Heft 5/6, 1997, S. 628.

471 Vgl. Hosmer, D. Lemeshow, S., Applied Logistic Regression, New York 1989, S. $138 \mathrm{f}$.

$472 \mathrm{Vgl}$. Urban, D., Logit-Analyse, a. a. O., S. $64 \mathrm{f}$.

473 Vgl. Hosmer, D. Lemeshow, S., Applied Logistic Regression, a. a. O., S. $140 \mathrm{ff}$.

$474 \mathrm{Vgl}$. Krafft, M., Der Ansatz der Logistischen Regression und seine Interpretation, a. a. O., S. 630.

$475 \mathrm{Vgl}$. McFadden, D., The Measurement of Urban Travel Demand, in: Journal of Public Economics, No. 3, 1974, S. 303-328. 
und 0,4 sind hierbei bereits als gut zu bezeichnen. ${ }^{476}$ Zusätzlich werden häufig Cox \& Snells- $\mathbf{R}^{2}$ und Nagelkerkes- $\mathbf{R}^{2}$ berechnet. Bei beiden Kriterien können Werte ab 0,4 als gut bezeichnet werden und zeugen damit von einer hohen Güte des Gesamtmodells. ${ }^{477}$

Als ein wichtiges Ziel der Logistischen Regression wird häufig auch die Vorhersagegenauigkeit mit Hilfe der sog. Klassifizierungsmatrix genannt. ${ }^{478}$ In dieser Matrix werden die auf Basis der Logistischen Regression vorhergesagten Werte den tatsächlich beobachteten Werten der Gruppenzugehörigkeit gegenübergestellt. Die als richtig klassifizierten Elemente werden auch als Trefferquote des Modells bezeichnet und sollten dabei höher liegen als aufgrund einer zufälligen Zuordnung. Da allerdings die Parameterschätzung sowie die Berechnung der Trefferquote auf ein und derselben Stichprobe basieren, ist bei einer Anwendung auf andere Stichproben mit niedrigeren Werten zu rechnen. ${ }^{479}$ Sollten die Gruppen, bspw. Bucher vs. Nicht-Bucher, gleich groß sein, wird daher anstelle der zufälligen Trefferquote (hier: $50 \%$ ) das „maximum chance criterium“ (MCC) verwendet, das dem Anteil der größeren Gruppe an der gesamten Stichprobe entspricht. ${ }^{480}$ Liegen zwei relativ unterschiedlich große Gruppen vor, ist das MCC allerdings weniger gut geeignet. ${ }^{481}$ In derartigen Fallen wird in der einschlägigen Literatur auf die Verwendung des ,proportional chance criterium“ (PCC) zurückgegriffen, welches der zufälligen Trefferquote von $\alpha^{2}+(1-\alpha)^{2}$ entspricht, wobei $\alpha$ für den Anteil einer der beiden Gruppen an der Gesamtzahl der Beobachtungen steht. ${ }^{482}$

Sollte die Beurteilung der Gesamtmodellgüte als genügend erkannt worden sein, stellt sich die Frage nach der Prüfung der unabhängigen Variablen hinsichtlich Signifikanz, Richtung und relativer Bedeutung. ${ }^{483}$ Die Prüfung der Signifi-

476 Vgl. Urban, D., Logit-Analyse, a. a. O., S. 62 f.; Backhaus, K. et al., Multivariate Analysemethoden. Eine anwendungsorientierte Einführung, 10. Aufl., Berlin u. a., S. $441 \mathrm{ff}$.

477 Vgl. Backhaus, K. et al., Multivariate Analysemethoden. Eine anwendungsorientierte Einführung, 10. Aufl., Berlin u. a., S. 448.

$478 \mathrm{Vgl}$. ebenda, S. $442 \mathrm{ff}$.

479 Dieser Stichprobeneffekt ist allerdings bei großen Stichproben als gering einzuschätzen. Vgl. Kraff, M., Der Ansatz der Logistischen Regression und seine Interpretation, a. a. O., S. 631.

$480 \mathrm{Vgl}$. Morrison, D. G., On the Interpretation of Discriminant Analysis, in: Journal of Marketing Research, No. 6, May 1969, S. 158.

481 Vgl. Krafft, M., Der Ansatz der Logistischen Regression und seine Interpretation, a. a. O., S. 632.

$482 \mathrm{Vgl}$. Morrison, D. G., On the Interpretation of Discriminant Analysis, a. a. O., S. 158.

483 Vgl. ebenda, S. 633. 
kanz des Einflusses der unabhängigen Variablen erfolgt bei metrischen Variablen mit Hilfe der Chi-Quadrat verteilten Wald-Statistik. Bei kategorial ausgeprägten Variablen erfolgt zudem noch eine Beachtung der Variablen Freiheitsgrade. ${ }^{484}$ Die Richtung des Einflusses kann hierbei durch das Vorzeichen des Logitkoeffizienten abgelesen werden. Negative Vorzeichen bedeuten, dass die Wahrscheinlichkeit $P_{i}$ sinkt, zu einer bestimmten Gruppe zu gehören, während positive Vorzeichen Ausdruck einer wachsenden Wahrscheinlichkeit darstellen. ${ }^{485}$

Während die Prüfung der Signifikanz und Richtung der unterschiedlichen unabhängigen Variablen relativ einfach bestimmt werden kann, gestaltet sich die Interpretation der Parameterschätzer als schwieriger. Dies liegt daran, dass nicht wie im Fall der linearen Regressionsanalyse der Koeffizient der absoluten Änderung der abhängigen Variablen entspricht, wenn sich das Ausgangsniveau der unabhängigen Variablen bspw. um eine Einheit ändert. ${ }^{486}$ Vielmehr stellt bei der Logistischen Regression der Koeffizient eine Änderung des Logit dar, der als natürlicher Logarithmus dem Verhältnis von Gewinnwahrscheinlichkeit zu Gegenwahrscheinlichkeit entspricht. ${ }^{487}$ In der Literatur werden daher verschiedene Verfahren diskutiert, die als Interpretationshilfe dienen können. Neben partiellen Ableitungen und Elastizitätsanalysen werden sog. Sensitivitätsanalysen vorgeschlagen, die sich vor dem Hintergrund der vorliegenden Fragestellung als besonders geeignet zur Interpretation erweist. Im Rahmen der Sensitivitätsanalyse wird die Reaktion der Wahrscheinlichkeit auf unterschiedliche Ausprägungen der unabhängigen Variablen untersucht. ${ }^{488}$ Durch die systematische Variation der einzelnen unabhängigen Variablen spiegelt sich so der Einfluss dieser als absoluter Effekt in der Höhe der Wahrscheinlichkeit wider. ${ }^{489}$ Die Schwankungsbreite

484 Vgl. Hosmer, D. Lemeshow, S., Applied Logistic Regression, a. a. O., S. 17.

$485 \mathrm{Vgl}$. Krafft, M., Der Ansatz der Logistischen Regression und seine Interpretation, a. a. O., S. 633

486 Vgl. Hair, J. F., Anderson, R. E., Tatham, R. L., Black, W. C., Multivariate Data Analysis with Readings, New York 1992, S. 61; DeMarris, A., Logit Modeling: Practical Applications, Newbury Park 1992, S. 44; Backhaus, K. et al., Multivariate Analysemethoden. Eine anwendungsorientierte Einführung, 10. Aufl., Berlin u. a., S. 433.

487 Vgl. Urban, D., Logit-Analyse: Statistische Verfahren zur Analyse von Modellen mit qualitativen Responsevariablen, a. a. O., S. $46 \mathrm{ff}$.

488 Vgl. Krafft, M., Der Ansatz der Logistischen Regression und seine Interpretation, a. a. O., S. $637 \mathrm{ff}$.

489 Vgl. Urban, D., Logit-Analyse: Statistische Verfahren zur Analyse von Modellen mit qualitativen Responsevariablen, a. a. O., S. $46 \mathrm{ff}$. 
der einzelnen unabhängigen Variablen lässt so Aussagen über deren relative $\mathrm{Be}$ deutung zu. ${ }^{490}$

\section{Ergebnisse der experimentellen Untersuchung}

\subsection{Einfluss endogener Determinanten des Direktmarketing-Einsatzes}

\subsubsection{Auswertung des Gesamtexperiments}

\subsubsection{Effektivitätsbeurteilung}

Im Rahmen der empirischen Untersuchung konnten insgesamt 2.800 Personen mit 28 verschiedenen Direktmarketing-Stimuli angesprochen werden, wobei die Stichprobengröße bei 100 Personen lag. Auf der Ebene der Gesamtaktion wurde hierbei eine Zustellquote von 98,4\% erreicht, was einer Zahl von 2.755 erfolgreich zugestellten Direktmarketing-Stimuli bzw. angesprochenen Personen entspricht. ${ }^{491}$ Insgesamt resultierten aus den verschiedenen DirektmarketingAktionen 780 Anrufe im Call-Center des Unternehmens, über das die Anfragen bzw. Buchungen abgewickelt wurden. Dieser Wert entspricht einer Responsequote von $27,9 \%$. Mehr als ein Viertel der reagierenden Personen (210 Personen) buchte letztendlich bei den Call-Center-Mitarbeitern mindestens einen Flug, was einer Buchungsquote von 7,5\% auf Gesamtaktionsebene gleichkommt. ${ }^{492}$ In diesem Zusammenhang zeigte sich, dass im Durchschnitt über sämtliche Zielgruppen und Angebote ca. vier Tickets pro Person und Buchungsvorgang verkauft wurden. Unabhängig davon ergab die Auswertung der Kontrollgruppe keinerlei Ticketverkäufe im Durchführungszeitraum der Experimente. ${ }^{493}$ Abb. 14 fasst die Ergebnisse der Experimente auf Gesamtebene zusammen.

490 Vgl. Krafft, M., Logistische Regression, a. a. O.; Kraff, M., Der Ansatz der Logistischen Regression und seine Interpretation, a. a. O.

491 Wurde bspw. ein Kunde mittels mehrerer Trägermedien auf ein Angebot hingewiesen, wurde dieser Vorgang nur als erfolgreiche Zustellung gewertet, wenn sämtliche Trägermedien erfolgreich zugestellt werden konnten.

492 Interessant erscheint in diesem Zusammenhang ein Vergleich zu herkömmlichen Aktionen, wie bspw. der regelmäßig stattfindenden Newsletter-Aktion. Hier liegt die Buchungsquote bei durchschnittlich 2,5\%.

493 Auf einen Vergleich der Ergebnisse der einzelnen Aktionen mit den Ergebnissen einer Analyse der Kontrollgruppe kann daher aufgrund der nicht vorgenommenen $\mathrm{Bu}$ chungen verzichtet werden. 


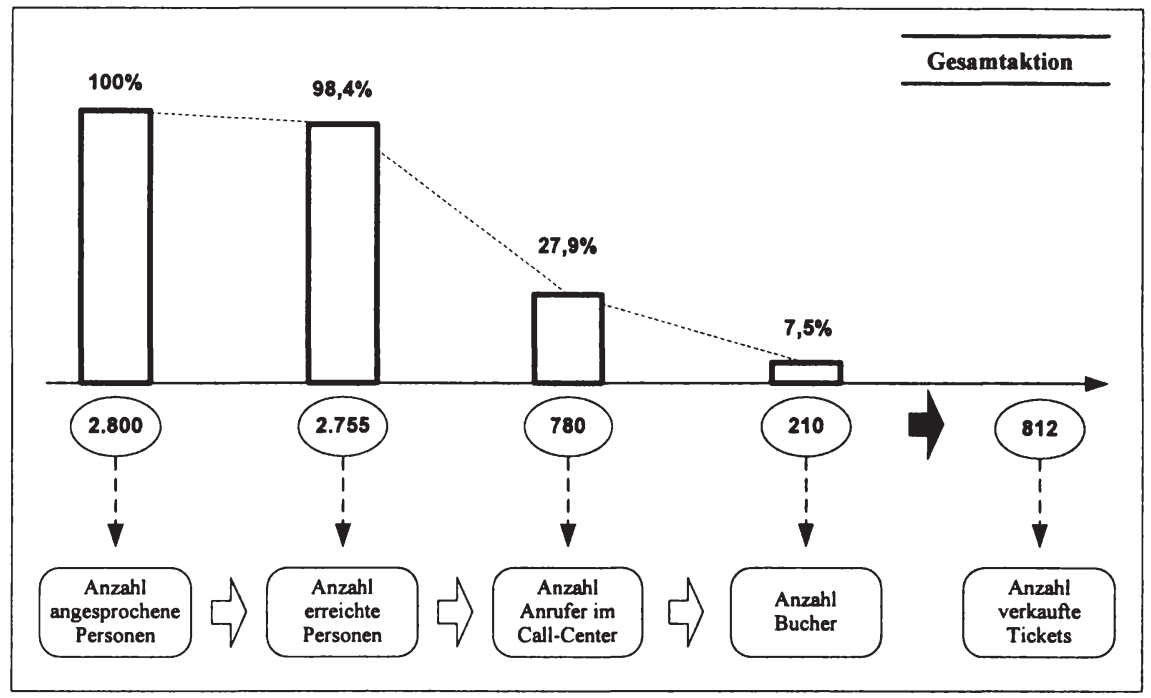

Abb. 14: Effektivitätskennzahlen der Experimente auf Gesamtaktionsebene

Neben den gemessenen Effektivitätsgrößen wurde das Informationsverhalten der Bucher im Call-Center näher analysiert. Auf Basis der vorgestellten dreistufigen Beurteilungsskala zeigte sich hierbei ein ausgeglichenes Bild (vgl. Tab. 9).

\begin{tabular}{|c|c|c|c|}
\hline & \multicolumn{3}{|c|}{$\begin{array}{l}\text { Informationsverhalten der Bucher auf } \\
\text { Gesamtaktionsebene }\end{array}$} \\
\hline Informationsverhalten & $\begin{array}{c}\text { gering } \\
(=1)\end{array}$ & $\begin{array}{c}\text { mittel }_{(=2)} \\
\end{array}$ & $\begin{array}{l}\text { viel } \\
(=3)\end{array}$ \\
\hline Anzahl absolut & 75 & 78 & 57 \\
\hline Mittelwert & \multicolumn{3}{|c|}{1,914} \\
\hline
\end{tabular}

Tab. 9: Informationsverhalten der Bucher auf Gesamtaktionsebene

Abschließend wurden die buchenden Kunden des Unternehmens nach ihrer Preisbereitschaft für das ihnen angebotene Ticket gefragt. Im Durchschnitt über sämtliche Zielgruppen und Angebotsformen ergab sich in diesem Zusammenhang ein Wert furr die maximale Preisbereitschaft i. H. v. 55,40 Euro, wobei als Minimalwert 19,00 Euro und als Maximalwert 200,00 Euro angegeben wurden. Die maximale Preisbereitschaft lag damit auf Gesamtaktionsebene deutlich uber dem realisierten durchschnittlichen Ticketpreis i. H. v. 27,05 Euro. 


\subsubsection{Effizienzbeurteilung}

Die Gesamtkosten der Durchfuhrung in Form von Konfigurations-, Werbemittelgestaltungs-, Versand-, Angebotsabwicklungskosten sowie Kosten des Adresskaufs beliefen sich kumuliert auf 5.423,99 Euro. Für die einzelnen Kostenkategorien ergaben sich dabei die folgenden Werte (vgl. Tab. 10):

\begin{tabular}{|l|c|}
\cline { 2 - 2 } \multicolumn{1}{c|}{} & Kosten auf Gesamtaktionsebene \\
\hline Konzeptionierungskosten & $1.392,40$ Euro \\
\hline Werbemittelkosten & $1.529,60$ Euro \\
\hline $\begin{array}{l}\text { Versandbezogene Abwick- } \\
\text { lungskosten }\end{array}$ & 165,14 Euro \\
\hline $\begin{array}{l}\text { Kundenbezogene Abwick- } \\
\text { lungskosten }\end{array}$ & 750,00 Euro \\
\hline Informationskosten & $1.586,85$ Euro \\
\hline Gesamtkosten & $5.423,99$ Euro \\
\hline
\end{tabular}

Tab. 10: Kostenkategorien der Experimente auf Gesamtaktionsebene

Die Gesamtkosten i. H. v. 5.423,99 Euro wurden entsprechend der jeweiligen Schlüsselung zur Berechnung der kostenorientierten Effizienzkennzahlen verwendet. Auffällig erscheint in diesem Zusammenhang die absolute Höhe der Kennzahlen „Cost per interest“ und „Cost per order“. Demnach kostete die „Generierung eines Anrufes“ im Call-Center das Unternehmen 6,95 Euro. Aufgrund der Tatsache, dass nur ca. jeder vierte Anrufer eine Buchung vornahm, ergab sich ein „Cost per order"-Wert i. H. v. 25,83 Euro. Dieser Wert erscheint im Verhältnis zu den Ticketpreisen, die beim standardisierten Angebot bspw. bei 19 Euro liegen, verhältnismäßig hoch. Zu bedenken ist hierbei allerdings, dass im Durchschnitt über sämtliche Bucher fast vier Tickets pro Buchungsvorgang verkauft wurden. Die kostenorientierten Effizienzkennzahlen auf Gesamtaktionsebene sind in der folgenden Tabelle dargestellt (vgl. Tab. 11).

\begin{tabular}{|l|c|}
\cline { 2 - 2 } \multicolumn{1}{c|}{} & $\begin{array}{c}\text { Kostenorientierte Effizienzkennzahlen auf } \\
\text { Gesamtaktionsebene }\end{array}$ \\
\hline Stückkosten & 1,67 Euro \\
\hline Cost per contact & 1,70 Euro \\
\hline Cost per interest & 6,95 Euro \\
\hline Cost per order & 25,83 Euro \\
\hline$*=$ ohne Auftragsbearbeitungskosten \\
\hline
\end{tabular}

Tab. 11: Kostenorientierte Effizienzkennzahlen auf Gesamtaktionsebene 
Insgesamt konnte im Rahmen der durchgeführten Direktmarketing-Aktionen 812 Tickets verkauft und ein Gesamtumsatz i. H. v. 21.964,00 Euro generiert werden, was unter Beachtung der angefallenen Kosten zu einem Deckungsbeitrag i. H. v. 16.540,01 Euro führte (vgl. Tab. 12). ${ }^{494}$

\begin{tabular}{|l|c|}
\cline { 2 - 2 } \multicolumn{1}{c|}{} & Deckungsbeitragsrechnung auf Gesamtaktionsebene \\
\hline Umsatz & $21.964,00$ Euro \\
\hline Kosten & $5.423,99$ Euro \\
\hline Deckungsbeitrag & $16.540,01$ Euro \\
\hline
\end{tabular}

Tab. 12: Deckungsbeitragsrechnung auf Gesamtaktionsebene

\subsubsection{Vergleichende Analyse von Neukundenakquisition und Bestandskunden- bearbeitung}

An erster Stelle der Entscheidungen im Hinblick auf den Direktmarketing-Einsatz steht die Frage, welche Zielgruppe mit dem Direktmarketing-Stimulus angesprochen werden soll. Mit Hilfe der empirischen Untersuchung soll daher im Folgenden analysiert werden, welchen Einfluss die Auswahl der Zielgruppe auf die Effektivität und Effizienz des Direktmarketing-Einsatzes besitzt. Zur Beantwortung dieser Frage wurden im Rahmen der experimentellen Untersuchung 700 Bestandskunden des Unternehmens und $\mathbf{7 0 0}$ Neukunden angesprochen. Die Adressaten in beiden Gruppen erhielten ein standardisiertes Angebot, welches ihnen über eine der sieben identifizierten Instrumentekombinationen zugesendet wurde. Jeweils 100 Personen pro 700er-Gruppe bildeten hierbei eine Zielgruppe, bei der das gleiche Trägermedium, bspw. eine Email, bzw. die gleichen Trägermedien, bspw. ein Mailing und eine SMS, zum Versand des Angebots eingesetzt wurde. ${ }^{495}$

494 Nicht berücksichtigt wurden hierbei sämtliche Kosten, die mit der eigentlichen Transportleistung, d. h. dem Flug, in Verbindung stehen. Die Kosten beziehen sich hierbei lediglich auf die im Rahmen des Direktmarketing-Einsatzes anfallenden Kosten sowie die verkaufsbedingten Abwicklungskosten durch das Call-Center. Der Umsatz kann in diesem Kontext unmittelbar den durchgeführten DirektmarketingAktionen zugeordnet werden, da die Telefonnummer der Buchungshotline ansonsten nicht freigeschaltet ist und auch bislang keiner Kundengruppe kommuniziert wurde.

495 In diesem Zusammenhang wurden 100 Personen mittels Email, 100 Personen mittels SMS und 100 Personen mittels Mailing angesprochen. Darüber hinaus erhielten jeweils 100 Personen die Angebote mittels einer Kombination aus zwei der genannten Instrumente im Rahmen des integrativen Einsatzes sowie 100 Personen mittels sämtlicher der drei Instrumente. 


\subsubsection{Effektivitätsbeurteilung}

Im Rahmen der Bestandskundenbearbeitung lag die Zustellquote bei 98,9\% der ausgesendeten Stimuli und bei $\mathbf{9 9 , 4} \%$ im Fall der Neukundenakquisition. Die Responsequote betrug bei den Bestandskunden 57,7\%, d. h. mehr als die Hälfte der Rezipienten riefen am Folgetag nach Erhalt des Stimulus im Call-Center des Unternehmens an, um weitere Informationen über das Angebot einzuholen bzw. eine Buchung vorzunehmen. Bei den Neukunden reagierten hingegen nur 7,3\% der angesprochenen Personen. Im Hinblick auf die Buchungsquote haben bei den Bestandskunden 15,9\% der angeschriebenen Personen Tickets gebucht. Bei den Neukunden waren es lediglich 1,1\%. Die folgende Abbildung zeigt die unterschiedlichen Effektivitätskennzahlen im direkten Vergleich der beiden Zielgruppen (vgl. Abb. 15).

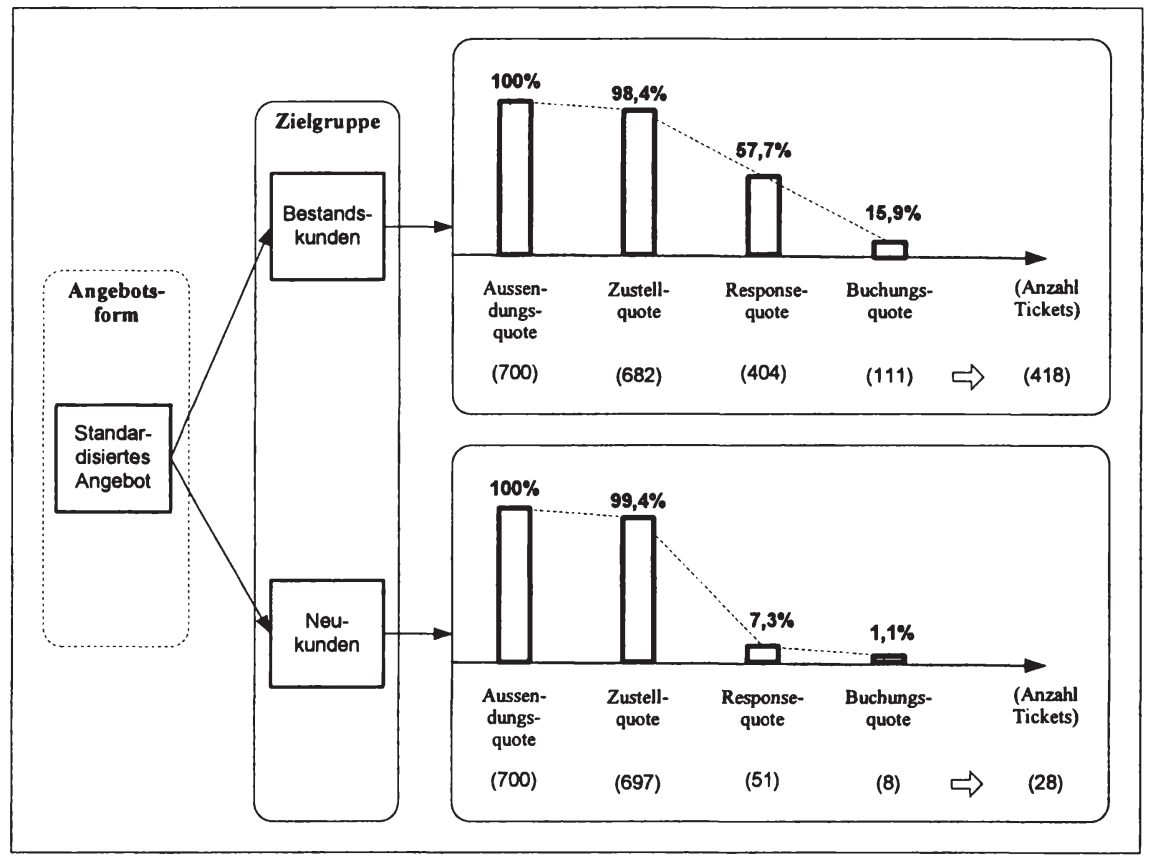

Abb. 15: Effektivitätsvergleich der Bestandskundenbearbeitung und der Neukundenakquisition

Aus der Analyse der Buchungsdaten konnten die Anzahl der pro Buchungsvorgang verkauften Tickets sowie die daraus resultierenden Umsätze ermittelt 
werden. Die durchschnittliche Ticketanzahl lag bei den Bestandskunden bei 3,77 Tickets pro Buchungsvorgang, bei den Neukunden bei 3,5 Tickets. Demnach buchte die Mehrzahl der Personen gleichzeitig vier Tickets innerhalb eines Buchungsvorgangs, was mit der häufigen Buchung von Hin- und Rückflug für zwei Personen erklärt werden konnte. ${ }^{496}$ Aus den Buchungen ergab sich aufseiten der Bestandskunden ein Umsatz i. H. v. 7.942 Euro (418 Tickets zum Preis von 19 Euro), während dieser Wert bei den Neukunden bei 532 Euro lag (28 Tickets zum Preis von 19 Euro).

Auf Basis dieser Werte konnte festgehalten werden, dass sich die konfigurierten Direktmarketing-Stimuli unter Effektivitätsgesichtspunkten sowohl zur Bearbeitung der Bestandskunden als auch zur Ansprache der Neukunden eigneten. Ein Vergleich der Effektivität dieser Gruppen macht allerdings deutlich, dass die eingesetzten Stimuli vorteilhafter hinsichtlich der Ansprache der Bestands- als der Neukunden eingesetzt werden können. Sowohl im Hinblick auf die Höhe des Responses als auch im Hinblick auf die Anzahl der Buchungen erweist sich die Bestandskundenbearbeitung gegenüber der Neukundenakquisition effektiver. ${ }^{497}$

Eine nähere Betrachtung der buchenden Personen hinsichtlich ihres Informationsverhaltens im Call-Center zeigt zudem weitere Unterschiede. So belegt die Analyse des Informationsverhaltens, dass die angesprochenen Bestandskunden einen tendenziell geringeren Informationsbedarf hatten als die angesprochenen Neukunden (vgl. Tab. 13).

\begin{tabular}{|l|c|c|c|c|c|c|}
\cline { 2 - 7 } \multicolumn{1}{c|}{} & \multicolumn{5}{c|}{ Informationsverhalten auf Zielgruppenebene } \\
Bestandskunden \\
\hline $\begin{array}{l}\text { Informations- } \\
\text { verhalten }\end{array}$ & $\begin{array}{c}\text { gering } \\
(=1)\end{array}$ & $\begin{array}{c}\text { mittel } \\
(=2)\end{array}$ & $\begin{array}{c}\text { viel } \\
(=3)\end{array}$ & $\begin{array}{c}\text { gering } \\
(=1)\end{array}$ & $\begin{array}{c}\text { mittel } \\
(=2)\end{array}$ & $\begin{array}{c}\text { viel } \\
(=3)\end{array}$ \\
\hline Anzahl absolut & 59 & 37 & 15 & 0 & 1 & 7 \\
\hline Mittelwert & \multicolumn{3}{c|}{1,604} & & 2,875 \\
\hline
\end{tabular}

Tab. 13: Informationsverhalten der Bestands- und Neukunden während des Buchungsvorgangs

Neben der Analyse des Informationsverhaltens wurden auch Unterschiede im Rahmen der maximalen Preisbereitschaft ermittelt. So erreichte die maximale Preisbereitschaft für das standardisierte Angebot bei den Bestandskunden einen

496 Die zeitgleiche Buchung von Hin- und Rückflug wurde auch durch die Ergebnisse der Ex-ante-Befragung unterstützt. Demnach buchten ca. $90 \%$ der Befragten Hinund Rück im Rahmen eines Buchungsvorgangs.

497 Auf weitere Effektivitätsunterschiede hinsichtlich der innerhalb eines Angebotes eingesetzten Instrumente wird im Rahmen der Betrachtung des medienbezogenen Einflusses näher eingegangen. 
Wert von 50,97 Euro, während dieser Wert bei den Neukunden bei lediglich 44,00 Euro lag. Auch bei der Betrachtung des Minimal- und des Maximalwertes - bezogen auf die Preisbereitschaft - zeigen sich deutliche Unterschiede (vgl. Tab. 14).

\begin{tabular}{|c|c|c|}
\hline & \multicolumn{2}{|c|}{$\begin{array}{l}\text { Realisierte Ticketpreise und maximale Preisbereit- } \\
\text { schaft auf Zielgruppenebene }\end{array}$} \\
\hline & Bestandskunden & Neukunden \\
\hline $\begin{array}{l}\text { Durchschnittlich realisier- } \\
\text { ter Ticketpreis }\end{array}$ & 19,00 Euro & 19,00 Euro \\
\hline $\begin{array}{l}\text { Durchschnittliche maxi- } \\
\text { male Preisbereitschaft }\end{array}$ & 50,97 Euro & 44,00 Euro \\
\hline Standardabweichung & 32,73 & 19,27 \\
\hline Minimalwert & 19,00 Euro & 19,00 Euro \\
\hline Maximalwert & 200,00 Euro & 79,00 Euro \\
\hline
\end{tabular}

Tab. 14: Durchschnittlich realisierte Ticketpreise sowie Preisbereitschaften in Abhängigkeit der unterschiedlichen Zielgruppen

\subsubsection{Effizienzbeurteilung}

Die Höhe der beschriebenen Effektivitătsgrößen lässt noch keine Schlüsse über die Effizienz der beiden Zielgruppenansprachen zu, da sich die Kosten für die Ansprache der unterschiedlichen Zielgruppen erheblich unterscheiden. Die Berechnung der kostenorientierten Kennzahlen erfolgt unter Hinzuziehung der Kosten für die Werbemittelgestaltung, den Versand, die Auftragsbearbeitung sowie die Kosten für den Kauf von Adressdaten im Fall der Neukundenbearbeitung.

\begin{tabular}{|c|c|c|}
\hline & $\begin{array}{r}\text { Gesamtkos } \\
\text { Bestandskunden }\end{array}$ & $\begin{array}{l}\text { ppenebene } \\
\text { Neukunden }\end{array}$ \\
\hline $\begin{array}{l}\text { Konzeptionierungs- } \\
\text { kosten }\end{array}$ & 319,18 Euro & 319,18 Euro \\
\hline Werbemittelkosten & 41,29 Euro & 41,29 Euro \\
\hline $\begin{array}{l}\text { Versandbezogene } \\
\text { Abwicklungskosten }\end{array}$ & 382,40 Euro & 382,40 Euro \\
\hline $\begin{array}{l}\text { Auftragsbearbeitungs- } \\
\text { kosten }\end{array}$ & 364,20 Euro & 50,93 Euro \\
\hline Informationskosten & 0 Euro & $1.586,85$ Euro \\
\hline $\begin{array}{l}\text { Gesamtkosten pro } \\
\text { Zielgruppe }\end{array}$ & 1.107,07 Euro & 2.380,65 Euro \\
\hline
\end{tabular}

Tab. 15: Gesamtkosten der Bestandskundenbearbeitung und der Neukundenakquisition 
Bei der Betrachtung der absoluten Höhe der einzelnen Kostengrößen fállt zunächst die identische Höhe der gestaltungs- und versandbezogenen Kosten auf, die angesichts gleicher Herstellungskosten und Stückzahlen zu erklären ist. Darüber hinaus existiert aufseiten der Neukunden mit den Kosten für die Adressanmietung ein zielgruppenspezifischer Kostenblock, der nicht in die Berechnung der Kostengrößen aufseiten der Bestandskunden einfließt. Tab. 15 gibt einen Überblick über die Höhe der einzelnen Kostenarten je Zielgruppe.

Im Rahmen der Betrachtung kostenorientierter Kennzahlen konnten auf Basis der erhobenen Daten verschiedene Effizienzkennzahlen berechnet werden. ${ }^{498}$ Die Gegenüberstellung zeigt in diesem Zusammenhang stark differierende Werte für die zwei untersuchten Zielgruppen. Auffällig sind hierbei die durchgängig höheren Werte bei der Zielgruppe der Neukunden, die sich primär aus den geringen Response- und Buchungsquoten verbunden mit den zusätzlichen Kosten für die Neukundenadressen ergeben (vgl. Tab. 16).

\begin{tabular}{|l|c|c|}
\cline { 2 - 3 } \multicolumn{1}{c|}{} & \multicolumn{2}{c|}{$\begin{array}{c}\text { Kostenorientierte Effizienzkennzahlen auf Zielgruppenebene } \\
\text { Bestandskunden }\end{array}$} \\
\hline Stückkosten & 1,02 Euro & 3,33 Euro \\
\hline Cost per contact & 1,03 Euro & 3,35 Euro \\
\hline Cost per interest & 2,61 Euro & 48,67 Euro \\
\hline Cost per order & 10,48 Euro & 283,18 Euro \\
\hline
\end{tabular}

Tab. 16: Kostenorientierte Effizienzkennzahlen der Bestandskundenbearbeitung und der Neukundenakquisition

Mit einem Wert von 283,18 Euro für die Generierung einer Buchung aufseiten der Neukunden erscheint die Neukundenakquisition mittels des hier vorgestellten Vorgehens nur ökonomisch sinnvoll, wenn der Verbleib eines neu gewonnenen Kunden im Unternehmen und eine damit verbundene regelmäßige Buchungstätigkeit nach der ersten Buchung sichergestellt werden kann. Selbst wenn die Adressdaten für die Neukundenakquisition, welche fast $66 \%$ der neukundenspezifischen Kosten ausmachen, dem Unternehmen kostenlos zur Verfügung gestanden hätten, läge der „Cost per order“-Wert immer noch bei rund 94,- Euro,

498 In diesem Zusammenhang gilt es zu beachten, dass die Umlage der Call-CenterKosten für die Berechung der "Cost per interest" und die Berechnung der „Cost per order" unterschiedlich ausfällt und jeweils neu berechnet wird. Der vorliegenden Berechnung liegt hierbei die Prämisse zugrunde, dass Nicht-Bucher, die aber trotzdem im Call-Center anrufen, die Ressourcen im Durchschnitt genauso intensiv belasten wie ein „,sich wenig informierender“ Bucher (Stufe 1, „wenig“). Die insgesamt angefallen Kosten i. H. v. 1.500,- Euro werden somit gemäß der Beanspruchung umgelegt. 
was angesichts der durchschnittlich realisierten Ticketpreise die Akquisitionskosten ggf. erst durch mehrere Buchungen des Kunden egalisieren würde. ${ }^{499}$

Auf Basis der erfassten Umsätze und Kosten lassen sich abschließend die durch die Direktmarketing-Experimente bei beiden Zielgruppen erzielten Deckungsbeiträge berechnen. Die Ergebnisse können Tab. 17 entnommen werden.

\begin{tabular}{|l|c|c|}
\cline { 2 - 3 } \multicolumn{1}{c|}{} & \multicolumn{2}{c|}{$\begin{array}{c}\text { Deckungsbeitragsrechnung auf Zielgruppenebene } \\
\text { Bestandskunden }\end{array}$} \\
\hline Umsatz & $7.942,00$ Euro & 532,00 Euro \\
\hline Kosten & $1.107,07$ Euro & $2.380,65$ Euro \\
\hline Deckungsbeitrag & $\mathbf{6 . 8 3 4 , 9 3 ~ E u r o ~}$ & $-1.848,65$ Euro \\
\hline
\end{tabular}

Tab. 17: Realisierte Deckungsbeiträge der Bestandskundenbearbeitung und der Neukundenakquisition

Während die Bestandskundenbearbeitung mit Hilfe des standardisierten Angebotes einen positiven Deckungsbeitrag realisiert, erzielt die Neukundenakquisition angesichts der hohen Kosten, der geringen Buchungsquote und der pro Buchung generierten Umsätze einen negativen Deckungsbeitrag. Zur Erreichung des Break-even-Points - bezogen auf die Neukundenakquisition - hätten 126 Tickets à 19 Euro verkauft werden müssen, was einer Buchungsquote von 18 $\%$ entsprochen hätte. Wie im Rahmen der bisherigen Ausfuhrungen deutlich wurde, erscheint damit die Bestandskundenbearbeitung nicht nur unter Effektivitäts- sondern auch unter Effizienzgesichtspunkten vorteilhafter als die Neukundenakquisition.

\subsubsection{Vergleichende Analyse verschiedener verkaufsorientierter Angebote im Rahmen der Bestandskundenbearbeitung}

An zweiter Stelle der Entscheidungen im Hinblick auf den DirektmarketingStimulus steht die Frage, welche Angebote den im ersten Schritt identifizierten Zielgruppen vor dem Hintergrund der beschriebenen Ziele unterbreitet werden sollen. ${ }^{500}$ Zur Untersuchung dieser Fragestellung wurden 2.100 Bestandskunden des Unternehmens mit unterschiedlichen Angeboten beworben und anschließend ihre Reaktionen auf den Stimulus gemessen. Als Angebotsformen kamen hierbei

499 An dieser Stelle sind weitere Kosten, wie z. B. Transportkosten sowie sonstige Abwicklungskosten am Terminal noch nicht enthalten.

500 Die Konfiguration des Angebots ist eng verbunden mit der Frage nach den Zielen des Direktmarketing-Einsatzes auf der einen und den beworbenen Zielgruppen auf der anderen Seite. 
ein standardisiertes 19-Euro-Angebot, ein rabattiertes sowie ein individualisiertes Angebot zum Einsatz. ${ }^{501}$ Angesichts der zugrunde liegenden Fragestellung erhielten drei Gruppen von jeweils 700 Personen eines der drei Angebote. Pro 700erGruppe kamen entsprechend der Berücksichtigung gleicher Strukturmerkmale wiederum sieben Gruppen à 100 Personen als Rezipienten zum Einsatz, wobei jede Testgruppe von 100 Personen mittels einer der sieben Instrumente- bzw. Instrumentekombinationen beworben wurde.

\subsubsection{Effektivitätsbeurteilung}

Während die Zustellquoten beim standardisierten Angebot mit 98,9\%, beim rabattierten Angebot mit 96,9\% und beim individualisierten Angebot mit 98,6\% ähnlich hohe Werte aufweisen, zeigt die Betrachtung der Responsequoten ein heterogenes Bild. Auf das rabattierte Angebot reagierten 16,4 \% der angesprochenen Personen, beim individualisierten Angebot lag dieser Wert sogar bei $30,0 \%$. Den höchsten Wert verzeichnete das standardisierte Angebot mit einer Responsequote von 57,7\%. ${ }^{502}$ Konsistenterweise zeigte auch die Buchungsquote ein divergierendes Niveau, welches entsprechend dem Verhältnis der Responsequoten der drei Angebote zueinander schwankte. Beim standardisierten Angebot lag der Wert bei $15,9 \%$, beim individualisierten bei $9,0 \%$ und beim rabattierten Angebot bei 4,0\%. Abb. 16 gibt einen Überblick über die Effektivităt der einzelnen Angebotsformen.

Insgesamt zeigte bereits die bisherige Auswertung, dass sich die konfigurierten Angebote unter Effektivitätsgesichtspunkten deutlich voneinander unterscheiden. So erscheint das standardisierte Angebot eine große Zahl von Personen anzusprechen, was sich in einer überdurchschnittlich hohen Response- und Buchungsquoten widerspiegelt. Im Vergleich zu den regelmäßig über den EmailNewsletter angebotenen Ticketkontingenten, welche in etwa dem standardisierten Angebot entsprechen, ist die Höhe der Buchungsquote als überdurchschnittlich zu beurteilen. So liegt die Buchungsquote bei derartigen Aktionen im Jahresdurchschnitt bei $2,5 \%$ pro Aktion.

Der durch die jeweilige Angebotsform generierte Umsatz differierte aufgrund der unterschiedlichen Anzahl verkaufter Tickets sowie der unterschiedlich hohen Verkaufspreise. Während im Rahmen des rabattierten Angebotes per se vier

$501 \mathrm{Vgl}$. Kap. C.2.2.2 zu den Ausführungen des experimentellen Inputs im Rahmen des Experiments.

502 Das verwendete standardisierte Angebot entsprach dem im Rahmen der vergleichenden Analyse des Einflusses der Zielgruppenauswahl verwendeten standardisierten Angebots, welches an die Bestandskunden des Unternehmens. 


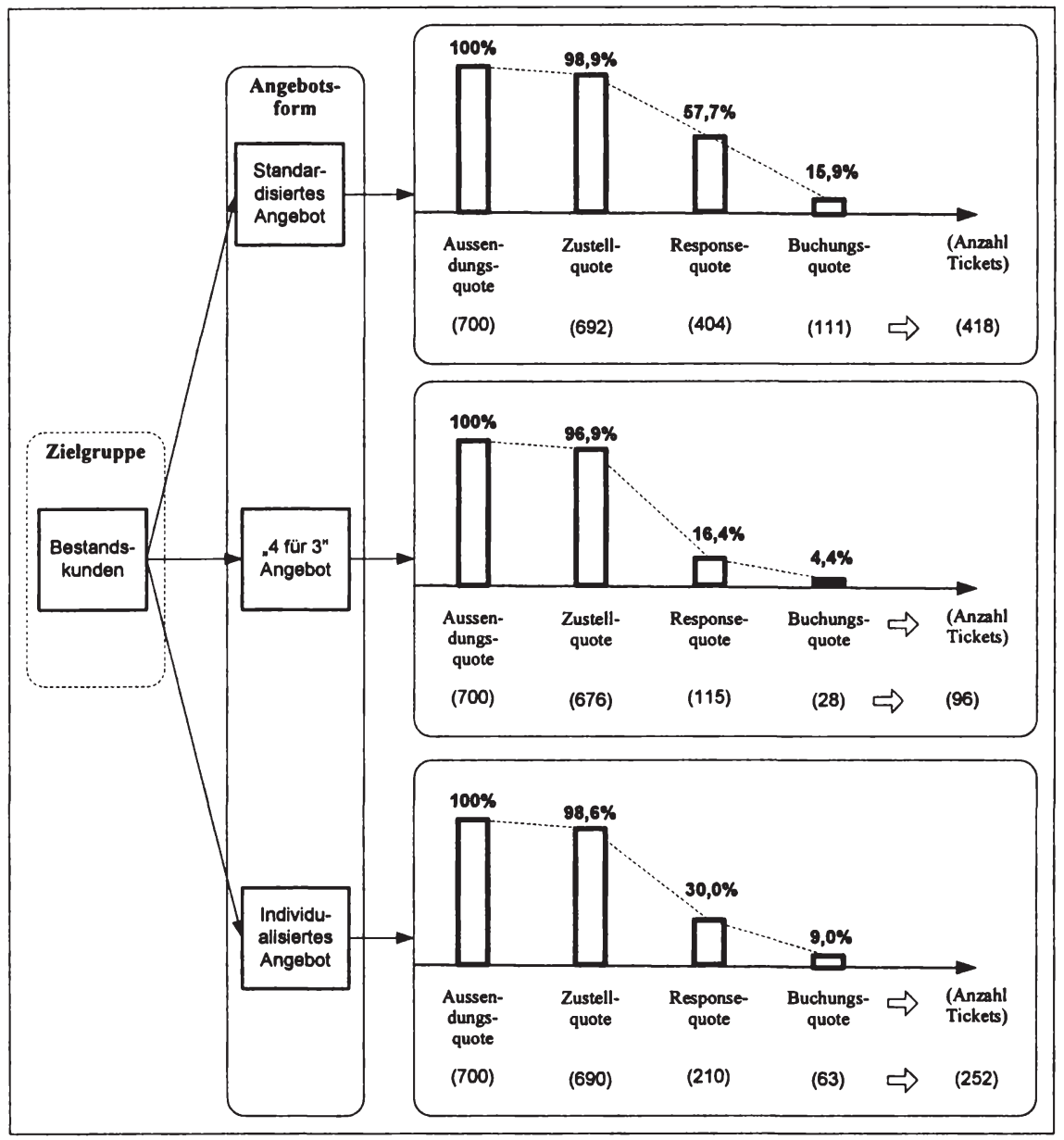

Abb. 16: Effektivitätsvergleich der drei verkaufsorientierten Angebote

Tickets gebucht wurden, lag dieser Wert auch im Rahmen des individualisierten Angebotes ebenfalls bei durchschnittlich vier Tickets pro Buchungsvorgang. Beim standardisierten Angebot wurden im Durchschnitt 3,77 Tickets pro Bucher verkauft. Im Ergebnis generierte das standardisierte Angebot einen Umsatz i. H. v. 7.942,00 Euro, das rabattierte Angebot einen Umsatz i. H. v. 3.186,00 Euro und das individualisierte Angebot einen Umsatz i. H.v. 10.304,00 Euro. 
Vor dem Hintergrund der Anfragen im Call-Center gingen den einzelnen Buchungen unterschiedlich intensive Informationsanfragen voraus, die zu einer unterschiedlichen Kapazitätsbeanspruchung des Call-Centers führten. Es zeigte sich, dass der geringste Informationsbedarf bei den standardisierten Angeboten bestand, während die nachgefragten Informationen beim rabattierten Angebot am größten waren (vgl. Tab. 18). ${ }^{503}$

\begin{tabular}{|c|c|c|c|c|c|c|c|c|c|}
\hline \multirow[b]{3}{*}{$\begin{array}{l}\text { Informationsver- } \\
\text { halten }\end{array}$} & \multicolumn{9}{|c|}{ Informationsverhalten auf Angebotsebene } \\
\hline & \multicolumn{3}{|c|}{$\begin{array}{c}\text { Standardisiertes } \\
\text { Angebot }\end{array}$} & \multicolumn{3}{|c|}{$\begin{array}{c}\text { Rabattiertes } \\
\text { Angebot }\end{array}$} & \multicolumn{3}{|c|}{$\begin{array}{c}\text { Individualisiertes } \\
\text { Angebot }\end{array}$} \\
\hline & $\begin{array}{c}\text { gering } \\
(=1)\end{array}$ & $\begin{array}{l}\text { mittel } \\
(=2)\end{array}$ & $\begin{array}{l}\text { viel } \\
(=3)\end{array}$ & $\begin{array}{c}\text { gering } \\
(=1)\end{array}$ & $\begin{array}{c}\text { mittel } \\
(=2)\end{array}$ & $\begin{array}{l}\text { viel } \\
(=3)\end{array}$ & $\begin{array}{c}\text { gering } \\
(=1)\end{array}$ & $\begin{array}{c}\text { mittel } \\
(=2)\end{array}$ & $\begin{array}{l}\text { viel } \\
(=3)\end{array}$ \\
\hline Anzahl absolut & 59 & 38 & 15 & 2 & 12 & 14 & 15 & 27 & 21 \\
\hline Mittelwert & \multicolumn{3}{|c|}{1,612} & \multicolumn{3}{|c|}{2,429} & \multicolumn{3}{|c|}{2,095} \\
\hline
\end{tabular}

Tab. 18: Informationsverhalten der buchenden Personen in Abhängigkeit des versendeten Angebots

Interessant erscheint in diesem Zusammenhang die Gegenüberstellung der angebotsspezifischen Preisbereitschaften mit den tatsächlich realisierten Ticketpreisen. So wurden die Bucher der verschiedenen Angebote nach Durchführung des Buchungsvorganges nach der maximalen Preisbereitschaft für das von ihnen gebuchte Angebot befragt. Hierbei zeigte sich erwartungsgemäß die höchste Preisbereitschaft beim individualisierten Angebot. Die größte Diskrepanz zwischen realisiertem Ticketpreis und maximaler Preisbereitschaft wies allerdings das standardisierte Angebot auf (vgl. Tab. 19).

\begin{tabular}{|l|c|c|c|}
\cline { 2 - 4 } \multicolumn{1}{c|}{} & \multicolumn{3}{c|}{$\begin{array}{c}\text { Realisierte Ticketpreise und maximale Preisbereitschaft auf } \\
\text { Zielgruppenebene }\end{array}$} \\
\cline { 2 - 4 } & $\begin{array}{c}\text { Standardisiertes } \\
\text { Angebot }\end{array}$ & $\begin{array}{c}\text { Rabattiertes } \\
\text { Angebot }\end{array}$ & $\begin{array}{c}\text { Individualisiertes } \\
\text { Angebot }\end{array}$ \\
\hline $\begin{array}{l}\text { Durchschnittlich reali- } \\
\text { sierter Ticketpreis }\end{array}$ & $\mathbf{1 9 , 0 0}$ Euro & $\mathbf{2 8 , 4 5}$ Euro & $\mathbf{4 0 , 8 9}$ Euro \\
\hline $\begin{array}{l}\text { Durchschnittliche } \\
\text { Preisbereitschaft }\end{array}$ & $\mathbf{5 0 , 9 7 \text { Euro }}$ & $\mathbf{5 7 , 0 0}$ Euro & $\mathbf{6 3 , 9 5}$ Euro \\
\hline Standardabweichung & 32,73 & $\mathbf{3 0 , 4 7}$ & 29,27 \\
\hline Minimalwert & 19,00 Euro & 25,00 Euro & 19,00 Euro \\
\hline Maximalwert & 200,00 Euro & 150,00 Euro & 153,00 Euro \\
\hline
\end{tabular}

Tab. 19: Durchschnittlich realisierte Ticketpreise sowie Preisbereitschaften in Abhängigkeit der unterschiedlichen Angebote

503 Entsprechend dieser Ergebnisse erfolgte eine Umlage der Call-Center-Kosten, welche im Rahmen der Effizienzbeurteilung von Bedeutung ist. 


\subsubsection{Effizienzbeurteilung}

Die Analyse der angefallenen Kosten zeigt bei den drei versendeten Angeboten grundsätzlich ähnliche Kostenstrukturen. Ein Unterschied hinsichtlich der betrachteten Angebote ergab sich bei der Höhe der Konfigurationskosten. Im Rahmen der Konfiguration des individualisierten Angebotes entstanden zusätzliche Kosten aufgrund zusätzlicher Arbeiten für die Aufbereitung der individualisierten Daten, (hauptsächlich für die Zusammenstellung von Destination, Preis und Anrede zu individuellen Angeboten). Die unterschiedliche Höhe der Auftragsbearbeitungskosten ergibt sich aufgrund der unterschiedlichen Beanspruchung der Call-Center-Kapazitäten in Abhängigkeit der versendeten Angebote. Im Vergleich zur Neukundenakquisition fielen bei allen drei Angeboten keine Adressenanmietungskosten an (vgl. Tab. 20).

\begin{tabular}{|l|c|c|c|}
\cline { 2 - 4 } \multicolumn{1}{c|}{} & $\begin{array}{c}\text { Standardisiertes } \\
\text { Angebot }\end{array}$ & $\begin{array}{c}\text { Rabattiertes } \\
\text { Angebot }\end{array}$ & $\begin{array}{c}\text { Individualisiertes } \\
\text { Angebot }\end{array}$ \\
\hline $\begin{array}{l}\text { Konfigurations- } \\
\text { kosten }\end{array}$ & 319,18 Euro & 319,18 Euro & 434,68 Euro \\
\hline Informationskosten & 0 Euro & 0 Euro & 0 Euro \\
\hline Werbemittelkosten & 41,29 Euro & 41,29 Euro & 41,29 Euro \\
\hline $\begin{array}{l}\text { Versandbezogene } \\
\text { Abwicklungskosten }\end{array}$ & 382,40 Euro & 382,40 Euro & 382,40 Euro \\
\hline $\begin{array}{l}\text { Auftragsbearbei- } \\
\text { tungskosten }\end{array}$ & 364,20 Euro & 119,60 Euro & 215,28 Euro \\
\hline $\begin{array}{l}\text { Gesamtkosten pro } \\
\text { Angebot }\end{array}$ & $\mathbf{1 . 1 0 7 , 0 7 ~ E u r o ~}$ & $\mathbf{8 6 2 , 4 7 \text { Euro }}$ & $\mathbf{1 . 0 7 3 , 6 5 \text { Euro }}$ \\
\hline
\end{tabular}

Tab. 20: Gesamtkosten der drei Angebote unterteilt nach den zentralen Kostenkategorien

Auf Basis der identifizierten Kosten erfolgte eine Berechnung der kostenorientierten Effizienzkennzahlen. Während sich keine größeren Unterschiede bei den Stück- und den Kontaktkosten abzeichneten, ergaben sich aufgrund der stark schwankenden Response- und Buchungsquoten relativ deutliche Unterschiede bei den Kennzahlen „Cost per interest“ und „Cost per order“. Insbesondere die hohen Kosten, die zur Generierung einer Buchung beim rabattierten Angebot notwendig waren, lassen den Einsatz dieses Angebottyps unter ökonomischen Gesichtspunkten fraglich erscheinen (vgl. Tab. 21). 


\begin{tabular}{|l|c|c|c|}
\cline { 2 - 4 } \multicolumn{1}{c|}{} & \multicolumn{3}{c|}{ Kostenorientierte Effizienzkennzahlen auf Angebotsebene } \\
\cline { 2 - 4 } \multicolumn{1}{c|}{} & $\begin{array}{c}\text { Standardisiertes } \\
\text { Angebot }\end{array}$ & $\begin{array}{c}\text { Rabattiertes } \\
\text { Angebot }\end{array}$ & $\begin{array}{c}\text { Individualisiertes } \\
\text { Angebot }\end{array}$ \\
\hline Stückkosten & 1,02 Euro & 1,06 Euro & 1,23 Euro \\
\hline Cost per contact & 1,03 Euro & 1,09 Euro & 1,24 Euro \\
\hline Cost per interest & 2,61 Euro & 7,66 Euro & 5,21 Euro \\
\hline Cost per order & 10,48 Euro & 31,29 Euro & 18,94 Euro \\
\hline
\end{tabular}

Tab. 21: Kostenorientierte Effizienzkennzahlen der drei Angebote im Vergleich

Aus der Versendung der 2.100 Angebote resultierte ein Gesamtumsatz i. H. v. 21.432 Euro. Letztendlich erzielte auch das rabattierte Angebot einen ausreichend hohen Umsatz, um - trotz der geringen Buchungsquote - einen positiven Deckungsbeitrag zu erwirtschaften. Eine differenzierte Betrachtung hinsichtlich der drei Angebotsformen ergab unter Berücksichtigung der entsprechenden Kostenblöcke folgendes Bild (vgl. Tab. 22).

\begin{tabular}{|c|c|c|c|}
\hline & \multicolumn{3}{|c|}{ Deckungsbeitrag auf Angebotsebene } \\
\hline & $\begin{array}{c}\text { Standardisiertes } \\
\text { Angebot }\end{array}$ & $\begin{array}{c}\text { Rabattiertes } \\
\text { Angebot }\end{array}$ & $\begin{array}{c}\text { Individualisiertes } \\
\text { Angebot }\end{array}$ \\
\hline Umsatz & 7.942,00 Euro & 3.186,00 Euro & $10.304,00$ Euro \\
\hline Kosten & $1.107,07$ Euro & 862,47 Euro & $1.073,65$ Euro \\
\hline Deckungsbeitrag & 6.834,93 Euro & 2.323,53 Euro & 9.230,35 Euro \\
\hline
\end{tabular}

Tab. 22: Realisierte Deckungsbeiträge der drei Angebote im Vergleich

Zusammenfassend erwies sich aufgrund der hohen durchschnittlichen Ticketpreise sowie der hohen Anzahl von Tickets pro Buchung das individuelle Angebot hinsichtlich der realisierten Deckungsbeiträge am vorteilhaftesten. Den Mehrkosten im Rahmen der Konfiguration standen insofern höhere realisierte Buchungspreise gegenüber.

\subsubsection{Vergleichende Analyse des Einsatzes verschiedener Instrumente und In- strumentekombinationen}

An dritter Stelle der Entscheidungen im Hinblick auf den DirektmarketingStimulus steht die Frage, welche Instrumente bzw. Instrumentekombinationen zur Ansprache der im ersten Schritt identifizierten Zielgruppen sowie der im zweiten Schritt festgelegten Angebote eingesetzt werden sollen. In diesem Zusammenhang galt es zunächst zu analysieren, welches Instrument sich unter Effektivitäts- und Effizienzgesichtspunkten am besten zur Ansprache der unterschied- 
lichen Zielgruppen eignet und ob durch eine Kombination mehrerer Instrumente beim Versand eines Angebots Effektivitäts- und Effizienzvorteile generiert werden können.

\subsubsection{Effektivitätsbeurteilung}

Werden die Ergebnisse der vorangegangenen Kapitel auf instrumentenspezifischer Ebene verfeinert, zeigt sich ein differenziertes Bild über die eingesetzten Instrumente hinsichtlich der unterschiedlichen Effektivitätskennzahlen. In Abhängigkeit der angesprochenen Zielgruppen sowie der verwendeten Angebotsform ergaben sich für die 28 verschiedenen Direktmarketing-Stimuli zusammenfassend die folgenden Ergebnisse, die übersichtsartig in Abb. 17 dargestellt sind:

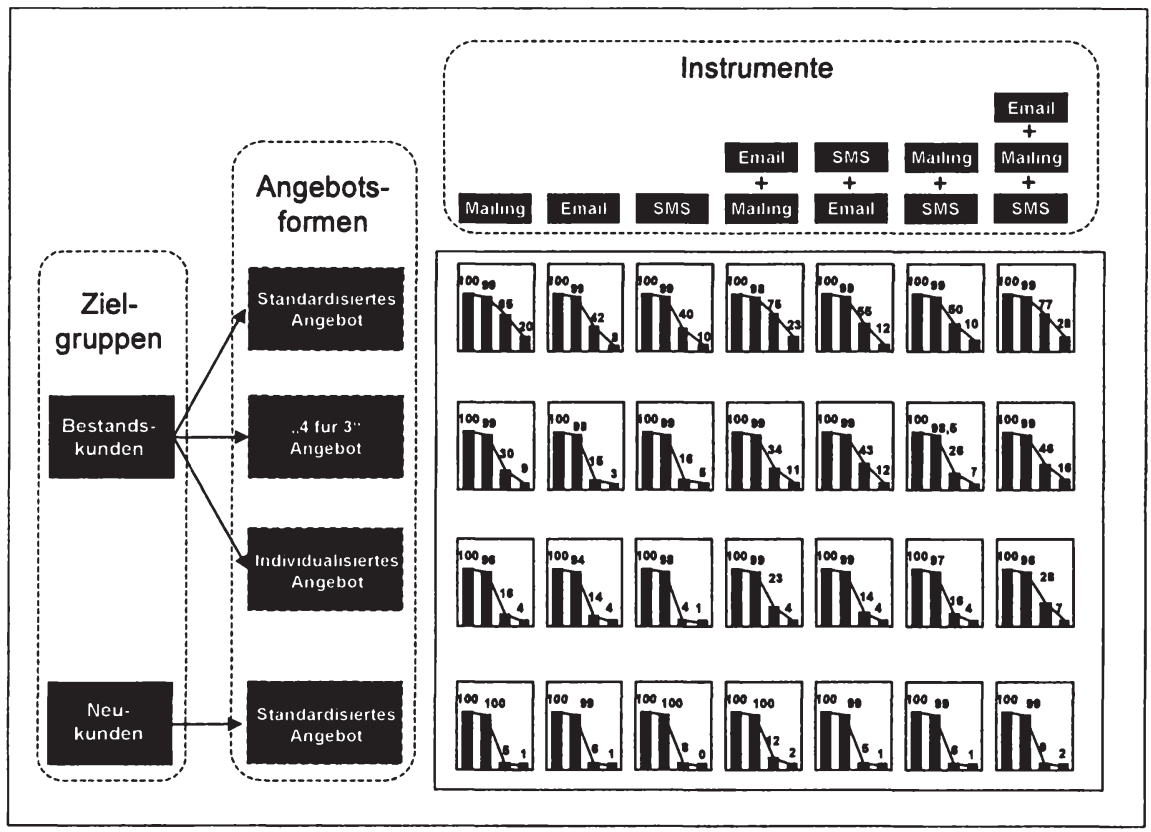

Abb. 17: Effektivitätskennzahlen der 28 versendeten Direktmarketing-Stimuli im Überblick

Während die Zustellquoten bei den Bestandskunden zwischen $94 \%$ und $99 \%$ lagen und keine signifikanten instrumentespezifischen Unterschiede erkennbar waren, konnten im Rahmen der Neukundenansprache durchgängig über sämtliche Instrumente und Instrumentekombinationen Zustellquoten zwischen 
$99 \%$ und $100 \%$ verzeichnet werden. Weitaus heterogener fielen die Höhen der Responsequoten aus. In Abhängigkeit des versendeten Angebots schwankten die durchschnittlichen Responsequoten erheblich. So zeigte sich im Rahmen des standardisierten Angebots, das eine durchschnittliche Responsequote von 57,7\% verzeichnen konnte, eine Schwankungsbreite auf instrumentespezifischer Ebene

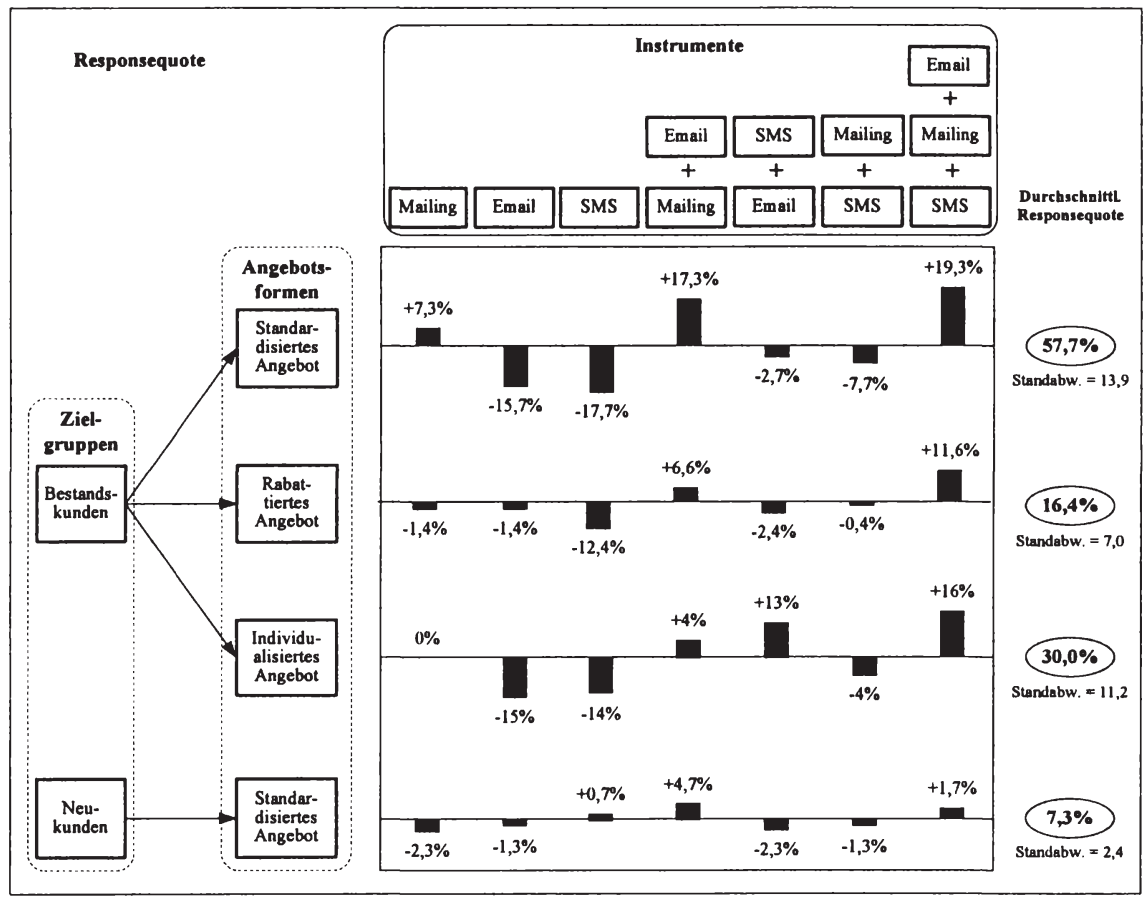

Abb. 18: Abweichung der Responsequote von der durchschnittlichen Responsequote der verschiedenen Zielgruppen und Angebote

zwischen $40 \%$ und $77 \%$. Im Rahmen des isolierten Einsatzes dominierte hierbei das Mailing mit einer Responsequote von $65 \%$. Im Rahmen von zwei integrativ eingesetzten Instrumenten war die Kombination von Mailing und Email mit einer Responsequote von $75 \%$ am effektivsten. Lediglich der integrative Einsatz aller drei Instrumente erreichte mit einer Responsequote von $77 \%$ einen noch höheren Wert. Im Rahmen der rabattierten und der individualisierten Angebotsform konnten ebenfalls die höchsten Responsequoten beim kombinierten Einsatz von drei Instrumenten verzeichnet werden. Einheitlich gestaltete sich darüber hinaus auch der Trend einer unterdurchschnittlichen Effektivität von Email und 
SMS im isolierten Einsatz, wenn auch angebotsspezifisch in unterschiedlicher Höhe. Vorteilhaft ist hingegen bei sämtlichen Angeboten die Kombination Email und Mailing im Vergleich zur durchschnittlichen Responsequote sämtlicher Instrumente zu bewerten (vgl. Abb. 18).

Die Responsequoten zeigten ähnlich der Bearbeitung der Bestandskunden auch bei der Neukundenakquisition eine erhebliche Varianz und schwankten in Abhängigkeit der eingesetzten Trägermedien zwischen $5 \%$ und $12 \%$. Den höchsten Wert beim isolierten Einsatz der Instrumente belegte mit $8 \%$ die SMS. Als effektivste Kombination zweier Instrumente zeichnete sich wie im Fall der Bestandskundenbearbeitung die Kombination aus Mailing und Email mit einer Responsequote von $12 \%$ aus. Hingegen erreichte der integrative Einsatz sämtlicher Instrumente keine höhere Responsequote (lediglich $9 \%$ ).

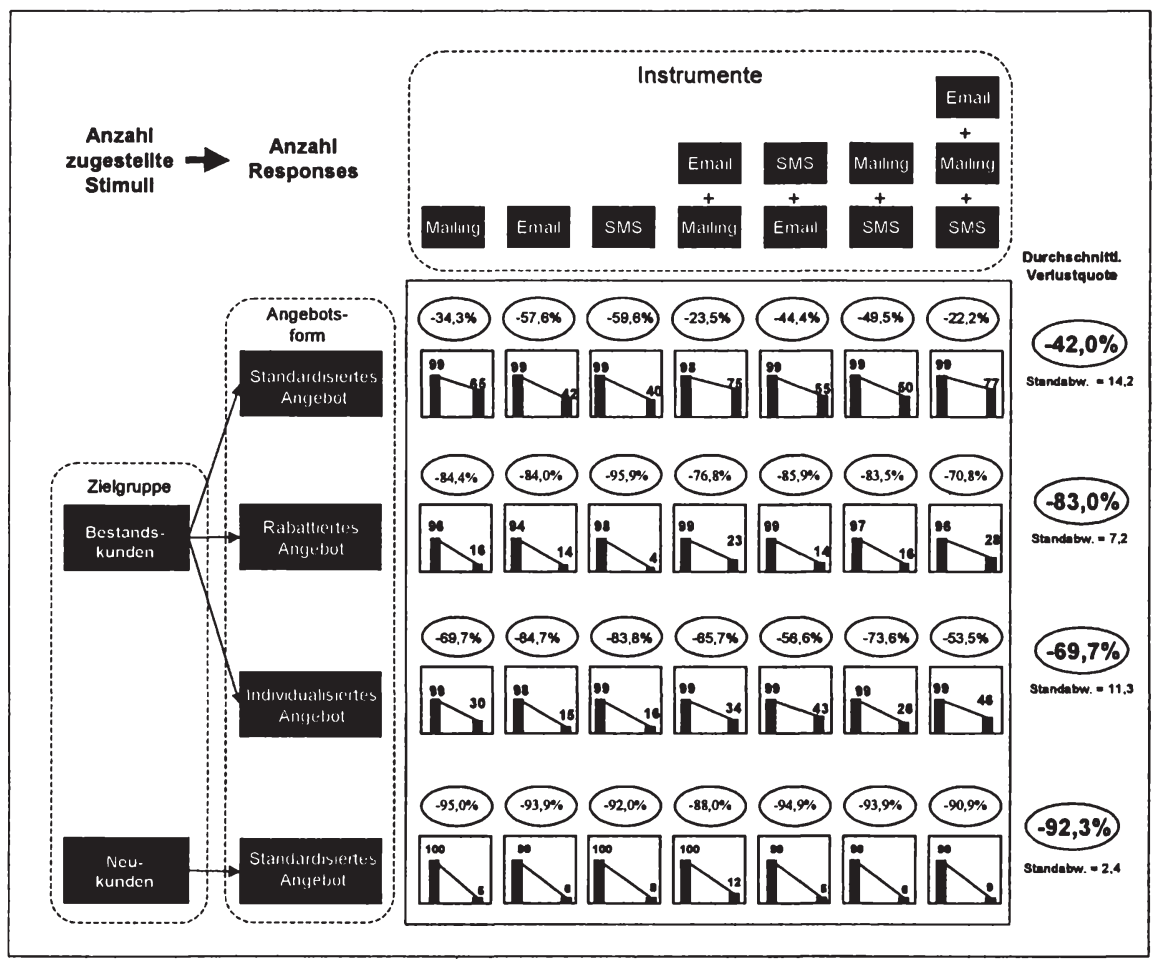

Abb. 19: Verlustquote von der Anzahl zugestellter Stimuli zur Anzahl Responses 
Spiegelbildlich zur Höhe der Responsequote ergibt sich die Abbruchquote zwischen der Zustellungs- und der Responsestufe, die als Differenz zwischen Zustell- und Responsequote berechnet wird. Bei der Bestandskundenbearbeitung lag dieser Wert im Rahmen des standardisierten Angebotes bei durchschnittlich $42 \%$, beim rabattierten Angebot bei $83 \%$ und beim individualisierten Angebot bei $69,7 \%$. Bei der Neukundenakquisition konnte eine durchschnittliche Abbruchquote von $92,3 \%$ ermittelt werden. Allerdings zeigte sich in Anlehnung an die vorangegangenen Ausführungen auf Basis der Instrumente und Instrumentekombinationen ein heterogenes Bild (vgl. Abb. 19).

Hinsichtlich der Responsequote konnten zudem Erkenntnisse bzgl. des kombinierten Einsatzes mehrerer Instrumente gewonnen werden. Insgesamt wurden zwölf der 28 versendeten Direktmarketing-Stimuli über ein isoliertes Trägermedium sowie 16 Stimuli über zwei oder drei Trägermedien an die Rezipienten versendet. Während die Analyse der Zustellquoten keine signifikanten Unterschiede zwischen dem isolierten und dem kombinierten Einsatz mehrerer Instrumente zeigte, zeichnete sich bei Auswertung der Responsequoten ab, dass der integrative Einsatz unabhängig von der angesprochenen Zielgruppe und dem erhaltenen Angebot im Durchschnitt zu höheren Responsequoten führte. Durchschnittlich generierte demnach der kombinierte Instrumenteeinsatz eine um etwa $10 \%$ höhere Responsequote als der isolierte Instrumenteeinsatz (vgl. Tab. 23).

\begin{tabular}{|c|c|c|c|c|c|c|c|c|c|}
\hline & \multicolumn{3}{|c|}{ Isolierter Einsatz } & \multicolumn{4}{|c|}{ Integrativer Einsatz } & \multirow[b]{3}{*}{ Differenz } & \multirow[b]{3}{*}{ Sign. } \\
\hline & Mailing & Email & SMS & $\begin{array}{l}\text { Mailing } \\
\text { + Email }\end{array}$ & $\begin{array}{c}\text { SMS + } \\
\text { Email }\end{array}$ & $\begin{array}{l}\text { Mailing } \\
\text { + SMS }\end{array}$ & $\begin{array}{c}\text { Email } \\
\text { Mailing } \\
\text { SMS }\end{array}$ & & \\
\hline $\begin{array}{l}\text { Durchschnittliche } \\
\text { Responsequote** }\end{array}$ & $28,5 \%$ & $19,5 \%$ & $17,0 \%$ & $36,0 \%$ & $29,3 \%$ & $24,5 \%$ & $40,0 \%$ & & \\
\hline Mittelwert & \multicolumn{3}{|c|}{$21,8 \%$} & \multicolumn{4}{|c|}{$32,4 \%$} & $10,6 \%$ & $0,00^{*}$ \\
\hline $\begin{array}{l}\text { Standard- } \\
\text { abweichung }\end{array}$ & \multicolumn{3}{|c|}{18,80} & \multicolumn{4}{|c|}{23,23} & & \\
\hline
\end{tabular}

Tab. 23: Vergleich der durchschnittlichen Responsequoten des isolierten und des integrativen Einsatzes der Direktmarketing-Instrumente

In Abhängigkeit der Anzahl der eingesetzten Instrumente im Rahmen des kombinierten Einsatzes zeigte sich, dass die Responsequote bei drei kombiniert eingesetzten Instrumente im Durchschnitt um 10,1\% über den Werten von zwei kombiniert eingesetzten Instrumenten liegt.

Die Betrachtung der Responsequoten lässt allerdings noch keine validen Aussagen über die Höhe der Buchungsquoten zu, jedoch sprechen tendenziell höhere 


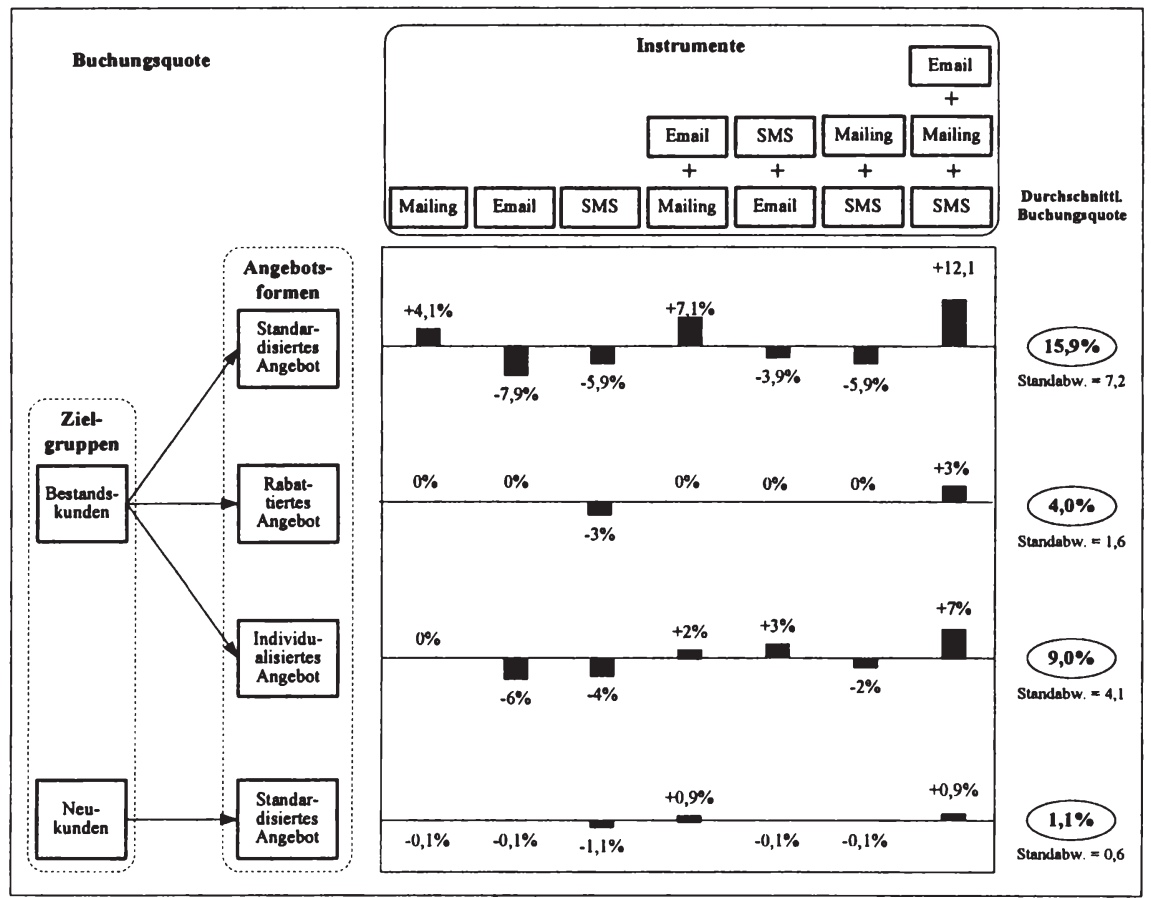

Abb. 20: Abweichung der Buchungsquoten der Instrumente und Instrumentekombinationen von der durchschnittlichen Buchungsquote

Responsequoten auch für höhere Buchungsquoten. So zeigt sich bzgl. der Buchungsquoten sowohl im Hinblick auf die Unterscheidung zwischen Bestandsund Neukunden als auch im Hinblick auf die eingesetzten Instrumente wiederum ein differenziertes Bild, das den Abweichungen der Responsequoten ähnelt. Vor diesem Hintergrund schwanken die Buchungsquoten bei den Bestandskunden zwischen $8 \%$ und $28 \%$. Im isolierten Instrumenteeinsatz beim standardisierten Angebot dominiert vor allem das Mailing mit einer Buchungsquote von $20 \%$, gefolgt von SMS (10\%) und Email (8\%). Im Rahmen des integrativen Einsatzes zweier Instrumente nimmt ein weiteres Mal die Kombination aus Mailing und Email die Spitzenposition ein (23\%). Die Effektivität im Hinblick auf Buchungen wird hierbei nur noch durch den integrativen Einsatz sämtlicher Instrumente mit einer Buchungsquote von $28 \%$ überboten. Im Vergleich der Buchungsquoten der einzelnen Instrumente zur durchschnittlichen Buchungsquote der anderen beiden Angebotsformen setzt sich der beobachtete Trend fort. Einheitlich gestaltet sich in diesem Zusammenhang die unterdurchschnittliche Buchungsquote 
beim isoliertem Einsatz von Emails und beim isoliertem Einsatz von SMS sowie beim integrativen Einsatz sämtlicher Instrumente. Ansonsten ist ein uneinheitlicher Trend zu konstatieren.

Im Rahmen der Neukundenakquisition lassen sich grundsätzlich Buchungsquoten zwischen $0 \%$ und $2 \%$ feststellen. Auf eine instrumentespezifische Betrachtung soll hierbei verzichtet werden. In diesem Zusammenhang bestätigt sich aber ebenfalls die Annahme, dass tendenziell höhere Responsequoten zu höheren Buchungsquoten führen (vgl. Abb. 20).

Wiederum spiegelbildlich verdeutlicht eine Betrachtung der Abbruchquoten von reagierenden Personen zu tatsächlichen Buchern, dass nahezu drei Viertel der Bestandskunden, die auf das Angebot reagiert haben, letztendlich keine Buchung vorgenommen haben. Bei den Neukunden lag dieser Wert mit $84 \%$ noch höher. Die folgende Abbildung zeigt die Abbruchquoten der einzelnen Zielgruppen sowie in Abhängigkeit der eingesetzten Instrumente und Instrumentenkombinationen (vgl. Abb. 21).

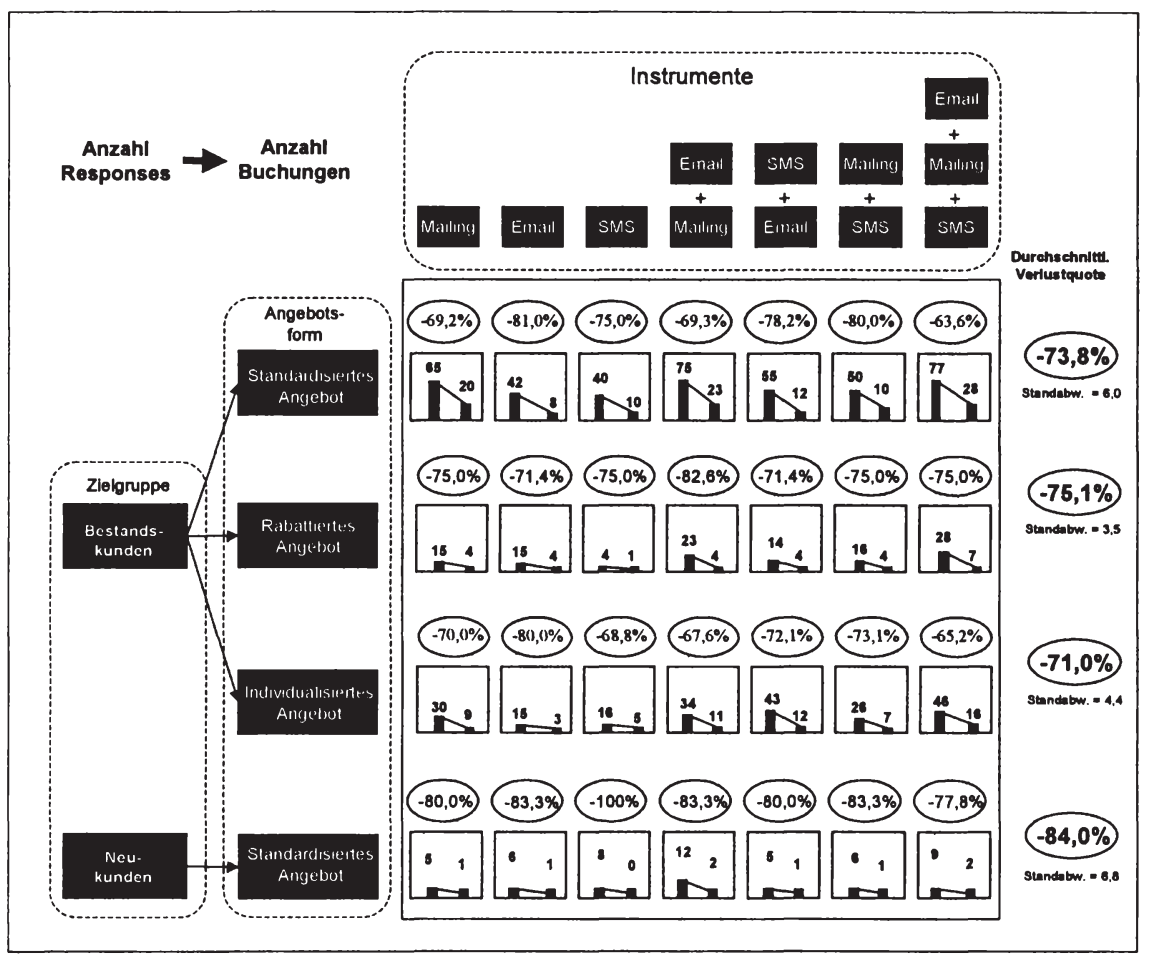

Abb. 21: Verlustquote von der Anzahl Responses zur Anzahl Buchungen 
Die Frage nach dem Einfluss des Trägermediums auf die Response- bzw. Buchungsquote kann an dieser Stelle noch nicht abschließend beantwortet werden. Es deutet sich jedoch an, dass aufgrund der geringeren Differenz der zweiten Abbruchquote von Response zur Buchung primär situative Gründe für den Abbruch des Buchungsvorgangs sprechen und nicht zielgruppen- oder angebotsbezogene Gründe. Zudem zeigt sich, dass sich die Abbruchquoten der beiden Zielgruppen annähern. Demzufolge wäre der Einfluss der unterschiedlichen Trägermedien primär auf der Ebene der Responsequote zu suchen.

Mit Blick auf die Buchungsquoten zeigte sich ebenfalls, dass der integrative Instrumenteeinsatz unter Effektivitätsgesichtspunkten vorteilhaft erscheint. Im Durchschnitt über sämtliche Angebote und Zielgruppen führte der integrative Einsatz mehrerer Direktmarketing-Instrumente zu einer 3,5\% höheren Buchungsquote (vgl. Tab. 24). ${ }^{504}$

\begin{tabular}{|c|c|c|c|c|c|c|c|c|c|}
\hline & \multicolumn{3}{|c|}{ Isolierter Einsatz } & \multicolumn{4}{|c|}{ Integrativer Einsatz } & \multirow[b]{3}{*}{ Diff. } & \multirow[b]{3}{*}{ Sign. } \\
\hline & Mailing & Email & SMS & $\begin{array}{l}\text { Mailing } \\
+ \text { Email }\end{array}$ & $\begin{array}{l}\text { SMS + } \\
\text { Email }\end{array}$ & $\begin{array}{l}\text { Mailing } \\
+ \text { SMS }\end{array}$ & $\begin{array}{c}\text { Email } \\
\text { Mailing } \\
\text { SMS }\end{array}$ & & \\
\hline $\begin{array}{l}\text { Durchschnittliche } \\
\text { Buchungsquote** }\end{array}$ & $8,5 \%$ & $4,0 \%$ & $4,0 \%$ & $10,0 \%$ & $7,3 \%$ & $5,5 \%$ & $13,3 \%$ & & \\
\hline Mittelwert & \multicolumn{3}{|c|}{$5,5 \%$} & \multicolumn{4}{|c|}{$9,0 \%$} & $3,5 \%$ & $0,00^{*}$ \\
\hline $\begin{array}{l}\text { Standard- } \\
\text { abweichung }\end{array}$ & \multicolumn{3}{|c|}{2,6} & \multicolumn{4}{|c|}{3,39} & & \\
\hline
\end{tabular}

Tab. 24: Vergleich der durchschnittlichen Buchungsquoten des isolierten und des integrativen Einsatzes der Direktmarketing-Instrumente

Weiterhin erlaubt eine differenzierte Betrachtung der integrativ eingesetzten Instrumente zusätzliche Erkenntnisse. So wurde mit Blick auf die Effektivität weiterhin untersucht, ob lediglich zwei oder sämtliche der drei DirektmarketingInstrumente zum Einsatz kamen. Es zeigte sich, dass die Buchungsquote um $5,7 \%$ höher liegt, wenn statt zwei drei Instrumente eingesetzt werden. ${ }^{505}$

Im Zusammenhang mit den eingesetzten Instrumenten stellt sich zudem die Frage, ob es $\mathrm{zu}$ instrumentespezifischen Unterschieden im Informationsbedarf kommt. Abschließend soll daher untersucht werden, ob die Menge der abgefragten Informationen, die über ein spezifisches Direktmarketing-Instrument trans-

504 Die durchschnittlichen Buchungsquoten unterscheiden sich nach dem T-Test signifikant voneinander.

505 Die aufgezeigten Unterschiede sind ebenfalls signifikant auf einem $5 \%$-igen Niveau. 
portiert werden können, zu einem unterschiedlichen Informationsbedarf aufseiten der Rezipienten führt. Dabei ist insbesondere im Rahmen des Einsatzes von SMS zu vermuten, dass es aufgrund der begrenzten Darstellungsmöglichkeiten zu intensiveren Informationsnachfragen im Call-Center kommt, da weniger Informationen zu einem spezifischen Angebot transportiert werden können. Die Ergebnisse dieses Vergleichs können Tab. 25 entnommen werden.

\begin{tabular}{|c|c|c|c|c|c|c|c|c|c|c|}
\hline \multirow[b]{3}{*}{$\begin{array}{l}\text { Informations- } \\
\text { verhalten }\end{array}$} & \multicolumn{9}{|c|}{ Bestandskunden } & \multirow{3}{*}{ Sign* } \\
\hline & \multicolumn{3}{|c|}{ SMS } & \multicolumn{3}{|c|}{ Email } & \multicolumn{3}{|c|}{ Mailing } & \\
\hline & $\begin{array}{c}\text { gering } \\
(=1)\end{array}$ & $\begin{array}{c}=2) \\
(=2)\end{array}$ & $\begin{array}{l}\text { viel } \\
(=3)\end{array}$ & $\begin{array}{c}\text { gering } \\
(=1)\end{array}$ & $\begin{array}{c}=2) \\
\text { mittel }\end{array}$ & $\begin{array}{l}\text { viel } \\
(=3)\end{array}$ & $\begin{array}{c}\text { gering } \\
(=1)\end{array}$ & $\begin{array}{c}\text { mittel } \\
(=2)\end{array}$ & $\begin{array}{l}\text { viel } \\
(=3)\end{array}$ & \\
\hline Anzahl absolut & 3 & 3 & 10 & 6 & 6 & 3 & 17 & 15 & 1 & \\
\hline Mittelwert & \multicolumn{3}{|c|}{2,44} & \multicolumn{3}{|c|}{1,80} & \multicolumn{3}{|c|}{1,52} & 0,00 \\
\hline $\begin{array}{l}\text { Standard- } \\
\text { abweichung }\end{array}$ & \multicolumn{3}{|c|}{0,72} & \multicolumn{3}{|c|}{0,76} & \multicolumn{3}{|c|}{0,63} & \\
\hline
\end{tabular}

*T-Test, $95 \%$ Konfidenzintervall

Tab. 25: Informationsbedarf der Rezipienten beim isolierten Instrumenteeinsatz

Es zeigte sich ein signifikanter Unterschied im Informationsbedarf bezogen auf die drei eingesetzten Direktmarketing-Instrumente. Demnach führt nicht nur der Einsatz von SMS aufgrund der begrenzten Darstellungsmöglichkeiten zu intensiveren Informationsanfragen im Vergleich zu Emails und Mailings. Angesichts der Unterschiede zwischen Email und Mailing erweist sich der Informationsbedarf vor allem durch die Zusendung von Mailings im Hinblick auf ein bestimmtes Angebot aus Rezipientensicht als ausreichend. Es ergaben sich keine wesentlichen Unterschiede beim kombinierten Einsatz, so dass von einer weiteren Betrachtung abgesehen werden kann.

In diesem Kontext konnte zusätzlich die Werbeerinnerung der Rezipienten an das im Dezember 2003 stattgefundene Angebot untersucht werden. In diesem Zusammenhang wurden die Rezipienten in der Ex-post-Befragung sowohl nach dem Angebot als auch nach den eingesetzten Trägermedien befragt, welches ihnen im Dezember 2003 zugegangen ist. Es zeigte sich, dass Rezipienten, die über mehrere Trägermedien gleichzeitig mit dem Angebot in Kontakt kamen, sich besser an das zugesandte Angebot erinnerten. Insgesamt ergab sich hierbei folgendes Ergebnis (vgl. Tab. 26).

Eine mögliche Begründung für die insgesamt hohen Werte im Rahmen der Werbeerinnerung sowohl an das eingesetzte Trägermedium als auch an das Angebot kann in der Einmaligkeit der Experimente gesehen werden, die ihrerseits im Rahmen der Ex-ante-Befragung zudem noch angekundigt wurden. 


\begin{tabular}{|l|c|c|c|c|c|c|c|c|}
\cline { 2 - 8 } \multicolumn{1}{c|}{} & \multicolumn{3}{|c|}{ Isolierter Einsatz } & \multicolumn{4}{c|}{ Integrativer Einsatz } & \\
\hline Gesendeter Stimulus & Mailing & Email & SMS & $\begin{array}{c}\text { Mailing } \\
+ \text { Email }\end{array}$ & $\begin{array}{c}\text { SMS + } \\
\text { Email }\end{array}$ & $\begin{array}{c}\text { Mailing } \\
+ \text { SMS }\end{array}$ & $\begin{array}{c}\text { Email } \\
\text { Mailing } \\
\text { SMS }\end{array}$ & Sign. \\
\hline $\begin{array}{l}\text { Erinnerung an das } \\
\text { eingesetzte Instru- } \\
\text { ment }\end{array}$ & $94 \%$ & $95 \%$ & $93 \%$ & $99 \%$ & $95 \%$ & $95 \%$ & $95 \%$ & \\
\hline Mittelwert & \multicolumn{3}{|c|}{$94 \%$} & & $96 \%$ & & $\mathbf{0 , 0 2 *}$ \\
\hline $\begin{array}{l}\text { Erinnerung an das } \\
\text { versendete Angebot }\end{array}$ & $92 \%$ & $91 \%$ & $93 \%$ & $95 \%$ & $99 \%$ & $98 \%$ & $94 \%$ & \\
\hline Mittelwert & \multicolumn{7}{|c|}{$92 \%$} & \multicolumn{6}{c|}{$96,5 \%$} & $\mathbf{0 , 0 3 *}$ \\
\hline
\end{tabular}

Tab. 26: Werbeerinnerung beim isolierten und integrativen Instrumenteeinsatz

Hinsichtlich der maximalen Preisbereitschaft ergaben sich keine signifikanten Unterschiede im Hinblick auf die Unterscheidung zwischen dem isolierten und dem kobinierten Einsatze der Instrumente. Ebenso konnten keine Unterschiede der gebuchten Ticketanzahl in Abhängigkeit der Instrumente bzw. Instrumentekombinationen identifiziert werden, so dass ebenfalls auf eine weitere Betrachtung ebenfalls verzichtet werden soll.

\subsubsection{Effizienzbeurteilung}

Nach instrumentespezifischer Schlüsselung der Kosten konnten die verschiedenen kostenorientierten Effizienzkennzahlen berechnet werden. Der Vergleich der Stück- und Kontaktkosten (Cost per contact) der einzelnen Instrumente und Instrumentekombinationen zeigt einen Vorteil des isolierten Einsatzes der Instrumente unter Effizienzgesichtspunkten. ${ }^{506}$ So gestaltet sich vor allem der Einsatz von SMS im Rahmen der Bestands- und Neukundenansprache zur Kontaktschaffung effizient. ${ }^{507}$ Dies liegt zum einen an den geringen Stückkosten für den isolierten SMS-Einsatz, der ggü. dem Mailing-Einsatz um $50 \%$, ggü. dem EmailEinsatz um ca. $66 \%$ geringer ist. Unter Hinzuziehung der Responsequoten lassen sich die instrumentespezifischen Kosten berechnen, die zur Generierung eines In-

506 Dies liegt vor allem daran, dass einem Direktmarketing-Stimulus bestehend aus zwei unterschiedliche Trägermedien beide „Stückkosten“ angerechnet werden.

507 Auffällig erscheinen in diesem Zusammenhang die hohen Stückkosten der Email, die aus einem hohen Fixkostenanteil der Gesamtkosten für die Abwicklung (Projektmanagement) resultieren. Bei größeren Stückzahlen wird dieser Anteil entsprechend gering ausfallen, so dass die ohnehin geringen variablen Kosten zum Hauptbestandteil der Stückkosten werden. 
teressentenanrufes benötigt werden. Nach Betrachtung der „Cost per interest“Werte erweist sich allerdings aufgrund der hohen Responsequoten das Mailing im isolierten Einsatz als effizientestes Trägermedium im Rahmen sämtlicher Angebotsformen und Zielgruppen. Unter Beachtung der Buchungsquote setzt sich dieses Bild fort, so dass auch die Kennzahl „Cost per order“ am geringsten ausfällt. Das Mailing erweist sich auch hier als das effizienteste Trägermedium.

\begin{tabular}{|c|c|c|c|c|c|c|}
\hline \multicolumn{3}{|c|}{ Direktmarketing-Stimulus } & \multicolumn{4}{|c|}{ Effizienz } \\
\hline $\begin{array}{l}\text { Ziel- } \\
\text { gruppe }\end{array}$ & $\begin{array}{c}\text { Ange- } \\
\text { bot }\end{array}$ & Instrument & $\begin{array}{c}\text { Cost per } \\
\text { contact in } €\end{array}$ & $\begin{array}{c}\text { Cost per } \\
\text { interest in } €\end{array}$ & $\begin{array}{c}\text { Cost per order } \\
\text { in } €\end{array}$ & $\begin{array}{l}\text { Deckungs- } \\
\text { beitrag in } €\end{array}$ \\
\hline \multirow{21}{*}{ 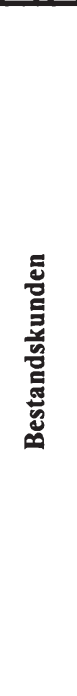 } & \multirow{7}{*}{ 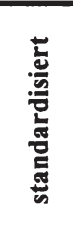 } & Email+Mailing+SMS & 1,88 & 3,32 & 9,14 & 1758,07 \\
\hline & & Email+Mailing & 1,58 & 3,02 & 9,83 & 1445,81 \\
\hline & & SMS+Email & 1,25 & 3,12 & 14,28 & 664,64 \\
\hline & & Mailing+SMS & 0,94 & 2,70 & 13,52 & 586,84 \\
\hline & & Email & 0,94 & 3,06 & 16,06 & 403,51 \\
\hline & & SMS & 0,31 & 1,79 & 7,14 & 650,58 \\
\hline & & Mailing & 0,63 & 1,82 & 5,93 & 1325,48 \\
\hline & \multirow{7}{*}{ 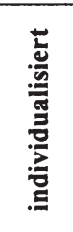 } & Email+Mailing+SMS & 2,17 & 5,69 & 16,36 & 2154,30 \\
\hline & & Email+Mailing & 1,67 & 5,93 & 18,31 & 1434,54 \\
\hline & & SMS+Email & 1,49 & 4,51 & 16,14 & 1878,26 \\
\hline & & Mailing+SMS & 1,18 & 5,50 & 20,41 & 1105,13 \\
\hline & & Email & 1,00 & 7,46 & 37,30 & 396,11 \\
\hline & & SMS & 0,50 & 4,25 & 13,59 & 872,04 \\
\hline & & Mailing & 0,68 & 3,14 & 10,47 & 1389,80 \\
\hline & \multirow{7}{*}{ 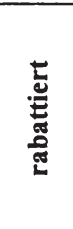 } & Email+Mailing+SMS & 1,93 & 7,65 & 30,61 & 784,73 \\
\hline & & Email+Mailing & 1,57 & 7,72 & 44,39 & 350,43 \\
\hline & & SMS+Email & 0,99 & 7,75 & 27,11 & 209,57 \\
\hline & & Mailing+SMS & 0,65 & 4,80 & 19,21 & 331,14 \\
\hline & & Email & 1,25 & 10,04 & 35,12 & 267,50 \\
\hline & & SMS & 0,31 & 8,79 & 35,15 & 51,85 \\
\hline & & Mailing & 0,96 & 6,86 & 27,42 & 328,31 \\
\hline \multirow{7}{*}{ 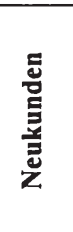 } & \multirow{7}{*}{ 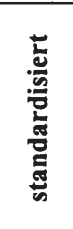 } & Email+Mailing+SMS & 5,88 & 65,74 & 295,84 & $-439,69$ \\
\hline & & Email+Mailing & 4,20 & 35,87 & 215,24 & $-316,47$ \\
\hline & & SMS+Email & 1,94 & 39,97 & 199,83 & $-123,83$ \\
\hline & & Mailing+SMS & 2,28 & 38,57 & 231,41 & $-155,41$ \\
\hline & & Email & 3,61 & 60,56 & 363,36 & $-287,36$ \\
\hline & & SMS & 1,63 & 21,31 & - & $-170,47$ \\
\hline & & Mailing & 3,92 & 78,68 & 393,40 & $-355,40$ \\
\hline
\end{tabular}

Tab. 27: Kostenorientierte Effizienzkennzahlen und Deckungsbeiträge in Abhängigkeit der eingesetzten Instrumente

Erst unter der Berücksichtigung der absoluten Höhe der mit dem Ticketverkauf erzielten Umsätze ergibt sich eine andere Reihenfolge. So werden die höchsten Deckungsbeiträge beim kombinierten Einsatz sämtlicher Instrumente verzeichnet, welche sich zunächst auf das individualisierte Angebot, aber auch auf das standardisierte Angebot beziehen. Die negativen Deckungsbeiträge sämtli- 
cher Instrumentekombinationen bestätigte die unter ökonomischen Gesichtspunkten kritisch zu beurteilende Neukundenakquisition mit Hilfe des Direktmarketing-Einsatzes. In diesem Zusammenhang zeigte die Analyse weiterhin, dass keine Instrumentekombination einen positiven Deckungsbeitrag realisierte und somit die Aussage instrumenteunabhängig Gültigkeit besitzt. Den geringsten negativen Deckungsbeitrag verursachte der Einsatz des Mailings. Die Tabelle 27 gibt einen Überblick über die versendeten 28 Direktmarketing-Stimuli.

Nachdem der positive Einfluss des integrativen Einsatzes mehrerer Instrumente auf die Effektivität im vorangegangenen Kapitel gezeigt wurde, soll auf die Analyse des kombinierten Einsatzes auch unter Effizienzgesichtspunkten näher eingegangen werden. In diesem Kontext stellt sich primär die Frage, ob die Steigerung der Effektivität zuungunsten der Effizienz ausfällt. Vor dem Hintergrund der Berechnung der bereits in den vorangegangenen Kapiteln verwendeten kostenorientierten Effizienzkennzahlen ergeben sich hierbei die folgenden durchschnittlichen Werte (vgl. Tab. 28).

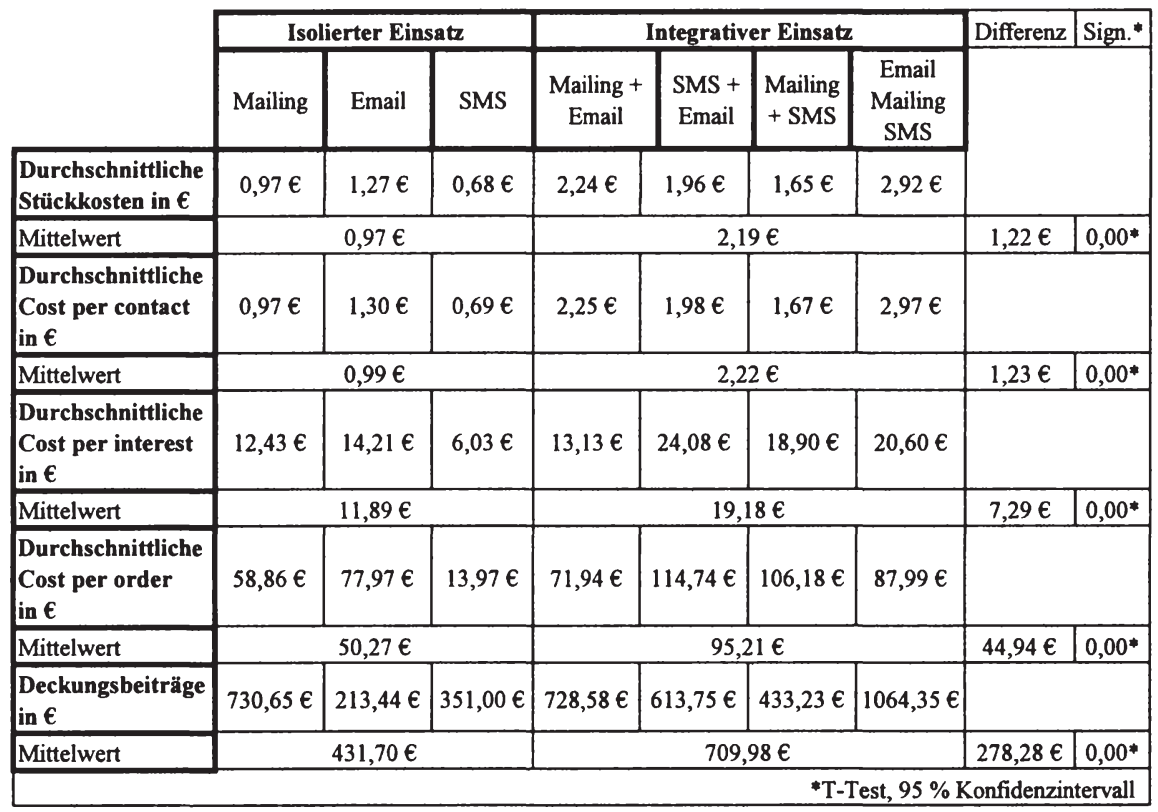

Tab. 28: Vergleich der Effizienzkennzahlen des isolierten und des integrativen Instrumenteeinsatzes 
Auf Basis dieser Auswertung beträgt der „Preisaufschlag“ zur Erreichung bestehender und potenzieller Kunden mittels des integrativen Einsatzes bei den einzelnen Kennzahlen zwischen $80 \%$ und $120 \%$. Eine durchschnittlich $10 \%$-ige Steigerung der Effektivităt, bspw. in Form einer verdoppelten Responsequote, wird demnach mit einer durchschnittlichen Verdoppelung der Kosten „erkauft“. Unter Berücksichtigung der Deckungsbeiträge ändert sich dieses Bild wiederum. So kann im Vergleich des isolierten und integrativen Einsatzes der Direktmarketing-Instrumente durchschnittlich ein um 278,28 Euro höherer Deckungsbeitrag im Rahmen des kombinieren Einsatzes generiert werden.

\subsubsection{Synoptische Darstellung der Ergebnisse der Effektivitäts- und Effizienzana- lyse}

In den vorangegangenen Experimenten konnten insgesamt 28 verschiedene Direktmarketing-Stimuli unter Effektivitäts- und Effizienzgesichtspunkten analysiert werden, wobei sich in Abhängigkeit der angesprochenen Zielgruppe, des versendeten Angebotes sowie der eingesetzten Trägermedien heterogene Werte ergaben. Vor diesem Hintergrund erscheint es fraglich, wie kongruent die Ergebnisse der Effektivitätsuntersuchung und der Effizienzuntersuchung im Vergleich zueinander sind. Von besonderem Interesse ist vor allem die Frage, ob sich auf Basis der verwendeten Größen ähnliche Gestaltungsempfehlungen treffen lassen (vgl. Tab. 29). ${ }^{508}$

Eine „gute Platzierung“ auf Basis einer Effektivitătskennzahl gewăhrleistet noch keinen hohen Rangplatz im Rahmen der Effizienzbetrachtung, wie bspw. das Beispiel von Stimulus Nr. 4 zeigt. Vielmehr kommt es je nach Priorisierung einer entsprechenden Kennzahl als Auswahlkriterium zu unterschiedlichen Empfehlungen hinsichtlich der Auswahl des einzusetzenden Stimulus. So erweist sich keiner der eingesetzten Direktmarketing-Stimuli als dominant.

508 Unter der Zielsetzung der Erreichung einer möglichst hohen Buchungsquote und freier Wahl sämtlicher Entscheidungsparameter hinsichtlich Zielgruppe, Angebotsform und Instrumenteeinsatz würde das Unternehmen bspw. Stimulus Nr. 1 auswählen. Unter dem Ziel der Deckungsbeitragsmaximierung wäre hingegen der Einsatz von Stimulus Nr. 6 empfehlenswert. 


\begin{tabular}{|c|c|c|c|c|c|c|c|c|c|c|c|}
\hline \multicolumn{4}{|c|}{ Zentrale Parameter } & \multicolumn{4}{|c|}{ Effektivität } & \multicolumn{4}{|c|}{ Effizienz } \\
\hline & & & & Rang & Rang & Rang & Rang & Rang & Rang & Rang & Rang \\
\hline $\begin{array}{c}\text { Stimulus } \\
\text { Nr. }\end{array}$ & Zielgruppe & Angebot & Instrument & $\begin{array}{c}\text { Zustell- } \\
\text { quote }\end{array}$ & $\begin{array}{l}\text { Response- } \\
\text { quote }\end{array}$ & $\begin{array}{l}\text { Buchungs- } \\
\text { quote }\end{array}$ & Umsatz & $\begin{array}{l}\text { Cost per } \\
\text { contact }\end{array}$ & $\begin{array}{l}\text { Cost per } \\
\text { interest }\end{array}$ & $\begin{array}{c}\text { Cost per } \\
\text { order }\end{array}$ & $\begin{array}{c}\text { Deckungs- } \\
\text { beitrag }\end{array}$ \\
\hline 1 & Bestandskunden & standardisiert & Email+Mailing+SMS & 2 & 1 & 1 & 3 & 20 & 8 & 3 & 3 \\
\hline 2 & Bestandskunden & standardisiert & Email+Mailing & 3 & 2 & 2 & 4 & 17 & 4 & 4 & 4 \\
\hline 3 & Bestandskunden & standardisiert & Mailing & 2 & 3 & 3 & 7 & 4 & 2 & 1 & 7 \\
\hline 4 & Bestandskunden & standardisiert & SMS+Email & 2 & 4 & 5 & 11 & 14 & 6 & 8 & 11 \\
\hline 5 & Bestandskunden & standardisiert & Mailing+SMS & 2 & 5 & 8 & 13 & 7 & 3 & 6 & 13 \\
\hline 6 & Bestandskunden & individualisiert & Email+Mailing+SMS & 2 & 6 & 4 & 1 & 23 & 13 & 11 & 1 \\
\hline 7 & Bestandskunden & individualisiert & SMS+Email & 2 & 7 & 6 & 2 & 15 & 10 & 10 & 2 \\
\hline 8 & Bestandskunden & standardisiert & Email & 2 & 8 & 11 & 14 & 8 & 5 & 9 & 14 \\
\hline 9 & Bestandskunden & standardisiert & SMS & 2 & 9 & 9 & 12 & 1 & 1 & 2 & 12 \\
\hline 10 & Bestandskunden & individualisiert & Email+Mailing & 2 & 10 & 7 & 5 & 19 & 14 & 12 & 5 \\
\hline 11 & Bestandskunden & individualisiert & Mailing & 2 & 11 & 10 & 6 & 6 & 7 & 5 & 6 \\
\hline 12 & Bestandskunden & rabbatiert & Email+Mailing+SMS & 5 & 12 & 12 & 9 & 21 & 17 & 17 & 10 \\
\hline 13 & Bestandskunden & individualisiert & Mailing+SMS & 2 & 13 & 13 & 8 & 12 & 12 & 14 & 8 \\
\hline 14 & Bestandskunden & rabbatiert & Email+Mailing & 2 & 14 & 15 & 15 & 16 & 18 & 21 & 16 \\
\hline 15 & Bestandskunden & individualisiert & SMS & 5 & 15 & 14 & 10 & 3 & 9 & 7 & 9 \\
\hline 16 & Bestandskunden & rabbatiert & Mailing & 5 & 15 & 17 & 17 & 9 & 15 & 16 & 18 \\
\hline 17 & Bestandskunden & rabbatiert & Mailing+SMS & 4 & 15 & 16 & 18 & 5 & 11 & 13 & 17 \\
\hline 18 & Bestandskunden & individualisiert & Email & 3 & 16 & 20 & 16 & 11 & 16 & 20 & 15 \\
\hline 19 & Bestandskunden & rabbatiert & Email & 6 & 17 & 19 & 19 & 13 & 21 & 18 & 19 \\
\hline 20 & Bestandskunden & rabbatiert & SMS+Email & 2 & 17 & 18 & 20 & 10 & 19 & 15 & 20 \\
\hline 21 & Neukunden & standardisiert & Email+Mailing & 1 & 18 & 21 & 22 & 27 & 23 & 23 & 26 \\
\hline 22 & Neukunden & standardisiert & Email+Mailing+SMS & 2 & 19 & 22 & 21 & 28 & 27 & 25 & 28 \\
\hline 23 & Neukunden & standardisiert & SMS & 1 & 20 & 28 & 28 & 18 & 22 & 28 & 24 \\
\hline 24 & Neukunden & standardisiert & Email & 2 & 21 & 24 & 26 & 25 & 26 & 26 & 25 \\
\hline 25 & Neukunden & standardisiert & Mailing+SMS & 2 & 21 & 23 & 25 & 24 & 24 & 24 & 23 \\
\hline 26 & Neukunden & standardisiert & Mailing & 1 & 22 & 26 & 27 & 26 & 28 & 27 & 27 \\
\hline 27 & Neukunden & standardisiert & SMS+Email & 2 & 22 & 25 & 24 & 22 & 25 & 22 & 22 \\
\hline 28 & Bestandskunden & rabbatiert & SMS & 3 & 23 & 27 & 23 & 2 & 20 & 19 & 21 \\
\hline
\end{tabular}

Tab. 29: Zusammenfassende Darstellung der Effektivität und Effizienz der eingesetzten Direktmarketing-Stimuli auf Basis von Rangplätzen 


\subsection{Einfluss exogener Determinanten des Direktmarketing-Einsatzes}

\subsubsection{Analyse des Einflusses personenbezogener Determinanten}

Im Folgenden sollen ausgewählte Zustands- und Verhaltensmerkmale der Rezipienten auf ihren Einfluss auf das im Anschluss an den Erhalt der versendeten Direktmarketing-Stimuli beobachtete Verhalten untersucht werden. Im Rahmen des in Kapitel C.2.4 beschriebenen dreistufigen Vorgehens wird jeweils der Zusammenhang zwischen einer unabhängigen exogenen Variablen und einer spezifischen Ausprägung der Verhaltensreaktionen der Rezipienten untersucht. Im ersten Schritt wird zunächst analysiert, ob die Variable einen Einfluss auf das grundsätzliche Responseverhalten hat (Analyse 1) und damit eine diskriminatorische Wirkung zur Unterscheidung von Nicht-Reagierern und Reagierern besitzt. In einem zweiten Schritt erfolgt die Untersuchung der Relevanz der entsprechenden Variablen im Rahmen des Buchungsverhaltens, allerdings ausschließlich bei Personen, die auf das Angebot reagiert haben (Analyse 2). Die Ergebnisse dieser Analyse erlauben damit Aussagen darüber, ob die entsprechende Variable bei denjenigen Personen von Bedeutung für die Buchungsentscheidung ist, die sich für das Angebot interessieren und im Call-Center des Unternehmens anrufen. Im dritten Analyseschritt sind die beiden ersten Untersuchungen implizit enthalten: so wird untersucht, ob ein genereller Zusammenhang zwischen der betrachteten Variablen und der Entscheidung, einen Flug zu buchen, besteht (Analyse 3). Zur Grundgesamtheit der Personen der dritten Analyse zählen neben den Buchern sämtliche Nicht-Reagierer. Um valide Aussagen darüber zu generieren, ob ein Zusammenhang zwischen einer personenbezogenen unabhängigen Variablen sowie der abhängigen Variablen in Form der Rezipientenreaktion besteht oder nicht, kommen je nach Skalierungsniveau der unabhängigen Variablen mit der Kontingenzanalyse, den T-Tests sowie den nicht-parametrischen Tests verschiedene statistische Analysemethoden zum Einsatz. ${ }^{509}$

509 Das bereits beschriebene dreistufige Vorgehen ermöglicht damit zunächst Aussagen darüber, welche soziodemographischen, psychographischen und verhaltensorientierten Personenmerkmale mit der jeweils betrachteten abhängigen Variablen zusammenhängen und damit ggf. über eine diskriminatorische Wirkung hinsichtlich der Verhaltensreaktion verfügen. In diesem Zusammenhang werden hierbei drei dichotome Ausprägungen untersucht: „,keine Reaktion“"vs. „Anruf im Call-Center“, „Anruf im Call-Center ohne Buchung“ vs. „Anruf im Call-Center mit Buchung“ sowie „keine Reaktion“ und „Anruf im Call-Center mit Buchung“. Zu einer detaillierten Darstellung der einzelnen Fragen im Rahmen der Ex-ante- und Ex-post-Befragung vgl. die im Anhang abgedruckten Fragebögen der empirischen Untersuchung. 
Vor dem Hintergrund der für die Untersuchung gebildeten zwei Altersklassen (,unter 35 Jahre“ und „über 35 Jahre“) konnte ein Anteil der „über 35-jährigen“ bei den Nicht-Reagierern von $55 \%$ und ein Wert von fast $70 \%$ bei den Reagierern verzeichnet werden. Demnach erscheint der durchschnittliche Reagierer auf die versendeten Direktmarketing-Aktionen tendenziell älter zu sein. ${ }^{510}$ Allerdings zeigt ein Vergleich der Alterstruktur der der Gruppe der Reagierer angehörenden Interessenten und Bucher, dass die Interessenten im Vergleich zu den Buchern im Durchschnitt ein höheres Alter aufweisen. ${ }^{511}$ Dennoch lässt sich im Rahmen der Analyse von Nicht-Reagierern und Buchern immer noch ein signifikant höherer Anteil der Altersklasse der ,über 35-Jährigen“ an den Buchern identifizieren. Damit kann der im Zuge der Bildung der strukturgleichen Stichproben angenommene positive Zusammenhang von höherem Alter und steigender $\mathrm{Bu}$ chungswahrscheinlichkeit tendenziell bestätigt werden.

Als weiteres demographisches Merkmal konnte darüber hinaus der Zusammenhang zwischen Geschlecht des Rezipienten und Verhaltensreaktion untersucht werden. ${ }^{512}$ Hierbei konnte im Vergleich von Nicht-Reagierern und Reagierern ein signifikant höherer Anteil an männlichen Personen bei den Reagierern ermittelt werden (58,8 \% im Verhältnis zu $55 \%$ ). Auch im Vergleich von Interessenten und Buchern wurde dieser Zusammenhang nachgewiesen $(56,7 \%$ bei den Interessenten und $59,8 \%$ bei den Buchern). Letztendlich erweist sich das Geschlecht auch signifikant trennend zwischen der Gruppe der Nicht-Reagierer und der Gruppe der Bucher, wobei tendenziell eher männliche Rezipienten gebucht haben.

Die Analyse der Ausbildung bzw. des Berufs hinsichtlich eines Zusammenhangs mit den beobachteten Rezipientenreaktionen führte zu heterogen Ergebnissen. Während die Art der Ausbildung und der damit verbundene Schul- oder Hochschulabschluss in keinem signifikanten Zusammenhang mit der Rezipientenreaktion steht, scheint die Art des Berufs einen Einfluss darauf zu besitzen, ob der Rezipient grundsätzlich auf das Angebot reagiert und im Call-Center anruft oder nicht. ${ }^{513}$ Hingegen kann kein Zusammenhang zwischen dem Beruf und dem Buchungsverhalten nachgewiesen werden. Auch das Einkommen kann als signi-

510 Das Durchschnittsalter der Nicht-Reagerer liegt bei 40 Jahren ggü. einem Durchschnittsalter der Reagierer von ungefähr 44 Jahren.

511 Auf Basis eines T-Tests, Signifikanzniveau von $5 \%$.

512 In der Ausgangssituation wurden sämtliche Stichproben mit 50 \% Frauenanteil quotiert.

513 Bspw. zeigte sich ein unterschiedlich hoher Anteil an selbständig berufstätigen Personen unter den Reagierern (26,2 \%) im Verhältnis zum Anteil bei den NichtReagierern (19,2\%), während dieser Anteil im Vergleich von Interessenten und Reagierern $(27,9 \%$ vs. $25,4 \%)$ tendenziell gleich ausfiel. 
fikant trennende Variable zwischen Reagierern und Nicht-Reagierern ermittelt werden. So lag der Anteil der Personen mit einem Monatseinkommen von > 3.000 Euro mit 45,9\% an der Gruppe der Reagierer signifikant höher als bei den Nicht-Reagierern (35,9\%). Ebenso trennt das Merkmal „Einkommen“ signifikant zwischen Interessenten und Buchern und hat demnach einen Einfluss auf die Buchungswahrscheinlichkeit von Anrufern im Call-Center. Die Ergebnisse der Analyse der demographischen Merkmale sind in der folgenden Tabelle noch einmal übersichtsartig zusammengefasst (vgl. Tab. 30).

\begin{tabular}{|c|c|c|c|}
\hline & $\begin{array}{c}\text { Analyse 1 } \\
\text { Nicht-Reagierer vs. } \\
\text { Reagierer }^{\mathbf{2}}\end{array}$ & $\begin{array}{l}\text { Analyse 2 } \\
\text { Interessenten vs. } \\
\text { Bucher }\end{array}$ & $\begin{array}{c}\text { Analyse 3 } \\
\text { Nicht-Reagierer vs } \\
\text { Bucher }\end{array}$ \\
\hline \multicolumn{4}{|c|}{ Soziodemographische Merkmale } \\
\hline \multicolumn{4}{|c|}{ Demographische Merkmale } \\
\hline Alter & signifikant * & signifikant * & signifikant * \\
\hline Geschlecht & signifikant * & signifikant * & signifikant * \\
\hline \multicolumn{4}{|c|}{ Sozioökonomische Merkmale } \\
\hline Ausbildung & n.s. & n.s. & n.s. \\
\hline Beruf & signifikant * & n.s. & n.s. \\
\hline Einkommen & signifikant * & signifikant * & signifikant * \\
\hline \multicolumn{4}{|c|}{$\begin{array}{l}\mathrm{a}=\text { die Gruppe der Reagierer beinhaltet die Gruppe der Interessenten und der Bucher } \\
*=\text { signifikant auf } 5 \% \text { Signifikanzniveau } \\
\text { n.s. = nicht signifikant }\end{array}$} \\
\hline
\end{tabular}

Tab. 30: Zusammenhang zwischen soziodemographischen Merkmalen und Rezipientenverhalten

Als zweiter Schwerpunkt der personenbezogenen Determinanten erfolgt eine Analyse ausgewählter psychographischer Personenmerkmale im Hinblick auf die Relevanz für das Response- und Buchungsverhalten. Als potenzielle Einflussfaktoren werden hierbei auf der einen Seite Einstellungen ggu. der klassischen Werbung, ggü. dem Direktmarketing im Allgemeinen sowie ggü. einzelnen Direktmarketing-Instrumenten im Speziellen angesehen. Auf der anderen Seite fallen in diesen Bereich auch auf das Unternehmen sowie dessen Leistungsangebot gerichtete Einstellungen ebenso wie Erfahrungen bzw. eine daraus resultierende Zufriedenheit mit Produkten und Dienstleistungen.

Als positive Treiber der Reaktionswahrscheinlichkeit konnten vor allem Einstellungen ggü. der Werbung im Allgemeinen aufgezeigt werden. ${ }^{514}$ Die Einfluss-

514 Abgefragt wurde in diesem Kontext die Zustimmung der Rezipienten zu den Aussagen, dass Werbung störend sei, es zuviel Werbung gebe bzw. diese in die Privatsphäre eindringe. Die Zustimmung wurde auf einer 5er Skala von ,stimme voll zu“ (=5) bis „stimme gar nicht zu“(=1) gemessen. 
richtung entspricht hierbei dem angenommenen positiven Zusammenhang, auf Basis dessen sich eine positive Einstellung ggü. Werbung bei den Reagierern als positiver Einflussfaktor auf das Reaktionsverhalten abzeichnet. Hinsichtlich der Einstellung ggü. einzelnen Trägermedien erweist sich vor allem die Einstellung ggü. Mailings als relevant, unabhängig davon, welches Trägermedium die Rezipienten im Rahmen des Experiments erhalten haben. Die analysierten $\mathrm{Zu}$ sammenhänge beziehen sich sowohl auf die Einstellung ggü. Mailings im Allgemeinen als auch auf die Einstellung ggü. dem Einsatz von Mailings im Rahmen der Marketing-Aktivitäten des betrachteten Unternehmens. ${ }^{515}$ Auffällig erscheint, dass die allgemeinen Einstellungen gegenüber den Instrumenten Mailing, Email und SMS generell schlechter ausfallen, als im Fall des Einsatzes durch das betrachtete Unternehmen. ${ }^{516}$ Neben den Einstellungen der Rezipienten ggü. Mailings sind zudem die Einstellungen ggü. SMS relevant. Allerdings ergibt sich zwischen der Beurteilung und dem grundsätzlichen Reaktionsverhalten nur dann ein signifikanter Zusammenhang, wenn die SMS vom Unternehmen als Kommunikationskanal eingesetzt werden. Zwischen den Einstellungen ggü. den verschiedenen Instrumenten und der Buchungswahrscheinlichkeit der Interessenten kann allerdings kein Zusammenhang hergestellt werden. Ein Zusammenhang zeigt sich vielmehr im Vergleich der Gruppe der Nicht-Reagierer und der Gruppe der Bucher.

Während sich die bisher betrachteten Einstellungen auf die eingesetzten Trägermedien bezogen, erscheint auch eine Analyse der Einstellungen ggü. dem Responsemedium geboten. Vor dem Hintergrund der Tatsache, dass mehr als $90 \%$ der Buchungen für gewöhnlich über die Unternehmenshomepage erfolgen, stellt sich die Frage nach einer vergleichenden Beurteilung des Responsekanals „Call-Center" ggü. dem bisherigen Responsekanal „Internet“" ${ }^{517}$ Im Rahmen der

515 In diesem Kontext wurde zwischen zwei Arten von Einstellungen ggü. Mailings unterschieden: zum einen wurden die Teilnehmer der Ex-ante Befragung um ihre Einstellung ggü. Mailings im Allgemeinen gebeten. Zum anderen wurden die Teilnehmer in der Ex-post-Befragung befragt, wie sie den Einsatz der unterschiedlichen Direktmarketing-Instrumente im Rahmen der Marketing-Aktivitäten des betrachteten Unternehmens beurteilten. Bei beiden Beurteilungen wurden die Einstellungen wiederum auf einer 5er Skala gemessen. Vgl. die beiden Fragebögen im Anhang. Ein höherer Wert sprach demnach für eine entsprechend positivere Beurteilung.

516 Vor dem Hintergrund der gemessenen hohen Sympathiewerte für das betrachtete Unternehmen (Der Aussage „Airline X finde ich sympathisch“ stimmten mehr als $80 \%$ der Befragten zu) sowie der hohen Zufriedenheit der befragten Kundengruppen hinsichtlich des Parameters Preis-/Leistungsverhältnis erscheinen diese Werte angemessen.

517 Der Begriff des Responsekanals gestaltet sich im Zusammenhang mit dem PullMedium Internet grundsätzlich kritisch. Die Verwendung des Begriffs „Responseka- 
Analyse konnte nämlich ein signifikanter Zusammenhang zwischen der Beurteilung des Call-Centers ggü. der Homepage und sämtlichen Verhaltensreaktionen aufgezeigt werden. Ausgangspunkt der Überlegungen bildete in diesem Kontext die Annahme, dass je positiver die Beurteilung des Call-Centers als Responsemedium im Vergleich zum Internet ist, desto höher die Wahrscheinlichkeit einer Reaktion bzw. einer Buchung eben über dieses Medium. ${ }^{518}$

Als wichtiger Bestandteil der psychographischen Rezipientenmerkmale erfolgte zudem eine Analyse des Zusammenhangs der Zufriedenheit der Rezipienten mit den Unternehmensleistungen sowie der Beurteilung des Unternehmens hinsichtlich der Erfüllung verschiedener Eigenschaften und dem Verhalten. Während hinsichtlich der Globalzufriedenheit bei sämtlichen der drei Analysen kein signifikanter Zusammenhang aufgezeigt werden konnte, bestehen zumindest zwischen einzelnen (Teil-)Zufriedenheiten und dem Rezipientenverhalten signifikante Zusammenhänge. Für die grundlegende Frage nach der Reaktion (NichtReagierer vs. Reagierer) erweist sich bspw. die Zufriedenheit mit dem Informationsmanagement und der Buchungsabwicklung sowie die Zufriedenheit mit Speisen und Getränken an Bord der Flugzeuge als relevant. ${ }^{519}$ Jedoch bezieht sich dies nur auf die Zufriedenheit mit der Buchungsabwicklung und dem Verhalten zwischen Nicht-Reagierern und Buchern, während die übrigen Zufriedenheitswerte keinen Einfluss besitzen. Für die Buchungswahrscheinlichkeit der Interessenten erscheint allerdings die Zufriedenheit mit dem Service bzw. den Mitarbeitern sowie mit Speisen und Getränken mitverantwortlich.

Hinsichtlich der Analyse des Zusammenhangs zwischen der Beurteilung des Unternehmens in Bezug auf die Eigenschaften ,preiswert“, „sympathisch“ und „vertrauenserweckend“ lassen sich - mit Ausnahme der ersten Eigenschaft -

nal“" soll vielmehr aufzeigen, dass über diesen Kommunikationskanal der Großteil der Rezipientenreaktion auf spezifische Marketing-Aktivitäten des Unternehmens über dieses Medium abgewickelt wird. Hierbei werden Preis- und Verfügbarkeitsanfragen im Internet für gewöhnlich durch den Kunden selbst durchgeführt. Nur in wenigen Fällen wird von Seiten des Kunden die Buchungshotline in Anspruch genommen.

518 Die Frage nach der Beurteilung des Call-Centers im Vergleich zum Internet erfolgte auf Basis einer 5er Skala. So wurden die Teilnehmer an der Ex-post-Befragung gebeten, den Responsekanal „Call-Center" im Vergleich zum bisherigen Buchungsmedium zu beurteilen, wobei ein Wert von „3“ für eine indifferente Beurteilung, Werte von ,4“ bzw. „5“ für eine positivere Beurteilung des Call-Centers standen.

519 Die Teilnehmer der Befragung wurden gebeten, die Zufriedenheit mit den Leistungen des Unternehmens zu beurteilen sowie ihre Zufriedenheit mit einzelnen Leistungskomponenten zu dokumentieren. Auf einer 5er Skala konnten Werte zwischen „sehr zufrieden“ ( $=5)$ und ,gar nicht zufrieden“ ( $(=1)$ angegeben werden. 


\begin{tabular}{|c|c|c|c|}
\hline & $\begin{array}{c}\text { Analyse 1 } \\
\text { Nicht-Reagierer vs. } \\
\text { Reagierer" }\end{array}$ & $\begin{array}{c}\text { Analyse } 2 \\
\text { Interessenten vs. } \\
\text { Bucher }\end{array}$ & $\begin{array}{c}\text { Analyse 3 } \\
\text { Nicht-Reagierer vs. } \\
\text { Bucher }\end{array}$ \\
\hline \multicolumn{4}{|c|}{ Psychographische Kriterien } \\
\hline \multicolumn{4}{|c|}{ Einstellungen } \\
\hline $\begin{array}{l}\text { Einstellung ggu. Wer- } \\
\text { bung („Werbung ist } \\
\text { storend“) }\end{array}$ & $\begin{array}{c}\text { signifikant ** } \\
3,42(\mathrm{NR}) \text { vs. } 3,59(\mathrm{R})\end{array}$ & $\begin{array}{l}\text { n.s. } \\
3,64 \text { (I) vs. } 3,56 \text { (B) }\end{array}$ & $\begin{array}{c}\text { signifikant }{ }^{* \star} \\
3,42 \text { (NR) vs. } 3,56 \text { auf } \\
\text { 5er Skala } \\
\end{array}$ \\
\hline $\begin{array}{l}\text { Einstellung gga. Wer- } \\
\text { bung („Es gibt zuviel } \\
\text { Werbung“) }\end{array}$ & $\begin{array}{c}\text { n.s. } \\
3,98 \text { (NR) vs. } 4,11 \text { (R) }\end{array}$ & $\begin{array}{c}\text { n.s. } \\
4,00 \text { (I) vs. } 4,16 \text { (B) }\end{array}$ & $\begin{array}{c}\text { signifikant ** } \\
3,98 \text { (NR) vs. } 4,16 \text { (B) }\end{array}$ \\
\hline $\begin{array}{l}\text { ggu. Wer- } \\
\text { bung dringt } \\
\text { läre ein") }\end{array}$ & $\begin{array}{c}\text { signifikant ** } \\
3,36(\mathrm{NR}) \text { vs. } 3,62(\mathrm{R})\end{array}$ & $\begin{array}{l}\text { n.s. } \\
3,77 \text { (I) vs. } 3,55 \text { (B) }\end{array}$ & $\begin{array}{c}\text { n.s. } \\
3,36 \text { (NR) vs. } 3,55 \text { (B) }\end{array}$ \\
\hline $\begin{array}{l}\text { g ggü. Post- } \\
\text { ungen im All- }\end{array}$ & $\begin{array}{c}\text { signifikant ** } \\
3,57(\mathrm{NR}) \text { vs. } 3,75(\mathrm{R})\end{array}$ & $\begin{array}{l}\text { n.s. } \\
3,74 \text { (I) vs. } 3,75 \text { (B) }\end{array}$ & $\begin{array}{c}\text { signifikant ** } \\
3,57 \text { (NR) vs. } 3,75 \text { (B) }\end{array}$ \\
\hline $\begin{array}{l}\text { Einstellung ggu. Wer- } \\
\text { be-SMS im Allgemei- } \\
\text { nen }\end{array}$ & $\begin{array}{c}\text { n.s. } \\
4,14 \text { (NR) vs. } 4,14 \text { (R) }\end{array}$ & $\begin{array}{l}\text { n.s. } \\
4,14 \text { (I) vs. } 4,13 \text { (B) }\end{array}$ & $\begin{array}{c}\text { n.s. } \\
4,14 \text { (NR) vs. } 4,13 \text { (B) }\end{array}$ \\
\hline $\begin{array}{l}\text { Einstellung ggu. Wer- } \\
\text { be-Emails im Allge- } \\
\text { meinen }\end{array}$ & $\begin{array}{c}\text { n.s. } \\
3,64 \text { (NR) vs. } 3,65(\mathrm{R})\end{array}$ & $\begin{array}{c}\text { signifikant ** } \\
3,9 \text { (I) vs. } 3,54 \text { (B) }\end{array}$ & $\begin{array}{c}\text { n.s. } \\
3,64 \text { (NR) vs. } 3,54 \text { (B) }\end{array}$ \\
\hline $\begin{array}{l}\text { Mai- } \\
\text { kanal } \\
\text { ens }\end{array}$ & $\begin{array}{c}\text { signifikant * } \\
3,87(\mathrm{NR}) \text { vs. } 4,17(\mathrm{R})\end{array}$ & $\begin{array}{l}\text { n.s. } \\
4,16 \text { (I) vs. } 4,17 \text { (B) }\end{array}$ & $\begin{array}{c}\text { signifikant * } \\
3,87(\mathrm{NR}) \text { vs. } 4,17(\mathrm{~B})\end{array}$ \\
\hline $\begin{array}{l}\text { S. SMS } \\
\text { lal des }\end{array}$ & $\begin{array}{c}\text { signifikant * } \\
2,67(\mathrm{NR}) \text { vs. } 3,28(\mathrm{R})\end{array}$ & $\begin{array}{c}\text { n.s. } \\
3,32 \text { (I) vs. } 3,23 \text { (B) }\end{array}$ & $\begin{array}{c}\text { signifikant * } \\
2,67(\mathrm{NR}) \text { vs. } 3,23 \text { (B) }\end{array}$ \\
\hline $\begin{array}{l}\text { gga. } \\
\text { nal des } \\
\text { nens }\end{array}$ & $\begin{array}{c}\text { n.s. } \\
4,41 \text { (NR) vs. } 4,45 \text { (R) }\end{array}$ & $\begin{array}{l}\quad \text { n.s. } \\
4,41 \text { (I) vs. } 4,48 \text { (B) }\end{array}$ & $\begin{array}{c}\text { n.s. } \\
4,41 \text { (NR) vs. } 4,48 \text { (B) }\end{array}$ \\
\hline $\begin{array}{l}\text { Beurteilung des Call- } \\
\text { Centers im Vergleich } \\
\text { zur Homepage }\end{array}$ & $\begin{array}{c}\text { signifikant * } \\
2,29 \text { (NR) vs. } 2,93(\mathrm{R})\end{array}$ & $\begin{array}{c}\text { signifikant * } \\
2,68 \text { (I) vs. } 3,13 \text { (B) }\end{array}$ & $\begin{array}{c}\text { signifikant * } \\
2,29(\mathrm{NR}) \text { vs. } 3,13(\mathrm{~B})\end{array}$ \\
\hline \multicolumn{4}{|c|}{$\begin{array}{l}\text { a = die Gruppe der Reagierer beinhaltet die Gruppe der Interessenten und der Bucher } \\
*=\text { signifikant auf } 5 \% \text { Signifikanzniveau } \\
\text { ** = signifikant auf } 10 \% \text { Signifikanzniveau } \\
\text { n.s. = nicht signifikant } \\
\text { (NR) = durchschnittlicher Zustimmungs- bzw. Beurteilungswert der Gruppe der Nicht-Reagierer } \\
\text { (R) = durchschnittlicher Zustimmungs- bzw. Beurteilungswert der Gruppe der Reagierer } \\
\text { (I) = durchschnittlicher Zustimmungs- bzw. Beurteilungswert der Gruppe der Interessenten } \\
\text { (B) = durchschnittlicher Zustimmungs- bzw. Beurteilungswert der Gruppe der Bucher }\end{array}$} \\
\hline
\end{tabular}

Tab. 31: Zusammenhang zwischen psychographischen Merkmalen und Rezipientenverhalten 
keine signifikanten Zusammenhänge identifizieren. ${ }^{520}$ Für weitere Unterscheidungen von Rezipientengruppen ist eine Verwendung der drei Variablen nicht geeignet. Die folgende Tabelle zeigte die Ergebnisse der vorangegangenen Analyse der psychographischen Personenmerkmale (vgl. Tab. 31). ${ }^{521}$

Den dritten Schwerpunkt im Rahmen der Untersuchung der personenbezogenen Variablen bilden die veraltensorientierten Merkmale, die sich auf verschiedene Bereiche des allgemeinen Reise- und Mediennutzungsverhaltens des Rezipienten zum einen und das Informations- und Buchungsverhalten zum anderen beziehen.

Der einzige signifikante Zusammenhang im Hinblick auf die abgefragten Merkmale des Reiseverhaltens konnte zwischen der Anzahl der in den letzten sechs Monaten mit dem Unternehmen getătigten Flüge und der Reaktionsbereitschaft gemessen werden sowie zwischen dem primären Reisegrund und dem Buchungsverhalten der Anrufer im Call-Center. Weitere Merkmale des allgemeinen Reiseverhaltens wie die Größe der Reisegruppe, das Vorhandensein eines Vielfliegerstatus bzw. der Besitz einer Miles \& More-Karte sowie Flüge mit anderen Low Cost Airlines in der Vergangenheit stehen grundsätzlich in keinem signifikanten Zusammenhang zum Rezipientenverhalten. Als einziges Merkmal im Rahmen der Analyse von Interessenten und Buchern zeigte sich neben der Anzahl der Flüge mit dem besagten Unternehmen der Reisegrund als „schwach signifikant“. ${ }^{522}$ Damit erscheint auch das einzige Kriterium, welches Nicht-Reagierer und Bucher bzgl. des Reiseverhaltens voneinander trennt, in der Anzahl der Flüge mit dem besagten Unternehmen zu liegen. ${ }^{523}$

Das dem Reiseverhalten vorgelagerte Informations- und Buchungsverhalten ist ebenfalls Gegenstand der Analyse. Irrelevant fur das Rezipientenverhalten gestaltet sich auf Basis der Untersuchung demnach die Frage, ob der Rezipient für den Email-Newsletter des Unternehmens eingetragen ist oder nicht.

520 Eine Erhebung der Beurteilung des Unternehmens erfolgte über die Zustimmung (von ,stimme voll zu“ $(=5)$ bis ,stimme gar nicht zu“ $(=1)$ ) zu verschiedenen, mit der Unternehmensmarke assoziierten Eigenschaften.

521 Neben der Aussage, ob ein signifikanter Zusammenhang zwischen den exogenen Variablen und der Verhaltensreaktion besteht, werden die durchschnittlichen Beurteilungswerte der Befragung dargestellt. Die Werte beziehen sich auf die bereits erwähnte 5er Skala, wobei hohe Werte eine hohe Zustimmung signalisieren bzw. eine positive Beurteilung.

522 Als „schwach signifikant trennend“ werden Zusammenhänge zwischen einer unabhängigen und einer abhängigen Variablen bezeichnet, die auf einem Niveau von $10 \%$ signifikant sind.

523 Während Nicht-Reagierer in den letzten sechs Monaten im Durchschnitt 2,9 Flüge mit dem betrachteten Unternehmen getätigt haben, liegt dieser Wert bei den Buchern bei durchschnittlich 2,6 Flügen. 
Dieser Zusammenhang zeigte sich auch für die Trennung zwischen den beiden weiteren Unterscheidungen Interessenten vs. Bucher sowie Nicht-Reagierer vs. Bucher als relevant. Hingegen erscheint für das gemessene Verhalten der Reagierer die Frage nach dem Ort des Erstkontakts mit dem Unternehmen (über Freunde, Zeitung, Werbung etc.) von Interesse, jedoch ebenfalls lediglich für die grundsätzliche Reaktionsbereitschaft des Rezipienten. Ein Zusammenhang zwischen dem Erstkontakt sowie der Frage nach der Buchung des versendeten Angebots konnte statistisch nicht nachgewiesen werden.

Im Rahmen des Buchungsverhaltens konnte jedoch der furr gewöhnlich gewählte Buchungsort (Homepage, Call-Center, Reisebüro, Flughafen etc.) als signifikant trennend zwischen sämtlichen Reaktionsgruppen ermittelt werden. In diesem Zusammenhang zeichnete sich ab, dass insbesondere andere, vom Internet verschiedene Buchungskanäle, wie bspw. das unternehmenseigene Call-Center sowie externe Reisebüros, häufiger von Interessenten und Buchern genutzt und Anfragen bzgl. Verfügbarkeit und Preis von Tickets abgewickelt werden. Als nicht relevant erwies sich allerdings die Frage, ob der Rezipient des Direktmarketing-Stimulus für gewöhnlich Selbstbucher ist oder eine Buchung über ein Reisebüro bzw. im Falle primär beruflicher Flüge über das Travel Management der jeweiligen Firma erfolgt.

In diesem Kontext konnten zudem potenziell für die Buchungsentscheidung relevante Einflussfaktoren detaillierter untersucht werden. Eine Analyse hinsichtlich der Relevanz zur Trennung der verschiedenen Rezipientenreaktionen ergab, dass die Bedeutung des Preises im Rahmen der Buchungsentscheidung zwar nicht dafur relevant zu sein schein, ob der Rezipient auf den DirektmarketingStimulus reagiert oder nicht. ${ }^{524}$ Trotzdem erweist sich dieser Faktor als signifikant trennend zwischen Interessenten und Buchern. ${ }^{525}$ Damit wird der endgültige Preis erst nach der Entscheidung, auf das versendete Direktmarketing-Angebot zu reagieren, relevant. Hierbei hat sich gezeigt, dass die Bedeutung des Preises generell von Buchern höher beurteilt wird als von Interessenten. Dies gilt auch für die Bedeutung einer nach der Buchung vorhandenen flexiblen Umbuchungsmöglichkeit der Flüge. Eine derartige Option wird von Buchern als signifikant wichtiger eingeschätzt als von Interessenten. Hingegen zeigte sich bzgl. der bekundeten Bedeutung von Sicherheitsaspekten während des Fluges, dass diese nur für die Unterscheidung zwischen Reagierern und Nicht-Reagierern geeignet

524 Die Bedeutung einzelner Komponenten für die Buchungsentscheidung erfolgte auf Basis von 5er-Skalen, bei denen ein Wert von 5 für ,sehr wichtig“ für die Buchungsentscheidung und ein Wert von 1 für ,sehr unwichtig“ stand.

525 Im Durchschnitt beurteilten die Interessenten den Preis für die Buchungsentscheidung mit einem Wert von 4,59 gegenüber einem durchschnittlichen Wert bei der Gruppe der Bucher von 4,74. 
erscheint, aber nicht auf anderen Stufen eine signifikante Trennung der Gruppen, d. h. das Rezipientenverhalten, ermöglicht.

Wie bereits im Rahmen der Betrachtung der Relevanz des Erhalts des EmailNewsletters für die Reaktions- bzw. Buchungswahrscheinlichkeit angedeutet wurde, scheinen regelmäßig stattfindende Direktmarketing-Aktionen die Wahrscheinlichkeit einer Reaktion auf einen weiteren Direktmarketing-Stimulus nicht explizit zu fördern. Diese Annahme wird bekräftigt durch die Analyse des $\mathrm{Zu}$ sammenhangs zwischen der Teilnahme an unternehmensbezogenen Direktmarketing-Aktionen in der Vergangenheit sowie der Reaktion auf die hier versendeten Stimuli, die sich als ebenfalls nicht signifikant trennend erweisen. ${ }^{526}$ Ebenso kann der Einfluss weiterer, das konkrete Buchungsverhalten betreffende Einflussfaktoren als nicht relevant bezeichnet werden. Dies bezieht sich zunächst auf die Frage nach der simultanen Buchung von Hin- und Rückflug, welche von über $95 \%$ der Befragten - unabhängig vom Reaktionsmuster - grundsätzlich vorgenommen wird. In diesem Kontext ist auch die Zeitdauer zwischen Buchung und Abflug sowie die Zeitdauer zwischen Hin- und Rückflug bei sämtlichen Rezipientengruppen ähnlich ausgeprägt, so dass kein signifikanter Zusammenhang zwischen diesen Variablen und der Rezipientenreaktion ersichtlich ist. Während die Zeitdauer zwischen Buchung und Abflug im Durchschnitt 4 Wochen beträgt, liegt die Zeitdauer zwischen Hin- und Rückflug i. d. R. zwischen sieben und acht Tagen.

Als weiterer Aspekt der verhaltensorientierten Merkmale wurden ausgewählte Aspekte des allgemeinen Mediennutzungsverhaltens der Rezipienten analysiert. Signifikant trennend erwies sich in diesem Kontext vor allem das persönliche Nutzungsverhalten von Emails, welches sich - unabhängig davon, über welches Trägermedium bzw. über welche Trägermedien das Direktmarketing-Angebot zugesendet wurde - unterschiedlich bei Nicht-Reagierern und Reagierern gestaltete. ${ }^{527}$ Ebenfalls zeigte sich bei diesem Kriterium ein Zusammenhang bzgl. der Buchungswahrscheinlichkeit von Interessenten. Neben der allgemeinen Mediennutzung wurde zudem das Verhalten der Rezipienten hinsichtlich Werbe-

526 Durchschnittlich waren über die Hälfte der Befragten der verschiedenen Gruppen für den

Email-Newsletter eingeschrieben.

527 Der beschriebene Zusammenhang gestaltete sich auf einem Niveau von $5 \%$ signifikant. Demnach zeichnen sich insbesondere Bucher durch ein intensives EmailNutzungsverhalten aus, während Nicht-Reagierer am wenigsten dieses Medium verwenden. 
Mailings, Werbe-Emails und Werbe-SMS untersucht. ${ }^{528}$ Zwischen Interessenten und Buchern trennt vielmehr, ob Werbe-SMS gelesen werden oder nicht, unabhängig davon, ob diese im vorliegenden Experiment als Trägermedium beim entsprechenden Rezipienten eingesetzt wurden. Darüber hinaus konnte nachgewiesen werden, dass Reagierer interessante Angebote in Form von Werbe-Emails bzw. Werbe-SMS tendenziell weniger oft an Freunde und Bekannte weiterleiten als Nicht-Reagierer. ${ }^{529}$ Die Relevanz der verschiedenen exogenen Variablen ist zusammenfassend in der folgenden Tabelle dargestellt (vgl. Tab. 32). ${ }^{530}$

\begin{tabular}{|c|c|c|c|}
\hline & $\begin{array}{c}\text { Analyse } 1 \\
\text { Nicht-Reagierer vs. } \\
\text { Reagierer" }\end{array}$ & $\begin{array}{c}\text { Analyse } 2 \\
\text { Interessenten vs. } \\
\text { Bucher }\end{array}$ & \begin{tabular}{l}
\multicolumn{1}{c}{ Analyse 3} \\
Nicht-Reagierer vs. \\
Bucher
\end{tabular} \\
\hline \multicolumn{4}{|c|}{ Verhaltensorientierte Merkmale } \\
\hline \multicolumn{4}{|c|}{ Informationsverhalten } \\
\hline $\begin{array}{l}\text { Erstkontakt mit dem Un- } \\
\text { ternehmen }\end{array}$ & signifikant * & n.s. & n.s. \\
\hline $\begin{array}{l}\text { Erhalt des Email- } \\
\text { Newsletters }\end{array}$ & $\begin{array}{c}\text { n.s. } \\
55,0 \%(\mathrm{NR}) \text { vs. } \\
58,5 \%(\mathrm{R}) \\
\end{array}$ & $\begin{array}{c}\text { n.s. } \\
57,3 \% \text { (I) } 59,1 \% \text { (B) }\end{array}$ & $\begin{array}{l}\text { n.s. } \\
55,0 \% \text { (NR) } 59,1 \% \\
\text { (B) }\end{array}$ \\
\hline \multicolumn{4}{|l|}{ Buchungsverhalten } \\
\hline Buchungsort & signifikant ** & signifikant * & signifikant * \\
\hline Selbstbucher & $\begin{array}{c}\text { n.s. } \\
\begin{array}{c}90,9 \% \text { (NR) vs. } 91,8 \\
\%(R)\end{array} \\
\end{array}$ & $\begin{array}{l}\text { n.s. } \\
95,1 \% \text { (I) vs. } 90,1 \% \\
\text { (B) }\end{array}$ & $\begin{array}{c}\text { n.s. } \\
90,9 \% \text { (NR) vs. } \\
90,1 \% \text { (B) } \\
\end{array}$ \\
\hline $\begin{array}{l}\text { Bedeutung des Preises } \\
\text { für die Buchung }\end{array}$ & $\begin{array}{c}\text { n.s. } \\
4,71 \text { (NR) vs. } 4,71(\mathrm{R})\end{array}$ & $\begin{array}{c}\text { signifikant * } \\
4,59 \text { (I) vs. } 4,76 \text { (B) }\end{array}$ & $\begin{array}{c}\text { n.s. } \\
4,71 \text { (NR) vs. } 4,71 \\
\text { (B) }\end{array}$ \\
\hline $\begin{array}{l}\text { Bedeutung der Sicher- } \\
\text { heit des Fluges }\end{array}$ & $\begin{array}{c}\text { signifikant }{ }^{\star \star} \\
4,60(\mathrm{NR}) \text { vs. } 4,70(\mathrm{R})\end{array}$ & $\begin{array}{l}\text { n.s. } \\
4,63 \text { (I) vs. } 4,70 \text { (B) }\end{array}$ & $\begin{array}{l}\text { signifikant }{ }^{*} \\
4,60(\mathrm{NR}) \text { vs. } 4,70 \\
\text { (B) }\end{array}$ \\
\hline
\end{tabular}

528 In diesem Zusammenhang wurde wiederum auf einer 5er Skala die Zustimmung oder Ablehnung der Rezipienten zu verschiedenen, die einzelnen Direktmarketing-Medien betreffende Aussagen gemessen.

529 Ebenfalls auf einer 5er Skala wurde abgefragt, mit welcher Frequenz verschiedene Medien genutzt werden bzw. ob Werbe-Emails bzw. Werbe-SMS an Freunde oder Bekannte weitergeleitet werden. Hierbei konnten grundsätzlich Werte zwischen „sehr häufig“ $(=5)$ und ,nie" $(=1)$ angegeben werden.

530 Die Tabelle enthält neben dem Hinweis auf einen signifikanten bzw. nichtsignifikanten Zusammenhang für die metrisch-skalierten Daten den Mittelwert der jeweiligen Gruppe, bspw. für die Gruppe der Nicht-Reagierer (NR). Im Falle kategorial-ausgeprägter Variablen wurde bei dichotomen Ausprägungen der Anteil der jeweils zutreffenden Antwort im Hinblick auf die einzelnen Gruppen dargestellt. Ein Wert von $55 \%$ (NR) bedeutet bspw. hinsichtlich der Frage nach dem EmailNewsletter, dass $55 \%$ der Nicht-Reagierer für den Email-Newsletter des Unternehmens eingeschrieben sind. Für die nicht-dichotomen Variablen wurde auf eine differenzierte Betrachtung verzichtet. 


\begin{tabular}{|c|c|c|c|}
\hline & $\begin{array}{c}\text { Analyse 1 } \\
\text { Nicht-Reagierer vs. } \\
\text { Reagierer } \\
\end{array}$ & $\begin{array}{c}\text { Analyse } 2 \\
\text { Interessenten vs. } \\
\text { Bucher } \\
\end{array}$ & \begin{tabular}{l}
\multicolumn{1}{c}{ Analyse 3 } \\
Nicht-Reagierer vs. \\
Bucher \\
\end{tabular} \\
\hline \multicolumn{4}{|l|}{ Buchungsverhalten } \\
\hline $\begin{array}{l}\text { Bedeutung einer flexib- } \\
\text { len Umbuchungsmog- } \\
\text { lichkeit }\end{array}$ & $\begin{array}{c}\text { n.s. } \\
3,83(\mathrm{NR}) \text { vs. } 3,86(\mathrm{R})\end{array}$ & $\begin{array}{c}\text { signifikant * } \\
4,00 \text { (I) vs. } 3,79 \text { (B) }\end{array}$ & $\begin{array}{c}\text { n.s. } \\
3,83 \text { (NR) vs. } 3,79 \\
\text { (B) }\end{array}$ \\
\hline $\begin{array}{l}\text { Teilnahme an Sonder- } \\
\text { aktionen des Unterneh- } \\
\text { mens }\end{array}$ & $\begin{array}{c}\text { n.s. } \\
27,3 \% \text { (NR) vs. } \\
30,5 \%(\mathrm{R}) \\
\end{array}$ & $\begin{array}{l}\text { n.s. } \\
28,8 \% \text { (I) vs. } 31,4 \% \\
\text { (B) }\end{array}$ & $\begin{array}{c}\text { n.s. } \\
27,3 \% \text { (NR) vs. } \\
31,4 \%(\mathrm{~B}) \\
\end{array}$ \\
\hline $\begin{array}{l}\text { Gleichzeitige Buchung } \\
\text { von Hin- und Ruckflug }\end{array}$ & $\begin{array}{c}\text { n.s. } \\
95,6 \%(\mathrm{NR}) \text { vs. } \\
97,0 \%(\mathrm{R}) \\
\end{array}$ & $\begin{array}{c}\text { n.s. } \\
96,1 \% \text { (I) vs. } 97,5 \% \\
\text { (B) }\end{array}$ & $\begin{array}{c}\text { n.s. } \\
95,6 \% \text { (NR) vs. } \\
97,5 \% \text { (B) } \\
\end{array}$ \\
\hline $\begin{array}{l}\text { Zeitdauer zwischen } \\
\text { Buchung und Abflug }\end{array}$ & $\begin{array}{c}\text { n.s. } \\
25 \text { Tage (NR) vs. } \\
30 \text { Tage (R) }\end{array}$ & \begin{tabular}{|c} 
n.s. \\
29 Tage (I) vs. 30 Tage \\
(B)
\end{tabular} & $\begin{array}{c}\text { n.s. } \\
25 \text { Tage (NR) vs. } 30 \\
\text { Tage (B) }\end{array}$ \\
\hline $\begin{array}{l}\text { Zeitdauer zwischen Hin- } \\
\text { und Ruckflug }\end{array}$ & $\begin{array}{l}\text { n.s. } \\
9 \text { Tage (NR) vs. } 7 \text { Tage } \\
\text { (R) }\end{array}$ & $\begin{array}{l}\text { n.s. } \\
7 \text { Tage (I) vs. } 6 \text { Tage } \\
\text { (B) }\end{array}$ & $\begin{array}{c}\text { n.s. } \\
9 \text { Tage (NR) vs. } \\
6 \text { Tage (B) }\end{array}$ \\
\hline \multicolumn{4}{|l|}{ Reiseverhalten } \\
\hline $\begin{array}{l}\text { Anzahl Fluge in den } \\
\text { letzten } 6 \text { Monaten }\end{array}$ & $\begin{array}{c}\text { signifikant * } \\
2,6(\mathrm{NR}) \text { vs. } 2,9(\mathrm{R})\end{array}$ & $\begin{array}{c}\text { signifikant * } \\
2,8 \text { (I) vs. } 3,0 \text { (B) }\end{array}$ & $\begin{array}{c}\text { signifikant }{ }^{* *} \\
2,6(\mathrm{NR}) \text { vs. } 3,0(\mathrm{~B})\end{array}$ \\
\hline Große der Reisegruppe & n.s. & n.s. & n.s. \\
\hline Primärer Reisegrund & & signifikant * & n.s. \\
\hline Vielflieger & $\begin{array}{c}\text { n.s. } \\
21,3 \% \text { (NR) vs. } \\
20,1 \%(\mathrm{R}) \\
\end{array}$ & $\begin{array}{c}\text { n.s. } \\
\begin{array}{c}20,2 \% \text { (I) vs. } 20,1 \% \\
\text { (B) }\end{array}\end{array}$ & $\begin{array}{c}\text { n.s. } \\
21,3 \% \text { (NR) vs. } 20,1 \\
\text { (B) }\end{array}$ \\
\hline $\begin{array}{l}\text { Besitz einer Miles \& } \\
\text { more-Karte }\end{array}$ & $\begin{array}{c}\text { n.s. } \\
31,2 \% \text { (NR) vs. } \\
30,9 \% \text { (B) } \\
\end{array}$ & $\begin{array}{c}\text { n.s. } \\
27,7 \% \text { (I) vs. } 32,5 \% \\
\text { (B) }\end{array}$ & $\begin{array}{c}\text { n.s. } \\
31,2 \%(\mathrm{NR}) \text { vs. } \\
32,5 \%(\mathrm{~B}) \\
\end{array}$ \\
\hline $\begin{array}{l}\text { Fluge mit anderen Low- } \\
\text { Cost-Airlines }\end{array}$ & $\begin{array}{c}\text { n.s. } \\
54,4 \% \text { (NR) vs. } \\
60,1 \%(\mathrm{R}) \\
\end{array}$ & $\begin{array}{l}\text { n.s. } \\
58,7 \% \text { (I) vs. } 60,9 \% \\
\text { (B) }\end{array}$ & $\begin{array}{c}\text { n.s. } \\
54,4 \% \text { (NR) vs. } 60,9 \\
\% \text { (B) }\end{array}$ \\
\hline \multicolumn{4}{|l|}{ Mediennutzung } \\
\hline $\begin{array}{l}\text { Nutzung von Emails im } \\
\text { Alltag }\end{array}$ & $\begin{array}{c}\text { signifikant }{ }^{* *} \\
4,27(\mathrm{NR}) \text { vs. } 4,14(\mathrm{R}) \\
\end{array}$ & $\begin{array}{c}\text { signifikant }{ }^{\star} \\
3,89 \text { (D) vs. } 4,27 \text { (B) }\end{array}$ & $\begin{array}{c}\text { n.s. } \\
4,27 \text { (NR) vs. } 4,27 \text { (B) }\end{array}$ \\
\hline $\begin{array}{l}\text { Nutzung von SMS im } \\
\text { Alltag }\end{array}$ & $\begin{array}{c}\text { n.s. } \\
3,52 \text { (NR) vs. } 3,50(\mathrm{R})\end{array}$ & $\begin{array}{c}\text { n.s. } \\
3,52 \text { (I) vs. } 3,50 \text { (B) }\end{array}$ & $\begin{array}{c}\text { n.s. } \\
3,52 \text { (NR) vs. } 3,50 \text { (B) }\end{array}$ \\
\hline Lese Postwurfsendungen & $\begin{array}{c}\text { signifikant }{ }^{\star \star} \\
2,26(\mathrm{NR}) \text { vs. } 2,12(\mathrm{R}) \\
\end{array}$ & $\begin{array}{c}\text { n.s. } \\
2,09 \text { (I) vs. } 2,12 \text { (B) }\end{array}$ & $\begin{array}{c}\text { n.s. } \\
2,26 \text { (NR) vs. } 2,12 \text { (B) } \\
\end{array}$ \\
\hline Lese Werbe-Emails & $\begin{array}{c}\text { n.s. } \\
2,01 \text { (NR) vs. } 1,95(\mathrm{R})\end{array}$ & $\begin{array}{c}\text { n.s. } \\
1,84 \text { (I) vs. } 2,00 \text { (B) } \\
\end{array}$ & $\begin{array}{c}\text { n.s. } \\
2,01 \text { (NR) vs. } 2,00 \text { (B) }\end{array}$ \\
\hline Lese Werbe-SMS & $\begin{array}{c}\text { n.s. } \\
1,52 \text { (NR) vs. } 1,55(\mathrm{R}) \\
\end{array}$ & $\begin{array}{c}\text { signifikant ** } \\
1,39 \text { (I) vs. } 1,63 \text { (B) }\end{array}$ & $\begin{array}{c}\text { n.s. } \\
1,52 \text { (NR) vs. } 1,63 \text { (B) }\end{array}$ \\
\hline $\begin{array}{l}\text { Leite Werbe-Emails / } \\
\text { Werbe-SMS weiter }\end{array}$ & $\begin{array}{c}\text { signifikant ** } \\
1,26(\mathrm{NR}) \text { vs. } 1,06(\mathrm{R}) \\
\end{array}$ & $\begin{array}{c}\text { n.s. } \\
1,22 \text { (I) vs. } 1,20 \text { (B) } \\
\end{array}$ & $\begin{array}{c}\text { signifikant }{ }^{*} \\
1,26(\mathrm{NR}) \text { vs. } 1,20 \text { (B) }\end{array}$ \\
\hline \multicolumn{4}{|c|}{ Erfahrungen mit dem Unternehmen / Beurteilung des Unternehmens } \\
\hline $\begin{array}{l}\text { (Global-) Zufriedenheit } \\
\text { mit dem Unternehmen }\end{array}$ & \begin{tabular}{c|} 
n.s. \\
4,33 (NR) vs. $4,34(\mathrm{R})$ \\
\end{tabular} & $\begin{array}{c}\text { n.s. } \\
4,43 \text { (I) vs. } 4,29 \text { (B) } \\
\end{array}$ & $\begin{array}{c}\text { n.s. } \\
4,33 \text { (NR) vs. } 4,29 \text { (B) }\end{array}$ \\
\hline $\begin{array}{l}\text { Zufriedenheit mit dem } \\
\text { Preis }\end{array}$ & $\begin{array}{c}\text { n.s. } \\
4,28 \text { (NR) vs. } 4,26(\mathrm{R})\end{array}$ & $\begin{array}{c}\text { n.s. } \\
4,32 \text { (I) vs. } 4,23 \text { (B) }\end{array}$ & $\begin{array}{c}\text { n.s. } \\
4,28 \text { (NR) vs. } 4,23 \text { (B) }\end{array}$ \\
\hline
\end{tabular}




\begin{tabular}{|c|c|c|c|}
\hline & $\begin{array}{c}\text { Analyse } 1 \\
\text { Nicht-Reagierer vs. } \\
\text { Reagierer }\end{array}$ & $\begin{array}{c}\text { Analyse 2 } \\
\text { Interessenten vs. } \\
\text { Bucher }\end{array}$ & \begin{tabular}{|l}
\multicolumn{1}{c}{ Analyse 3 } \\
Nicht-Reagierer vs. \\
Bucher \\
\end{tabular} \\
\hline \multicolumn{4}{|c|}{ Erfahrungen mit dem Unternehmen / Beurteilung des Unternehmens } \\
\hline $\begin{array}{l}\text { Zufriedenheit mit dem } \\
\text { Informationsmanage- } \\
\text { ment }\end{array}$ & $\begin{array}{c}\text { signifikant } \star * \\
3,64(\mathrm{NR}) \text { vs. } 3,65(\mathrm{R})\end{array}$ & $\begin{array}{c}\text { n.s. } \\
3,78 \text { (I) vs. } 3,60 \text { (B) }\end{array}$ & $\begin{array}{c}\text { n.s. } \\
3,64 \text { (NR) vs. } 3,60 \text { (B) }\end{array}$ \\
\hline & $\begin{array}{l}n+28(\mathrm{R}) \\
4,2\end{array}$ & $\begin{array}{c}\text { n.s. } \\
4,37 \text { (I) vs. } 4,23 \text { (B) }\end{array}$ & $\begin{array}{c}\text { signifikant * } \\
4,37 \text { (NR) vs. } 4,23 \text { (B) }\end{array}$ \\
\hline & $\begin{array}{c}\text { n.s. } \\
4,24 \text { (NR) vs. } 4,27 \text { (R) }\end{array}$ & $\begin{array}{c}\text { signifikant * } \\
4,46 \text { (I) vs. } 4,17 \text { (B) }\end{array}$ & $\begin{array}{c}\text { n.s. } \\
4,24 \text { (NR) vs. } 4,17 \text { (B) }\end{array}$ \\
\hline & $\begin{array}{c}\text { signifikant } \star \star \\
3,55 \text { (NR) vs. } 3,67(R)\end{array}$ & $\begin{array}{c}\text { signifikant * } \\
3,86 \text { (I) vs. } 3,58 \text { (B) }\end{array}$ & $\begin{array}{c}\text { n.s. } \\
3,55 \text { (NR) vs. } 3,58 \text { (B) }\end{array}$ \\
\hline & $\begin{array}{l}\text { ikant }{ }^{\star \star} \\
\text { vs. } 4,38(R)\end{array}$ & $\begin{array}{c}\text { n.s. } \\
4,37 \text { (I) vs. } 4,38 \text { (B) }\end{array}$ & $\begin{array}{c}\text { n.s. } \\
4,46 \text { (NR) vs. } 4,38 \text { (B) }\end{array}$ \\
\hline a- & $\begin{array}{c}\text { n.s. } \\
4,10 \text { (NR) vs. } 4,12(\mathrm{R})\end{array}$ & $\begin{array}{c}\text { n.s. } \\
4,14 \text { (I) vs. } 4,10 \text { (B) }\end{array}$ & $\begin{array}{c}\text { n.s. } \\
4,10 \text { (NR) vs. } 4,10 \text { (B) }\end{array}$ \\
\hline $\begin{array}{l}\text { Beurteilung des Unter- } \\
\text { nehmens als vertrauens- } \\
\text { erweckend }\end{array}$ & $\begin{array}{c}\text { n.s. } \\
4,04 \text { (NR) vs. } 4,12 \text { (R) }\end{array}$ & $\begin{array}{c}\text { n.s. } \\
4,12 \text { (I) vs. } 4,12 \text { (B) }\end{array}$ & $\begin{array}{c}\text { n.s. } \\
4,04 \text { (NR) vs. } 4,12 \text { (B) }\end{array}$ \\
\hline \multicolumn{4}{|c|}{$\begin{array}{l}\text { a = die Gruppe der Reagierer beinhaltet die Gruppe der Interessenten und der Bucher } \\
\text { * signifikant auf } 5 \% \text { Signifikanzniveau } \\
\text { ** = signifikant auf } 10 \% \text { Signifikanzniveau } \\
\text { n.s. = nicht signifikant } \\
\text { (NR) = durchschnittlicher Zustimmungs-, Beurteilungs- oder Anteilswert der Gruppe der Nicht-Reagierer } \\
\text { (R) = durchschnittlicher Zustimmungs-, Beurteilungs- oder Anteilswert der Gruppe der Reagierer } \\
\text { (I) = durchschnittlicher Zustimmungs-, Beurteilungs- oder Anteilswert der Gruppe der Interessenten } \\
\text { (B) = durchschnittlicher Zustimmungs-, Beurteilungs- oder Anteilswert der Gruppe der Bucher }\end{array}$} \\
\hline
\end{tabular}

Tab. 32: Zusammenhang zwischen verhaltensorientierten Merkmalen und dem Rezipientenverhalten

\subsubsection{Analyse des Einflusses situationsbezogener Determinanten}

Im Rahmen der konzeptionellen Ausführungen der vorliegenden Arbeit wurde neben den personenbezogenen Determinanten als weitere Kategorie der exogenen Einflussfaktoren der Bereich der situationsbezogenen Determinanten auf Relevanz hinsichtlich des Direktmarketing-Einsatzes analysiert. ${ }^{531}$ Als zentrale Kategorien der situationsbezogenen Determinanten zeigten sich vor allem vier Bereiche: Störeinflüsse während der Botschaftsaufnahme durch andere Personen, andere Medien, Lärm etc., die zeitlichen Umstände der Botschaftsaufnahme, der physische Ort des Werbekontaktes sowie die situationsbedingte subjektive Ent-

531 Vgl. Steffenhagen, H., Wirkungen der Werbung, a. a. O., S. 13; Dallmer, H., Erfolgsbedingungen der Kommunikation im Direct-Marketing, a. a. O., S. 73. 
scheidungssituation des Rezipienten. ${ }^{532}$ Aufgrund der aufgezeigten Erhebungsproblematik des situativen Umfeldes während des Stimulus-Kontaktes kann eine Analyse des potenziellen Einflussniveaus einzelner situationsbezogener Determinanten i. d. R. nur durch eine nachträgliche Befragung der Rezipienten erfolgen. ${ }^{533}$ Aus diesem Grund wurde eine direkte Befragung der Rezipienten im Anschluss an die Direktmarketing-Aktionen vorgenommen. ${ }^{534}$

Bereits im Rahmen der konzeptionellen Analyse des Einflusses der situationsbezogenen Determinanten konnte aufgezeigt werden, dass das Reaktionsverhalten des Rezipienten grundsätzlich mit dessen Bedarf (an Flügen) zum Zeitpunkt des Kommunikationskontaktes zusammenhängt. Dieser Zusammenhang konnte auch in der vorliegenden Untersuchung zwischen der mit Hilfe der Expost-Befragung erhobenen rezipientenindividuellen Bedarfssituation und dem Rezipientenverhalten nachgewiesen werden. Ebenso zeigte sich, dass die situativen Umstände während des Kommunikationskontaktes mit dem Direktmarketing-Stimulus ebenfalls einen signifikanten Zusammenhang zum Rezipientenverhalten aufweisen. ${ }^{535}$ Je günstiger die Beurteilung des situativen Umfelds durch den Rezipienten, desto wahrscheinlicher eine Reaktion bzw. eine Buchung.

In diesem Kontext erwies es sich allerdings als irrelevant, ob und ggf. welche Tätigkeit während des Kommunikationskontaktes vom Rezipienten ausgeführt wurde. Es besteht daher zwischen dem Verhalten und der ausgeführten Tätigkeit kein signifikanter Zusammenhang. Die folgende Abbildung zeigt die Ergebnisse der Analyse des Zusammenhangs situationsbezogener Determinanten und dem Rezipientenverhalten (vgl. Tab. 33).

532 Vgl. Belk, R. W., Situational Variables and Consumer Behavior, in: Journal of Consumer Research, Vol. 2, 1975, S. 159. Zur deutschen Übersetzung vgl. Mühlbacher, H., Ein situatives Modell der Motivation zur Informationsaufnahme und verarbeitung bei Werbekontakten, a. a. O., S. $85 \mathrm{f}$.

533 Vgl. Lasslop, I., Effektivität und Effizienz von Events, a. a. O., S. 11, S. 59.

534 Diese Ex-post-Befragung fand drei Monate nach der Versendung der verschiedenen Direktmarketing-Aktionen statt. Umso erstaunlicher ist der hohe Rücklauf von insgesamt knapp 800 Fragebögen.

535 Die Abfrage der situativen Umstände erfolgte auf Basis einer Selbsteinschätzung der Befragten, die ausweisen sollten, wie günstig sie den Zeitpunkt des Erhalts des Direktmarketing-Stimulus insgesamt beurteilten. Die Rezipienten wurden gebeten, ihre Beurteilung Auf einer 5er-Skale zu dokumentieren, wobei der Wert 5 für ,sehr günstig“ bzw. der Wert 1 für ,sehr ungünstig“ standen. 


\begin{tabular}{|c|c|c|c|}
\hline & $\begin{array}{c}\text { Analyse 1 } \\
\text { Nicht-Reagierer vs. } \\
\text { Reagierer }\end{array}$ & $\begin{array}{c}\text { Analyse } 2 \\
\text { Interessenten vs. Bu- } \\
\text { cher }\end{array}$ & $\begin{array}{c}\text { Analyse } 3 \\
\text { Nicht-Reagierer vs. } \\
\text { Bucher }\end{array}$ \\
\hline \multicolumn{4}{|c|}{ Situationsbezogene Determinanten } \\
\hline $\begin{array}{l}\text { Bedarf zum Kontakt- } \\
\text { zeitpunkt }\end{array}$ & $\begin{array}{c}\text { signifikant * } \\
35,9 \%(\mathrm{NR}) \text { vs. } \\
53,6 \%(\mathrm{R}) \\
\end{array}$ & $\begin{array}{c}\text { n.s. } \\
49,4 \% \text { (I) vs. } 57,7 \% \\
\text { (B) }\end{array}$ & $\begin{array}{c}\text { signifikant * } \\
35,9 \%(\mathrm{NR}) \text { vs. } \\
57,7 \%(\mathrm{~B})\end{array}$ \\
\hline $\begin{array}{l}\text { Tatigkeit wahrend des } \\
\text { Erhaltes / Betrachtung } \\
\text { des Direktmarketing- } \\
\text { Stimulus ausgeführt }\end{array}$ & $\begin{array}{c}\text { n.s. } \\
39,1 \% \text { (NR) vs. } \\
39,6 \% \text { (R) }\end{array}$ & $\begin{array}{l}\text { n.s. } \\
40,4 \% \text { (I) vs. } 37,5 \% \\
\text { (B) }\end{array}$ & $\begin{array}{c}\text { n.s. } \\
39,1 \% \text { (NR) vs. } \\
37,5 \% \text { (B) }\end{array}$ \\
\hline $\begin{array}{l}\text { Art der ausgefuhrten } \\
\text { Tatigkeit }\end{array}$ & n.s. & n.s. & n.s. \\
\hline $\begin{array}{l}\text { Personliche Umstănde } \\
\text { wăhrend des Erhaltes / } \\
\text { Betrachtung des } \\
\text { Direktmarketing- } \\
\text { Stimulus }\end{array}$ & 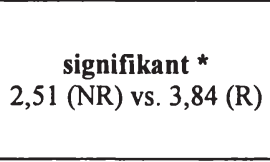 & $\begin{array}{c}\text { signifikant * } \\
3,08 \text { (I) vs. } 4,44 \text { (B) }\end{array}$ & $\begin{array}{c}\text { signifikant * } \\
2,51(\mathrm{NR}) \text { vs. } 4,44(\mathrm{~B})\end{array}$ \\
\hline \multicolumn{4}{|c|}{$\begin{array}{l}\text { a = die Gruppe der Reagierer beinhaltet die Gruppe der Interessenten und der Bucher } \\
\text { * = signifikant auf } 5 \% \text { Signifikanzniveau } \\
\text { ** = signifikant auf } 10 \% \text { Signifikanzniveau } \\
\text { n.s. = nicht signifikant } \\
\text { (NR) = durchschnittlicher Zustimmungs-, Beurteilungs- oder Anteilswert der Gruppe der Nicht-Reagierer } \\
\text { (R) = durchschnittlicher Zustimmungs-, Beurteilungs- oder Anteilswert der Gruppe der Reagierer } \\
\text { (I) = durchschnittlicher Zustimmungs-, Beurteilungs- oder Anteilswert der Gruppe der Interessenten } \\
\text { (B) = durchschnittlicher Zustimmungs-, Beurteilungs- oder Anteilswert der Gruppe der Bucher }\end{array}$} \\
\hline
\end{tabular}

Tab. 33: Analyse des Zusammenhangs zwischen situationsbezogenen Determinanten und Rezipientenverhalten

\subsubsection{Synoptische Darstellung der Ergebnisse der Analyse ausgewählter exoge- ner Determinanten}

Insgesamt ermöglichte das hier verwendete dreistufige Vorgehen hinsichtlich der Analyse verschiedener personen- und situationsbezogener Einflussfaktoren einen grundlegenden Überblick über potenzielle exogene Einflussfaktoren des Response- und Buchungsverhaltens. Es zeigte sich, dass einige der betrachteten Variablen für sämtliche Verhaltensstufen relevant sind, während dies bei anderen Variablen nur auf eine oder zwei Ausprägungen der abhängigen Variablen zutrifft. In der folgenden Abbildung werden die Ergebnisse der Analyse zusammenfassend dargestellt (vgl. Abb. 22). Demnach zeigt sich, dass bspw. das Alter, das Geschlecht, die Beurteilung des Rückkanalmediums, der gewöhnliche Buchungsort, die Bedarfssituation sowie die situativen Umstände während des Stimulus-Kontaktes in Zusammenhang mit sämtlichen der drei dichotomen Ausprägungen der abhängigen Variablen von Relevanz sind. 


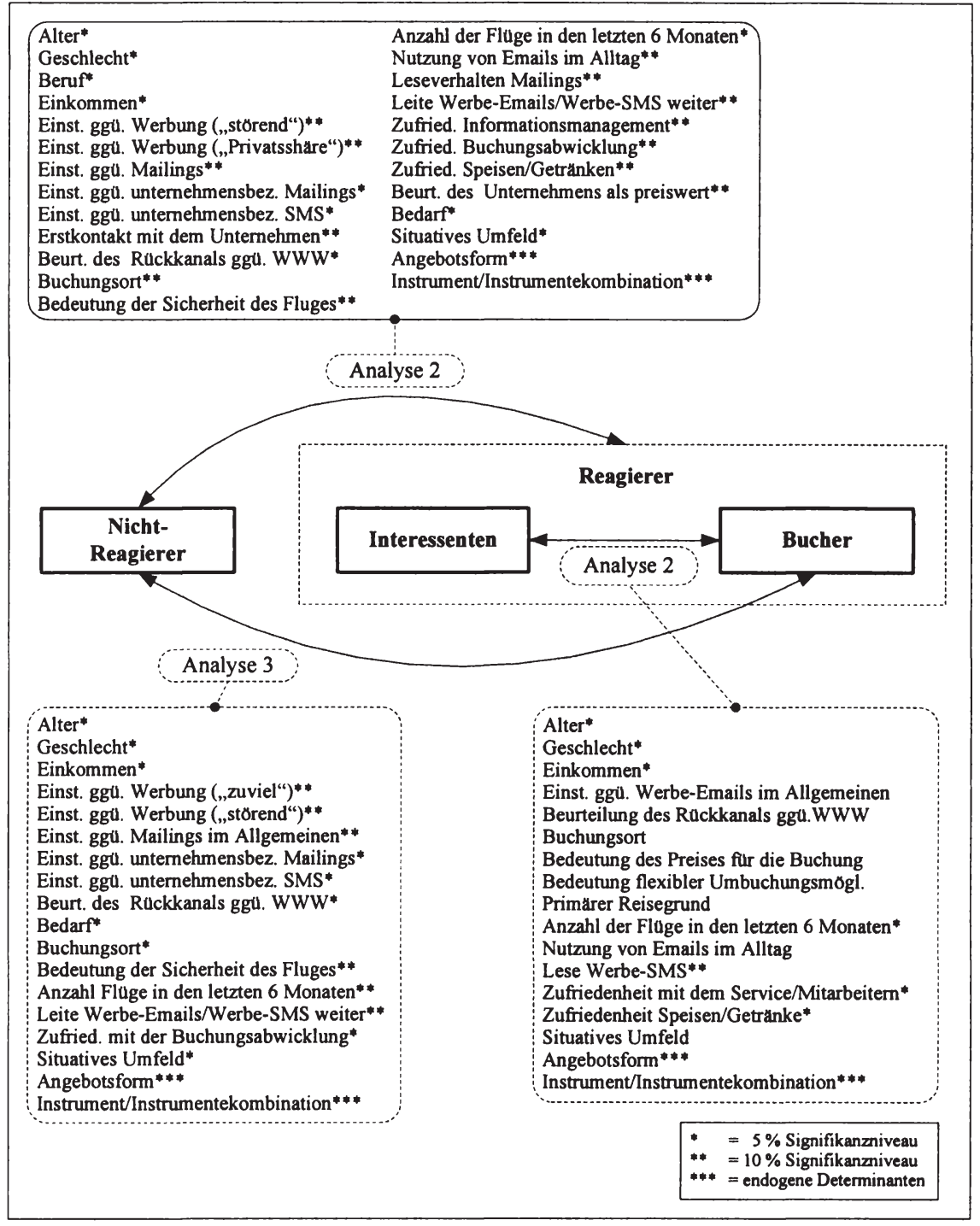

Abb. 22: Synoptische Darstellung der Analyse des Einflusses ausgewählter exogener Determinanten auf das Rezipientenverhalten 


\subsection{Integrative Analyse des Einflusses ausgewählter endogener und exogener Determinanten auf das Rezipientenverhalten}

Eine umfassende Analyse der relativen Bedeutung der endogenen und exogenen Determinanten im Verhältnis zueinander ist auf Basis der bisher verwendeten Analysemethoden nicht möglich. Aus diesem Grund wird zur weiteren Analyse das Verfahren der Logistischen Regression eingesetzt, das die Einflussrichtung und -stärke sowohl metrisch als auch kategorial ausgeprägter unabhängiger Variablen im Zusammenspiel hinsichtlich einer abhängigen Variable untersucht. Das im vorangegangenen Kapitel durchgeführte dreistufige Vorgehen konnte in diesem Zusammenhang erste Anhaltspunkte für die weitere Analyse aufzeigen und neben den endogenen Faktoren mehrere personen- und situationsbezogene Determinanten aufzeigen, die in Zusammenhang mit den beobachteten Verhaltensreaktionen des Rezipienten stehen. So wurde auf der ersten Stufe untersucht, ob einer der ausgewählten Faktoren das Eintreten einer Reaktion auf den versendeten Direktmarketing-Stimulus beeinflusst oder nicht. In einem zweiten Schritt wurde analysiert, ob die betrachtete Variable für das Buchungsverhalten verantwortlich ist. Hinter dieser Fragestellung steht die Motivation an einer Identifikation der zentralen Treiber, die Personen, die ein grundlegendes Interesse an einem Angebot bekundet haben, zum Kauf desselben zu bewegen. Der letzte Untersuchungsschritt bezog sich auf den Vergleich von Nicht-Reagierern und Buchern und die Analyse der diskriminierenden Wirkung der einzelnen Faktoren. Erkenntnisse in diesen Bereichen erscheinen insbesondere aus Unternehmenssicht für die Steigerung der Effektivität ihrer Verkaufsbemühungen mit Hilfe des Direktmarketing relevant.

Vor dem Hintergrund dieser drei differenzierten Analysen wird das Verfahren der Logistischen Regression eingesetzt, um die Stärke des Einflusses derjenigen Determinanten in Relation zueinander zu berechnen, die für die Beeinflussung des Übergangs von einer Verhaltensstufe zur nächsten von signifikanter Bedeutung sind. ${ }^{536}$ Neben den identifizierten exogenen Determinanten sollen auch endogene Determinanten als unabhängige Variablen eingesetzt werden. Während die Auswahl der exogenen Determinanten aufgrund der im vorangegangenen Kapitel durchgefuhrten Signifikanztests erfolgte, wurde die Relevanz der endogenen Determinanten „Angebotsform“ und „Instrument/Instrumentekombination“ auf

536 In diesem Kontext werden nur Variablen zugelassen, die sich auf einem Niveau von $5 \%$ als signifikant erwiesen. Gleichwohl wurden die drei Modelle auf Basis der Logsitischen Regression unter Einschluss sämtlicher, d. h. auch schwach signifikanter, Variablen durchgefuhrt, was allerdings zu einer, bereits im Vorfeld angenommenen schlechterten Modellgüte führte. Gleichzeitig war eine Anwendung der Logistischen Regression aufgrund zu geringer Fallzahlen z. T. nicht mehr möglich. 
Basis der stark differierenden Ergebnisse der Effektivitätsanalyse abgeleitet. Eine Untersuchung der endogenen Determinanten „Zielgruppe“ war wegen einer zu geringen Stichprobe der Neukunden nicht möglich. ${ }^{537}$

\subsubsection{Vergleich von Nicht-Reagierern und Reagierern}

Die vorangegangenen Ausführungen haben gezeigt, dass weder einzelne endogene Determinanten, wie bspw. die gewählte Absatzform, noch einzelne exogene Determinanten das Verhalten des Rezipienten als alleiniger Faktor determinieren. Vielmehr kann angenommen werden, dass vor allem das Zusammenspiel verschiedener endogener und exogener Determinanten die Wahrscheinlichkeit einer Rezipientenreaktion erhöht. In der folgenden Untersuchung wurde das Verfahren der Logistischen Regression verwendet, um den Einfluss verschiedener unabhängiger endogener und exogener Variablen auf die abhängige Variable mit den zwei Ausprägungen „keine Reaktion“ und „Anruf im Call-Center“ zu untersuchen. Entsprechend der vorliegenden Ergebnisse wurden 11 der exogenen Determinanten sowie die zwei endogene Determinanten in die Analyse miteinbezogen (vgl. Abb. 23).

Von den insgesamt 798 Beobachtungen konnten 482 Fälle für die Analyse auf Basis der Logistischen Regression verwendet werden. ${ }^{538} 290$ der ausgewählten Fälle $(60,2 \%)$ sind den sog. Nicht-Reagierern zuzuordnen, während 192 Fälle $(39,8 \%)$ der Gruppe der Reagierer angehören. In diesem Kontext wurden sämtliche Beobachtungen von der weiteren Betrachtung ausgeschlossen, die fehlende Werte bei einer der 13 unabhängigen Variablen aufwiesen. Mittels dieses Vorgehens wurden letztendlich 316 Fälle ausgeschlossen, so dass sich die Anzahl der Beobachtungen um ca. $40 \%$ der Fälle reduzierte. Als notwendige Voraussetzung zur Durchführung einer Logistischen Regression erfolgte eine Analyse der unabhängigen Variablen auf Multikolliniearität. In diesem Kontext wurden kei-

537 Zum notwendigen Stichprobenumfang bei der Logistischen Regression vgl. Backhaus et al., Multivariate Analysemethoden. Eine anwendungsorientierte Einfuhrung, 9. Aufl., Berlin u a. 2000, S. 137 ff. Als Mindestwert der Fallzahl pro Gruppe, d. h. pro Ausprägung der unabhängigen Variablen, gilt ein Wert von 25 Beobachtungen.

538 Voraussetzung für die Einbeziehung einer Beobachtung in die Analyse ist das Vorliegen eines Fragebogens sowohl aus der Ex-post-Befragung als auch aus der Exante-Befragung, da aus beiden Fragebögen Angaben benötigt werden. Die Zahl von 798 Beobachtungen ergibt sich durch die Anzahl von Personen, die beide Fragebögen ausgefüllt haben. Die entsprechende Reduzierung auf 482 Fälle ergibt sich durch den Ausschluss von Beobachtungen mit fehlenden Werten. 
ne Korrelationen festgestellt. ${ }^{539}$ Auf eine detaillierte Darstellung der Ergebnisse soll an dieser Stelle verzichtet werden.

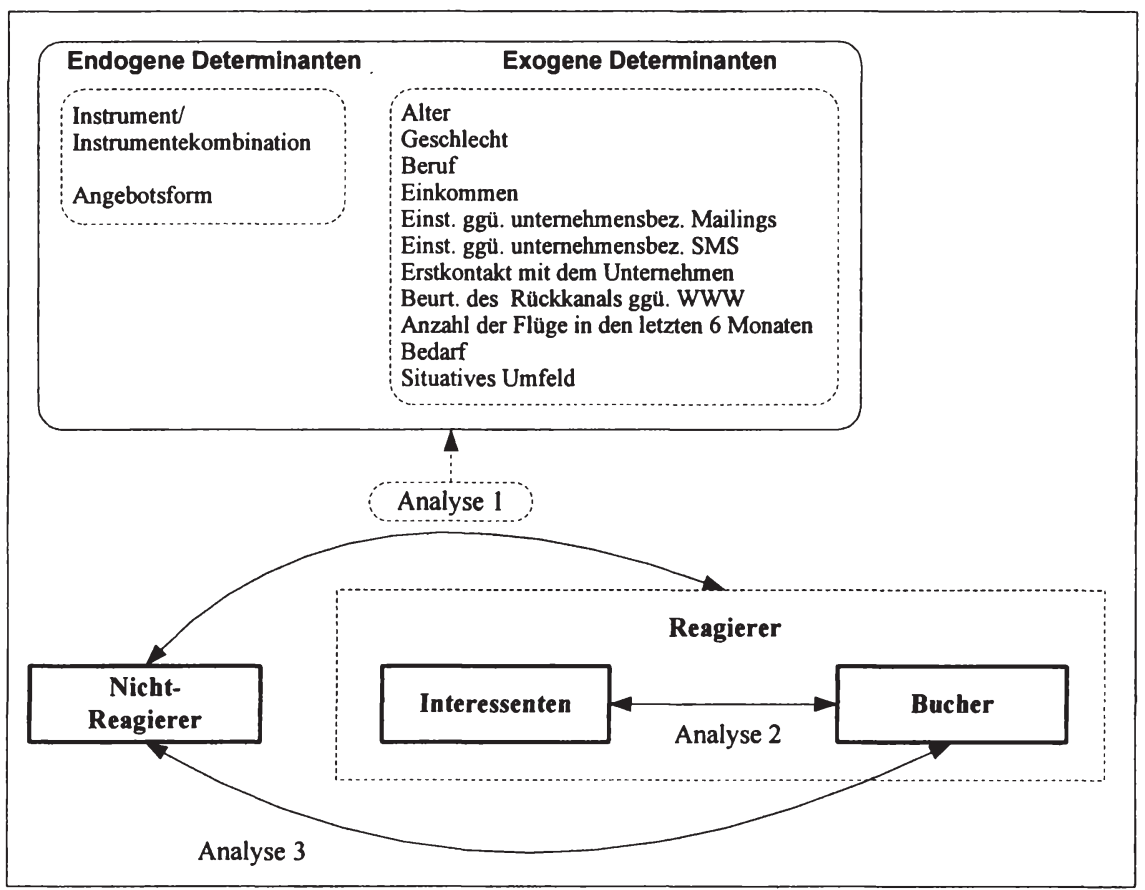

Abb. 23: Analysierte endogene und exogene Variablen im Hinblick auf das Responseverhalten (Analyse l)

Auf Basis der sog. Einschluss-Methode wurden sämtliche der unabhängigen Variablen gleichzeitig in die Untersuchung mit aufgenommen. Vor dem Hintergrund des explorativen Charakters der vorliegenden Untersuchung hätten aufgrund sachlogischer Zusammenhänge auch iterative Verfahren, wie etwa die Vorwärts- oder Rückwärtsmethode, zum Einsatz kommen können, um schrittwei-

539 Die Analyse der Korrelationen zwischen zwei metrisch-skallierten Variablen wurde hierbei auf Basis des Pearson'schen Korrelationskoeffizienten untersucht. Korrelationen zwischen nominal-skalierten Variablen konnten mittels des Konfidenzkoeffizienten abgebildet werden. Auch hierbei wurden keine kritischen Korrelationen festgestellt. Der Zusammenhang zwischen metrisch-skalierten und nominal-skalierten Variablen konnte auf Basis von Plausbilitätsüberlegungen zum größten Teil ausgeschlossen werden. Vgl. Janssen, J., Laatz, W., Statistische Datenanalyse mit SPSS für Windows, a. a. O., S. $234 \mathrm{ff}$. 
se nicht signifikante Variablen zu eliminieren. ${ }^{540}$ Jedoch soll diesem Vorgehen nicht gefolgt werden, da iterative Verfahren die Aussagekraft von F-Tests des gesamten Modells sowie t-Tests der Koeffizienten einschränken. Zudem impliziert ein derartiges Vorgehen ein hohes Maß an Willkür. ${ }^{541}$ In diesem Zusammenhang weist Krafft darauf hin, dass das Einschluss-Verfahren im Vergleich zu einem „trial-and-error"-Verfahren i. d. R. zu einer geringeren Gute des Gesamtmodells führt, die so gewonnenen Ergebnisse jedoch willkürfrei sind und auch hinsichtlich ihrer statistischen Güte konservativen Ansprüchen genügen. ${ }^{542}$

Bevor mit der Interpretation der Koeffizienten begonnen werden kann, erfolgt zunächst eine Überprüfung der Güte des auf Basis des reduzierten Datensatzes berechneten Gesamtmodells. Für das Gesamtmodell ergibt sich eine Devianz von 91,136. Der Likelihood-Quotienten-Test deutet hierbei auf eine gute Anpassung des Modells hin. Nach Einbeziehung sämtlicher unabhängiger Variablen sinkt die Devianz des Null-Modells von 648,129 auf 479,902. Die absolute Differenz der Devianz von 168,227 bei einer Irrtumswahrscheinlichkeit von $<1 \%$ ist signifikant, was bedeutet, dass die unabhängigen Variablen eine signifikant verbesserte Modellgüte ggü. dem Nullmodell ermöglichen. Durch Division der Devianz des Nullmodells bzw. des Gesamtmodells durch -2 und Einsetzen in die Gleichung für McFaddens- $\mathbf{R}^{2}$ ergibt sich ein Wert von 0,2604 , der als sehr gut anzusehen ist. ${ }^{543}$ Auch hinsichtlich der zwei weiteren Gütemaße werden gute Modellanpassungen aufgezeigt. So liegt der Wert furr Cox $\&$ Snells-R ${ }^{2}$ bei 0,295 bzw. der Wert von Nagelkerkes- $\mathbf{R}^{2}$ bei 0,398 . In beiden Fällen wird damit ebenfalls eine akzeptable bis gute Modellgüte impliziert. ${ }^{544}$

Die Klassifikationsmatrix der vorliegenden Logistischen Regression zeigt eine korrekte Zuordnung von 75,5\% der Fälle, was eine deutliche Steigerung ggü. einer zufallsbasierten Klassifikation darstellt. Der Definition des Maximal Chance Criterium (MCC) folgend wird der größere Anteil als ein möglicher Referenzpunkt zur Beurteilung der Klassifizierungskraft des Modells hinzugezogen. Mit $62,5 \%$ und $84,1 \%$ liegen beide Klassifizierungswerte oberhalb des Wertes

540 Vgl. Draper, N., Smith, H., Applied Regression Analysis, 2. Aufl., New York u. a., 1981, S. $305 \mathrm{ff}$

$541 \mathrm{Vgl}$. Krafft, M., Außendienstentlohnung im Licht der Neuen Institutionenlehre, Wiesbaden 1995, S. $289 \mathrm{f}$.

542 Vgl. ebenda, S. 290.

543 Grundsätzlich wird bei Werten zwischen 0,2 und 04 bereits von einer guten Modellanpassung gesprochen. Vgl. Urban, D., Logit-Analyse: Statistische Verfahren zur Analyse von Modellen mit qualitativen Responsevariablen, a. a. O., S. 62 f.

$544 \mathrm{Vgl}$. Backhaus, K. et al., Multivariate Analysemethoden. Eine anwendungsorientierte Einführung, 10. Aufl., Berlin u a., S. 448. 
des MCC i. H. v. 60,2\%. ${ }^{545} \mathrm{Da}$ aber u. a. die richtige Klassifizierung beider Gruppen das Ziel der empirischen Untersuchung sein sollte, erscheint das MCC als alleiniger Referenzpunkt nur begrenzt geeignet. ${ }^{546}$ Aus diesem Grund wird zusätzlich das Proportional Chance Criterium (PCC) angewendet. So konnten insgesamt $39,8 \%$ der Stichprobe als Reagierer und die übrigen als NichtReagierer identifiziert werden. Damit erreicht das PCC einen Wert von 52,08\%. Im Vergleich zu diesem Wert erscheint die Prognosekraft des vorliegenden Modells von einer relativ hohen Modellgüte gekennzeichnet zu sein. Sowohl das MCC als auch das PCC werden von beiden Klassifikationswerten übertroffen. Die folgende Tabelle beinhaltet die Klassifikationsmatrix, die die prognostizierte Gruppenzugehörigkeit der tatsächlich beobachteten Gruppenzugehörigkeit gegenüber stellt (vgl. Tab. 34).

\begin{tabular}{|l|c|c|c|}
\hline & \multicolumn{2}{|c|}{ Prognostizierte Gruppenzugehörigkeit } & \\
\hline $\begin{array}{l}\text { Beobachtete Grup- } \\
\text { penzugehörigkeit }\end{array}$ & Nicht-Reagierer & Reagierer & $\begin{array}{c}\text { Korrekte Klassi- } \\
\text { fizierung }\end{array}$ \\
\hline Nicht-Reagierer & 244 & 46 & $84,1 \%$ \\
\hline Reagierer & 72 & 120 & $62,5 \%$ \\
\hline Insgesamt & & & $83,1 \%$ \\
\hline
\end{tabular}

Tab. 34: Klassifikationsmatrix des Modells zum Vergleich von Nicht-Reagierern und Reagierern

Angesichts der Tatsache, dass sich das vorliegende Modell der Logistischen Regression als gut hinsichtlich sämtlicher Gütekriterien erwiesen hat, kann mit der Interpretation der Ergebnisse auf Basis der Koeffizienten begonnen werden. Vor diesem Hintergrund können die Logit-Koeffizienten hinsichtlich des Signifikanzniveaus sowie der Richtung ihres Einflusses direkt interpretiert werden, wobei ein positives Vorzeichen ein Indikator für einen positiven Einfluss auf die Wahrscheinlichkeit ist, auf den Direktmarketing-Stimulus zu reagieren. Im Falle metrischer Variablen bedeutet dies, dass eine Erhöhung des jeweiligen Wertes die Wahrscheinlichkeit auf eine positive Reaktion erhöht. Bei kategorial ausgeprägten Variablen tritt eine Erhöhung der Wahrscheinlichkeit ein, wenn bspw.

545 Im vorliegenden Fall entspricht der Wert des MCC 60,2\%, welches dem Anteil der Reagierer an der zugrunde liegenden Stichprobe entspricht.

$546 \mathrm{Vgl}$. Kraff, M., Außendienstentlohnung im Licht der Neuen Institutionenlehre, a. a. O., S. 316. 


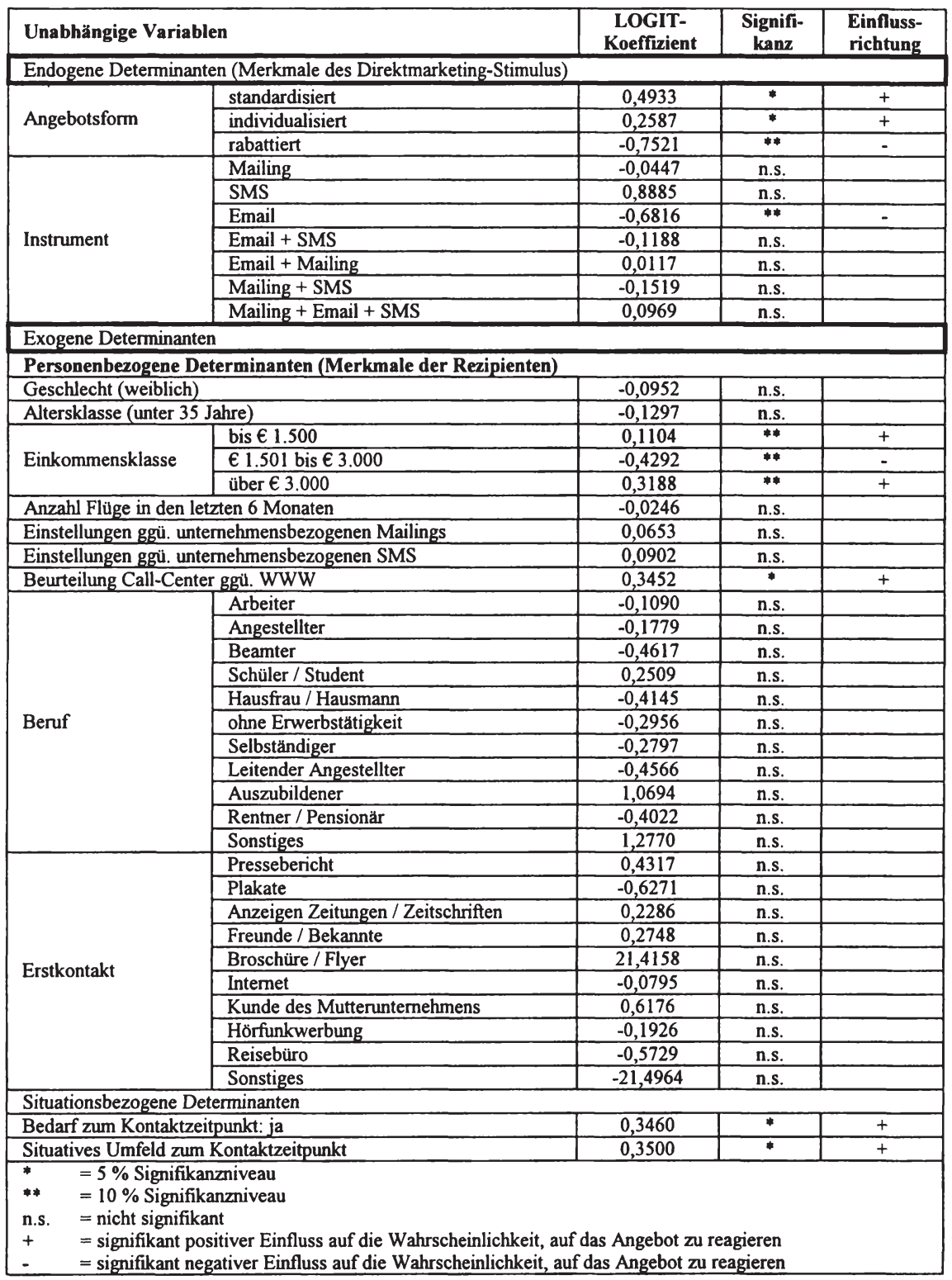

Tab. 35: Empirische Analyse des Responseverhaltens auf Basis der Logistischen Regression (Analyse I) 
die entsprechende Person die betrachtete Eigenschaft besitzt. ${ }^{547}$ Aussagen hinsichtlich der Einflussstärke der signifikanten Variablen bedürfen allerdings einer detaillierten Analyse, welche im Rahmen der Sensitivitätsanalyse vorgenommen wird. Die folgende Tabelle zeigt daher zunächst die Ergebnisse der Logistischen Regression für das untersuchte Responseverhalten („keine Reaktion“ vs. „Anruf im Call-Center"). Insgesamt werden von den 13 eingeschlossenen unabhängigen Variablen sechs als signifikant für die abhängige Variable mit den Ausprägungen „keine Reaktion“ und „Anruf im Call-Center" identifiziert (vgl. Tab. 35). ${ }^{548}$

Auf Basis dieser Analyse konnte zunächst der Einfluss der Merkmale des Direktmarketing-Stimulus, d. h. der unterschiedlichen Ausprägungen der beiden endogenen Determinanten, auf das Reaktions- oder Responseverhalten untersucht werden. Hinsichtlich der Angebotsform zeigte sich, dass sowohl das ,standardisierte" als auch das ,individualisierte“ Angebot einen positiven Einfluss auf die Wahrscheinlichkeit besitzen, dass der Rezipient des Direktmarketing-Stimulus auf das Angebot reagiert und im Call-Center anruft. Hingegen wirken sich rabattierte Angebote eher negativ auf die Reaktionswahrscheinlichkeit des Rezipienten aus.

Neben der Angebotsform wurde als zweites Merkmal des DirektmarketingStimulus der Einfluss des Trägermediums analysiert. In diesem Zusammenhang konnte festgestellt werden, dass sich die Wahrscheinlichkeit einer Rezipientenreaktion lediglich durch die Auswahl des Trägermediums „Email im isolierten Einsatz" beeinflussen lässt. Die Einflussrichtung erweist sich hierbei als negativ, d. h. der isolierte Einsatz von Emails beeinflusst das gewünschte Reaktionsverhalten negativ. ${ }^{549}$

Eine Reihe der im Rahmen der Analyse des Zusammenhangs zwischen einzelnen exogenen personenbezogenen Determinanten und der Rezipientenreak-

547 Die Berechnung der Logit-Koeffizienten erfolgte bei einzelnen Ausprägungen der kategorialen Variablen durch Summenbildung der übrigen Regressionskoeffizienten. Aufgrund der Effektcodierung der Variablen in der vorliegenden Untersuchung ist dieses Vorgehen möglich.

548 Im vorliegenden Fall können sich, wie bspw. im Fall der Einkommensklasse, auch lediglich einzelne Ausprägungen der Variablen als signifikant erweisen. Vgl. bspw. auch die Arbeit von Wulf, K. de, Hoekstra, J. C., Commandeur, H. R., The Opening und Reading Behavior of Business-to-Business Direct Mail, a. a. O., S. 133-145.

549 Vor dem Hintergrund der durchgeführten experimentellen Analyse der unterschiedlichen Trägermedien erscheint dieses Ergebnis verwunderlich, zumal sich im Rahmen des Feldexperiments in Abhängigkeit der unterschiedlichen eingesetzten Instrumente und Instrumentekombinationen stark schwankende Responsequoten zeigten. Entscheidend furr die Analyse ist allerdings die relative Bedeutung des Einflusses von Emails im Rahmen des gesamten Responseprozesses zu sehen, welcher auf Basis der Sensitivitätsanalyse erfolgt. 
tion nachgewiesenen Korrelationen konnte allerdings nicht bestätigt werden. So wurde kein Einfluss der untersuchten (sozio-)demographischen Merkmale Geschlecht, Alter und Beruf festgestellt. Ebenso erwies sich der Einfluss von Einstellungen ggü. Mailings und SMS des betrachteten Unternehmens als nicht signifikant. Hingegen konnte ein Zusammenhang zwischen der Einkommensklasse und dem Responseverhalten bestätigt werden. So zeigt die Zugehörigkeit zur Einkommensklasse des Rezipienten „bis $€ 1.500$ “ einen positiven Einfluss auf die Wahrscheinlichkeit, dass dieser auf den versendeten Direktmarketing-Stimulus reagiert. Hingegen erweist sich dieser Zusammenhang im Falle eines Einkommens von „ $€ 1.500$ bis $€ 3.000$ “ als negativ. Darüber hinaus zeigte sich ein signifikanter positiver Einfluss der Beurteilung des Call-Centers als Responsekanal im Vergleich zum Internet. Demnach beinhaltet eine bessere Bewertung von CallCentern ggü. dem Internet einen positiven Effekt auf die Reaktionswahrscheinlichkeit.

Im Vergleich zu den personenbezogenen Determinanten konnte der Einfluss sämtlicher situationsbezogener Einflussfaktoren auf einem Signifikanzniveau von $5 \%$ bestätigt werden. So konnte zwischen der konkreten Bedarfssituation und dem Responseverhalten ein positiver Zusammenhang festgestellt werden. Gleichzeitig bestätigt sich ein positiver Zusammenhang zwischen der Beurteilung des situativen Umfelds. Je positiver die situativen Umstände während des Kontaktes mit dem Direktmarketing-Stimulus beurteilt wird, desto wahrscheinlicher ist eine positive Reaktion des Rezipienten.

Während das Vorhandensein eines grundsătzlichen Einflusses einer unabhängigen Variablen auf die abhängige Variable durch Betrachtung der Signifikanzniveaus und Logit-Koeffizienten möglich ist, gestaltet sich die Interpretation der Koeffizienten hinsichtlich ihrer Einflussstärke schwieriger. Dies liegt daran, dass von der absoluten Höhe des Koeffizienten - wie bspw. im Fall der linearen Regressionsanalyse - nicht auf die Stärke des Einflusses geschlossen werden kann. Aus diesem Grund wird eine Sensitivitätsanalyse der signifikanten Variablen durchgeführt, im Rahmen derer unter Konstanthaltung der Ausprägungen aller übrigen unabhängigen Variablen die systematische Variation einer einzelnen unabhängiger Variablen erfolgt. ${ }^{550}$ Mittels dieses Verfahrens erfolgt die systematische Variation der signifikanten Variablen und es lässt sich der absolute Effekt von unterschiedlichen Ausprägungen dieser auf die Wahrscheinlichkeit able-

550 Eine detaillierte Beschreibung des Verfahrens der Sensitivitätsanalyse findet sich bei LeClere und Urban. Vgl. LeClere, M., The Interpretation of Coefficients in Models with Qualitative Dependent Variables, in: Decision Sciences, Vol 23, 1992, S. 722 f.; Urban, D., Logit-Analyse: Statistische Verfahren zur Analyse von Modellen mit qualitativen Responsevariablen, a. a. O., S. $46 \mathrm{ff}$. 
sen. ${ }^{551}$ Damit zeigt die Differenz der ursprünglich geschätzten und der aus der Variation resultierenden neuen Wahrscheinlichkeit den Einfluss veränderter Ausprägungen eben dieser Variablen und damit deren Relevanz. ${ }^{552}$

Vor der Analyse des Einflusses einzelner Variablen auf die Responsewahrscheinlichkeit ist das Ausgangsniveau der metrischen und kategorialen endogenen und exogenen Variablen festzulegen. Hinsichtlich der endogenen Determinanten soll als Ausgangspunkt der folgenden Überlegungen ein rabattiertes Angebot dienen, das per Email an die Rezipienten verschickt wird. Im Bereich der exogenen Determinanten entspricht das Ausgangsniveau der metrischen Variablen dem Durchschnittswert der Gruppe der Nicht-Reagierer. Im Fall der kategorialen Variablen wird das Ausgangsniveau auf dem Wert der am häufigsten genannten Kategorie festgelegt. ${ }^{553}$ Werden diese Werte in die Regressionsgleichung eingesetzt, so ergibt sich eine Wahrscheinlichkeit i. H. v. 37,03\%, dass der Rezipient auf das Angebot mit einem Anruf im Call-Center reagiert.

Im Rahmen der Sensitivitätsanalyse werden in einem ersten Schritt die signifikanten exogenen Variablen in ihrer Ausprägung variiert. Für die metrischen Variablen wird dazu der durchschnittliche Wert der Reagierer-Stichprobe eingesetzt. ${ }^{554}$ Die Werte der kategorialen Variablen werden entsprechend der als signifikant identifizierten Ausprägungen variiert. In einem zweiten Schritt erfolgt die Variation des Direktmarketing-Stimulus hinsichtlich der als signifikant identifizierten Ausprägungen der zwei unabhängigen Variablen „Angebotsform“ und "Trăgermedium“. Wăhrend bei der Angebotsform sowohl das individualisierte als auch das rabattierte Angebot „eingesetzt“ wird, erscheint eine Variation der Variablen „Trägermedium“ lediglich in Richtung „kein Email“ sinnvoll, da sämtliche anderen Ausprägungen dieser Variablen als nicht signifikant ermittelt wurden. Die sich aus der Variation des Ausprägungsniveaus ergebenden Differenzen der absoluten Responsewahrscheinlichkeit sind in der folgenden Abbildung dargestellt (vgl. Abb. 24).

551 Ein derartiges Vorgehen ist auf Basis der im vorangegangenen Kapitel 3.2 und der dort verwendeten Analysemethoden nicht möglich.

$552 \mathrm{Vgl}$. Krafft, M., Außendienstentlohnung im Licht der Neuen Institutionenlehre, a. a. O., S. 298.

553 Damit entspricht der Rezipient in diesem Fall einem „typischen“ Nicht-Reagierer, bei dem sämtliche unabhängige Variablen die durchschnittlichen Ausprägungen der Gruppe der Nicht-Reagierer beinhalten.

554 Der mittlere Wert aus Nicht-Reagierer und Reagierer-Stichprobe wurde hierbei als ungewichtetes Mittel berechnet. 


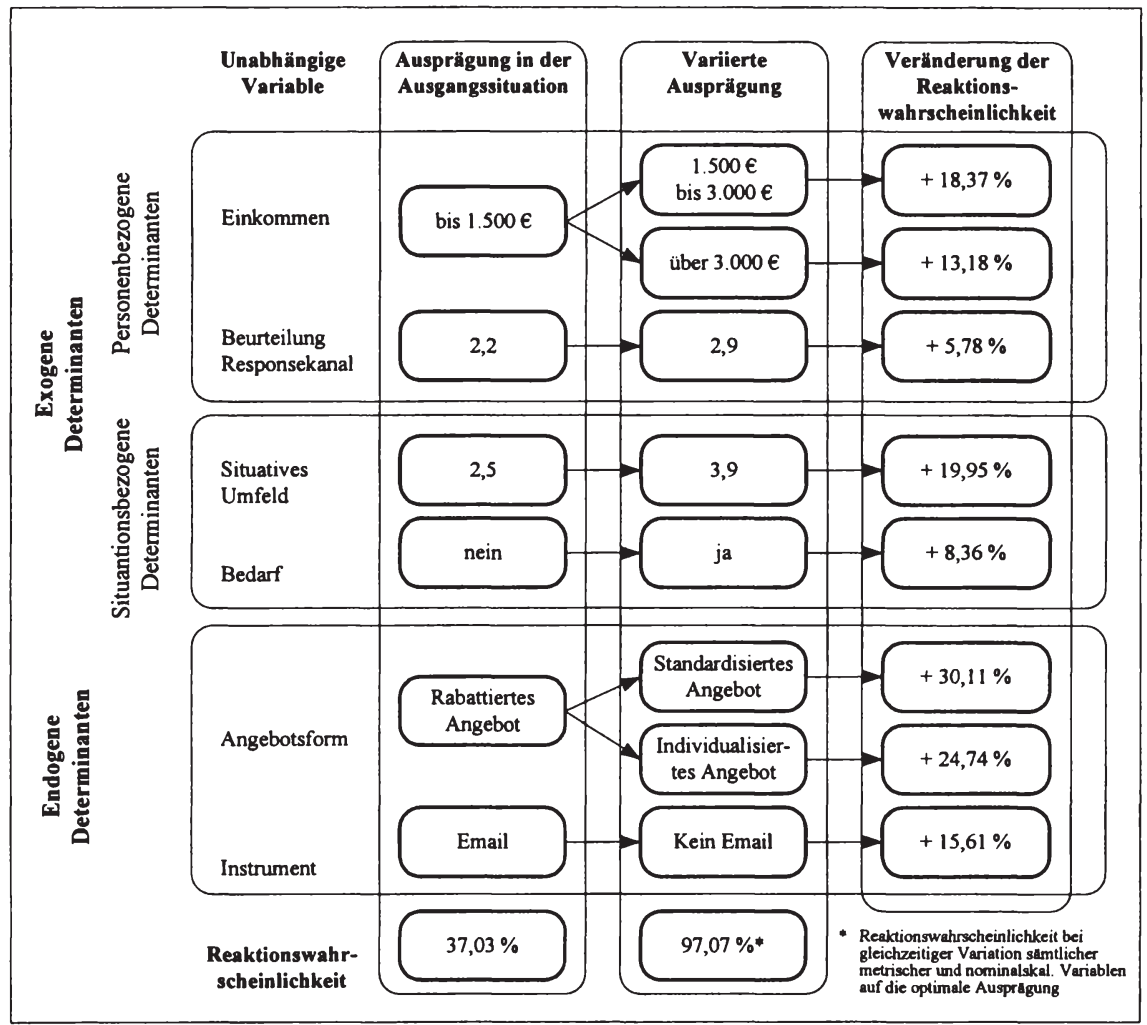

Abb. 24: Sensitivitätsanalyse der signifikanten Variablen im Rahmen der ersten Analyse

Auf Basis der Ergebnisse der Sensitivitätsanalyse ist ersichtlich, dass die Variable „Angebotsform“ mit einer positiven Differenz von 30,11\% den größten Einfluss auf die Wahrscheinlichkeit besitzt, dass ein Rezipient eines Direktmarketing-Stimulus auf diesen auch reagiert. Im Falle des Einsatzes eines individualisierten Angebots anstelle des rabattierten Angebots wächst die Reaktionswahrscheinlichkeit immerhin noch um $24,74 \%$. Wird anstelle des eingesetzten Trägermediums „Email“ ein anderes Trägermedium ausgewählt, kommt es im Durchschnitt zu einem positiven Effekt i. H. v. 15,61\%. Bei den metrisch skalierten Determinanten erweist sich insbesondere die Beurteilung des situativen Umfelds als äußerst relevant. Wird der Wert der durchschnittlichen Beurteilung aus Sicht der Nicht-Reagierer durch die durchschnittliche Beurteilung der Reagierer-Gruppe ersetzt, führt dies zu einer absoluten positiven Veränderung der Wahrscheinlichkeit um 19,95\%. 
Entsprechend dieser Analyse ist der Einfluss der Beurteilung des Responsekanals auf die Wahrscheinlichkeit am geringsten. Unabhängig vom Einfluss der exogenen Determinanten ist der Einfluss der endogenen Determinanten im Vergleich der absoluten Differenzen deutlich höher ausgefallen. So wirkt sich eine Variation der Angebotsform vom rabattierten in ein standardisiertes Angebot in nahezu einer Verdopplung der Reaktionswahrscheinlichkeiten aus. Werden in die Gleichung der Logistischen Regression jeweils die abgebildeten optimalen Ausprägungen der verschiedenen Variablen gleichzeitig eingesetzt $-\mathrm{d}$. h. simultan variiert - steigt die Wahrscheinlichkeit einer Reaktion auf 97,07\%. Dies bedeutet, dass das betrachtete Unternehmen, wenn es ein standardisiertes Angebot über ein „email-verschiedenes“ Trägermedium an Bestandskunden der mittleren Einkommensklasse versendet, die zum Zeitpunkt des Kommunikationskontaktes beabsichtigten, in nächster Zeit einen Flug zu buchen und die hinsichtlich der Beurteilung des Responsekanals „Call-Center“ keine Vorurteile besitzen und deren situatives Umfeld zu diesem Zeitpunkt als relativ ruhig angesehen wird, mit einer Wahrscheinlichkeit von über $97 \%$ auf das Angebot reagieren und einen Anruf über die Buchungshotline tätigen.

Die Ergebnisse zeigen einen mittleren Einfluss des Trägermediums auf das Reaktionsverhalten der Rezipienten, während angebotsbezogene Komponenten einen starken Einfluss besitzen. Diese Erkenntnisse bestätigen einige der Tendenzaussagen zur relativen Bedeutung der unterschiedlichen endogenen Determinanten, die sich bereits auf Basis der Response- und Buchungsauswertung des Feldexperiments abzeichneten. So deutete sich bereits dort an, dass die Variation der Angebotsform zu stark unterschiedlichen durchschnittlichen Responsequoten führte. Innerhalb einer Angebotsformenkategorie erwiesen sich die Varianzen zwischen den einzelnen Instrumente und Instrumentekombinationen allerdings als nicht so stark schwankend.

\subsubsection{Vergleich von Interessenten und Buchern}

In der folgenden zweiten Untersuchung wird - wiederum basierend auf dem Verfahren der Logistischen Regression - der Einfluss verschiedener unabhängiger endogener und exogener Variablen auf die abhängige Variable mit den zwei Ausprägungen „keine Buchung“ und „Buchung“ untersucht. Als Grundgesamtheit der Untersuchung dient hierbei die Gruppe der Reagierer, die sich aus Interessenten zusammensetzt, die im Call-Center angerufen, aber dort nicht gebucht haben („,keine Buchung“) sowie Personen, die im Call-Center angerufen und dort eine oder mehrere Buchungen vorgenommen haben (,Bucher"). Ziel der folgenden Ausführungen soll es daher sein, die Einflussstärke der endogenen Determinanten „Instrument bzw. Instrumentekombination“ und „Angebotsform“ im Zu- 
sammenspiel mit den als relevant erachteten exogenen Determinanten aufzuzeigen und damit die zentralen Treiber zu identifizierten, von denen abhängt, ob ein Anrufer im Call-Center das ihm zugesandte Angebot bucht. Entsprechend der Ergebnisse der Signifikanztests aus dem Kapitel 3.2 werden hierbei 14 exogene sowie zusätzlich zwei endogene Determinanten in die Analyse miteinbezogen (vgl. Abb. 25).

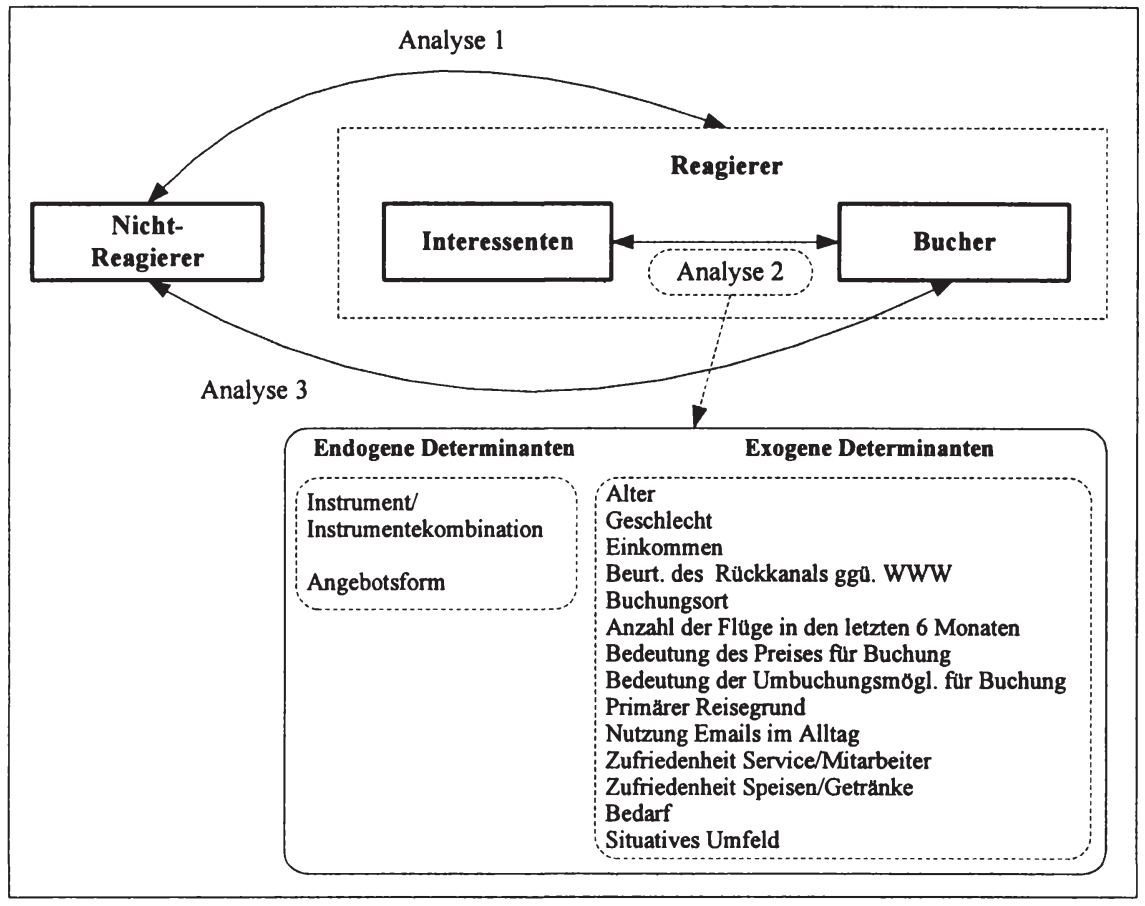

Abb. 25: Analysierte endogene und exogene Variablen im Hinblick auf das Buchungsverhalten (Analyse 2)

Von den insgesamt 798 Beobachtungen konnten 128 Fälle für die Analyse auf Basis der Logistischen Regression verwendet werden. ${ }^{555} 60$ der ausgewählten

555 Voraussetzung für die Einbeziehung einer Beobachtung in die Analyse ist das Vorliegen eines Fragebogens sowohl aus der Ex-post-Befragung als auch aus der Exante-Befragung, da aus beiden Fragebögen Angaben benötigt werden. Die Zahl von 798 Beobachtungen ergibt sich durch die Anzahl von Personen, die beide Fragebögen ausgefüllt haben. Die entsprechende Reduzierung auf 128 Fälle ergibt sich durch den Ausschluss von Beobachtungen mit fehlenden Werten. 
Fälle $(46,9 \%)$ sind den Interessenten zuzuordnen, die angerufen, aber nicht gebucht haben, während 68 Fälle $(53,1 \%)$ der Gruppe der Bucher angehören. Wiederum wurden sämtliche Beobachtungen von der weiteren Betrachtung ausgeschlossen, die fehlende Werte bei einer der 16 unabhängigen Variablen aufwiesen. Als notwendige Voraussetzung zur Durchführung einer Logistischen Regression wurden die unabhängigen Variablen wiederum auf Multikolliniearität getestet. In diesem Kontext wurden keine Korrelationen festgestellt. ${ }^{556}$ Auf eine detaillierte Darstellung der Ergebnisse soll an dieser Stelle verzichtet werden. Auch beim zweiten Modell wurde die Einschluss-Methode gewählt und somit sämtliche der unabhängigen Variablen in die Berechnung aufgenommen. ${ }^{557}$

Bevor mit der Interpretation der Koeffizienten begonnen werden kann, erfolgt zunächst wiederum eine Überprüfung der Güte des auf Basis des reduzierten Datensatzes berechneten Gesamtmodells. Für das Gesamtmodell ergibt sich eine Devianz von 93,914. Vor dem Hintergrund des Signifikanzniveaus spricht dieser relativ niedrige Wert ebenfalls für eine hohe Modellgüte. Wie im vorherigen Modell auch unterstreicht der Likelihood-Quotienten-Test die gute Anpassung des Modells. Nach Einbeziehung sämtlicher unabhängiger Variablen sinkt die Devianz des Null-Modells von 176,945 auf 93,914. Die absolute Differenz der Devianz beträgt damit 83,031 und ist bei einer Irrtumswahrscheinlichkeit von < $1 \%$ signifikant. Dies bedeutet, dass die unabhängigen Variablen eine signifikant verbesserte Modellgüte ggü. dem Nullmodell ermöglichen. Wird die Devianz des Nullmodells bzw. des Gesamtmodells durch -2 dividiert und in die Gleichung für Pseudo- $\mathbf{R}^{2}$ eingesetzt, ergibt sich ein Wert von 0,4692 , der als sehr gut anzusehen ist. ${ }^{558}$ Auch hinsichtlich der weiterhin verwendeten Gütemaße wird eine hohe Modellgüte aufgezeigt. So liegt der Wert für Cox $\&$ Snells- $\mathbf{R}^{2}$ bei 0,477 und für Nagelkerkes- $\mathbf{R}^{2}$ bei 0,637 . Beide Werte sind als sehr gut anzusehen.

Auch die Klassifikationsmatrix der vorliegenden zweiten Logistischen Regression zeigt eine hohe korrekte Zuordnung i. H. v. 83,6\% der Fälle. Sowohl das Maximal Chance Criterium (MCC) mit einem Wert von 53,10\% als auch das Proportional Chance Criterium (PCC) mit einem Wert von 50,19\% werden

556 Die Analyse der Korrelationen zwischen metrisch-skallierten Variablen wurde hierbei auf Basis des Pearson'schen Korrelationskoeffizienten untersucht. Korrelationen zwischen nominal-skalierten Variablen konnten mittels des Konfidenzkoeffizienten abgebildet werden. Auch hierbei wurden keine kritischen Korrelationen festgestellt. Der Zusammenhang zwischen metrisch-skalierten und nominal-skalierten Variablen konnte auf Basis von Plausbilitätsüberlegungen zum größten Teil ausgeschlossen werden. Vgl. Janssen, J., Laatz, W., Statistische Datenanalyse mit SPSS für Windows, a. a. O., S. 234 ff.

$557 \mathrm{Vgl}$. zur Begründung für ein derartiges Vorgehen die Ausführungen im vorangegangenen Kapitel.

558 Vgl. Urban 1993, S. 62 f. 
hinsichtlich beider Klassifizierungswerte übertroffen, wodurch die gute Prognosekraft des vorliegenden Modells sowie die allgemeine Modellgüte unterstrichen wird. In der folgenden Abbildung ist die Klassifikationsmatrix ersichtlich, die die prognostizierte Gruppenzugehörigkeit zu der tatsächlich beobachteten Gruppenzugehörigkeit ins Verhältnis setzt (vgl. Tab. 36).

\begin{tabular}{|l|c|c|c|}
\hline & \multicolumn{2}{|c|}{$\begin{array}{c}\text { Prognostizierte Gruppenzugehörig- } \\
\text { keit }\end{array}$} & \\
\hline $\begin{array}{l}\text { Beobachtete Grup- } \\
\text { penzugehörigkeit }\end{array}$ & Interessenten & Bucher & $\begin{array}{c}\text { Korrekte Klas- } \\
\text { sifizierung }\end{array}$ \\
\hline Interessenten & 49 & 11 & $81,7 \%$ \\
\hline Bucher & 10 & 58 & $85,3 \%$ \\
\hline Insgesamt & & & $83,6 \%$ \\
\hline
\end{tabular}

Tab. 36: Klassifikationsmatrix des Modells zum Vergleich von Interessenten und Buchern

Angesichts der hohen Modellgüte des vorliegenden zweiten Modells kann in einem nächsten Schritt die Interpretation der Ergebnisse erfolgen. In diesem Zusammenhang können die Logit-Koeffizienten hinsichtlich des Signifikanzniveaus sowie der Richtung ihres Einflusses zunächst direkt interpretiert werden. Die folgende Tabelle zeigt die Ergebnisse der Logistischen Regression für das Buchungsverhalten. Insgesamt werden von den 16 eingeschlossenen unabhängigen Variablen fünf als signifikant für die abhängige Variable („Buchung ja/nein“) identifiziert (vgl. Tab. 37).

Auffällig erscheint im Rahmen der Analyse der endogenen Determinanten zunächst, dass im Vergleich zur vorherigen Untersuchung zum Responseverhalten, das ursprünglich zur Übermittlung des Angebotes eingesetzte Trägermedium auf die Buchungsentscheidung keinen Einfluss besitzt. Vielmehr zeigt sich wiederum der signifikante Einfluss der Angebotsform auf die abhängige Variable. Ebenso wie in der ersten Logistischen Regression sind hierbei sämtliche Angebotsformen relevant. In diesem Kontext bestätigen sich zwei der in der ersten Analyse aufgezeigten Einflussrichtungen: zum einen erweist sich die Auswahl eines standardisierten Angebots als positiver Einflussfaktor auf die Buchungswahrscheinlichkeit, zum anderen besitzt das rabattierte Angebot wiederholt einen negativen Einfluss.

Die Analyse des Einflusses der exogenen personenbezogenen Determinanten zeigt ebenfalls ein heterogenes Bild. So wies die Untersuchung der demographischen Merkmale Alter und Geschlecht ebenfalls keinen signifikanten Einfluss zum Buchungsverhalten auf. Ebenso konnte kein Einfluss zwischen der Emailnutzung und dem Buchungsverhalten nachgewiesen werden. Hingegen steht ein 


\begin{tabular}{|c|c|c|c|c|}
\hline \multicolumn{2}{|c|}{ Unabhăngige Variablen } & $\begin{array}{c}\text { LOGIT- } \\
\text { Koeffizient }\end{array}$ & $\begin{array}{c}\text { Signifi- } \\
\text { kanz }\end{array}$ & $\begin{array}{l}\text { Einfluss- } \\
\text { richtung }\end{array}$ \\
\hline \multicolumn{5}{|c|}{ Endogene Determinanten (Merkmale des Direktmarketing-Stimulus) } \\
\hline \multirow{3}{*}{ Angebotsform } & standardisiert & 1,8092 & * & + \\
\hline & individualisiert & $-0,7210$ & *** & - \\
\hline & rabattiert & $-1,0883$ & * & - \\
\hline \multirow{7}{*}{ Instrument } & Mailing & 0,3224 & n.s. & \\
\hline & SMS & 2,4494 & n.s. & \\
\hline & Email & $-0,7653$ & n.s. & \\
\hline & Email + SMS & $-1,3947$ & n.s. & \\
\hline & Email + Mailing & 0,7965 & n.s. & \\
\hline & Mailing + SMS & $-1,9930$ & n.s. & \\
\hline & Mailing + Email + SMS & 0,5847 & n.s. & \\
\hline \multicolumn{5}{|c|}{ Exogene Determinanten } \\
\hline \multicolumn{5}{|c|}{ Personenbezogene Determinanten (Merkmale der Rezipienten) } \\
\hline \multicolumn{2}{|c|}{ Geschlecht (weiblich) } & 0,2862 & n.s. & \\
\hline \multicolumn{2}{|c|}{ Altersklasse (unter 35 Jahre) } & 0,5198 & n.s. & \\
\hline \multicolumn{2}{|c|}{ Emailnutzung im Alltag } & $-0,8344$ & ** & - \\
\hline \multicolumn{2}{|c|}{ Anzahl Fluge in den letzten 6 Monaten } & $-0,0460$ & n.s. & \\
\hline \multicolumn{2}{|c|}{ Zufriedenheit Service / Mitarbeiter } & 0,5759 & n.s. & \\
\hline \multicolumn{2}{|c|}{ Zufriedenheit Speisen / Getrănke } & 0,6722 & ** & + \\
\hline \multicolumn{2}{|c|}{ Beurteilung Call-Center ggü. WWW } & $-0,3393$ & n.s. & \\
\hline \multicolumn{2}{|c|}{ Bedeutung des Preises für Buchung } & $-0,4656$ & n.s. & \\
\hline \multicolumn{2}{|c|}{ Bedeutung der Umbuchungsmöglichkeit fur Buchung } & 0,3512 & n.s. & \\
\hline \multirow{3}{*}{ Reiseklasse } & privat & 0,4794 & n.s & \\
\hline & privat \& beruflich & 0,0428 & n.s. & \\
\hline & beruflich & $-1,0420$ & n.s. & \\
\hline \multirow{3}{*}{ Einkommensklasse } & bis $€ 1.500$ & $-1,7608$ & $*$ & - \\
\hline & $€ 1.501$ bis $€ 3.000$ & 0,5513 & n.s. & \\
\hline & uber $€ 3.000$ & 1,2096 & * & + \\
\hline \multirow{5}{*}{ Buchungsort } & Call-Center & $-19,8630$ & n.s. & \\
\hline & Newsletter & $-1,0759$ & n.s. & \\
\hline & Homepage & $-0,8860$ & n.s. & \\
\hline & am Flughafen & 23,5090 & n.s. & \\
\hline & Call Center 1 & $-19,8630$ & n.s. & \\
\hline \multicolumn{5}{|c|}{ Situationsbezogene Determinanten } \\
\hline \multicolumn{2}{|c|}{ Bedarf zum Kontaktzeitpunkt: ja } & 0,7115 & * & + \\
\hline \multicolumn{2}{|c|}{ Situatives Umfeld zum Kontaktzeitpunkt } & 0,7767 & * & + \\
\hline $\begin{array}{ll}* & =5 \% \text { Signifik } \\
* * & =10 \% \text { Signifi } \\
\text { n.s. } & =\text { nicht signifi } \\
+ & =\text { signifikant } \mathrm{p} \\
- & =\text { signifikant } \mathrm{n}\end{array}$ & $\begin{array}{l}\text { veau } \\
\text { hiveau } \\
\text { er Einfluss auf die Wahrsch } \\
\text { ver Einfluss auf die Wahrsch }\end{array}$ & $\begin{array}{l}\text { ichkeit, das } \\
\text { lichkeit, das }\end{array}$ & $\begin{array}{c}\text { ot } \mathrm{zu} \\
\text { bot } \mathrm{zu}\end{array}$ & \\
\hline
\end{tabular}

Tab. 37: Empirische Analyse des Buchungsverhaltens auf Basis der Logistischen Regression (Analyse 2) 
Ergebnis in besonderem Einklang mit dem fehlenden Einfluss des Trägermediums für die Wahrscheinlichkeit der Buchung: nachdem in der vorherigen Analyse festgestellt wurde, dass eine positivere Beurteilung des Call-Centers als Responsekanal im Vergleich zum Internet auch einen positiven Einfluss auf das Responseverhalten besitzt, erweist sich die Relevanz dieser Variablen für die Wahrscheinlichkeit einer Buchung als nicht signifikant. Damit besitzen weder das Trägermedium noch der Responsekanal einen Einfluss darauf, ob Personen, die im Call-Center anrufen, letztendlich auch buchen.

Darüber hinaus konnte kein signifikanter Einfluss zwischen der Bedeutung einzelner Komponenten des Leistungsangebotes des Unternehmens und dem Buchungsverhalten festgestellt werden. So gestaltet sich weder die rezipientenseitige Beurteilung der Relevanz des Preises für den Buchungsabschluss noch die rezipientenseitige Bedeutung einer flexiblen Umbuchungsmöglichkeit als relevant für das Buchungsverhalten. Im Zusammenspiel der verschiedenen endogenen und exogenen Determinanten erweist sich diese Variable als nicht relevant. Im Gegensatz dazu besteht wiederum ein signifikanter Einfluss zwischen dem Einkommen und der Buchungswahrscheinlichkeit: während allerdings bei der Untersuchung des Responseverhaltens ein geringeres Einkommen tendenziell zu einer höheren Responsewahrscheinlichkeit führte, erweist sich ein niedrigeres Einkommen als negativer Einflussfaktor auf die Buchungswahrscheinlichkeit et vice versa.

Als weiterhin signifikante Einflussfaktoren auf die Buchungswahrscheinlichkeit erweisen sich die Erfahrungen der Rezipienten mit den Leistungen des Unternehmens. Erstaunlich erscheint hierbei, dass die Zufriedenheit mit Speisen und Getränken, nicht aber die Zufriedenheit mit der Buchungsabwicklung an sich von Relevanz ist. ${ }^{559}$ Ebenfalls irrelvant für das Buchungsverhalten ist die Anzahl der in den letzten sechs Monaten mit dem Unternehmen getätigten Flüge sowie der primäre Buchungsort der Rezipienten.

Neben ausgewählten personenbezogenen Determinanten konnte zudem der Einfluss der beiden untersuchten situationsbezogenen Einflussfaktoren wiederum auf einem Signifikanzniveau von $5 \%$ bestätigt werden. Demnach besitzt sowohl die Bedarfssituation des Rezipienten als auch eine positive Beurteilung des situativen Umfelds einen positiven Einfluss auf die Buchungswahrscheinlichkeit.

Um die Einflussstärke der verschiedenen endogenen und exogenen Determinanten im Zusammenspiel auf das Buchungsverhalten zu untersuchen, reicht eine oberflächliche Betrachtung der Koeffizienten nicht aus. Vielmehr ist eine gezielte Variation der einzelnen signifikanten Variablen sowie der Messung der Auswir-

559 Vor dem Hintergrund des No-Frills-Konzepts des betrachteten Unternehmens müssen Speisen und Getränke an Bord der Flugzeuge käuflich erworben werden. 
kungen auf die Buchungswahrscheinlichkeit im Rahmen einer Sensitivitätsanalyse notwendig. ${ }^{560}$ Wie im Rahmen der ersten Logistischen Regression ebenfalls durchgeführt werden die nicht variierten metrischen und kategorialen Variablen auf einem spezifischen Niveau konstant gehalten. ${ }^{561}$ In einem ersten Schritt erfolgt eine Variation der einzelnen signifikanten exogenen Variablen gemäß dem vorherigen Vorgehen: bei den metrischen Variablen werden die durchschnittlichen Werte der Interessenten in der Ausgangskonfiguration eingesetzt und durch den durchschnittlichen Wert der Reagierer-Stichprobe ersetzt. ${ }^{562}$ Die kategorialen Variablen werden entsprechend ihrer signifikanten Ausprägungen variiert. In einem zweiten Schritt erfolgt eine Variation der signifikanten endogenen Determinanten - in diesem Fall lediglich eine Variation der Variablen „Angebotsform". Als Ausgangspunkt soll in diesem Zusammenhang ein rabattiertes Angebot dienen, das mit Hilfe des Einsatzes von SMS an die Rezipienten verschickt wurde und auf das diese bereits reagiert haben. Die Wahrscheinlichkeit, dass ein „typischer" Interessent, d. h. eine Person, die sich hinsichtlich sämtlicher Variablen der Interessenten-Stichprobe auf dem Durchschnittsniveau bewegt, das Angebot bucht, beträgt weniger als ein Prozent (vgl. Abb. 26). ${ }^{563}$

Auf Basis der vorliegenden Untersuchung erscheinen mit der Angebotsform sowie mit der Einkommensklasse jeweils eine endogene und eine exogene Variable als besonders relevant zur Steigerung der Buchungswahrscheinlichkeit. Werden statt der bisherigen Zielgruppe nicht Personen mit einem Einkommen von unter $€ 1.500$ angesprochen, sondern Personen mit mehr als $€ 3.000$ Einkommen, so erhöht sich die Buchungswahrscheinlichkeit um mehr als $15 \%$. Ebenso führt die Verwendung eines standardisierten Angebotes anstelle des eingesetzten rabattierten Angebots zu einer Erhöhung der Buchungswahrscheinlichkeit um einen ähnlich hohen absoluten Betrag (+14,13\%). Die übrigen Variablen erweisen sich in diesem Zusammenhang als relativ unsensitiv. Ein Angleichen bspw. des Emailnutzungsverhaltens der Interessenten an das der „typischen“ Bucher hat sogar einen negativen Einfluss auf die Buchungswahrscheinlichkeit $(-0,26 \%)$. Von zwar geringem aber - in Anlehnung an die Ergebnisse der vorhe-

560 Auf die Gründe für das Vorgehen soll nicht wiederholt eingegangen werden. Vgl. hierzu das vorherige Kapitel.

561 Die metrischen Variablen werden zunächst auf dem durchschnittlichen Niveau der Interessenten konstant gehalten. Die kategorialen Variablen werden auf dem Niveau der häufigsten Ausprägung ,fixiert“.

562 Für andere Ausgangssituationen ergeben sich entsprechend der Ausprägungen der kategorialen Variablen andere Wahrscheinlichkeitswerte.

563 In Anlehnung an die Ergebnisse des Feldexperiments verwundert dieser geringe Wert nicht, da es sich bei der betrachteten Person um einen „typischen“ Interessenten handelt. So wurde in $75 \%$ der betrachteten Anrufe keine Buchung vorgenommen. 
rigen Analyse - wiederum positivem Einfluss gestalten sich demnach die Bedarfssituation und das situative Umfeld des Rezipienten.

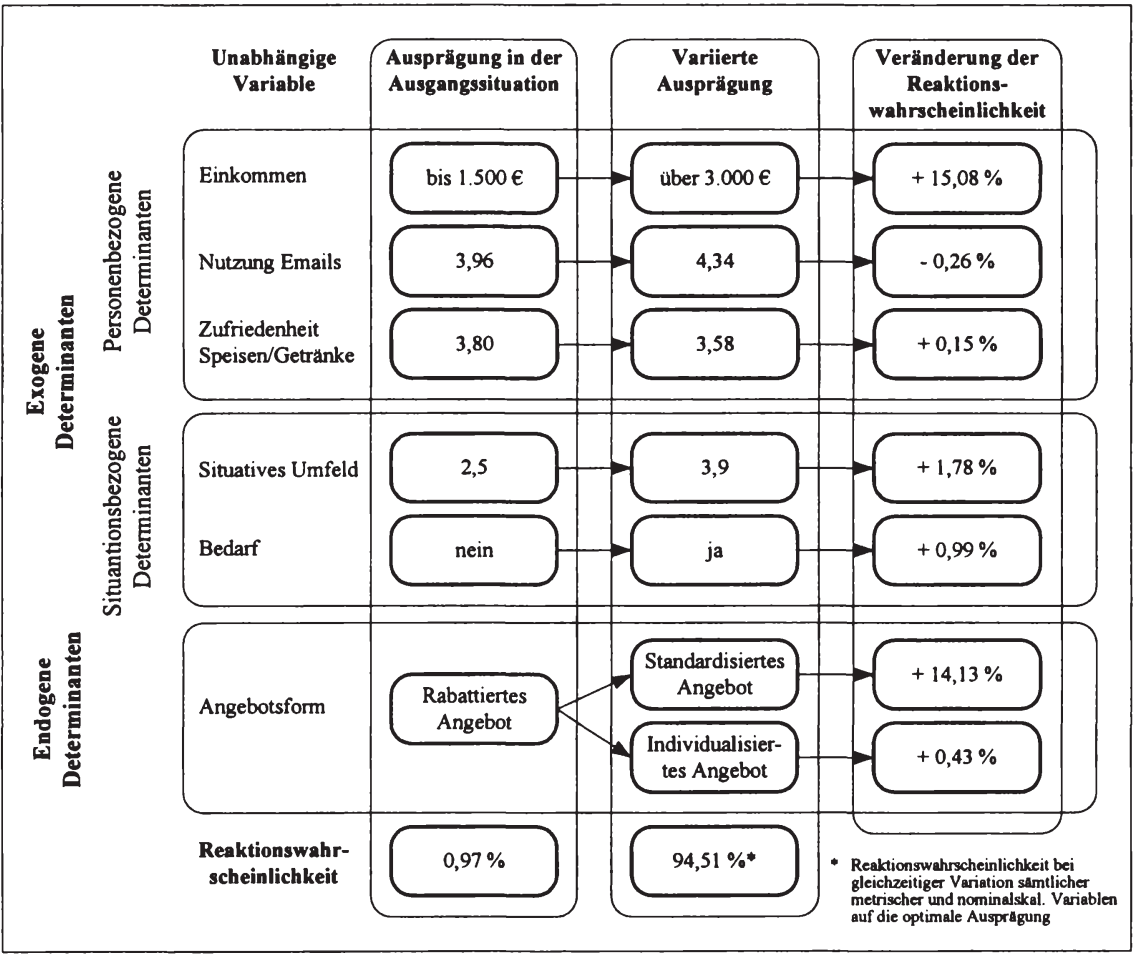

Abb. 26: Sensitivitätsanalyse der signifikanten Variablen im Rahmen der zweiten Analyse

Insgesamt zeigt sich, dass die Wahrscheinlichkeit, dass Interessenten, die im Call-Center anrufen auch eine Buchung vornehmen, primär von der Angebotsform und den finanziellen Verhältnissen in Form der Einkommenssituation der Anrufer determiniert wird, während die übrigen Faktoren eine untergeordnete Rolle spielen. Wird abschließend eine Variation sämtlicher signifikanter Variablen hinsichtlich der für die Buchungswahrscheinlichkeit „optimalen“ Ausprägung vorgenommen, steigt die Wahrscheinlichkeit einer Buchung auf fast $95 \%$.

\subsubsection{Vergleich der Nicht-Reagierern und Buchern}

Die dritte Untersuchung beschäftigt sich ebenfalls mit der Analyse des Einflusses verschiedener unabhängiger endogener und exogener Variablen auf eine abhän- 
gige Variable, die die Ausprägungen „keine Reaktion“ oder „Buchung“ aufweist. Ziel der dritten Analyse ist es, die zentralen Einflussfaktoren auf das Buchungsverhalten sowie deren Einflussstärke in Relation zueinander aufzuzeigen. Im Rahmen dieser Analyse soll bewusst auf eine Analyse der Zwischenstufe „Response, aber ohne Buchung" verzichtet werden. Aus Unternehmenssicht erscheint primär die Frage relevant, welche Treiber nicht reagierende Personen zu einer Buchung veranlassen. Als Grundgesamtheit der Untersuchung kommen sämtliche Personen in Frage, die der Gruppe der „Nicht-Reagierer“, d. h. der Personen, die nicht im Call-Center angerufen haben, um sich zu informieren bzw. um zu buchen, sowie der Gruppe der „Bucher“ angehören. Auf Basis der Ergebnisse der Signifikanztests aus Kapitel 3.2 werden hierbei insgesamt zehn exogene Determinanten sowie zwei endogene Determinanten in die Analyse einbezogen (vgl. Abb. 27).

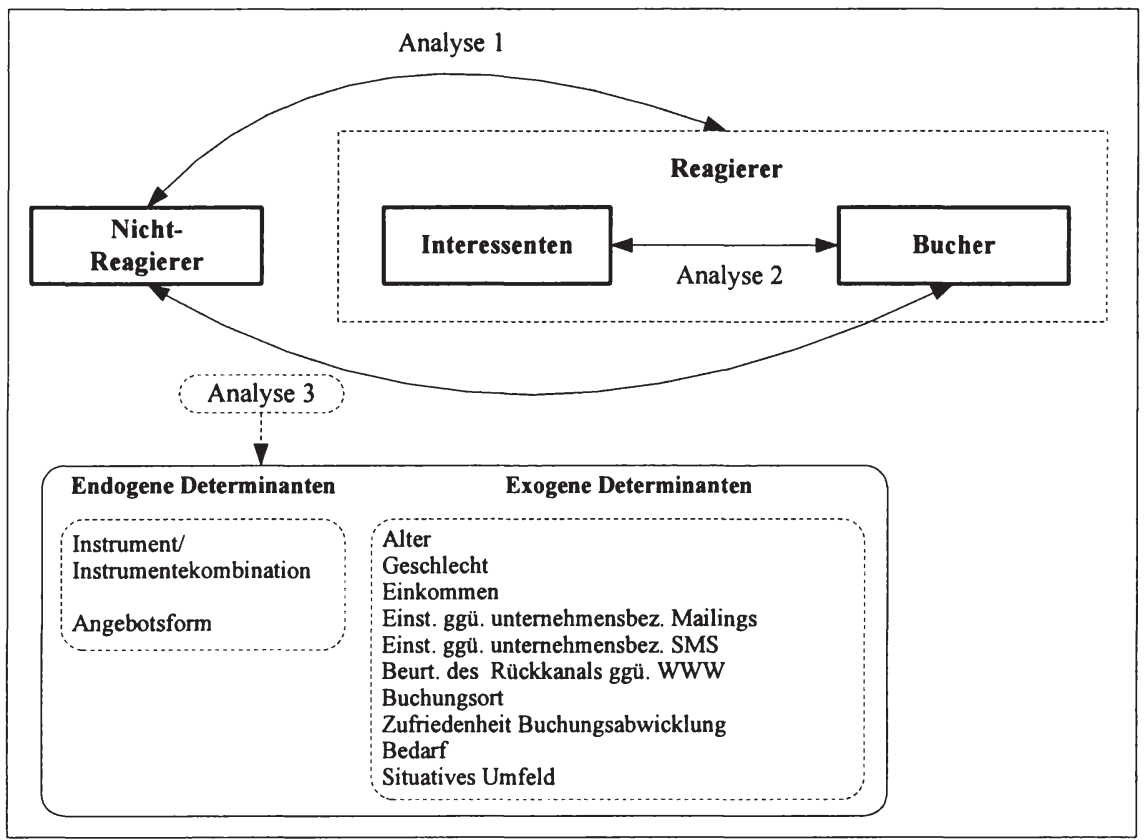

Abb. 27: Analysierte endogene und exogene Variablen im Hinblick auf das Buchungsverhalten (Analyse 3) 
Von den insgesamt 798 Beobachtungen konnten in der dritten Logistischen Regression 377 Fälle verwendet werden. ${ }^{564} 284$ der ausgewählten Fälle (75,3 \%) sind hierbei der Gruppe Nicht-Reagierer zuzuordnen, die weder im Call-Center angerufen noch gebucht haben, während 93 Fälle $(24,7 \%)$ der Gruppe der Bucher zuzuordnen sind. Wiederum wurden sämtliche Beobachtungen von der weiteren Betrachtung ausgeschlossen, die fehlende Werte bei einer der zwölf unabhängigen Variablen aufwiesen. Mittels dieses Vorgehens wurden letztendlich ca. $53 \%$ der Fälle ausgeschlossen. Als notwendige Voraussetzung zur Durchfuhrung einer Logistischen Regression wurden die unabhängigen Variablen wiederum auf Multikolliniearität getestet. In diesem Kontext wurden keine Korrelationen festgestellt. ${ }^{565}$ Auf eine detaillierte Darstellung der Ergebnisse soll an dieser Stelle verzichtet werden. ${ }^{566}$ Auch beim dritten Modell auf Basis der Logistischen Regression wurde die Einschluss-Methode gewählt und somit sämtliche der unabhängigen Variablen gleichzeitig in die Untersuchung aufgenommen. ${ }^{567}$

Im Vorfeld der Interpretation der einzelnen Koeffizienten erfolgt wiederum eine Überprüfung der Güte des Gesamtmodells. Für das Gesamtmodell ergibt sich eine Devianz von 246,536. Wie im vorherigen Modell unterstreicht der Likelihood-Quotienten-Test die gute Anpassung des Modells. Nach Einbeziehung sämtlicher unabhängiger Variablen sinkt die Devianz des Null-Modells von 421,232 auf 246,536. Die absolute Differenz der Devianz beträgt 174,696 und ist bei einer Irrtumswahrscheinlichkeit von $<1 \%$ signifikant. Dies bedeutet, dass die unabhängigen Variablen wiederum eine signifikant verbesserte Modellgüte ggü. dem Nullmodell ermöglichen. Wird die Devianz des Nullmodells bzw. des Gesamtmodells durch -2 dividiert und in die Gleichung für Pseudo- $\mathbf{R}^{2}$ eingesetzt,

564 Voraussetzung für die Einbeziehung einer Beobachtung in die Analyse ist das Vorliegen eines Fragebogens sowohl aus der Ex-post-Befragung als auch aus der Exante-Befragung, da aus beiden Fragebögen Angaben benötigt werden. Die Zahl von 798 Beobachtungen ergibt sich durch die Anzahl von Personen, die beide Fragebögen ausgefüllt haben. Die entsprechende Reduzierung auf 377 Fälle ergibt sich durch den Ausschluss von Beobachtungen mit fehlenden Werten.

565 Die Analyse der Korrelationen zwischen metrisch-skallierten Variablen wurde hierbei auf Basis des Pearson'schen Korrelationskoeffizienten untersucht. Korrelationen zwischen nominal-skalierten Variablen konnten mittels des Konfidenzkoeffizienten abgebildet werden. Auch hierbei wurden keine kritischen Korrelationen festgestellt. Der Zusammenhang zwischen metrisch-skalierten und nominal-skalierten Variablen konnte auf Basis von Plausbilitätsüberlegungen zum größten Teil ausgeschlossen werden. Vgl. Janssen, J., Laatz, W., Statistische Datenanalyse mit SPSS für Windows, a. a. O., S. $234 \mathrm{ff}$.

566 In diesem Zusammenhang konnte auf Ergebnisse verschiedener Tests der Korrelationen aus den vorangegangenen Untersuchungen zurückgegriffen werden.

$567 \mathrm{Vgl}$. zur Begründung für ein derartiges Vorgehen die Ausfuhrungen im vorangegangenen Kapitel. 
ergibt sich ein Wert von 0,4147 , der als sehr gut anzusehen ist. ${ }^{568}$ Auch hinsichtlich der weiterhin verwendeten Gütemaße werden gute Modellanpassungen aufgezeigt. So liegt der Wert für Cox $\&$ Snells- $\mathbf{R}^{2}$ bei 0,371 und von Nagelkerkes$\mathbf{R}^{2}$ bei 0,551. Der letztgenannte Wert deutet sogar auf ein sehr gutes Modell hin. ${ }^{569}$

Auch die Klassifikationsmatrix der vorliegenden zweiten Logistischen Regression zeigt eine hohe korrekte Zuordnung i. H. v. 85,1 \% der Fälle. Sowohl das Maximal Chance Criterium (MCC) mit einem Wert von $75,3 \%$ als auch das Proportional Chance Criterium (PCC) mit einem Wert von $62,8 \%$ werden übertroffen, was die gute Prognosekraft des vorliegenden Modells sowie die allgemeine Modellgüte unterstreicht. In der folgenden Abbildung ist die Klassifikationsmatrix ersichtlich, die die prognostizierte Gruppenzugehörigkeit zu der tatsächlich beobachteten Gruppenzugehörigkeit ins Verhältnis setzt (vgl. Tab. 38).

\begin{tabular}{|l|l|l|l|}
\hline & Prognostizierte Gruppenzugehörigkeit & \\
\hline $\begin{array}{l}\text { Beobachtete Grup- } \\
\text { penzugehörigkeit }\end{array}$ & Nicht-Reagierer & Bucher & $\begin{array}{l}\text { Korrekte Klassi- } \\
\text { fizierung }\end{array}$ \\
\hline Nicht-Reagierer & 262 & 22 & $92,3 \%$ \\
\hline Bucher & 34 & 59 & $63,4 \%$ \\
\hline Insgesamt & & & $85,1 \%$ \\
\hline
\end{tabular}

Tab. 38: Klassifikationsmatrix des Modells zum Vergleich von Nicht-Reagierern und Reagierern

Da auch das dritte Modell auf Basis der Logistischen Regression als gut hinsichtlich sämtlicher Gütekriterien beurteilt werden kann, ist als nächster Schritt eine Interpretation der Ergebnisse möglich. In diesem Zusammenhang können die Logit-Koeffizienten hinsichtlich des Signifikanzniveaus sowie der Richtung ihres Einflusses wiederum direkt interpretiert werden. Die folgende Tabelle zeigt die Ergebnisse der Logistischen Regression für das Buchungsverhalten. Insgesamt werden von den zwölf eingeschlossenen unabhängigen Variablen sechs Variablen als signifikant für die abhängige Variable („Buchung ja/nein“) identifiziert (vgl. Tab. 39).

Auf Basis dieser Analyse konnte zunächst der Einfluss einzelne Ausprägungen der beiden endogenen Determinanten nachgewiesen werden: demnach gestaltet sich auch im Rahmen einer weiteren Untersuchung des Buchungsverhaltens die Angebotsform mit den Ausprägungen „standardisiertes Angebot“ sowie ,ra-

568 Vgl. Urban 1993, S. 62 f.

$569 \mathrm{Vgl}$. Backhaus, K. et al., Multivariate Analysemethoden. Eine anwendungsorientierte Einführung, 10. Aufl., a. a. O., S. 448. 
battiertes Angebot" als signifikant relevant im Zusammenspiel sämtlicher Variablen. Während von der Auswahl eines standardisierten Angebots ein positiver Impuls in Richtung des Buchungsverhaltens ausgeht, beeinflusst die Auswahl eines rabattierten Angebotes - wenn auch nur schwach signifikant - die Buchungswahrscheinlichkeit negativ.

\begin{tabular}{|c|c|c|c|c|}
\hline \multicolumn{2}{|c|}{ Unabhăngige Variablen } & $\begin{array}{c}\text { LOGIT- } \\
\text { Koeffizient }\end{array}$ & $\begin{array}{c}\text { Signifi- } \\
\text { kanz }\end{array}$ & $\begin{array}{l}\text { Einfluss- } \\
\text { richtung }\end{array}$ \\
\hline \multicolumn{5}{|c|}{ Endogene Determinanten (Merkmale des Direktmarketing-Stimulus) } \\
\hline \multirow{3}{*}{ Angebotsform } & standardisiert & 0,7139 & * & + \\
\hline & individualisiert & $-0,0444$ & n.s. & \\
\hline & rabattiert & $-0,6695$ & $* *$ & - \\
\hline \multirow{7}{*}{ Instrument } & Mailing & 2,7042 & n.s. & \\
\hline & SMS & $-15,5579$ & n.s. & \\
\hline & Email & 2,1838 & n.s. & \\
\hline & Email + SMS & 2,7823 & n.s. & \\
\hline & Email + Mailing & 3,2755 & n.s. & \\
\hline & Mailing + SMS & 1,5419 & n.s. & \\
\hline & Mailing + Email + SMS & 3,0702 & ** & + \\
\hline \multicolumn{5}{|c|}{ Exogene Determinanten } \\
\hline \multicolumn{5}{|c|}{ Personenbezogene Determinanten (Merkmale der Rezipienten) } \\
\hline \multicolumn{2}{|c|}{ Geschlecht (weiblich) } & $-0,0892$ & n.s. & \\
\hline \multicolumn{2}{|c|}{ Altersklasse (unter 35 Jahre) } & 0,2538 & n.s. & \\
\hline \multicolumn{2}{|c|}{ Einstellungen ggü. unternehmensbezogenen Mailings } & $-0,0517$ & n.s. & \\
\hline \multicolumn{2}{|c|}{ Einstellungen ggu. unternehmensbezogenen SMS } & $-0,0611$ & n.s. & \\
\hline \multicolumn{2}{|c|}{ Zufriedenheit Buchungsabwicklung } & 0,4129 & ** & \\
\hline \multicolumn{2}{|c|}{ Beurteilung Call-Center ggü. WWW } & 0,4768 & * & + \\
\hline \multirow{3}{*}{ Einkommensklasse } & bis $€ 1.500$ & $-0,6434$ & * & - \\
\hline & $€ 1.501$ bis $€ 3.000$ & 0,4548 & ** & + \\
\hline & uber $€ 3.000$ & 0,1886 & *** & \\
\hline \multirow{6}{*}{ Buchungsort } & Call Center & 4,9019 & n.s. & \\
\hline & Newsletter & 6,9749 & n.s. & \\
\hline & Homepage & 6,0031 & n.s. & \\
\hline & am Flughafen & $-10,3554$ & n.s. & \\
\hline & im Reiseburo & 5,2969 & n.s. & \\
\hline & sonstiges & $-6,6378$ & n.s. & \\
\hline \multicolumn{5}{|c|}{ Situationsbezogene Determinanten } \\
\hline \multicolumn{2}{|c|}{ Bedarf zum Kontaktzeitpunkt: ja } & 0,4768 & * & + \\
\hline \multicolumn{2}{|c|}{ Situatives Umfeld zum Kontaktzeitpunkt } & 1,0780 & $*$ & + \\
\hline \multicolumn{5}{|c|}{$\begin{array}{ll}* & =5 \% \text { Signifikanzniveau } \\
* * & =10 \% \text { Signifikanzniveau } \\
\text { n.s. } & =\text { nicht signifikant } \\
+ & =\text { signifikant positiver Einfluss auf die Wahrscheinlichkeit, das Angebot zu buchen } \\
- & =\text { signifikant negativer Einfluss auf die Wahrscheinlichkeit, das Angebot zu buchen }\end{array}$} \\
\hline
\end{tabular}

Tab. 39: Empirische Analyse des Buchungsverhaltens auf Basis der Logistischen Regression (Analyse 3) 
Hinsichtlich des Einflusses des Trägermediums konnte in diesem Zusammenhang der auf Basis der Auswertung der feldexperimentellen Untersuchung aufgezeigte positive Einfluss des kombinierten Einsatzes mehrerer Direktmarketing-Instrumente auf die Buchungsquote bestätigt werden. Demnach erscheint insbesondere die Kombination sämtlicher der drei ausgewählten Direktmarketing-Instrumente einen positiven Einfluss auszuüben.

Hinsichtlich der Beurteilung des Responsekanals „Call-Center“ ggü. dem herkömmlichen Buchungsweg Internet zeigt sich ein signifikant positiver Einfluss zur Buchungswahrscheinlichkeit und unterstreicht damit den insgesamt wichtigen Einfluss der Trägermedien hinsichtlich der Buchungswahrscheinlichkeit. Dieser Aspekt korrespondiert mit dem Einfluss der Zufriedenheit mit der Buchungsabwicklung und dem Buchungsverhalten. Je positiver die Zufriedenheit mit der bisherigen Buchungsabwicklung, desto wahrscheinlicher die Buchung. Insgesamt bestätigt sich der postulierte Zusammenhang im Hinblick auf die unabhängige Variable „Einkommen“ und die abhängige Variablen „Buchung“, auf Basis derer mit steigendem Einkommen die Buchungswahrscheinlichkeit zunimmt. Hingegen besitzt der Buchungsort keinen signifikanten Einfluss auf das Rezipientenverhalten.

Ein drittes Mal konnte auch in dieser Analyse der signifikante Einfluss verschiedener situationsbezogener Determinanten nachgewiesen werden. Es wurde wiederholt nachgewiesen, dass die Bedarfssituation des Buchers dessen Bereitschaft determiniert, Flüge zu buchen. Darüber hinaus ist der Einfluss des situativen Umfelds auf die Buchungswahrscheinlichkeit wiederholt gegeben. Je positiver die Einschätzungen hinsichtlich des situativen Umfelds, desto höher die Wahrscheinlichkeit.

In der abschließenden Sensitivitätsanalyse erfolgte wiederum die gezielte Variation des Ausprägungsniveaus der signifikanten unabhängigen Variablen mit dem Ziel, die relative Einflussstärke dieser hinsichtlich der abhängigen Variablen sowie in Relation zueinander aufzuzeigen. Das Ausgangsniveau der Buchungswahrscheinlichkeit wurde wiederum auf Basis eines spezifischer Direktmarketing-Stimulus sowie entsprechender Merkmale des Rezipienten berechnet. Dieser Direktmarketing-Stimulus - bestehend aus einem individualisierten Angebot, welches mittels des Trägermediums Emails an die Rezipienten verschickt wurde - führt bei einem typischen „Nicht-Reagierer“ zu einer Buchungswahrscheinlichkeit von $6,41 \%$. Die signifikanten Variablen mit metrischen Skalenniveau wurden dabei durch die durchschnittlichen Werte der Bucher-Stichprobe ersetzt; die kategorialen Variablen wurden entsprechend der signifikanten Ausprägungen variiert. Die folgende Abbildung zeigt die Ergebnisse der Sensitivitätsanalyse (vgl. Abb. 28). 


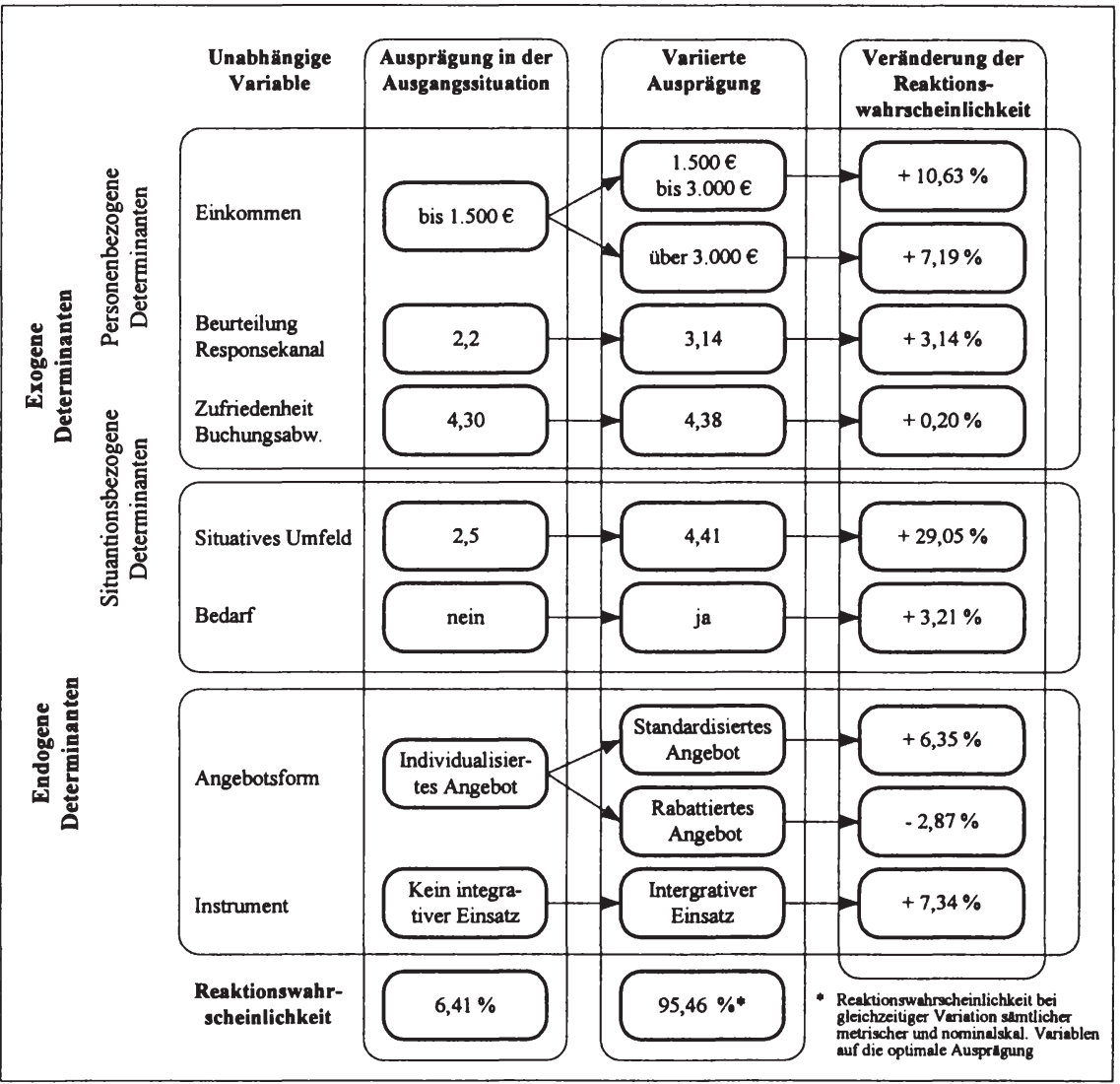

Abb. 28: Sensitivitätsanalyse der abhängigen Variablen im Rahmen der dritten Analyse

Auf Basis der Sensitivitătsanalyse zeigt sich der größte Einfluss auf die Buchungswahrscheinlichkeit - mit deutlichem Abstand vor der Variablen „Einkommensklasse“ - hinsichtlich des situativen Umfelds. An dritter Stelle erweist es sich als relevant, ob der Instrumenteeinsatz in kombinierter Form erfolgt. So steigt die Buchungswahrscheinlichkeit um mehr als $7 \%$, wenn anstelle des isolierten oder gepaarten Einsatzes von zwei Instrumenten sämtliche der drei Instrumente zum Einsatz kommen. Von untergeordneter Bedeutung erweisen sich hingegen tendenziell die personenindividuelle Bedarfssituation zum Zeitpunkt des Kommunikationskontaktes sowie die Zufriedenheit mit der Buchungsabwicklung. In diesem Zusammenhang besitzt die Angebotsform in Form des 
standardisierten Angebots einen positiven in Form des rabattierten Angebots hingegen einen negativen Einfluss.

\subsubsection{Synoptische Darstellung der Ergebnisse der integrativen Analyse}

Die verschiedenen durchgeführten Logistischen Regressionen verfolgten das Ziel, die Signifikanz, Einflussrichtung und Einflussstärke potenziell relevanter endogener und exogener Determinanten des Direktmarketing-Einsatzes auf verschiedene Ausprägungen des Rezipientenverhaltens zu analysieren. Im Vergleich zu den bisherigen Aussagen der Kapitel 3.1. und 3.2. ermöglicht der Einsatz der

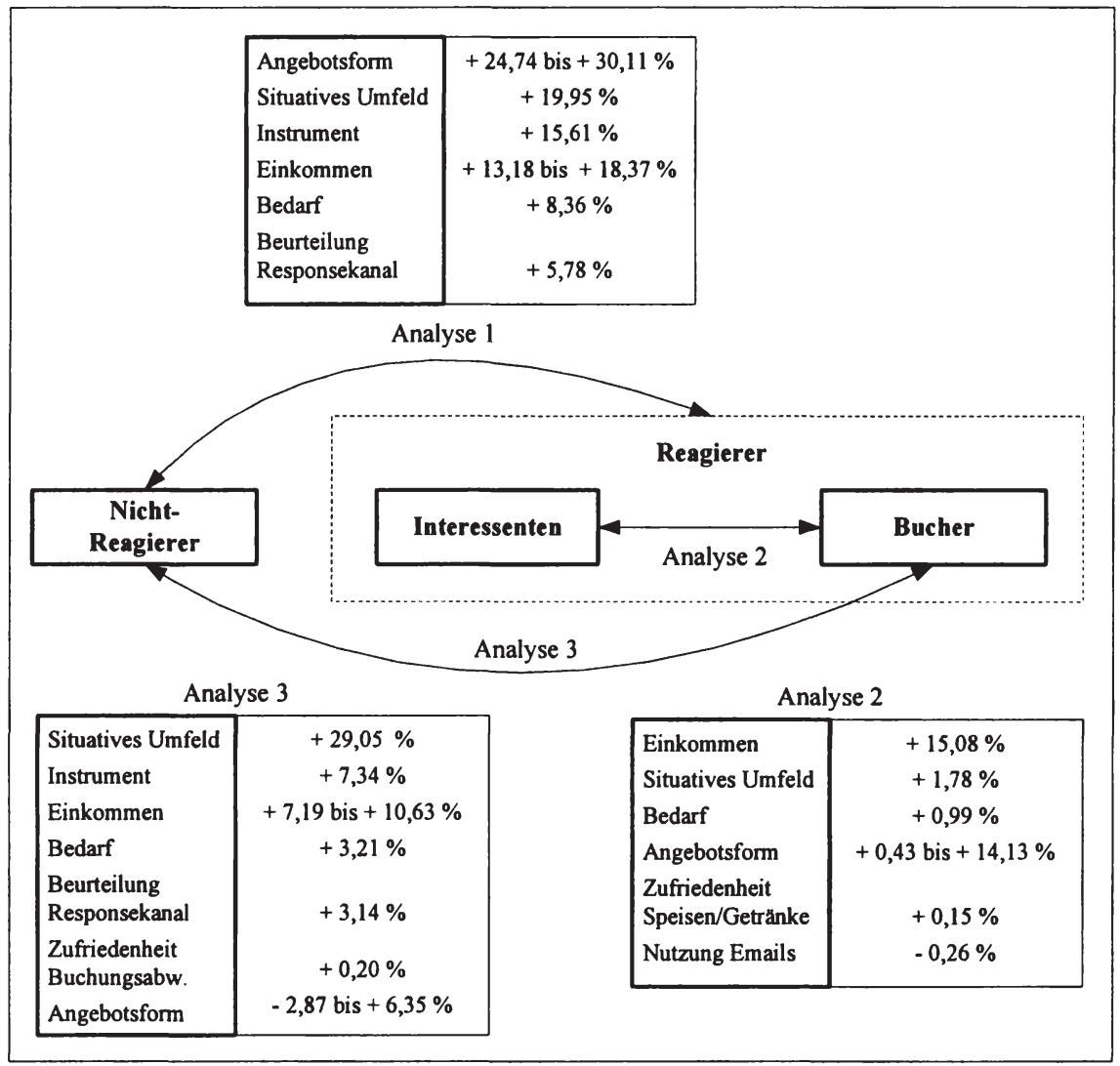

Abb. 29: Synoptische Darstellung der Einflussstärke der signifikanten endogenen und exogenen Determinanten auf Basis der drei Analysen 
Logistischen Regression Aussagen über die relative Bedeutung der verschiedenen Einflussfaktoren in Relation zueinander. Wenngleich die absoluten Höhen der drei durchgeführten Rechnungen auf Basis der Logistischen Regression aufgrund der unterschiedlichen Ausgangsniveaus der Reaktionswahrscheinlichkeit vor Durchführung der Signifikanztests nicht miteinander verglichen werden können, ermöglicht eine synoptische Darstellung der Untersuchungsergebnisse zumindest einen vergleichenden Blick auf die Bedeutung der unterschiedlichen Determinanten für die einzelnen Verhaltensreaktionen. In der Abbildung 29 sind die verschiedenen, als signifikant identifizierten, endogenen und exogenen Determinanten hinsichtlich ihrer Einflussstärke übersichtsartig dargestellt.

Zusammenfassend zeigte sich aufseiten der endogenen Determinanten der Einfluss der Angebotsform sowie aufseiten der exogenen Determinanten die Relevanz des Einkommens im Rahmen sämtlicher Analysen als relativ bedeutsam. Zwar schwankt die absolute Höhe des Einflusses der Angebotsform in den vorangegangenen drei Analysen erheblich, einheitlich zeichnet sich jedoch grundsätzlich der positive Einfluss der Verwendung der standardisierten Angebotsform auf die Reaktions- und Buchungswahrscheinlichkeit ab. 


\section{Zusammenfassung und Implikationen}

\section{Zusammenfassende Würdigung der Forschungsergebnisse}

Den Ausgangspunkt der vorliegenden Arbeit bildeten die strukturellen Veränderungen in der Kommunikationslandschaft der letzten Jahre und die damit verbundene Feststellung, dass die Leistungsfähigkeit der klassischen Mediawerbung hinsichtlich Wirksamkeit und Wirtschaftlichkeit stetig nachlässt. Als Konsequenz dieser Entwicklung hat sich der Einsatz des kommunikativen Instrumentariums zugunsten direkter Kommunikationsformen, insbesondere in Richtung von Emails, SMS und Postwurfsendungen, verschoben. So werden mittlerweile diese Direktmarketing-Instrumente aufgrund ihrer guten Skalierbarkeit von vielen Unternehmen nicht nur ergänzend zu den klassischen Kommunikationsinstrumenten im Rahmen von Sonderaktionen eingesetzt, sondern bilden immer häufiger einen zentralen Gestaltungsparameter des Marketing-Mix. In Verbindung mit der zum gegenwärtigen Zeitpunkt zu verzeichnenden branchenübergreifenden zunehmenden Wertorientierung des Direktmarketing und der gleichzeitig wachsenden wertmäßigen Anteile an den Kommunikationsbudgets stellt sich vermehrt die Frage nach der Effektivität und Effizienz beim Einsatz der verschiedenen Direktmarketing-Instrumente. Das übergeordnete Ziel der vorliegenden Arbeit bestand daher darin, einen Beitrag zur experimentellen Analyse der Wirkungen ausgewählter Direktmarketing-Instrumente zu leisten.

Bevor eine ökonomische Bewertung der durch den Einsatz der Direktmarketing-Instrumente erzielten Wirkungen beim Rezipienten möglich ist, erscheint eine detaillierte Auseinandersetzung mit dem Wirkungsprozess des Direktmarketing geboten. Als Grundlage dieser bedarf es zunächst einer begründeten Vorstellung darüber, welche Besonderheiten der Wirkungsprozess im Direktmarketing vor dem Hintergrund der unterschiedlichen Instrumente aufweist. Im Rahmen einer Bestandsaufnahme bisheriger Forschungsarbeiten zur Wirkungsweise der Direktmarketing-Instrumente wurde konstatiert, dass die vorliegenden konzeptionellen und empirischen Befunde lediglich Partialmodelle, jedoch keinen geschlossenen Rahmen zur Systematisierung, Erklärung und Beurteilung des Einflusses endogener, d. h. vom Unternehmen steuerbarer, sowie exogener Determinanten bereitstellen. Zudem fehlt es den bisherigen Arbeiten an einer konsistenten Verdichtung der zentralen Entscheidungstatbestände des DirektmarketingEinsatzes in einem integrierten Modell. 
Eine detaillierte Analyse der direktmarketingspezifischen Literatur machte darüber hinaus deutlich, dass in einigen zentralen Forschungsgebieten, die im Bereich des klassischen Marketing schon vielfältig untersucht wurden, im Direktmarketing bislang nur rudimentäre Erkenntnisse vorliegen. Zwar werden mit Hilfe von Intermediavergleichen Effektivitäts- und Effizienzunterschiede der klassischen Marketing-Instrumente und der Instrumente des Direktmarketing gegenübergestellt, jedoch gibt es noch immer Defizite bzgl. umfassender empirischer Intramediavergleiche mehrerer Direktmarketing-Instrumente, die sich auf mehr als eine effektivitätsbezogene Kennzahl, wie bspw. die Responsequote, beziehen. Gleichzeitig existieren gegenwärtig nur wenige Erkenntnisse zu den Wirkungen des kombinierten Einsatzes mehrerer Direktmarketing-Instrumente sowie den daraus resultierenden ökonomischen Konsequenzen. Und letztendlich lassen sich aus den bisherigen Arbeiten kaum Aussagen zur relativen Bedeutung endogener und exogener Determinanten hinsichtlich der Erreichung der gewünschten Wirkungen des Direktmarketing ableiten. Dabei könnten insbesondere valide empirische Aussagen zur relativen Bedeutung der verschiedenen Entscheidungstatbestände eine Orientierungshilfe darstellen, auf welche Felder sich die Optimierung des Direktmarketing-Einsatzes fokussieren sollte. In diesem Zusammenhang konzentrierten sich die folgenden Überlegungen und die empirische Untersuchung auf die Direktmarketing-Instrumente Postwurfsendung (Mailing), Email und SMS.

Trotz des Defizits bestehender Forschungsansätze konnten wichtige Hinweise hinsichtlich der Konzeption des zu entwickelnden Modells ermittelt werden. So konnte u. a. gefolgert werden, dass zur Modellierung der DirektmarketingWirkungen auf Erkenntnisse der klassischen Werbewirkungsforschung zurückzugreifen ist. Aus diesem Grund wurde ein theoretischer Bezugsrahmen mit dem Ziel abgeleitet, die Vielzahl der potenziellen Wirkungsgrößen zu systematisieren und eine Fokussierung auf die für das zu konstruierende Modell relevanten GröBen vorzunehmen. Diese Überlegungen wurden in Anlehnung an eine in der deutschsprachigen Literatur weit verbreitete Systematisierung von Kommunikationswirkungen und -determinanten von Steffenhagen konkretisiert. Hierzu wurde die ursprüngliche Unterteilung in momentane Gedächtniswirkungen, dauerhafte Gedächtniswirkungen und Verhaltenswirkungen in den Bereich des Direktmarketing übertragen und verfeinert. Vor dem Hintergrund des in der Praxis häufig zu konstatierenden aktionistischen Charakters des Direktmarketing-Einsatzes erschien es angemessen, die Kategorie der Verhaltenswirkungen weiterführend in momentane und dauerhafte Verhaltenswirkungen zu unterteilen, um den zumeist kurzfristigen Zeithorizont der Zielwirkungen des Direktmarketing-Einsatzes in besonderem Maße zu berücksichtigen. Den Bezugsrahmen der Wirkungsweise 
des Direktmarketing-Einsatzes komplettierten stimulus-, personen- und situationsbezogene Determinanten.

Im dem sich anschließenden Teil der Arbeit wurden die einzelnen Elemente des aufgezeigten Bezugsrahmens vertiefend dargestellt und hinsichtlich ihres funktionalen Zusammenhangs analysiert. Anhand verschiedener Kriterien wurde zunächst die Eignung der vier identifizierten Zielgrößen des Wirkungsmodells als Effektivitätsmaßstab des Direktmarketing-Einsatzes diskutiert. Als Ergebnis des Analyseprozesses wurden zum einen dauerhafte Gedächtnis- und dauerhafte Verhaltenswirkungen aufgrund mangelnder Reagibilität und Steuerungskraft sowie zum anderen momentane Gedächtniswirkungen aufgrund ihres hohen Erhebungsaufwandes als untaugliche Zielsetzungen von der weiteren Betrachtung ausgeschlossen. Dagegen erfullten momentane Verhaltenswirkungen, bspw. in Form eines spontanen Response- oder Kaufverhaltens, die i. d. R. in kurzem zeitlichem Abstand zum Kontakt mit einem konkreten Direktmarketing-Stimulus stehen, sämtliche Anforderungen an geeignete Effektivitätsmaßstäbe und wurden daher als finale Zielgrößen der weiteren Arbeit ausgewăhlt.

Den momentanen Verhaltenswirkungen vorgelagert sind verschiedene Verarbeitungsstufen im Bereich der momentanen Gedächtniswirkungen, die jeweils als „Filterstufen“ mit Blick auf die Wirkung der drei Instrumente Mailing, Email und SMS interpretiert wurden. Zur Messung des Wirkungserfolgs der vom Unternehmen eingesetzten Direktmarketing-Stimuli, die zumeist aus einem konkreten Angebot bestehen und mit Hilfe eines direktmarketingspezifischen Trägermediums ubermittelt werden, existieren mit der Zustell-, der Response- und der Kaufquote verschiedene Kennzahlen, die sich entlang eines idealtypischen „Verhaltens-Funnels“ zur Messung der Effektivităt einsetzen lassen. In diesem Kontext wurde auch auf verschiedene Möglichkeiten zur Ermittlung der mit der Erreichung der verschiedenen Wirkungsstufen verbundenen Effizienz eingegangen. Als Effizienzmaße wurden die durch eine Belegung mit Kostengrößen zuvor identifizierten Effektivitätskennzahlen (Cost per contact, Cost per interest und Cost per order) sowie gewinn- und rentabilitätsbezogene Größen festgehalten. Als Besonderheit des Direktmarketing-Einsatzes konnte hierbei insbesondere die Möglichkeit zur Berechnung kundenindividueller Effektivitäts- und Effizienzkennzahlen herausgearbeitet werden.

In den sich anschließenden Ausführungen der Untersuchung erfolgte eine Analyse der endogenen Determinanten sowie ihres Einflusses im Rahmen des Wirkungsprozesses. Um die Vielzahl vom Unternehmen steuerbarer Determinanten systematisch darzustellen und ihre Relevanz im Rahmen des Wirkungsprozesses aufzuzeigen, wurden zunächst die einzelnen Faktoren auf Basis eines operativ ausgerichteten Direktmarketing-Entscheidungsprozesses dargestellt, dessen Ziel die Konfiguration eines möglichst effektiven und effizienten Direkt- 
marketing-Stimulus bildet. Anhand der identifizierten Entscheidungsstufen wurde der Einfluss zielgruppen-, angebots-, medien- sowie gestaltungsbezogener Determinanten auf die Wirkungsweise der drei ausgewählten Instrumente diskutiert.

Als besonders relevant für die empirische Untersuchung der endogenen Determinanten erwies sich hinsichtlich der potenziellen Zielgruppe eines Direktmarketing-Stimulus die Unterscheidung in Bestands- und Neukunden. Aufgrund der primär angestrebten momentanen Verhaltenswirkungen bei Bestands- und Neukunden wurde der Fokus möglicher angebotsbezogener Determinanten auf den Bereich des verkaufsorientierten Direktmarketing-Einsatzes gelegt, der den kurzfristigen Absatz von Produkten und Dienstleistungen anstrebt. Als relevante Ausprägungen für die empirische Analyse wurden mit den standardisierten, den individualisierten sowie den rabattierten Angeboten drei zentrale Angebotsformen abgeleitet, die im Direktmarketing zu den häufigsten Erscheinungsformen zählen. Im Rahmen der Untersuchung medienbezogener Determinanten wurde der Einfluss des isolierten und kombinierten Einsatzes der drei Trägermedien sowie des Responsekanals auf die Effektivität und Effizienz des DirektmarketingEinsatzes analysiert.

Nach der konzeptionellen Untersuchung der endogenen Determinanten des Direktmarketing-Einsatzes erfolgte die Analyse des Einflusses exogener Determinanten. $\mathrm{Zu}$ diesem Zweck wurde auf personen- und situationsbedingte Einflussfaktoren vertiefend eingegangen. Neben soziodemographischen Faktoren, wie Alter, Geschlecht, Beruf und Einkommen konnte bei der Betrachtung personenbedingter Faktoren der Direktmarketing-Wirkung detailliert auf die Rolle psychographischer und verhaltensorientierter Rezipientenmerkmale eingegangen werden. Ergänzt wurde die Untersuchung der exogenen Determinanten durch die Analyse des Einflusses des situativen Umfelds während der Botschaftsaufnahme und-verarbeitung.

Die gewonnenen Erkenntnisse über die Zusammenhänge der einzelnen Zielwirkungen, Teilwirkungen und Wirkungsdeterminanten wurden abschließend in einem integrierten Modell zur Beurteilung der Effektivität und Effizienz des Direktmarketing zusammengefuhrt. Insgesamt ergab sich auf Basis der vorangegangenen Ausführungen ein Modell, das den komplexen Wirkungsmechanismus des Direktmarketing in einer transparenten Struktur widerspiegelte und hierbei die zentralen Einflussfaktoren berücksichtigt.

Auf der Grundlage der konzeptionellen Überlegungen bestand ein weiteres Ziel der Arbeit darin, im Rahmen einer (feld-)experimentellen Untersuchung die Wirkungen der drei ausgewählten Direktmarketing-Instrumente zu untersuchen und Erkenntnisse hinsichtlich der identifizierten Forschungsdefizite zu gewinnen. Im Mittelpunkt der empirischen Untersuchung stand die Analyse der Effektivität- 
und Effizienz verschiedener Direktmarketing-Aktionen eines Unternehmens aus dem Verkehrsdienstleistungsbereich, das dem Segment der sog. Low Cost Airlines zuzuordnen ist. In verschiedenen Direktmarketing-Aktionen wurden insgesamt 2.800 Personen mit realen Angeboten des Unternehmens beworben und die Reaktionen dieser im Hinblick auf die unterschiedlichen Stimuli gemessen. Ergänzt wurde die Untersuchung durch die Auswertung zweier Befragungswellen, die sich auf die besagten Direktmarketing-Aktionen bezogen. In diesem $\mathrm{Zu}-$ sammenhang konnten zusätzliche Daten über mögliche Wirkungszusammenhänge zum einen durch eine Ex-ante-Befragung, an der ca. 3.300 Personen im Vorfeld der Experimente teilnahmen, zum anderen durch eine Ex-post-Befragung von ca. 800 der teilnehmenden Personen generiert werden und in die Analyse einfließen.

Die Auswertung der empirischen Untersuchung basierte auf einem dreigeteilten Vorgehen. In einem ersten Schritt erfolgte die Analyse der Rezipientenreaktionen auf 28 versendete Direktmarketing-Stimuli, die jeweils an eine Gruppe von 100 Rezipienten verschickt wurden. Als zentrale Stellhebel der Konfiguration der einzelnen Stimuli lagen mit der Zielgruppenauswahl (Bestandskunden vs. Neukunden), mit der Angebotsform (standardisiertes vs. individualisiertes vs. rabattiertes Angebot) sowie hinsichtlich der eingesetzten Trägermedien (Mailing, Email und/oder SMS) drei Entscheidungstatbestände vor, die es als experimentellen Input der Untersuchung zu variieren galt. Der Schwerpunkt der Untersuchung lag gemäß den Zielsetzungen der vorliegenden Arbeit auf der Analyse der drei eingesetzten Instrumente, wobei neben dem isolierten Einsatz auch der paarweise bzw. der kombinierte Einsatz sämtlicher Instrumente analysiert wurde. Die Zusammensetzung der 28 Testgruppen erfolgte anhand von drei Strukturmerkmalen, von denen angenommen wurde, dass sie einen Einfluss auf die Buchungswahrscheinlichkeit besitzen. Durch die Berücksichtigung dieser Merkmale konnten personenbezogene Einflussfaktoren auf die Wirkungen der einzelnen Stimuli reduziert und damit die beobachteten residual gemessenen Wirkungsunterschiede primär der Variation eines der drei Stellhebel zugeschrieben werden.

Insgesamt zeigte die Auswertung der Reaktionen im Rahmen der Effektivitätsanalyse der angesprochenen Personen auf die versendeten DirektmarketingStimuli ein im Vergleich mit herkömmlichen Direktmarketing-Aktionen überdurchschnittlich hohes Response- und Buchungsverhalten. Innerhalb der Analyse des Einflusses der Zielgruppenselektion fiel auf, dass sich sowohl die Bestandskundenbearbeitung als auch die Neukundenakquisition mit Hilfe des Direktmarketing-Einsatzes grundsätzlich effektiv gestalten lassen, $d$. h. die betrachteten Aktionen zu den gewünschten Rezipientenreaktionen führen. In einem direkten Vergleich der beiden angesprochenen Zielgruppen stellte sich allerdings die Bestandskundenbearbeitung als wesentlich effizienter heraus. Aufgrund der niedri- 
geren Response- und Buchungsquoten bei einer höheren Kostenbelastung, die primär aus dem Zukauf von Neukundenadressen resultierte, wurde im Rahmen der Neukundenakquisition sogar ein negativer Deckungsbeitrag realisiert.

Obwohl das standardisierte Angebot im Rahmen der Effektivitätsanalyse im Vergleich zum rabattierten und zum individualisierten Angebot höhere Responseund Buchungsquoten verzeichnete, gestaltete sich das individualisierte Angebot unter Effizienzgesichtspunkten - trotz höherer Konfigurationskosten - am vorteilhaftesten. In diesem Zusammenhang wurde zudem offensichtlich, dass die Preisbereitschaft im Rahmen des standardisierten Angebotes noch nicht ausgeschöpft scheint.

Die Ergebnisse der Analyse der Zielgruppen und der angebotsbezogenen Determinanten konnten hinsichtlich der eingesetzten Instrumente und Instrumentekombinationen verfeinert werden und in einen umfangreichen Intramediavergleich einfließen. Dabei erwies sich unabhängig von der Angebotsform das Mailing im isolierten Einsatz als das effektivste Trägermedium, während die SMS das effizienteste Trägermedium darstellte. Im integrierten Einsatz zweier Instrumente dominierte unter Effektivitätsgesichtspunkten die Kombination von Mailing und Email, die nur durch den Einsatz sämtlicher Instrumente übertroffen wurde. Insgesamt ergab die Analyse des Einflusses des integrativen Einsatzes mehrerer Direktmarketing-Instrumente, dass von einem koordinierten Einsatz mehrerer Direktmarketing-Instrumente ein positiver Einfluss auf die Responseund in der Folge auch auf die Buchungsquote ausgeht und durchschnittlich zu einer $10 \%$-igen Steigerung der Response- und in der Folge zu einer 3,5\%-igen Steigerung der Buchungsquote fürt. Mit den Effektivitätsvorteilen in besagter Höhe gingen allerdings durchschnittliche Kostensteigerungen zwischen $80 \%$ und $120 \%$ einher, was den isolierten Einsatz im Blick auf die kostenorientierten Effizienzkennzahlen zunächst vorteilhafter erscheinen ließ. Die Analyse der durchschnittlich erwirtschafteten Deckungsbeiträge zeigte jedoch, dass mit Hilfe des kombinierten Einsatzes mehrerer Instrumente ein durchschnittlich um $64 \%$ höherer Deckungsbeitrag erwirtschaft wurde. Zusammenfassend konnte im Rahmen des Intramediavergleichs kein dominantes Trägermedium, das sowohl unter Effektivităts- als auch unter Effizienzgesichtspunkten vorteilhafter ist, identifiziert werden.

Der zweite Schritt der empirischen Untersuchung widmete sich der Analyse des Einflusses exogener Determinanten in Form von personen- und situationsbezogenen Determinanten auf die Wirkungen des Direktmarketing-Einsatzes. In diesem Zusammenhang bestätigte sich der im Rahmen der Konfiguration der strukturgleichen Testgruppen postulierte Zusammenhang zwischen den Rezipientenmerkmalen Alter, Einkommen bzw. Reiseanlass und dem Response- bzw. Buchungsverhalten. Gleichzeitig konnten mittels verschiedener statistischer Auswer- 
tungsverfahren isolierte Zusammenhänge zwischen ausgewählten exogenen Determinanten und unterschiedlichen Ausprägungen des Response- und Buchungsverhaltens aufgezeigt werden. Als signifikant erwiesen sich einzelne demographische Merkmale wie Geschlecht, zahlreiche psychographische Personenmerkmale in Form von Einstellungen sowie allgemeine verhaltensorientierte Merkmale des Mediennutzungs-, des Informations-, Buchungs-, und Reiseverhaltens der Rezipienten. Ebenfalls konnte ein Zusammenhang zwischen verschiedenen situationsbezogenen Determinanten, wie bspw. der Bedarfssituation sowie dem situativen Umfeld des Rezipienten zum Zeitpunkt des Kommunikationskontaktes, und dem Response- und Buchungsverhalten nachgewiesen werden.

In einem dritten Schritt erfolgte auf Basis des Rechenansatzes der Logistischen Regressionen eine Untersuchung der relativen Bedeutung der zuvor detailliert analysierten endogenen und exogenen Determinanten des Response- und Buchungsverhaltens. Mit dem Ziel, die Signifikanz einzelner Faktoren im $\mathrm{Zu}-$ sammenspiel der wichtigsten endogenen und exogenen Determinanten sowie deren Einflussrichtung und -stärke hinsichtlich des Response- und Buchungsverhaltens aufzuzeigen, wurden die im zweiten Schritt der empirischen Untersuchung als signifikant identifizierten exogenen Determinanten sowie die endogenen Determinanten „Angebotsform“ und „Instrument bzw. Instrumentekombination" als unabhängige Variablen der einzelnen Modelle verwendet. Es zeigte sich, dass hinsichtlich der Entscheidung eines Rezipienten, auf einen ihm zugesandten Direktmarketing-Stimulus zu reagieren, insbesondere die Angebotsform, das Einkommen, das eingesetzte Instrument sowie das situative Umfeld von wesentlicher Bedeutung für die Reaktionswahrscheinlichkeit sind. Hinsichtlich der Frage, von welchen Faktoren abhängt, dass ein „Reagierer“, d. h. eine Person, die für ein konkretes Direktmarketing-Angebot bereits dem Unternehmen sein Interesse bekundet hat, letztendlich zum „Bucher“ wird, konnte ermittelt werden, dass dies wiederum in besonderem Maße von der Angebotsform sowie dem Einkommen des Rezipienten abhăngt. Andere Determinanten sind in diesem Prozess nur von untergeordneter Bedeutung. So spielen die verwendeten Instrumente ebenso wenig eine Rolle für die Entscheidung wie die Bedarfssituation oder situative Einflüsse. In der abschließenden Analyse der positiven Treiber, welche die Buchungswahrscheinlichkeit von Personen erhöht, die grundsätzlich der Gruppe der Nicht-Reagierer zuzuordnen sind, konnten die Angebotsform, das situative Umfeld sowie der dreifach kombinierte Einsatz der Direktmarketing-Instrumente identifiziert werden. Die so gewonnenen Erkenntnisse ermöglichen letztendlich eine Einordnung der durchgeführten Effektivitäts- und Effizienzvergleiche und verdeutlichen gleichermaßen die Relevanz exogener, zumeist nicht beeinflussbarer Wirkungsdeterminanten auf das Response- und Buchungsverhalten. 
Zusammenfassend kann konstatiert werden, dass mit den konzeptionellen Ausführungen zu einem integrierten Modell einem bisherigen Defizit der Direktmarketing-Forschung begegnet wurde. Die Stärke des vorliegenden Modells kann in einer Systematisierung vielfältigster Einflussfaktoren gesehen werden. Darüber hinaus konnten im Rahmen der empirischen Untersuchung zentrale endogene und exogene Determinanten der Effektivität und Effizienz des Direktmarketing-Einsatzes untersucht werden, was bis dato in einer (feld-)experimentellen Untersuchung in dieser Weise noch nicht erfolgte. Insbesondere der vorliegende empirische Intramediavergleich, der neben dem isolierten Einsatz der Instrumente auch die Integration mehrerer Trägermedien sowie zielgruppen- und angebotsbezogener Einflüsse berücksichtigte, liegt so noch nicht vor. Weiterhin ist zu erwähnen, dass im Gegensatz zu zahlreichen veröffentlichten experimentellen Wirkungsstudien keine wirklichkeitsferne Laborsituation, sondern eine Feldstudie unter realen Wirkungsbedingungen durchgeführt wurde. Zudem ist festzuhalten, dass durch die Konstanthaltung verschiedener, den Wirkungsprozess beeinflussender endogener und exogener Determinanten die gemessenen Verhaltenswirkungen zum Großteil auf den experimentellen Input sowie ausgewählte exogene Determinanten zurückzuführen sind. Neben der Bildung von strukturgleichen Testgruppen konnte bei keiner Person einer ebenfalls strukturgleichen Kontrollgruppe, die zeitlich parallel zu den Experimenten keinerlei Direktmarketing-Stimulus erhalten hatte, eine Buchung verzeichnet werden. Vielmehr wurde das zeitliche Umfeld der Aktionen so gewählt, dass ein möglichst geringes Einflusspotenzial durch weitere Direktmarketing-Aktivitäten gegeben war, was weiterhin die Validität der gemessenen Ergebnisse erhöht. Die abschließende Untersuchung der relativen Einflussstärke der betrachteten endogenen und exogenen Determinanten ermöglicht zusammenfassend ein umfassendes Bild des direktmarketing-spezifischen Wirkungsprozesses der drei ausgewählten Direktmarketing-Instrumente.

Im Rahmen einer kritischen Würdigung lassen sich ebenfalls Schwächen der konzeptionellen und empirischen Untersuchung identifizieren. Als Einschränkung hinsichtlich des Umfangs der einbezogenen Parameter muss konstatiert werden, dass nicht sämtliche, der im Rahmen des konzeptionellen Modells identifizierten Determinanten durch die zur Verfügung stehende Datenbasis analysiert werden konnten. Gleichzeitig erfolgte keine reale Messung der dem Verhalten vorgelagerten psychographischen Wirkungsprozesse, sondern lediglich eine Befragung der Rezipienten, was die Validität teilweise einschränkt. Ebenso besitzen die Aussagen zur Effizienz des Direktmarketing-Einsatzes in einigen Teilen keine Allgemeingültigkeit, da sich die Kostenstruktur des Direktmarketing-Einsatzes von Unternehmen zu Unternehmen unterschiedlich gestaltet. So können in diesem Zusammenhang lediglich Tendenzaussagen getroffen werden. 


\section{Spezifische Implikationen für die Optimierung des Direktmarketing- Einsatzes im untersuchten Unternehmen}

Im Anschluss an die Analyse der durchgeführten Direktmarketing-Aktionen stellt die Identifikation von spezifischen Maßnahmen, die zu einer Verbesserung der Marktbearbeitung mit Hilfe des Direktmarketing in dem untersuchten Unternehmen führen, einen ersten Teilschritt auf dem Weg zur Generierung allgemeiner Handlungsempfehlungen dar. In diesem Zusammenhang ermöglicht die Analyse der relativen Bedeutung der einbezogenen endogenen und exogenen Determinanten hinsichtlich des Response- und des Buchungsverhaltens zunächst eine Priorisierung der Handlungsfelder, auf die sich die folgenden Implikationen beziehen sollen.

Als wichtiger Treiber des Response- und des Buchungsverhaltens erwies sich aus Unternehmenssicht insbesondere die Angebotsform der versendeten Direktmarketing-Stimuli. Wie aus den Ergebnissen der Effektivitäts- und Effizienzanalyse ersichtlich wurde, gestaltete sich das standardisierte Angebot am responseund buchungsstärksten, was nicht zuletzt an der Vertrautheit der Bestandskunden mit dieser Angebotsform gelegen haben dürfte. Die bisherige Angebotspolitik sollte sich daher auch weiterhin dieser Angebotsform bedienen. Es zeigte sich allerdings auch, dass die maximale Preisbereitschaft für das standardisierte Angebot deutlich oberhalb der durchschnittlich realisierten Ticketpreise lag. Zur Steigerung des Umsatzes wäre demnach über eine Erhöhung der Ticketpreise bei den kurzfristig-verkaufsorientierten Angeboten - insbesondere für das standardisierte Angebot - nachzudenken, wobei allerdings nicht die Signalwirkung der 19-EuroAngebote bzgl. der Positionierung des Unternehmens übersehen werden darf.

Aufgrund der aufgezeigten ökonomischen Attraktivität der individualisierten Angebotsform, die sich im höchsten Deckungsbeitrag sämtlicher Angebotsformen niederschlug, ist weiterhin über eine dauerhafte Aufnahme dieser Angebotsform in das Leistungsangebot des Unternehmens nachzudenken. Eine Erhöhung der Preise im Rahmen der individualisierten Angebote erscheint im Vergleich zur standardisierten Angebotsform vor dem Hintergrund der maximalen Preisbereitschaft, die ebenfalls deutlich über den durchschnittlichen Ticketpreisen lag, tendenziell unproblematisch, da bei dieser Angebotsform keine eindeutige Preistransparenz gegeben ist. Für die dauerhafte Einführung individualisierter Angebote spricht zudem, dass die für die Konfiguration eines individualisierten Angebotes notwendigen Informationen (Anrede, Zieldestination, Preis), die im vorliegenden Experiment in der Ex-ante-Befragung erhoben wurden, auch ohne Durchführung einer Befragung der Kunden auf Basis der Personendaten sowie der kundenindividuellen Buchungshistorie aus der Datenbank des Unternehmens ermittelt und so ohne großen Aufwand berücksichtigt werden können. Die Höhe des 
Preises dieser individualisierten Angebote könnte sich nach spezifischen personenindividuellen Merkmalen richten, die sich als kaufverhaltensrelevant und im Zeitablauf stabil erwiesen haben. In diesem Zusammenhang wäre über eine Verwendung der Einkommensklasse des Rezipienten als Referenzgröße nachzudenken. Dies erscheint insbesondere deshalb sinnvoll, da der im Rahmen der Konfiguration der strukturgleichen Testgruppen angenommene Zusammenhang von Einkommen und Response- bzw. Buchungswahrscheinlichkeit in sämtlichen Analyseschritten nachgewiesen werden konnte. Für eine zukünftige Segmentierung der Bestandskunden sowie eine darauf aufbauende zielgruppenspezifische Angebotspolitik könnte daher in Abhängigkeit der identifizierten Einkommensklassen eine weitere Differenzierung der Leistungen erfolgen. Fraglich erscheint hierbei lediglich die Angabe des Einkommens durch den Rezipienten, insbesondere auf freiwilliger Basis.

Im Hinblick auf eine segmentspezifische Marktbearbeitung ist auch die in der vorliegenden Untersuchung verfolgte Strategie der Neukundengewinnung mit Hilfe des Direktmarketing kritisch zu analysieren. Aufgrund der hohen Kosten für die Adressanmietung in Verbindung mit einer geringen Buchungsquote bei gleichzeitig niedrigen Ticketpreisen resultierten aus der Neukundenakquisition lediglich geringe Umsätze sowie ein negativer Deckungsbeitrag. Die Zahl der Bucher blieb insgesamt gesehen mit acht neuen Kunden deutlich hinter den Erwartungen des Managements zurück. ${ }^{570}$ Unter ökonomischen Gesichtspunkten erscheint daher der Einsatz des Direktmarketing zur Ansprache neuer Kundengruppen mit dem Ziel des kurzfristigen Verkaufs von Tickets im untersuchten Unternehmen nicht geeignet. ${ }^{571}$ Soll an dem Ziel der Neukundenakquisition mittels des Einsatzes verkaufsorientierter Direktmarketing-Angebote festgehalten werden, ist über alternative Akquisitionsmaßnahmen nachzudenken. Ein erster Ansatzpunkt könnte in der Suche nach einer kostengünstigeren Möglichkeit zur Gewinnung von Neukundenadressen für den Einsatz unterschiedlicher Direktmarketing-Instrumente bestehen, da sich die Kosten für die Adressanmietung im Verhältnis zu den übrigen Kostenarten als unangemessen hoch darstellten. ${ }^{572} \mathrm{Ei}$ -

570 Intern hatte die Unternehmensleitung mit einer Buchungsquote von $10 \%$ kalkuliert.

571 Im Rahmen der durchgeführten Experimente kamen im Rahmen des Informationsmanagements sowohl selbst erhobene als auch gekaufte Adressdaten zum Einsatz. Während die Erstgenannten mit Hilfe der Ex-ante-Befragung erhoben wurden, erfolgte die Bereitstellung der zweiten Informationsbasis durch Kauf bei einem Adressanbieter.

572 In diesem Zusammenhang zeigte sich, dass auch die selbst erhobenen Daten im Rahmen der Ex-ante-Befragung - nach dem Durchlaufen unternehmensinterner Plausibilitätschecks - über eine nahezu identische Validität verfügten wie die gekauften Daten. Dies zeigte sich in ähnlich hohen Zustellquoten, d. h. erfolgreich versendeten und zugestellten Direktmarketing-Stimuli. So konnte mit den im Unternehmen aufbe- 
ne Sammlung von Adressen kann bspw. mit Hilfe von Promotionsaktionen wie Aktionsständen an Flughäfen oder mittels prämienbasierter Werber-Programme (,Kunden werben Kunden") erfolgen. Gleichzeitig ist darüber nachzudenken, ob sich das eingesetzte standardisierte Angebot grundsätzlich überhaupt zur Ansprache von Neukunden eignet. So ist es durchaus denkbar, dass die Versendung von konkreten Angeboten bei Unkenntnis des Absenders eher abschreckend wirkt. Als Alternative könnten bspw. Rabattgutscheine zum Einsatz kommen, die per Mailing, SMS oder Email an die Neukunden versendet werden. ${ }^{573}$

Neben dem nachgewiesenen hohen Einfluss der Angebotsform, besitzt auch die Auswahl des geeigneten Instruments eine relative hohe Bedeutung für die Response- und Buchungswahrscheinlichkeit. Der nachgewiesene negative Einfluss von Emails fuihrt allerdings lediglich zu der Empfehlung, im Rahmen des isolierten Einsatzes über eine alternative Verwendung von SMS oder Mailing nachzudenken. Grundsätzlich sollte sich die Auswahl und Gestaltung der Instrumente im Rahmen des kurzfristig-verkaufsorientierten Direktmarketing an den spezifischen Informations- und Buchungsbedürfnissen der Zielgruppen und an der Art der zu versendenden Angebote orientieren, aber auch die ermittelten Effektivitäts- und Effizienzunterschiede berücksichtigen. Je nach Priorisierung des Auswahlkriteriums empfiehlt sich daher der Einsatz unterschiedlicher Instrumente bzw. Instrumentekombinationen. Vor dem Hintergrund verschiedener Intramediavergleiche konnten in diesem Kontext erhebliche Effektivitäts- und Effizienzunterschiede der eingesetzten Instrumente Mailing, Email und SMS festgestellt werden. Aus diesem Grund lassen keine allgemeingültigen Aussagen zum isolierten Einsatz treffen. Hingegen bestätigte sich im Rahmen der logistischen Regression der positive Einfluss des integrativen Einsatzes sämtlicher der drei ausgewählten Direktmarketing-Instrumente. Vor dem Hintergrund der verschiedenen Kosten für die drei eingesetzten Trägermedien ist dabei zu prüfen, ob gegebenenfalls der mehrmalige Einsatz des gleichen Trägermediums ähnliche wirkungsverstärkende Effekte aufweist. Dies könnte u. U. zu Kostendegressionseffekten führen und damit die Effizienz erhöhen. Hierbei wäre auch das Timing des integrierten Einsatzes genauer zu untersuchen, um zusätzliche nicht genutzte Wirkungspotenziale zu identifizieren.

reiten Daten, die durch die Ex-ante-Befragung erhoben wurden, eine durchschnittliche Zustellquote von 98,9\% realisiert werden. Im Rahmen der empirischen Untersuchung ließ sich aufgrund der ohnehin gewährleisteten hohen Validität der gekauften Adressen im Rahmen der Neukundengewinnung sogar eine Zustellquote von 99,4 \% erreichen. Vgl. Kapitel C.2.2.1.1.

573 Der Vorteil gegenüber dem bisherigen Vorgehen würde darin bestehen, dass Gutscheine einen „echten“ monetären Vorteil suggerieren. Zudem kann der Konsument den Gutschein auf den von ihm präferierten Strecken zeitlich individuell einlösen. 
Gleichzeitig ist die Auswahl der einzusetzenden Trägermedien mit Blick auf die Ergebnisse der Analyse des Informations- und Buchungsverhaltens der Kunden zu modellieren. So ergibt sich aufseiten der Konsumenten ein dem Buchungsverhalten vorgelagerter mehr oder weniger intensiver Informationsprozess, der normalerweise über die Homepage des Unternehmens erfolgt. ${ }^{574}$ Aufgrund der hohen Personalkosten ist der alleinigen Einsatz eines Call-Centers - wie im Rahmen des vorliegenden Feldexperiments - als primäres Informationsmedium im „normalen“ Betrieb nicht anzustreben. Um trotzdem die in Zusammenhang mit dem rabattierten und dem individualisierten Angebot aufgetretenen Fragen beantworten zu können, sollte die Option auf eine persönliche Beratung bei dauerhafter Integration dieser Angebotsformen hingegen auch weiterhin angeboten werden. Alternativ wäre auch eine Erweiterung der Homepage um einen passwortgeschützten Bereich denkbar, bei dem der Kunde nach dem Vorbild verschiedener internetbasierter Informationsportale seinen Zugang zu Unternehmens- und Angebotsinformationen (Abflugzeiten, Abflugorte, Preise) nach persönlichen Präferenzen konfigurieren kann und auf diese Weise individuelle Informationen und Angebote vorfindet. Dieser Schritt würde die angestrebte Individualisierung der Angebots- und Informationspolitik ebenfalls kostengünstiger gestalten. Einen weiteren Ansatzpunkt im Rahmen des Informationsmanagements bietet die Erweiterung des vierzehntägig erscheinenden Email-Newsletter, der in standardisierter Form aktuelle Angebote an sämtliche der Abonnenten verschickt. Hier erscheint ebenfalls die Möglichkeit der Individualisierung der Inhalte geboten, um Angebot und Nachfrage besser als bisher zu synchronisieren und somit kurzfristige Buchungen zu forcieren. So wäre bspw. denkbar, eine Individualisierung des Newsletters auf Basis des gewünschten Flugziels, einer möglichen Preisobergrenze sowie einer bestimmten Personenanzahl sowie gegebenenfalls zusätzlich relevanter Reiseinformationen bzgl. Hotels, Mietwagen etc. vorzunehmen. ${ }^{575}$

Die bisherigen Ausführungen bezogen sich primär auf den kurzfristig-verkaufsorientierten Einsatz des Direktmarketing. Demgegenüber stehen verschiedene Möglichkeiten, die Instrumente des Direktmarketing vor dem Hintergrund langfristig-bindungsorientieter Ziele zu verwenden. Den meisten Kundenbin-

574 So informieren sich über $90 \%$ der Kunden auf der unternehmenseigenen Homepage, die damit das mit Abstand am meisten verwendete Informationsmedium darstellt.

575 Sollte die Ausweitung des Leistungsangebotes des Unternehmens um die beschriebenen Reiseleistungen erfolgen, ist der Einsatz der im Rahmen des Experiments verwendeten Trägermedien kritisch zu diskutieren, da mit dem Verkauf weiterer Leistungen das Informationsbedürfnis des Rezipienten potenziell steigt. Wie im Rahmen der empirischen Untersuchung gezeigt, erscheint der Einsatz von SMS daher ggf. gar nicht oder nur in Kombination mit den anderen Trägermedien geboten. 
dungsmaßnahmen ist allerdings gemein, dass sie im Gegensatz zu den kurzfristigverkaufsorientierten Angeboten i. d. R. keinen direkten Einfluss auf die Erlössituation des Unternehmens besitzen, sondern zunächst lediglich Kosten verursachen. Ihr Erfolgsbeitrag liegt in den meisten Fällen in der positiven Beeinflussung der der emotionalen Bindung an das Unternehmen vorgelagerten psychographischen Größen, wie etwa der Zufriedenheit mit Unternehmensleistungen oder Einstellungen gegenüber dem Unternehmen. Darüber hinaus kann sich Kundenbindung auch in faktischer Kundenbindung manifestieren, die sich bspw. in mit einem Wechsel des Unternehmens verbundenen Wechselkosten niederschlägt.

Der letztgenannte Aspekt verdeutlicht zunächst Ansatzpunkte des Direktmarketing-Einsatzes der klassischen Airlines und erfordert eine Diskussion der Übertragbarkeit in den Bereich der Low Cost Airlines. Zu den wichtigsten Kundenbindungsinstrumenten zählt hierbei der Einsatz von kartengebundenen Bonusprogrammen nach der Art des Miles \& More-Programms der Deutschen Lufthansa AG. Der Anreiz aus Sicht des Kunden besteht in der Sammlung so genannter Bonusmeilen, die ihm bei jedem Flug auf seinem Meilenkonto gutgeschrieben werden und die der Inhaber des Kontos nach Erreichen bestimmter Meilengrenzen in Produkt- oder Dienstleistungsprämien umtauschen kann. Aus Sicht des untersuchten Unternehmens läge ein Vorteil des Einsatzes einer derartigen Kundenkarte zum einen in dem beabsichtigten Bindungsaspekt, der sich für gewöhnlich durch derartige Bonussysteme einstellt, sowie zum anderen in einer vereinfachten Sammlung, Speicherung und Auswertung kundenindividueller Informationen. Hinsichtlich eines Einsatzes im untersuchten Unternehmen wäre allerdings die Vorteilhaftigkeit eines derartigen Programms unter ökonomischen Gesichtspunkten zu prüfen, insbesondere vor dem Hintergrund notwendiger Investitionen in Informationstechnologien sowie Prämien. ${ }^{576}$

576 Auf Basis der auf den Karten gespeicherten zusätzlichen Informationen bzgl. des Buchungs- und Reiseverhaltens in Verbindung mit den persönlichen Angaben bei Beantragung einer Karte entstehen Ansatzpunkte für eine differenzierte Bearbeitung der Bestandskunden. Würde ein derartiges Programm eingeführt, ließen sich durch entsprechende Kooperationen mit anderen Unternehmen die Einsatzmöglichkeiten der Karten erweitern und eine Meilensammlung auch durch Käufe bei anderen Unternehmen möglich sein. Dies könnte den Bindungsaspekt ebenso positiv beeinflussen. Neben der Bestandskundenbindung kann dieser Aspekt auch auf die Neukundenakquisition übertragen werden. So werden häufig in Kooperation mit Kreditkartengesellschaften Bonusprogramme kombiniert und den Kunden unabhängig davon, ob sie bereits Kunden des untersuchten Unternehmens sind, kostenlos zur Verfügung gestellt. Für den Einsatz eines Vielfliegerprogramms nach Art von Miles \& More spricht ebenfalls die relativ geringe Diffusion von Bonusprogrammen anderer Airlines unter den Bestandskunden des Unternehmens. So besitzen lediglich $35 \%$ der befragten Kunden eine Lufthansa Miles \& More-Karte. 
Im Rahmen der durchgeführten Experimente kam mit dem rabattierten Angebot bereits ein Angebot zum Einsatz, dass den Kauf von vier Tickets durch einen Rabatt von $25 \%$ incentivierte. Es zeigte sich, dass die durchschnittlich gekaufte Ticketanzahl ohnehin bei ca. vier Tickets pro Bucher lag, da die meisten Kunden zu zweit reisen und $99 \%$ der Kunden i. d. R. die Tickets für Hin- und Rückflug zeitgleich erwerben. Zu diskutieren ist in diesem Zusammenhang eine Erhöhung der Ticketanzahl, bspw. von vier auf acht Tickets, welche ggf. mit einem Mengenrabatt von $30 \%$ verbunden sein könnte. Die aus der längerfristigen Prädisposition der Flüge resultierende ,zeitliche“ Bindung an das Unternehmen könnte für weitere, bspw. auf Cross-Selling abzielende Aktivitäten genutzt werden.

Darüber hinaus ist der langfristig-bindungsorientierte Einsatz des Direktmarketing im Bereich des Informationsmanagements zu diskutieren. In Zusammenhang mit entsprechenden Buchungsinformationen lassen sich mit Hilfe der Instrumente des Direktmarketing zusätzliche Informationsservices anbieten, die bereits bei anderen Airlines bzw. in anderen Branchen zum Einsatz kommen. So kann der Kunden auf Wunsch eine bestimmte Zeit vor dem Abflug eine Bestätigungsnachricht über die genaue Flugzeit und das Abflugterminal erhalten oder auf verspätete Flüge hingewiesen werden. Prädestiniert für derartige Informationsservices ist der Einsatz von SMS, da die permanente Erreichbarkeit der Personen gegeben ist und der Erhalt der Nachricht nicht an einen Computer o. ä. gebunden ist.

Aufgrund der hohen Bedeutung situativer Komponenten sind weitere Informationen zur Empfangssituation der Rezipienten zu erheben, die im Rahmen der vorliegenden Untersuchung aus forschungsökonomischen Gründen nicht beachtet werden konnten. Dies betrifft zum einen eine qualitative Analyse der situativen Umstände während des Empfangs des Direktmarketing-Stimulus (insbesondere bei SMS und Emails) und zum anderen eine Analyse der Bedeutung des Timings des Direktmarketing-Einsatzes hinsichtlich Uhrzeit, Wochentag, Monat, Jahreszeit etc. Derartige Erkenntnisse sind im Rahmen weiterer unternehmensbezogener Tests zu eruieren und in die Aktionsplanung einzubeziehen. Fraglich bleibt die Relevanz gestalterischer Aspekte im Gesamtzusammenhang der Untersuchungsergebnisse, die ebenfalls mit Hilfe von Tests untersucht werden sollte. 


\section{Allgemeine Implikationen für den Direktmarketing-Einsatz und die Effek- tivitäts- und Effizienzmessung}

Während die Ableitung spezifischer Implikationen, die streng genommen nur für das betrachtete Unternehmen Gültigkeit besitzen, primär auf die empirischen Befunde der experimentellen Untersuchung rekurrierte, basieren die allgemeinen Implikationen im Wesentlichen auf den konzeptionellen Überlegungen der Untersuchung sowie auf dem eingesetzte Methodenspektrum.

Die bisherige Direktmarketing-Forschung liefert eine Vielzahl von empirischen Ergebnissen zur Analyse der Effektivität und Effizienz des Direktmarketing-Einsatzes auf Basis unterschiedlicher experimenteller Untersuchungen. Während die Erkenntnisse der verschiedenen Untersuchungen zum Entstehen zahlreicher partialanalytischer Modelle beitrugen, erweitert die vorliegende Arbeit den Fokus. So ermöglichen die Untersuchungsergebnisse nicht nur den Vorteilhaftigkeitsvergleich im Hinblick auf einzelne endogene Entscheidungstatbestände, sondern stellen einem Direktmarketing einsetzenden Unternehmen darüber hinaus auch ein Systematisierungsraster direktmarketingbezogener Entscheidungen bereit. Beim Einsatz des Direktmarketing sind demnach nicht nur Entscheidungen hinsichtlich der einzusetzenden Instrumente und deren gestalterischer Umsetzung zu treffen, sondern auch und insbesondere hinsichtlich zielgruppenund angebotsspezifischer Determinanten. Aufgrund des identifizierten hohen Einflusses personenbezogener Determinanten erweist sich - neben der Auseinandersetzung mit demographischen Merkmalen der Zielgruppe - eine Berücksichtigung psychographischer und verhaltensorientierter Kriterien unabdingbar für einen erfolgreichen Einsatz des Direktmarketing. Nur eine entsprechend ganzheitliche Sicht des Direktmarketing erlaubt dabei eine zielführende Marktbearbeitung.

Die Analyse des Zusammenspiels zentraler endogener und exogener Determinanten - wie sie in der vorliegenden Arbeit durchgeführt wurde - ermöglicht Aussagen über die Einflussstärke einzelner Determinanten innerhalb des direktmarketingspezifischen Wirkungsprozesses. In Verbindung mit der aufgezeigten kontakt- und wirkungsbasierten Effektivitäts- und Effizienzmessung können Direktmarketing einsetzenden Unternehmen Probleme oder Störfaktoren im Wirkungsprozess identifizieren und entsprechend der jeweiligen Relevanz im Rahmen des Entscheidungsprozesses fokussieren.

Die Verwendung eines derartigen Kennzahlensystems erscheint jedoch nur sinnvoll, wenn ein Unternehmen über eine adäquate Datenbasis verfügt. Neben der kontinuierlichen Erhebung direktmarketingspezifischer Aktions- und Reaktionsdaten im Sinne eines umfassenden Responsemanagements, erfordert dies eine grundlegende Untersuchung der branchenspezifschen Charakteristika des Direkt- 
marketing-Wirkungsprozesses. Im Vorfeld des kontinuierlichen Einsatzes eines derartigen Systems bedarf es allerdings der Konfiguration eines robusten Modells, das die Einflussrichtung und Einflussstärke zentraler endogener und exogener Determinanten beinhaltet. Hierbei stellen die Durchführung von feldexperimentellen Untersuchungen, in der verschiedene Direktmarketing-Stimuli auf ihren Wirkungseinfluss hin untersucht werden, sowie eine Befragung der Rezipienten zur Erhebung der wichtigsten exogenen Determinanten in Form personenund situationsspezifischer Einflussfaktoren eine notwendige Voraussetzung des Einsatzes dar.

Während laborbezogenen Untersuchungen zumeist ein mangelnder Realitätsbezug vorgeworfen wird, erweist sich aber auch die Durchführung von Feldexperimenten nicht per se realitätsnäher. So gestaltet sich nicht nur die exakte Definition des experimentellen Inputs als entscheidender Parameter der Validität der Ergebnisse, sondern auch die Kontrolle der übrigen Einflussfaktoren als herausfordernd. Der Aufwand des vorliegenden Feldexperiments hat in diesem Zusammenhang aber gezeigt, dass die Ergebnisse als nahezu typisches Rezipientenverhalten anzusehen sind sowie keiner künstlichen Versuchssituation entstammen und damit einen hohen Praxisbezug aufweisen. Für direktmarketing-affine Unternehmen erweisen sich daher die Informationen derartiger (feld-)experimenteller Untersuchungen als überaus hilfreich bei der Gestaltung zukünftiger Direktmarketing-Aktionen. Aus Unternehmenssicht ist folglich eine zunehmende Verwendung sinnvoll, nicht zuletzt aufgrund der Tatsache, dass die ausgelösten Reaktionen reale Kaufakte darstellen können. Allerdings ist gleichzeitig darauf hinzuweisen, dass eine zu offensive „Test“-Politik des Unternehmens auch zu Verwirrung aufseiten der Konsumenten führen kann und daher nach situativer Abwägung einzusetzen ist.

In Zusammenhang mit der Analyse der Einflussstärke der verschiedenen endogenen und exogenen Faktoren im Rahmen des aufgezeigten Wirkungsprozesses erweist sich das Verfahren der logistischen Regression als zweckdienliche Analysemethode. So eignet sich das Verfahren insbesondere zur detaillierten Untersuchung dichotom ausgeprägter abhängiger Variablen. Der Einsatz der logistischen Regression in der Unternehmenspraxis setzt allerdings auch ein entsprechendes Methodenwissen sowie das Vorliegen valider empirischer Daten voraus.

\section{Ansatzpunkte für weiterführende Forschungsarbeiten}

Aufbauend auf den Erkenntnissen der vorliegenden Arbeit sollen abschließend Ansatzpunkte für weiterführende Forschungsarbeiten aufgezeigt werden. In Anbetracht ihrer theoretischen Konzeption und empirischen Anlage kommt der vor- 
liegenden Arbeit insbesondere hinsichtlich der betrachteten Zielwirkungen und Einflussfaktoren der Charakter einer Basisuntersuchung zu. Die Beantwortung vertiefender Fragestellungen muss weiteren, auf den dargestellten Ergebnissen und Implikationen aufbauenden Studien vorbehalten bleiben. Ausgehend von dieser Einschätzung lassen sich verschiedenen Ansatzpunkte identifizieren:

- Bei der Konstruktion des integrierten Modells zur Beurteilung der Effektivität und Effizienz des Direktmarketing-Einsatzes wurde hinsichtlich der Anzahl einzubeziehender Elemente nicht der Anspruch erhoben, sämtliche relevanten Ziel- und Einflussgrößen abzubilden. Eine Berücksichtigung weiterer psychographischer und verhaltensorientierter Konstrukte erscheint daher als Gegenstand zukünftiger Forschung wünschenswert.

- Im Rahmen der konzeptionellen Analyse der Zielwirkungen wurde zudem der Einsatz des Direktmarketing zur Beeinflussung dauerhafter Gedächtnisinhalte diskutiert, allerdings wurde aus forschungsökonomischen Gründen auf eine empirische Analyse verzichtet. Trotz der Existenz einzelner Forschungsansätze auf diesem Gebiet fehlt es an umfassenden empirischen intramedialen Vergleichen zu den Besonderheiten der Wirkungsweise verschiedener Direktmarketing-Instrumente. Das Ausmaß der Beeinflussbarkeit dauerhafter Gedächtnisinhalte sollte zudem auch in Forschungsarbeiten zur Effizienz des Direktmarketing-Einsatzes vertiefend untersucht werden.

- Im Zuge der empirischen Untersuchung konnte der Einfluss zentraler endogenen Determinanten auf die Effektivität und Effizienz des DirektmarketingEinsatzes mit Ausnahme der Gestaltung untersucht werden. Obgleich eine Vielzahl von Forschungsarbeiten zu diesem Themenbereich zu finden ist, fehlt es an fundierten Aussagen, welche relative Bedeutung die Optimierung gestalterischer Elemente gegenüber der Zielgruppe-, Angebotsform- und Instrumenteauswahl besitzt. Somit besteht die Notwendigkeit vertiefender Analysen zur Wirkung der unterschiedlichen endogenen Einflussfaktoren und ihrer Bedeutung für die Zielerreichung des Direktmarketing-Einsatzes, um begründete Empfehlungen für die Bedeutung der Gestaltung in der Praxis treffen zu können.

- Die konzeptionelle und empirische Analyse der Effektivität und Effizienz des Direktmarketing-Einsatzes bezog sich ausschließlich auf die drei Instrumente Mailing, Email und SMS. Die Berücksichtigung weiterer von der Untersuchung ausgeklammerter Instrumente wie aktivem Telefonmarketing, Couponing oder interaktiven TV-Sendungen (DRTV) stellt vor dem Hintergrund der durchgeführten intramedialen Effektivitäts- und Effizienzvergleiche ein noch weitestgehend offenes Forschungsfeld dar, welches im Zuge zukünftiger Forschungsbemühungen vertiefend behandelt werden sollte. Gleichzeitig gilt es, kritisch zu prüfen, ob die verwendeten Zielgrößen in der 
vorgestellten Form auch im Rahmen derartiger Intramediavergleiche eingesetzt werden können oder weitere Beurteilungskriterien bereitgestellt werden sollten.

- Aufgrund der hohen Dynamik der technologischen Entwicklungen und der raschen empfängerseitigen Adaption neuer Technologien insbesondere im Bereich des Mobilfunks ist mit der steten Ausweitung der Anwendungsmöglichkeiten - insbesondere von SMS und Email - zu rechnen. Die Analyse der wirkungstheoretischen Grundlagen bleibt hierbei auch in Zukunft von elementarer Bedeutung, sollen hinreichend praxisrelevante Aussagen generiert werden.

- In den von der Betrachtung weitestgehend ausgeklammerten mehrstufigen Direktmarketing-Aktionen werden in Literatur und Praxis einstimmig zusätzliche Wirkungspotenziale vermutet, die in zukünftigen Untersuchungen zum Direktmarketing eine stärkere Berücksichtigung finden sollten. In diesem Zusammenhang kann auf Teile der vorliegenden Forschungsergebnisse zum integrativen Einsatz mehrerer Direktmarketing-Instrumente der vorliegenden Arbeit zurückgegriffen werden.

- Die Verallgemeinerungsfähigkeit der Effizienzuntersuchung sollte durch weitere Untersuchungen permanent weiter beleuchtet werden. So hängen die Aussagen zur Wirtschaftlichkeit des Direktmarketing-Einsatzes nicht zuletzt von einer Vielzahl von Faktoren ab, die eine Vergleichbarkeit zwischen verschiedenen Unternehmen erschweren. Gleichzeitig ist fraglich, welche Gültigkeitsdauer derartige Aussagen besitzen, da bspw. durch die Entwicklung neuer Produktionstechniken die Kosten zur Herstellung der Mailings bzw. die Versandkosten von Emails und SMS permanent sinken.

- Im Zuge der Analyse der Effizienz kommunikationspolitischer Maßnahmen werden immer häufiger auch mathematische Optimierungsverfahren wie die Data Envelopment Analyse (DEA) diskutiert, die eine Verdichtung verschiedener In- und Outputgrössen in eine eindimensionale Effizienzgröße zum Ziel haben. ${ }^{577}$ Wenngleich durch derartige Verfahren wertvolle Erkenntnisse zur Effizienzbeurteilung im Falle mehrerer Ziele und Zielgruppen gewonnen werden können, ist ihr Anwendungspotenzial für den Bereich des Direktmarketing kritisch zu überprüfen.

- Die vorgelegten empirischen Ergebnisse basieren auf der Untersuchung verschiedener Direktmarketing-Aktivitäten eines einzelnen Unternehmens und

577 Vgl. Luo, X., Donthu, N., Benchmarking Advertising Efficiency, in: Journal of Advertising Research, Jg. 41, Nr. 6, 2001, S. 7ff.; Greißinger, P., Wirtschaftlichkeitsanalysen im Gesundheitswesen. Analyse und beispielhafte Anwendung der Data Envelopment Analysis, Wiesbaden 2000. 
weisen somit den Charakter einer Einzellfallstudie auf. Dies gilt umso mehr, als dass das untersuchte Unternehmen aufgrund der Branche und der angebotenen Dienstleistung einen Spezialfall des Direktmarketing-Einsatzes darstellt. Zur Verfestigung und Verallgemeinerung der hier gewonnenen Erkenntnisse kann die Durchfuhrung ähnlicher Experimente mit dem Fokus auf anderen Produktklassen und Branchen zusätzliche Erkenntnisse liefern.

Als Ausblick bleibt festzuhalten, dass die Frage nach Effektivität und Effizienz des Marketing nicht nur in der klassischen Kommunikation, sondern vor allem auch im Direktmarketing vermehrt an Bedeutung gewinnt. Wissenschaft und Praxis haben diese Herausforderung erkannt und bieten in Folge des steigenden Bedarfs an Know-how eine Vielzahl von Veröffentlichungen, Konferenzen und Beratungsangeboten zum Themenbereich der Effektivitäts- und Effizienzanalyse im Direktmarketing. Zum aktuellen Zeitpunkt ist ein Ende der wachsenden Bedeutung des Direktmarketing nicht abzusehen. Nicht zuletzt aufgrund der Ergebnisse der vorliegenden Arbeit ist davon auszugehen, dass sich der Schwerpunkt von der gestalterischen Umsetzung des Direktmarketing zunehmend auf andere endogene und exogene Determinanten mit Einfluss auf die Effektivität und Effizienz des Direktmarketing-Einsatzes verschiebt. Vor diesem Hintergrund wird das Direktmarketing mit seinen heterogenen Instrumenten allerdings auch in $\mathrm{Zu}$ kunft beweisen müssen, dass es im Wettbewerb um knapper werdende Budgets eine sinnvolle Alternative zu klassischen Werbeformen darstellt. Die Forschung ist im Dialog mit der Praxis aufgefordert, weitere Anhaltspunkte zur begründeten Beantwortung dieser Fragestellung bereitzustellen. 
Michael Ahrens - 978-3-631-75048-3

Downloaded from PubFactory at 01/11/2019 08:52:24AM

via free access 


\section{Anhang}

\section{Anhang 1: Ex-ante-Fragebogen}

\begin{tabular}{|c|c|c|c|}
\hline $\operatorname{MCM} \frac{\pi}{5}$ & \multicolumn{3}{|c|}{$\begin{array}{l}\text { Woatfalische Wilhelma. Universitat Munster } \\
\text { Centrum für interaktives Marketing und Medienmanagement, } \\
\text { Prof. Dr. Dr. h. c. muk. H. Meffert }\end{array}$} \\
\hline \multicolumn{4}{|c|}{ Forschungeprojekt } \\
\hline \multicolumn{4}{|c|}{ "Direktmarketing im Verkehredienetleistungebereich" } \\
\hline \multicolumn{4}{|c|}{ 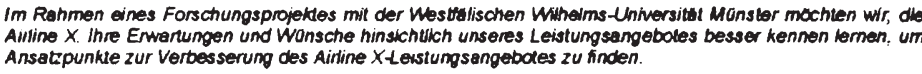 } \\
\hline \multicolumn{4}{|c|}{ Die Beentwortung des vorliegenden Fragebogens wird ca. 15 - 20 Min. beanspructen. } \\
\hline \multicolumn{4}{|c|}{$\begin{array}{l}\text { Der Fragebogen wird kurz vor Ende des fluges von den Intervewern wieder eingesammell, oder aber Sie } \\
\text { belassen thn an Ihrem Plalz. Sie konnen uns den Fragebopen aber gerne auch per Post zusenden. }\end{array}$} \\
\hline \multicolumn{4}{|c|}{ Bei Ruckfragen stehen Ithnen die Interviewer gerne zur Vertugung. } \\
\hline \multicolumn{4}{|c|}{$\begin{array}{l}\text { Fur alle Teilnehmer der Befragung hat Aintine } X \text { eine Uberraschung: Jeder Gast, oer einen Fragebogen } \\
\text { ausfülth, nimmt nicht nur an der Vertosung von }\end{array}$} \\
\hline \multicolumn{4}{|c|}{$5 \times 2$ Frelfiugen } \\
\hline \multicolumn{4}{|c|}{ nach London, Rom, Paris oder Mailand } \\
\hline \multicolumn{4}{|c|}{ 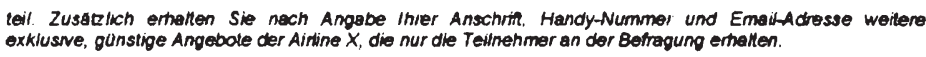 } \\
\hline \multicolumn{4}{|c|}{$\begin{array}{l}\text { Bilte beechten Sie, dass wir thre Handy-Nummer for die Gewinnbenachrichtigung benotigen. Wir werden Ihre } \\
\text { Daten selbstiverstandlich vertraulich behandeln. }\end{array}$} \\
\hline \multicolumn{4}{|c|}{ Dalenschutzhinwotse: } \\
\hline \multicolumn{4}{|c|}{$\begin{array}{l}\text { Alte Angaben sind freiwillig und werden ausschlieblich zur Verbesserung des Leistungsangebotes und der } \\
\text { Kundenbelreuung der Airtine } X \text { Gmbtt verarbeitet und genutzt }\end{array}$} \\
\hline \multicolumn{4}{|c|}{ Herzlichen Dank für Ihre Mithilfe! } \\
\hline \multirow{5}{*}{\multicolumn{4}{|c|}{ 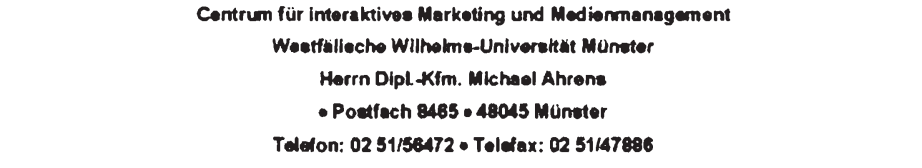 }} \\
\hline & & & \\
\hline & & & \\
\hline & & & \\
\hline & & & \\
\hline \multicolumn{4}{|c|}{ Vom Interviower auszuflallen: } \\
\hline Interviewer: & Datum: & Fhugnummer: & \\
\hline Rolsestrecke: von & Nach & Eingabe von: & Eingabedotum: \\
\hline
\end{tabular}


Zunächst möchten wr Ihnen elnige Fragen zu Ihren Erfahrungen mit Alrine X stelien!

1. Wodurch haben Sle zum ersten Mal vom Angebot von Alrline $X$ erfahren?

P Presseberichte

Reisemessen/Ausstellungen

Airline X Broschüe/Flyer

Plakate

Anzeigen in Zeitungen/Zeitschriften

Internet

a Freunde oder Bekannte

früher bereits Kunde der Eurowings $\mathrm{GmbH}$

a Hörunkwerbung

sonstiges

2. Wie oft sind Sle in den letzten 6 Monaten mit Alriline $X$ geflogen?

ce. mal

3. Welchen Prels haben Sle In etwa Im Durchschnitt für einen Alrline X-Flug (elnfache Strecke) bezahit?

ca. $\epsilon$

4. Wenn Sle regelmäßig mit Alrline $X$ filegen, welches waren die drel häuflasten Zlelorte?

1.

2.

3.
Anzahl Reisen (je einfacher Flug) ca.

Anzahl Reisen (je einfacher Flug) ca.

Anzahl Reisen (je einfacher Flug) $\mathbf{c a}$.

5. Nutzen Sie die Angebote von Airline $X$ Im Allgemeinen privat oder geschäftlich?

$\begin{array}{llll}\text { nur } & \text { eher } & \text { beides eher } & \text { nur } \\ \text { privat } & \text { privat } & \text { gleich häufig geschäftich geschäftich }\end{array}$

6. Aus welchen Gründen relsen Sle mit Alrilne $X$ (Mehrfachantworten moglich)?
U Urlaub (mehr als 3 Übernachtungen)
Kurzurlaub (1-3 Übernachtungen)
D Besuch von Verwandten/Bekannten
sonstiges:

Geschăftsreise

Fernpendler (z.B. Famille im Ausland)

7. Welches sind für Sle dle drel wichtlgsten Gründe, warum Sle slch für einen Flug mit AIrllne $X$ entschelden? Bltte vergeben Sie dle Rangplätze 1-3 (1 =wichtigster Grund, 2=zweitwichtigster Grund, 3=drittwichtigster Grund)!

_ gunstiger Preis

— gutes Preis- /Leistungsverhältnis

- kurze Anreise zum Flughafen

- gute Abllug- und Ankuntszeiten

- interessante Zielorte

- Service / Freundlichkeit der Mitabeiter

- Image

- einfache Abwicklung der Buchung und des Ticketerhalts

- habe bereits gute Erfahrungen gemacht

— sonstiges 
8. Auf welchen Wegen informieren Sie sich in der Regel vor dem Flug?

\begin{tabular}{|c|c|c|}
\hline Airline X Info-Schalter am Flughafen & $\begin{array}{c}\text { überwiegend } \\
\square\end{array}$ & $\begin{array}{c}\text { teilweise } \\
\square\end{array}$ \\
\hline Reisebáros am Flughafen Koln $/$ Bonn & 日 & 口 \\
\hline Sonstige Reiseburos & 口 & a \\
\hline Arlin $X$ Call Center & a & ä \\
\hline Airline X Homepage (www.Airline Xde) & a & $\bar{a}$ \\
\hline Airline X Newsietter I Emails & $\overline{0}$ & 미 \\
\hline Airline X Broschuren / Flugpläne / Flyer & a & ב \\
\hline Bel Freunden / Bekannten / Verwandten & $\bar{a}$ & a. \\
\hline Sonstige & ם & ם \\
\hline
\end{tabular}

9. Was Ist für Sle Im Rahmen der Informationssuche besonders wichtig? (Mehrfachantworten möglich)
a Schnelligkeit der Informationssuche
- Komfort (bspw. von Zuhause aus)
$\square$ personliche Beratung
Kostengünstigkeit der Informationssuche
口 Übersichtlichkeit des Mediums
$\checkmark$ jederzeitiger Zugriff (bspw. über das Internet)
a sonstiges

10. Sind Sie für den Airline $X$ Emaill-Newsletter eingeschrieben?
$\square$ ja
$\square$ nein

11. Wie lange vor Antritt der Reise buchen Sie für gewöhnlich Ihren Flug mit Airline $X$ ?

ca. Tage vorher

12. Buchen Sie Ihre Flüge selber?
व ich buche selbst
口 Travel Management der Firma / ein Reiseburo

13. Wo buchen Sie für gewöhnlich Ihren Airline X-Flug?
$\square$ uber das Airline X Call Center
$\square$ am Flughafen
$\square$ uber den Airline X Newsletter
a im Reiseburo
$\checkmark$ auf der Airline X Homepage (www.Airline X.de)
a sonstiges:

14. Was Ist für Sle Im Rahmen der Buchung besonders wichtlg? (Mehrantworten möglich!)
Z Zeitersparnis
Einfachheit der Abwicklung
ㅁ persönliche Beratung
geringe Abwicklungskosten
$\checkmark$ Schnelligkeit der Abwicklung
- Sicherheit der Buchung
Vertrautheit des Buchungsvorgangs
口 Abfrage der Verfügbarkeit freier Plätze
口 sonstiges

15. Nehmen Sie regelmäßig an der Airline $X$ „Crazy Night“ teil?
व ja
$\square$ nein

Nun möchten wir Ihnen elnige Fragen zu Ihren Relseverhalten Im Allgemeinen stellen!

16. Buchen Sie in der Regel einen einfachen Flug oder Hin- und Rückflug zusammen?
$\square$ einfacher Flug
a Hin- und Rackflug

17. Reisen Sie für gewöhnlich alleine zu Zweit oder in einer Gruppe von mehr als zwel Personen?
ㅁ als Einzelreisender
ㅁ zu Zweit
$\square$ in einer Gruppe 
18. Wie viel Zeit llegt für gewöhnlich zWischen Ihrem Hiln-und Rückflua?

ca. Tage

19. Wenn Sie mit Alrilne $X$ filegen, welche Unterkunn haben Sie für gewöhnlich am Zlelort (Mehrfachnennungen moglich)?
Dotel/Pension
U Unterkunt bei Verwandten/Bekannten
a keine Übernachtung am Zielort
sonstiges

[ Ferienhaus/Ferienwohnung (gemietet)

$\square$ eigenes Ferienhaus/eigene Ferienwohnung

$\square$ eigene Hauptwohnung/eigene Pendlerwohnung

Im Folgenden möchten Wr Ihnen einige Fragen zu Thren Elnschätzungen von Alline XIm Vergleich zu anderen Billigillegern stellen!

20. Sind Sie berelts mit anderen Billigfliegern, wie bspw. Ryan Air, Hapa-Lloyd Express oder Deutsche BA, geflogen?
$\square$ ja
a nein

21. Melleicht haben Sle in den letzten Wochen und Monaten schon einmal erwägt, mit Airline $X z u$ fllegen, haben dles letztendlich aus elnem oder mehreren Gründen nicht getan. Welches waren für Sie die drei wichtigsten Gründe, warum Sle dies nicht getan haben? Bitte vergeben Sle die Rangplätze 1.3 (1 =wichtigster Grund, 2=zweitwichtigster Grund, 3=drittwichtigster Grund)!

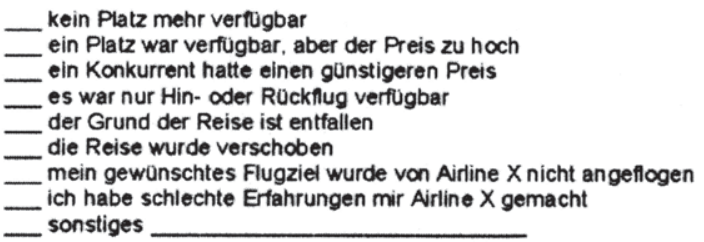

22. We beurteilen Sie die Preise von Airtine X Im Vergleich zu anderen Low-Cost Carriern?

$\begin{array}{ccccc}\text { viel } & \text { etwas } & \text { gleich } & \text { etwas } & \text { viel } \\ \text { billiger } & \text { billiger } & \begin{array}{c}\text { teuer } \\ \text { teurer }\end{array} & \text { teurer } \\ \square & \square & \square & \square & \square\end{array}$

Der nun folgende Abschnitt dient dazu, Thre Wünsche und Erwartungen an Airline X Im Hinblick auf unsere Kommunikation mit unseren Kunden kennen zu lernen.

23. Im Folgenden möchten wir gerne von Ihnen Wissen, welche Bedeutung einige Bestandtelle einer Flugreise Im Allgemeinen für Sle besitzen.

Fur meine Entscheidung, eine Flugreise bei einer bestimmten Airline zu buchen ist

\begin{tabular}{|c|c|c|c|c|c|}
\hline ... der Preis & $\begin{array}{c}\text { sehr } \\
\text { wichtig } \\
\square\end{array}$ & $\begin{array}{c}\text { wichtig } \\
\square\end{array}$ & $\begin{array}{l}\text { weder } \\
\text { noch unwichtig } \\
\square\end{array}$ & $\begin{array}{c}\text { sehr } \\
\text { unwichtig } \\
\square\end{array}$ & $\begin{array}{c}\text { keine } \\
\text { Angabe } \\
\square\end{array}$ \\
\hline w die Ankunits - und Ablugzelten & a & Q & a & a & $\overline{\mathbf{g}}$ \\
\hline ... die Sicherheit des Fluges & $\square$ & 口 & $\square$ & a & a \\
\hline E. der an Bord angebolene Komifort & 0 & a & e & $\bar{\square}$ & a \\
\hline ... das kostenlose Angebot von Getrănken & $\overline{0}$ & a & $\bar{a}$ & $\overline{0}$ & 0 \\
\hline 2. das kostenlose. Angobot von Spaisen & D & Q & a & a. & a \\
\hline ... die Auswahl moglicher Zielorte & $\square$ & $\bar{a}$ & a & $\overline{0}$ & 0 \\
\hline 6. das Unterhaltun gsprocramm an Bord & a & a & घ) & $\overline{\mathbf{a}}$ & $\overline{\mathbf{a}}$ \\
\hline ... die Punktlichkeit der Fluge & 口 & $\square$ & $\square$ & a & 口 \\
\hline W. des Angebot von Duty-Free Waron & $\bar{a}$ & $\overline{0}$ & $\bar{a}$ & $\overline{\mathbf{a}}$ & $\overrightarrow{0}$ \\
\hline
\end{tabular}


... die Mőglichkeit, umbuchen zu können

... die Fluglinie selbst bzw. die Marke

... die Freundlichkeit der Mitarbeiter

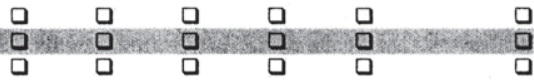

24. Wr möchten Sle bltten, uns elnige Informationen über dle Bedeutung einzelner Kommunikationsmedien in Ihrem Alltag zu geben.

Ich nutze das Internet.
ICh schrelbe/erhalte Emails

25. Wenn Sie regelmäßig Produkte und/oder Dienstleistungen über das Internet kaufen bzw. buchen, um welche Artikelgruppen handelt es sich dabel?

26. Wir möchten Sie bitten, uns einige Informationen zu Ihren Enstellungen gegenüber verschiedenen Medlenformen zu geben. Stimme Sle den folgenden Aussagen eher zu oder nicht? Grundsătzlich gibt es zu viel Werbung

Die Werbung dringt mittlerweile in meine Privatsphäre ein Fluge uber ein Call-Center zu buchen. ist sicher

Ich kaufe/buche im Allgemeinen gerne Sonderangebote

stimme
voll zu

gar nicht zu Angabe

Werbung von gunstigen Aogeboten empfinde ich nicht als störend

Werbe-Emails empfinde ich grundsătzlich als störend

Werbe-Emails von Airline $\mathrm{X}$ empfinde Ich informativ

Werbe-SMS empfinde ich grundsătzlich als störend

Postwurfsendungen empfinde lch als storend

Flage úber das Internet zu buchen, ist sicher

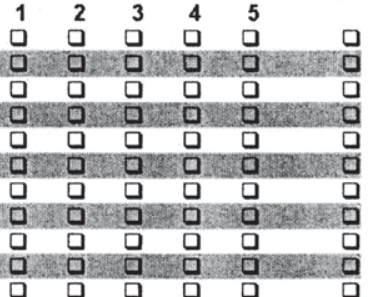

27. Wr möchten Sle nun bitten, uns einige Enschätzungen Airline X Im Allgemeinen zu geben. Stimme Sle den folgenden Aussagen eher zu oder nlcht?

Wenn ich die Marke Airline $X$ beschreiben sollte, dann ist sie unter anderem...

$\begin{array}{lccccc} & \text { stimme } & & \text { stimme } & \text { keine } \\ \text { gar nicht zu Angabe }\end{array}$


28. Können Sie sich grundsätzlich vorstellen, exkluslve günstlge Angebote von Alrline $X$ neben der Ihnen bekannten Form als Emall-Newsletter zu erhalten?

$\square$ ja $\square$ nein

29. Wenn Ja, auf welche Art und Welse solite Alrline $X$ Ihnen Informationen zu exklusiven günstlgen Angeboten zukommen lassen?
per Post
per Annf aus dem Call Center
per SMS
per Emall

30. Können Sie sich vorstellen, lhre Buchung über elnen anderen Weg als bisher vorzunehmen?
$\square$ ja
nein

31. Wenn Ja, auf welche Art und Welse solite Alrline $X$ Ihnen eine Buchung der Flüge anbleten?
$\square$ per Post
$\square$ per SMS
sonstiges:

32. Wie beurtellen Sle dle folgenden Aussagen Im Hinblick auf Emalls, SyS und Psotwurfsendungen? .Die Entscheidung, ob ich eine mir zugeschickte Emall zu lesen, hangt davon ab...

... ob ich personlich mit Namen angesprochen werde

... ob die Email zum richtigen Zeitpunkt kommt

... ob ich meine Erlaubnis zum Bewerben vorher gegeben habe

... ob die Email grafisch ansprechend aufbereitet ist

... ob ich den Absender kenne

... ob ich die Absender sympathisch finde

... ob die Inhalte auf mich zugeschnitten sind

... ob ich die Email erwartet habe

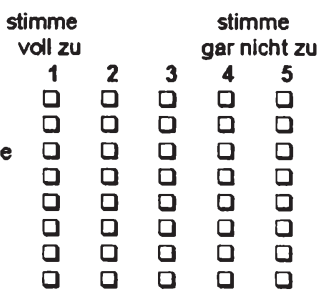

keine Angabe

Die Entscheidung, eine mir zugeschickte SMS zu lesen, hängt davon ab ...

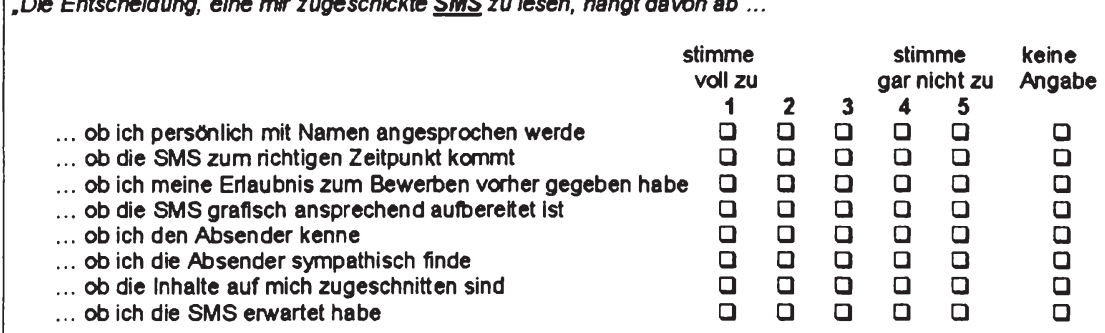


.Die Entscheidung, eine mir zugeschickte Postwurfsendung zu lesen, hängt davon ab ...

... ob ich persönlich mit Namen angesprochen werde

... ob die Postmurfsendung zum richtigen Zeitpunkt kommt

... ob ich meine Erlaubnis zum Bewerben vorher gegeben habe

... ob die Sendung grafisch ansprechend aufbereltet ist

... ob ich den Absender kenne

... ob ich die Absender sympathisch finde

... ob die Inhalte auf mich zugeschnitten sind

... ob ich die Postwurfsendung erwartet habe

\begin{tabular}{|c|c|c|c|c|c|}
\hline $\begin{array}{l}\text { stimme } \\
\text { voll zu }\end{array}$ & & & $\begin{array}{c}\text { stim } \\
\text { gar n }\end{array}$ & $\begin{array}{l}\text { me } \\
\text { cht zu }\end{array}$ & $\begin{array}{l}\text { keine } \\
\text { Angabe }\end{array}$ \\
\hline $\begin{array}{l}1 \\
0 \\
0 \\
0 \\
0 \\
0 \\
0 \\
0 \\
0\end{array}$ & $\begin{array}{l}2 \\
0 \\
0 \\
0 \\
0 \\
0 \\
0 \\
0 \\
0\end{array}$ & $\begin{array}{l}3 \\
0 \\
0 \\
0 \\
0 \\
0 \\
0 \\
0 \\
0\end{array}$ & $\begin{array}{l}4 \\
0 \\
0 \\
0 \\
0 \\
0 \\
0 \\
0 \\
0\end{array}$ & $\begin{array}{l}5 \\
0 \\
0 \\
0 \\
0 \\
0 \\
0 \\
0 \\
0\end{array}$ & $\begin{array}{l}\text { 口 } \\
\text { 口 } \\
\text { 口 } \\
\text { 口 } \\
\text { 口 } \\
\text { 口 }\end{array}$ \\
\hline
\end{tabular}

33. Wonn Alrine X Ihnen anbletet, Ste wahlwelse über elnen, zwol oder drel Informatlonskanäle mit Angeboten zu Informieren? Über welches Medlum bzw. welche Medlenkomblnatlon würden Sle gerne über aktuelle Angebote informlert werden (Mehrfachantworten möglich)?
per Email
$\square$ per SMS
per Postwurfsendung

34. In wolchen zeltllchen Abständen wollten Sle, wenn möglich, über dle von Ihnen bevorzugten Medlen bzW. Medienkombination informiert werden?
T Táglich
worchentlich
a alle 2 bis 3 Tage
monatlich
alle 14 Tage
sonstiges:

Der nun folgende Abschnitt dlent dazu, Thre Wünsche und Erwartungen an das zukünftige Angebot Alrline $X$ näher kennenzulemen.

35. Stellen Sie sich vor, Sle fllegen mit Airilne $X$ an elnen Ort, an dem Sie mehr als elnen Tag blelben möchten. Könnten Sie sich vorstellen, bel der Buchung des Fluges bei Alrilne $X$ auch gleichzeltig andere Dienstlelstungen und Produkte zu buchen?

$\square$ nein, ich wurde alle weiteren Leistungen selbst organisieren

$\square$ ja, und zwar

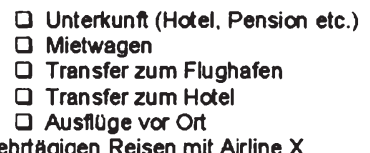

$\square$ nein, ich unternehme keine mehrtägigen Reisen mit Airline $X$

36. Falls Sle dle vorherlgen Frage mit ja beantwortet haben, welche Voraussetzungen müssten erfüllt seln, damit Sle dlese Lelstungen, bspw. elnen Hotelaufenthalt oder einen Mlotwagen, ebenfalls bel Alrllne $X$ buchen würden (Mehrfachnennungen möglich)?
günstige Preise
bekannter Kooperation spartner
口 Hohe Qualitat
a zusátzliche Informationen
personliche Beratung
Einfachheit der Buchung
Prospekte
geringe Abwicklungskosten
Schnelligkeit der Abwicklung
口 Sicherheit der Buchung
Q Vertrautheit des Buchungsvorgangs
Informationen im Internet
- Abfrage der Verfugbarkeit freier Platze
$\square$ passend zum Markenimage von Airline $X$

sonstiges 
37. Könnten Sle sich vorstellen, ein speziell auf Sie zugeschnittenes, exkluslves Angebot bel Airline $X$ zu buchen?

ja

38. Abschlleßend möchten Wr Sle bitten, uns einlge Angaben zu Ihrer Zufrledenhelt mit den Lelstungen von AIrline $X$ zu machen.

Wie zutrieden sind Sle insgesamt mit Airline $X$ ?

insgesamt

\begin{tabular}{|c|c|c|c|c|}
\hline $\begin{array}{c}\text { sehr } \\
\text { zufrieden }\end{array}$ & & & $\begin{array}{l}\text { ga } \\
\text { nich } \\
\text { fried }\end{array}$ & $\begin{array}{c}\text { keine } \\
\text { Angabe }\end{array}$ \\
\hline 12 & 3 & 4 & 5 & \\
\hline 口 & 口 & a & 口 & 口 \\
\hline
\end{tabular}

Wie zufrieden sind Sie mit einzelnen Leistungen von Airline $X$ ?

Preis

Ankunfts- und Abflugzeiten

Auswahl der Zielorte

Unterhaltungsprogramm an Bord

Angebot von Duty-Free Waren

die Möglichkelt, flexibel umbuchen zu können

Informationsmanagement

Informationen im Email-Newsletter

Buchungsabwicklung

Abfertigung am Boden

Service und Freundlichkeit der Mitarbeiter

Speisen und Getranke an Bord

Punktlichkeit

Komfort an Bord

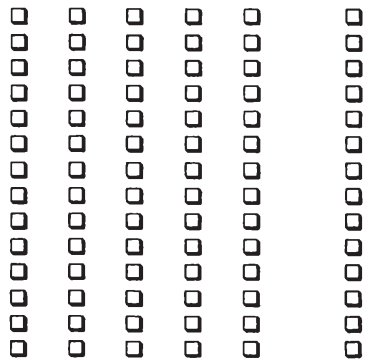

Abschlleßend folgen einlge statlstische Fragen:

39. Welches Geschlecht haben Sie? weiblich $\square$ männlich $\square$

40. Sind Sie Velfileger (Fllegen Sle mehr als 50.000 Mellen pro Jahr unabhänglg von der Arline)?

Ja nein $\square$

41. Besitzen Sie eine Lufthansa Miles \& More-Card?

ja 0 nein $\square$

42. We groB ist Ihr Wohnort? (Elnwohner)
口 $<20.000$
ㄴ $20.001-50.000$
口 $50.001-100.000$
$100.001-200.000$
$200.001-500.000$
$500.001-1.000 .000$
a $>1.000 .000$

43. Wie lautet dle Postleitzahl Ihres Wohnortes?

44. Welchen Beruf üben Sie aus?

a Arbeiter/Facharbeiter/Handwerker

Angestellter

Beamter

S Schüler/Student

- Hausfrau/Hausmann

z.Zt. ohne Erwerbstătigkeit

Q Selbständiger/Freiberuffer

$\square$ Leitender Angestellter

口 Soldat/Zivildienstleistender

Auszubildender

Rentner/Pensionär

$\square$ Sonstiges 
45. Welche Ausbildung haben Sie?
- Hauptschulabschluß
$\square$ Lehre
Fachabitur
Fachhochschulabschluss
Promotion

46. Wie alt sind Sie?

\author{
Realschulabschluss \\ Meister \\ Abitur \\ Universitätsabschluss
}

Jahre

47. Wie hoch ist Ihr monatliches Haushaltsnettoeinkommen In EURO ungefähr?
a unter $500 \epsilon$
口 $1.501-2.000 €$
口 $500-1.000 €$
ㅁ $2.001-2.500 €$
口 $3.001-3.500 €$
व $1.001-1.500 €$
口 $2.501-3.000 €$
uber $3.500 €$

Alrline $\mathrm{X}$ möchte In den nächsten Monaten auch welterhin dle Kommunlkation und Interaktion mit seinen Kunden verbessern. Wir möchten Sle daher fragen, ob Sle damit elnverstanden sind, an einer Testphase unserer neuen Kommunlkationsstrategle tellzunehmen. Neben der Verlosung von Frelflügen, an der Sie automatisch teilnehmen, wenn Sle den Fragebogen ausgefüllt haben, erhalten Sle In den nächsten Monaten exklusive Sonderangebote von Airline $X$, dle Sie direkt buchen können.

Bitte beachten Sle, dass wr Ihre Handy-Nummer benötigen, da elne nur auf dlesem Wege erfolgen kann. Wir werden Ihre Daten selbstverständlich vertraulich behandeln.

- Ich bin damit elnverstanden, von der Alrline $X$ exklusive Sonderangebote über die oben genannten Informationskanäle (SMS, Email und Malling) zu erhalten.

Aktuelle Anschrift:

Name:

Anschrift:

Aktuelle Handy-Nummer:

Aktuelle Emalladresse:

\section{Herzlichen Dank für Ihre Mithilfe!}




\section{Anhang 2: Ex-post-Fragebogen}

Bitte zurücksenden an:

Fax-Nr. $0251-47886$

1. Als Dank für dle Tellnahme an unserer großen Unfrage vom Oktober / November 2003 haben wr lhnen kurz vor Welhnachten eln exklusives Angebot zugeschickt. Was hat Ihnen Airline $X$ hlerbel konkret angeboten?

a oin $\in$ 19-Angebo

4 Tickets zum Preis tor 3

$\square$ eine ausgewăhlte Strecke zu einem

Ich erinnere mich nicht mehr vorgegebenen Preis

2. Auf wolchem Weg haben Sle dleses Angebot erhalten?
a SMS
口 Postwurfsendung + Email
Emal
Email + SMS
Q Postwurfsendung + SMS
Dosturifsendung
Email + SMS + Postwurtsendung
Ich erinnere mich nicht mehr

3. Ungeachtet des Angebotes vom Dezember 2003, hatten Sie zum Zeltpunkt des Erhalts ohnehin vor, elnen Flug zu buchen?
$\square$ ja. und zwar bei Airline $X$
nein, ich hatte keinen Bedarf
a ja, allerdings bel oiner anderen Arrline
b ja. ich hatte das chnehin vor, aber ich
neln, aber der Bedarf wurde durch das
Angebot geweckt wusste noch nicht, bel welcher Arline
a Sonstiges:

4. Wie haben Sle auf unser Angebot reaglert?

a ich habe die angegebene Hottline angerufen und einen bzw. mehrere Fluge gebucht

bitte weiter mit Frage 5 a

Ich habe die angegebene Hotline angerufen. aber keine Fluge gebucht

bitto weiter mit Frage $5 b$

- Ich habe auf das Angebot gar nicht reagiert

bitte weiter mit Frage $5 \mathrm{c}$

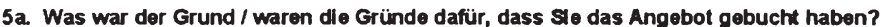
(Mehrfachantworten möglich)
Gutes Preis- $/$ eistungsverhältnis
Bessere Leistung als bei der Konkurrenz
a Angebot kam genau zur richtigen Zeit

口 Vertugbarkelt der Tickets

B Besserer Preis als bei der Konkurrenz

Sonstiges:

5b. Was war der Grund / waren dle Gründe dafür, dass Sle dle angegebene Hotlline angerufen, aber kelne Flüge gebucht haben? (Mehrfachantworten moglich)
- Ich hatte das Angebot falsch verstanden
Die Buchung am Telefon war umständlich
Der Preis fur den Ruckilug war zu hoch
Unfreundiche Mitarbeiter im Call-Center
Es waren keine frelen Plätze

an meinem Wunschtermin verfogbar

口 Sonstiges:

5c. Was war der Grund / waren dle Gründe dafir, dass Sle nicht auf das Angebot reaglert haben? (Mehrfachantworten moglich)

I Ich hatte zu diesem Zeitpunkt keinen Bedarf an Flogen

- Es fehte an finanzielien Mitteln fur eine solche Reise

口 Ich wollte mich zuerst auf der Airline $X$-Homepage nach anderen Angeboten umsehen

Der Preis war zu hoch

- Ich konnte mich nicht so schnell entscheiden

a Mein(e) Partner(in) V Freunde und ich waren uns wegen der Reiseplanung unsicher

口 Es fehlte mir an zusátzilichen Informationen

Sonstiges: 
6. Wer hat bel Ihnen die Entscheldung getrofien, Flüge bzw. keine Flüge zu buchen?
Ich allein
mein(e) Partner(in) und ich
meine Familie und ich
sonstige Personen:
meine Freunde und ich

7. We attraktiv fanden Sle unser Angebot Insgesamt?

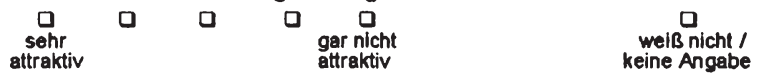

8. We urmfangrelch empfanden Sle die Informationen in unserem Angebot zu den angebotenen Leistungen?

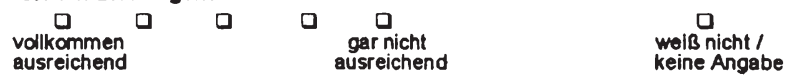

9. We empfanden Sie den Zeltpunkt, an dem Sle das Angebot von uns erhalten haben?

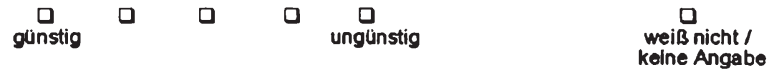

10. Was haben Sle zu dlesem Zeltpunkt gerade getan?

문

weiß nicht /

keine Angabe

11. Sle haben unser Angebot über einen Kanal oder mehrere Kanäle erhalten. Wie attraktlv empfanden Sle den bzW. die von uns ausgewähiten Kommunlkatlonskanäle, auf denen wr Ihnen das Angebot haben zukommen lassen?

\begin{tabular}{|c|c|c|c|c|c|c|}
\hline & $\begin{array}{c}\text { sehr } \\
\text { attraktiv }\end{array}$ & & & & $\begin{array}{l}\text { gar nicht } \\
\text { attraktiv }\end{array}$ & $\begin{array}{l}\text { welB nicht / } \\
\text { keine Angabe }\end{array}$ \\
\hline $\begin{array}{l}\text { Email } \\
\text { SMS } \\
\text { Postwurfsendung }\end{array}$ & $\begin{array}{l}\square \\
\square \\
\square\end{array}$ & $\begin{array}{l}0 \\
0 \\
0\end{array}$ & $\begin{array}{l}0 \\
0 \\
0\end{array}$ & $\begin{array}{l}\square \\
0 \\
\square\end{array}$ & $\begin{array}{l}0 \\
0 \\
0\end{array}$ & $\begin{array}{l}\text { 口 } \\
\square \\
0\end{array}$ \\
\hline
\end{tabular}

12. Wie beurtellen Sle die Buchungsmöglichkelt über unser Call-Center im Verglelch zu einer Online-Buchung?
besser
a $\underset{\text { schlechter }}{a}$ weiß nicht /
keine Angabe

13. Möchten Sie noch elnmal auf dlesem Weg eln solches Angebot von Alrline $X$ erhalten?
口 Ja
Nein, und zwar weil:

Fur die Teilnahme an unserer Verlosung benotigen wir entweder Ihren Namen oder Ihre EmailAdresse, die Sie bei Airline $X$ angegeben haben:

Name gder Emall-Adresse:

Vielen Dank für die Teilnahme an unserer Befragung! 
Michael Ahrens - 978-3-631-75048-3

Downloaded from PubFactory at 01/11/2019 08:52:24AM

via free access 


\section{Literaturverzeichnis}

Adam, D., Produktionsmanagement, 9. Aufl., Wiesbaden 1998.

Ahrens, M., Münstermann, M., Schneider, H., Direktmarketing in der wissenschaftlichen Diskussion, Arbeitspapier des Centrum für interaktives Marketing und Medienmanagement, Münster 2002.

Akaah, I. P., Korgaonkar, P. K., Lund, D., Direct Marketing Attitudes, in: Journal of Business Research, Vol. 34, No. 3, 1995, S. 211-219.

Alba, J. W., Hutchinson, J., Dimensions of Consumer Expertise, in: Journal of Consumer Research, Vol. 13, No. 4, 1987, S. 411-454.

Aldrich, J., Cnudde, C. F., Probing the Bounds of Conventional Wisdom: A Comparison of Regression, Probit, Discriminant Analysis, in: American Journal of Political Science, No. 19, August 1975, S. 571-608.

Aldrich, J., Nelson, F. D., Linear Probability, Logit and Probit Models, Beverly Hills, London 1984.

Allensbacher Computer- und Technik-Analyse (ACTA 2003), Ungebrochene Dynamik der Internetnutzung, Institut für Demokopie, Allensbach 2003.

Andersen, H., Andreasen, M. D., Jacobsen, P. Ø., The CRM Handbook, Hellerup 1999.

Aschoff, M., Professionelles Direkt- und Dialogmarketing per E-Mail, München, Wien 2002.

Bachem, Ch., Erfolgskontrolle im E-Commerce, in: Thexis, 17. Jg., Nr. 3, 2000, S. 59.

Bachem, Ch., Marketingcontrolling im E-Commerce, in: Reinecke, S., Tomczak, T., Geis, G. (Hrsg.), Handbuch Marketingcontrolling: Marketing als Motor für Wachstum und Erfolg, Frankfurt a. M., Wien 2001.

Bachmann, M., Adressvalidierung und Adressanreicherung via Internet, in: Dallmer, H. (Hrsg.), Direct Marketing \& More, 8. Aufl., Wiesbaden 2002, S. 673-688.

Backhaus, K. et al., Multivariate Analysemethoden. Eine anwendungsorientierte Einführung, 9. Aufl., Berlin u. a. 2000.

Backhaus, K. et al., Multivariate Analysemethoden. Eine anwendungsorientierte Einführung, 10. Aufl., Berlin u. a. 2003.

Bamler, G., Zur Wirkung von Direkt-Werbung im Industriegüterbereich, Frankfurt a. M. u. a. 1987. 
Bauer, H. H., Mäder, R., Fischer, C., Determinanten der Werbewirkung von Markenhomepages, Arbeitspapier des Instituts für Marktorientierte Unternehmensführung, Mannheim 2001.

Bawa, K., Influences on Consumer Response to Direct Mail Coupons: An Integrative Review, in: Psychology and Marketing, Vol. 13, No. 2, 1996, S. 129156.

Beard, J. D., Williams, D. L., Kelly, J. P., The Long versus the Short Letter, in: Journal of Direct Marketing, Vol. 5, 1991, S. 27-34.

Behrens, G., Werbewirkungsanalyse, Opladen 1976.

Behrens, AK. Ch., Absatzwerbung, 2. Aufl., Wiesbaden 1976.

Belk, R. W., An Explanatory Assessment of Situational Effects in Buyer Behavior, in: Journal of Marketing Research, Vol. 11, 1974, S. 156-163.

Belz, C., Strategisches Direktmarketing, Wien 1997.

Berekoven, L., Eckert, W., Ellenrieder, P., Marktforschung. Methodische Grundlagen und praktische Anwendung, Wiesbaden 1999.

Bessler, H., Bledjian, F., Systematik der Massenkommunikationsforschung, München, Basel 1967.

Bezjian-Avery, A., Calder, B., Iacobucci, D., New Media Interactive Advertising vs. Traditional Advertising, in: Journal of Advertising Research, No. 38, JulyAugust 1998, S. 23-32.

Bird, D., Commonsense Direct Marketing, Landsberg/Lech, New York 1990.

Bluedorn, A. C., Cutting the Gordian Knot: A Critique of the Effectiveness Tradition in Organizational Research, in: Sociology and Social Research, Vol. 64, No. 4, 1980, S. 477-496.

Böhler, H., Marksegmentierung auf Basis eines Direct-Marketing-Konzepts, in: Dallmer, H. (Hrsg.), Direct Marketing \& More, 8. Aufl., Wiesbaden 2002, S. 921-937.

Bonoma, T. V., Clark, B. H., Marketing Performance Assessment, Boston 1988. Broadbent, D. E., Persecption and Communication, London 1958.

Broverman, D. M, Klaiber, E., Kobayashi, Y., Vogel, W., Roles of Activation and Inhibition in Sex Differences in Cognitive Abilities, in: Psychological Review, Vol. 75, No. 1, 1968, S. 23-50.

Brown, H. E., Buskirk, B., Readings \& Cases in Direct Marketing, Lincolnwood, Chicago 1989.

Brown, S., Customer Relationship Marketing: A Strategic Imperative in the World of E-business, Ontario 2000.

Brucks, M., The Effect of Product Class Knowledge on Information Search Behavior, in: Journal of Consumer Research, Vol. 12, No. 1, 1985, S. 1-16.

Bruhn, M., Kommunikationspolitik, Munchen 1997.

Bruns, J., Direktmarketing, Ludwigshafen 1998. 
Bünting, H. F., Organisatorische Effektivität von Unternehmen. Ein zielorientierter Ansatz, Wiesbaden 1995.

Burmann, Ch., Erfolgskontrolle im Eventmanagement, IST-Studieninstitut für Kommunikation GmbH (Hrsg.), Düsseldorf 1998.

Busch, R., Dögl, R., Unger, F., Integriertes Marketing. Strategie - Organisation Instrumente, Wiesbaden 1995.

Ceyp, M., Erfolgskontrollen im Direktmarketing, in: Direkt Marketing. Zeitschrift für Dialogmarketing und Integrierte Kommunikation, Nr. 4, 2002, S. 26-31.

Chittenden, L., Rettie, R., An evaluation of e-mail marketing and factors affecting response, in: Journal of Targeting, Measurement and Analysis for Marketing, Vol. 11, No. 3, S. 203-217.

Church, A. H., Estimating the Effect of Incentives on Mail Survey Resonse Rates: A Meta-Analysis. Public Opinion Quarterly, Vol. 57, No.1, 1993, S. 6279.

Corkindale, D., Newall, J., Advertising thresholds and wearout, in: European Journal of Marketing, Vol. 12, No. 5, 1978, S. 327-378.

Cox, E. P., Marketing Research, New York u. a. 1979.

Craik, F. I. M., Byrd, M., Aging and cognitive deficits: The role of attentional resources, in: Craik, F. I. M., Trehub, S. E. (Hrsg.), Aging and cognitive processes, New York 1982, S. 191-221.

Crux, R., Scherfke, A., Chancen und Risiken im CRM-gestützten Direct Marketing, in: Dallmer, H. (Hrsg.), Direct Marketing \& More, 8. Aufl., Wiesbaden 2002, S. 1121-1135.

Cunningham, J. B., Approaches to the Evaluation of Organizational Effectiveness, in: Academy of Management Review, Vol. 2, July 1977, S. 463-474.

Dallmer, H., Erfolgsbedingungen der Kommunikation im Direct-Marketing, Wiesbaden 1979.

Dallmer, H., Direct-Marketing, in: Bruhn, M. (Hrsg.), Handbuch des Marketing, München 1989.

Dallmer, H. (Hrsg.), Handbuch Direct Marketing, 6. Aufl. Wiesbaden 1991.

Dallmer, H. (Hrsg.), Handbuch Direct Marketing, 7. Aufl., Wiesbaden 1997.

Dallmer, H. (Hrsg.), Direct Marketing \& More, 8. Aufl., Wiesbaden 2002.

Dallmer, H., Thedens, R., (Hrsg.), Handbuch des Direct-Marketing, Wiesbaden, Darmstadt 1975.

Dallmer, H., Thedens, R., (Hrsg.), Handbuch des Direct-Marketing, Wiesbaden 1981.

Danaher, P. J., Green, B. J., A Comparison of Media Factors that influence the Effectiveness of Direct Response Television, in: Journal of Direct Marketing, Vol. 11, No. 2, 1997, S. 46-58. 
Danne, S., Messebeteiligungen von Hochschulen: Ziele und Erfolgskontrolle, Frankfurt a. M. 2000.

Darley, W. K., Smith, R. E., Gender differences in information processing strategies: An empirical test of the Selectivity Model in Advertising Response, in: Journal of Advertising, Vol. 24, No. 1, 1995, S. 41-56.

Dellmann, K., Eine Systematisierung der Grundlagen des Controlling, in: Spremann, K., Zur, E. (Hrsg.), Controlling, Wiesbaden 1992.

DeMarris, A., Logit Modeling: Practical Applications, Newbury Park 1992.

Dettmar, H., Thema Mediamix wird wichtiger, in: Horizont, o. Jg., Nr. 30, 1998, S. 41.

Deutscher Direktmarketing Verband (Hrsg.), Facts \& Figures, Wiesbaden 2000.

Deutscher Direktmarketing Verband (Hrsg.), eMail-Marketing - Dialog pur, DDV Best Practice Guide Nr. 4, 2003.

Deutscher Direktmarketing Verband (Hrsg.), Mobile-Marketing - Dialog on Tour, DDV Best Practice Guide Nr. 7, Mainz 2003.

Deutsche Post AG (Hrsg.), Verbraucher und Direktwerbung - Verkaufen per Post 1995, Direkt Marketing Monitor, Studie 2, Bonn 1995.

Deutsche Post AG (Hrsg.), Werbeerfolgsmessung von Werbesendungen, Direkt Marketing Monitor, Studie 4, Bonn 1997.

Deutsche Post AG (Hrsg.), Verbraucherbefragung zur Akzeptanz von Produktproben, unveröffentlichte Studie, Bonn 2000.

Deutsche Post AG (Hrsg.), Direktmarketing Deutschland 2003 - Fakten, Trends und Hintergründe zum Direktmarketing 2002/2003, Direkt Marketing Monitor, Studie 15, Bonn 2003.

Di Ianni, A., The e-business enterprise and the "Web First" principle of emarketing, in: Journal of Interactive Marketing, Vol. 2, No. 3, 2000, S. 158170.

Diamond, W. D., Noble, S. M., Defensive Responses to Charitable Direct Mail Solutions, in: Journal of Interactive Marketing, Vol. 15, No. 3, Summer 2001, S. 2-12.

Dichter, E., A Psychological View of Advertising Effectiveness, in: Journal of Marketing, Vol. 14, No. 1, 1949, S. 61-66.

Dögl, R., Unger, F., Taschenbuch der Werbepraxis, Heidelberg 1995.

Donelly, L., Geradi, A., Direkt-Marketing ist nicht Versandhauswerbung, in: D \& G Direkt Marketing, Ettlingen 1987.

Dorner, B., Versandhandelsmarketing. Ansätze zur Kundengewinnung und Kundenbindung, Wiesbaden 1999.

Doubleclick, eMail Trend-Report December 2003, www.doubleclick.net, Abruf 15.12.2003. 
Draper, N., Smith, H., Applied Regression Analysis, 2. Aufl., New York u. a., 1981.

Eggert, A., Fassot, G. (Hrsg.), eCRM - Electronic Customer Relationship Management, Stuttgart 2001.

Elsner, R., Optimiertes Direkt- und Database-Marketing unter Einsatz mehrstufiger dynamischer Modelle, Dissertation, Wissenschaftliche Hochschule fur Unternehmensführung (WHU), Vallendar 2003.

Ernst, O., Meinungen über die Werbung - was bedeuten sie?, in: ZV + ZV, 71 . Jg., Nr. 19/20, 1974, S. 514-526.

Esch, F.-R., Wirkung integrierter Kommunikation. Ein verhaltenswissenschaftlicher Ansatz für die Werbung, Wiesbaden 1998.

Evans, M., O`Malley, L., Patterson, M., Direct Marketing: rise and rise or rise and fall, in: Marketing Intelligence and Planning, Vol. 13, No. 6, 1995, S. 1623.

Fahrmeir, L., Hamerle, A., Grundlegende multivariate Schätz- und Testprobleme, in: Fahrmeir, L, Hamerle, A. (Hrsg.), Multivariate statistische Verfahren, Berlin, New York 1984.

Fahrmeir, L, Hamerle, A. (Hrsg.), Multivariate statistische Verfahren, Berlin, New York 1984.

Farley, J. U.; Harvey, P. D., Assessing a "Real World" Mail Order Experiment, Current Issues \& Research in Advertising, Vol. 7, No. 1, 1984, S. 123-139.

Fassot, G., eCRM-Instrumente: Ein beziehungsorientierter Überblick, in: Eggert, A., Fassot, G. (Hrsg.), eCRM - Electronic Customer Relationship Management, Stuttgart 2001, S. 131-157.

Feinberg, R. A., Eastlick, M. A., Direct Marketing in the USA: past failures and future promises, in: International Journal of Retail and Distribution Management, Vol. 25, No. 8, 1997, S. 256-261.

Fiore, A. M., Yu, H., Effects of imagery copy and product samples on responses towards the product, in: Journal of Interactive Marketing, Vol. 15, No. 2, 2001, S. 37-47.

Fleiss, J. L., Statsitical Methods for Rates and Proportions, 2nd edition, NewYork 1981.

Forrester (Hrsg.), M-Commerce in 2003, unveröffentlichte Studie, New York 2003.

Fraser-Robinson, J., The Secret of Effective Direct Mail, London 1989.

Frazier, G. et al. (Hrsg.), 1988 AMA Educators Proceedings. Efficiency and Effectiveness in Marketing, Chicago 1988.

Frese, E. (Hrsg.), Handwörterbuch der Organisation, 3. Aufl., Stuttgart 1992.

Gabler Wirtschaftslexikon, Wiesbaden 1997. 
Geller, L. K., Response! Die unbegrenzten Möglichkeiten des Direktmarketing, Landsberg/Lech 1997.

Gerardi, A., zit. nach Kirchner, G., Sobeck, S., Lexikon des Direktmarketing, Landsberg/Lech 1989.

Gerth, N., Die Bedeutung des Online Marketing fur die Distributionspolitik, in: Link, J. (Hrsg.), Wettbewerbsvorteile durch Online Marketing. Die strategischen Perspektiven elektronischer Märkte, 2. Aufl., Berlin, Heidelberg, New York 2000, S. 149-195.

GfK (Hrsg.), GfK Direktmarketing Panel, in: GfK Newsletter 02/2003, Nürnberg 2003.

GfK, Finanzdienstleister verzeichnen Anstieg, in: DDV (Hrsg.), Dialog, Nr. 7, 2003, S. 9.

Gierl, H., Koncz, J., Customer Lifetime Value, in: Dallmer, H. (Hrsg.), Direct Marketing \& More, 8. Aufl., Wiesbaden 2002, S. 939-956.

Gill, K. H., Optimierung durch Media-Mix-Strategien, in: Horizont, o. Jg., Nr. 24, 1997, S. 37.

Gillett, P. L., A Profile of Urban In-home Shoppers, in: Journal of Marketing, Vol. 34, Heft 3, 1970, S. 40-45.

Gleser, G., Gottschalk, L. A., Gottschalk, J. W., The Relationship of Sex and Intelligence to Choice of Words: A Normative Study of Verbal Behavior, in: Journal of Clinical Psychology, Vol. 15, No. 2, 1959, S. 182-191.

Godin, S., Permission Marketing. Turning strangers into friends, and friends into customers, New York 1999.

Gordon, P. J., Tellerman, B. J., Impact of prompt-Response and Quantity Premiums on Direct-Mail Response Rates, in: Journal of Direct Marketing, Vol. 4, No. 3, S. 41-45.

Göttgens, O., Wastl, S., Kein Marketing - Kein Konsum - Kein Wachstum, Insight, 4/2003, S. 51-60.

Gottschling, St., Rechenbauer, H. O., Direktmarketing, München 1994.

Grabatin, G., Effizienz von Organisationen, Berlin 1981.

Green, P. E., Tull, D. S., Methoden und Techniken der Marketingforschung, 4. Aufl., Stuttgart 1982.

Greff, G., Telefonmarketing und Call-Center, in: Dallmer, H. (Hrsg.), Direct Marketing \& More, 8. Aufl., Wiesbaden 2002, S. 251-264.

Greißinger, P., Wirtschaftlichkeitsanalysen im Gesundheitswesen. Analyse und beispielhafte Anwendung der Data Envelopment Analysis, Wiesbaden 2000.

Grimm, C., Tietz, W., Die Einstellung von Werbeempfängern zur Direktwerbung, Arbeitspapier Nr. 83, Lehrstuhl für Marketing der Universität Erlangen-Nürnberg, Nürnberg 1999. 
Gunn, E. P., Marketers keen on enriched email, in: Advertising Age, Vol. 71, No. 43, 2000, S. 512-514.

Gzuk, R., Messung der Effizienz von Entscheidungen, Tübingen 1975.

Hair, J. F., Anderson, R. E., Tatham, R. L., Black, W. C., Multivariate Data Analysis with Readings, 2. Aufl., New York 1992.

Hatch, D., The Ultimate Direct Mail Checklist, in: Target Marketing, Vol. 12, No. 8, 1998, S. 34-35.

Heinemann, Ch., Werbung im interaktiven Fernsehen, Wiesbaden 1998.

Heinen, E., Einführung in die Betriebswirtschaftslehre, 9. Aufl., Wiesbaden 1985.

Helgesson, J. G., Voss, K. E., Terpening, W. D., Determinants of Mail-Survey Response: Survey Design Factors and Respondent Factors, in: Psychology \& Marketing, Vol. 19, No. 3, S. 302-328.

Hennessy, H. D., Comparing Personalized Name to Title Only Labels in a Business Mailing, in: Journal of Direct Marketing, Vol. 1, No. 2, 1987, S. 99-102.

Hermanns, A., Konsument und Werbewirkung - Das phasenorientierte Werbewirkungsmodell, Bd. 14 der Schriftenreihe: Schwerpunkt Marketing, Bielefeld, Köln 1979.

Hilke, W., Kennzeichnung und Instrumente des Direkt-Marketing, in: Hilke, W. (Hrsg.), Direkt-Marketing, Schriften zur Unternehmensführung, Bd. 47, Wiesbaden 1993, S. 5-30.

Hilke, W. (Hrsg.), Direkt-Marketing, Schriften zur Unternehmensführung, Bd. 47, Wiesbaden 1993.

Hill, R. J., Bonjean, C. M., News Diffusion: A Test of the Regularity Hypothesis, in: Journalism Quarterly, Vol. 41, Summer 1964, S. 336-342.

Hinrichs, Ch., Lippert, I., Kosten und Wirkungen mobiler Werbung, in: Silberer, G., Wohlfahrt, J., Wilhelm, T. (Hrsg.), Mobile Commerce. Grundlagen, Geschäftsmodelle, Erfolgsfaktoren, Wiesbaden 2002, S. 265-278.

Hitchcock, T., Ragosin, Y., Creating Power Pictures, in: Target Marketing, Vol. 21, No. 6, 1998, S. 54-57.

Holder, D., Davies, D., Stone, M., Part of IDM Diploma course material, Institute of Direct Marketing, Teddington, Richmond-upon-Thames 1992.

Holland, H., Direktmarketing, München 1992.

Holland, H., Direktmarketing-Aktionen professionell planen, Wiesbaden 2001.

Holland, H. (Hrsg.), Das Mailing - Planung, Gestaltung, Produktion, Wiesbaden 2002.

Holland, H., Direktmarketing, 2. Aufl., München 2004.

Hölscher, U., Entscheidungsprozess bei der Anmietung von Adressen im Business-Bereich, in: Dallmer, H. (Hrsg.), Direct Marketing \& More, 8. Aufl., Wiesbaden 2002, S. 559-568. 
Horváth, P., Controlling, 4. Aufl., München 1991.

Hosmer, D., Lemeshow, S., Applied Logistic Regression, New York 1989.

Hünerberg, R., Bedeutung von Online-Medien für das Direktmarketing, in: Link, J. (Hrsg.), Wettbewerbsvorteile durch Online Marketing. Die strategischen Perspektiven elektronischer Märkte, 2. Aufl., Berlin, Heidelberg, New York 2000, S. 121-147.

Hünerberg, R., Heise, G. (Hrsg.), Multi-Media und Marketing. Grundlagen und Anwendungen, Wiesbaden 1995.

Hünerberg, R., Mann, A., Einsatzgebiete und -probleme des Internet im Marketing, in: Industrie Management, Nr. 1, 1999, S. 31-35.

IMR (Hrsg.), Bedeutung und Nutzeffekt der Direktwerbung, IMR (Hrsg.), Bedeutung und Nutzeffekt der Direktwerbung, in: Direkt-Marketing, 10. Jg., Nr. 1, 1975, S. 376-377, zitiert bei Dallmer, H., Erfolgsbedingungen der Kommunikation im Direct-Marketing.

Iyer, R. T., Hill, J. S., International direct marketing strategies: a US-European comparison, in: European Journal of Marketing, Vol. 30, No. 3, 1996, S. 6583.

Jain,C. L., Broadcast support to newspaper ads, in: Journal of Advertising Research, Vol. 15, No. 5, 1975, S. 69-72.

James, E. L.; Li, H., Why Do Consumers Open Direct Mail?, in: Journal of Direct Marketing, Vol. 7, No. 2, S. 34-40.

Janis, I. L., Kaye, D., Kirschner, P., Facilitating Effects of "Eating-WhileReading" on Responsiveness to Persuasive Communications, in: Journal of Personality and Social Psychology, Vol. 1, 1965, S. 181-186.

Janssen, J., Laatz, W., Statistische Datenanalyse mit SPSS für Windows, 4. Aufl., Berlin u. a. 2003.

Jardine, F., The Power of Promotional Stamps and Donors through these Powerful Response Boosters, in: Target Marketing, Vol. 20, No. 10, 1997, S. 23-29. Joachimsthaler, E. A., Aaker, D. A., Aufbau von Marken im Zeitalter der PostMassenmedien, in: Esch, F. R. (Hrsg.), Moderne Markenführung, 3. Aufl., Wiesbaden 2001, S. 539-563.

Kahn, H. L., How to Get Customers to Read What You Send Them, in: Sales and Marketing, Vol. 16, No. 7, 1991, S. 23.

Kaiser, A., Werbung - Theorie und Praxis werblicher Beeinflussung, München 1980.

Kehl, R. E., Controlling mit Database Marketing - Effizienzmessung absatzpolitischer Instrumente, Ettlingen 2000.

Kent, R., Brandal, H., Improving email response in a permission marketing context, in: International Journal of Market Research, Vol. 45, No. 3, 2003. 
Kinnear, T. C., Taylor, J. R., Marketing Research. An Applied Approach, New York 1983.

Kirchner, G., Die neue Praxis der Direktwerbung. Wie Sie Ihre Verkaufs- und Werbeprobleme selbst lösen, Wiesbaden 1991.

Kirchner, G., Sobeck, S., Lexikon des Direktmarketing, Landsberg/Lech 1989.

Köhler, R., Der Einfluß des Kommunikators auf die Wirkung direkter Marktkommunikation, in: Dallmer, H. (Hrsg.), Direct Marketing, 7. Aufl., Wiesbaden 1997, S. 149-170.

Kortzfleisch, G. v. (Hrsg.), Wissenschaftsprogramm und Ausbildungsziele der Betriebswirtschaftslehre, Berlin 1971.

Kotler, Ph., Development and Tendencies of Marketing in the 1990's, in: Marketing - Quo vadis?, in: Meffert, H., Wagner, H. (Hrsg.), Arbeitspapier Nr. 49 der Wissenschaftlichen Gesellschaft für Marketing und Unternehmensführung e. V., Münster 1988.

Kotler, Ph., Bliemel, F., Marketing-Management. Analyse, Planung, Umsetzung und Steuerung, 8. Aufl., Stuttgart 1995.

Krafft, M., Außendienstentlohnung im Licht der Neuen Institutionenlehre, Wiesbaden 1995.

Krafft, M., Der Ansatz der Logistischen Regression und seine Interpretation, in ZfB, 67. Jg. Heft 5/6, 1997, S. 625-642.

Krafft, M., Logistische Regression, in: Herrmann, A., Homburg, Ch. (Hrsg.), Marktforschung. Methoden, Anwendungen, Praxisbeispiele, Wiesbaden, 2. Aufl., 2000, S. 237-264.

Kreutzer, R. T., Die Basis für den Dialog, in: Absatzwirtschaft, Nr. 4, 1990, S. 106.

Kroeber-Riel, W., Marketingtheorie - eine verhaltensorientierte Erklärung von Marktreaktionen, Köln 1972.

Kroeber-Riel, W., Konsumentenverhalten, München 1975.

Kroeber-Riel, W., Informationsüberlastung durch Massenmedien und Werbung in Deutschland, in: Die Betriebswirtschaft, Jg. 47, Nr. 3, 1987, S. 257-264.

Kroeber-Riel, W., Esch, F.-R., Strategie und Technik der Werbung, Verhaltenswissenschaftliche Ansätze, 5. Aufl., Stuttgart 2000.

Kroeber-Riel, W., Weinberg, P., Konsumentenverhalten, 5. Aufl., München 1992.

Kwon, Y.-H., Paek, S. L., Arzeni, M., Catalog vs. non-catalog shoppers of apparel: Perceived risks, shopping orientations, demographics, and motivations, in: Clothing and Textiles Research Journal, Vol. 10, No. 1, 1991, S. 13-19.

Lasslop, I., Effektivität und Effizienz von Events - Wirkungstheoretische Analyse und empirische Befunde, Wiesbaden 2004. 
LeClere, M., The Interpretation of Coefficients in Models with Qualitative Dependent Variables, in: Decision Sciences, Vol 23, No. 3, 1992, S. 770-776.

Lehr, G., Entscheidungsprozesse bei der Anmietung von Adressen im ConsumerBereich, in: Direct Marketing \& More, 8. Aufl., Wiesbaden 2002, S. 509-543. Levin, N., Zahavi, J., Continuous predictive modeling, in: Journal of Interactive Marketing, Vol. 12, No. 2, 1998, S. 5-22.

Link, J. (Hrsg.), Wettbewerbsvorteile durch Online Marketing. Die strategischen Perspektiven elektronischer Märkte, 2. Aufl., Berlin, Heidelberg, New York 2000.

Link, J, Gerth, N., eCRM als strategische und organisatorische Herausforderung, in: Eggert, A., Fassot, G. (Hrsg.), eCRM - Electronic Customer Relationship Management, Stuttgart 2001, S. 305-328.

Link, J., Schleuning, C., Das neue interaktive Direktmarketing: die neuen elektronischen Möglichkeiten der Kundenanalyse und Kundenbindung, Ettlingen 1999.

Löffler, H., Scherfke, A., Praxishandbuch Direct Marketing, Berlin 2000.

Loro, L., No, Direct Mail is not Dead, in: Business Marketing, Vol. 82, No. 10, S. 27-29.

Luo, X., Donthu, N., Benchmarking Advertising Efficiency, in: Journal of Advertising Research, Vol. 41, No. 6, 2001, S. 7-18.

Malthouse, E., Assessing the performance of direct marketing scoring models, in: Journal of Interactive Marketing, Vol. 15, No. 1, 2001, S. 49-62.

Mayer, H., Werbewirkung und Kaufverhalten. Unter ökonomischen und psychologischen Aspekten, Stuttgart 1990.

Mayer, H., Illmann, T., Markt- und Werbepsychologie, Stuttgart 2000.

Mayer, H., Reitmeier, A.-F., Empfängerabhängige Bedingungen werblicher Kommunikation, in: GfK (Hrsg.), Jahrbuch der Absatz- und Verbrauchsforschung, Jg. 27, Heft 3, 1981, S. 197-239

McFadden, D., The Measurement of Urban Travel Demand, in: Journal of Public Economics, Vol. 3, No. 3, 1974, S. 303-328.

McGuire, W. J., Nature of attitudes and Attitude Change, in: Lindzey, G., Aronson, E. (Hrsg.), Handbook of Social Psychology, London 1969, S. 372-398.

MediaTransfer AG Netresearch \& Consulting (Hrsg.), Status quo und Trends im Kommunikations-Markt in Europa und Nord-Amerika, Hamburg 2003.

Meffert, H., Die Flexibilität in betriebswirtschaftlichen Entscheidungen, unveröffentlichte Habilitationsschrift, München 1968.

Meffert, H., Die Leistungsfähigkeit der entscheidungs- und systemorientierten Marketinglehre, in: Kortzfleisch, G. v. (Hrsg.), Wissenschaftsprogramm und Ausbildungsziele der Betriebswirtschaftslehre, Berlin 1971, S. 167-187. 
Meffert, H., Direct Marketing und marktorientierte Unternehmensführung, in: Dallmer, H. (Hrsg.), Direct Marketing \& More, 8. Aufl., Wiesbaden 2002, S. 33-55.

Meffert, H., Marketingforschung und Käuferverhalten, 2. Aufl., Wiesbaden 1992. Meffert, H., Marketing: Grundlagen marktorientierter Unternehmensführung, 9. Aufl., Wiesbaden 2000.

Meffert, H., Konsumgütermarketing: Vom Massen- zum Individualmarketing, in: Frankfurter Allgemeine Zeitung, 15. April 2002, Nr. 87, S. 25.

Meffert, H., Burmann, C., Koers, M. (Hrsg.), Markenmanagement. Grundlagen der identitätsorientierten Markenführung, Wiesbaden 2002.

Meffert, H., Bruhn, M., Dienstleistungsmarketing, 3. Aufl., Wiesbaden 2003.

Meffert, H., Giloth, M., Aktuelle markt- und unternehmensbezogene Herausforderungen an die Markenfuhrung, in: Meffert, H., Burmann, C., Koers, M. (Hrsg.), Markenmanagement. Grundlagen der identitätsorientierten Markenführung, Wiesbaden 2002, S. 99-132.

Meffert, H., Kirchgeorg, M., Marketing - Quo Vadis? - Herausforderungen und Entwicklungsperspektiven des Marketing aus Unternehmenssicht, in: Meffert, H., Wagner, H., Backhaus, K. (Hrsg.), Arbeitspapier Nr. 89 der Wissenschaftlichen Gesellschaft für Marketing und Unternehmensfuhrung e. V., Münster 1994.

Meffert, H., Wagner, H. (Hrsg.), Marketing - Quo Vadis?, Arbeitspapier Nr. 49 der Wissenschaftlichen Gesellschaft für Marketing und Unternehmensführung e. V., Münster 1988.

Mercer Management Consultants (Hrsg.), Communications Benchmark 2003, München 2003, Abruf am 15.12.2003 unter www.presseportal.de Meyer, P. W., Die Werbeerfolgskontrolle, Düsseldorf 1963.

Morrison, D. G., On the Interpretation of Discriminant Analysis, in: Journal of Marketing Research, No. 6, May 1969, S. 156-163.

Morwitz, V. G., Schmittlein, D., Using Segmentation to Improve Sales Forecasts Nased on Purchase Intent. Which "Intenders" Actually Buy?, in: Journal of Marketing Research, Vol. 12, No. 2, 1992, S. 391-405.

Mühlbacher, H., Ein situatives Modell der Motivation zur Informationsaufnahme und -verarbeitung bei Werbekontakten, in: Marketing ZFP, 10. Jg., Heft 2, 1988, S. 85-94.

Müller, H., Khazaka, D., Stimmungseinflüsse auf die Wirkung informativer und emotionaler Werbung, in: Marketing ZFP, 17. Jg., Heft 3, 1995, S. 186-194.

Münstermann, M., Stand der Direktmarketing-Forschung und -Praxis in den USA, Dokumentation einer Forschungsreise zur DMA Annual Conference and Exhibition 2002 und DMEF Educators' Conference 2002, Arbeitspapier des Centrum für interaktives Marketing und Medienmanagement, Münster 2002. 
Nash, E. L., Direct Marketing - Strategy, Planning, Execution, 4th edition, New York 2000.

Nemetz, K., Wie lernt der Konsument? Lernen aus Werbung und/oder Produktverwendung, in: Marketing Journal, 25. Jg., 1993, S. 152-157.

Nielsen S + P 1996, zitiert bei: Bruhn, M., Kommunikationspolitik, München 1997.

Pepels, W., Lexikon des Marketing, München 1996.

Pickhardt, M., Mehrwert durch Personalisierung, in: Dallmer, H. (Hrsg.), Direct Marketing \& More, Wiesbaden 2002, S. 969-981.

Picot, A., Neuburger, R., Mobile Business - Erfolgsfaktoren und Voraussetzungen, in: Reichwald, R., Mobile Kommunikation. Wertschöpfung, Technologien, neue Dienste, Wiesbaden 2002, S. 55-69.

Piller, T., Kundenindividuelle Massenproduktion. Die Wettbewerbsstrategie der Zukunft, München, Wien 1998.

Phillips, C. F., A critical Analysis of recent Literature dealing with Marketing Efficiency, in: Journal of Marketing, Vol. 5, No. 4, 1941, S. 360-366.

Press, S. J., Wilson, S., Choosing Between Logistic Regression and Discriminant Analysis, in: Journal of the American Statistical Association, No. 73, 1978, S. 699-705.

Price, J. L., The Study of Organizational effectiveness, in: The Sociological Quarterly, Vol. 13, No. 1, 1972, S. 3-15.

Ray, M. L., Sawyer, A. G., Repetition in media models. A laboraty technique, in: Journal of Market Research, No. 2, 1971, S. 20-29.

Reichheld, F., Sasser, W. E., Zero-Migration: Dienstleister im Sog der Qualitätsrevolution, in: Harvard Business Manager, 13. Jg., Nr. 4, 1991, S. 108-116.

Reinecke, S., Tomczak, T., Geis, G. (Hrsg.), Handbuch Marketingcontrolling: Marketing als Motor für Wachstum und Erfolg, Frankfurt a. M., Wien 2001.

Reinecke, S., Tomzcak, T., Einsatz von Instrumenten und Verfahren des Marketingcontrollings in der Praxis, in: Reinecke, S., Tomczak, T., Geis, G. (Hrsg.), Handbuch Marketingcontrolling: Marketing als Motor für Wachstum und Erfolg, Frankfurt a. M., Wien 2001.

Riedmüller, F., Der Einfluss situationsspezifischer Faktoren auf die Werbequalität, in: Transfer. Werbeforschung \& Praxis, Vol. 1, 2000, S. 22-26.

Roberts, M. L., Expanding the role of direct marketing database, in: Journal of Interactive Marketing, Vol. 11, No. 4, 1997, S. 26-35.

Roberts, M. L., Berger, P. D., Direct Marketing Management, 2nd edition, Upper Saddle River, New Jersey 1999.

Rogers, J. L., Consumer Response to Advertising Mail, in: Journal of Advertising Research, Vol. 29, No. 6, 1989, S. 18-24.

Rogge, H.-J., Werbung, 4. Aufl., Ludwigshafen 1996. 
Roman, E., Integrated Direct Marketing, Techniques and Strategies for Success, New York 1988.

Ronneberger, F., Sozialisation durch Massenkommunikation, in: Ronneberger, F. (Hrsg.), Sozialisation durch Massenkommunikation - Der Mensch als soziales und personales Wesen (Band IV), Stuttgart 1971, S. $73 \mathrm{ff}$.

Ronneberger, F. (Hrsg.), Sozialisation durch Massenkommunikation - Der Mensch als soziales und personales Wesen (Band IV), Stuttgart 1971.

Ruland, J., Werbeträger: Einführung in die Praxis des Werbeträgereinsatzes, Bad Homburg 1972.

Salthouse, T. A., A theory of cognitive aging, New York 1985.

Schaller, G., Organisation der Erfolgskontrolle im Direct Marketing, in: Dallmer, H. (Hrsg.), Handbuch Direct Marketing, 7. Aufl., Wiesbaden 1997, S. 580589.

Schefer, D., Medien des Direct Marketing im Intermedia-Vergleich, in: Dallmer, H. (Hrsg.), Direct Marketing \& More, 8. Aufl., Wiesbaden 2002, S. 105-123. Schefer, D., Schuler, R., Adressgenerierung im Internet, in: Dallmer, H. (Hrsg.), Direct Marketing \& More, 8. Aufl., Wiesbaden 2002, S. 583-608.

Schlosser, A. E., Shavitt, S., Kanfer, A., Survey of Internet users' attitudes toward Internet advertising, in: Journal of Interactive Marketing, Vol. 13, No. 3, 1999, S. 1-21.

Schnäbele, P., Mass Customized Marketing - Effiziente Individualisierung von Vermarktungsobjekten und -prozessen, Wiesbaden 1997.

Schoenleber, H., Response-Building Reply Devices - more than 30 ways to make your reply mechanism work harder, in: Target Marketing, Vol. 20, No. 12, 1997, S. 42-45.

Scholz, C., Effektivität und Effizienz, organisatorische, in: Frese, E. (Hrsg.), Handwörterbuch der Organisation, 3. Aufl., Stuttgart 1992, S. 533-552.

Scholzen, J. N., Schubert, A., Richtlinien für eine erfolgreiche Kundenanalyse, in: Dallmer, H. (Hrsg.), Direct Marketing \& More, 8. Aufl., Wiesbaden 2002, S. 705-718.

Schramm, W., Roberts, P. (Hrsg.), The process and effects of mass communication, Urbana 1970.

Schultz, D. E., Kitchen, P. J., Integrated Marketing Communications in U.S. Advertising Agencies: An Exploratory Study, in: Journal of Advertising Research, Vol. 37, No. 5, 1997, S. 7-18.

Schulz, D. E., Kitchen, P. J., A Multi-country comparison of the drive for IMC, in: Journal of Advertising Research, Vol. 39, No. 1, 1999, S. 21-37.

Schwarz, T., Permission Marketing macht Kunden süchtig, 2. Aufl., Würzburg 2000 . 
Schweiger, A., Wilde, K.-D., Database-Marketing - Aufbau und Management, in: Hilke, W. (Hrsg.), Direkt-Marketing, Wiesbaden 1993, S. 89-125.

Schweiger, G., Grundlagen der Streuplanung, in: Kaiser, A., Werbung - Theorie und Praxis werblicher Beeinflussung, München 1980. S. $156 \mathrm{ff}$.

Seaver, B. L., Simpson, E., Mail Order Catalog Design and Consumer Response Behavior: Experimentation and Analysis, in: Journal of Direct Marketing, Vol. 9, No. 3, Summer 1995, S. 8-20.

Sherif, C. W., Sherif, M., Nebergall, R. E., Attitude and Attitude Change, Philadelphia, London 1965.

Sherman, E., Greene, J. N., Plank, R. E., Exploring Business-to Business Direct Mail Campaigns: Comparing One-Sided, Two-Sided and Comparative Message Structures, in: Journal of Direct Marketing, Vol. 5, No. 2, 1991, S. 2530.

Simmel, L. L., Berger, P. D., The art of the ask: Maximizing verbal compliance in telefundraising, in: Journal of Interactive Marketing, Vol. 14, No. 3, 2000, S. 12-40.

Sistenich, F., Eventmarketing. Ein innovatives Instrument zur Metakommunikation in Unternehmen, Wiesbaden 1999.

Smith, J., Berger, P. D., The Impact of Direct Marketing Appeals on Charitable Marketing Effectiveness, in: Journal of the Academy of Marketing Science, Vol. 24, No. 3, 1996, S. 219-231.

Spremann, K., Zur, E. (Hrsg.), Controlling, Wiesbaden 1992.

Springer-Verlag (Hrsg.), Lernprozesse im Test, aus der Reihe „Marken im Bild“, o. J., o. O.

StarWap AG, unveröffentlichte Studie 2003, S. 1-3.

Steffenhagen, H., Wirkungen absatzpolitischer Instrumente: Theorie und Messung der Marktreaktion, Stuttgart 1978.

Steffenhagen, H., Wirkungen der Werbung. Konzepte - Erklärungen - Befunde, Aachen, Mainz 2000.

Stone, B., Jacobs, R., Successful Direct Marketing Methods, 7th edition, New York 2001.

Striegl, T., Effizientes Direktmarketing - Mit der richtigen Email-Strategie Absatz fördern, Kunden binden, Kosten senken, Bonn 2003.

Sujan, M., Consumer Knowledge: Effects on Evaluation Strategies Mediating Consumer Judgments, in: Journal of Consumer Research, Vol. 12, No. 1, 1985, S. 31-46.

Szugger, A., Text im Mailing, in: Holland, H. (Hrsg.), Das Mailing - Planung, Gestaltung, Produktion, Wiesbaden 2002, S. 89-112.

Tapp, A., Principles of Direct and Database Marketing, 2nd edition, Harlow, Es$\operatorname{sex} 2000$. 
TNS Emnid, unveröffentlichte Studie im Auftrag des Deutschen Direktmarketing Verbandes, 2003.

Tran, T., How to trap into the teen marketing, in: Advertising \& Marketing to Children, July-September 2003, S. 31-38.

Ueding, R., Management von Messebeteiligungen, Frankfurt a. M. 1998.

Unger, F. et al., Mediaplanung, Methodische Grundlagen und praktische Anwendungen, 3. Aufl., Heidelberg 2002.

Urban, D., Logit-Analyse: Statistische Verfahren zur Analyse von Modellen mit qualitativen Responsevariablen, Stuttgart 1993.

Vakratsas, D., Ambler, T., How Advertising Works: What Do We Really Know, in: Journal of Marketing, Vol. 63, No. 1, 1999, S. 26-43.

VATM (Hrsg.), Neue Zahlen zum Mobilfunkmarkt. Mehr Kunden nutzen immer mehr SMS, Pressemitteilung vom 18.03.2004, Abruf am 12.06.2004 unter www.vatm.de.

Vögele, S., 99 Erfolgsregeln des Direktmarketing, 5. Aufl., Landsberg/Lech 2003.

Vögele, S., Bidmon, R. K., Psychologische Aspekte der Dialogmethode, in: Dallmer, H. (Hrsg.), Direct Marketing \& More, 8. Aufl., Wiesbaden 2002, S. 435-457.

Vögele, S., Handbook of Direct Mail,: The Dialogue Method of Direct Written Sales Communication, New York 1992.

Vriens, M., van der Scheer, H. R., Hoekstra, J. C., Bult, J. R., Conjoint experiments for direct mail response optimization, in: European Journal of Marketing, Vol. 32, No. 3, 1998, S. 323-339.

Weaver-Lariscy, R. A., Tinkham, S. F., Use of impact of direct mail in the context of integrated marketing communications: U.S. Congressional campaigns in 1982 and 1990. in: Journal of Business Research, Vol. 37, No. 3, 1996, S. 233-244.

Weinhold-Stünzi, H., Grundsätze des Kundenstamm-Marketing, in: Thexis, Heft 2, 1987, S. 8-14.

Weis, H. Ch., Steinmetz, P., Marktforschung, Ludwigshafen, Kiel 1991.

Weitkunat, R., Weitkunat, T., Effektivität von Direktmarketing: Sozialpsychologische Ursachen der Verweigerung von Prospekten durch Konsumenten, in: GfK (Hrsg.), Jahrbuch der Absatz- und Verbrauchsforschung, 47. Jg., 2001, S. 406-419.

Wheaton, J., How demographic and psychographic Database overlays can boost your Response, in: Target Marketing, Vol. 21, No. 10, 1998, S. 107-111.

Wiedemann, R., Schwab, K., Erfolgsmessung und -optimierung von OnlineMarketing-Aktivitäten, in: Dallmer, H. (Hrsg.), Direct Marketing \& More, 8. Aufl., Wiesbaden 2002, S. 845-864. 
Wimmer, R.-M., Wiederholungswirkungen der Werbung - Eine empirische Untersuchung zur Auswirkung von Kontaktwiederholungen bei emotionaler Werbung, Saarbrücken 1979.

Witt, F. H., Theorietradition der betriebswirtschaftlichen Forschung, Wiesbaden 1995.

Wolf, J., Marktforschung: praktische Anwendung mit zahlreichen Arbeitsblättern, Checklisten und Fallbeispielen, Landsberg/Lech 1988.

Wulf, K. de, Hoekstra, J. C., Commandeur, H. R., The Opening und Reading Behavior of Business-to-Business Direct Mail, in: Industrial Marketing management, Vol. 29, No. 2, S. 133-145.

Yoon, S.-J., Kim, J.-H., Is the Internet More Effective than Traditional Media? Factors Affecting the Choice of Media, in: Journal of Advertising Research, Vol. 41, No. 6, 2001, S. 53-60.

Zentralverband der deutschen Werbewirtschaft (Hrsg.), Werbung in Deutschland 2003, Bonn 2003.

Zorn, D., Integrierte Kommunikation - Grundlagen und zukünftige Entwicklung, in: Dallmer, H. (Hrsg.): Handbuch Direct Marketing. 6. Auflage, Wiesbaden 1991, S. 51-64. 


\section{SCHRIFTEN ZUM MARKETING}

Band 1 Friedrich Wehrle: Strategische Marketingplanung in Warenhäusern. Anwendung der Portfolio-Methode. 1981. 2. Auflage. 1984.

Band 2 Jürgen Althans: Die Übertragbarkeit von Werbekonzeptionen auf internationale Märkte. Analyse und Exploration auf der Grundlage einer Befragung bei europaweit tätigen Werbeagenturen. 1982.

Band 3 Günter Kimmeskamp: Die Rollenbeurteilung von Handelsvertretungen. Eine empirische Untersuchung zur Einschätzung des Dienstleistungsangebotes durch Industrie und Handel. 1982.

Band 4 Manfred Bruhn: Konsumentenzufriedenheit und Beschwerden. Erklärungsansätze und Ergebnisse einer empirischen Untersuchung in ausgewählten Konsumbereichen. 1982.

Band 5 Heribert Meffert (Hrsg.): Kundendienst-Management. Entwicklungsstand und Entscheidungsprobleme der Kundendienstpolitik. 1982.

Band 6 Ralf Becker: Die Beurteilung von Handelsvertretern und Reisenden durch Hersteller und Kunden. Eine empirische Untersuchung zum Vergleich der Funktionen und Leistungen. 1982.

Band 7 Gerd Schnetkamp: Einstellungen und Involvement als Bestimmungsfaktoren des sozialen Verhaltens. Eine empirische Analyse am Beispiel der Organspendebereitschaft in der Bundesrepublik Deutschland. 1982.

Band 8 Stephan Bentz: Kennzahlensysteme zur Erfolgskontrolle des Verkaufs und der MarketingLogistik. Entwicklung und Anwendung in der Konsumgüterindustrie. 1983.

Band 9 Jan Honsel: Das Kaufverhalten im Antiquitätenmarkt. Eine empirische Analyse der Kaufmotive, ihrer Bestimmungsfaktoren und Verhaltenswirkungen. 1984.

\section{SCHRIFTEN ZU MARKETING UND MANAGEMENT}

Band 10 Matthias Krups: Marketing innovativer Dienstleistungen am Beispiel elektronischer Wirtschaftsinformationsdienste. 1985.

Band 11 Bernd Faehsler: Emotionale Grundhaltungen als Einflußfaktoren des Käuferverhaltens. Eine empirische Analyse der Beziehungen zwischen emotionalen Grundhaltungen und ausgewählten Konsumstrukturen. 1986.

Band 12 Ernst-Otto Thiesing: Strategische Marketingplanung in filialisierten Universalbanken. Integrierte Filial- und Kundengruppenstrategien auf der Grundlage erfolgsbeeinflussender Schlüsselfaktoren. 1986.

Band 13 Rainer Landwehr: Standardisierung der internationalen Werbeplanung. Eine Untersuchung der Prozeßstandardisierung am Beispiel der Werbebudgetierung im Automobilmarkt. 1988.

Band 14 Paul-Josef Patt: Strategische Erfolgsfaktoren im Einzelhandel. Eine empirische Analyse am Beispiel des Bekleidungsfachhandels. 1988. 2. Auflage. 1990.

Band 15 Elisabeth Tolle: Der Einfluß ablenkender Tätigkeiten auf die Werbewirkung. Bestimmungsfaktoren der Art und Höhe von Ablenkungseffekten bei Rundfunkspots. 1988.

Band 16 Hanns Ostmeier: Ökologieorientierte Produktinnovationen. Eine empirische Analyse unter besonderer Berücksichtigung ihrer Erfolgseinschätzung. 1990.

Band 17 Bernd Büker: Qualitätsbeurteilung investiver Dienstleistungen. Operationalisierungsansätze an einem empirischen Beispiel zentraler EDV-Dienste. 1991.

Band 18 Kerstin Ch. Monhemius: Umweltbewußtes Kaufverhalten von Konsumenten. Ein Beitrag zur Operationalisierung, Erklärung und Typologie des Verhaltens in der Kaufsituation. 1993. 
Band 19 Uwe Schürmann: Erfolgsfaktoren der Werbung im Produktlebenszyklus. Ein Beitrag zur Werbewirkungsforschung. 1993.

Band 20 Ralf Birkelbach: Qualitätsmanagement in Dienstleistungscentern. Konzeption und typenspezifische Ausgestaltung unter besonderer Berücksichtigung von Verkehrsflughäfen. 1993.

Band 21 Simone Frömbling. Zielgruppenmarketing im Fremdenverkehr von Regionen. Ein Beitrag zur Marktsegmentierung auf der Grundlage von Werten, Motiven und Einstellungen. 1993.

Band 22 Marcus Poggenpohl: Verbundanalyse im Einzelhandel auf der Grundlage von Kundenkarteninformationen. Eine empirische Untersuchung von Verbundbeziehungen zwischen Abteilungen. 1994.

Band 23 Kai Bauche: Segmentierung von Kundendienstleistungen auf investiven Mărkten. Dargestellt am Beispiel von Personal Computern. 1994.

Band 24 Ewald Werthmöller: Räumliche Identität als Aufgabenfeld des Städte- und Regionenmarketing. Ein Beitrag zur Fundierung des Placemarketing. 1995.

Band 25 Nicolaus Müller: Marketingstrategien in High-Tech-Märkten. Typologisierung, Ausgestaltungsformen und Einflußfaktoren auf der Grundlage strategischer Gruppen. 1995.

Band 26 Nicolaus Henke: Wettbewerbsvorteile durch Integration von Geschäftsaktivitäten. Ein zeitablaufbezogener wettbewerbsstrategischer Analyseansatz unter besonderer Berücksichtigung des Einsatzes von Kommunikations- und Informationssystemen (KIS). 1995.

Band 27 Kai Laakmann: Value-Added Services als Profilierungsinstrument im Wettbewerb. Analyse, Generierung und Bewertung. 1995.

Band 28 Stephan Wöllenstein: Betriebstypenprofilierung in vertraglichen Vertriebssystemen. Eine Analyse von Einflußfaktoren und Erfolgswirkungen auf der Grundlage eines Vertragshändlersystems im Automobilhandel. 1996.

Band 29 Michael Szeliga: Push und Pull in der Markenpolitik. Ein Beitrag zur modellgestūtzten Marketingplanung am Beispiel des Reifenmarktes. 1996.

Band 30 Hans-Ulrich Schröder: Globales Produktmanagement. Eine empirische Analyse des Instrumenteeinsatzes in ausgewählten Branchen der Konsumgüterindustrie. 1996.

Band 31 Peter Lensker: Planung und Implementierung standardisierter vs. differenzierter Sortimentsstrategien in Filialbetrieben des Einzelhandels. 1996.

Band 32 Michael H. Ceyp: Ökologieorientierte Profilierung im vertikalen Marketing. Dargestellt am Beispiel der Elektrobranche. 1996.

Band 33 Mark Unger: Die Automobil-Kaufentscheidung. Ein theoretischer Erklärungsansatz und seine empirische Überprüfung. 1998.

Band 34 Ralf Ueding: Management von Messebeteiligungen. Identifikation und Erklärung messespezifischer Grundhaltungen auf der Basis einer empirischen Untersuchung. 1998.

Band 35 Andreas Siefke: Zufriedenheit mit Dienstleistungen. Ein phasenorientierter Ansatz zur Operationalisierung und Erklärung der Kundenzufriedenheit im Verkehrsbereich auf empirischer Basis. 1998.

Band 36 Irene Giesen-Netzer: Implementierung von Rücknahme- und Recyclingsystemen bei Gebrauchsgütern. 1998.

Band 37 Frithjof Netzer: Strategische Allianzen im Luftverkehr. Nachfragerorientierte Problemfelder inrer Gestaltung. 1999.

Band 38 Silvia Danne: Messebeteiligungen von Hochschulen. Ziele und Erfolgskontrolle. 2000.

Band 39 Martin Koers: Steuerung von Markenportfolios. Ein Beitrag zum Mehrmarkencontrolling am Beispiel der Automobilwirtschaft. 2001. 
Band 40 Frank Wolter: Koordination im internationalen Umweltmanagement. Dargestellt an Beispielen aus der Automobilindustrie. 2002.

Band 41 Mirko Caspar: Cross-Channel-Medienmarken. Strategische Optionen, Ausgestaltungsmöglichkeiten und nachfragerseitige Bewertung. 2002.

Band 42 Andreas Seifert: Typologie des Marketing-Management. Theoretisch-konzeptionelle Grundlagen und internationale empirische Befunde. 2002.

Band 43 Markus Dömer: Ressourcenbasierte Erschließung neuer Märkte. Dargestellt am Beispiel der Textilindustrie. 2002.

Band 44 Jens Röder: Europäische Markterschließungsstrategien im Schienenverkehrsdienstleistungsbereich am Beispiel des Personenverkehrs der Deutschen Bahn AG. 2003.

Band 45 Andreas Bierwirth: Die Führung der Unternehmensmarke. Ein Ansatz zum zielgruppenorientierten Corporate Branding. 2003.

Band 46 Mathias Giloth: Kundenbindung in Mitgliedschaftssystemen. Ein Beitrag zum Kundenwertmanagement - dargestellt am Beispiel von Buchgemeinschaften. 2003.

Band 47 Fabian Hieronimus: Persönlichkeitsorientiertes Markenmanagement. Eine empirische Untersuchung zur Messung, Wahrnehmung und Wirkung der Markenpersönlichkeit. 2003.

Band 48 Marcel Kranz: Die Relevanz der Unternehmensmarke. Ein Beitrag zum Markenmanagement bei unterschiedlichen Stakeholderinteressen. 2004.

Band 49 Bastian Grunberg: Zeitbezogene Nutzenkomponenten von Verkehrsdienstleistungen. Erklärung und Wirkung am Beispiel von Bahnreisen. 2004.

Band 50 Christian Ebert: Identitätsorientiertes Stadtmarketing. Ein Beitrag zur Koordination und Steuerung des Stadtmarketing. 2004.

Band 51 Michael Ahrens: Experimentelle Analyse der Wirkungen ausgewählter Direktmarketing-Instrumente. Ein Beitrag zur Effektivitäts- und Effizienzmessung im Direktmarketing. 2007.

www.peterlang.de 


\section{Zielgruppenselektion für Direktmarketingkampagnen}

\section{Scoringmodellierung bei unterrepräsentierter Zielgruppe unter Verwendung supplementierender Datenbestände}

Frankfurt am Main, Berlin, Bern, Bruxelles, New York, Oxford, Wien, 2005.

322 S., zahlr. Abb., Tab. und Graf.

Informationsmanagement und strategische Unternehmensführung.

Herausgegeben von Franz Schober und Johannes Ruhland. Bd. 9

ISBN 3-631-54157-0 • br. € 56.50*

Aus einer Liste potentiell kontaktierbarer Personen die Zielgruppe auszuwählen entscheidet über den Erfolg von Direktmarketingkampagnen. Für die Zieigruppenselektion werden so genannte Scoringmodelle genutzt, die man mithilfe von Lernfällen kalibriert. Ideale Lernfälle sind Personen, die bereits an einer gleichartigen Kampagne teilnahmen und darauf z. B. durch (Nicht-)Kauf reagierten. Durch Produktvielfalt und Zielgruppendifferenzierung liegen oft zu wenige oder keine idealen Lernfälle vor. Dieses Buch illustriert, wie Hilfsdatenbestände die fehlenden Lernfälle ergänzen bzw. ersetzen. Experimente mit Automobildaten zeigen, auf welche Weise man mittels halbüberwachtem Lernen, Variablentransformation und Analogieschluss geeignete Hilfsdatenbestände auswählt und somit das Scoringmodell und letztlich die Zielgruppenselektion für eine Kampagne optimiert.

Aus dem Inhalt: Direktmarketing als Instrument zur Neukundengewinnung . Zielgruppenselektion als Erfolgsfaktor im Direktmarketing · Scoringmodelle basierend auf Data Mining und Statistik-Verfahren - Gründe für fehlende Lernfälle zur Kalibrierung eines Scoringmodells · Handlungsalternativen bei fehlenden Lernfällen · Halbüberwachtes Lernen, Analogieschluss und Variablentransformation zur Auswahl von Hilfsdatenbeständen bei fehlenden Lernfällen - Experimente basierend auf Automobildaten und mikrogeografischen Informationen mit logistischer Regression, Naive BayesKlassifikator, Entscheidungsbaum und Neuronalem Netz zum Nutzen von Hilfsdatenbeständen

Frankfurt am Main - Berlin - Bern - Bruxelles - New York · Oxford · Wien

Auslieferung: Verlag Peter Lang AG

Moosstr. 1, $\mathrm{CH}-2542$ Pieterlen

Telefax 0041 (0) 32/3761727

*inklusive der in Deutschland gültigen Mehrwertsteuer

Preisänderungen vorbehalten

Homepage http://www.peterlang.de 